\title{
DE89 014375
}

ARGONNE NATIONAL LABORATORY

9700 South Cass Avenue

Argonne, Illino is 60439-4801

\section{KAOS/LIB-V: A LIBRARY OF NUCLEAR RESPONSE FUNCTIONS GENERATED BY KAOS- $V$ CODE FROH ENDF/B-V AND OTHER DATA FILES}

\section{by}

Y. Farawila*, Y. Gohar, and C. Maynard**

\author{
Work supported by \\ Office of Fusion Energy \\ U.S. Department of Energy \\ Under Contract W-31-109-Eng-38
}

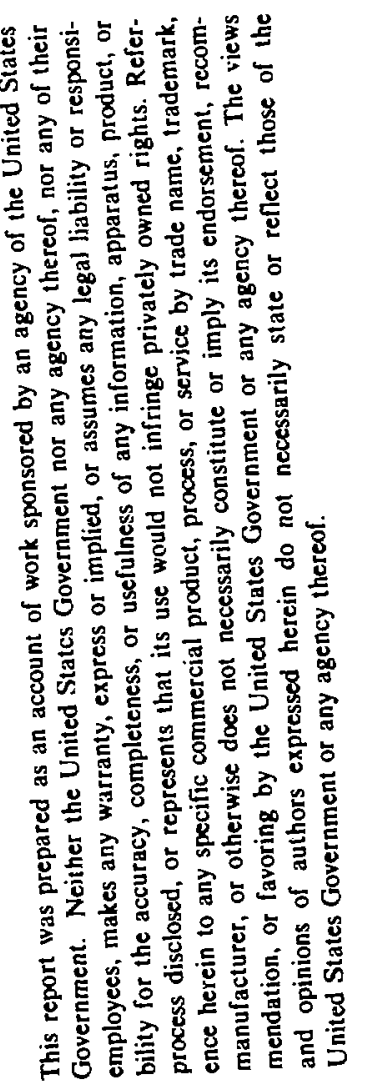

* Nuclear Engineering and Health Physics Programs, Georgia Institute of Technology, Atlanta, Georgia 30332.

* Nuclear Engineeririg and Engineering Physics Department, University of Wisconsin-Madison, Madison, Wisconsin 53706. 


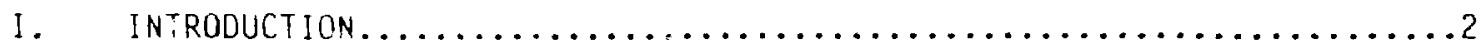

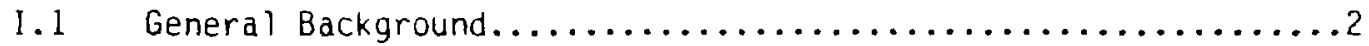

I.2 Kerma Factor Evaluation Procedure.....................

II. DESCRIPTION OF THE KAOS/LIB-V LIBRARY CONTENTS $\ldots \ldots \ldots \ldots \ldots \ldots \ldots \ldots$

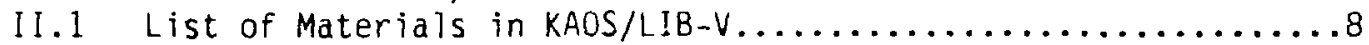

II.2 Nuclear Response................................

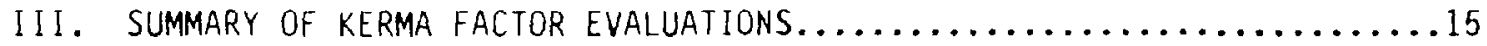

IIl.1 Single-Isotope Materials.................................

III.2 Kerma Factor Evaluation Summary for Natural Elements.........30

III.3 Fissionable Materials................................50

IV. GRAPHICAL REPRESENTATION OF KAOS/LIB-V RESPONSE FUNCTIONS..........51

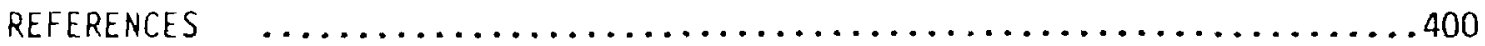

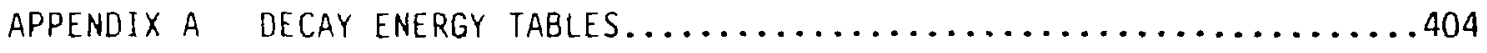

APPENDIX B VITAMIN-E 174 GROUP STRUCTURE........................ 411 


\section{KAOS/LIB-V: A LIBRARY OF NUCLEAR RESPONSE FUNCTIONS GENERATED BY KAOS- $Y$ CODE \\ FROM ENDF/B-V AND OTHER DATA FILES}

by

Y. Farawila, Y. Gohar, and C. Maynard

\section{ABSTRACT}

KAOS/LIB-V: A library of processed nuclear responses for neutronics analyses of nuclear systems has been generated. The library was prepared using the KAOS-V code and nuclear data from ENDF/B-V. The library includes kerma (kinetic energy released in materials) factors and other nuclear response functions for all materials presently of interest in fusion and fission applications for 43 nonfissionable and 15 fissionable isotopes and elements. The nuclear response functions include gas production and tritiumbreeding functions, and all important reaction cross sections. KAOS/LIB-V employs the VITAMIN-E weighting function and energy group structure of 174 neutron groups.

Auxiliary nuclear data bases, e.g., the Japanese evaluated nuclear data library JENDL-2 were used as a source of isotopic cross sections when these data are not provided in ENDF/B-V files for a natural element. These are needed mainly to estimate average quantities such as effective Q-values for the natural element. This analysis of local energy deposition was instrumental in detecting and understanding energy balance deficiencies and other problems in the ENDF/B-V data. Pertinent information about the library and a graphical display of the main nuclear response functions for all materials in the library are given. 


\section{INTRODUCTION}

KAOS/LIB-V: a library of processed nuclear responses is described in this report. The library includes neutron kerma-factors, gas production, tritium breeding cross sections, and all important reaction cross sections, for a large number of materials of interest in fusion and fission systems. The library was generated with the KAOS-V code employing basic nuclear data from ENDF/B-V.

The kerma (kinetic energy released in materials) factors are the key nuclear responses in the library because of the impcrtance of the nuclear heating. The kerma factor evaluation procedure, description of the KAOS/LIB-V contents and summary of kerma factor evaluations are given in this report. Also, a graphical display of the main nuclear response functions for materials in the library are shown to help in the nuclear design analysis.

\section{I.1 General Background}

Heating is an important parameter of any nuclear system. The energy multiplication in a fusion reactor blanket is a very important factor in the assessment of the economic performance of the power plant. More important are the severe design constraints due to the undesirable heating effects. This is particularly true for the plasma facing components where excessive heating is expected. Other fusion reactor components where heating effects are important are the superconducting magnets. The accurate calculation of heating in the magnet due to the leaking neutrons and photons is a key factor in the design of the cryogenic heat removal systems. Accurate neutron kerma factors are necessary for heating calculations and are also in increasing demand for biomedical applications. Direct experimental measurements of kerma factors are limited to a few isotopes and over the small range of high incident 
neutron energies of particular importance to radiation therapy. The microscopic cross section data bases, especially the ENOF/B files 11 / provide the means for computing kerma factors for many materials and for incident neutron energies up to $20 \mathrm{MeV}$. The computed kerma factors from microscopic cross sections however, suffer from large uncertainties that cruelly manifest themselves sometimes as negative values $|2,3|$. It is generally believed justifiably so - that the fundamental improvement in the computed kerma factors rests on improving the nuclear data files. The huge inertia which is involved in adjusting the massive nuclear data files and the fact that these are generally satisfactory for their main purpose - transport calculations make it difficult to take this route.

Thus, there is a need for improved methods in calculating nuclear heating parameters from the existing data. This work is a response to this need, especially for the fusion community. 


\section{I.2 Kerma Factor Evaluation Procedure}

The code KAOS-V (Kerma And Other Stuff) was written to be used as the main kerma factor evaluation tool. A brief code description along with a user's guide are given in reference [4]. The evaluation of the recommended neutron kerma factors for a material depends largely on the individual characteristics of that material and the particularities of its evaluated nuclear data. The kerma factor evaluation procedure for each of the 64 materials covered in this work is briefly described in section III. There is however a general procedure that applies to most of these evaluations. This general procedure is described as follows:

* The ENDF/E-V data for the material under study is reviewed. The nuclear structure, excitation levels, and decay modes for the target and product nuclei are reviewed using the Table of Isotopes. The reaction Q-values are also checked. This review helps primarily in planning the next steps.

* For materials where resonance parameters are given in ENDF/B-V file 2, an NJOY run $[5,6]$ was made to reconstruct the cross sections and perform Doppler broadening at 300 degree Kelvin. The NJOY module HEATR is also run [6] to make comparisons with subsequent KAOS-V runs. The NJOY PENDF file is kept for these purposes.

* A KAOS $-V$ run is made in the Direct Energy Balance mode. The partial and total kerma factors and gamma energy production cross section are printed and plotted. The intermediate files in which these results are stored are saved for subsequerit comparison tests. The total kerma factors produced in this stage are in good agreement with the NJOY (HEATR) results for most materials. * A KAOS $V$ run is made in the kinematics mode. Again, the partial and total kerma factors are printed, plotted, and saved for the subsequent comparisors. 
* The total kerma factors produced by Direct Energy Balance and Kinematics are compared. Good agreement is expected for materials for which the ENDF/B-V files include detailed gamma production for each reaction. On the other hand, if substantial disagreement was found, further testing and comparison is made in the partial kerma factor level. The interactive mode of the KAOS-V code is frequently used in this stage which facilitates detailed analysis of partial kerma factors and provides the means for performing non-standard tests.

* Partial kerma factors for each reaction produced by the Direct Energy Balance and Kinematics methods are compared. The gamma energy production is also compared with the excitation energy of the residual nuclei, a common test to identify energy balance problems.

* For natural elements, the energy-dependent Q-values are estimated using additional data sources as described earlier. The Direct Energy Balance and Kinematics runs are repeated using the variable Q-values. The sensitivity of the total kerma factors to the introduction of the variable Q-values is studied. Depending on this sensitivity, more effort was made in estimating the more important Q-values, and the use of variable Q-values was dropped altogether for some other reactions.

* After making the necessary data corrections (if any), the information collected through the above testing and comparisons is used to make the final decision on the recommenced set of prompt kerma factors. In general, at low incident neutron energies, kerma factors are dominated by radiative capture and elastic scattering. The methods for calculating kerma from these two processes are identical for both Direct Energy Balance and Kinematics approaches. At higher incident neutron energies, the inelastic scattering is 
turned on. If the ENDF/B-V file 12 is used to represent inelastic gammas in a manner consistent with file 3 cross sections, the agreement between the two methods will be extended to cover the range of incident neutron energies below the thresholds of other nonelastic reactions. In this incident energy range, the total kerma factors using Direct Energy Balance for many materials experiences a minimum and becomes more sensitive to gamma energy imbalance or representation problems and is often negative, and the Kinematics method is therefore used. For incident neutron energies high enough to produce excited residuals following the emission of charged particles, Direct Energy Balance is generally preferred. The reasons for this preference are the fact that the Kinematics method is not equipped to account for residual excitation and generally overestimates kerma in this range, and also because the magnitude of kerma factors is higher and the sensitivity to gamma errors is therefore reduced. To preserve the continuity of the final recommended set of kerma factors, the connection between the results from the two methods is made at an incident neutron energy at which the two results are in agreement.

* The decay of the unstable products is then studied. The decay heat is included to produce additional kerma response functions as detailed in later sections. 


\section{DESCRIPTION OF THE KAOS/LIB-V LIBRARY CONTENTS}

The nuclear response library KAOS/LIB-V (Kerma And Other Stuff) is generated by using the code KAOS-V [4] as an evaluation tool. The evaluated nuclear data files ENDF/B-V are the chief source of data used in these nuclear response evaluations. Doppler broadening at $300^{\circ} \mathrm{K}$ was performed for materials where resonance parameters are provided. The effect of Doppler broadening on the group values was very small ( $<2.0 \%$ in most cases). The library contains 22 response functions for 64 materials. The responses are presented in Vitamin-E 174 group structure, where the first group refers to the lowest neutron energy. The nuclear responses are presented in the units of (ev.barn) for kerma factor responses and gamma energy production, and (barn) for all the other responses. The library is saved in a readable text file. The materials are ordered such that those with lower atomic number are listed first, and lighter isotopes are also presented before the heavier isotopes of the same element. For each material, 22 titled responses are given. FIDO format [7] was used to fill the zero groups before thresholds, and also to zero the responses that do not apply or are not provided for a certain material. This helped reduce the storage size needed for the library. An interactive program, RETRIEVE, was written to retrieve selected responses and perform group collapsing from the Vitamin-E [8] 174 group values. 
II. L List of Materials in KAOS/LIB-V

$\begin{array}{rlll}\text { No. } & \text { Material } & \text { ENDF/B-V } & \text { KAOS/LIB-V } \\ & \text { Name } & \text { MAT number } & \text { Symbol } \\ 1 & \text { Hydrogen } & 1301 & \\ 2 & \text { Deuterium } & 1302 & \mathrm{~h}-1 \\ 3 & \text { Helium } & 1270 & \text { he- } \\ 4 & \text { Lithium-6 } & 1303 & \text { li-6 } \\ 5 & \text { Lithium-7 } & 1397 & \text { li-7 } \\ 6 & \text { Beryllium } & 1304 & \text { be-9 } \\ 7 & \text { Boron-10 } & 1305 & \text { b-10 } \\ 8 & \text { Boron-11 } & 1160 & \text { b-11 } \\ 9 & \text { Carbon } & 1306 & \mathrm{c}-12 \\ 10 & \text { Nitrogen } & 1275 & \mathrm{n}-14 \\ 11 & \text { Oxygen } & 1276 & 0-16 \\ 12 & \text { Fluorine } & 1309 & \mathrm{f}-19 \\ 13 & \text { Sodium } & 1311 & \text { na-23 } \\ 14 & \text { Magnesium } & 1312 & \text { mg-nat } \\ 15 & \text { Aluminum } & 1313 & \text { al-27 } \\ 16 & \text { Silicon } & 1314 & \text { si-nat } \\ 17 & \text { Phosphorus } & 1315 & \text { p-31 } \\ 18 & \text { Sulfur } & 1347 & \text { s-nat } \\ 19 & \text { Chlorine } & 1149 & \text { cl-nat } \\ 20 & \text { Potassium } & 1150 & \text { k-nat } \\ 21 & \text { Calcium } & 1320 & \text { ca-nat } \\ 22 & \text { Titanium } & 1322 & \text { ti-nat } \\ 23 & \text { Vanadium } & 1323 & \text { V-nat } \\ 24 & \text { Chromium } & 1324 & \text { cr-nat } \\ 25 & \text { Manganese } & 1325 & \text { mn-55 } \\ 26 & \text { Iron } & 1326 & \text { fe-nat } \\ 27 & \text { Cobalt } & 1327 & \text { co-59 } \\ 28 & \text { Nickel } & 1328 & \text { ni-nat } \\ 29 & \text { Copper } & 1329 & \text { cu-nat } \\ & & & \end{array}$




$\begin{array}{llll}\text { Nc. } & \text { Material } & \text { ENDF/B-V } & \text { KAOS/LIB-V } \\ & \text { Name } & \text { MAT number } & \text { Symbol } \\ & & & \\ 30 & \text { Zirconium-90 } & 1385 & \text { zr-90 } \\ 31 & \text { Zirconium-91 } & 1386 & \text { Zr-91 } \\ 32 & \text { Zirconium-92 } & 1387 & \text { zr-92 } \\ 33 & \text { Zirconium-94 } & 1388 & \text { zr-94 } \\ 34 & \text { Zirconium-96 } & 1389 & \text { zr-96 } \\ 35 & \text { Niobium } & 1189 & \text { nb-93 } \\ 36 & \text { Molybdenum } & 1321 & \text { mo-nat } \\ 37 & \text { Hafnium-174 } & 1374 & \text { hf-174 } \\ 38 & \text { Hafnium-176 } & 1376 & \text { hf-176 } \\ 39 & \text { Hafnium-177 } & 1377 & \text { hf-177 } \\ 40 & \text { Hafnium-178 } & 1378 & \text { hf-178 } \\ 41 & \text { Hafnium-179 } & 1383 & \text { hf-179 } \\ 42 & \text { Hafnium-180 } & 1384 & \text { hf-180 } \\ 43 & \text { Tantalum } & 1285 & \text { ta-181 } \\ 44 & \text { Tungsten-182 } & 1475 & \text { w-182 } \\ 45 & \text { Tungsten-183 } & 1476 & \text { w-183 } \\ 46 & \text { Tungsten-184 } & 1477 & \text { w-184 } \\ 47 & \text { Tungsten-186 } & 1478 & \text { w-186 } \\ 48 & \text { Lead } & 1382 & \text { pb-nat } \\ 49 & \text { Bismuth } & 1375 & \text { bi-209 } \\ 50 & \text { Thorium-232 } & 1390 & \text { th-232 } \\ 51 & \text { Protactinium-233 } & 1391 & \text { pa-233 } \\ 52 & \text { Uranium-233 } & 1393 & \text { u-233 } \\ 53 & \text { Uranium-234 } & 1394 & \mathrm{u}-234 \\ 54 & \text { Uranium-235 } & 1395 & \mathrm{u}-235 \\ 55 & \text { Uranium-236 } & 1396 & \mathrm{u}-236 \\ 56 & \text { Uranium-238 } & 1398 & \mathrm{u}-238 \\ 57 & \text { Neptunium-237 } & 1337 & \mathrm{np-237} \\ 58 & \text { Plutonium-238 } & 1338 & \text { pu-238 } \\ & & & \end{array}$


No. Material

Name

59 Plutonium-239

60 Plutonium-240

61 Plutonium-241

62 Plutonium-242

63 Americium-241

64 Americium-243
ENDF/B-V

MAT number

1399

1380

1381

1342

1361

1363
KAOS/LIB-V

Symbol

pu-239

pu-240

pu-241

pu-242

am-241

am-243 


\section{II.2 Nuclear Responses}

There are 22 nuclear response function tables for each material in the library. These responses are outlined below:

1- Recommended Prompt Kerma Factor: The recommended prompt kerma factor can be either calculated by direct energy balance, kinematics, or a combination of these methods in different energy ranges according to the description in the summary provided in section III. This response is provided for all the materials included in this library. The response is given in units of (ev.barn).

2- Recommended Kerma Factor with Charged Particle Decay Heat: In this response, the decay heat deposited locally via the emitted charged particles are added to the recommended set of prompt kerma factors. This response is provided for all materials. In case there is no charged particle decay heat (within the half-life cutoff of 1 day), this response will be identical to the recommended prompt kerma.

3- Recommended Kerma Factor Plus Total Decay heat: In this response, the total decay heat including energy carried by charged particles and gamma rays are added to the recommended prompt kerma factor response.

4- Direct Energy Balance Prompt Kerma Factor: The prompt kerma factors produced by the direct energy balance method in units of (ev.barn) are listed. Notice that in the case gamma files are not provided in ENDF/B-V, the Direct Energy Balance Prompt kerma factor includes the gamma energy as deposited locally. It is possible to find negative kerma factors in this list. These can be used for comparison purposes and in special cases to account for a global energy balance in large systems. This response is presented for most but not all materials, where zeros are entered instead. 
5- Direct Energy Balance Kerma Factor with Charged Particle Decay Heat: Charged Particle decay heat is added to the direct energy balance prompt kerma in the same way described for the recommended set. If the prompt direct energy balance was zeroed out, this response will be also zeroed out. 6- Direct energy Balance Kerma Factor Plus Total Decay Heat: The total decay heat is added to the direct energy balance kerma in the same way described for the recommended set.

7- Kinematics Prompt Kerma Factor: The prompt kerma factor produced by the kinematics method is listed. This response is provided for most, but not all materials.

8- Kinematics Kerma Factor with Charged Particle Decay Heat: The charged particle decay heat is added to the prompt kinematic kerma factor in the same way described for the recommended set. This response will be zeroed out in the case the prompt kinematic kerma factor is not provided.

9- Kinematics Kerma Plus Tocal Decay Heat: The total decay heat is added to the kinematics prompt kerma in the same way described for the third response. Agäin, this response will be zeroed out if the prompt kinematic set is not provided, and it will be identical to the prompt set if decay heat (within the 1 day half-life cutoff) is zero.

10- Prompt Gamma Energy Production: The total prompt gamma energy production is given in the units of (ev.barn). With the exception of Zirconium isotopes, where gamma files are not provided in the ENDF/B- $V$, this response is not calculated from kinematics, but rather by direct processing of ENDF/B-V files. In a few cases, interpolation and other minor corrections were made. 
11- Hydrogen-1 Production Cross Section: In this response the $\mathrm{H}-1$ gas production cross section is given in barns. The response is computed by summing up all proton-producing reaction cross sections multiplied by the respective number of emitted protons. For gas production responses, the delayed component of gas production due to radioactive decay is also added. 12- Deuterium Production cross Section: The production of $\mathrm{H}-2$ gas is presented in the same way as in response 11.

13- Tritium Production Cross Section: The production of $\mathrm{H}-3$ is presented in this response in the same way as response 11 .

14- Helium-3 Production Cross Section: See response 11.

15- Helium-4 Production Cross Section: See response 11.

16- Total cross section: The total cross section is computed from the ENDF/B-V file 2 resonance parameters (if any) and MT=1 file 3 data.

17- Elastic Scattering Cross Section: Computed from ENDF/B-V resonance file 2 (if any) and $M T=2$ file 3 data.

18- Total Inelastic Cross Section: This response is equivalent to the data presented in ENDF/B-V file $3 M T=4$. The $(n, n x)$ and $(n, x n)$ reactions, where $x$ represents a charged particle e.g. proton or alpha particle, are not included if they are not contained in MT=4 (and equivalently MT=51-91 series).

19- Radiative Capture Cross Section: Computed from ENDF/B-V file 2 resonance parameters (if any) and file $3 \mathrm{MT}=102$ data.

20- Total $(n, 2 n)$ Cross Section: All non-redundant reactions producing two neutrons are added to form this response. These include sequential representation $(M T=6-9)$, direct reaction $(M T=16),(n, 2 n \alpha)$ reaction $(M T=24)$, and $(n, 2 n 2 a)$ reaction $(M T=30)$. 
21- Total $(n, 3 n)$ Cross Section: Reactions producing 3 neutrons are added to form this response. These include $(n, 3 n)$ and $(n, 3 n a)$ reactions (MT=17, MT $=25$ ) .

22- Number of Fission Neutrons * Cross Section: In this response, the total number of fission neutrons (prompt plus delayed) is given. It is computed by multiplying the total number of neutrons produced per fission obtained from ENDF/B-V file 1 by the fission cross section. The fission cross section is computed from file 2 resonance pranameters and file 3 MT=18 data. 


\section{II. SUMMARY OF KERMA FACTOR EVALUATIONS}

In this chapter, the kerma factor evaluation procedure will be summarized for the processed 64 materials included in the KAOS/LIB-V library. The degree of details will vary from one material to another according to the complexity of the analysis that was needed and the amount of data substitution from sources other than ENDF/B-V general files. Since this kind of analysis depends on the critical review of the nuclear data, comments about the data problems will be embedded in the kerma factor evaluation description. The kerma factor evaluation summary of the single-isotope materials will be presented first, followed by that for natural elements, and finally the kerma factor evaluation for fissionable materials.

The abbreviations KIN and DEB are used throughout this summary to stand for KINEMATICS and DIRECT ENERGY BALANCE respectively. Reactions producing chargec particles, e.g. $(n, p)$ and $(n, a)$ reactions are referred to as $(n, x)$ reactions. The kerma factors are grouped in Vitamin-E structure, and are ordered such that the first group refers to the group with the lowest neutron energy . 


\section{I Single-Isotope Materials}

$1-\mathrm{H}-1$

This hydrogen isotope has only two reactions, elastic scattering and radiative capture which simplifies the nuclear, response calculation. The calculated kerma factors using the direct energy balance and kinematics are equivalent.

$1-\mathrm{H}-2$

There are only elastic scattering, radiative capture, and $(n, 2 n)$ reactions for this material. The elastic and capture reactions receive the same treatment in both kinematics and direct energy balance procedures. However, the different treatment of $(n, 2 n)$ reaction resulted in a KIN total kerma factor greater by a maximum of $34 \%$ than the DEB kerma (at $20 \mathrm{MeV}$ incident neutron energy). The DEB result was recommended and adopted for the KAOS/LIB-V library. The KIN result is less reliable because of the indistinguishability error between the first and second secondary neutron energies in ENDF/B-V file 5. This error is proportional to $1 / A$ which is particularly large for deuterium where the nuclear mass ratio $A=2$ is small.

\section{2-He-4}

Elastic scattering is the only energy deposition mechanism below $20 \mathrm{MeV}$. The DEB and KIN results are therefore equivalent.

\section{$3-L i-6$}

Gamma production is limited to radiative capture photons, and the 3.562 MeV inelastic photon. The inelastic gamma production is represented in 
ENDF/B-V file 12, and the KIN kerma factor is therefore equivalent to that produced by DEB.

The nuclear data for Li-6 was evaluated by I. Bondarenko and Eh. Petrov [9] for the purpose of calculating kerma factors. They published their kerma factor result as a log-scale graph, which made the comparison rather approximate. Their kerma factors were found to be larger than those computed from ENDF/B-V by roughly $20 \%$ above the incident neutron energy of $3 \mathrm{MeV}$.

\section{$3-L i-7$}

Gamma production is limited to radiative capture photons, and the 0.4776 MeV inelastic photon. The inelastic photon is represented in ENDF/B-V file 12 which preserves energy balance. The DEB and KIN kerma results are therefore equivalent.

The nuclear data of $\mathrm{Li}-\mathrm{T}$ was evaluated by $\mathrm{I}$. Bondarenko and Eh. Petrov [9] for the purpose of calculating kerma factors. Comparison was made between our results and theirs which were published as a log-scale graph in the incident neutron energy range 0.001-16. MeV. In the keV region, the ENDF/B-V kerma factors including decay neat was found to be in good agreement with that of Bondarenko and Petrov. Above $4 \mathrm{MeV}$ however, their kerma factors were found to be higher than ours by roughly $20 \%$.

\section{4-Be-9}

Special care was taken to compute the $(n, 2 n)$ reaction kerma factor which contributes a significant portion of the total energy deposition. The reaction is represented in ENDF/B- $V$ as a sequence of two events resulting in the formation of the unstable Be-8. The problem lies in the absence of any 
information about the possible excitation energy of the product Be- 8 before it splits into two alpha particles. The computed KIN kerma factors ignoring the excitation of Be-8 should be underestimated and was actually found to be less than the DEB result at high incident neutron energies. The second neutron's energy obtained from ENDF/B-V file 5 for low incident neutron energy was found to be larger than the kinematic prediction assuming isotropic emission. The reason can be attributed to the anisotropic angular distribution of the second neutron and its correlation to the first one.

DEB method, on the other hand, requires neither intermediate nucleus excitation energy nor angular distribution information. Furthermore, the qualitative analysis of KIN results indicates the superiority and justifies the use of DEB. Although the ENDF/B-V representation of the $(n, 2 n)$ reaction considers Be-8 as the reaction product, the immediate decay of the unstable Be-8 to two alpha particles was taken to contribute to the prompt kernia.

\section{$5-B-10$}

The DEB results for prompt kerma factcrs are equal to those for KIN to $0.1 \%$ accuracy. This is largely due to the level $(n, x)$ reaction representation in ENDF/B-V which is ideal for consistent gamma file representation. However there is still a need to make some comments about the data representation:

1. For $\left(n, a_{0}\right)$ and $\left(n, a_{1}\right)$ reactions (MT $=780$ and 781 respectively) the ENDF/B-V Q-values were found to be zeros. These were corrected to 2.79 and $2.312 \mathrm{MeV}$ respectively.

2. The tenth inelastic level (MT $=60$ ) is flagged to decay by alpha emission $(L R=22)$. There is however a $6.029 \mathrm{MeV}$ photon in file 13 which indicates a 
decay of the same level by gamma emissions. The cross section for this photon's production is too small to worry about the overall accuracy of kerma factors and gas production response functions, but the need to account for branching in the data representation is recognized. The resulting error is significant for other materials, e.g., F-19.

3. The two $0.511 \mathrm{MeV}$ gammas produced due to the decay of the $4^{\text {th }}$ level $\left(n, P_{4}\right)$ by pair production $(L R=40)$ were not accounted for in the gamma files. The resulting error in kerma factors was found to be negligible.

\section{$5-B-11$}

There are no gamma files in ENDF/B-V for this material and kinematics method is the only option. It was not necessary to use an accurate value for $\eta$-factor to account for the capture gamma spectrum on the recoil energy. The reason is that the capture kerma is dominated by the decay energy of B-12 with the short half life of $0.02 \mathrm{sec}$, and $\eta=1$ was used.

\section{$6-C-12$}

The ENDF/B-V gamma files represent only the photons produced in the inelastic scattering and capture reactions. This makes KIN and DEB resuits equivalent and the ENDF/B-V is self-consistent. The alpha particle-producing reactions are particularly important at high incident energies. The $\left(n, a_{0}\right)$ Be-9 reaction [18] leaves the Be-9 nucleus in the ground state, and was adequately evaluated by studying the reciprocal reaction $B e-9(a, n) C-12$. The contribution from $\left(n, a_{0}\right)$ reaction calculated from ENDF/B-V was found to be in agreement with the experimental measurement at $14.1 \mathrm{MeV}$ [11]. The $2.43 \mathrm{MeV}$ excited state of Be-9 decays by disintegration to a neutron and Be-8 which is 
unstable in the ground state and splits into two alpha particles. The secondary neutron average energy was calculated from the excitation level and mass difference assuming that all events are isotropic in the center-of-mass. The resulting secondary neutron average energy increases linearly with the incident neutron energy as:

$E_{n^{\prime}}=0.401+0.0375 \mathrm{E} \mathrm{MeV}$, which is always larger than the $0.6 \mathrm{MeV}$ computed from the incident energy-independent evaporation spectrum given in ENDF/B-V. The resulting effect on total kerma factor was too small to account for the discrepancies with experimental results. Because of its importance in health physics and medical applications, direct measurement of the carbor, kerma factor were carried out above $14 \mathrm{MeV}$. Results by Barschall, DeLuca and others [10-13] are systematically lower than the computed kerma by $5-25 \%$. These results reveal an overestimation [22] of the evaluated $\left(n, n^{\prime} 3 a\right)$ cross section listed in ENDF/B-V file. The experimental kerma factors were not used in the KAOS/LIB-V library pending confirmation of the diagnosis of the problem and the subsequent modification of data files in the coming version ENDF/B-VI. This is consistent with the methodology adopted here for calculating kerma factors for heating purposes consistent with transport calculations. Other data sources, experimental or evaluated, are used only in the absence of corresponding ENDF/B-V information but are not used to replace them.

\section{7-N-14}

The primary capture photon of $10.835 \mathrm{MeV}$ is not isotropically emitted. However, the anisotropy begins at a high incident neutron energy of $1 \mathrm{MeV}$ at which the recoil energy is dominated by the incident neutron impact not the 
recoiling photon. The isotropic photon assumption is therefore used without compromising accuracy. In addition, the low energy kerma for this material is dominated by the $(n, p)$ reaction, not capture. Due to the use of ENDF/B-V reaction numbers $M T=700$ 's series to represent the $(n, p),(n, t)$ and $(n, a)$ reactions, a good match (to $4 \%$ ) between the KIN and DEB results was obtained. The KIN result was adopted because of the possible neglect of $(n, 2 n)$ gammas which will result in a slightly overestimated DEB kerma.

\section{$8-0-16$}

With the detailed gamma file, it was possible to obtain matching KIN and DEB kerma factors that differ by no more than $0.3 \%$.

\section{$9-F-19$}

The prompt kerma factors at low incident neutron energy were found to be much smaller than those computed by the NJOY module HEATR. This discrepancy is due to the absence of an ENDF/B- $V$ gamma file 12 for radiative capture, and HEATR was not able to perform the kinematic evaluation of capture kerma factors using the alternative file 13, MT $=102$. DEB and KIN methods results were calculated and compared. The kerma factor values using both methods are almost identical below an incident neutron energy of 1-5 MeV (grcups 1 through 120). Between 5.0 and $6.0 \mathrm{MeV}$ incident energy the oscillating error did not exceed a maximum of $20 \%$. Above $6 \mathrm{MeV}$ incident energy, the kerma factors computed by the two methods diverged considerably where DEB results were as large as three times the KIN values. A comparison between gamma energy production from file 13 and excitation energy from file 3 was made. It was found that excitation energy is much larger than gamma production 
energy above $6 \mathrm{MeV}$ incident neutron energy - a result cited earlier by MacFarlane [23]. The ENDF/B-V data evaluation was reviewed and the error was found. The inelastic de-excitation flag LR was set to zero for all levels and continuum which indicates decay by gamma emission. Levels of excitation energy of $4.683 \mathrm{MeV}$ and above decay also by alpha and proton eimission [24]. The adjustment of the LR flag to indicate parti'cle rather than gamma emission for MT=64 through 91 [25] should solve the problem. The binding energy of the alpha is $4.01 \mathrm{MeV}$ which is much lower than the $7.99 \mathrm{MeV}$ proton binding energy. Also, up to the highest energy level in the Table of Isotopes [24] of $10.411 \mathrm{MeV}$, no proton emission was indicated. From the above argument, the decay of levels $4.683 \mathrm{MeV}$ and above is dominantly by alpha emission. The flag $L R$ is therefore adjusted to $L R=22$ for $M T=64-91$. After making the $L R$ and the accompanying Q-value changes, the gap between KIN and DEB kerma factor values were greatly narrowed. The new KIN results were found to be larger than the DEB kerma factors by a maximum of $27 \%$. This difference is not large. It also carries the correct sign since KIN kerma factors are expected to be larger than DEB kerma factors at high incident neutron energy when detailed gamma production is not provided and KIN procedures ignore the possible emission of gammas following charged particle reactions. The new DEB kerma factors are therefore recommended for the KAOS/LIB-V library. The change of LR flag does not change the transport cross sections and is still consistent with the model's commitment in that regard. However, users of the KAOS/LIB-V library should be aware, that the helium gas production response function is affected. 


\section{$11-\mathrm{Na}-23$}

Below the incident neutron energy of $2 \mathrm{MeV}$, the kerma factors produced by KIN and DEB methods are identical. The difference between the two methods oscillates between $2 \mathrm{Me} !$ and $6.5 \mathrm{MeV}$ incident eriergies with an amplitude of nearly $30 \%$. This error can be attributed to the representation of gamma production as a function of incident neutron energy using too few points, although the spectral data are adequately represented. Above the incident neutron energy of $6.5 \mathrm{MeV}$, the ratio between DEB to KIN kerma factors increases gradually until it approaches 2 near $20 \mathrm{MeV}$ incident neutron energy. The problem is less severe but qualitatively similar to the F-19 case described earlier. The $Q$-value for $\left(n, n^{\prime} p\right)$ and $\left(n_{1}, n^{\prime} \alpha\right)$ reactions are $-8.79 \mathrm{MeV}$ and $-10.47 \mathrm{MeV}$ respectively. Allowing $1 \sim 2 \mathrm{MeV}$ for the Coulomb barrier, these reactions are energetically possible above an incident neutron energy of about $10 \mathrm{MeV}$. Unlike F-19, the above reactions are not considered in ENDF/B-V data for $\mathrm{Na}-23$. Until the possibility for these reactions is quantitatively determined in the entire incident neutron energy range, the KIN results were chosen as the recommended kerma factors. The DEB kerma factors are also kept in the KAOS/LIB-V library. If the $\left(n, n^{\prime} x\right)$ reactions were considered, the resulting kerma should be between the present KIN and DEB values but closer to the KIN estimation. This was verified at the incident neutron energy of $14.6 \mathrm{MeV}$ using the measurement of $\left(n, n^{\prime} p\right)$ cross section $\sigma=270 \pm 30 \mathrm{mb}$ by Yu Kozy et a1. [26]. The overestimated DEB kerma factor was therefore reduced by $\sigma|Q|$, i.e., reduced from $5.86 \times 10^{6}$ ev.barn to $3.49 \times 10^{6} \mathrm{eV} \cdot \mathrm{barn}$. The excitation energy of $\mathrm{Na}-23$ prior to the proton emission was estimated to be $11 \mathrm{MeV}$. The KIN kerma factor should be increased by $\sigma .\left(E^{*}-|Q|\right)$, i.e., increased from $2.98 \times 10^{6}$ eV.barn to $3.53 \times 10^{6}$ 
ev.barn. The two new results are different by only $1 \%$ which is a surprisingly good agreement.

\section{$13-A 1-27$}

Detailed gamma production for each reaction is provided in ENDF/B-V. The DEB and KIN methods are therefore equivalent for all the reactions except for the $(n, 2 n)$ reaction. The $(n, 2 n)$ reaction is far from dominating the energy deposition. The KIN and DEB evaluations of kerma factors are found to be close. The DEB result is accepted for KAOS/LIB-V library.

\section{$15-P-31$}

The relative difference between DEB and KIN kerma factors is as small as 1\% for incident neutron energies below $1.5 \mathrm{MeV}$. Between 1.5 MeV and 2.3 MeV incident neutron energy, the DEB kerma factors are less than the KIN kerma factors by a maximum of $18 \%$.

Above the incident neutron energy of $2.3 \mathrm{MeV}$, the relative difference between the two methods increases to a maximum of $57 \%$ indicating underestimated gamma production or an overlooked inelastically excited level decay by particle emission. The inelastic scattering is represented only by a continuum reaction due to the scarcity of experimental data [27]. The KIN results were adopted for prompt kerma factors.

\section{$25-M n-55$}

The KIN evaluation of kerma factors is adopted for the KAOS/LIB-V library. The KIN evaluation was judged to be superior to the DEB evaluation because of the problems with the gamma files. The capture gamma energy 
production was $36-53 \%$ too high compared to the available energy. Although gamma heating will be significantly overestimated for low incident neutron energies, the neutron heating is not affected as much. This is particularly valid for this material because capture kerma is dominated by the decay heat of the residual Mn-56. For groups 75 through $105(E=0.13-0.67$. MeV), the DEB values are much larger than the KIN ones. This underestimation of gamma production between the incident neutron energy of 0.13 and $0.2 \mathrm{MeV}$ is purely due to the neglect of the $1.258 \times 10^{5} \mathrm{eV}$ first level inelastic photon. Between the incident energy of $0.2 \mathrm{MeV}$ and $0.67 \mathrm{MeV}$, the gamma file which represents capture photons does not contain the inelastic photon because the spectrum is truncated at $0.3 \mathrm{MeV}$ gamma energy. Gamma production is overestimated between the incident neutron energies of $0.57 \mathrm{MeV}$ and $10.0 \mathrm{MeV}$ resulting not only in DEB kerma factors much lower than the corresponding KIN results, but also negative DEB kerma in 40 out of 55 groups in this incident neutron energy range. Above the incident neutron energy of $10 \mathrm{MeV}$, the DEB kerma factors are found to be larger than the KIN equivalent indicating gamma production underestimation. The evaluated gamma production deviates considerably from the experimental data used for the evaluation [28] and was not helpful in evaluating kerma factors.

\section{$27-\operatorname{Co}-59$}

The kerma factors produced by DEB for groups 116 through 120 and 167 through 173 (incident neutron energy between $1.2-1.5 \mathrm{MeV}$, and $14-17 \mathrm{MeV}$ ) were found to be negative. The observed negative group kerma factors are due to overestimated gamma production. DEB grouped kerma factors much larger than the KIN counterparts were also observed. The overall gamma files are 
not as terrible as it may sound, for the absolute gamma production error is only $\sim 20 \%$. Due to the kerma sensitivity to gamma production errors and the inevitable inconsistency when gamma production is evaluated independently from the neutron files, the KIN method was used in the entire incident neutron energy range.

\section{$40-Z r-90,91,92,94$, and 96}

There are no gamma files for any of the zirconium isotopes in ENDF/B-V evaluations. The KIN method had to be used to compute the neutron kerma factors recommended for the KAOS/LIB-V library. Thermal capture gamma spectra and multiplicity for natural zirconium are obtained from the ENDL-2 files. The $\eta$-factor of 2.327 was computed from the above data and used in computing capture kerma factors for each zirconium isotope. The resulting capture kerma is better than that obtained using the single-photon approximation even though the individual isotopic $\eta$-factors were not available.

Depending on the particular heating application, the DEB kerma factors with gamma energy assumed to be deposited locally could be also used. It was necessary to correct capture Q-values for each isotope, since a single Qvalue of $7.98 \mathrm{MeV}$ was given for all the isotopes in ENDF/B-V. The new Qvalues for $\mathrm{Zr}-90, \mathrm{Zr}-91, \mathrm{Zr}-92, \mathrm{Zr}-94$ and $\mathrm{Zr}-96$ are $7.1973,8.635,6.732$, 6.4709 and $5.5809 \mathrm{MeV}$ respectively.

\section{$41-\mathrm{Nb}-93$}

Between the inelastic scattering threshold of $0.0293 \mathrm{MeV}$ and $0.1 \mathrm{MeV}$, the $0.029 \mathrm{MeV}$ photon is not represented in the gamma files. The resulting 
error in DEB kerma was confined to $0.4 \%$ because energy deposition by elastic scattering is dominant in this energy range. Above the incident neutron energy of $0.1 \mathrm{MeV}$ and up to $1 \mathrm{MeV}$ however, all the 43 group DEB kerma factors are negative. Gamma production in that incident neutron energy range is obtained by interpolating spectral and production cross section data given at 0.1 and $1 \mathrm{MeV}$. This poorly-represented gamma production would be further overestimated if the provided interpolation schemes were used directly due to the parabolic interpolation problem. Between the incident neutron energy of $1.0-4.0 \mathrm{MeV}$, the error oscillates due to having too few gamma points. The 4 garma points in file 13 at 1 . MeV intervals are not enough to produce the details required for 28 groups. The KIN method was therefore recommended for incident neutron energies up to $4.0 \mathrm{MeV}$. Above $4 \mathrm{MeV}$ however, 12 gamma points at intervals of 1 or 2 MeV were provided in files 13 and 15 and provide better details for the remaining 33 groups. The DEB method kerma factors were found to be less than the KIN counterparts by an average of $30 \%$ above $4 \mathrm{MeV}$ incident neutron energy. This could be explained as due to the subtraction of the $(n, x)$ gamma not accounted for in the KIN method. The recommended kerma factors are computed by the KIN method for the first 141 groups, and by the DEB method for groups 142 through 174 .

\section{$72-\mathrm{HF}-174,176,177,178,179$, and 180}

There are no gamma files for any of the Hafnium isotopes in ENDF/B-V evaluations. The KIN method had to be used to compute the neutron kerma factors recommended for the KAOS/LIB-V library. Thermal capture gamma spectra and multiplicity for natural Hafnium are obtained from the ENDL-2 files. The $\eta$-factor of 2.446 was computed from the above data and used in 
computing capture kerma factors for each Hafnium isotope. The resulting capture kerma factor is better than that obtained using the single-photon approximation even though the individual isotopic $\eta$-5actors were not availabie.

Depending on the particular heating application, the DEB kerma factors with gamma energy assumed to be deposited locally could be also used. It was necessary to correct capture Q-values for each isotope, since a single Qvalue of $7.18 \mathrm{MeV}$ was given for all the isotopes in ENDF/B-V. The new Qvalues for $\mathrm{Hf-174}, \mathrm{Hf}-176, \mathrm{Hf}-17 \mathrm{7}, \mathrm{Hf}-178, \mathrm{Hf}-179$, and $\mathrm{Hf}-180$ are 6.789, $6.383,7.627,6.099,7.388$, and $5.695 \mathrm{MeV}$ respectively.

\section{$73-T a-181$}

The KIN method is accepted in the entire incident neutron energy range. The DEB results suffer from the gamma inaccuracy that resulted - when overestimated - in 34 negative kerma groups. Other positive DEB kerma factor group values are generally overestimated by an order of magnitude.

$74-W-182,183,184$, and 186

The ENDF/B-V data file (MAT=1474) for natural tungsten is produced by combining isotopic data files using the NJOY module MIXER. The DEB method was used to calciliate kerma factors for natural tungsten (MAT=1474). The data files (MAT=1475, 1476, 1477 and 1478) were used to calculate the individual isotopic kerma factors. The kerma factors for natural tungsten are then cbtained from the isotopic kerma by abundance-weighted averaging. rerma factors obtained for MAT=1474 were found to be negative for groups 110 trruugh 112 and 120 through 123. Isotopic kerma factors for these groups are 
all positive (with the exception of $W-184$ group 122) and the weighted natural tungsten kerma factors are all positive. This discrepancy is rather odd because natural element kerma factors calculated with a single Q- value per reaction tends to be over-estimated. The error - if at all explainable - can be due to the reduced availability of level inelastic series where 40 levels $(M T=51-90)$ were provided for the natural file, compared to a total of 60 levels for the 4 isotopes. The individual isotope approach was therefore chosen. Detailed gamma production is provided for each isotope for inelastic, capture, $(n, 2 n)$, and $(n, 3 n)$ reactions. The DEB kerma factor groups 149-151 for $W-183$ and group 122 for $W-184$ are negative. No $(n, p)$ or $(n, a)$ gammas were considered which leaves the DEB method without merit relative to the KIN method. The KIN method was therefore used to calculate kerma factors for all the 4 isotopes in the entire incident neutron energy range.

\section{3-Bi-209}

Many negative DEB kerma factor groups were found. The problems with gamma files - spotted by MacFarlane [23] are generally confirmed here. With almost $50 \%$ chance of producing the isomeric state of $\mathrm{Bi}-210$ in the radiative capture reaction, MacFarlane suggestion of increasing capture gamma multiplicity by $10 \%$ needs to be slightly reduced to only $6.5 \%$. The KIN method was used to produce the recommended kerma factors in the entire incident neutron energy range. 


\section{III.2 Kerma Factor Evaluation Summary For Natural Elements}

\section{2-Mg-nat}

The energy-dependent Q-values are evaluated as follows:

$(n, 2 n)$ Q-value

Below an incident neutron energy of $12 \mathrm{MeV}$, the only participating isotope is $\mathrm{Mg}-25$, therefore

$Q(E)=-7.33 \mathrm{MeV}, \quad$ Threshold $<E<12.0 \mathrm{MeV}$.

Between 12 and $17 \mathrm{MeV}$, abundance weighting of Q-values of Mg-25 and Mg26 results in:

$Q(E)=-9.2 \mathrm{MeV}, \quad$ for $12.0<E<17.0 \mathrm{MeV}$.

Above an incident neutron energy of $17 \mathrm{MeV}$, the cross section $\sigma_{24}$ for the more abundant isotope $\mathrm{Mg}-24$ is taken from Reference [29] and used to get:

$$
Q(E)=\left(\sigma_{24} \text { a24 Q24 }+\left(\sigma_{\text {nat }}-\sigma_{24} \text { a24 }\right) Q^{\star}\right) / \sigma_{\text {nat }}
$$

where $a_{24}=0.786$ is the abundance of Mg-24, and Q24 is its $(n, 2 n)$ Qvalue. $Q^{*}=-9.2 \mathrm{MeV}$ is the Q-value of the Mg-25 and Mg-26 isotopes weighted by their respective abundances. $\sigma_{\text {nat }}$ is the $(n, 2 n)$ reaction cross section for natural Magnesium provided in ENDF/B-V.

$(n, p)$ Q-value

Threshold energies for the (n,p) reactions for Mg-24 and Mg-26 are estimated from:

$$
E_{\text {th }}=|Q|(A+1) / A+0.1 z Z \mathrm{MeV}
$$


These threshold energies are used to divide the incident neutron energy range where one, two, or three isotopes undergo the $(n, p)$ reaction. In each region, the Q-value is obtained by abundance weighting. The resulting Qvalue is tabulated as follows:

$\begin{array}{ll}E(\mathrm{MeV}) & Q(\mathrm{MeV}) \\ 4.95 & -3.079 \\ 6.7 & -3.3 \\ 7.0 & -4.54 \\ 9.8 & -4.54 \\ 10.0 & -4.9 \\ 20.0 & -4.9\end{array}$

\section{$(n, a)$ Q-value}

The energy dependence of the $(n, a)$ Q-value is obtained by the same technique used for $(n, p)$ reaction. The resulting $Q$-value is tabulated as follows:

$\begin{array}{ll}E(\mathrm{MeV}) & Q(\mathrm{MeV}) \\ 2.11 & 0.4818 \\ 5.0 & 0.4818 \\ 6.0 & -2.16 \\ 8.0 & -2.16 \\ 9.0 & -2.5 \\ 20.0 & -2.5\end{array}$

\section{$(n, n p)$ and $(n, n a)$ Q-values}

The most abundant isotope $\mathrm{Mg}-24$ has the largest Q-value and therefore dominates these reactions. Other isotopes' (O-values are not much smaller than the $M g-24$ Q-value. The ENDF/B-V Q-value is used directly where the slight overestimation of the resulting kerma is expected to be minimal. 


\section{Decay Heat}

Abundance-weighted cross sections are used to calculate the decay kerma factor. The proper adjustments for threshold energies are also made. The $(n, 2 n)$ cross section for $M g-24$ used previously to compute the Q-value is again used for decay calculations.

\section{Recommended Prompt Kerma Factors}

The DEB and KIN calculations were made with and without the energy dependent Q-values. Two negative DEB kerma factor groups (121 and 126) were found for both constant and variable Q-values. The use of energy-dependent Q-values resulted in lowering kerma by a maximum of $25 \%$. The KIN evaluation with variable Q-values is recommended for the entire incident neutron energy range.

\section{4-Si-nat}

Reaction Q-values provided in ENDF/B-V are equal to those for the most abundant isotope, Si-28 $(92 \%)$, except for the $(n, 2 n)$ reaction where the Qvalue for Si-29 with $4.7 \%$ abundance was used. Because of the high threshold energy for $\mathrm{Si}-28(n, 2 n)$ reaction of nearly $18 \mathrm{MeV}$, the effect of the less abundant isotopes on the Q-value needs to be considered for the DEB calculations. Between the threshold group 157 and group 165, the $(n, 2 n)$ DEB kerma factors using the ENDF/B-V Q-value were found to be negative, while the last 4 groups were found to be much larger than the KIN counterparts. The use of a variable Q-value should bring these overestimated groups closer to the KIN value, but the problem with the negative groups remains unchanged. The KIN kerma factors are formally independent of the Q-value and much less 
sensitive to errors in the secondary neutron energy, and is therefore preferred. Energy distributions of secondary proton and alpha particles from $\left(n, n^{\prime} p\right)$ and $\left(n, n^{\prime} \alpha\right)$ reactions are provided in the ENDF/B-V file 5, MT=719 and 799 respectively. One should expect these data to appear in file 25 . This is the only material however, that a KIN treatment was possible for charged particle-producing reactions. A good agreement between DEB and KIN results was observed, especially at lower energies. The KIN kerma is larger than DEB by a maximum of $28 \%$ for the $\left(n, n^{\prime} \alpha\right)$ reaction and by $15 \%$ for the $\left(n, n^{\prime} p\right)$ reaction. Errors in inelastic gamma production result in negative DEB inelastic kerma for groups 140 and 170 through 174. Between groups 142 and 165. the underestimated gamma production results in overestimated DEB inelastic kerma by a maximum of over $100 \%$. The $(n, p)$ reaction was treated by both DEB and KIN methods. The KIN treatment was possible for this material because the level and continum cross sections are given and the continum proton energy is given in file 5 MT $=718$. The maximum difference between the two results did not exceed $14 \%$.

The $(n, a)$ reaction was examined in a similar manner to the $(n, p)$ reaction. It was found that the KIN kerma factors exceed the DEB kerma factors by $\sim 80 \%$ for the first 4 groups (group 136 threshold to 140), but the absolute value of this partial kerma factor is small in that range of higher incident neutron energies. However, the maximum difference does not exceed $23 \%$. The prompt kerma factors produced by the KIN method are recommended.

Decay energy production was computed only for the most abundant isotope $\mathrm{Si}-28$. The decay of $\mathrm{Si}-27$ formed by the $(n, 2 n)$ reaction is ignored due to the high reaction threshold of $\sim 18 \mathrm{MeV}$. Only the $\beta$ - decay of Al-28 produced by the $(n, p)$ reaction is considered. 
In addition to the natural element evaluation provided in the general ENDF/B-V files under material number MAT $=1347$, a separate ENDF/B-V evaluation is provided in the dosimetry files for S-32, the most abundant sulfur isotope $(95 \%)$. These data were used to separate the, less abundant isotopes' cross sections for the purpose of estimating the variation of the elemental Qvalues with incident neutron energy.

\section{$(n, 2 n)$ Q-values}

Between the threshold and $11.5 \mathrm{MeV}$ incident neutron energy, the $(n, 2 n)$ reaction is energetically possible only with the isotope S-33 and its Q-value of -8.642 is used. In the incident neutron energy range of $11.5-15.1 \mathrm{MeV}$, the abundance-weighted $Q$-value of $S-33$ and $S-34, Q^{*}=-11.0$ MeV is taken. Above $15.1 \mathrm{MeV}$, the cross section of $\mathrm{S}-32$ and the combined cross section of S-33 and S-34 and their abundances are used to obtain the energy-dependent Qvalue as:

$$
Q(E)=\left(\sigma_{32} \text { a32 Q32 }+\left(\sigma_{\text {nat }}-\sigma_{32} \text { a32 }\right) Q^{\star}\right) / \sigma_{\text {nat }}
$$

where $\mathrm{a}_{32}=0.9502$ is the abundance of $S-32, \sigma_{32}$ and $\sigma_{\text {nat }}$ are the $(n, 2 n)$ microscopic cross sections for $\mathrm{S}-32$ and natural sulfur respectively, $Q_{32}=-15.0424 \mathrm{MeV}$ is the Q-value for $S-32$, and $Q^{*}=-11.0 \mathrm{MeV}$ is the $Q$ - value of the $S-33$ and $S-34$ isotopes obtained by abundance weighting.

\section{$\left(n, n^{\prime} a\right)$ Q-value}

The reaction is not included in the ENDF/B-V (MAT $=1316$ ) evaluation for $\mathrm{S}-32$. The $Q$-value of the most abundant $S-32$ is the largest in addition to 
being close in magnitude to the other isotopes' Q-values. No energydependent Q-value was used for this reaction.

\section{$(n, n p)$ Q-value}

The combined abundance-weighted cross section of S-33 and S-34 was found to oscillate between small positive and negative values when computed from the ENDF/B-V data for natural sulfur and S-32, MAT=1347 and MAT=1316 respectively. The minor isotopes' participation is therefore minimal and the single Q-value for S-32 was kept for kerma factor evaluation.

\section{$(n, p)$ Q-value}

Below the S-32 threshold of $1.6 \mathrm{MeV}$, the S-33 Q-value of $0.53 \mathrm{MeV}$ was used. The Q-value then linearly decreases with incident energy up to $2.0 \mathrm{MeV}$ above which the -0.928 MeV Q-value for S-32 was used.

\section{$(n, d)$ Q-value}

The reaction is not considered for S-32 evaluation. The Q- value provided for the elemental evaluation is equal to that of the abundant S-32 and therefore accepted.

\section{$(n, t) Q$-value}

The S-33 Q-value was used for incident neutron energies below the 13.09 MeV threshold of S-32. The S-32 Q-value is taken above 14 MeV with a linear transition allowed between the threshold and $14 \mathrm{MeV}$. 


\section{$(n, a)$ Q-value}

Below $1.4 \mathrm{MeV}$ incident energy, the reaction is energetically possible only in S-32 and S-33 isotopes. With the exception of the low energy end where the S-nat evaluation gives a zero cross section while the S-32 evaluation provides a positive value, the two evaluations provided for a formally complete determination of the elemental Q-value by abundance and

cross section weighting. Above $1.5 \mathrm{MeV}$, the Q-value for the most abundant isotope S-32 was used because the two ENDF/B-V evaluations resulted in a combined $\mathrm{S}-33$ and $\mathrm{S}-34$ cross section with many negative points.

\section{$(n, 2 p)$ Q-value}

The reaction is not represented in the $\mathrm{S}-32$ evaluation. The $\mathrm{S}-32$ threshold of $9.9 \mathrm{MeV}$ marked the transition between the S-33 Q-value of -9.00 MeV below the threshold and the S-32 Q-value of $-9.57 \mathrm{MeV}$ above the threshold.

\section{Decay Heat}

The S-32 evaluation was used to compute decay heat due to $S-32(n, 2 n) S-$ 31 and S-32 (n,t) P-30 reactions. The S-nat (MAT 1347) evaluation was used with abundance and threshold corrections to compute the decay heat from S-34 $(n, p) P-34, S-34(n, a) S i-31$, and $S-32(n, 2 p)$ Si-31 reactions.

\section{Prompt Kerma Factors}

A correction to ENDF/B-V $($ MAT $=1347, M F=12, M T=102)$ was made by adding a point $\left(8.99 \times 10^{5} \mathrm{eV}, 2.82297\right)$ to the capture gamma multiplicity table. This corrected the obviously unintended linear decline of the 
multiplicity to zero at the $0.9 \mathrm{MeV}$ cutoff. This correction resulted in improving the gamma production but less significant improvement to the capture kerma. The introduction of energy-dependent Q-values resulted in the reduction of the DEB prompt kerma factors by a maximum of $40 \%$. Gamma production is found to be underestimated between 0.9 and $10 \mathrm{MeV}$ incident energy. The KIN prompt kerma factors are accepted for incident neutron energies up to $10 \mathrm{MeV}$. Above $10 \mathrm{MeV}$, the DEB method was used to account for possible $(n, x)$ gammas.

\section{$17-C l-n a t$}

Variable Q-values were not needed for this material. The maximum error in the total prompt kerma due to using a single $Q$-value for the $(n, 2 n)$ reaction does not exceed $1 \%$. Cross sections for the $c l-35(n, p)$ and $C l-35$ $(n, a)$ reactions [29] are about an order of magnitude larger than those for Cl-37. This justified keeping ENDF/B-V Q-values which are equal to those of the most abundant isotope $c l-35$ for these two reactions. For the $\left(n, n^{\prime} p\right)$ and $\left(n, n^{\prime} a\right)$ reactions, the $Q$-values of the two isotopes are different by $24 \%$ and $11 \%$ respectively, but the combined error in total kerma due to using the single Cl-35 Q-value provided in ENDF/B-V is less than $1 \%$. The KIN prompt kerma factors were found to agree with the DEB kerma factors with a maximum error of $15 \%$ below the incident neutron energy of $6.4 \mathrm{MeV}$. Above $6.4 \mathrm{MeV}$, the DEB kerma factors become increasingly larger than the KIN counterparts up to a factor of 3 near the incident neutron energy of $20 \mathrm{MeV}$. Only inelastic and capture photons are represented in the gamma files. The underestimation of gamma production for incident neutron energies above 6 MeV could be due to neglecting other nonelastic gammas. The KIN method is used to produce the 
recommended kerma factors. The abundance-weighted isotopic cross sections for decay heat calculations are obtained by adjusting the ENDF/B-V cross sections for the isotopic thresholds.

\section{$19-K-$ nat}

The utilization of variable Q-values was not necessary because of the high abundance of $K-39(93.3 \%)$. An attempt to separate the $k-39(n, p)$ cross section was made by subtracting the abundance weighted cross section for the K-41 $(n, p)$ reaction found in the partial evaluation ENDF/B-V MAT=7191. The resulting K-39 cross section contained many negative points and was discarded. Level inelastic gamma production between the $2.59 \mathrm{MeV}$ threshold of $\mathrm{K}-39$ and $6.8 \mathrm{MeV}$ matches the inelastic excitations computed from neutron files. Between the incident neutron energy of 1.01 and $6.8 \mathrm{MeV}$, the continuum inelastic scattering cross section represents the reaction in the less abundant isotope $\mathrm{K}-41$. No inelastic gamma production was represented in that range. Above $6.8 \mathrm{MeV}$ incident energy, the inelastic gamma was underestimated. It seems like the inelastic contribution of K-41 was entirely ignored resulting in overestimating the DEB kerma factors by an average of $10 \%$ and a maximum of $39 \%$. The KIN method was therefore used to produce recommended prompt kerma factors. For decay heat calculations, abundance ratios were used tu obtain isotopic cross sections from elemental $(n, 2 n),(n, \gamma)$ and $(n, a)$ cruss sections. The $(n, a)$ cross section was trimmed at the threshold of $K-41$. The partial evaluation MAT $=7191$ was used to compute decay heat resulting from the $K-41(n, p) \operatorname{Ar}-41$ reaction. 20-Ca-nat

The most abundant isotope ca-40 with abundance $96.9 \%$ dominates the ENDF/B-V elemental evaluation. Its Q-values are used for all reactions 
except for the $(n, 2 n)$ reaction where the higher Q-value of Ca-48 was used to include a small contribution from this isotope. This overestimated Q-value did not affect the accuracy of the total kerma. With the detailed gamma production provided in ENDF/B-V file 12 , the relative difference between the DEB and KIN results did not exceed $1 \%$ for most groups, and the DEB kerma factors are accepted to fill the recommended kerma factor field in the KAOS/LIB-V library. The small decay heat contribution from Ca-40 $(n, 2 n)$ Ca39 and $\mathrm{Ca}-40(n, t)$ K-38 reactions was computed. The threshold energy for the $(n, 2 n)$ reaction was restored to $16.0 \mathrm{MeV}$ for the purpose of the decay heat calculations.

\section{2-Ti-nat}

In addition to the natural element evaluation in ENDF/B- $V$, MAT $=1322$, there are partial evaluations for $T i-46, T i-47, T i-48$, and $T i-50$ represented by material numbers MAT $=7226,7227,7228$, and 7220 respectively. With no dominating isotope with large abundance, the estimation of the variation of the elemental Q-values becomes necessary. These are evaluated as follows:

\section{$(n, 2 n)$ Q-value}

Prompt DEB kerma factors were found to be sensitive to changes in the $(n, 2 n)$ Q-values at high incident neutron energy. An initial estimation of the energy dependence of the Q-value was made. This estimation depended on approximating the weighting isotopic cross sections with magnitudes proportional to their abundances as a ramp function that starts at their respective threshold energies. A better estimation of the energy-dependent Q-value was made by taking the elemental cross section function weighted by 
abundance and translating it to begin at each isotope's threshold. This latter estimation of the Q-value variation with energy was found to be in good agreement with that obtained by using the evaluated isotopic cross sections from reference $[30]$. The $(n, 2 n)$ reaction $Q$-value used in this evaluation is tabulated below:

$\begin{array}{ll}E(\mathrm{MeV}) & Q(\mathrm{MeV}) \\ \text { threshold } & -8.14 \\ 11.0 & -8.4 \\ 12.0 & -8.6 \\ 13.0 & -9.6 \\ 16.0 & -10.5 \\ 20.0 & -11.0\end{array}$

$(n, p)$ Q-value

The ENDF/B-V zero Q-value was replaced. The isotopic cross sections are available only for $\mathrm{T} i-46, \mathrm{~T} i-47$, and $\mathrm{T} i-48$ from ENDF/B-V partial evaluations. Since the Ti-49 Q-value is close to that of $\mathrm{Ti}-46$, both abundances were combined and the modified abundances along with the three available cross sections are used to compute the reaction Q-value before the threshold of $\mathrm{Ti}$ 50. The effect of Ti-50 on the Q-value was assumed to be small and the previous evaluation was extended to the incident neutron energy of $20 \mathrm{MeV}$.

$(n, a)$ Q-value

The ENDF/B-V zero Q-value was replaced. The new energy-dependent Qvalue was constructed using threshold energies and abundance weighting. The resulting Q-value is tabulated below: 


$\begin{array}{ll}E(\mathrm{MeV}) & Q(\mathrm{MeV}) \\ 1.0 \mathrm{E}-11 & 1.357 \\ 3.0 & 1.357 \\ 4.0 & 0.79 \\ 5.0 & 0.7 \\ 6.0 & 0.0 \\ 7.0 & -1.0 \\ 7.5 & -1.2 \\ 10.0 & -1.3 \\ 20.0 & -1.35\end{array}$

Other reaction Q-values were not changed. The reason is that the reaction cross section is small or the variation of isotopic Q-values is sufficiently small.

Isotopic cross sections for producing radioactive decay heat were obtained as follows: the Ti-46 $(n, 2 n)$ cross section is obtained from reference [31]. Threshold-adjusted, abundance-weighted elemental cross sections were used to compute decay heat from the other reactions as needed. The DEB and KIN methods were used to compute prompt kerma factors with and without the variable Q-values. There is an oscillating DEB/KIN error for groups 114 through 148 . There are also five DEB negative groups (129 through 133). The KIN result was accepted for groups 1 through 148 i.e., up to the incident neutron energy of $6.0 \mathrm{MeV}$. Above $6.0 \mathrm{MeV}$, DEB results were taken to account for the $(n, x)$ gamma production.

\section{3-V-NAT}

The only stable isotope is $\mathrm{V}-51$. Although the abundance of the long lived isotope $V-50$ is only $0.25 \%$, its Q-values for $(n, p)$ and $(n, a)$ reactions are larger than those for the principal isotope $V-51$ [32] and are listed in the ENDF/B-V evaluation. The adjusted Q-values are taken such that the $V-50$ 
Q-values are considered only below the $V$ - 51 threshold. The elemental Qvalue for $(n, p)$ reaction used in this evaluation is tabulated below:

$\begin{array}{lc}E(\mathrm{MeV}) & Q(\mathrm{MeV}) \\ 1.0 \mathrm{E}-11 & 2.995 \\ 1.717 & 2.995 \\ 1.8 & -1.6837 \\ 20.0 & -1.6837\end{array}$

The $(n, a)$ Q-value is similarly tabulated as follows:

$\begin{array}{lr}E(\mathrm{MeV}) & \mathrm{Q}(\mathrm{MeV}) \\ 1.0 \mathrm{E}-11 & 0.7577 \\ 2.096 & 0.7577 \\ 2.1 & -2.0546 \\ 20.0 & -2.0546\end{array}$

The comparison between the DEB and KIN prompt kerma factor results shows good agreement for groups 1 through 137, i. e., up to the incident neutron energy of $3.0 \mathrm{MeV}$. The removal of a parabolic interpolation overestimation error in gamma production reduced the number of negative DEB kerma factor groups from 5 to 4 (groups $139-142$ ). The difference between both calculations fluctuates to a maximum of $\pm 50 \%$ for groups 144 through 162 . The KIN results were therefore accepted up to the incident neucron energy of 12.0 $\mathrm{MeV}$ (groups 1 through 163). Above $12.0 \mathrm{MeV}$, the DEB kerma factors are less than the KIN counterparts by an average of $30 \%$ reflecting the consideration of $(n, x)$ gamma production, and is therefore accepted in that range. For decay heat calculation, the $(n, p)$ reaction cross section was set to zero before the threshold energy of $V-51$.

\section{4-Cr-nat}

The gamma production cross section in ENDF/B-V file $13, M T=3$ is zero at the incident neutron energy of $20 \mathrm{MeV}$. The situation was corrected by 
inserting the value of 2.4267 barn taken from reference [33]. Isotopic evaluations for the four chromium isotopes were found in the JENDL-2 library. These data are used to evaluate cross section and abundance-weighted Q-values for the $(n, p)$ and $(n, a)$ reactions. Threshold adjustments were needed to fit the principal ENDF/B- $V$ data source. Other reaction cross sections found in ENDF/B-V elemental evaluations were not accounted for in some or all JENDL isotopic evaluations. The overestimation of the DEB kerma factors due to use of the single ENDF/B-V Q-values for these reactions is expected to be small. The use of energy-dependent Q-values affect the KIN kerma factors above group 145. The largest reduction amounts to $24 \%$ in group 153. Large gamma production errors result in large errors in the DEB kerma factors with 33 negative groups. The KIN method was therefore used to produce the recommended prompt kerma factors in the entire incident neutron energy range. Abundance weighting is used for decay heat computations. This was done because not all the radioactivity-producing reactions are accounted for in the JENDL-2 evaluations, and also because of the large discrepancies between ENDF/B- $V$ and the combined elemental cross sections from JENDL-2.

\section{6-Fe-nat}

In addition to the elemental ENDF/B-V.2 evaluation, other data sources were considered for the kerma factor evaluation. Among those are the isotopic ENDF/B-V dosimetry files and INDL-V partial evaluations, JENDL-2 isotopic evaluations, and the Los Alamos evaluation [34] of Fe-54 and Fe-56.

\section{Variable Q-values}

The isotopic evaluations of JENDL-2 were used to construct the capture Q-value as a function of incident neutron energy. On the other hand, an 
abundence-weighted single Q-value was listed in the ENDF/B-V.2 evaluation. The maximum relative difference between the energy-dependent Q-value and the single ENDF/B-V.2 value was $2.65 \%$ in the $0.8-2.0 \mathrm{MeV}$ incident neutron energy range. This small difference does not account for the energy imbalance problems in that range contrary to the explanation provided by Larson (reference [35] page 25). The ENDF/B-V.2 capture Q-value was used. The $(n, p)$ Q-value listed in ENDF/B-V.2 is equal to that of the Fe-54 isotope with only a $5.8 \%$ abundance. The elemental $(n, p)$ cross section was constructed from JENDL-2, INDL-V, partial ENDF/B-V MAT $=6430$ and MAT $=6431$ evaluations, and the LoS Alamos Fe-54 and $\mathrm{Fe}-56$ evaluations. All the constructed elemental cross section are found to be in good agreement with the ENDF/B-V.2 value. Because JENDL-2 is the most complete of all data sources where all isotopic data were found, these data were used to evaluate the variable $(n, p) Q$-value. The maximum reduction in the total prompt kerma factor due to using a variable $(n, p)$ Q-value amounts to $19 \%$ at the incident neutron energy of $10 \mathrm{MeV}$. The $(n, a) \quad Q$ - value in ENDF/B-V.2 is also equal to that of Fe-54. Construction of the elemental $(n, a)$ cross section from the available isotopic data sources was done. The INDL-V data were not sufficient, whil: the JENDL-2 reconstructed elemental $(n, a)$ cross section was much larger than that of ENDF/B-V.2. The constructed elemental $(n, a)$ cross section from LoS Alamos data is also larger than that of ENDF/B-V.2 but with better agreement. The variable $(n, d)$ Q-value evaluated from the Los Alamos evaluation data was found to affect the total prompt kerma by no more thari $1 \%$ and was subsequently dropped. Other reaction Q-values were also studied and the listed ENDF/B-V.2 values were found adequate. Only the $(n, p)$ reaction variable Q-values needed to be considered. Gamma production files in the revision 2 of ENDF/B-V.2 data were modified in response to MacFarlane's work 
on energy imbalance $[23,35]$. The new gamma production was modified above 2 MeV to "reproduce" the estimated heating, which makes these gamma files particularly suspicious for the purpose of evaluating kerma factors. A thorough review of gamma production was therefore necessary. Apart from flaws irrelevant to energy balance, e.g., the branching ratios for MT $=57$ which are taken opposite to those of the Table of Isotopes, other energy balance problems were found. Inelastic gamma represented in file 12 are not only resulting from the excitation of the respective levels by inelastic scattering of the corresponding file 3 data, but also the excitation of these levels due to gamma decay of higher levels. Although this practice reduces data volume a little, the energy balance is no longer as strict as expected from file 12 representation. A comparison between excitation energy production and inelastic gamma production shows that the former was larger by a maximum of $9 \%$. In the incident neutron energy range $0.85-2.122 \mathrm{MeV}$, the gamma production in the ENDF/B-V files $M F=13, M F=15$ represents capture photons. As mentioned earlier, the energy imbalance that produced negative kerma facturs in that range can not be due to elemental Q-value problems. The elemental $(n, \gamma)$ cross section constructed from JENDL-2 data is in better qualitative agreement with the ENDF/B-V.2 capture gamma production than the ENOF/B-V.2 $(n, \gamma)$ cross section. The possibly underestimated ENDF/B- V.2 $(n, \gamma)$ cross section needs to be reviewed in this range, and more gamma resolution than the provided 4 point histogram is needed.

The difference between the DEB and KIN results alternates between positive and negative values for groups 110-146. The DEB kerma factor of group 136 is negative. Although the DEB problem below the incident neutron energy of $2.122 \mathrm{MeV}$ is due to a mismatch between neutron and gamma files, the 
coarse histogram gamma production representation is the source of the DEB error between the incident energy of 2.122 and $5.5 \mathrm{MeV}$. The KIN prompt kerma factors are therefore recommended below $5.5 \mathrm{MeV}$. Above $5.5 \mathrm{MeV}$, the DEB kerma factors are accepted to account for the $(n, x)$ gamma production.

For decay heat calculations, the isotopic cross sections from the JENDL2 files were normalized to reproduce the ENDF/B-V.2 cross sections. No radioactivity-producing reaction in $\mathrm{Fe}-57$ was found in JENDL-2 and considering its small abundance, its decay heat contribution was ignored. Fe-58 $(n, p)$ and $(n, a)$ reactions are the only radioactivity-producing reactions found for this isotope in JENDL-2 data and their small contribution was considered.

\section{8-Ni-nat}

In addition to the ENDF/B-V elemental evaluation, JENDL-2 isotopic evaluations for the 5 Nickel isotopes were used. Variable Q-values for $(n, p)$ and $(n, a)$ reactions were computed from JENDL-2 data. The maximum reduction of total kerma factors due to using these variable Q-values amounts to only $5 \%$. The effect of the other reactions' variable Q-values was assumed to be small and was not considered. The DEB and KIN results are in good agreement up to group 113 where the error does not exceed $3.8 \%$. Between group 114 and 141, the difference oscillates between positive and negative values with 3 negative DEB kerma factor groups 121, 122, and 127 . The reason for this DEB inaccuracy is the coarse histogram gamma production representation. In that range, there are only 6 histogram intervals which cio not provide the resolution needed for the 28 groups. Therefore for the first 141 groups, up to an incident neutron energy of $4.0 \mathrm{MeV}$, the KIN results are recommended. Above $4.0 \mathrm{MeV}$, there are 14 histogram intervals for gamma 
production which provide better resolution for the remaining 33 groups. To account for $(n, x)$ gamma production, the DEB kerma factors are recommended above $4.0 \mathrm{MeV}$.

Decay heat production is produced by reactions with the low-abundance isotopes $\mathrm{Ni}-61, \mathrm{Ni}-62$, and $\mathrm{Ni}-64$. The JENDL-2 cross sections for these isotopes were therefore used for decay heat production calculations directly without normalization to elemental ENDF/B-V values.

\section{9-Cu-nat}

There are only two copper natural isotopes Cu-63 and Cu-65 with abundances of $69.2 \%$ and $30.8 \%$ respectively. This made the unique determination of isotopic data possible if data for either isotope is available. ENDF/B-V activation files provide for partial isotopic evaluations wilich are prepared by the same evaluator, C. Fu, and therefore consistent with the elemental evaluation. The $\mathrm{Cu}-63(n, \gamma),(n, p)$ and $(n, d)$ cross sections were obtained from ENDF/B-V MAT $=7293$, while the Cu-65 $(n, 2 n)$ cross section was obtained from ENDF/B-V, MAT $=7295$. The $\left(n, n^{\prime} a\right)$ cross sections for both isotopes are taken from JENDL-2 and normalized to ENDF/B-V elemental values. The Cu-65 $\left(n, n^{\prime} p\right)$ cross section is taken from reference [36] and is tabulated below:

$\begin{array}{ll}\begin{array}{l}\text { Neutron Energy } \\ \text { E (MeV) }\end{array} & \begin{array}{l}\text { Cu-65 (n, n'p) cross } \\ \text { section (barn) }\end{array} \\ 8.0 & \\ 9.5 & 0.0 \\ 10.5 & 1.0 \mathrm{E}-3 \\ 12.0 & 6.0 \mathrm{E}-3 \\ 13.5 & 7.0 \mathrm{E}-3 \\ 14.5 & 1.7 \mathrm{E}-2 \\ 16.0 & 1.8 \mathrm{E}-2 \\ 20.0 & 3.0 \mathrm{E}-2 \\ & 6.0 \mathrm{E}-2\end{array}$


The isotopic cross section data mentioned above were used to compute variable $Q$-values for $(n, 2 n),\left(n, n^{\prime} a\right),\left(n, n^{\prime} p\right),(n, p)$, and $(n, a)$ reactions. Variable Q-values for $(n, d)$ and $(n, H e 3)$ reactions are not considered because their maximum effect can not exceed $7.0 \times 10^{3}$ and $6.0 \times 10^{3}$ eV.barn respectively. Away from the sensitive groups near the negative DEB kerma factor groups 169 and 170, the maximum reduction in DEB kerma factors due to variable Q-values amounts to $75 \%$ at the last group 174 . The DEB kerma factor is negative in the incident neutron energy range of 1.25 through $1.65 \mathrm{MeV}$ i. e. groups 118 through 122. Kerma factor groups 169 and 170 are also negative. Comparison with KIN results shows that the difference between kerma factors computed by the two methods oscillates between positive and negative values in the entire incident neutron energy range. The gamma production represents only capture, inelastic, and $(n, 2 n)$ photons, i. e. there is no representation for possible $(n, x)$ gammas. The KIN method is therefore used to produce the recommended kerma factors. The isotopic cross sections are used to compute decay heat production.

\section{2-Mo-nat}

In the elemental ENDF/B- $V$ evaluation, the only reactions represented are the elastic, continiuum inelastic, $(n, 2 n),(n, 3 n)$ and capture. While the $(n, p)$ and $(n, a)$ reactions are ignored in the ENDF/B-V evaluation, non-trivial cross sections of the order of $25 \mathrm{mb}$ and $10 \mathrm{mb}$ respectively are to be found in JENDL-2 evaluations. DEB kerma factors with constant Q-values are negative for 97 out of 174 groups. The use of variable Q-values would introduce more negative groups. The DEB kerma is particularly sensitive to gamma production errors because there are no $(n, x)$ reactions in the 
representation. The accuracy of gamma files can not be judged before detailed level inelastic, $(n, p)$, and $(n, \alpha)$ reactions are introduced to the neutron files. Since the KIN method is well suited to treat all the listed reactions in ENDF/B-V without the need of any Q-value information, this method was used to produce the recommended kerma factors.

For decay heat calculations, the Mo-92 $(n, 2 n)$ cross section was taken from JENDL-2 and normalized to ENDF/B-V values. Abundance weighting was used to compute heating from the Mo-100 $(n, \gamma)$ reaction.

\section{2-Pb-nat}

In the elemental ENDF/B-V evaluation, the only reactions represented are elastic and inelastic scattering, $(n, 2 n),(n, 3 n)$, and radiative capture. Small $\left(n, n^{\prime} p\right),\left(n, n^{\prime} a\right),(n, p)$, and $(n, a)$ reaction cross sections are found in the JENDL-2 evaluations. With constant Q- values, the DEB kerma factors were found to be negative for 26 groups. With variable Q-values generated from JENDL-2 for $(n, 2 n)$ and $(n, 3 n)$ reactions, 4 more groups became negative. The negative kerma error can be attributed to the DEB kerma factor sensitivity to gamma production because there are no charged particle-producing reactions in the representation. Improvements in that area is expected to have a good impact on DEB kerma factor accuracy. On the other hand, the KIN treatment of the represented reactions is complete and independent of Q-values. The KIN kerma factors are therefore recommended.

For decay heat computations, JENDL-2 isotopic cross sections normalized to elemental ENDF/B-V are used. 


\section{III.3 Fissionable Materials}

Kerna factors were evaluated for 15 fissionable materials listed in section II.1. The kerma factors for these materials are dominated by the contribution of the fission reaction. This is particularly true for fissile materials where thermal neutrons are able to induce fission. The other reactions whose cross sections are given in ,ENDF/B-V are capture, elastic scattering, inelastic scattering, $(n, 2 n)$, and $(n, 3 n)$ reactions. For fertile materials, the fission effective threshold energy is lower than that for the $(n, 2 n)$ and $(n, 3 n)$ reactions. Below the fission threshold, the kerma factor will be dominated by capture and scattering interactions. Since capture and scattering treatment is equivalent for both DEB and KIN general approaches, and the fission kerma factor computation method is not a kinematic one, it was appropriate to apply the DEB method to all fissionable materials. It should be noted however, that gamma files are not provided in the ENDF/B-V data for some materials. These materials include Pa-233, U-234, U-236, Np237, and Pu-238. In this case, the computed DEB kerma factors are larger than the KIN ones because they include the unwanted component of gamma energy from fission and other reactions. The KIN results are therefore recommended for the KAOS/LIB-V library for these materials. Both DEB and KIN kerma factors are saved for all the fissionable materials in the KAOS/LIB-V library.

The radioactive decay contribution to the local energy deposition was calculated from the delayed beta component of the fission energy release given in ENDF/B-V file 1. The charged particle decay energy from radioactive nuclei formed by non-fission reactions was also added. Similar treatment was given to gamma decay heat component. 


\section{GRAPHICAL REPRESENTATION OF KAOS/LIB-V RESPONSE FUNCTIONS}

In this section, graphs of all nuclear response functions are presented for all the materials included in the KAOS/LIB-V library.

Only the recommended set of prompt and total kerma factors are plotted. The log-scale graphs show the low incident neutron energy part in more detail. The linear scale graphs show the high incident neutron energy part more clearly. 


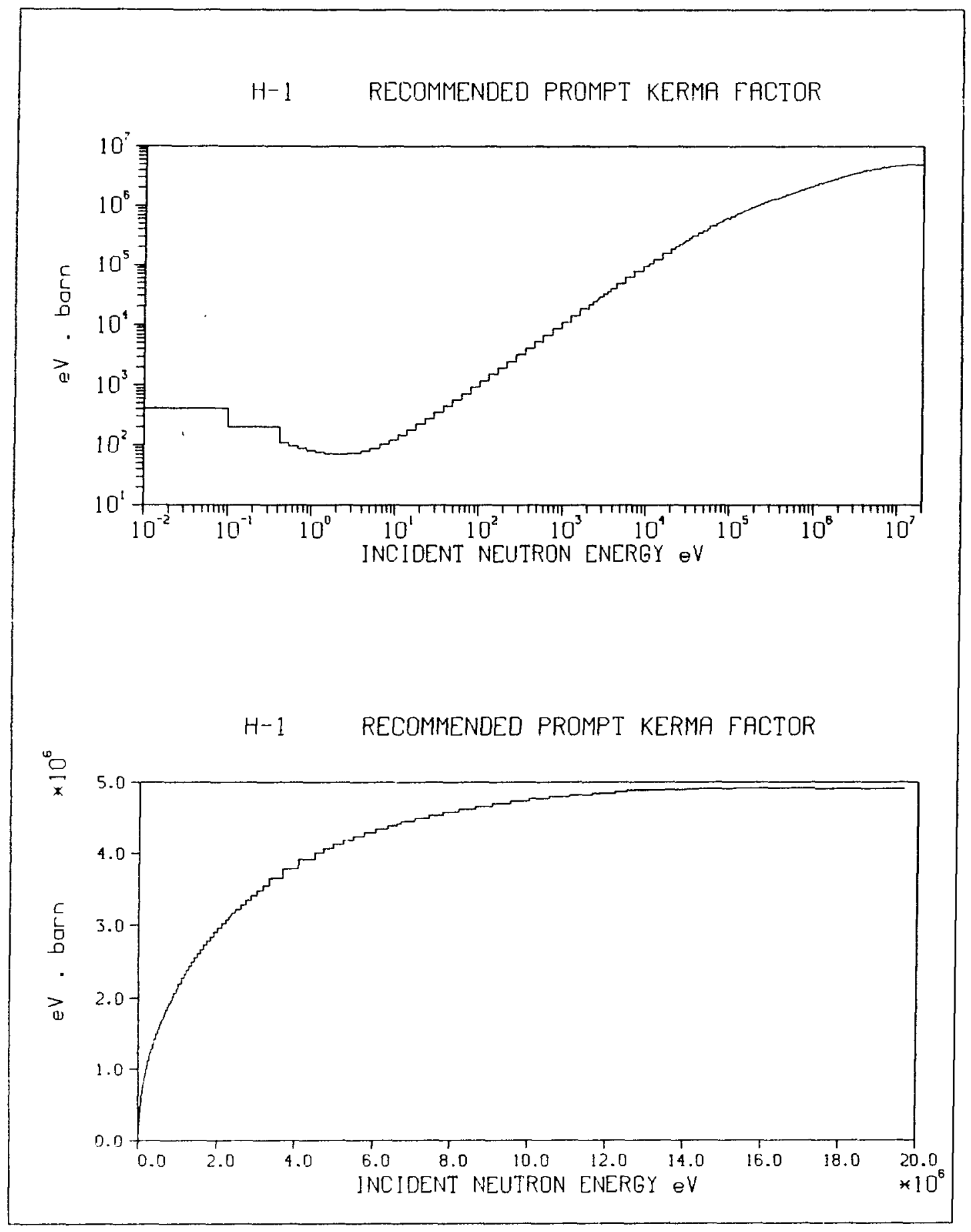




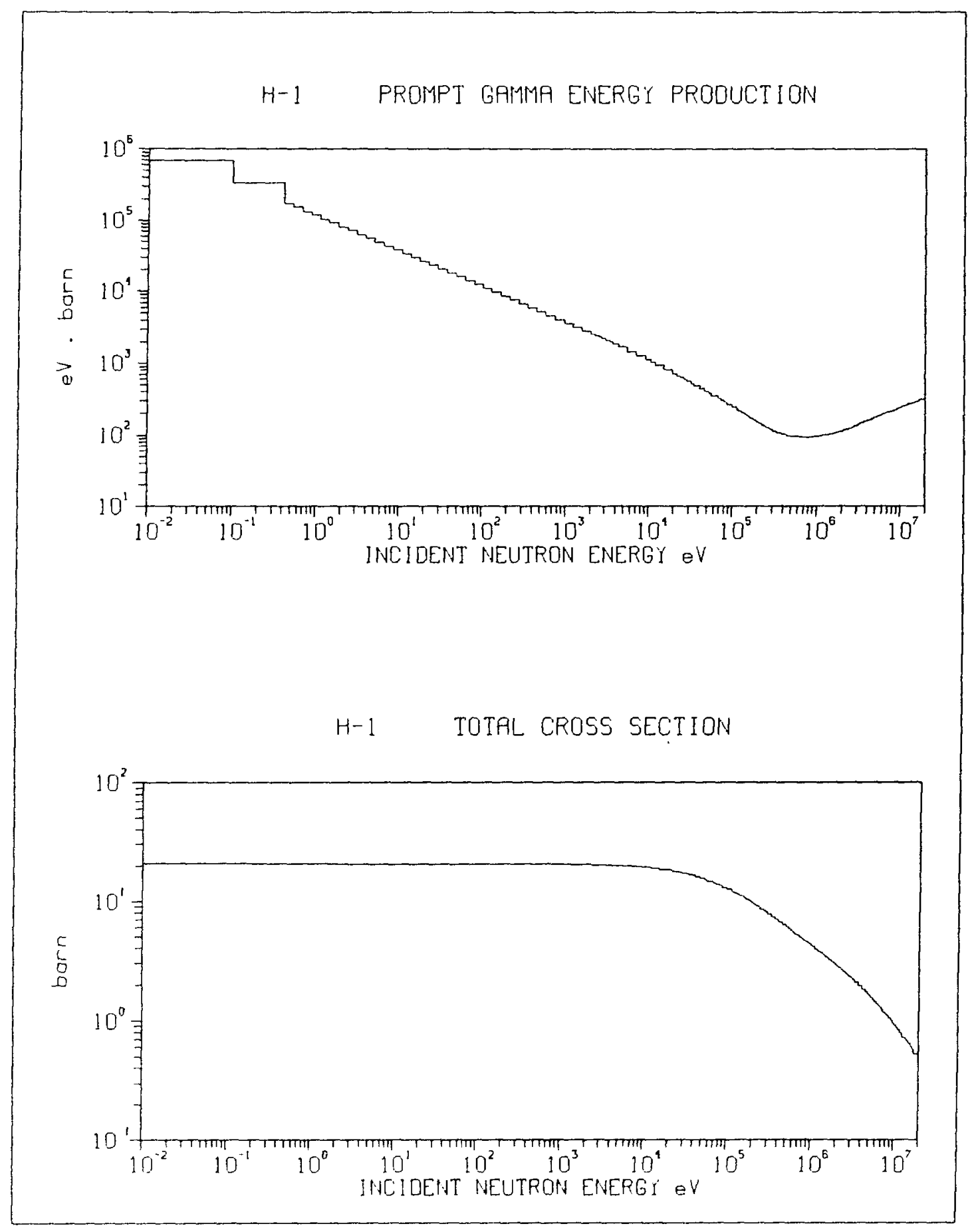




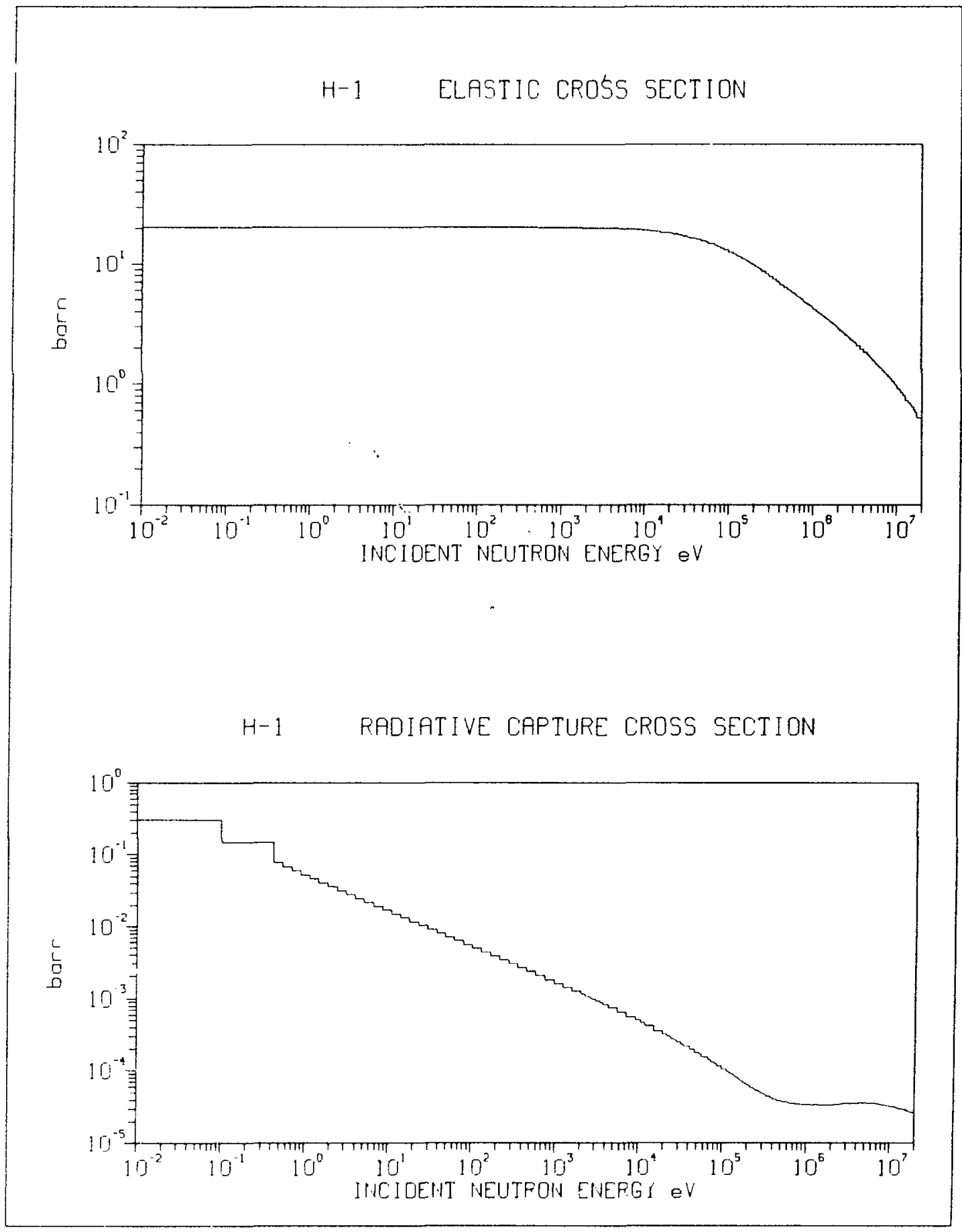




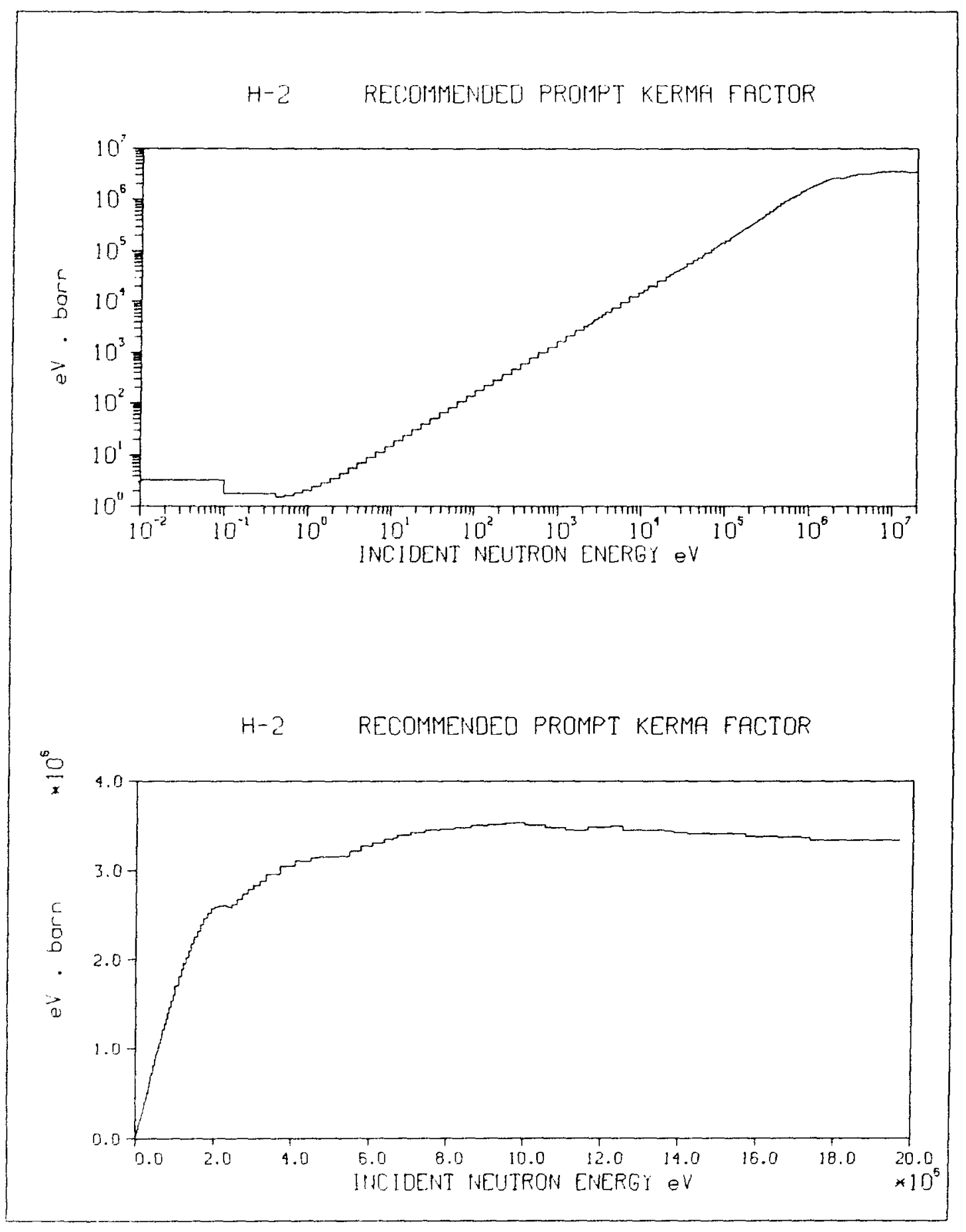




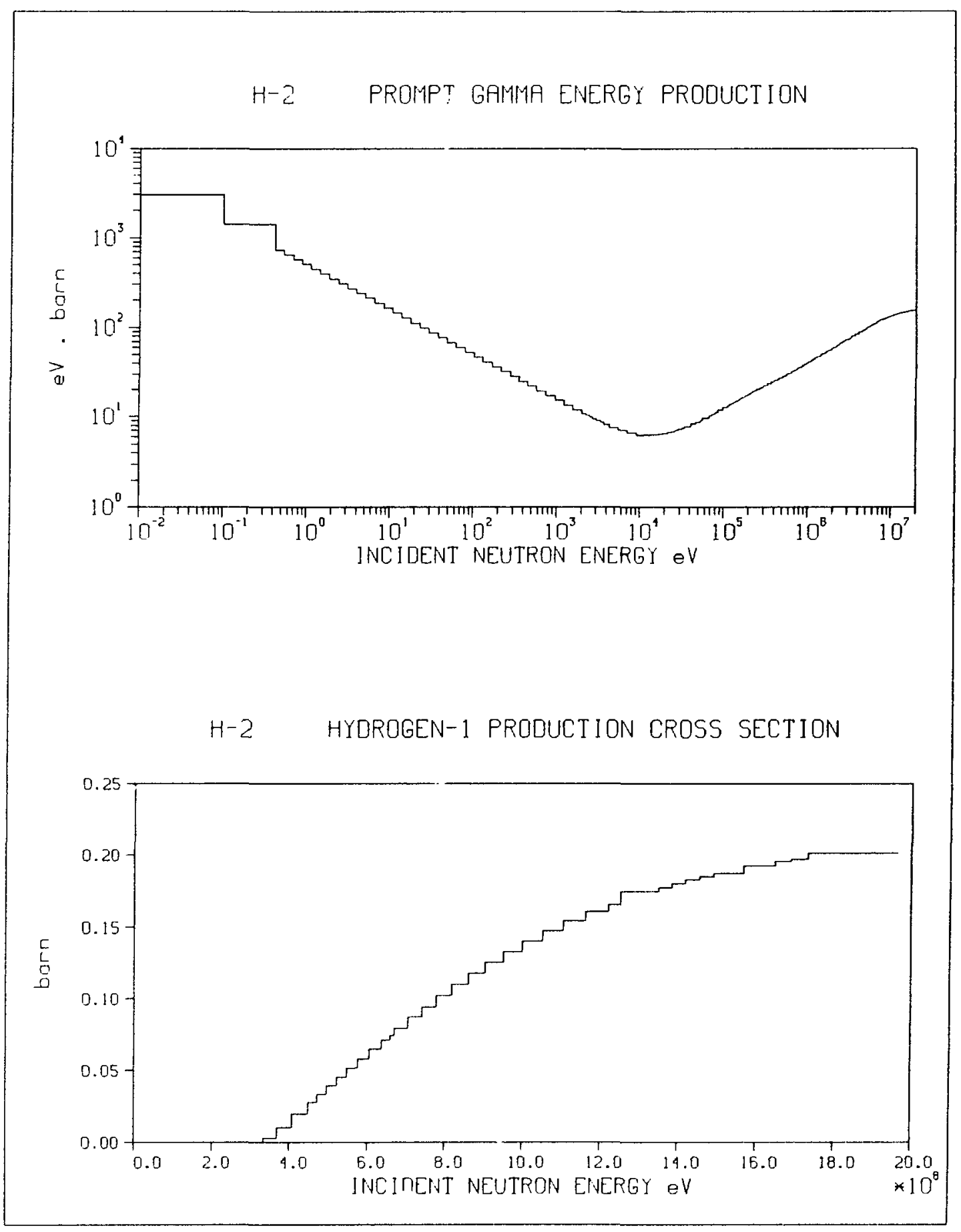




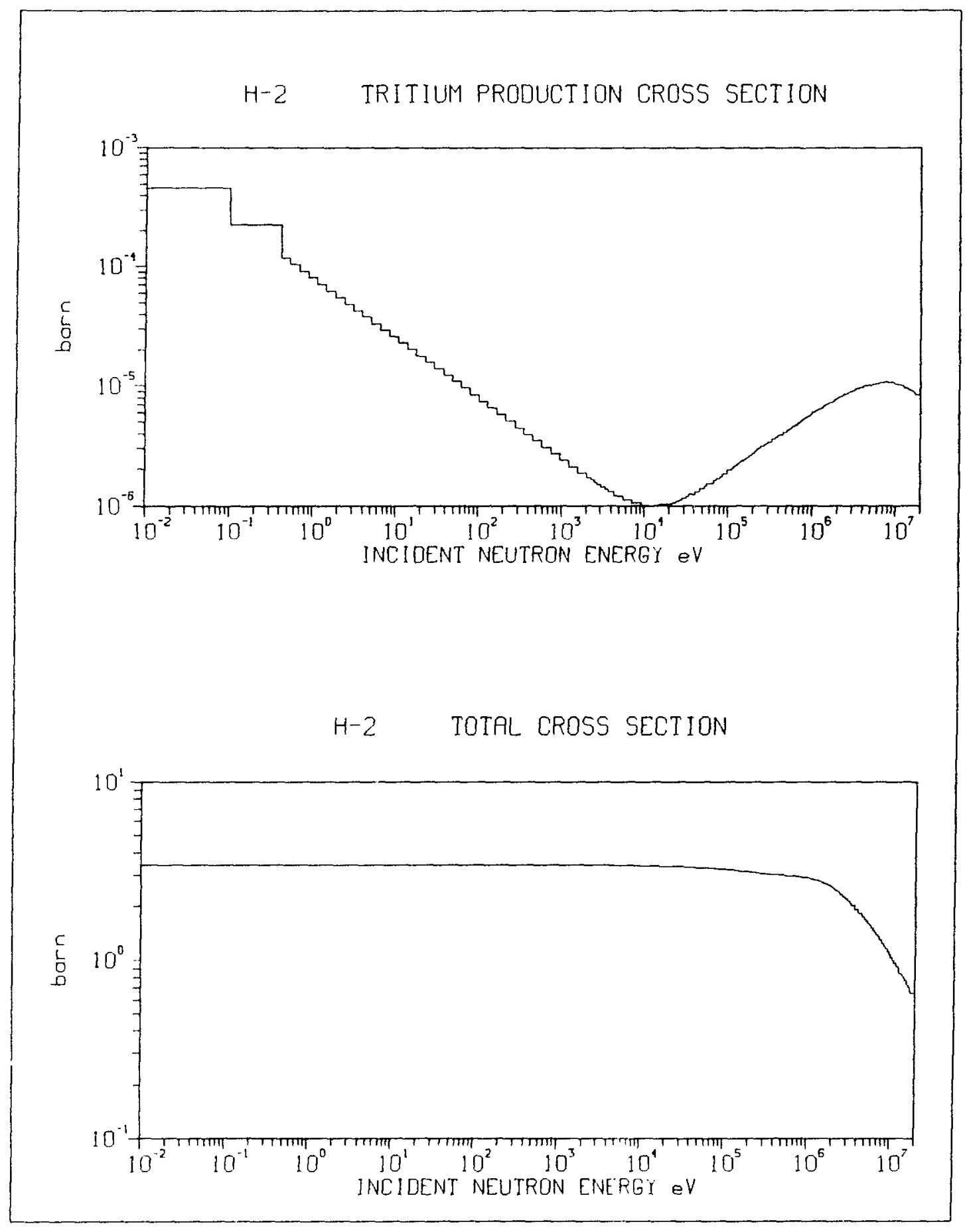




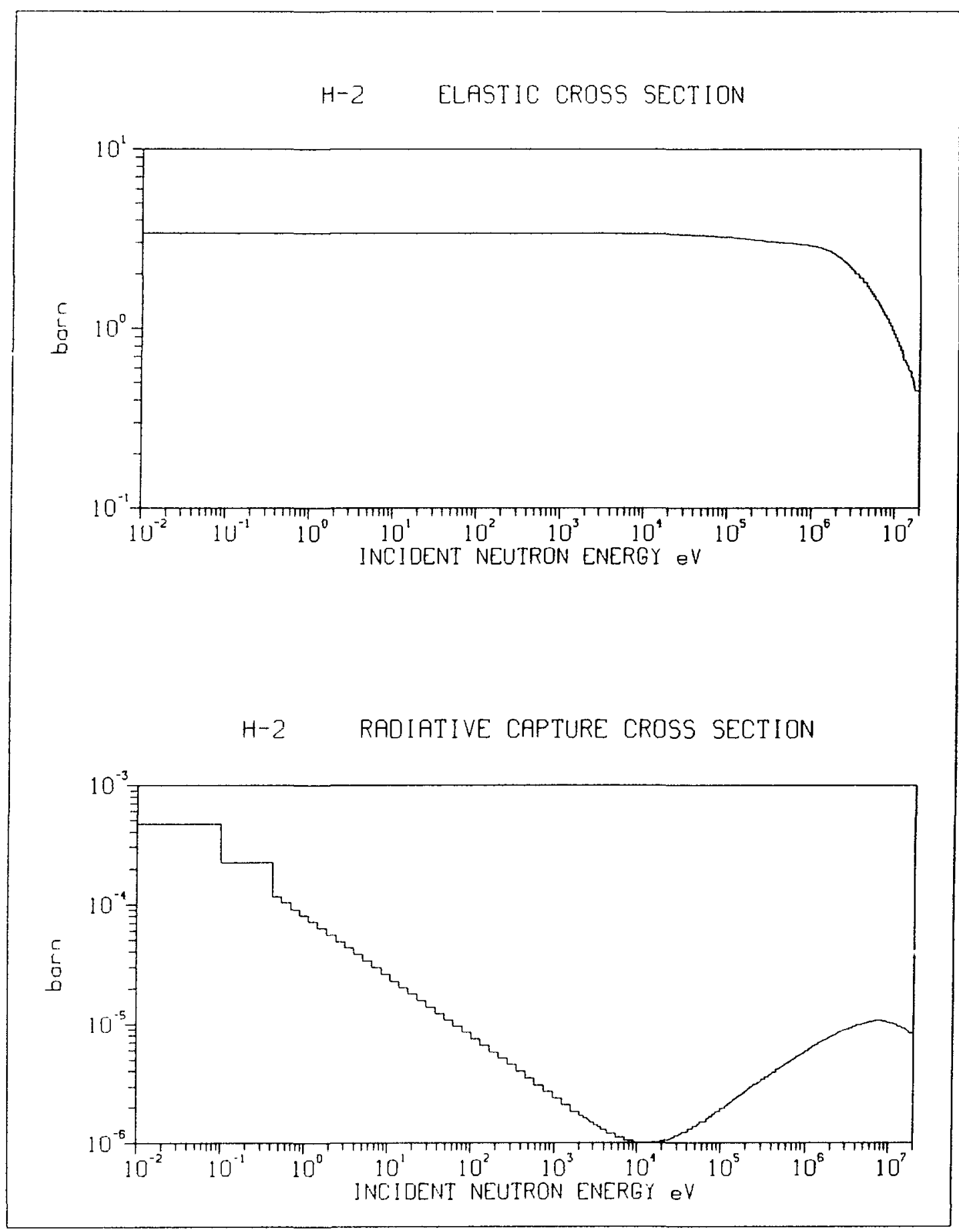




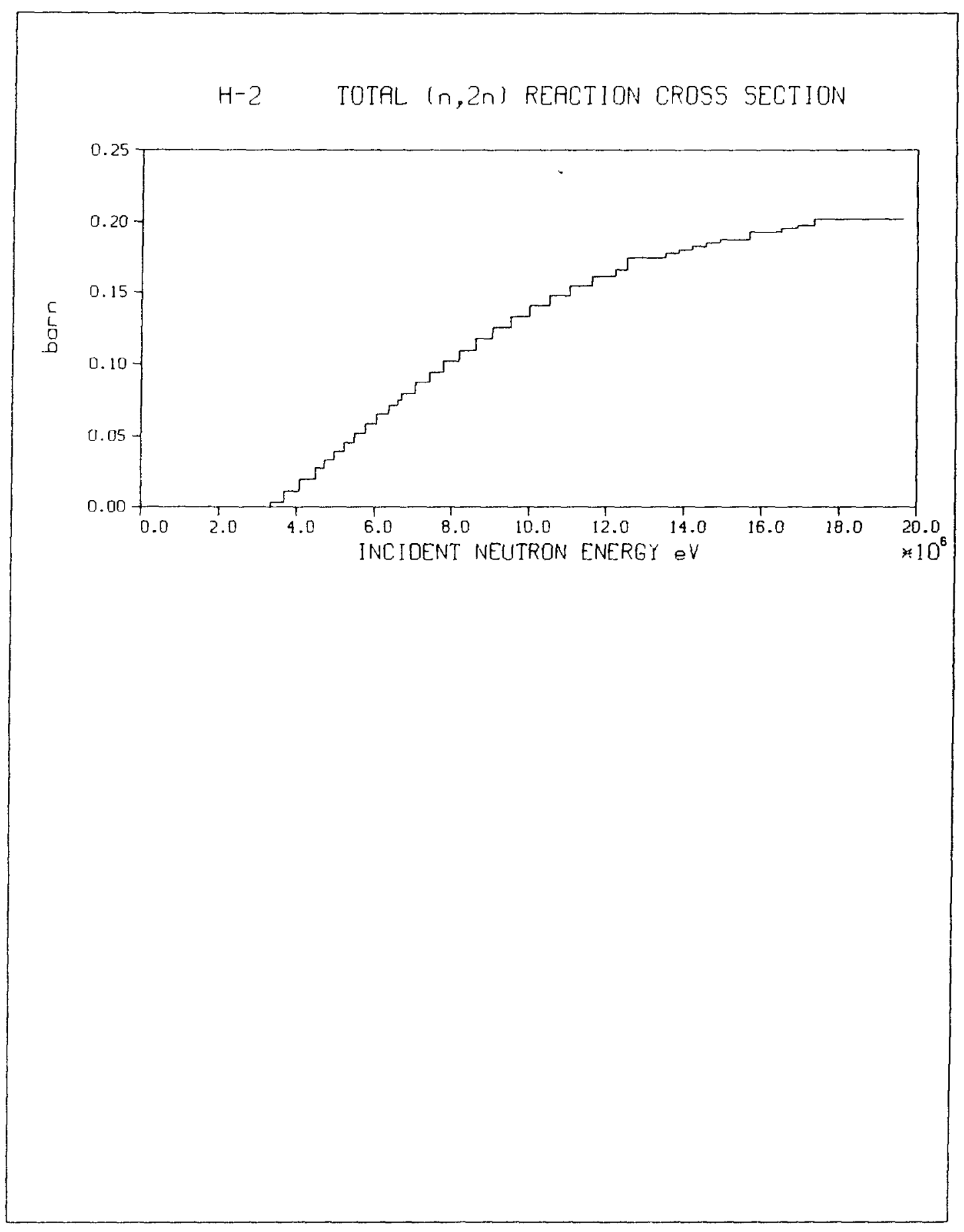




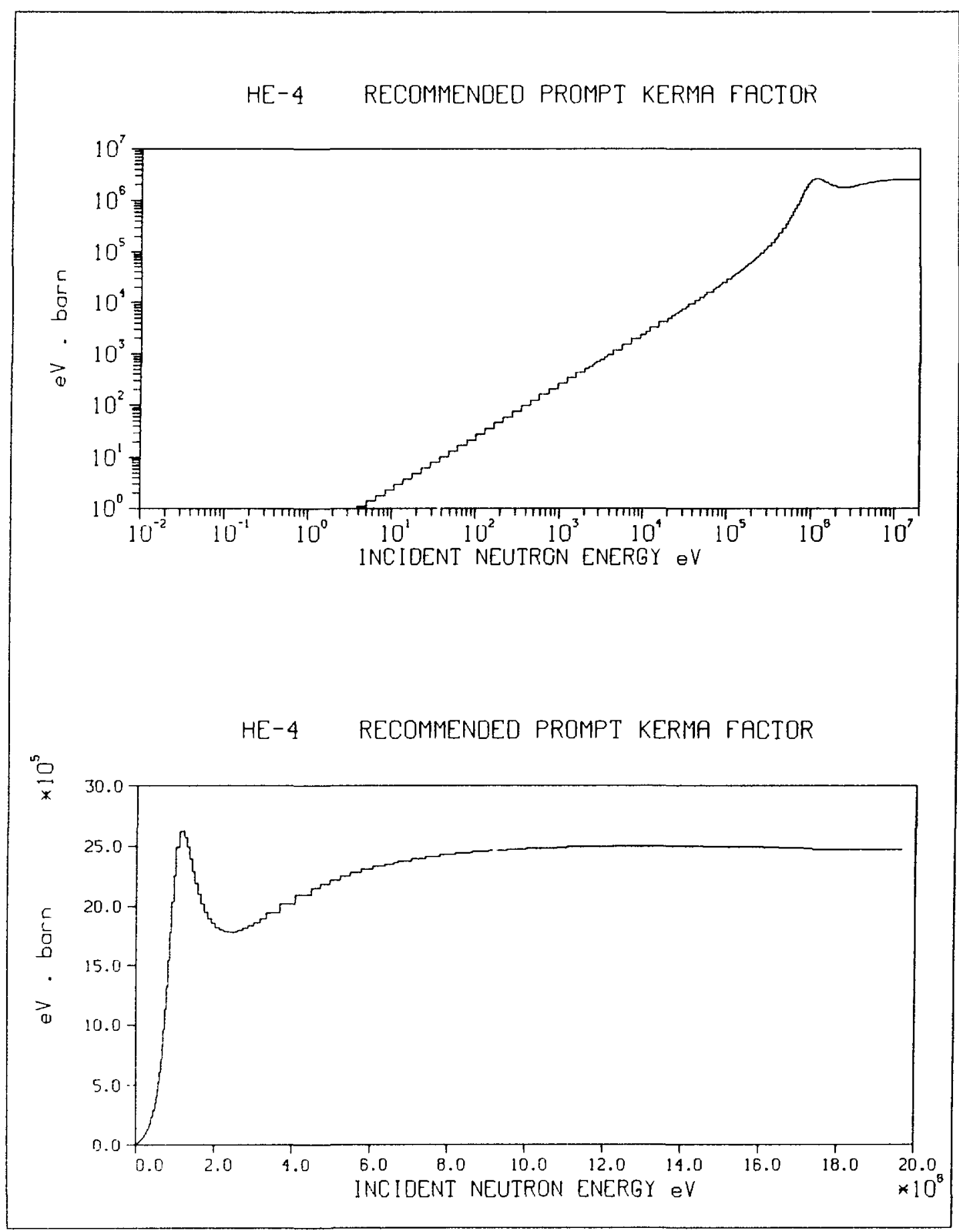


HE-4 TOTAL CROSS SECTION

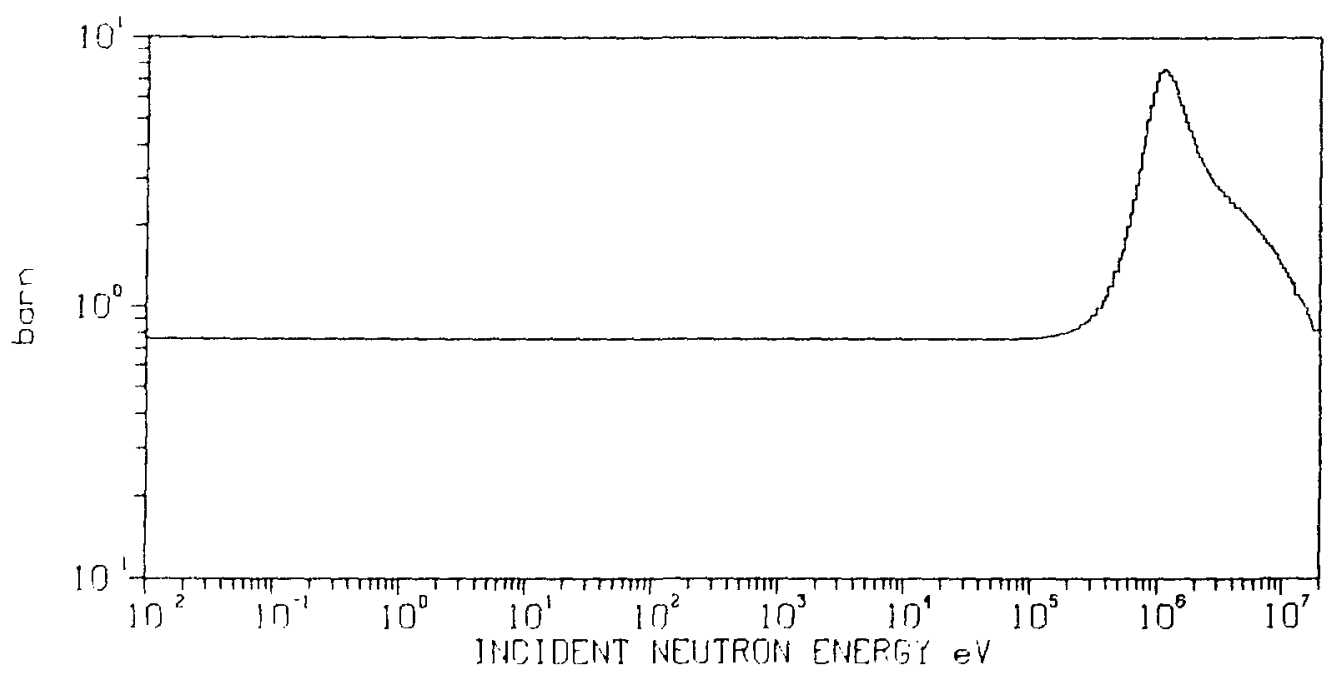

HE-4 ELASTIC CROSS SECTION

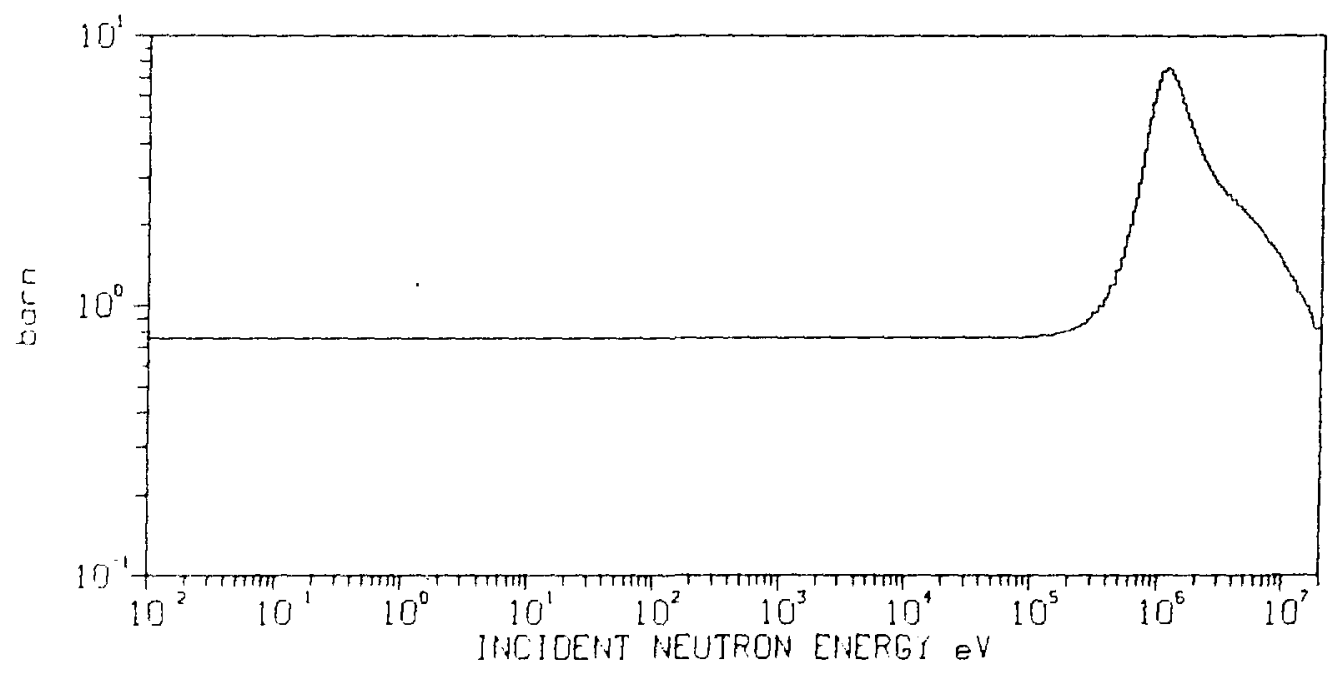




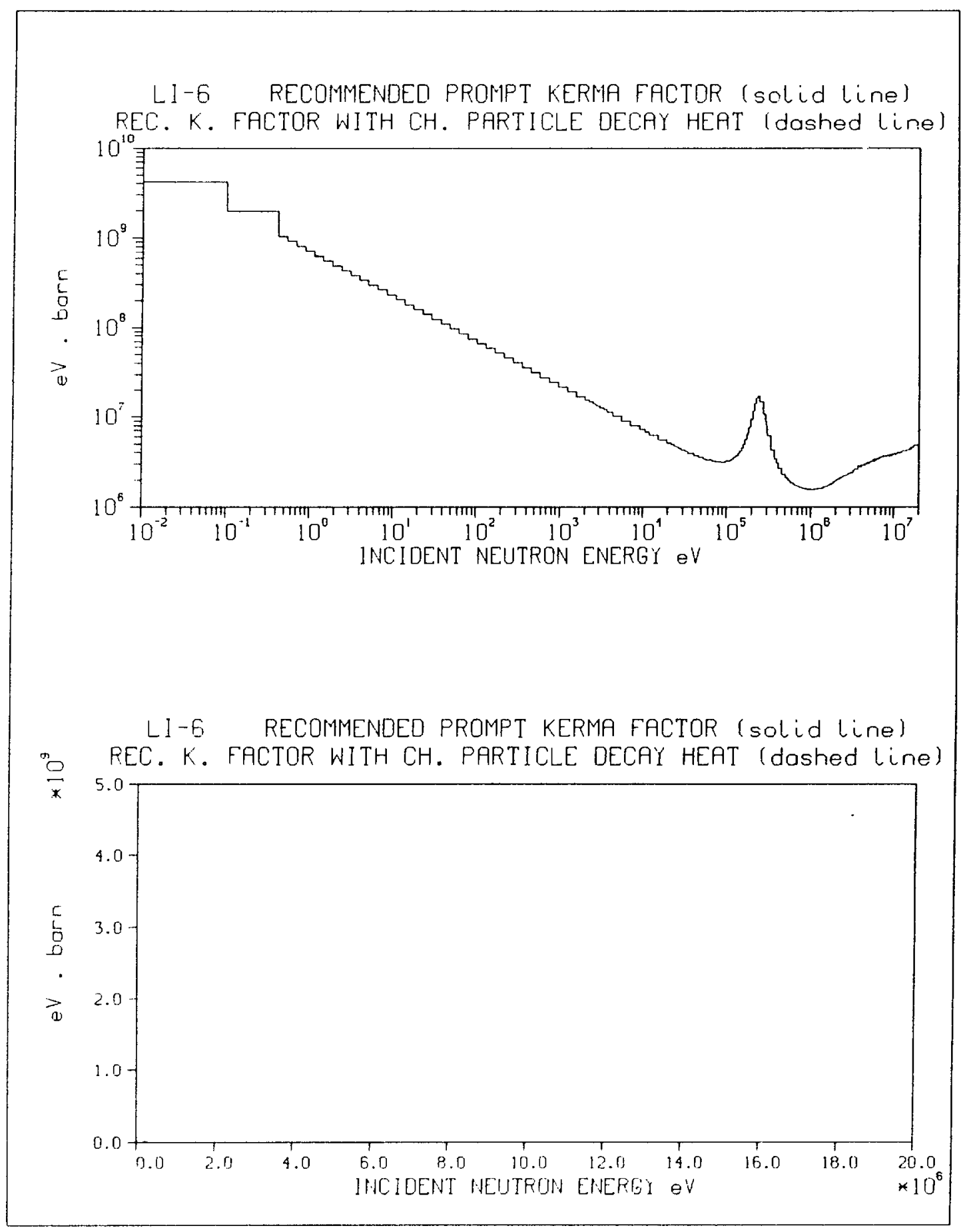




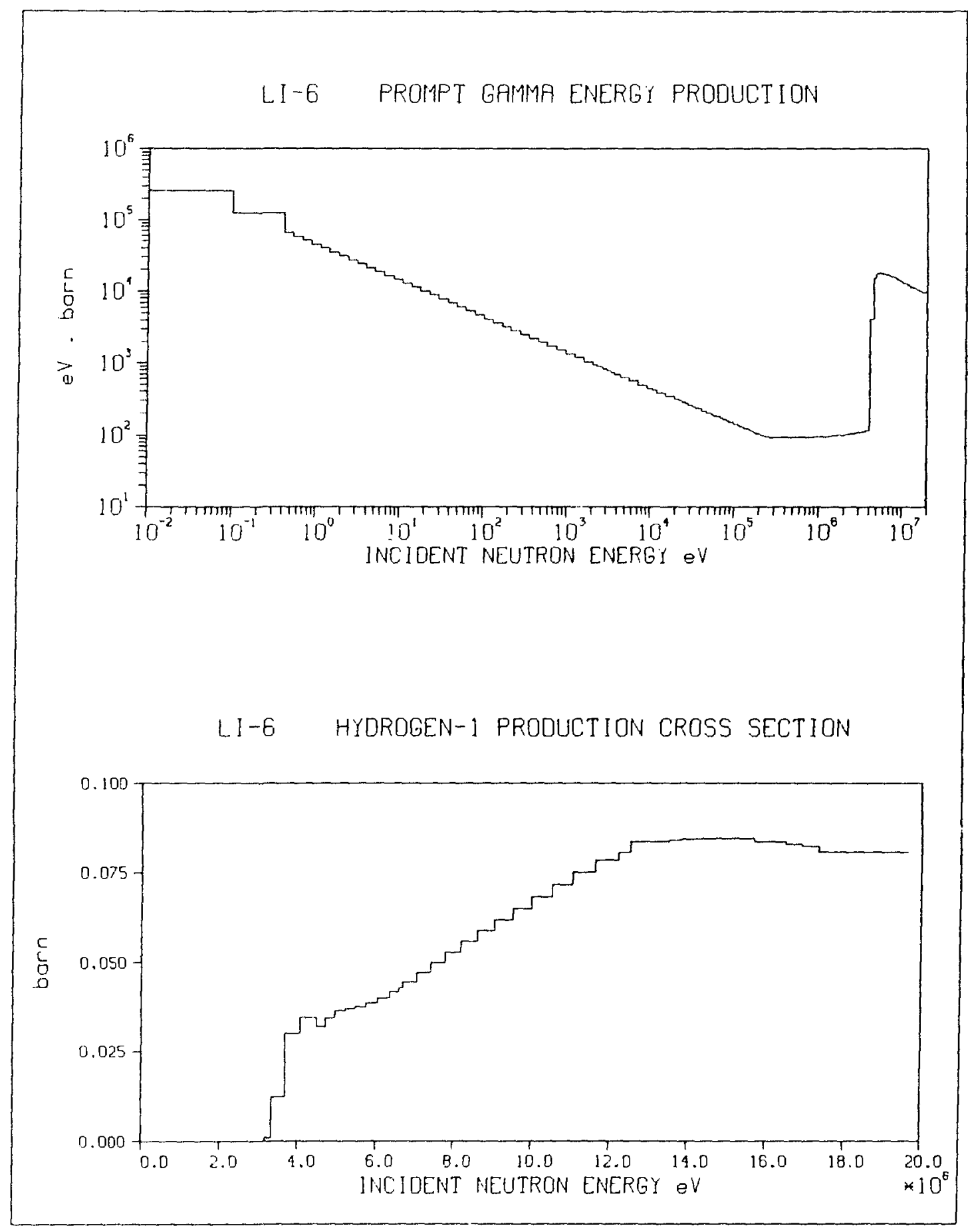




\section{LI-6 DEUTERIUM PRODUCTION CROSS SECTION}

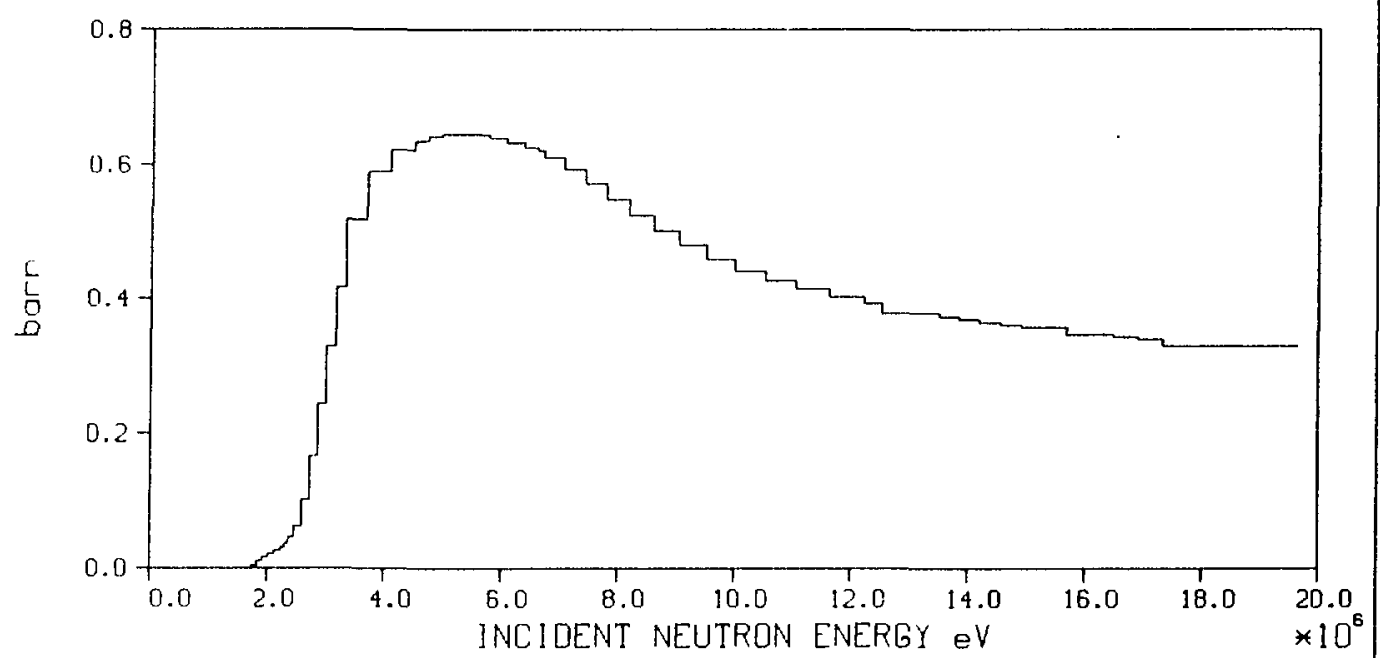

LI-6 TRITIUM PRODUCTION CROSS SECTION

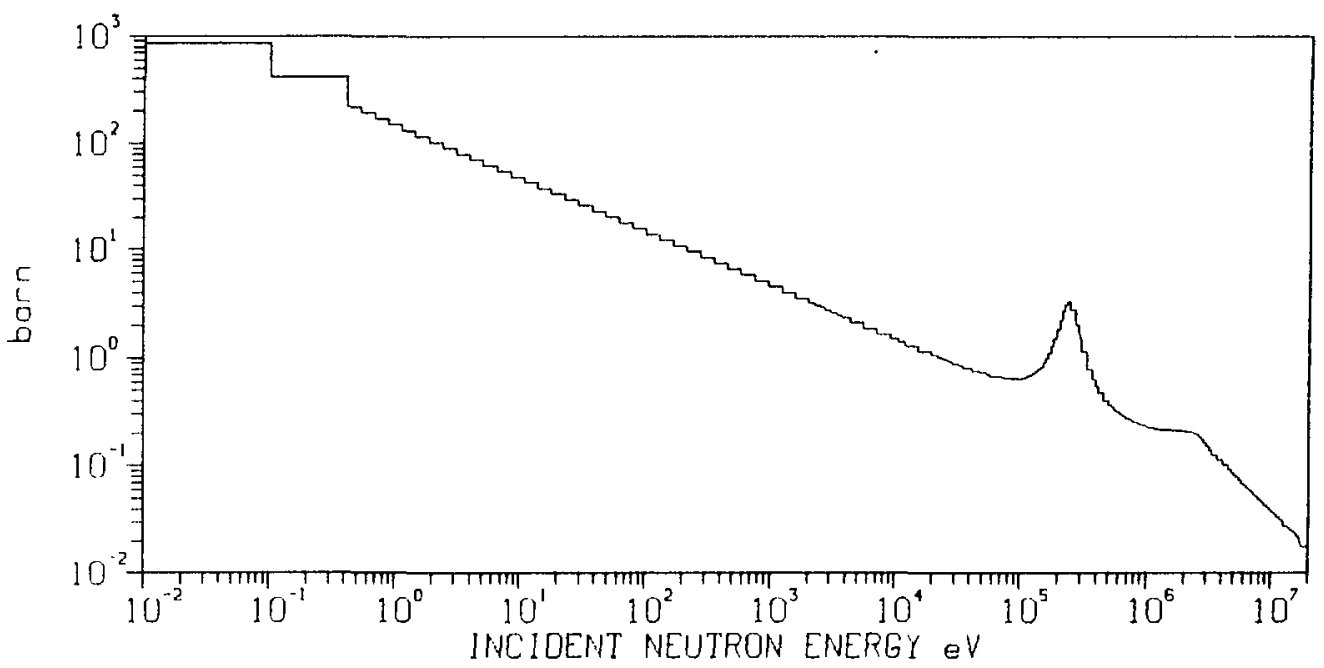




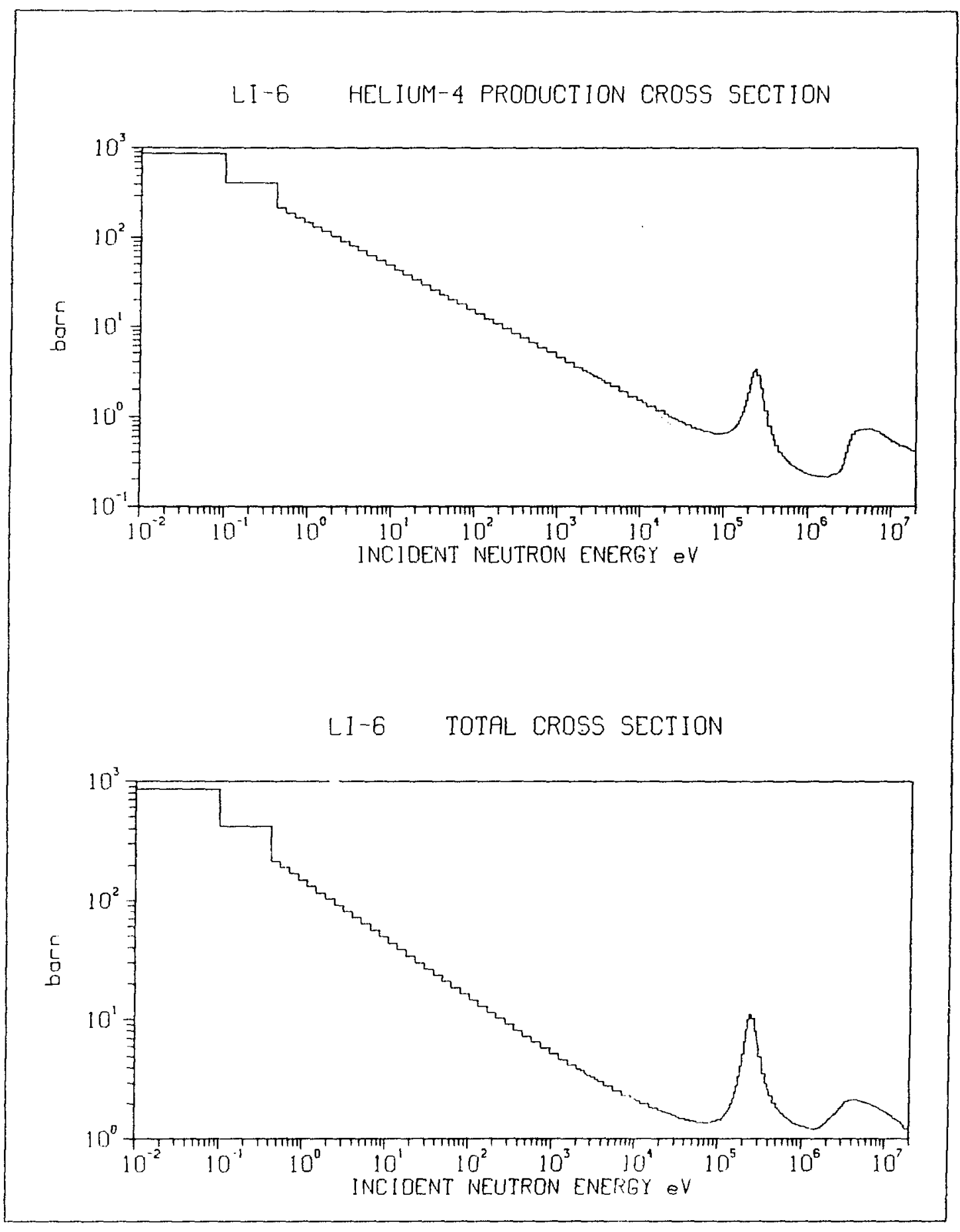




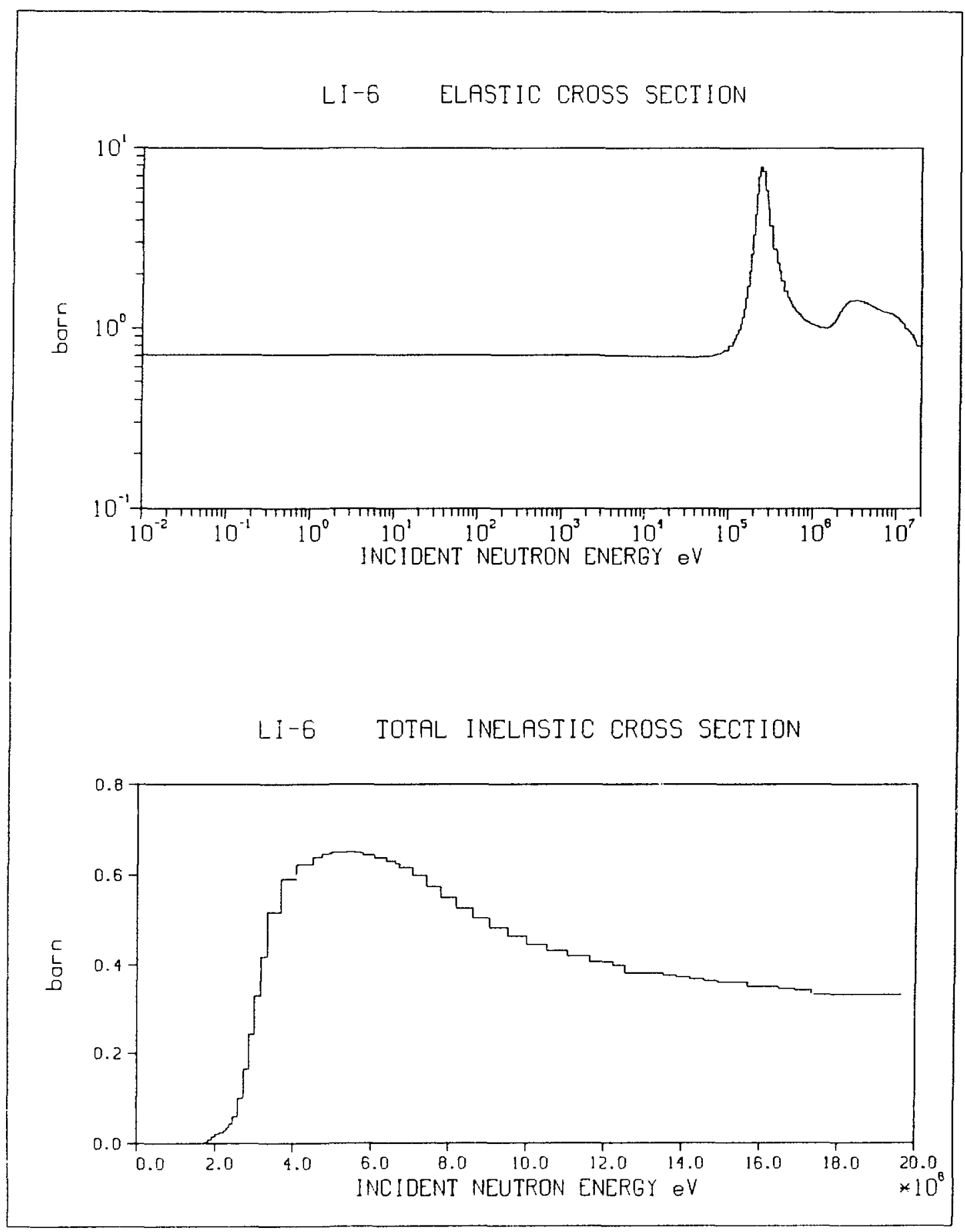




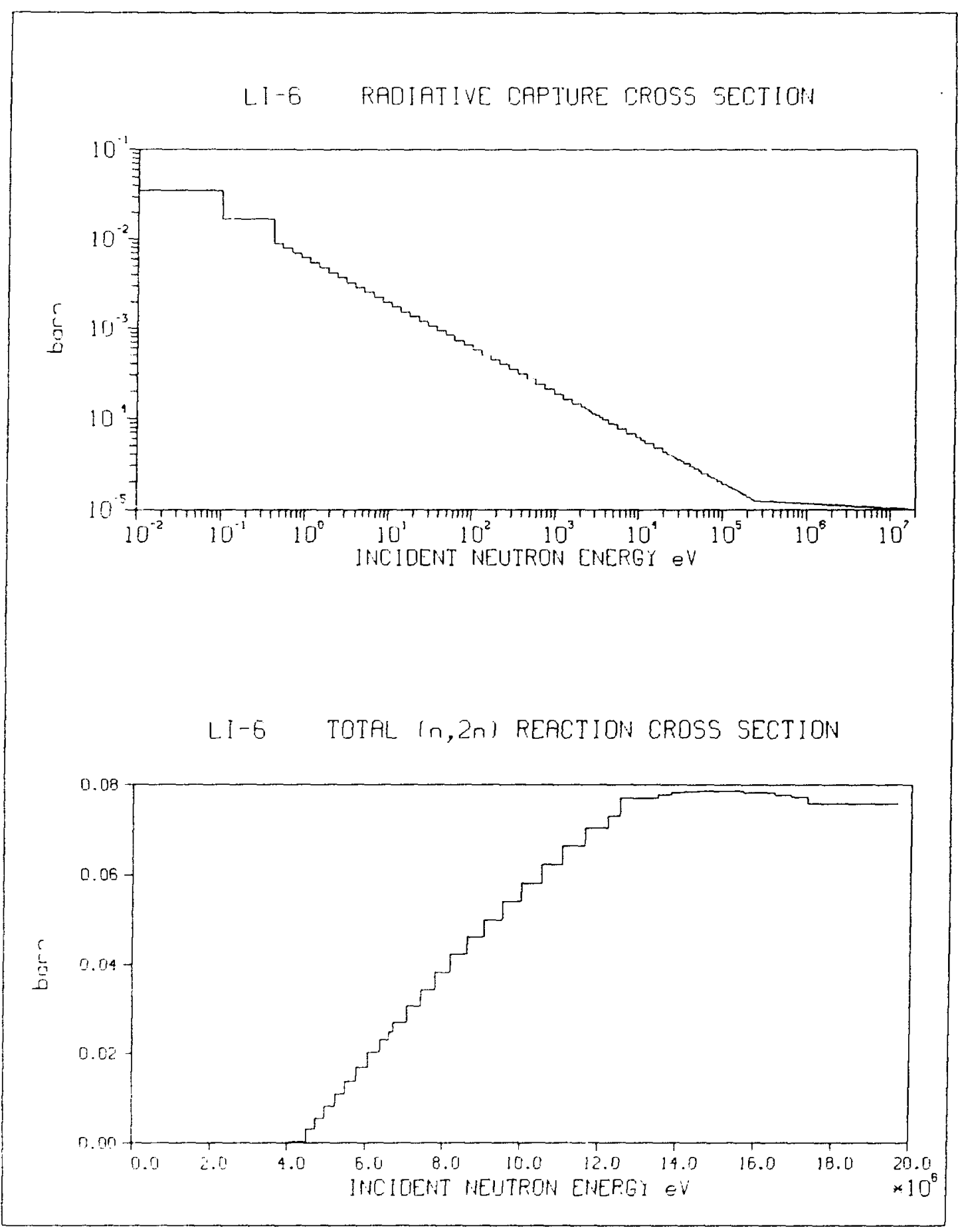




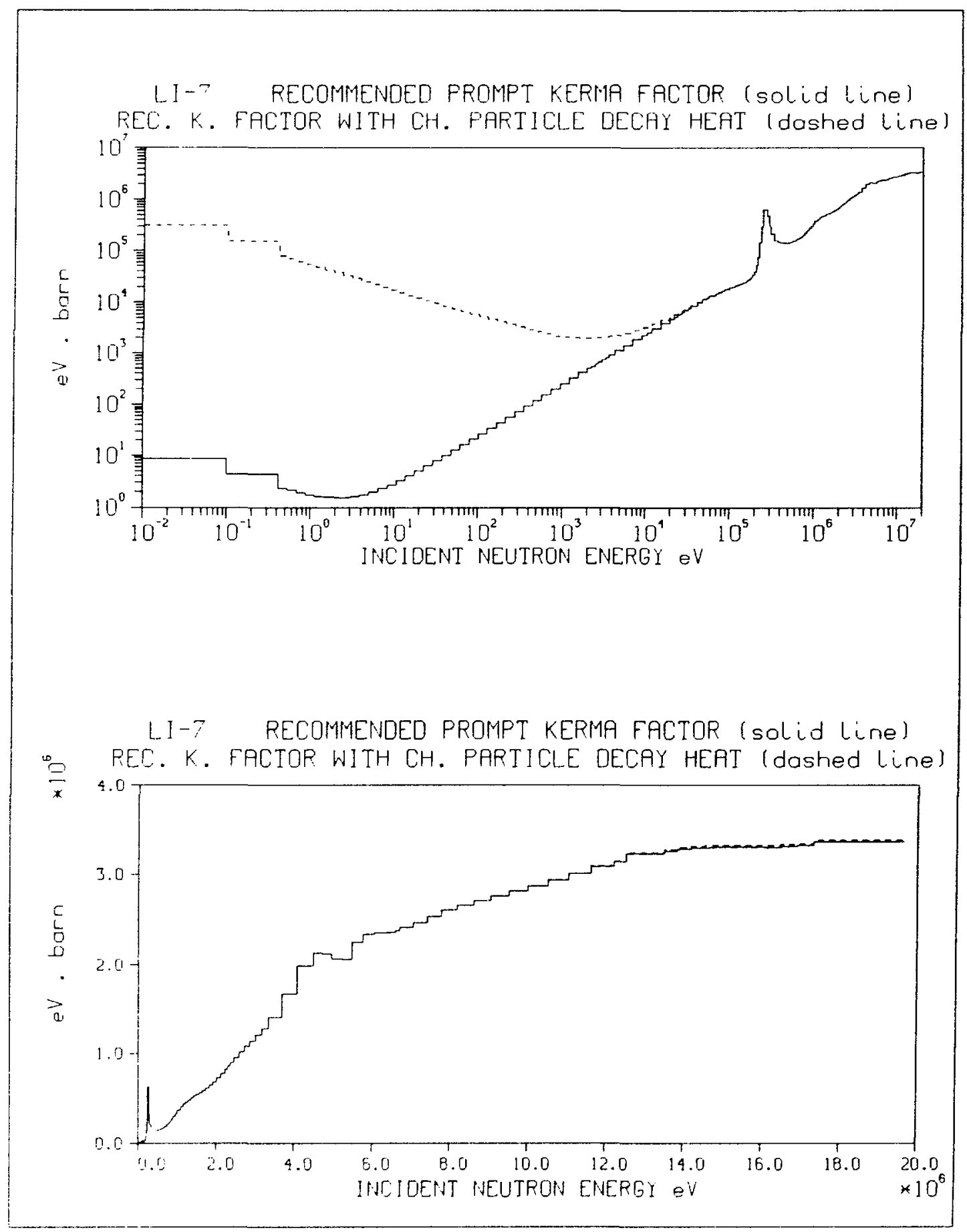




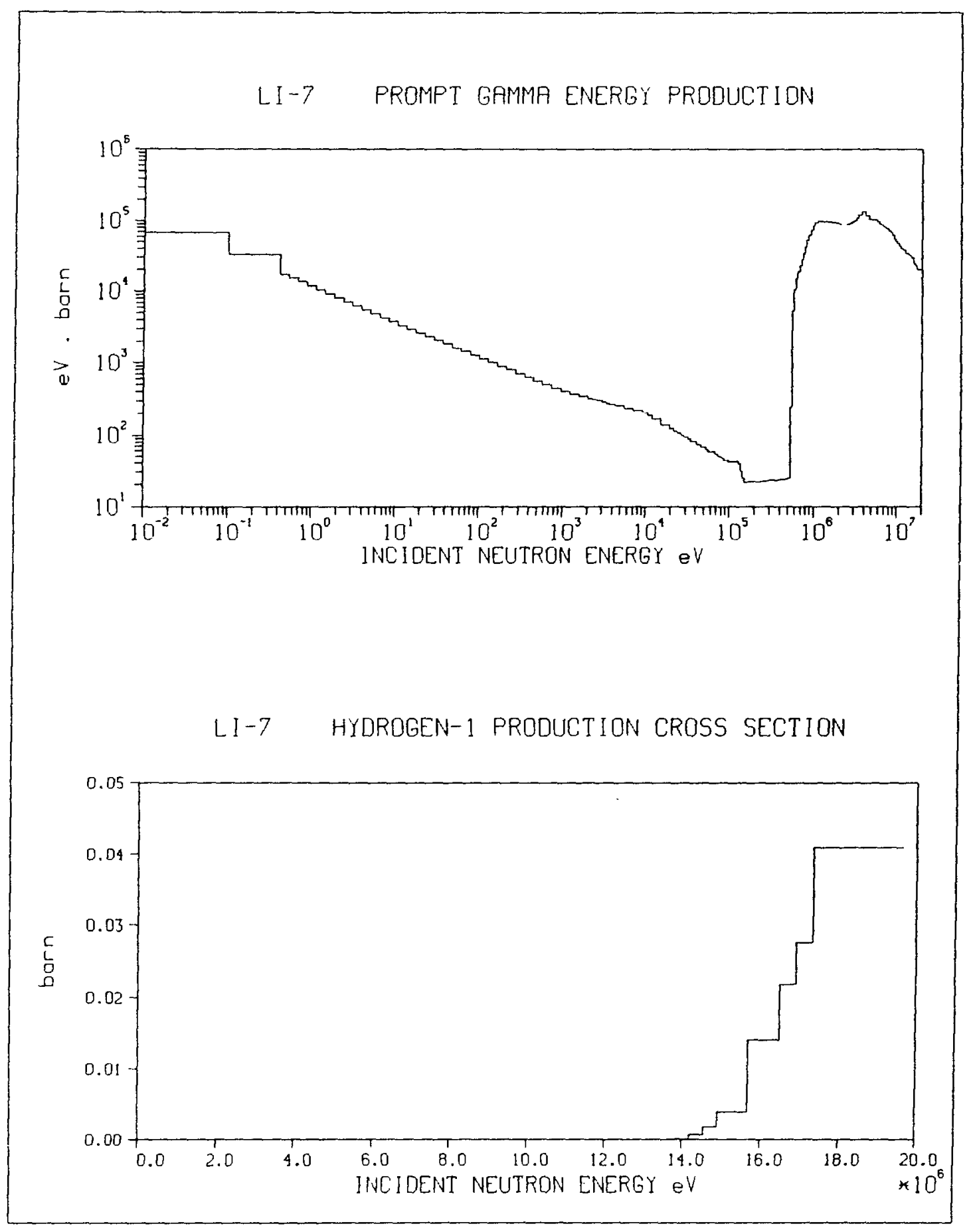




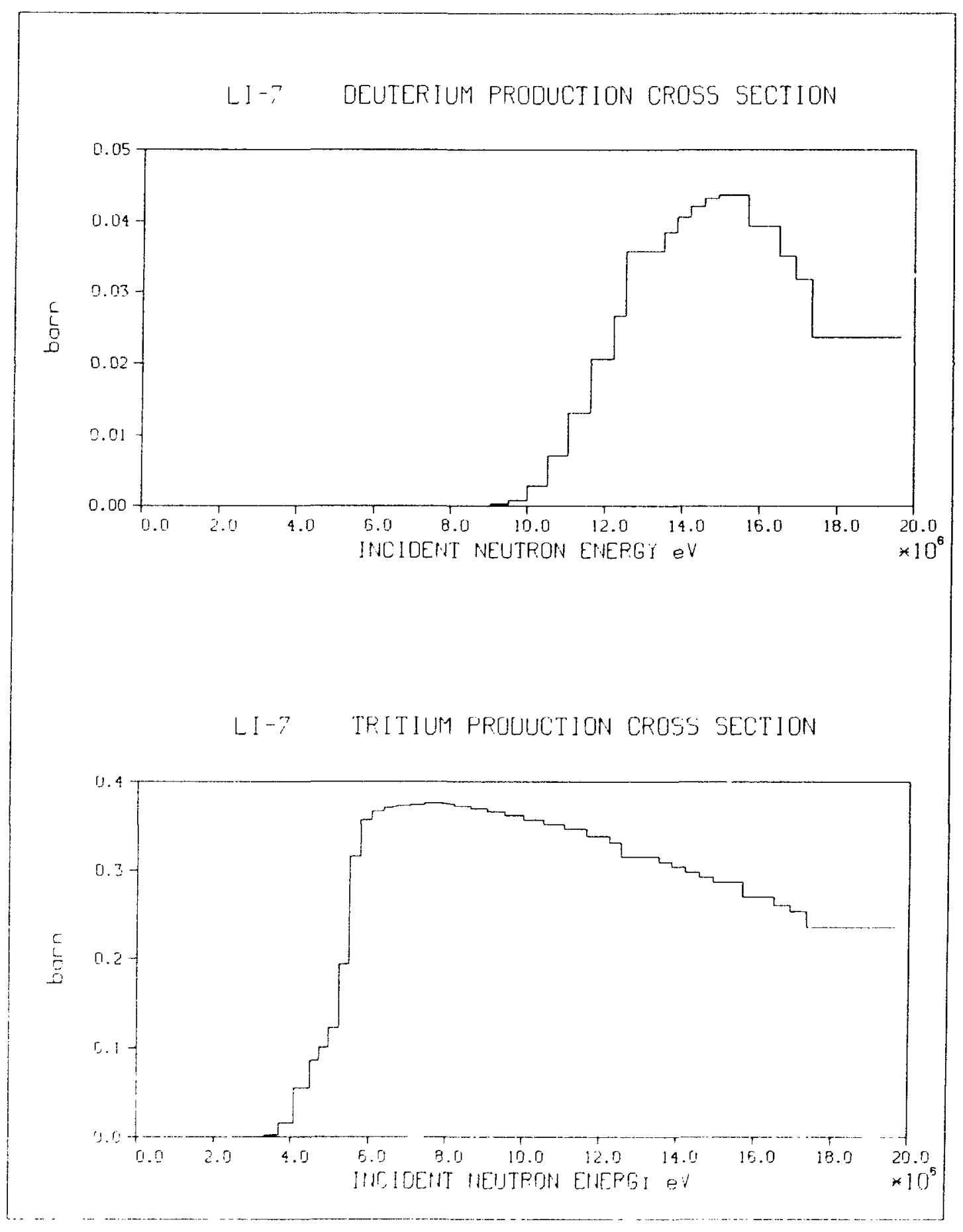




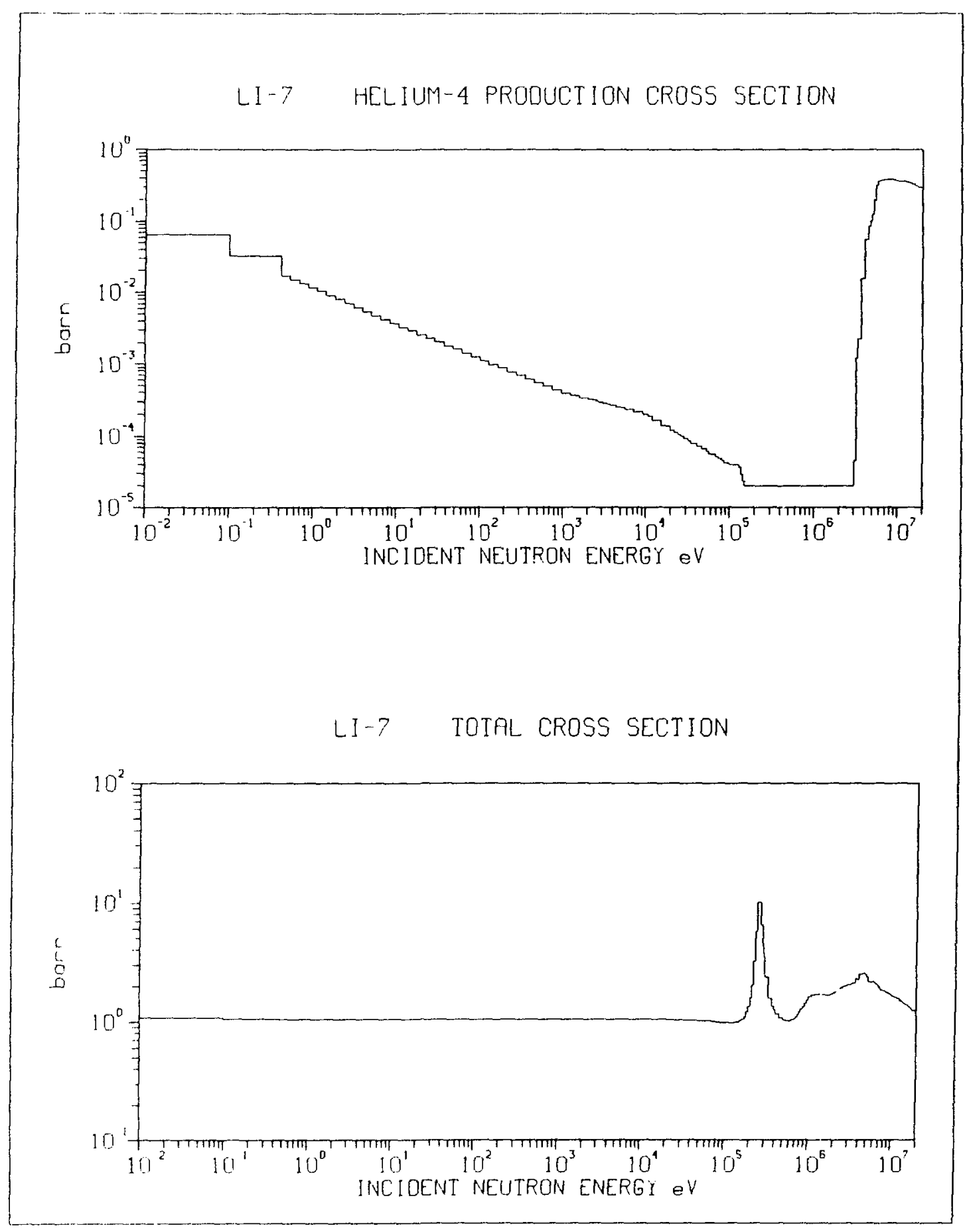


LIT ELASTIC CROSS SECTION

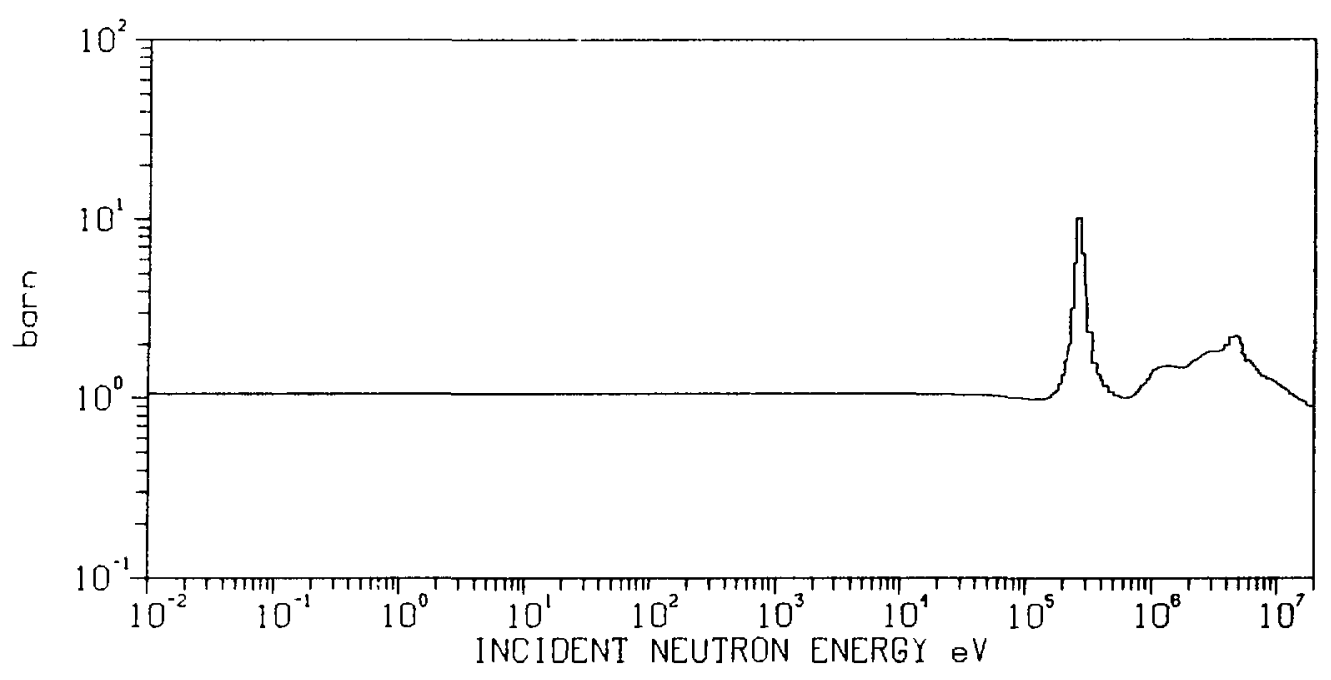

LI-7 TOTRL INELASTIC CROSS SECTION

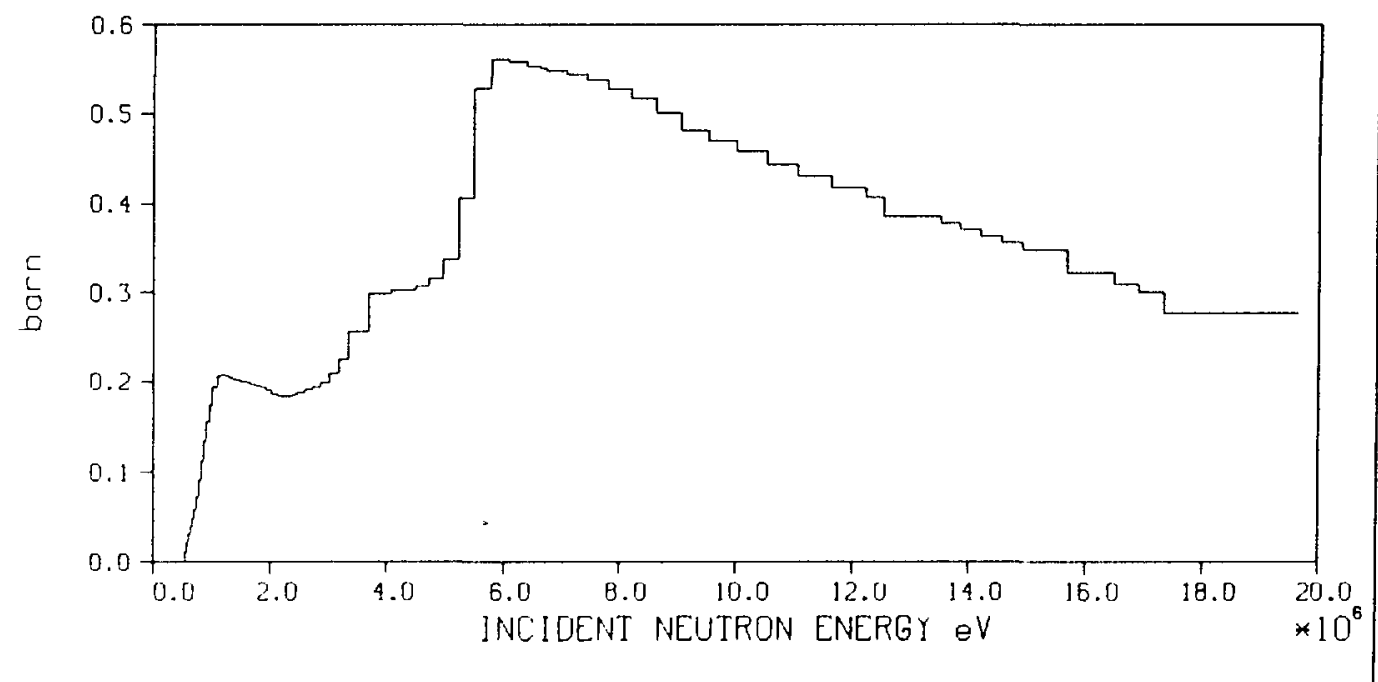




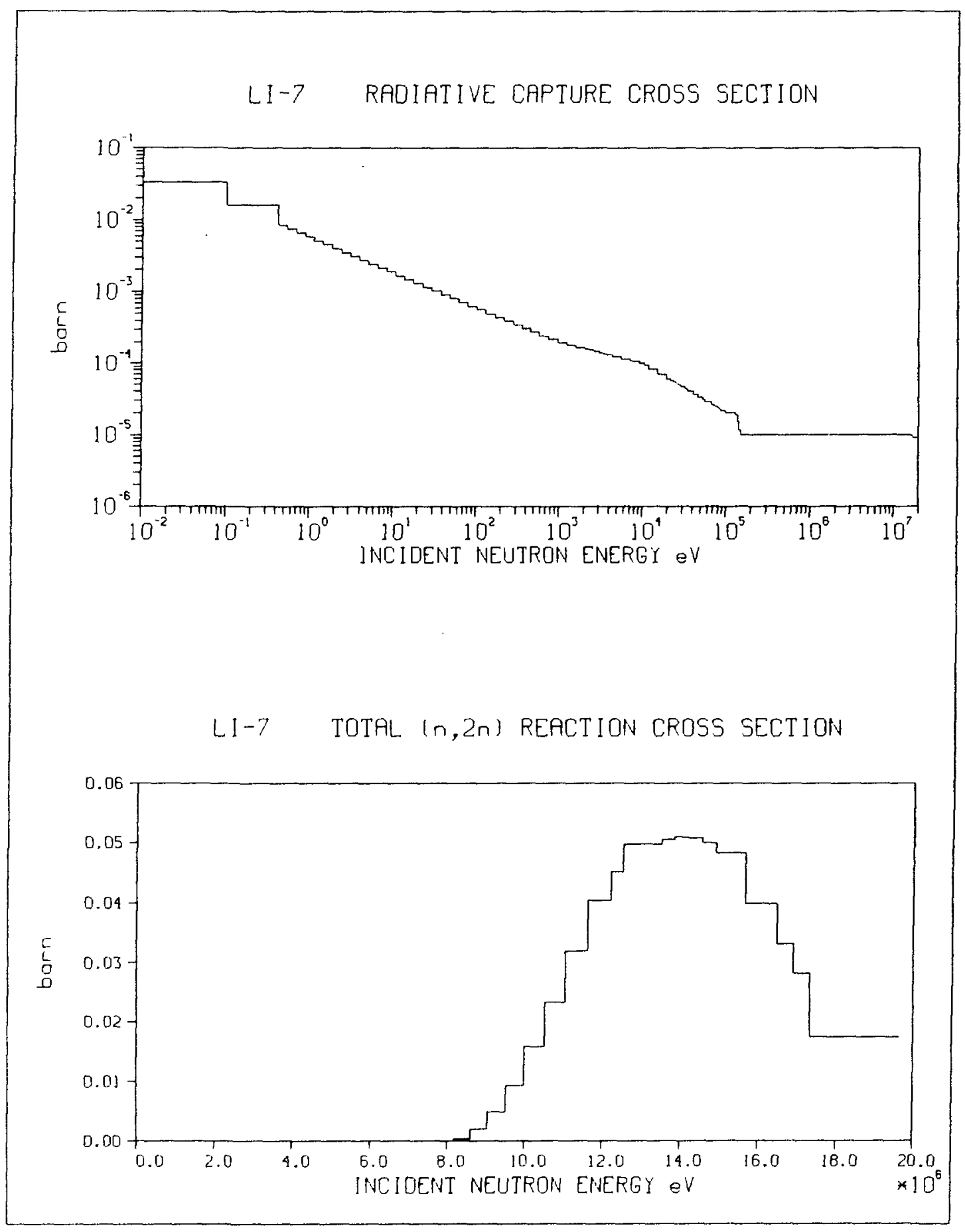




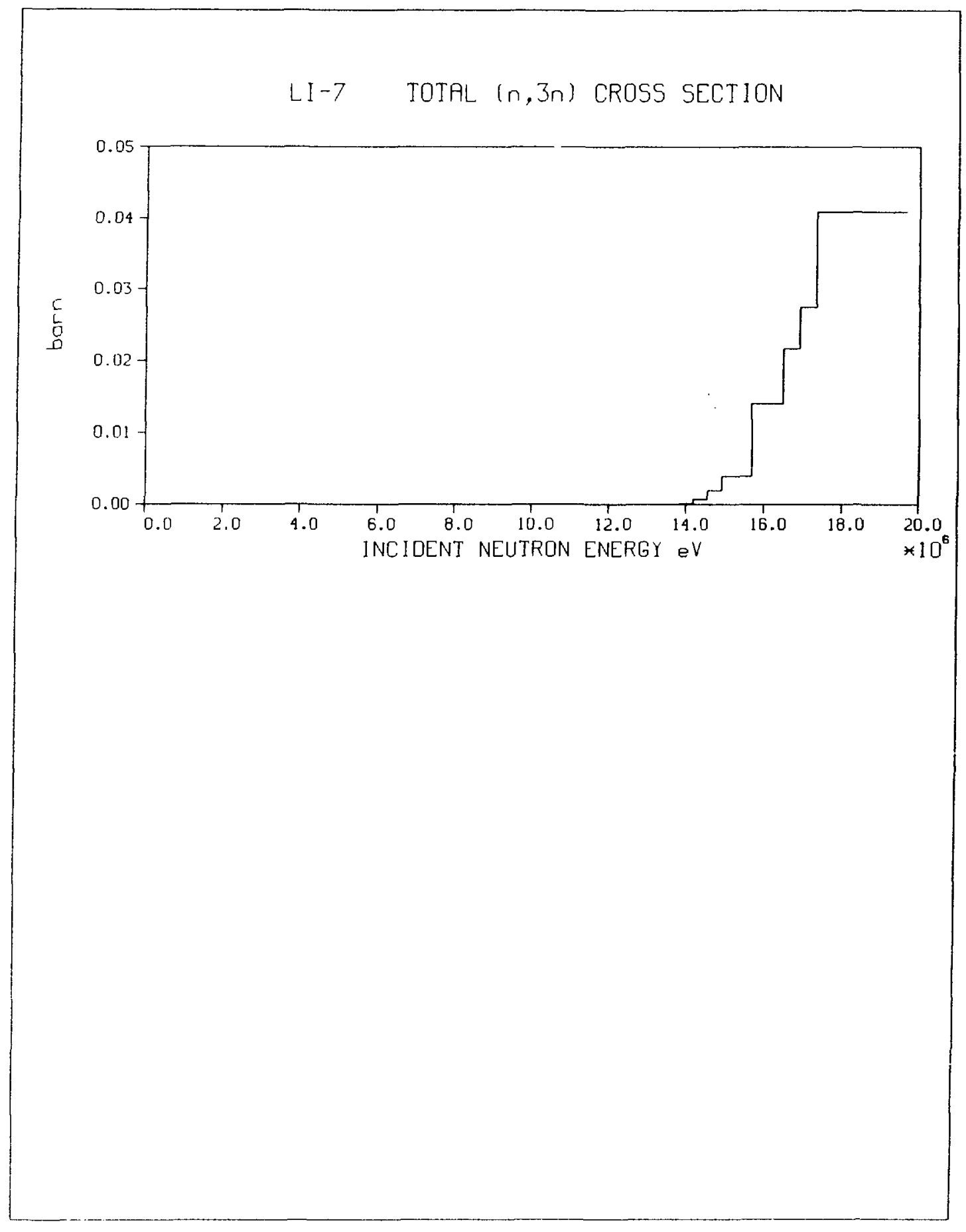




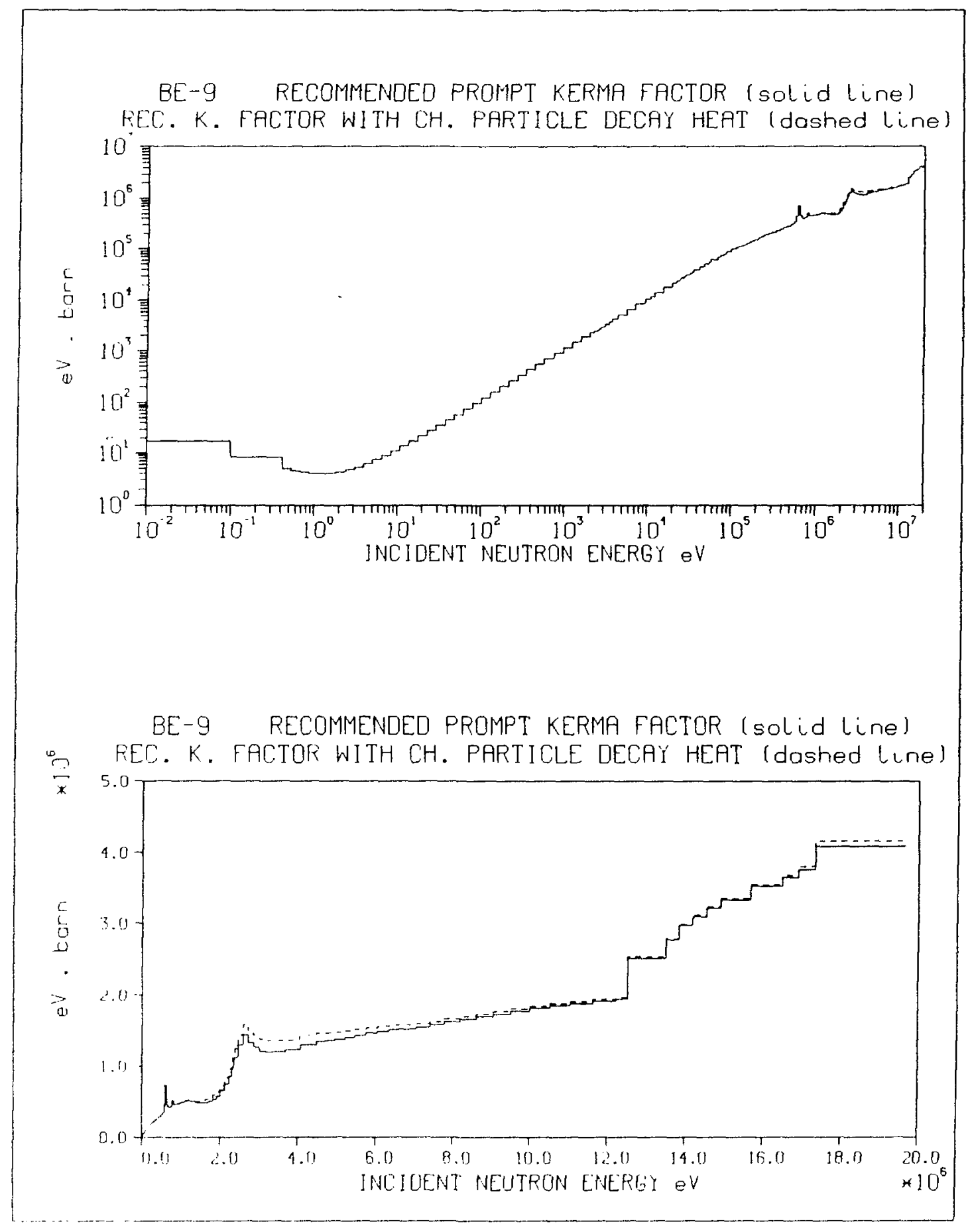




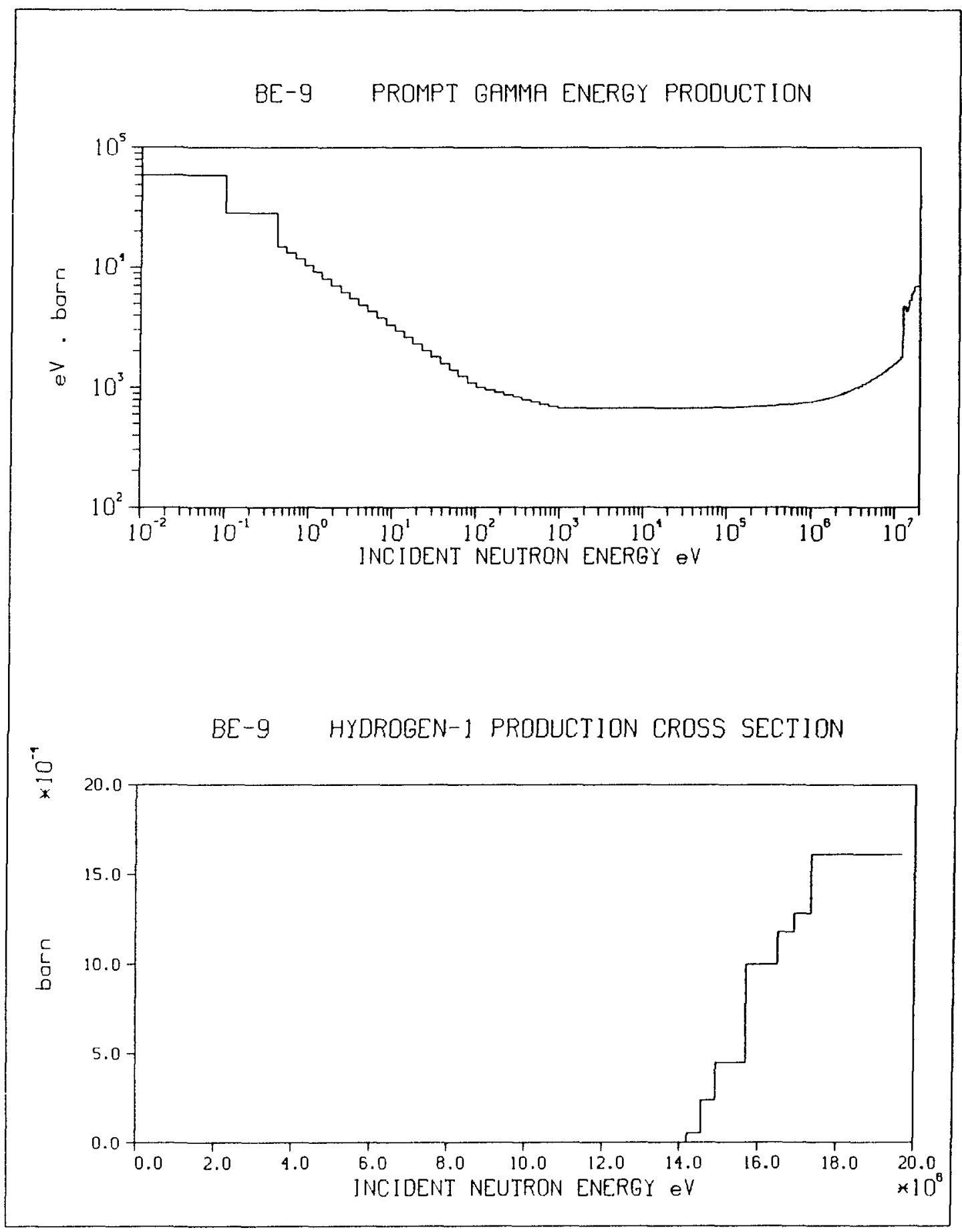




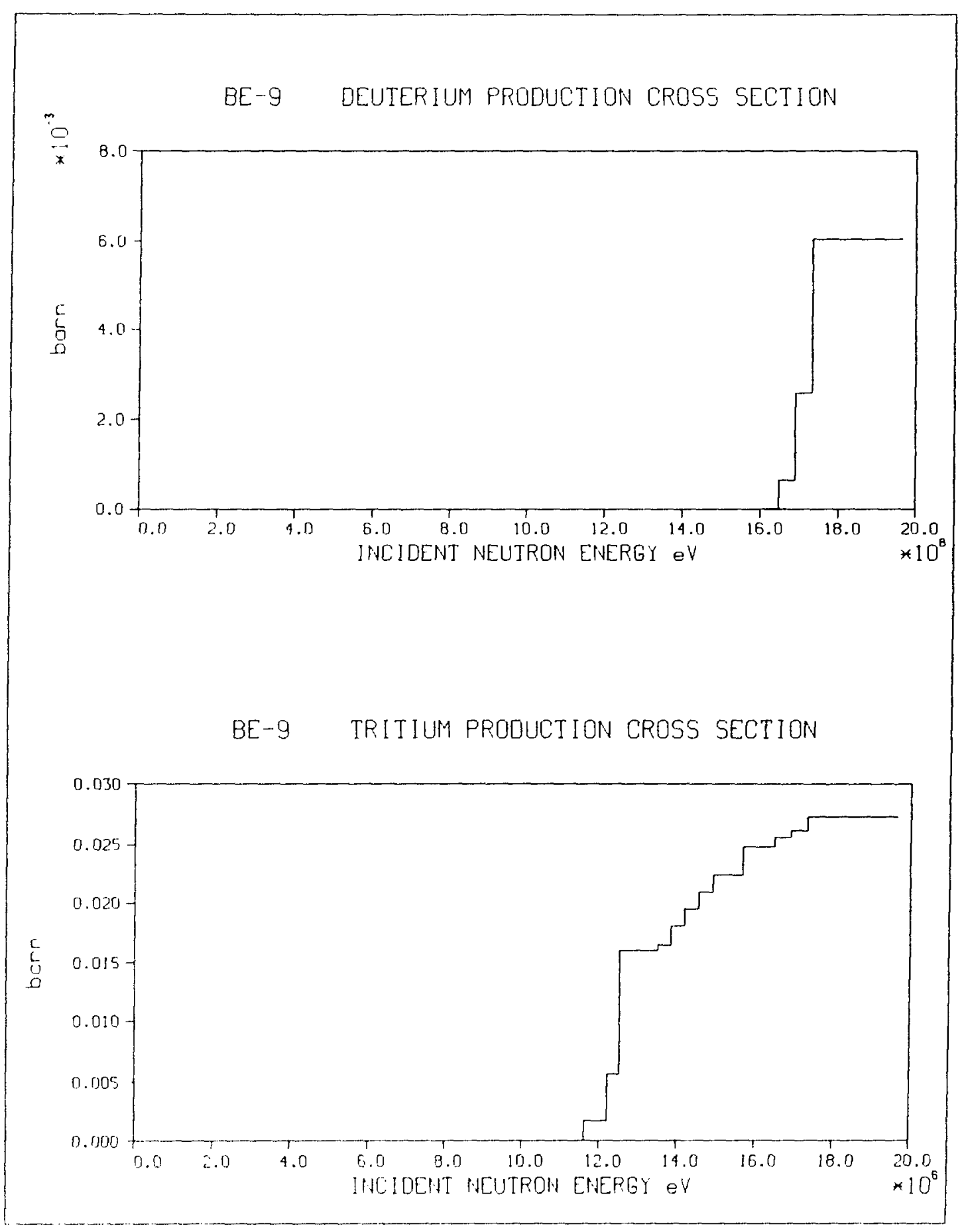




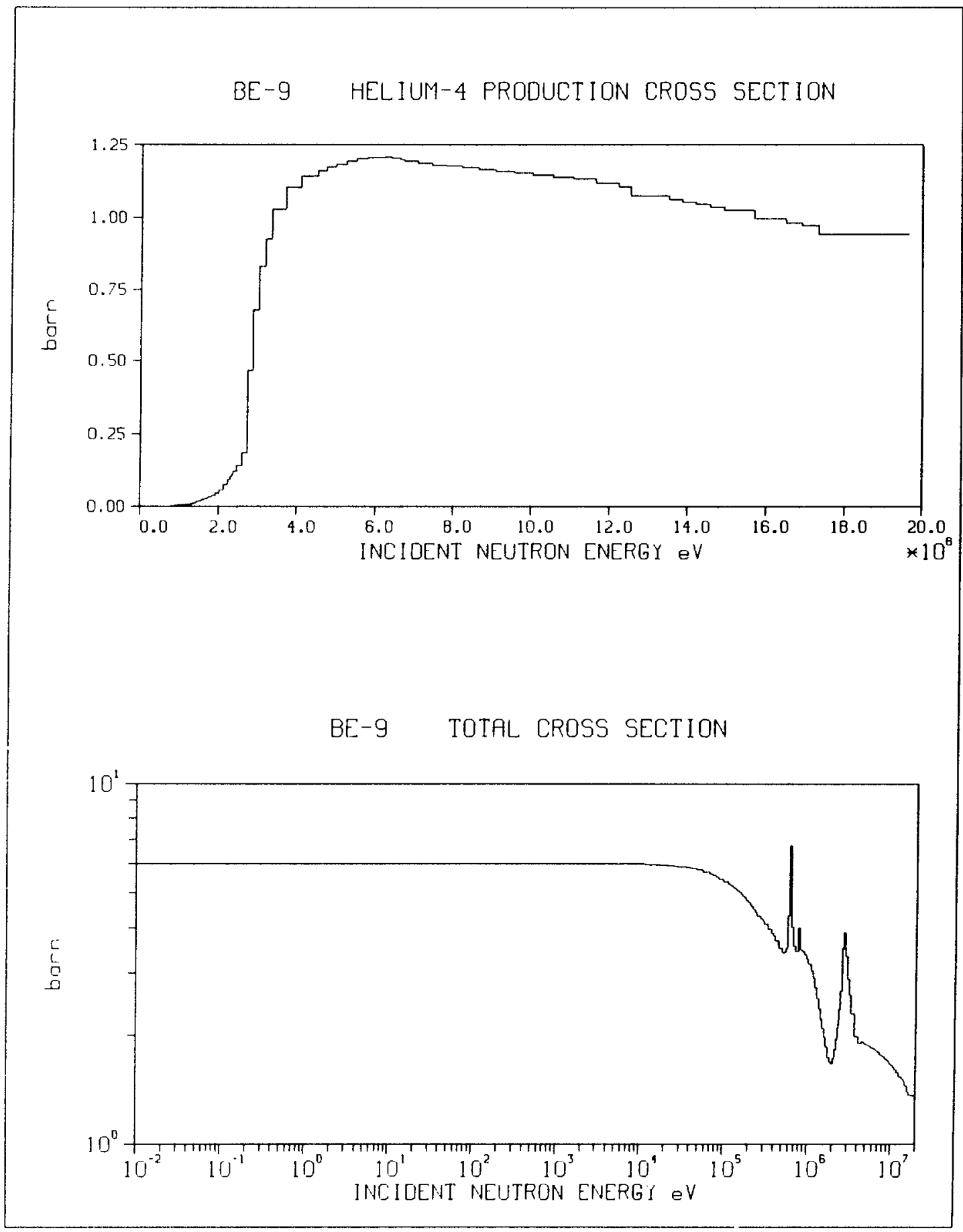




\section{BE-9 ELASTIC CROSS SECTION}

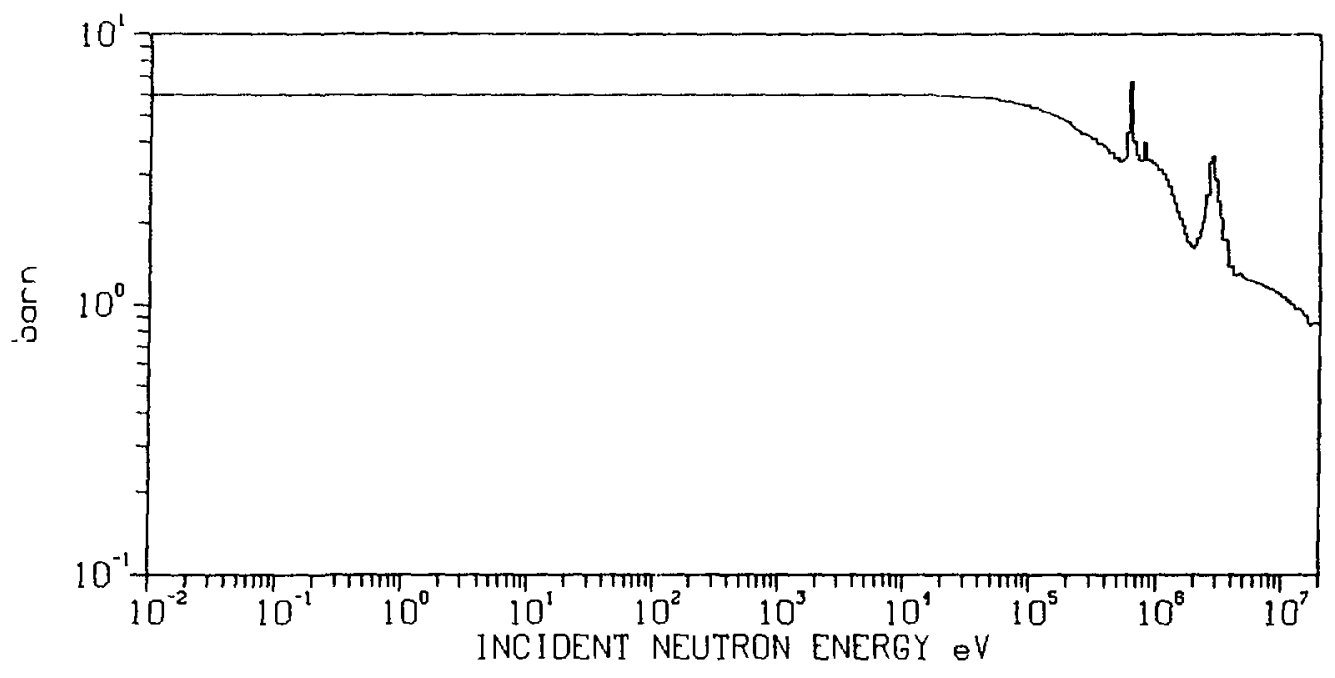

BE-9 RADIATIVE CAPTURE CROSS SECTION

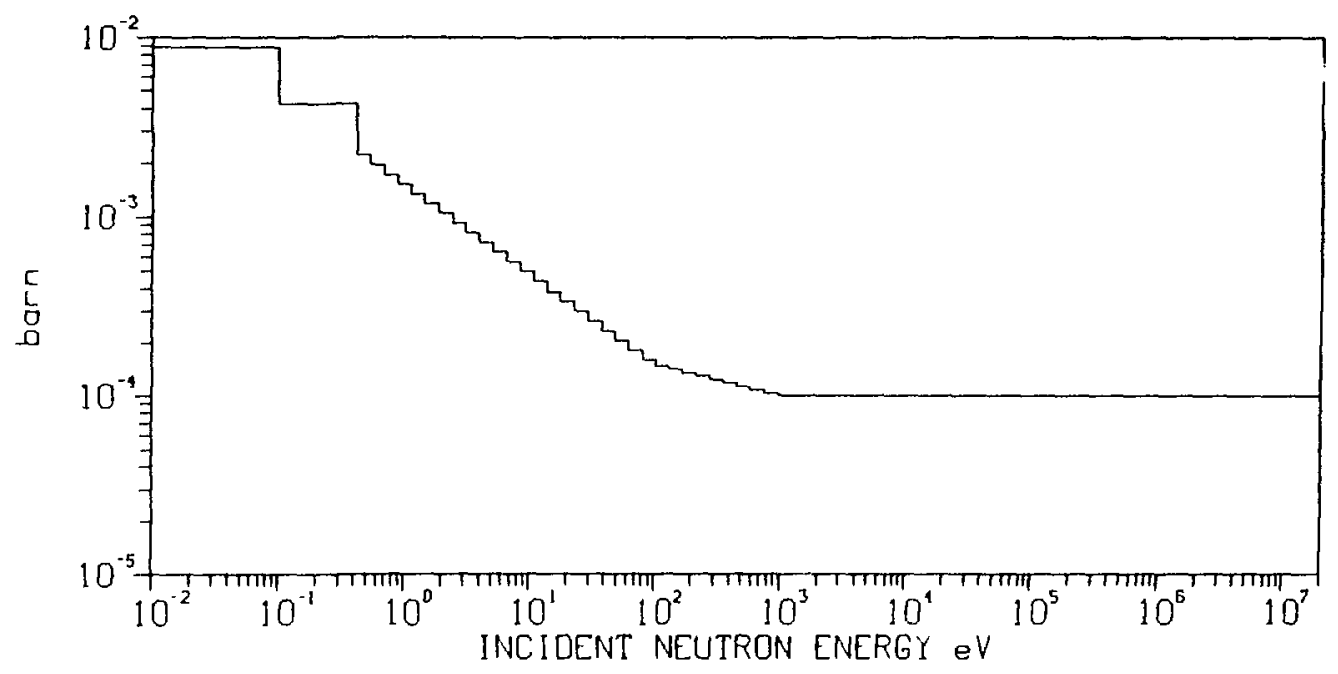




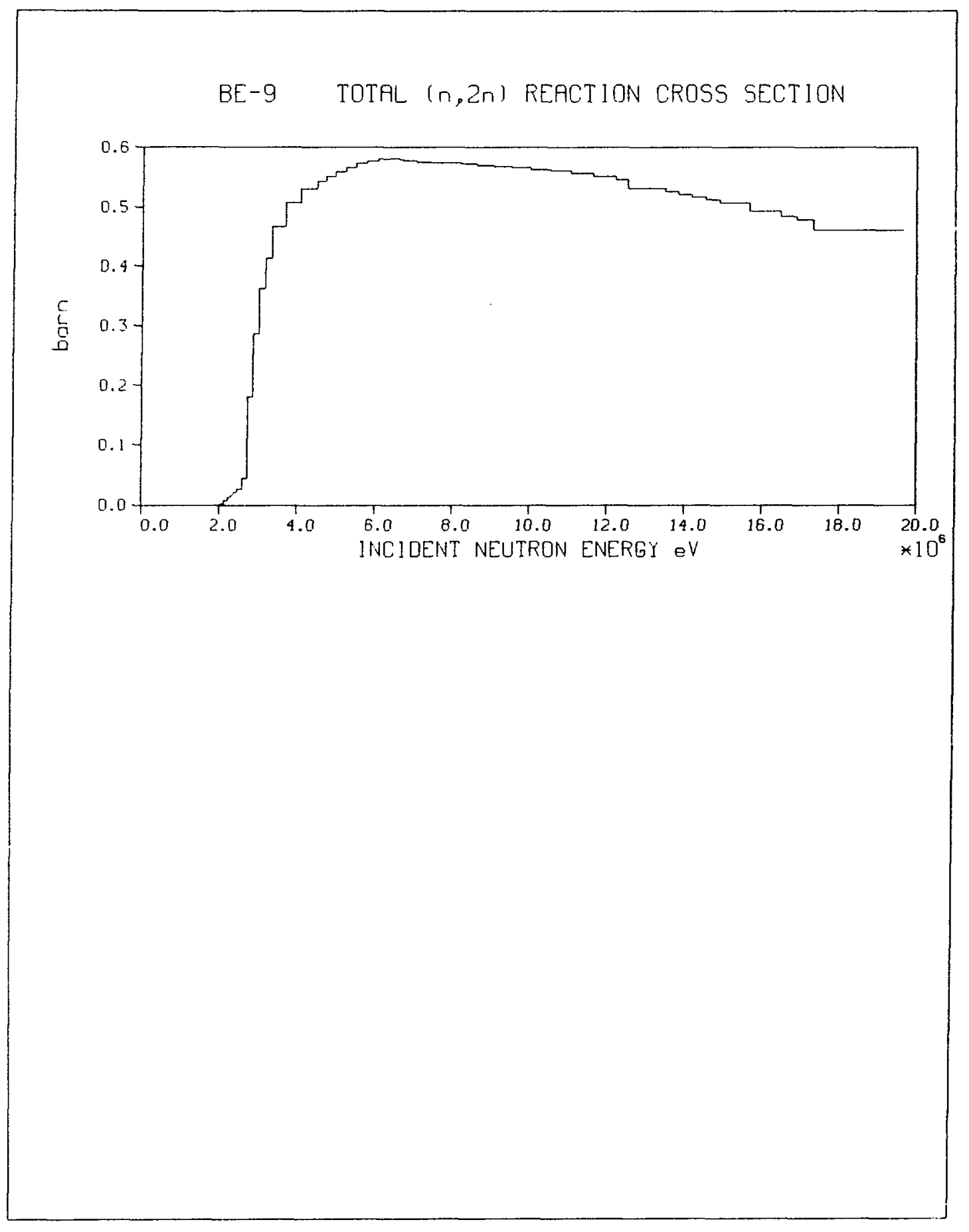




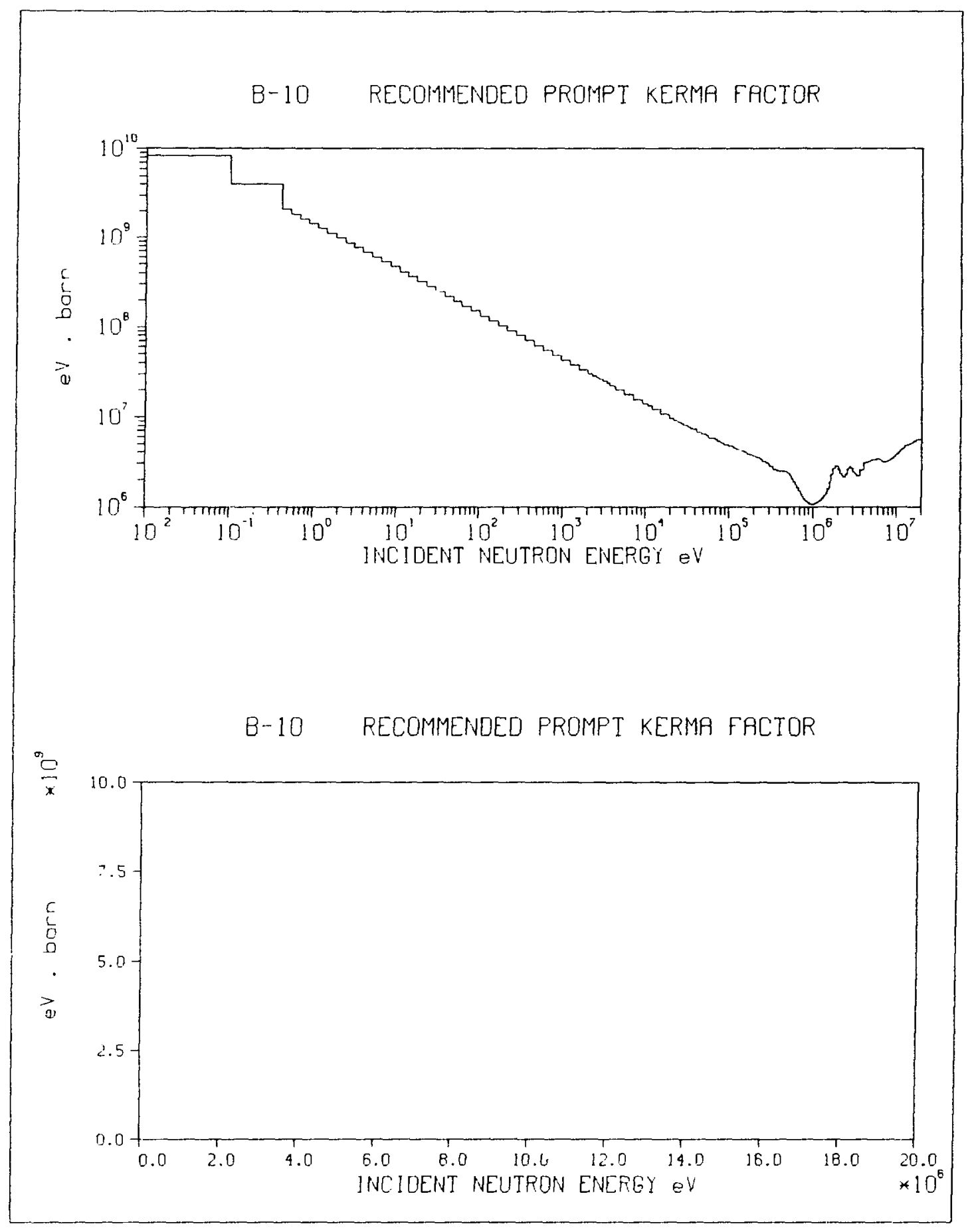




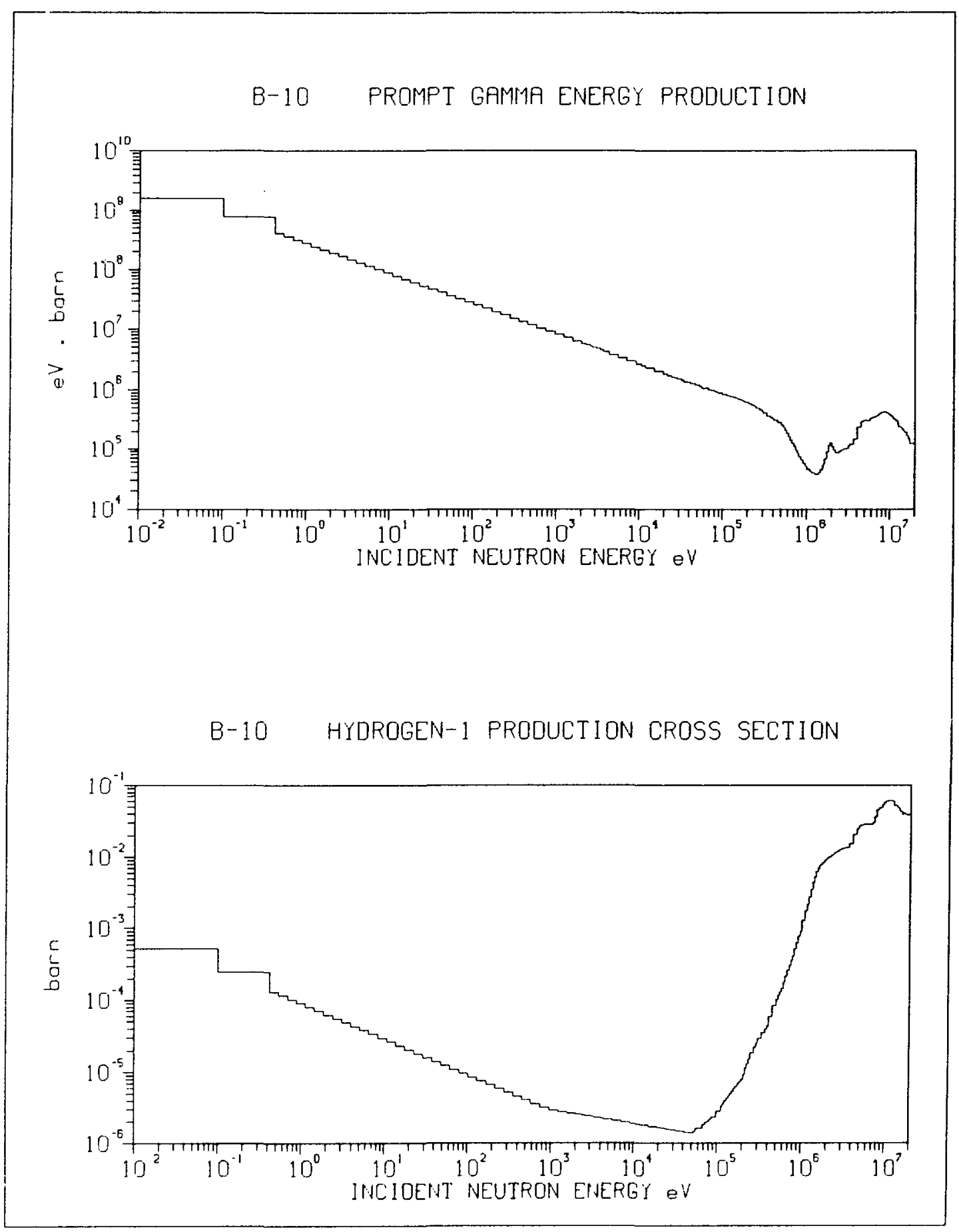




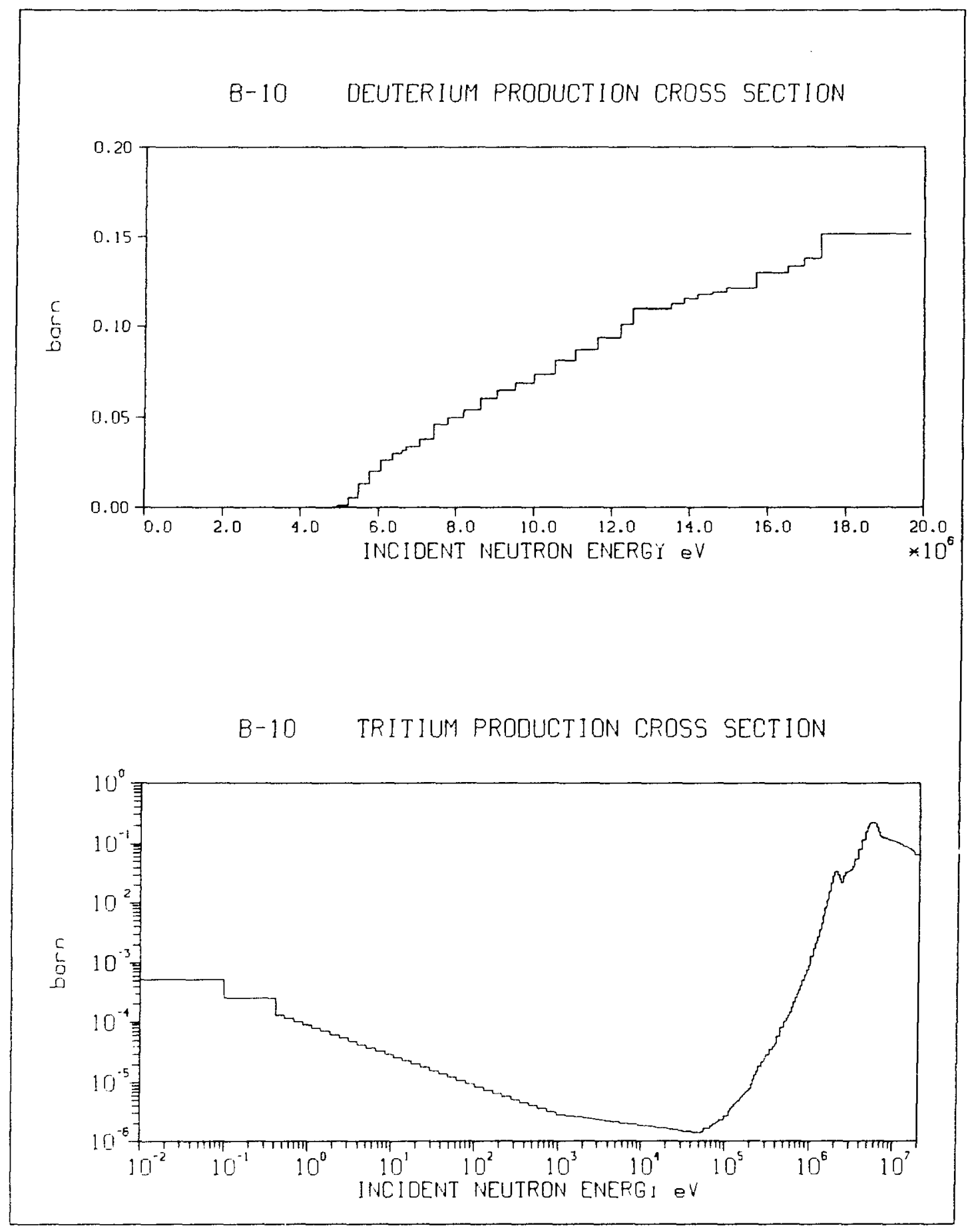




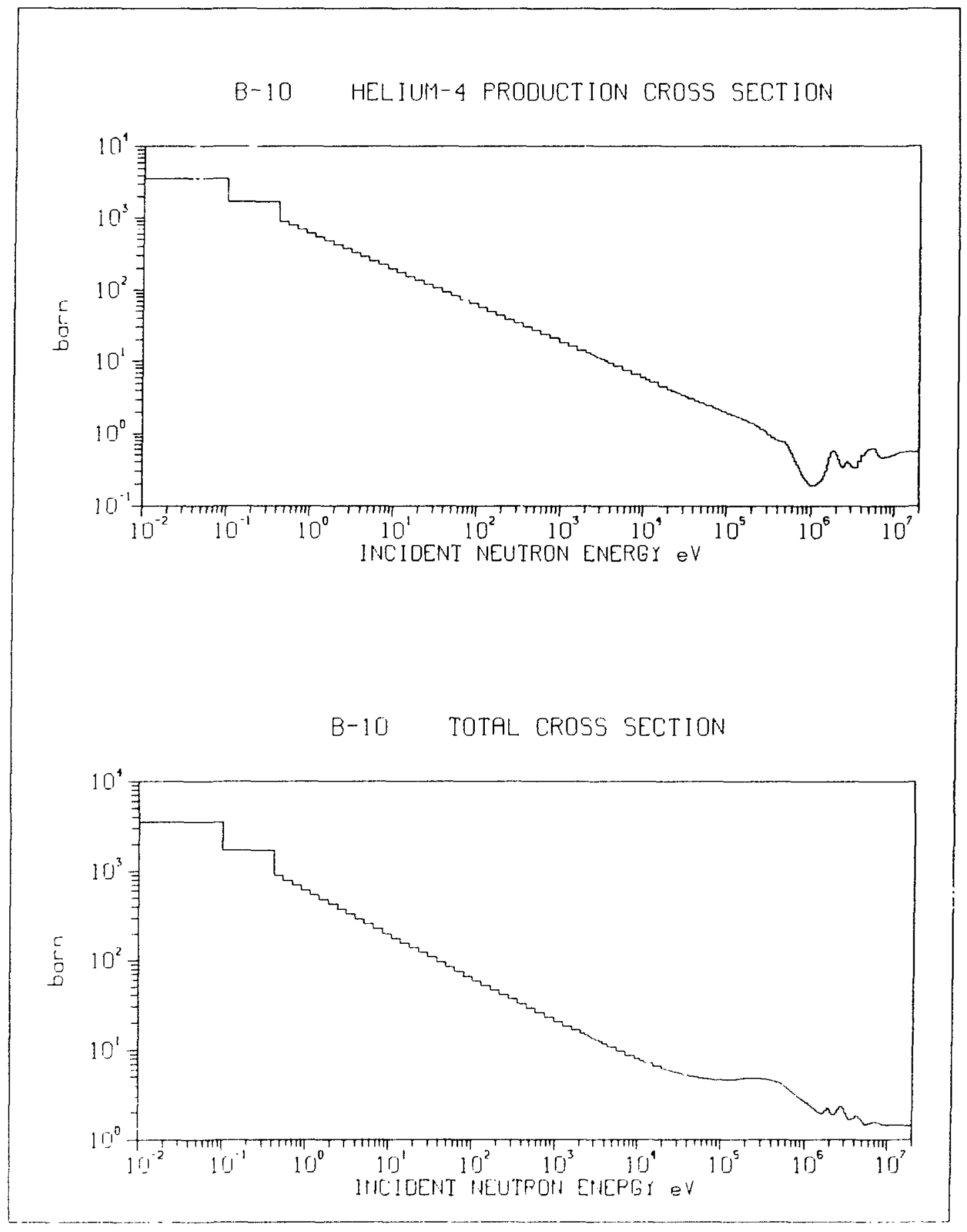




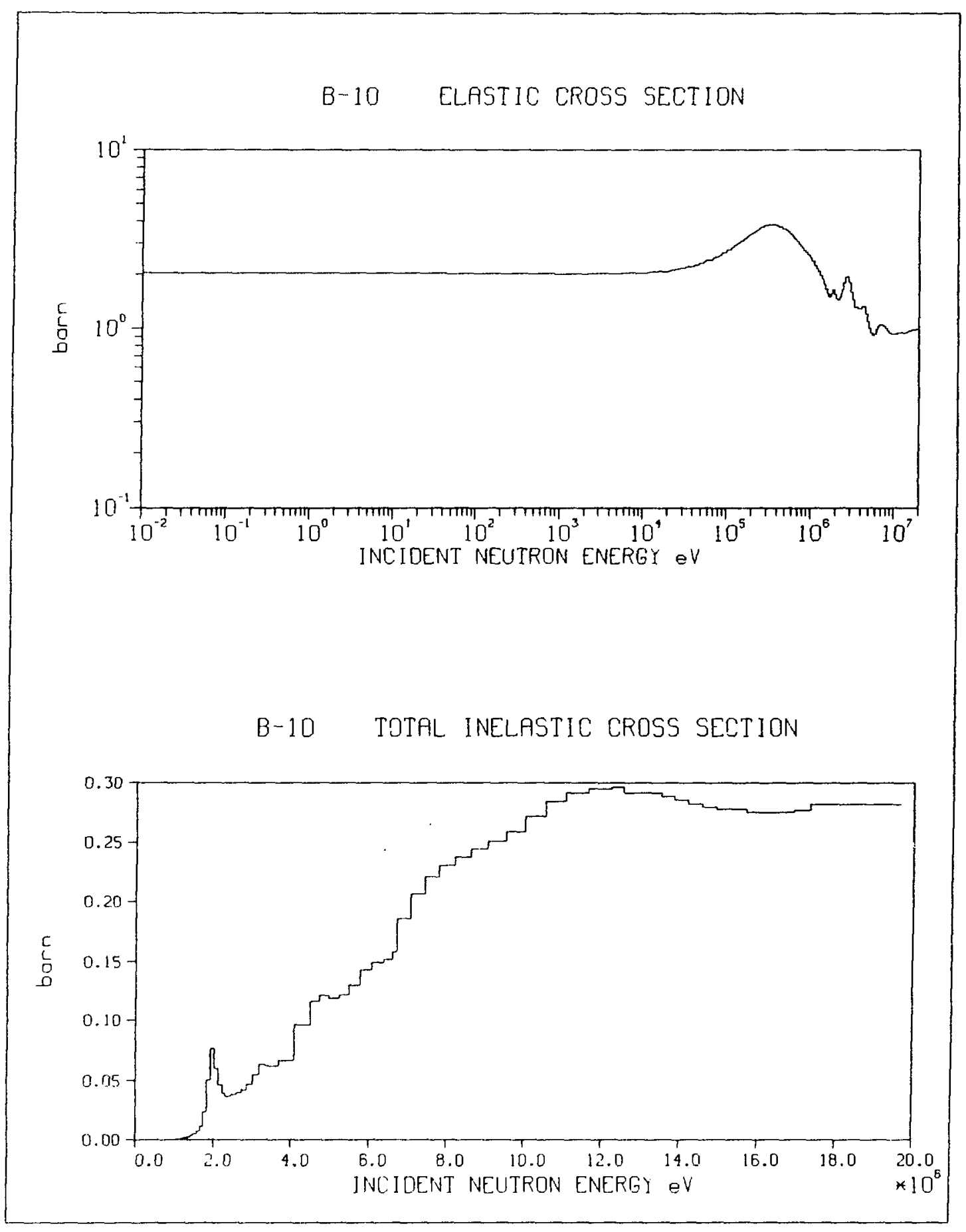




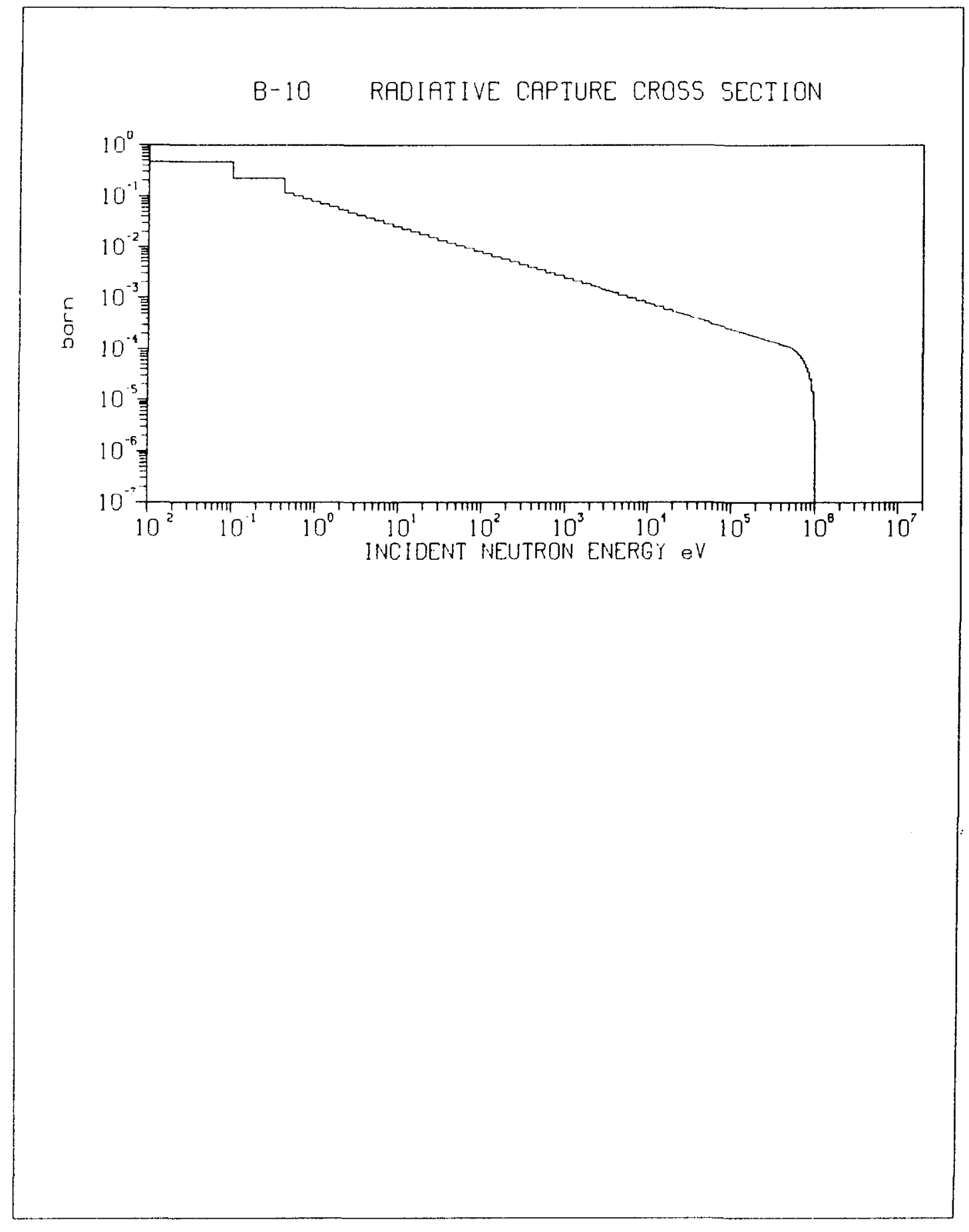




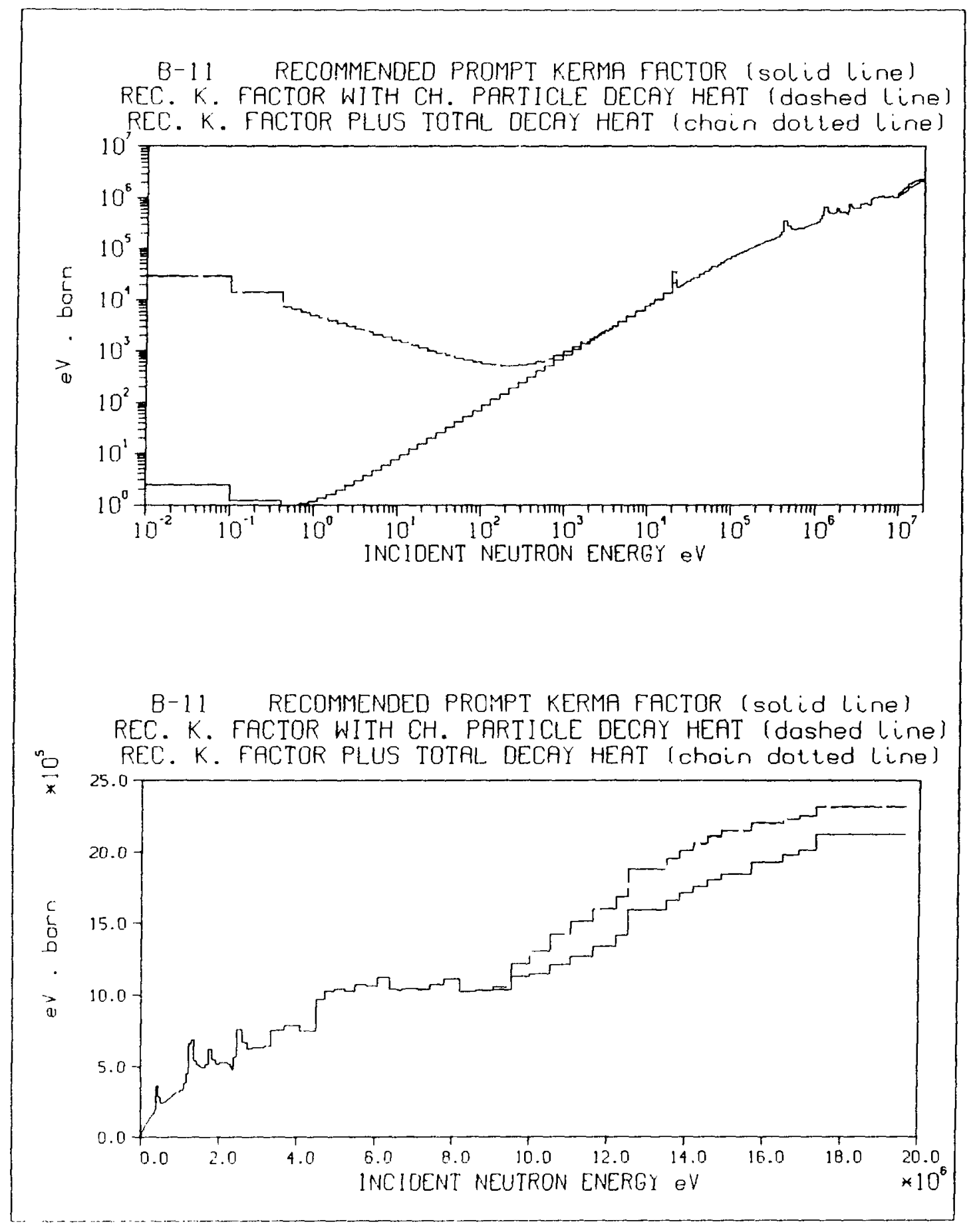




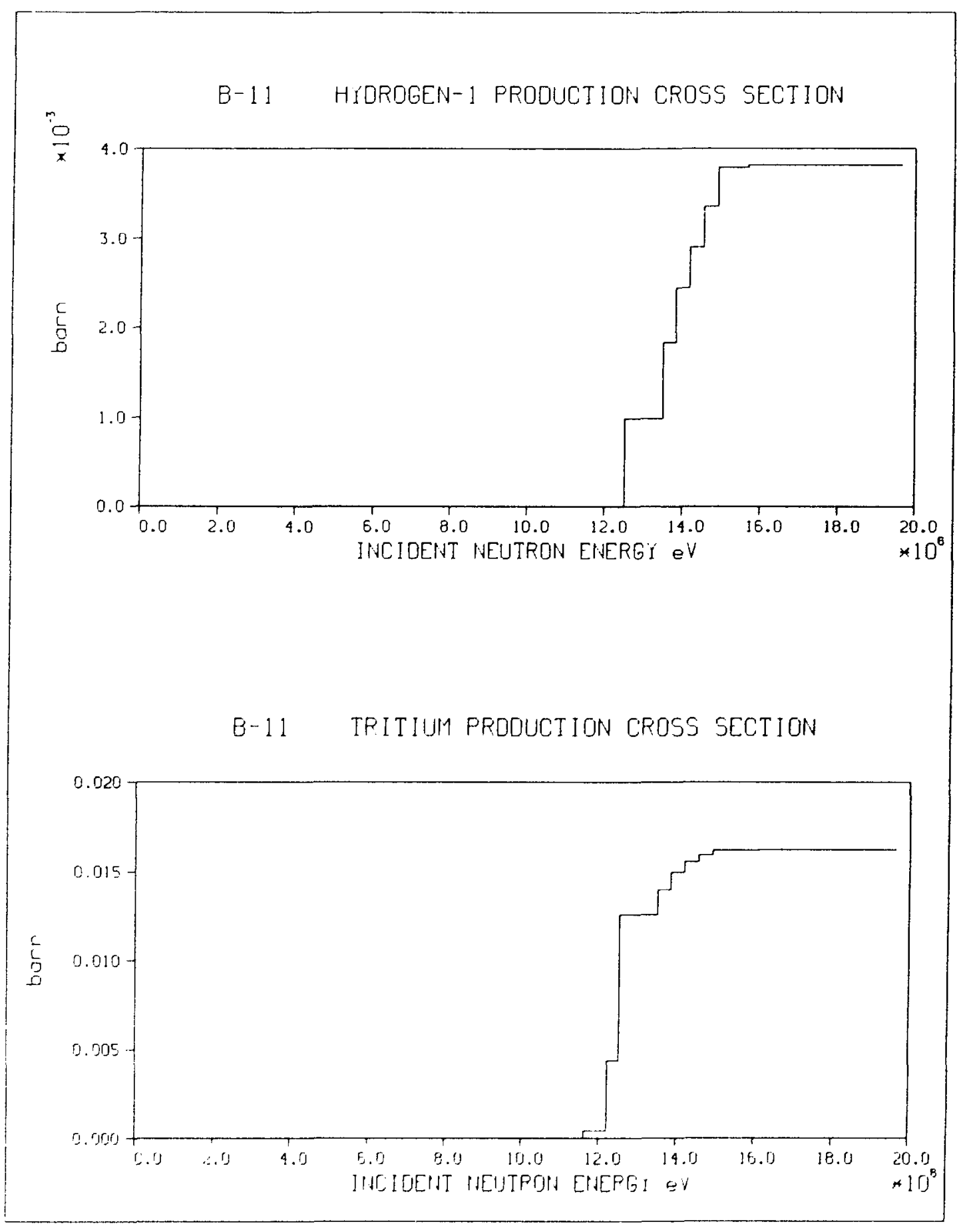




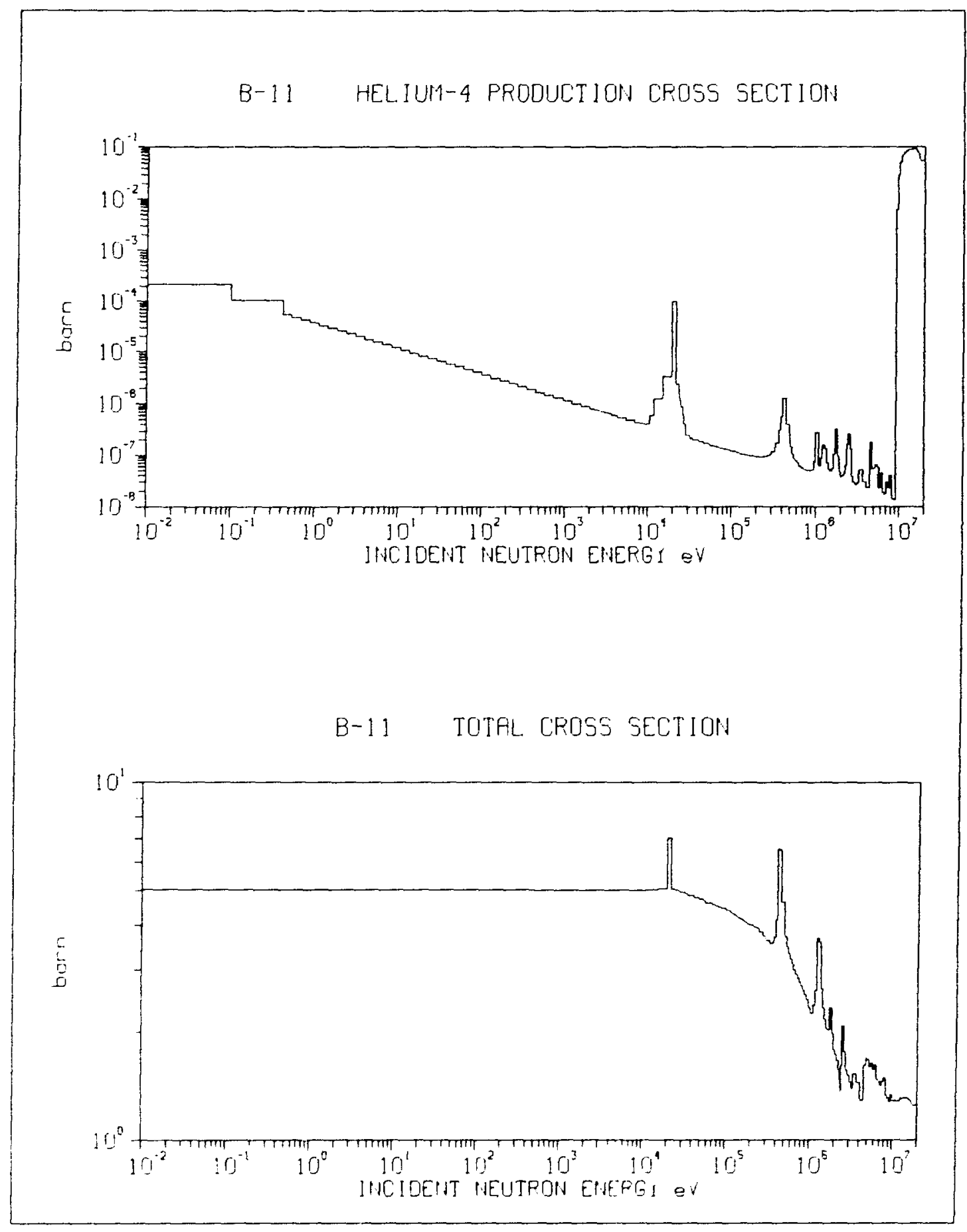




\section{B-11 ELASTIC CROSS SECTION}

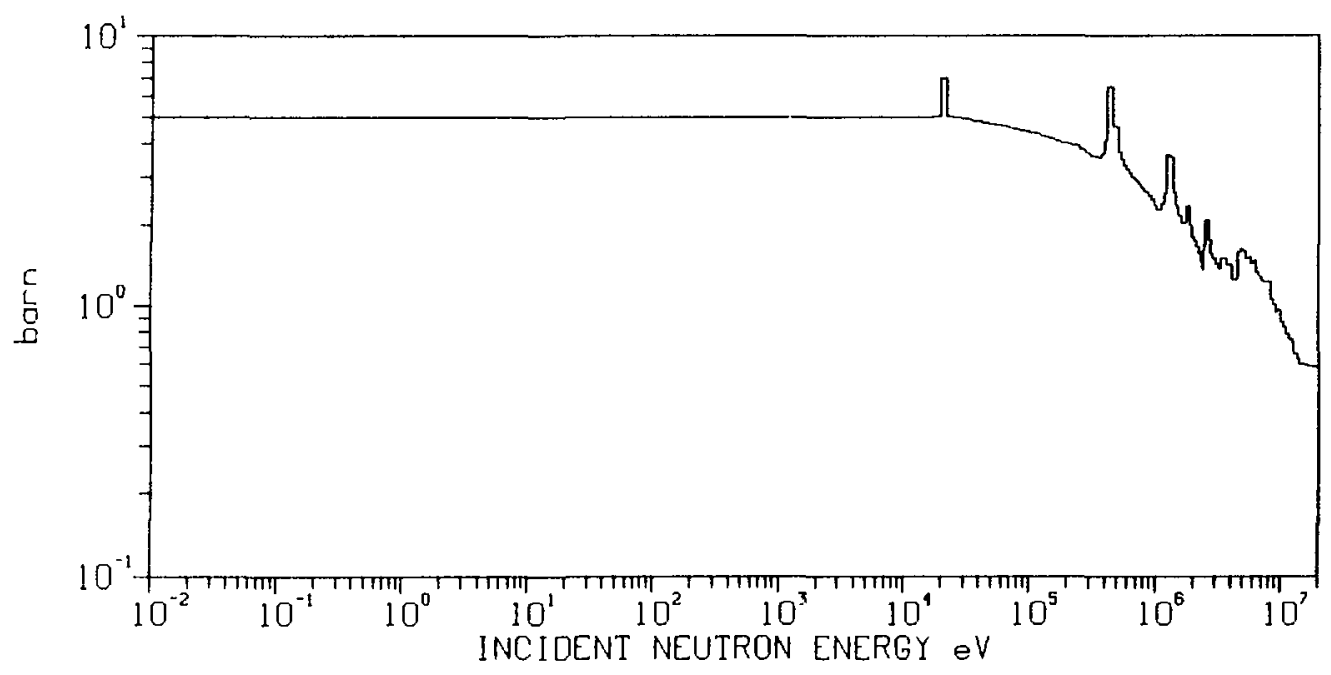

B-11 TOTAL INELASTIC CROSS SECTION

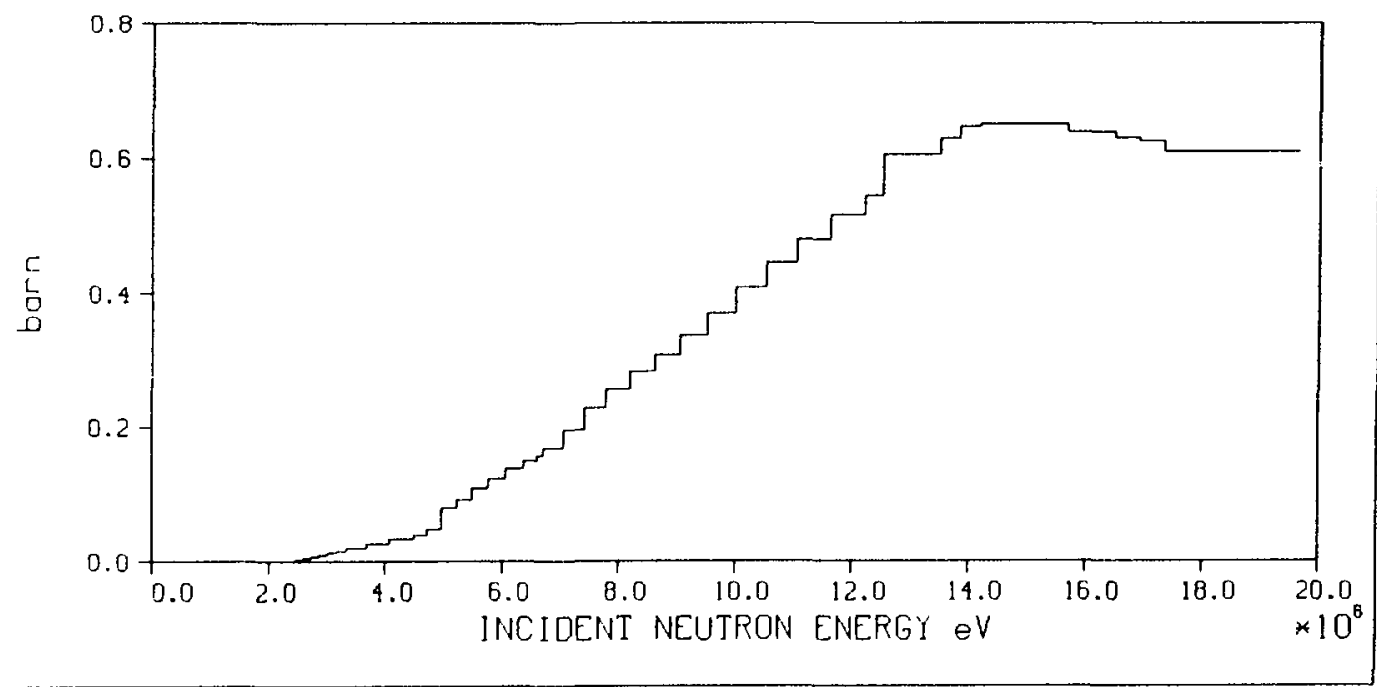




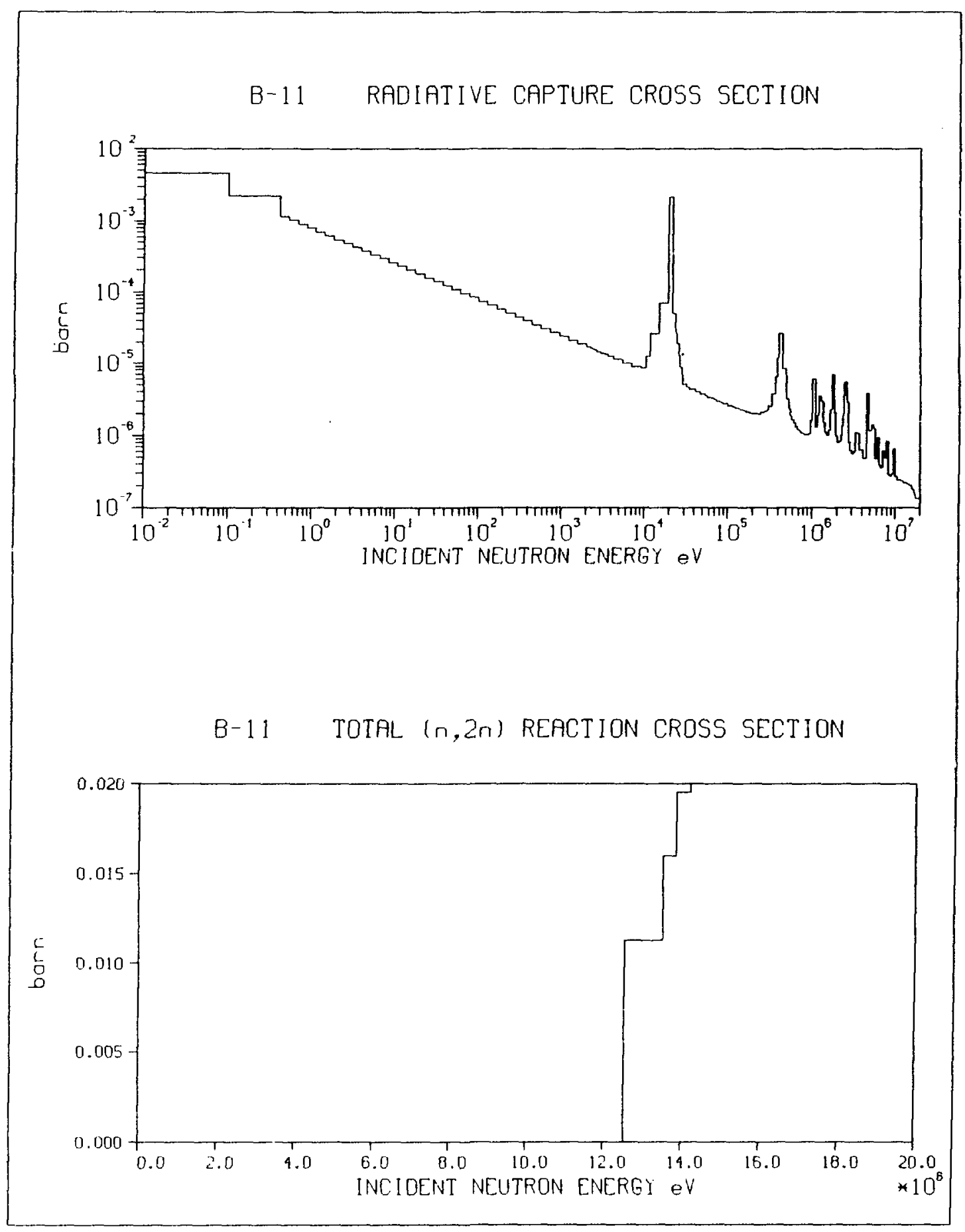




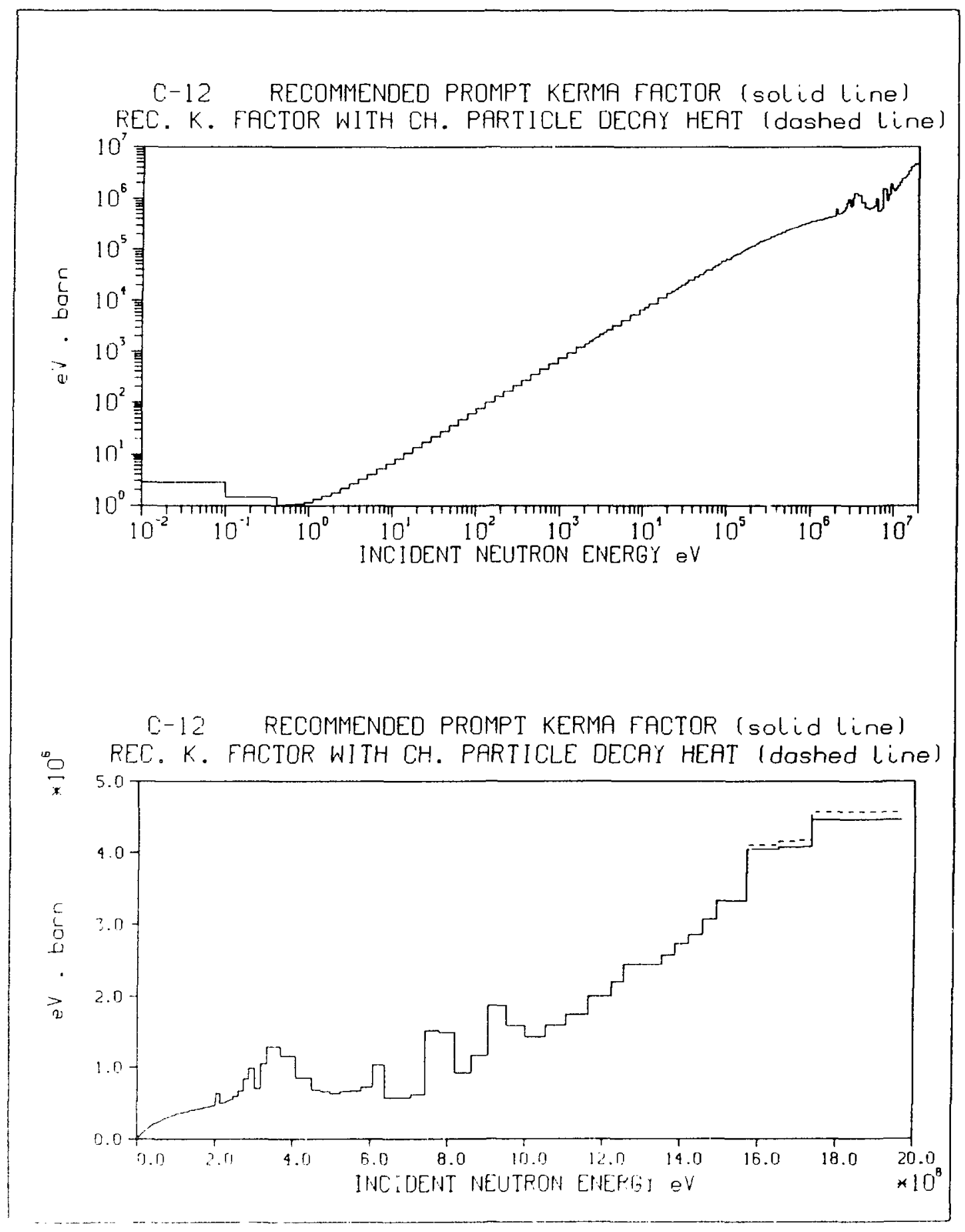




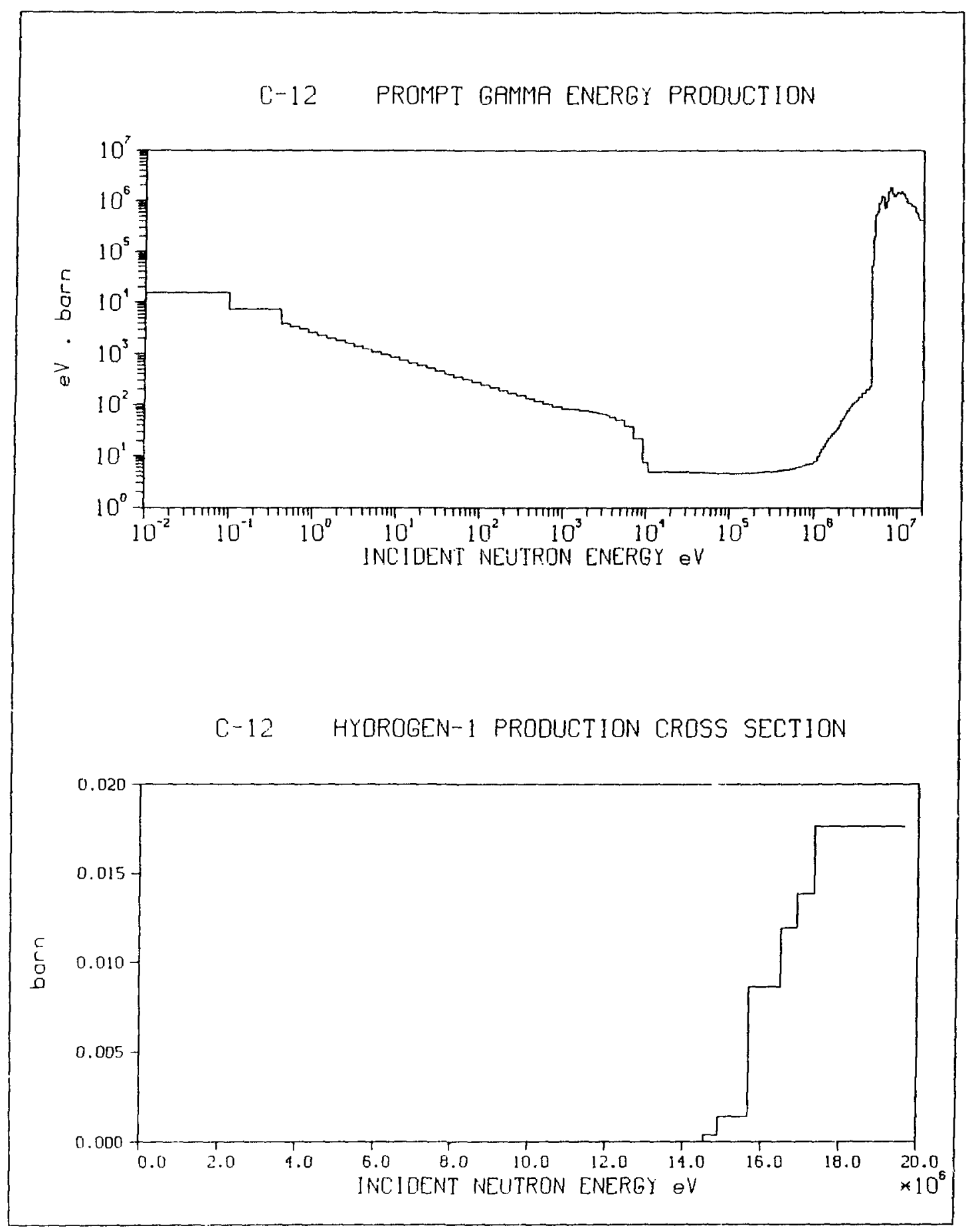




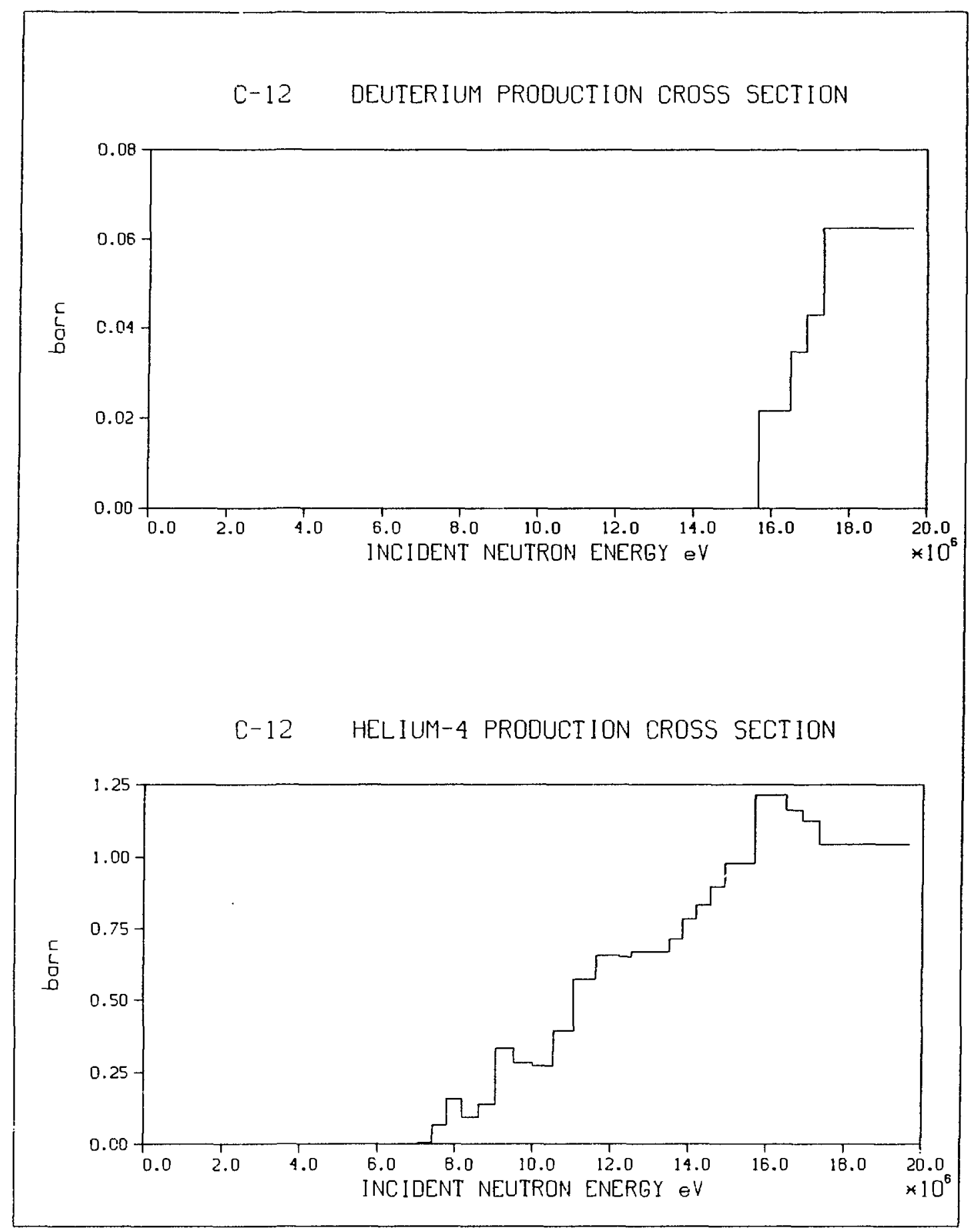




\section{C-12 TOTAL CROSS SECTION}

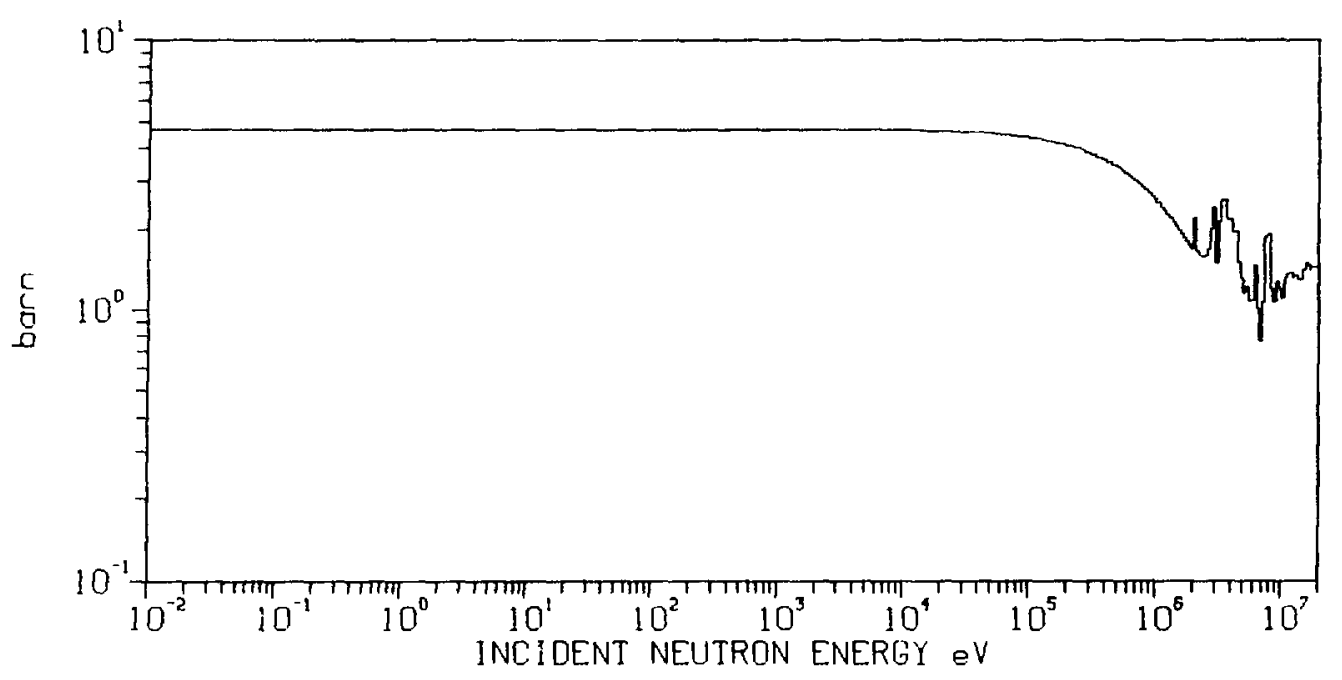

C-12 ELASTIC CROSS SECTION

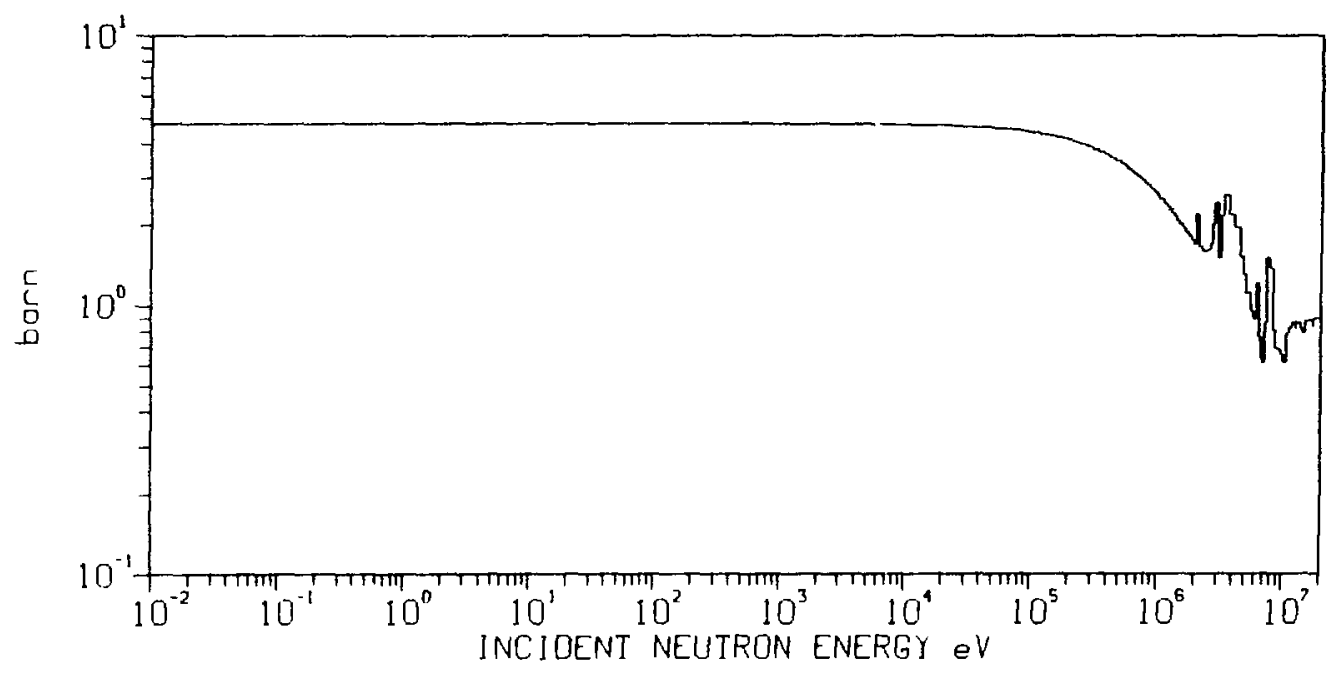




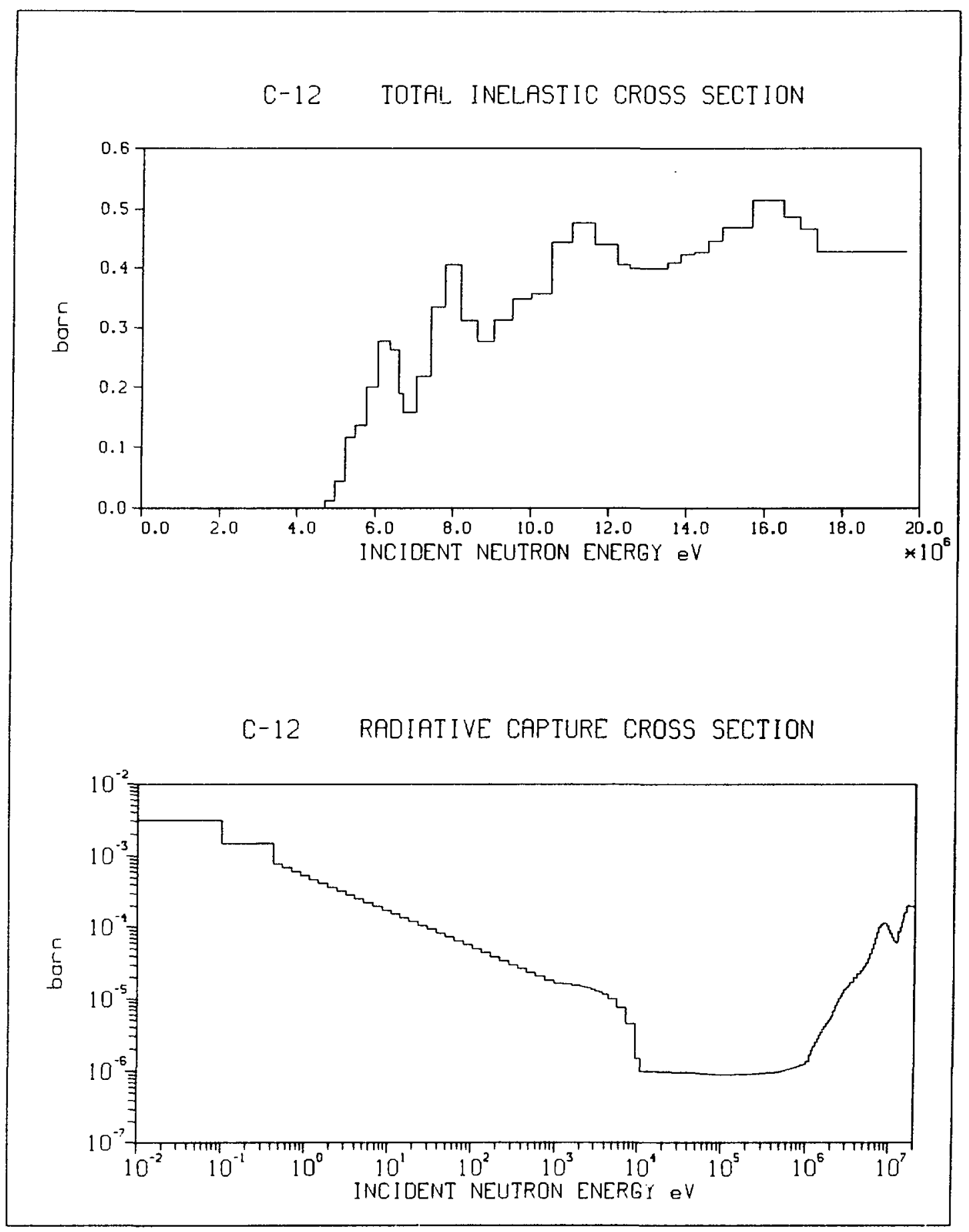




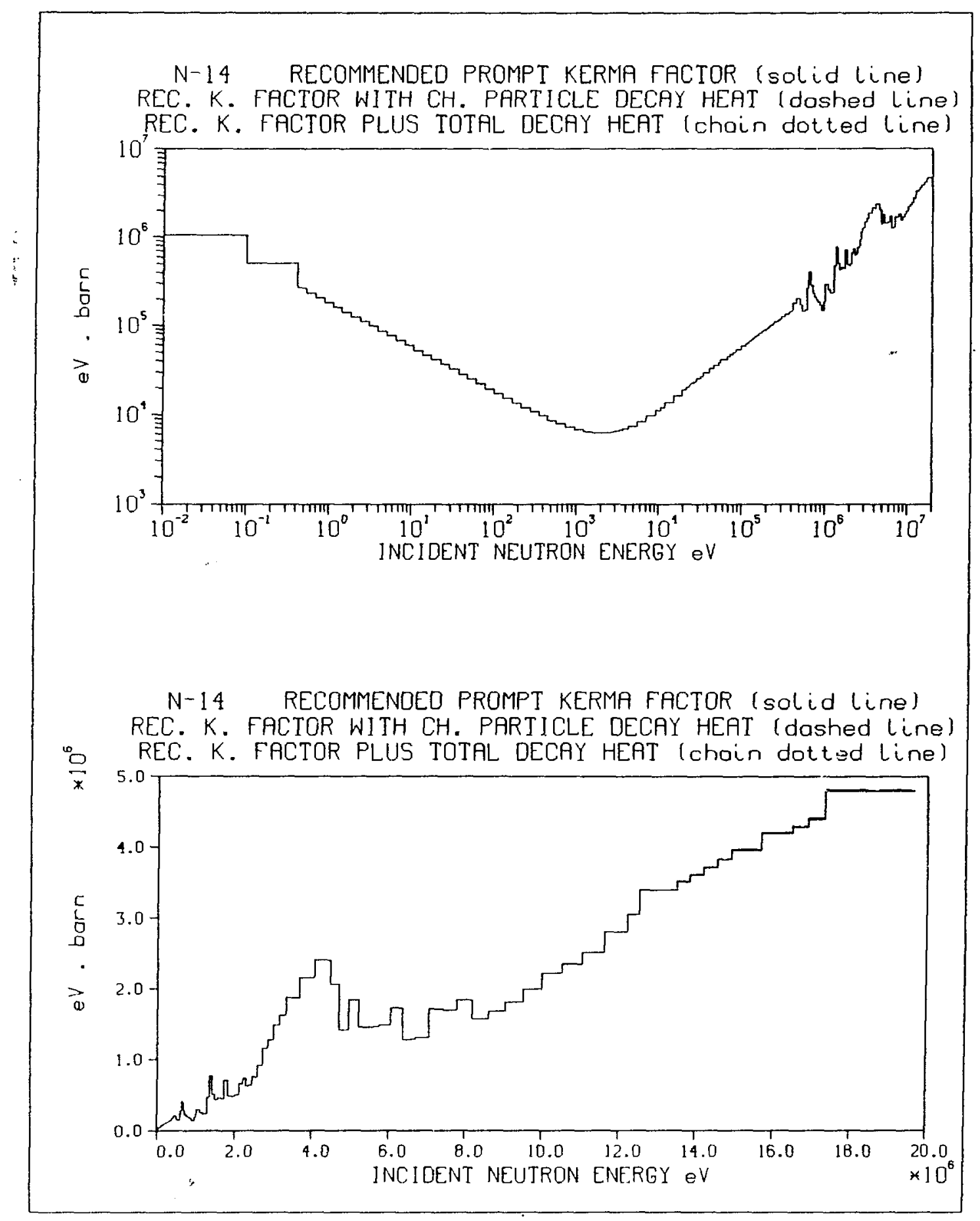




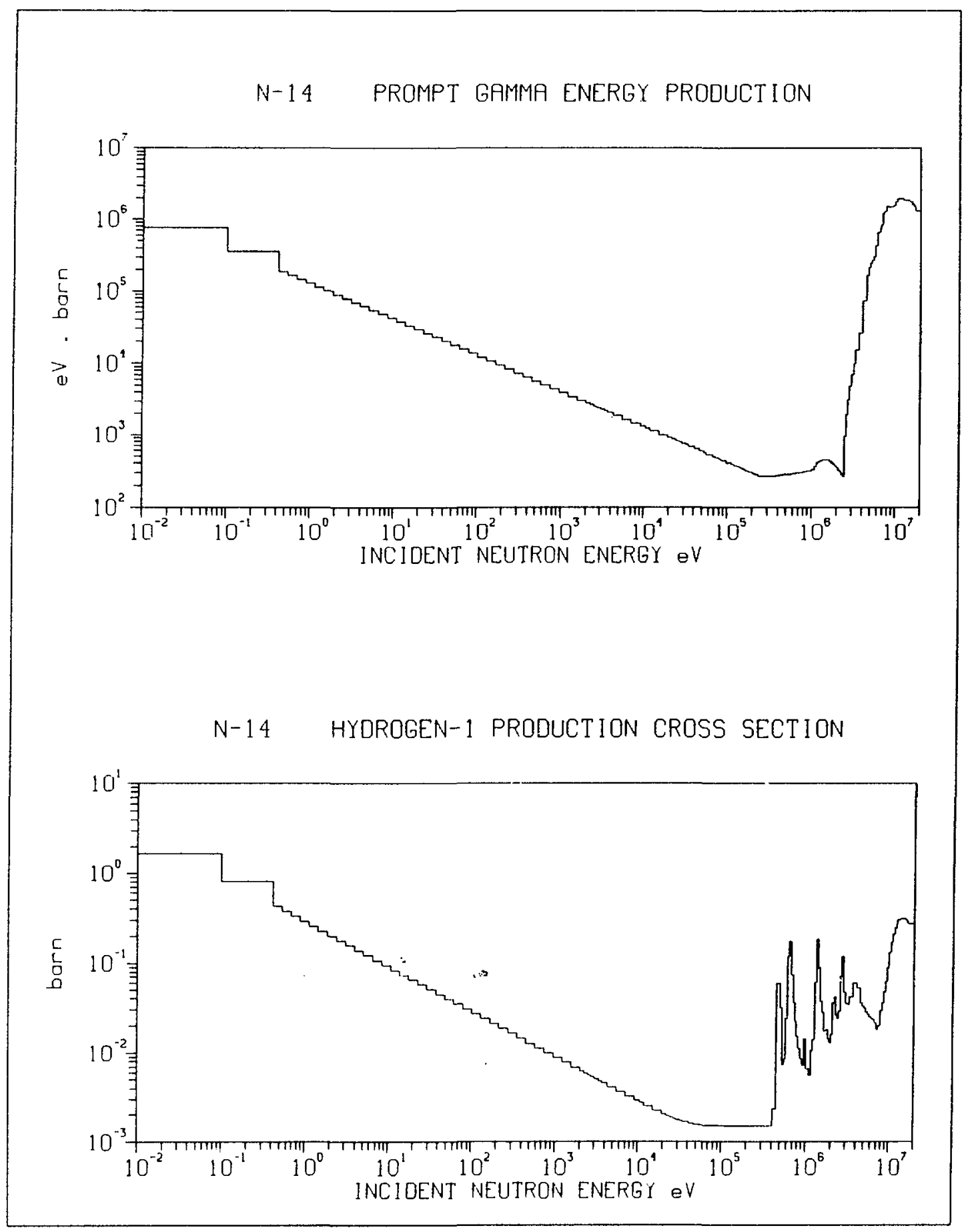




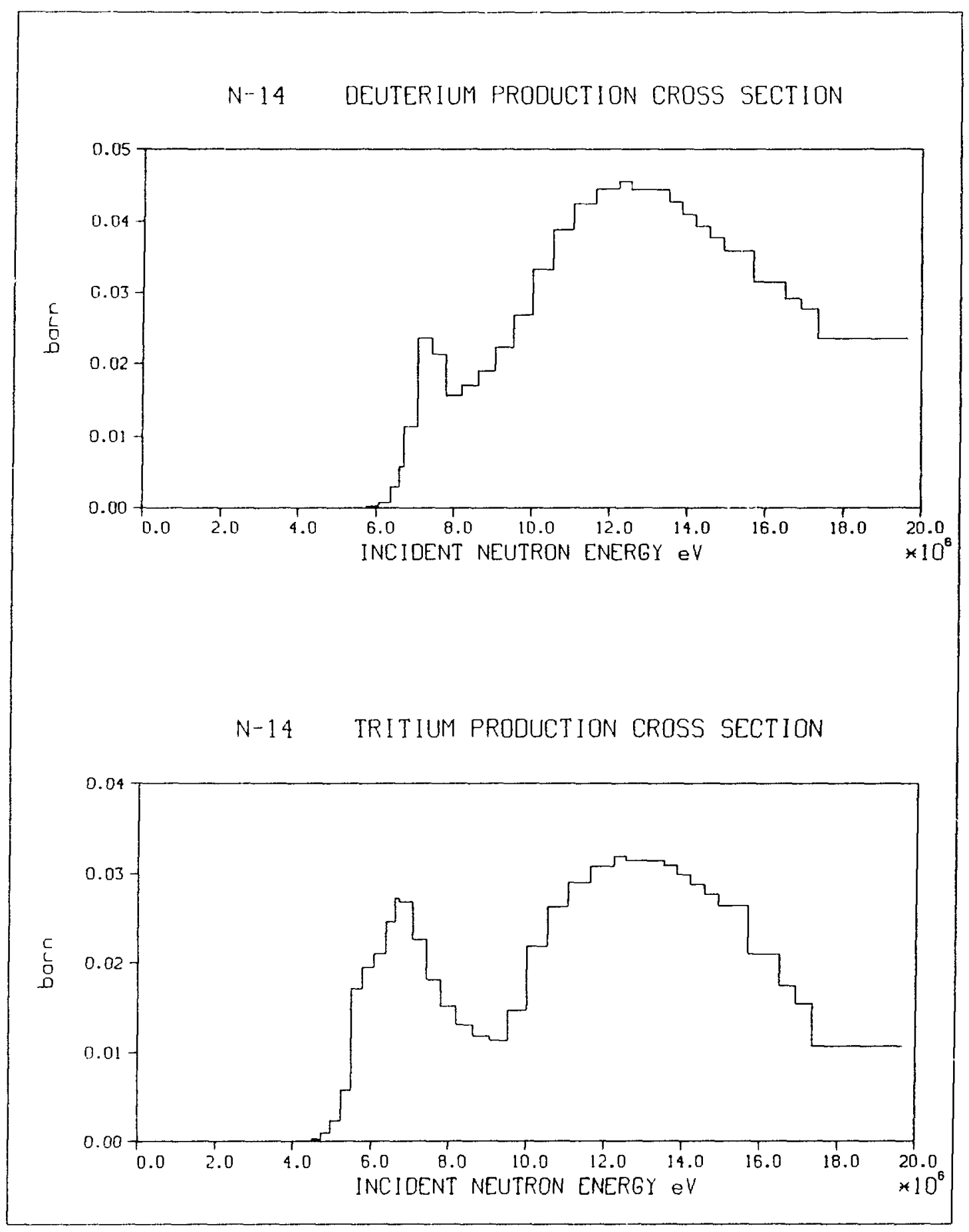




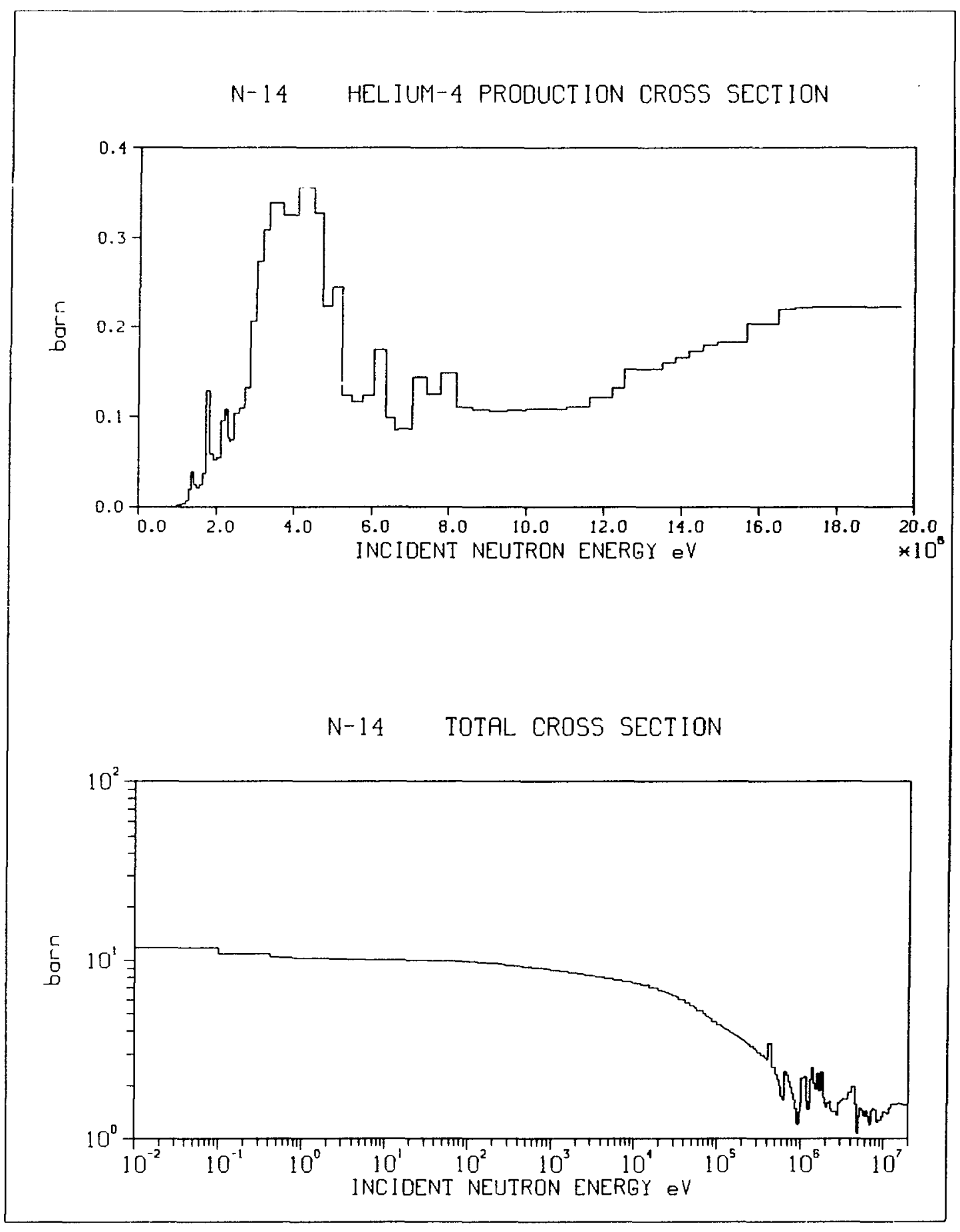




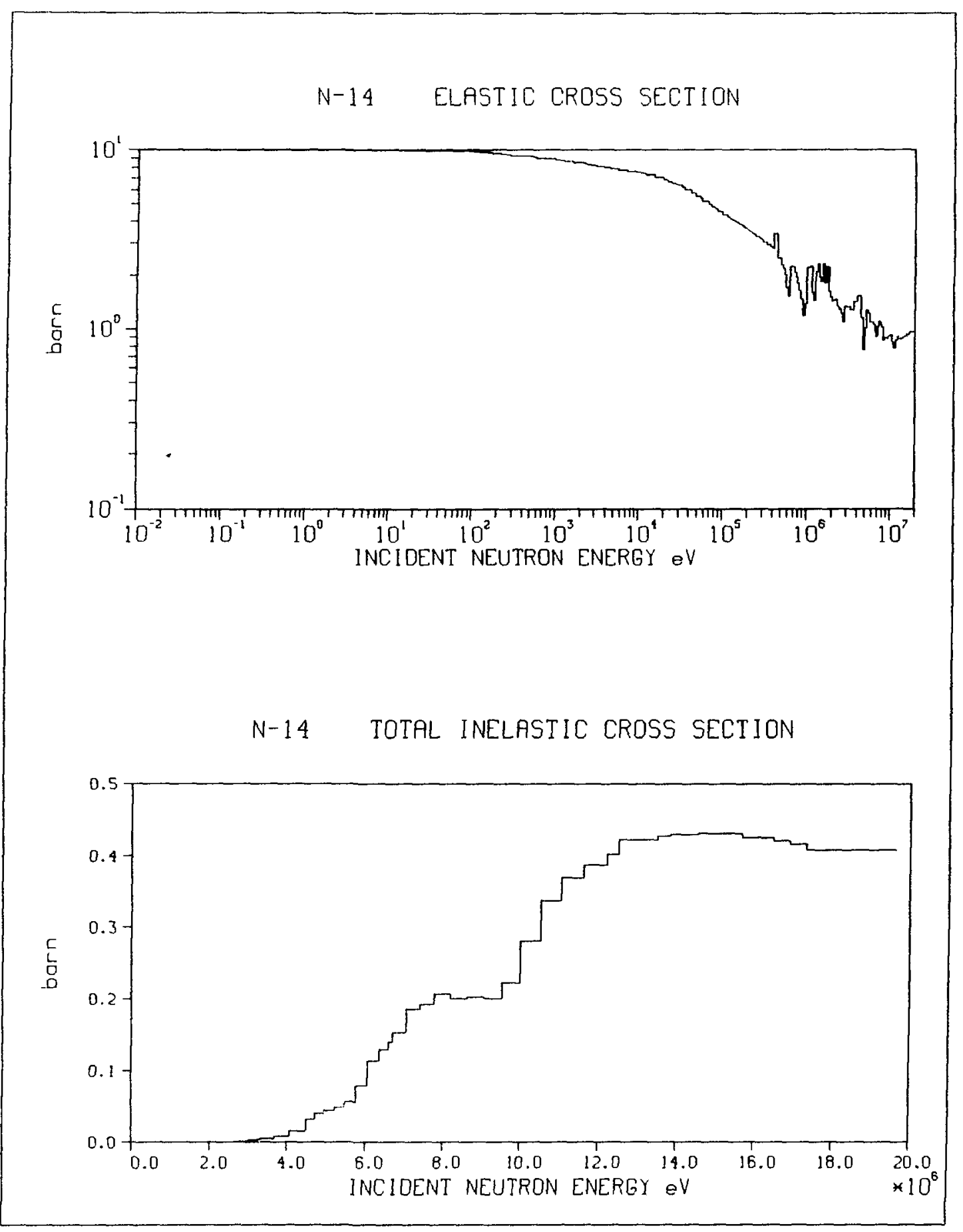


N-14 RADIATIVE CAPTURE CROSS SECTION

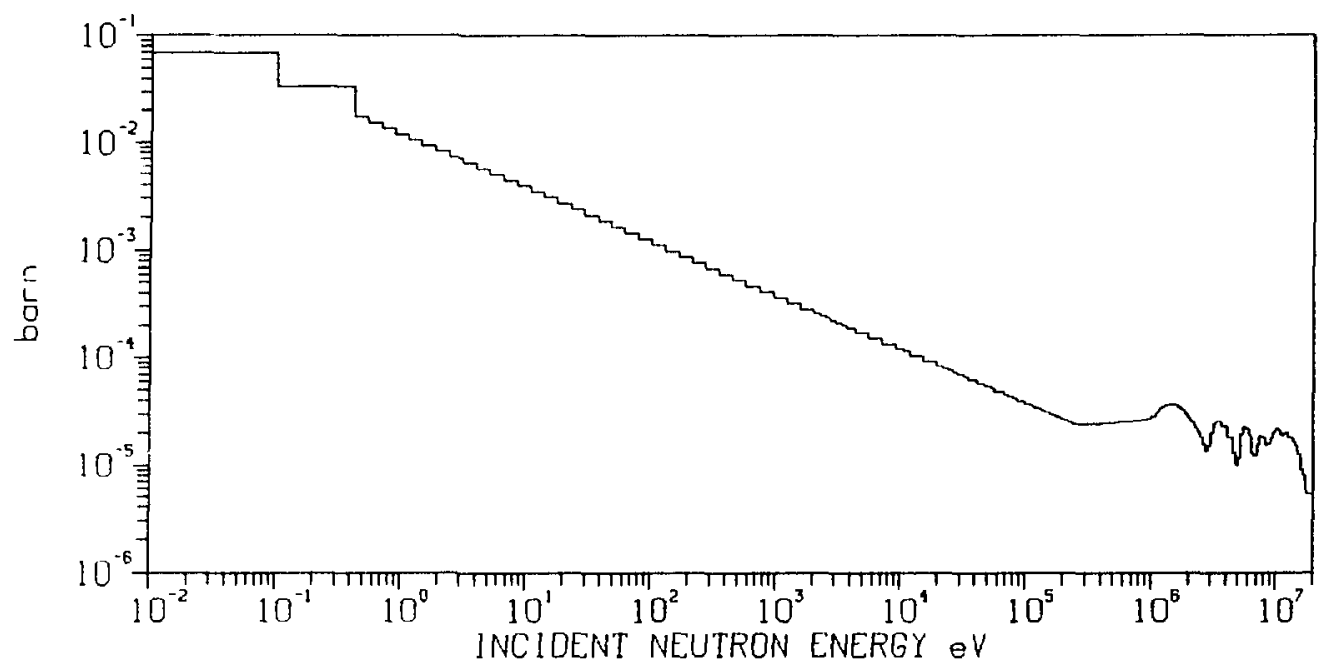

N-14 TOTAL $(n, 2 n)$ REACTION CROSS SECTION

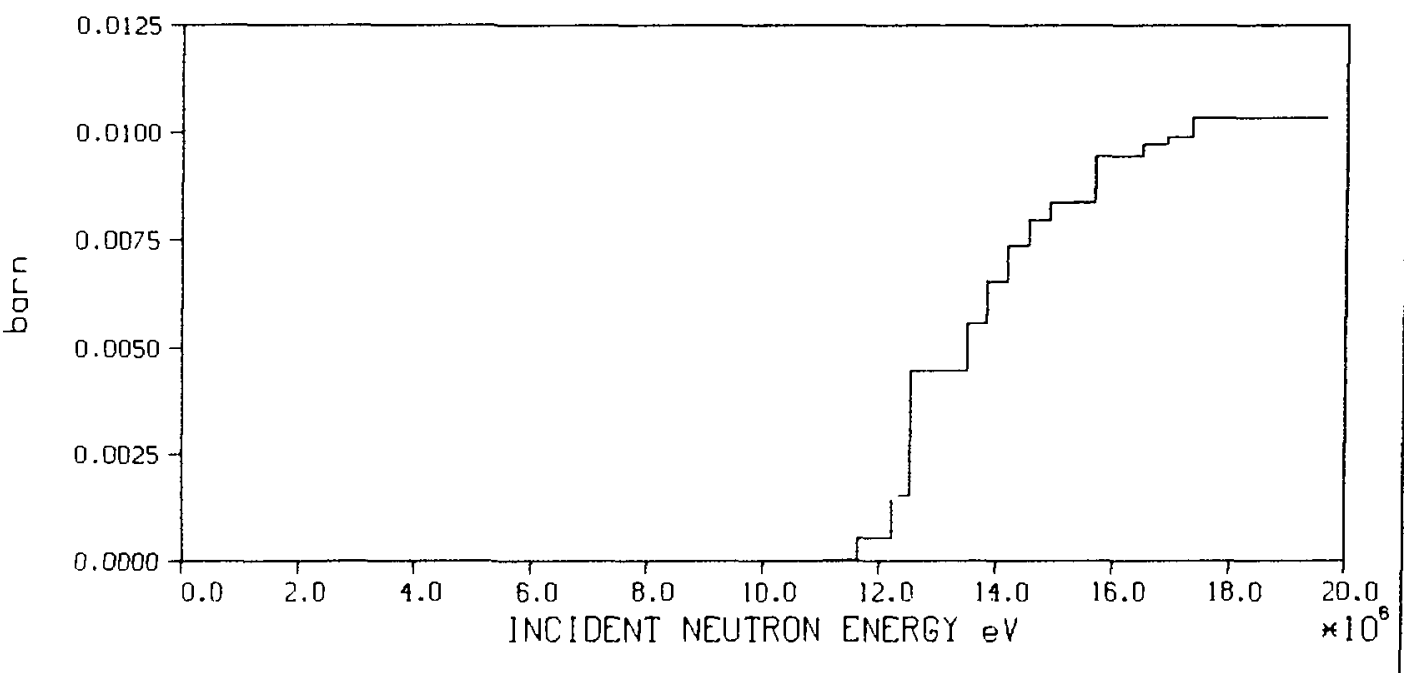




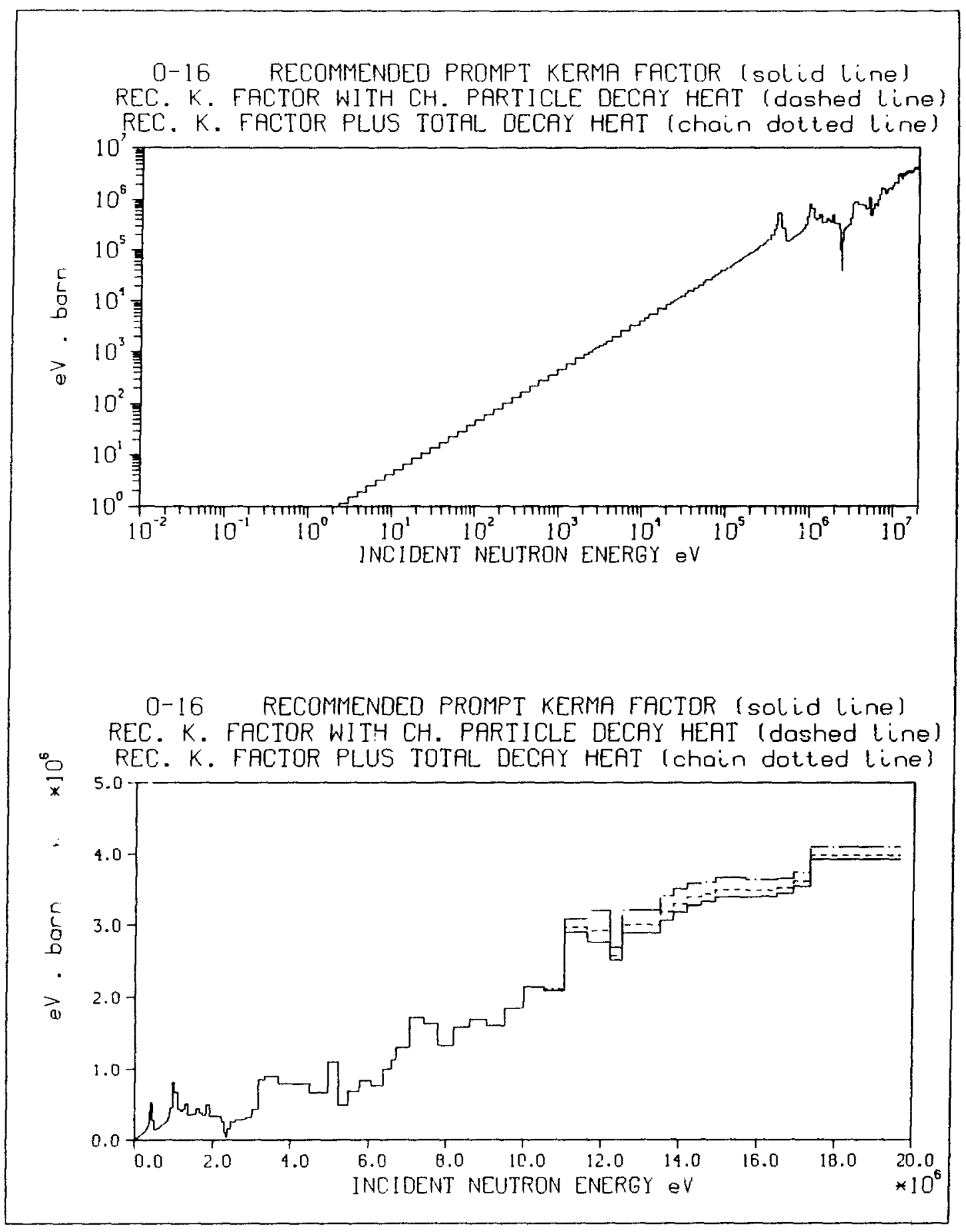




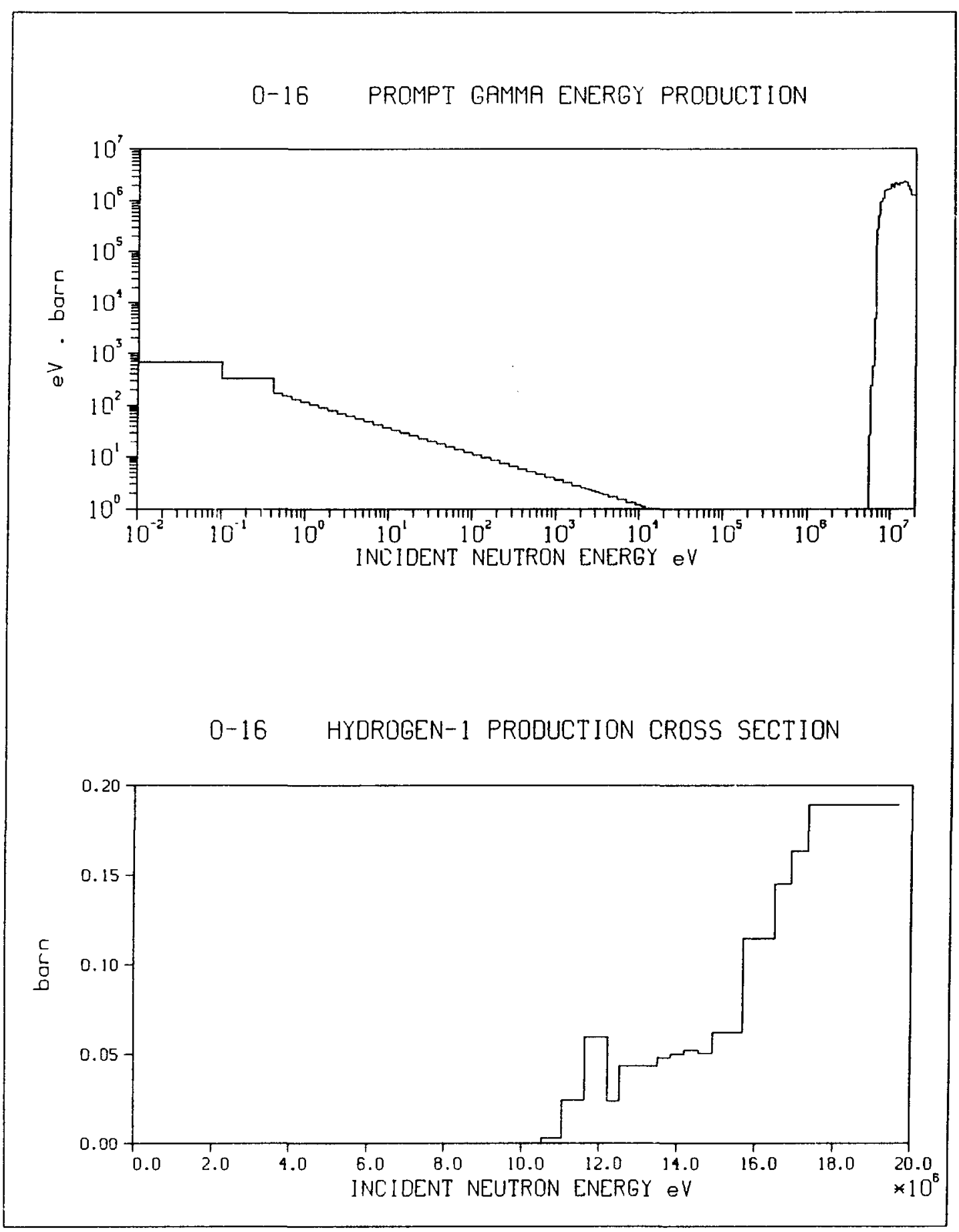




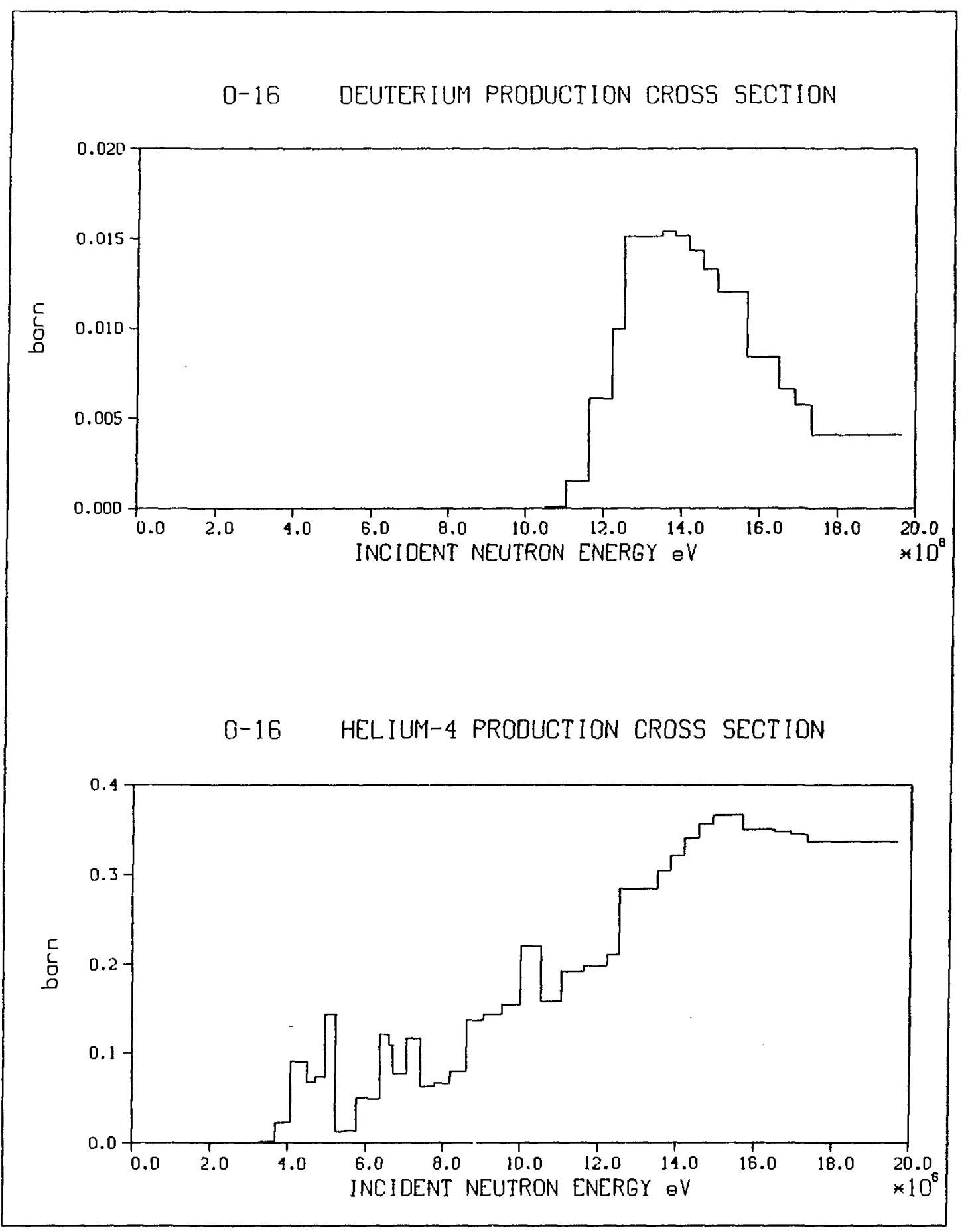




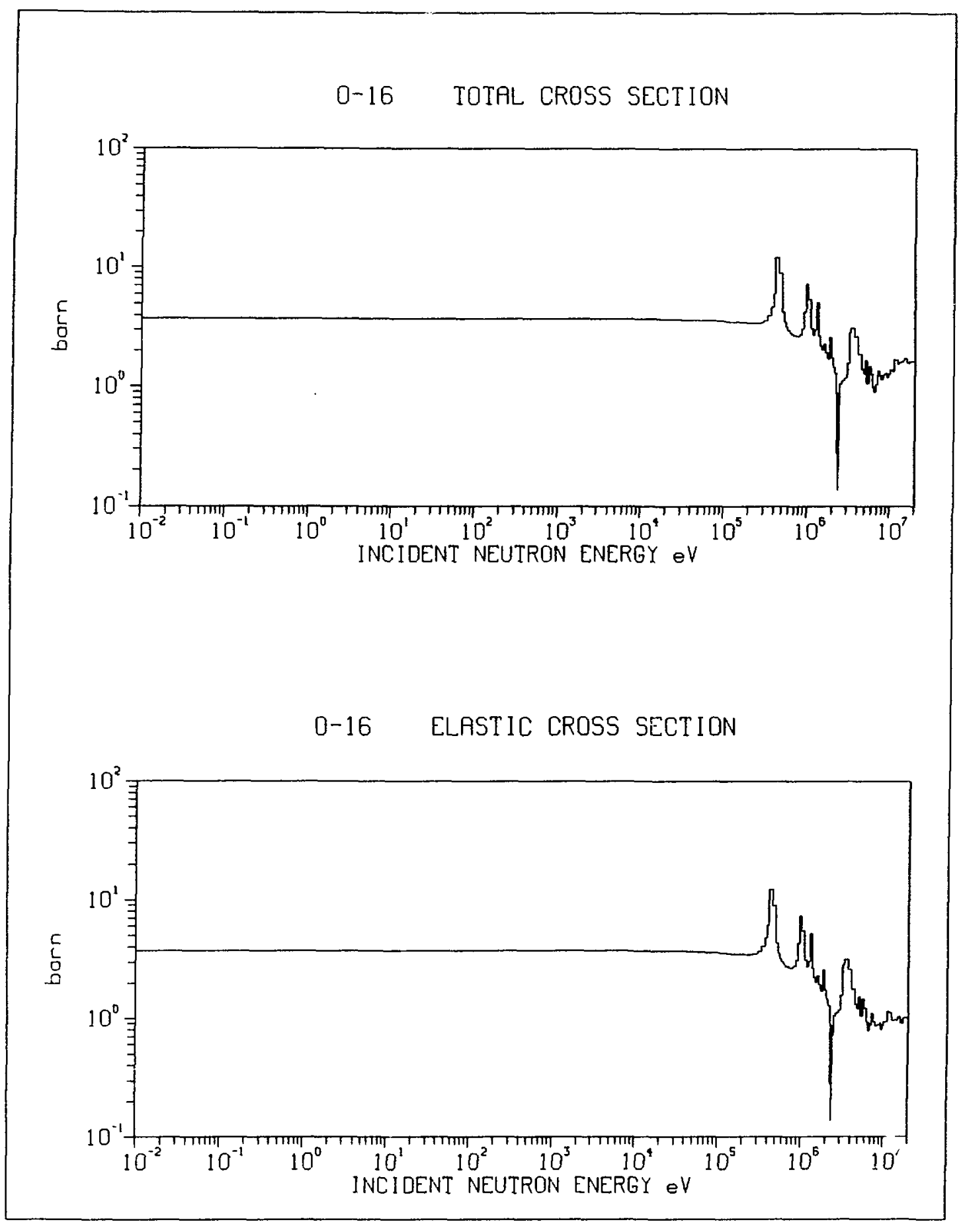




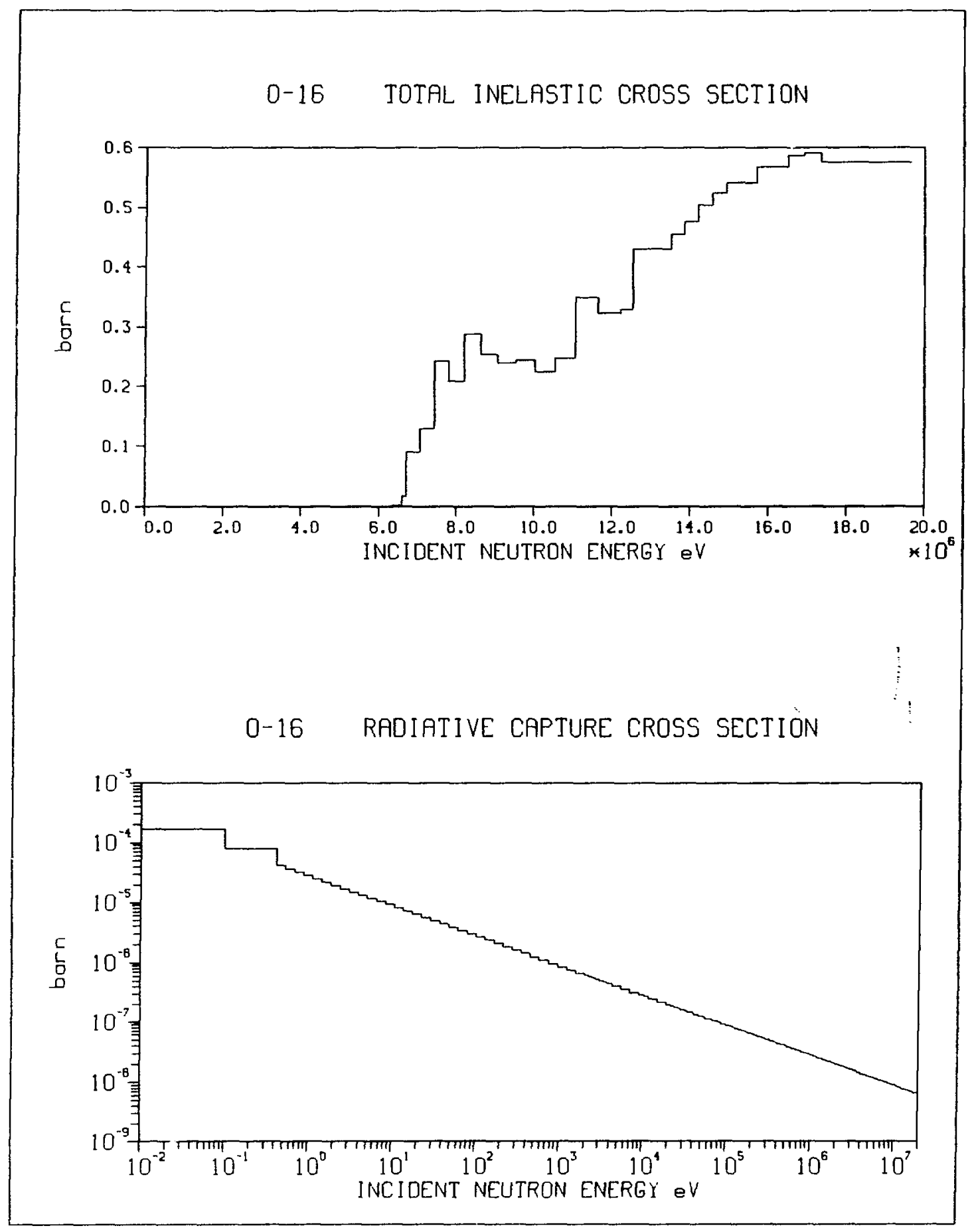




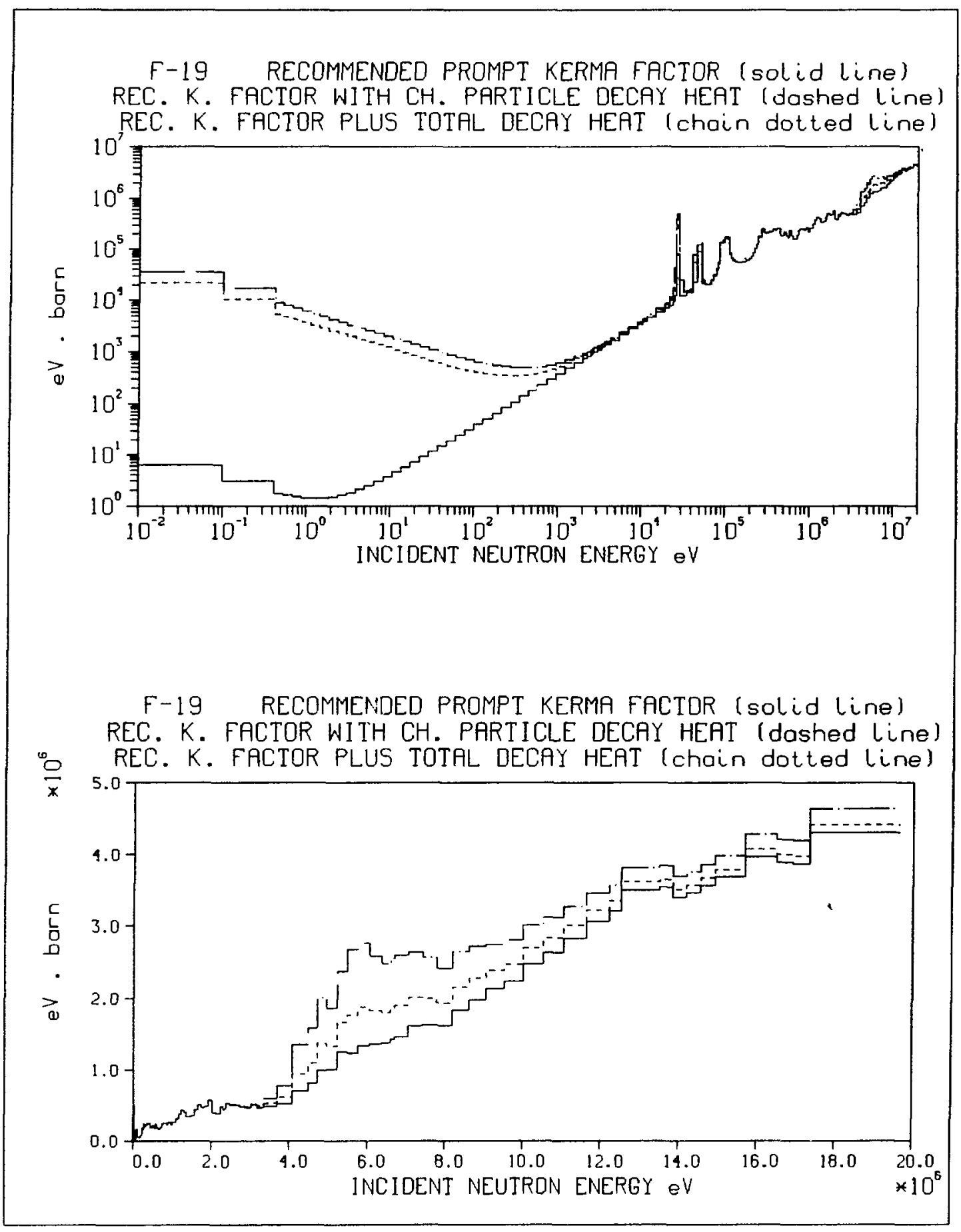




\section{F-19 PROMPT GAMMA ENERGY PRODUCTION}

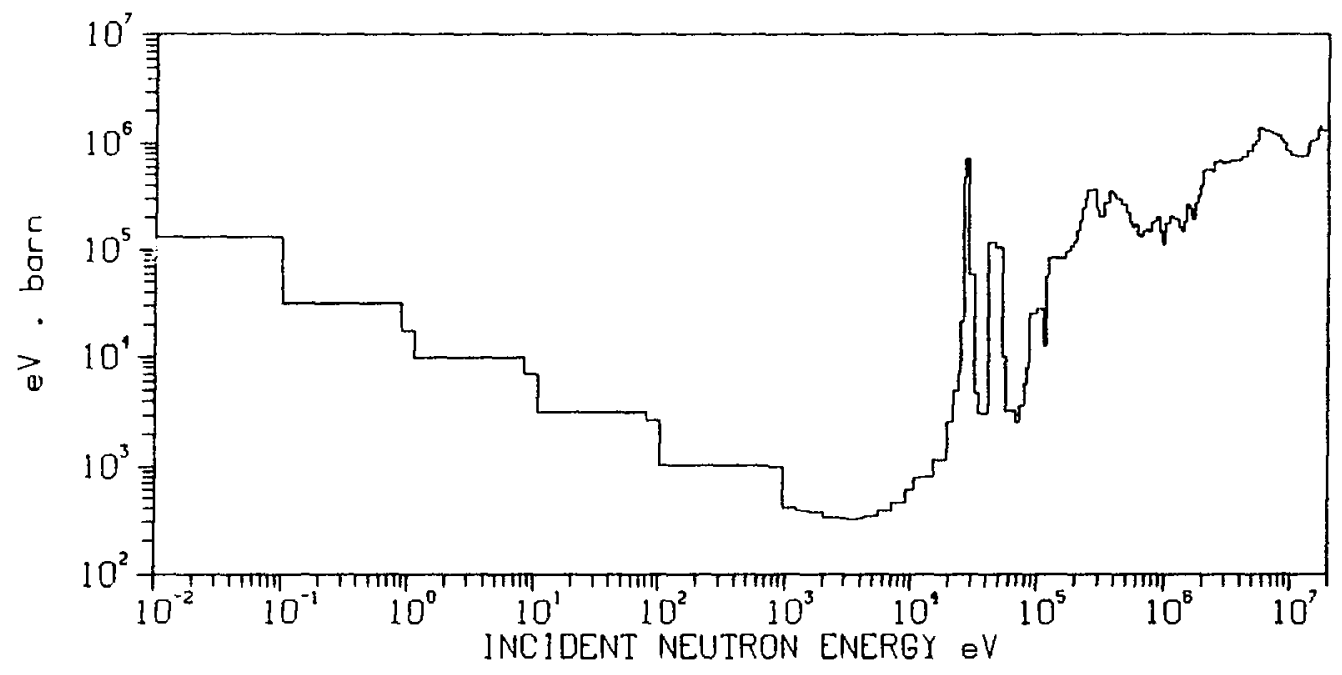

F-19 HYDROGEN-1 PRODUCTION CROSS SECTION

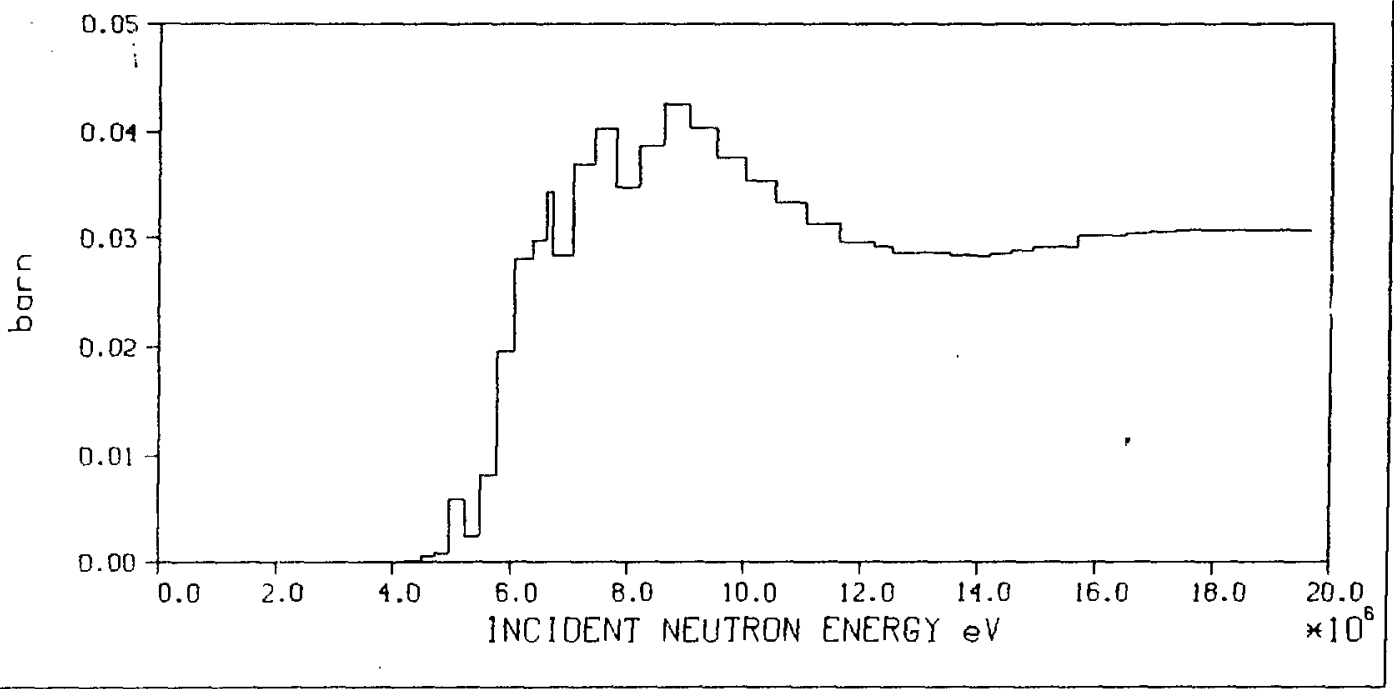




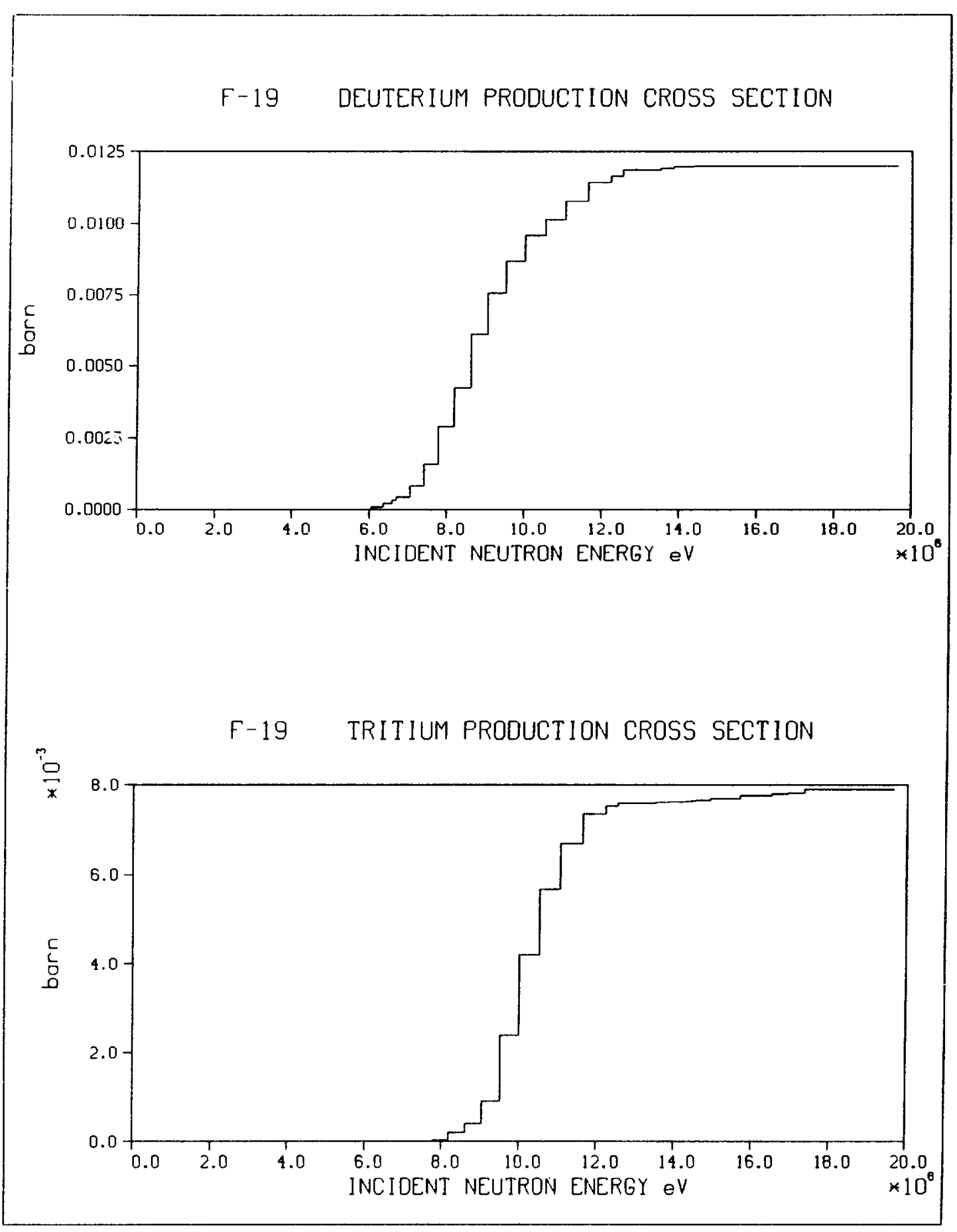




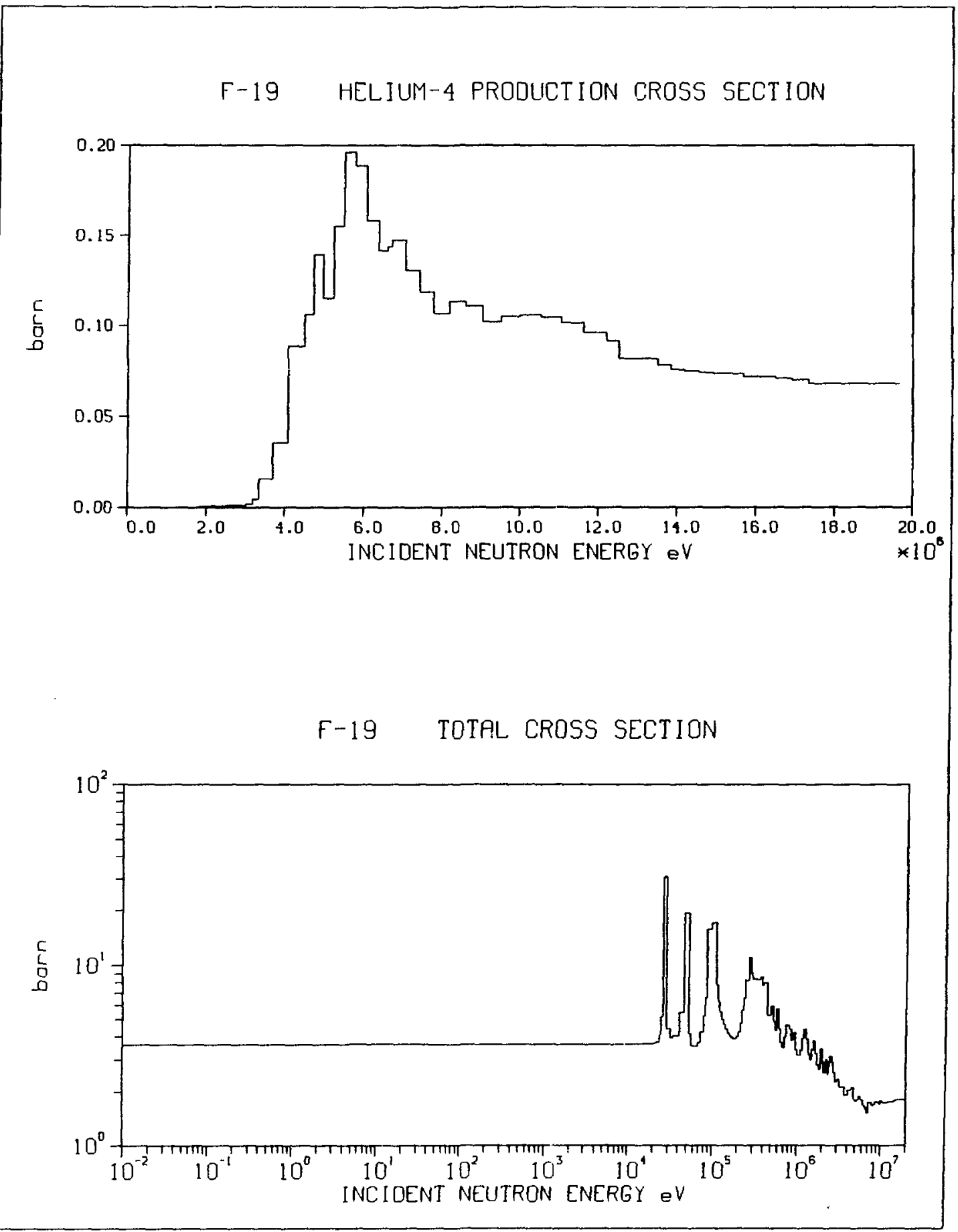




\section{F-19 ELASTIC CROSS SECTION}

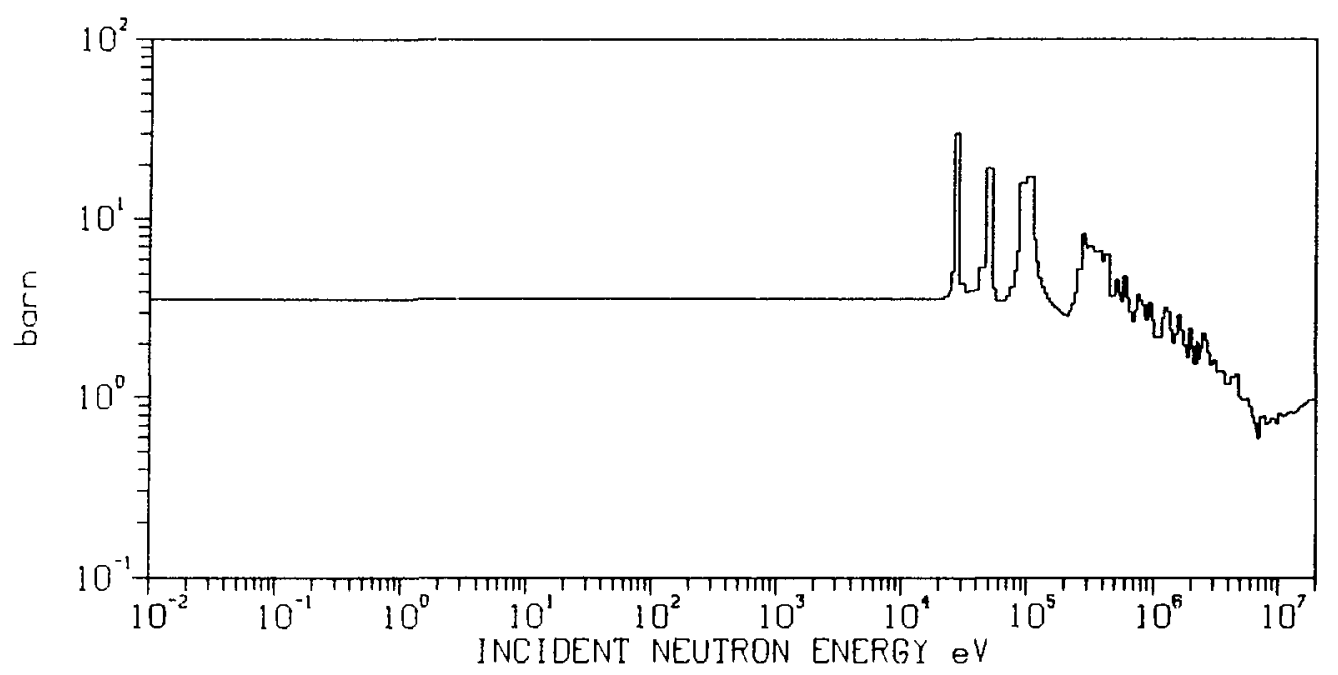

F-19 TOTAL INELASTIC CROSS SECTION

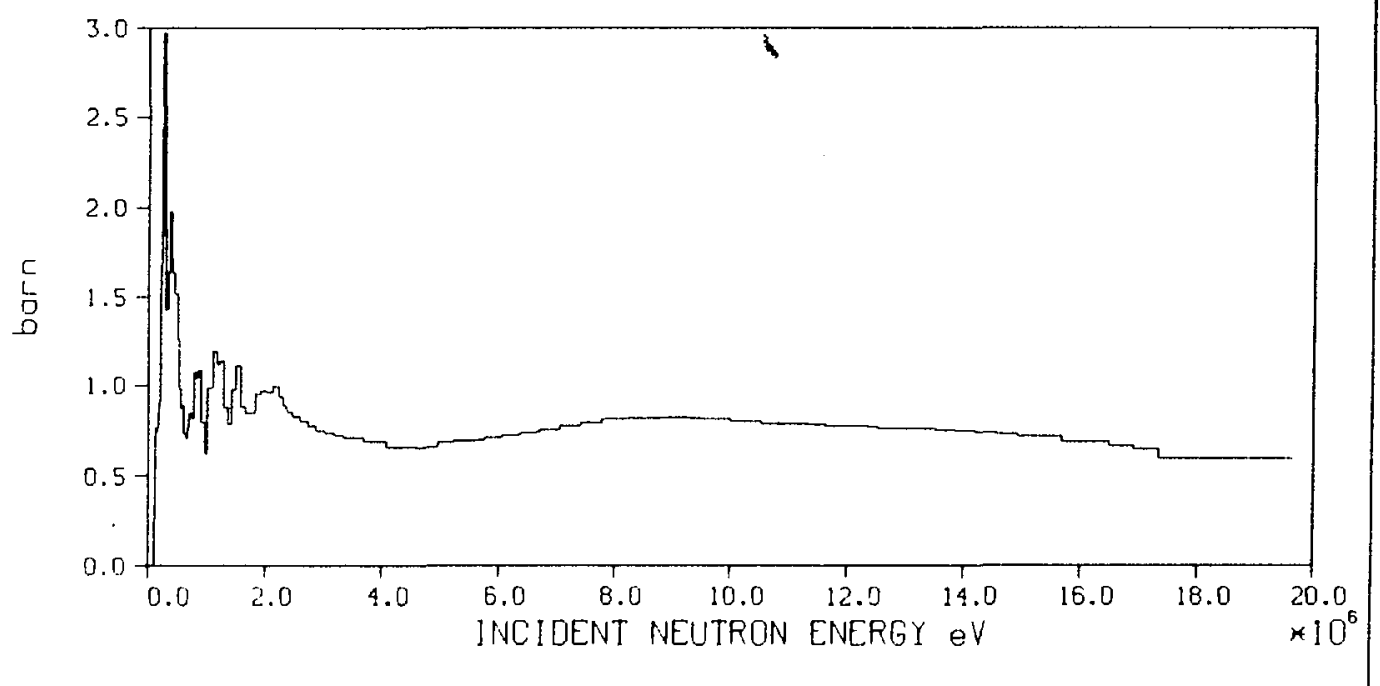




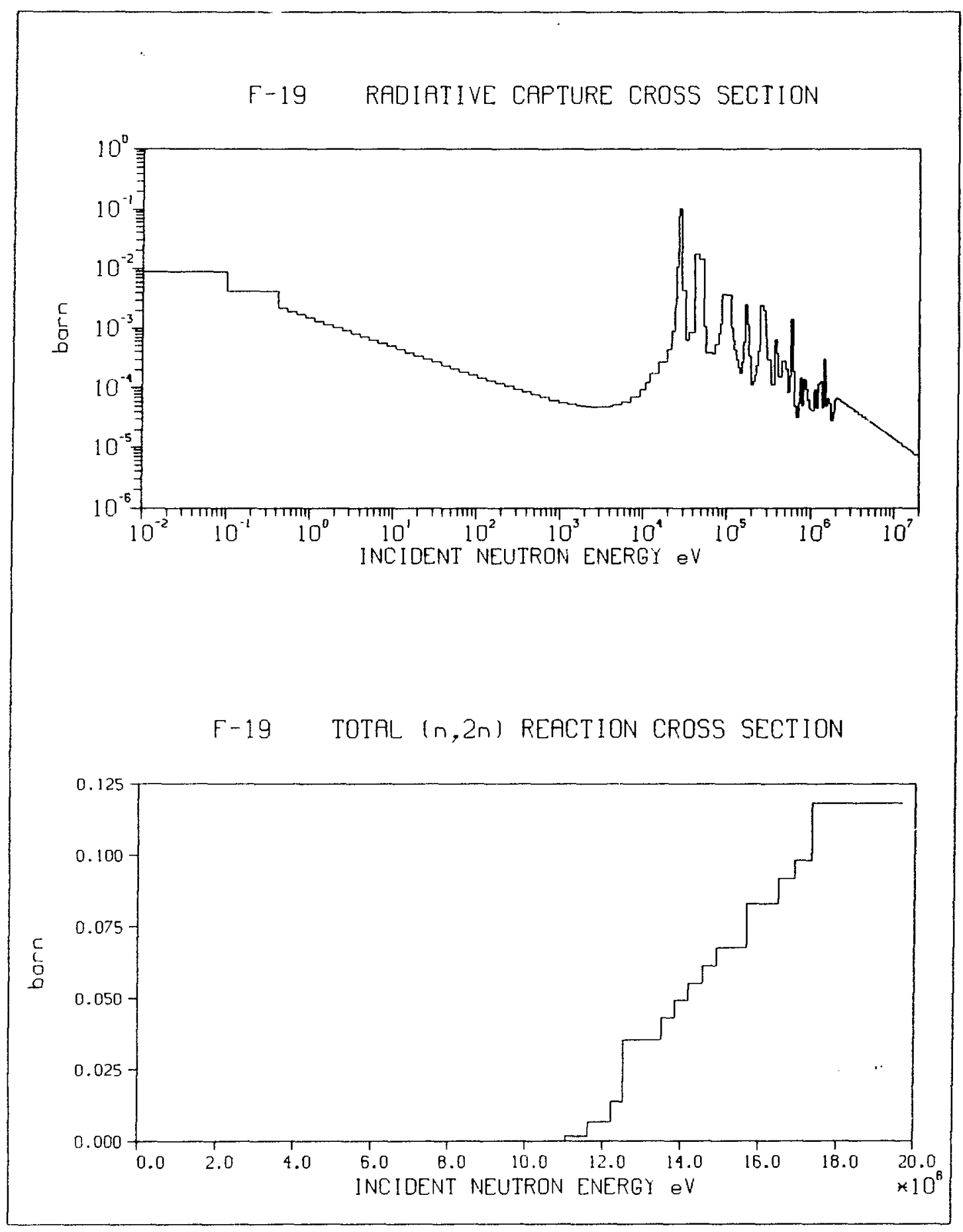


NA-23 RECOMMENDED PROMPT KERMA FACTOR (solid line) REC. K. FACTOR WITH CH. PARTICLE DECAY HEAT (dashed line)

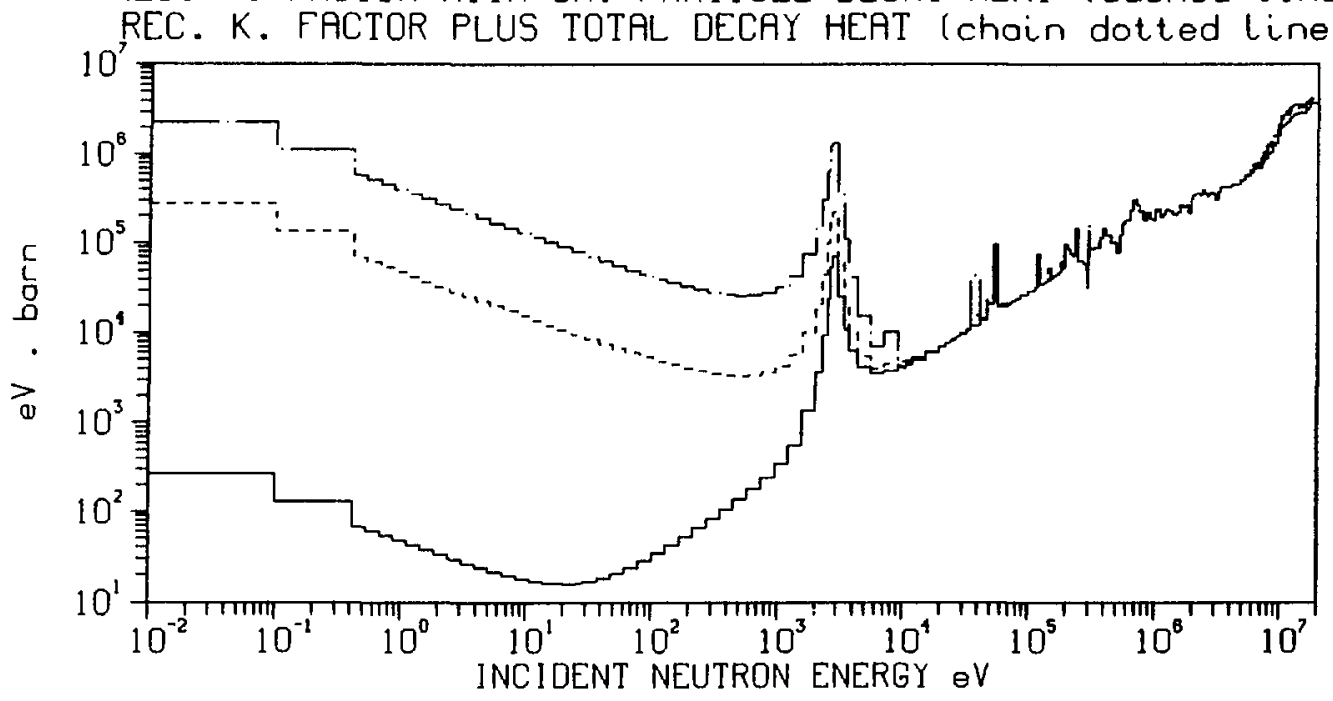

NA-23 RECOMMENDED PROMPT KERMA FACTOR (solid line) REC. K. FACTOR WITH CH. PARTICLE DECAY HEAT (dashed line) ¿ REC. K. FACTOR PLUS TOTAL DECAY HEAT (choin dotted line)

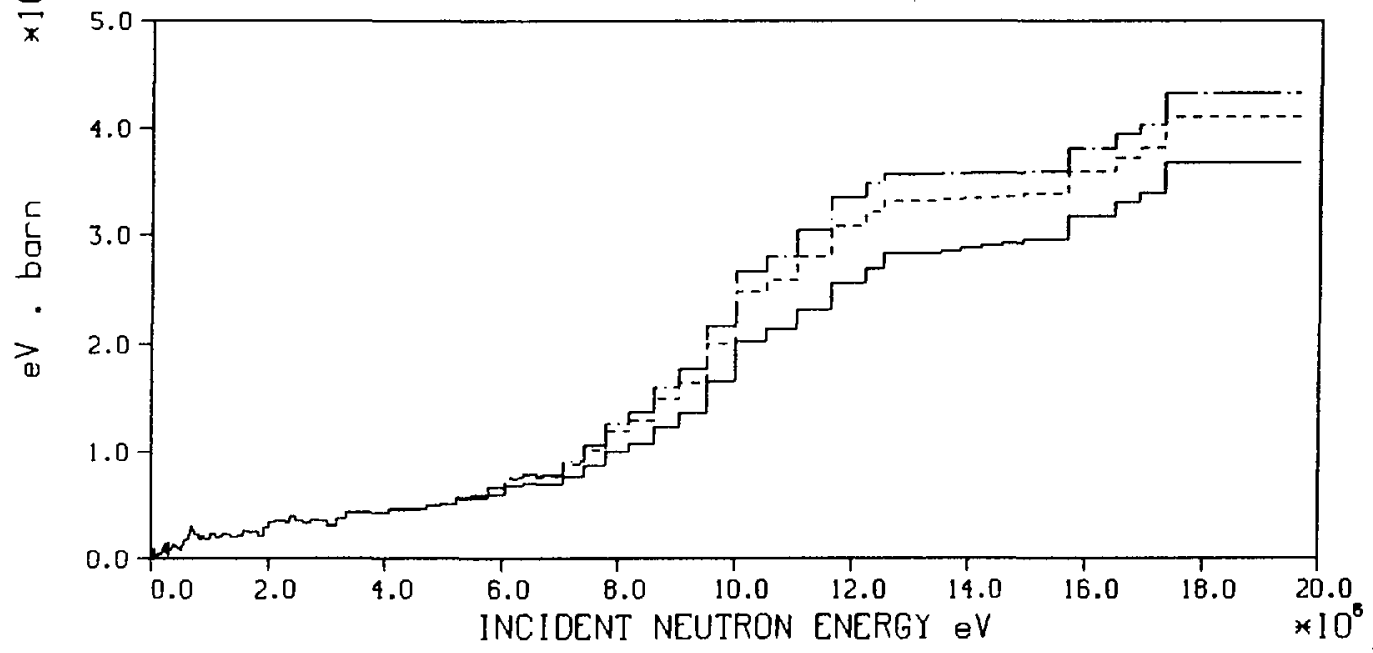




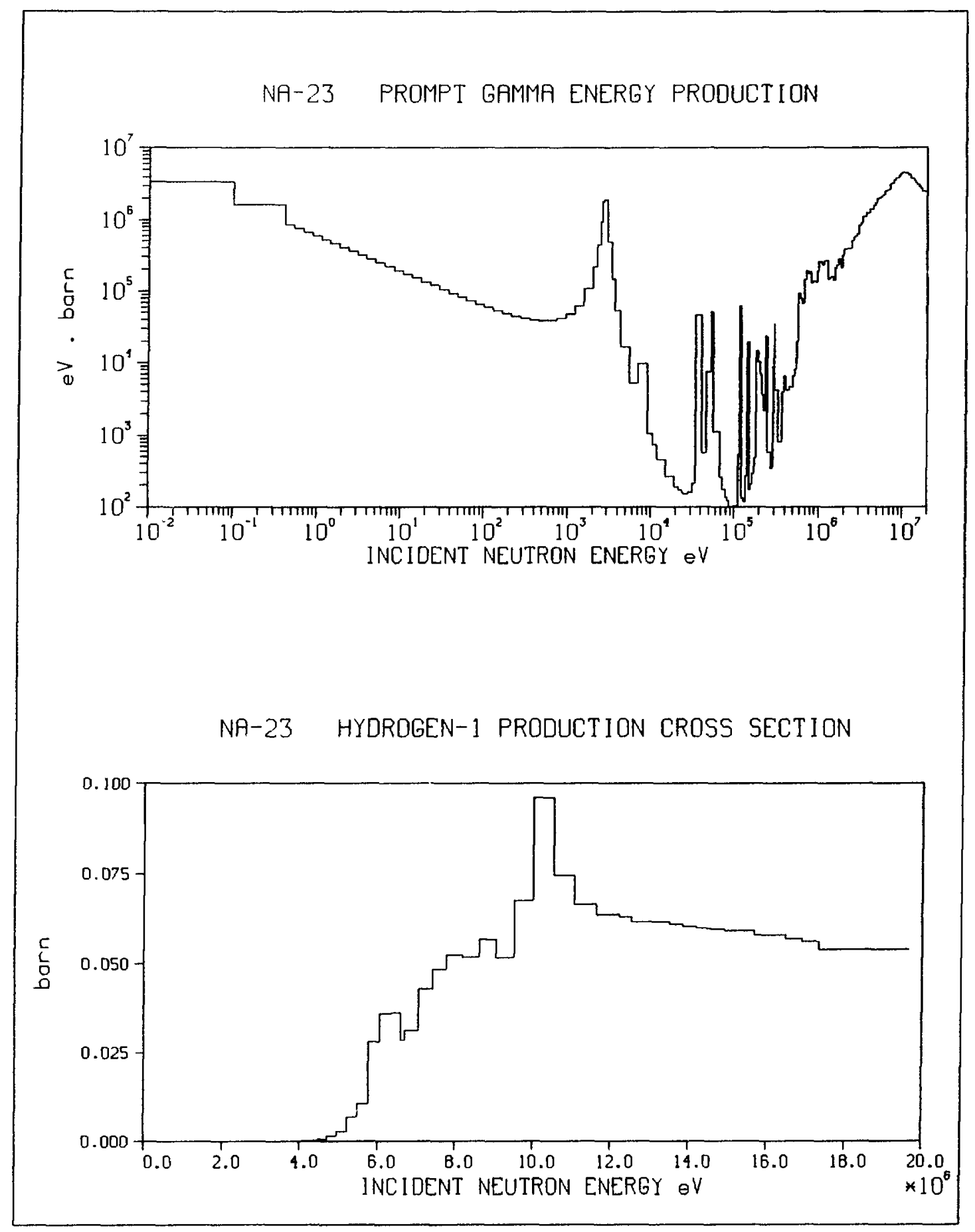




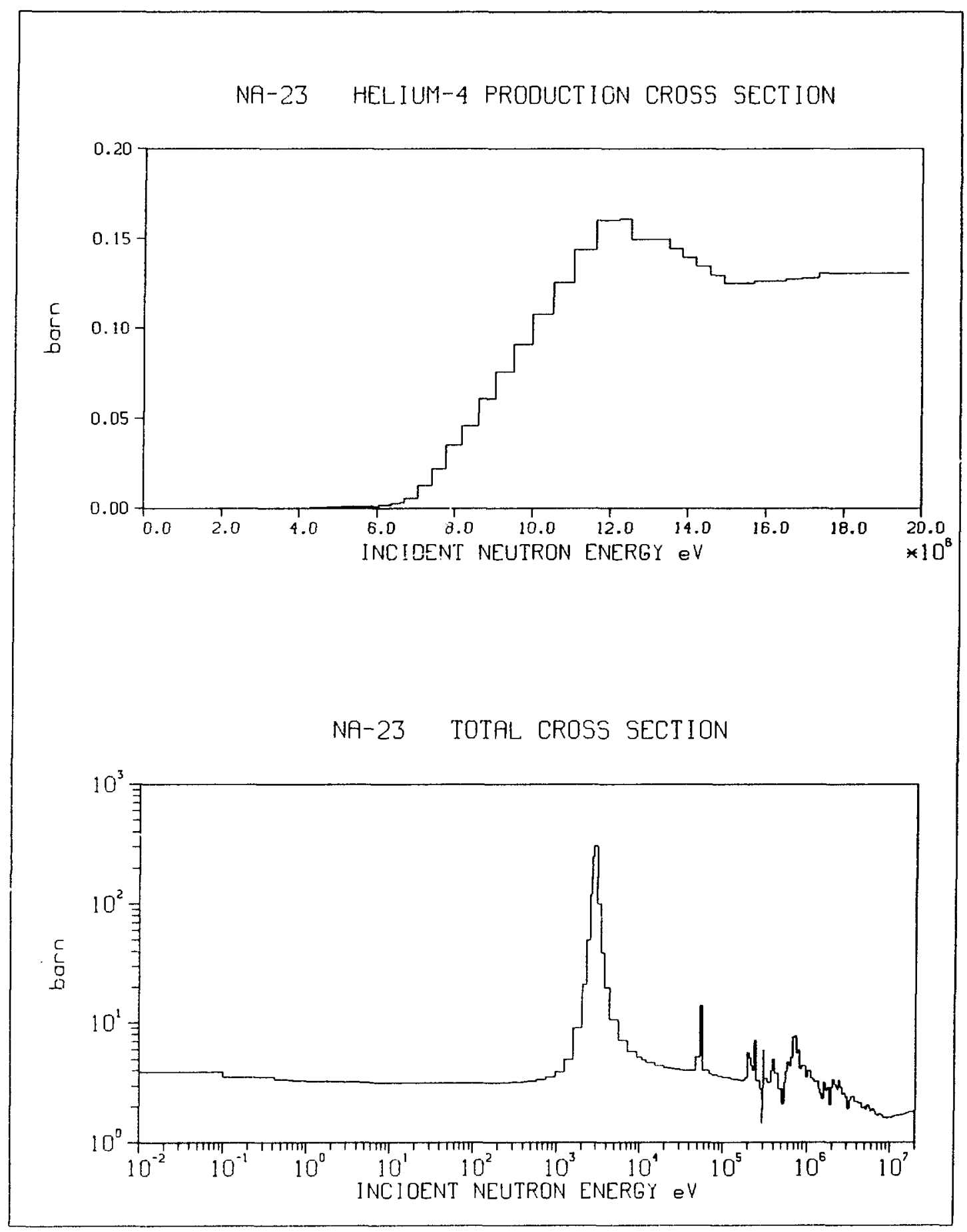




\section{NA-23 ELASTIC CROSS SECTION}

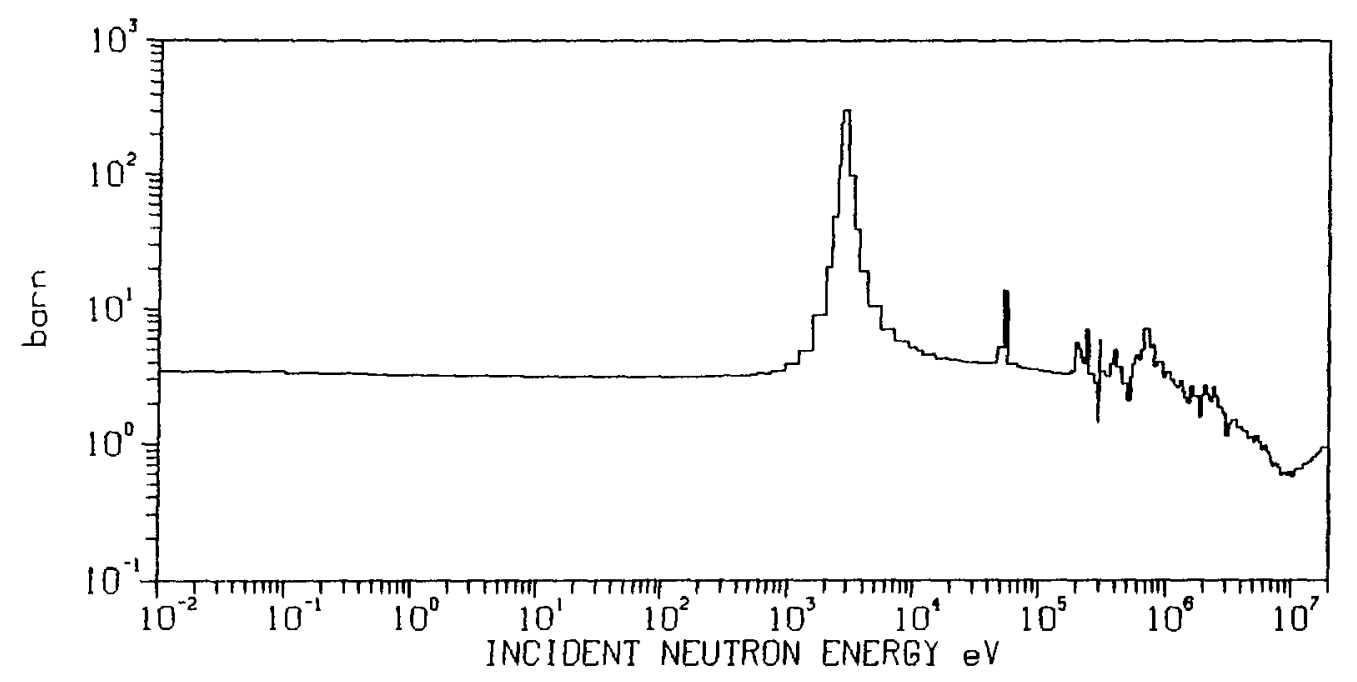

NA-23 TOTAL INELASTIC CROSS SECTION

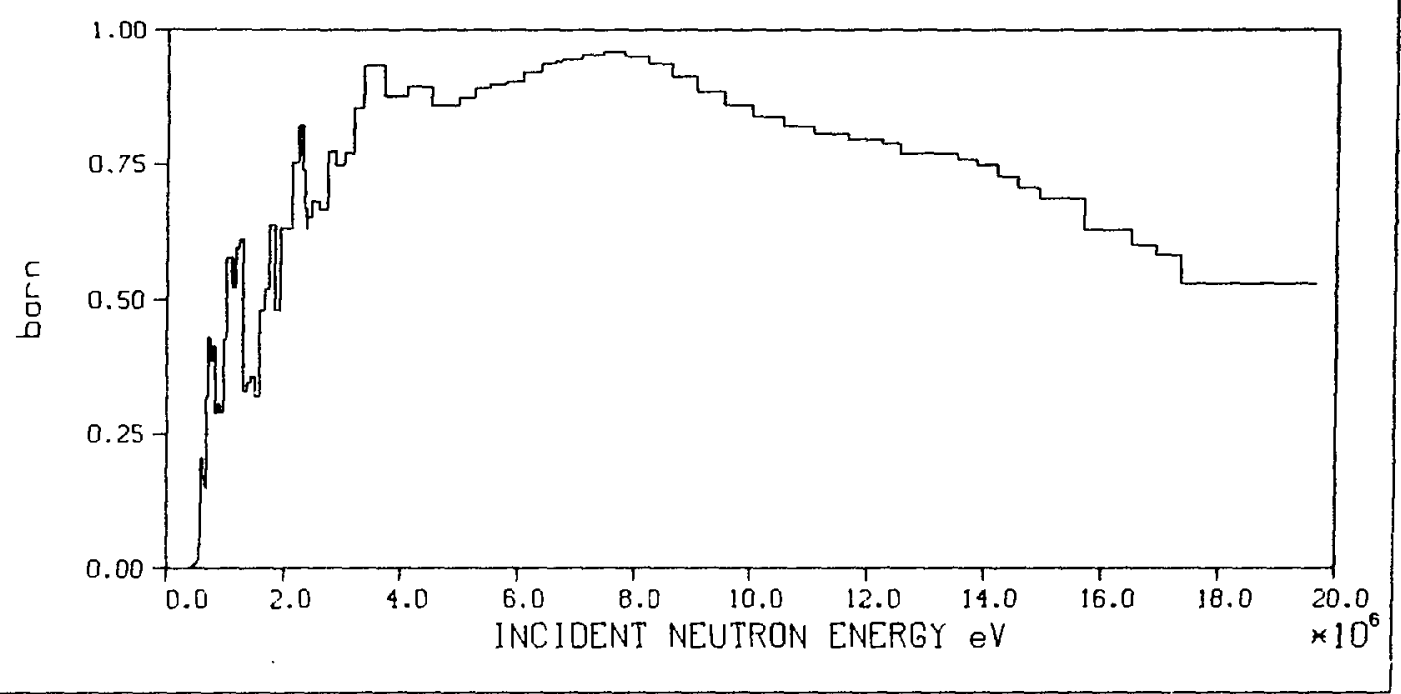




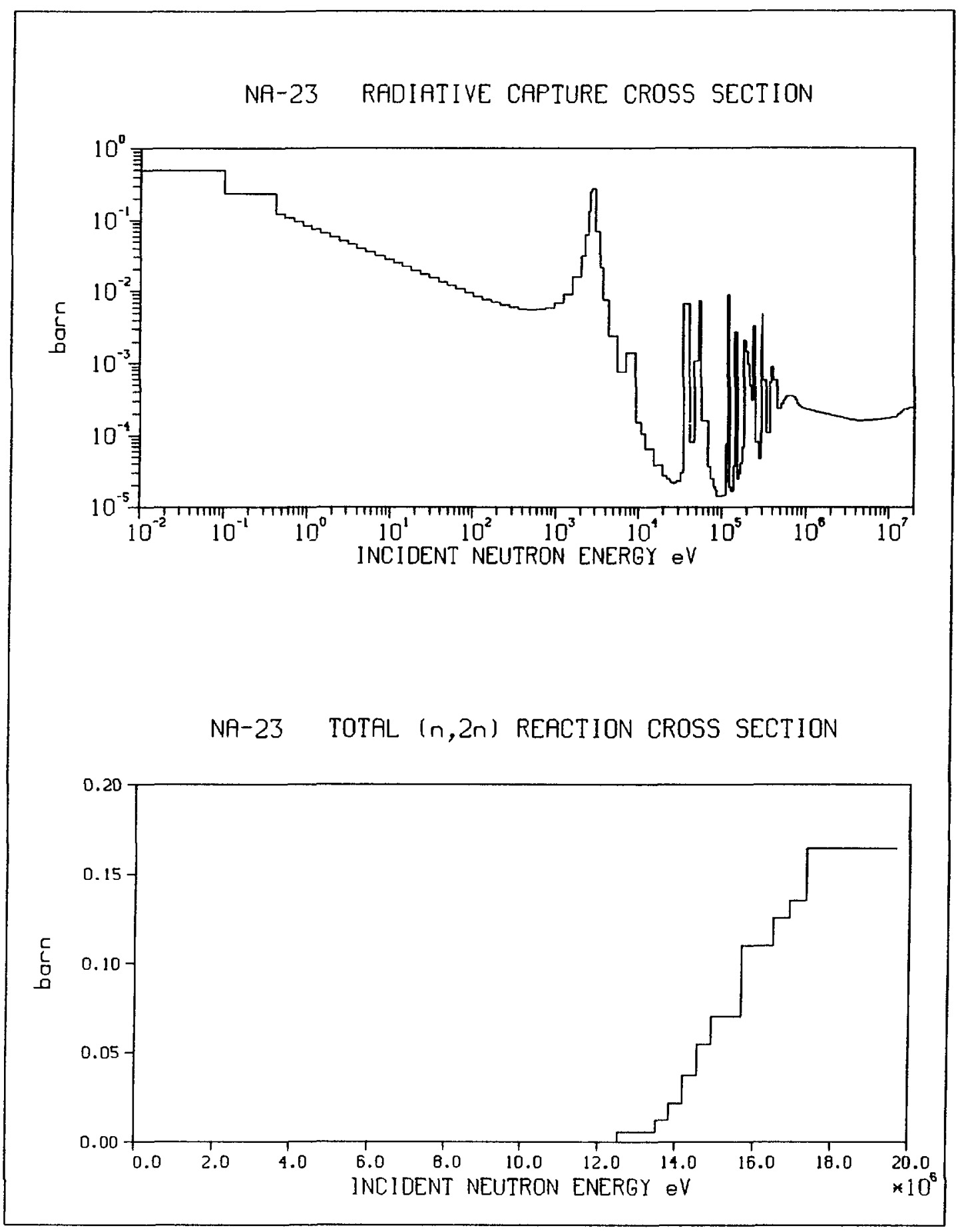




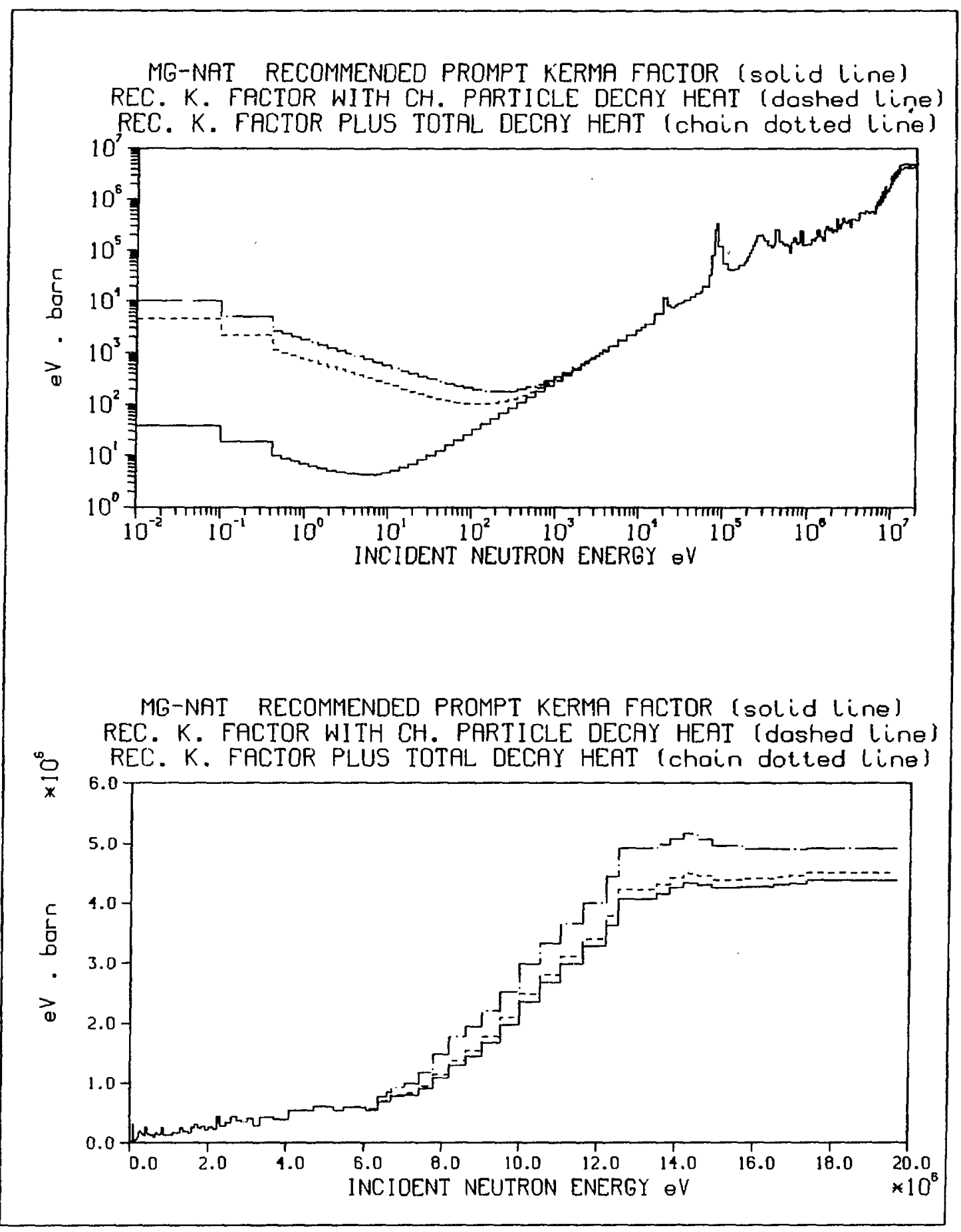


MG-NAT PROMPT GAMMA ENERGY PRODUCTION

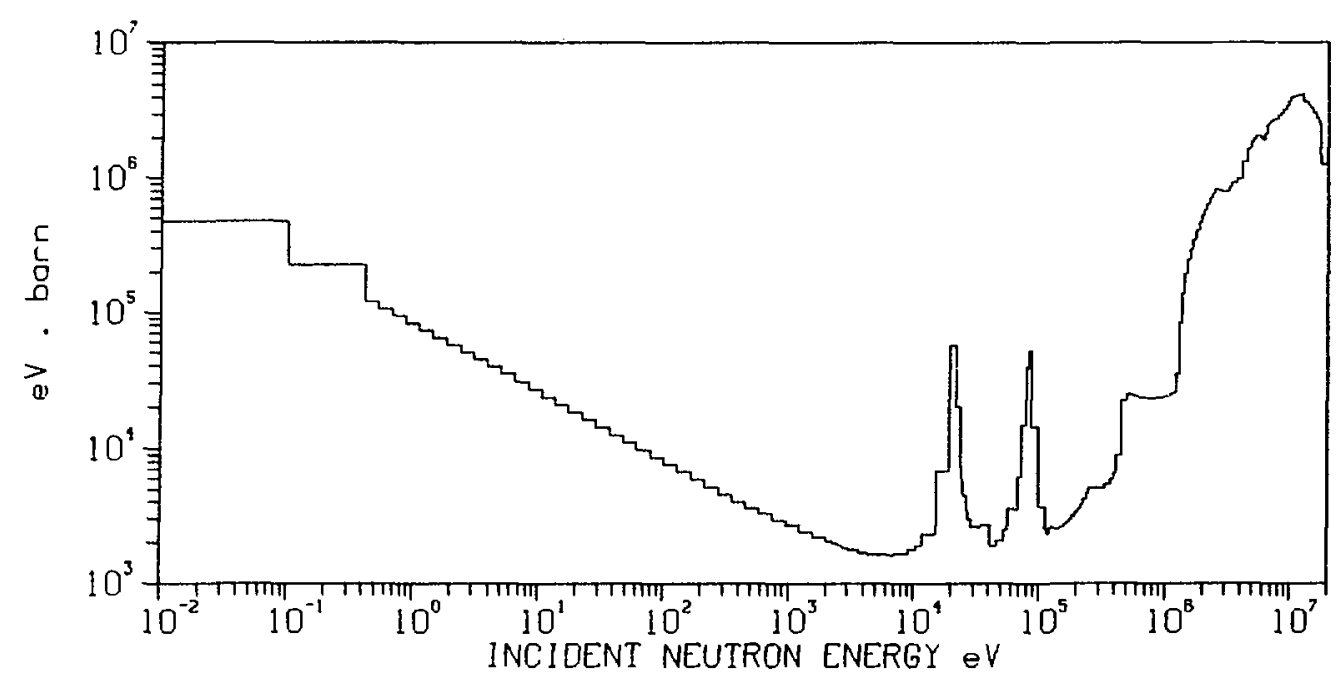

MG-NAT HYDRDGEN-1 PRODUCTION CROSS SECTION

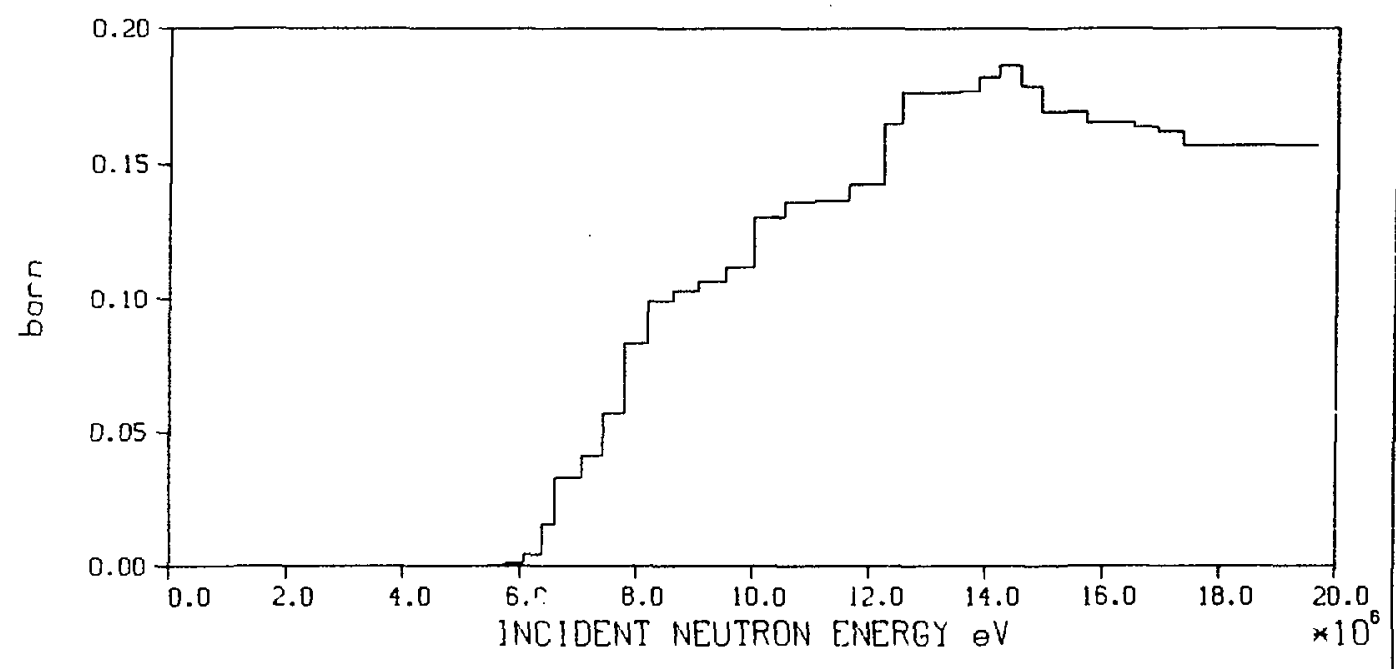




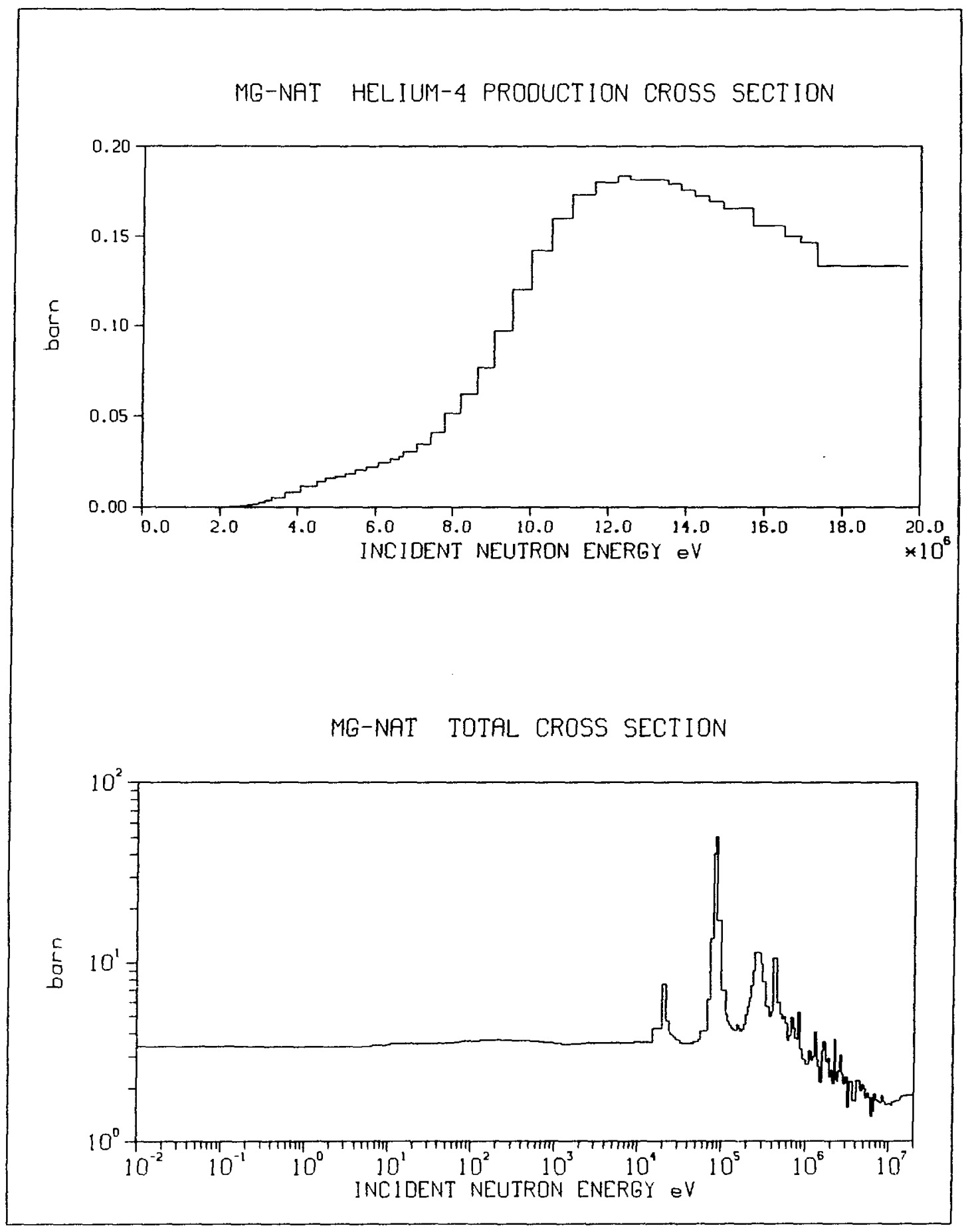




\section{MG-NAT ELASTIC CROSS SECTION}

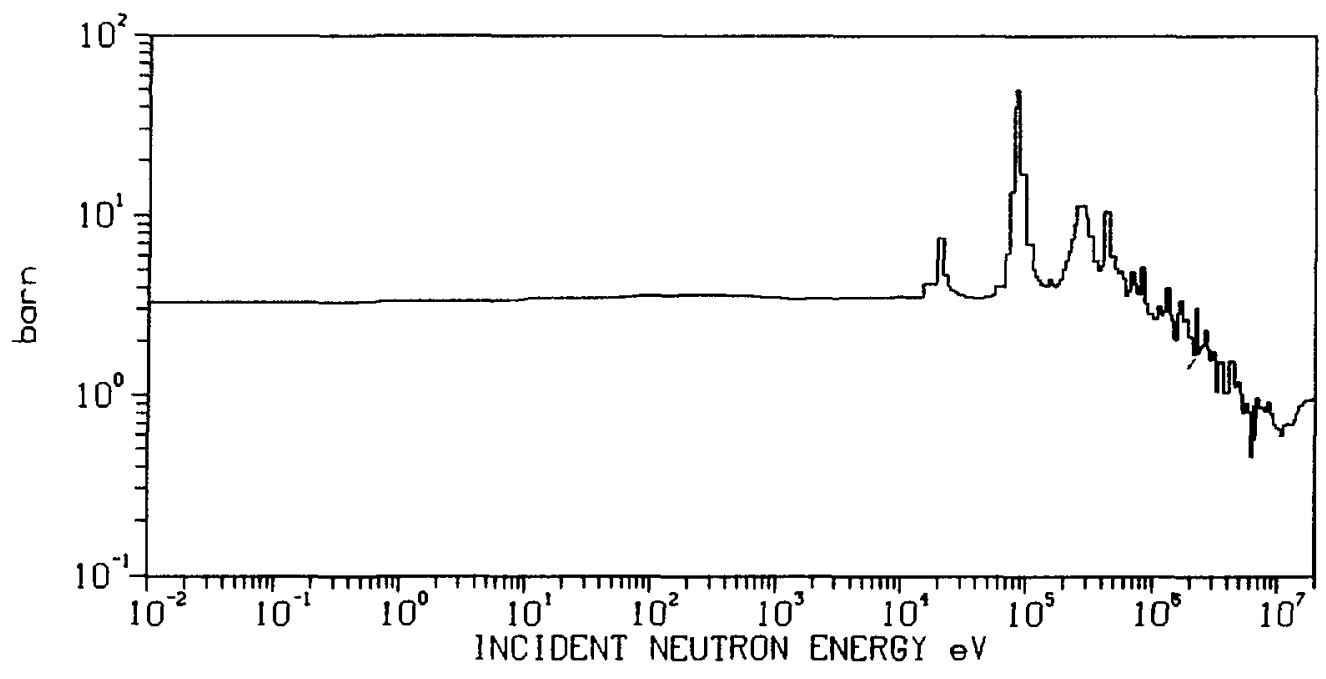

MG-NAT TOTAL INELASTIC CROSS SECTION

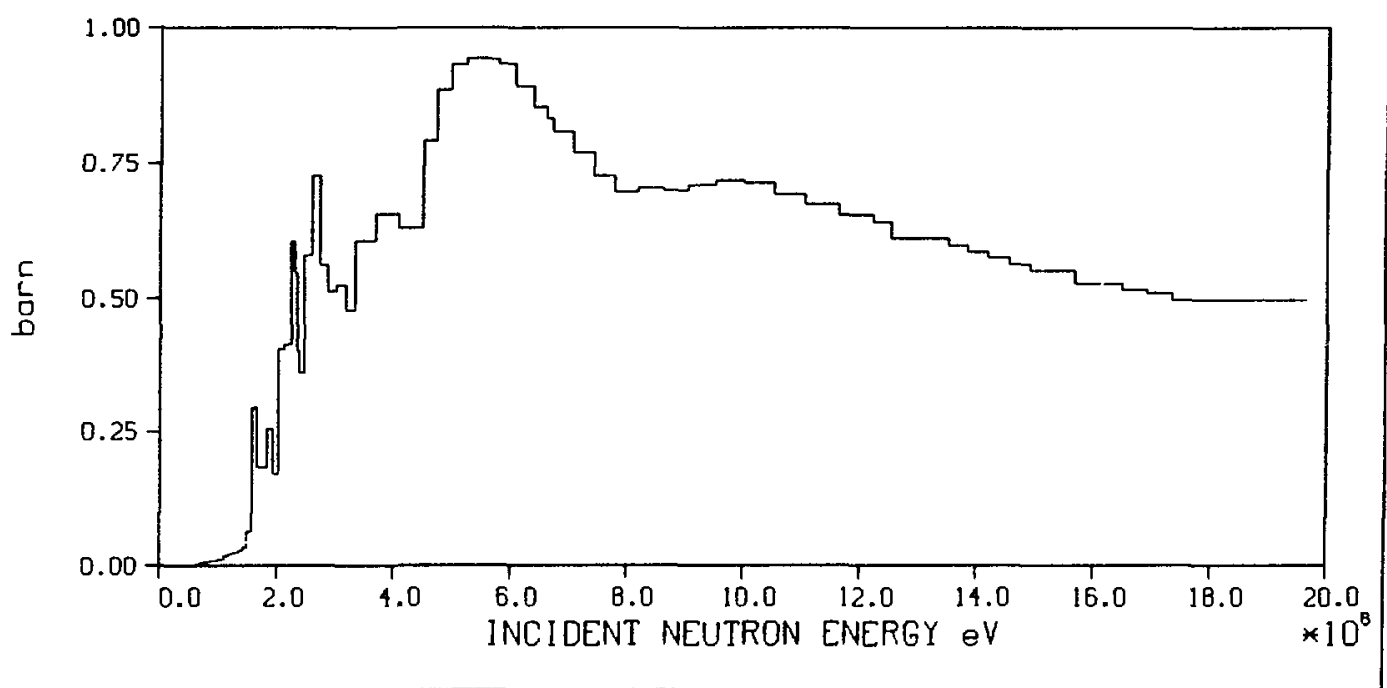




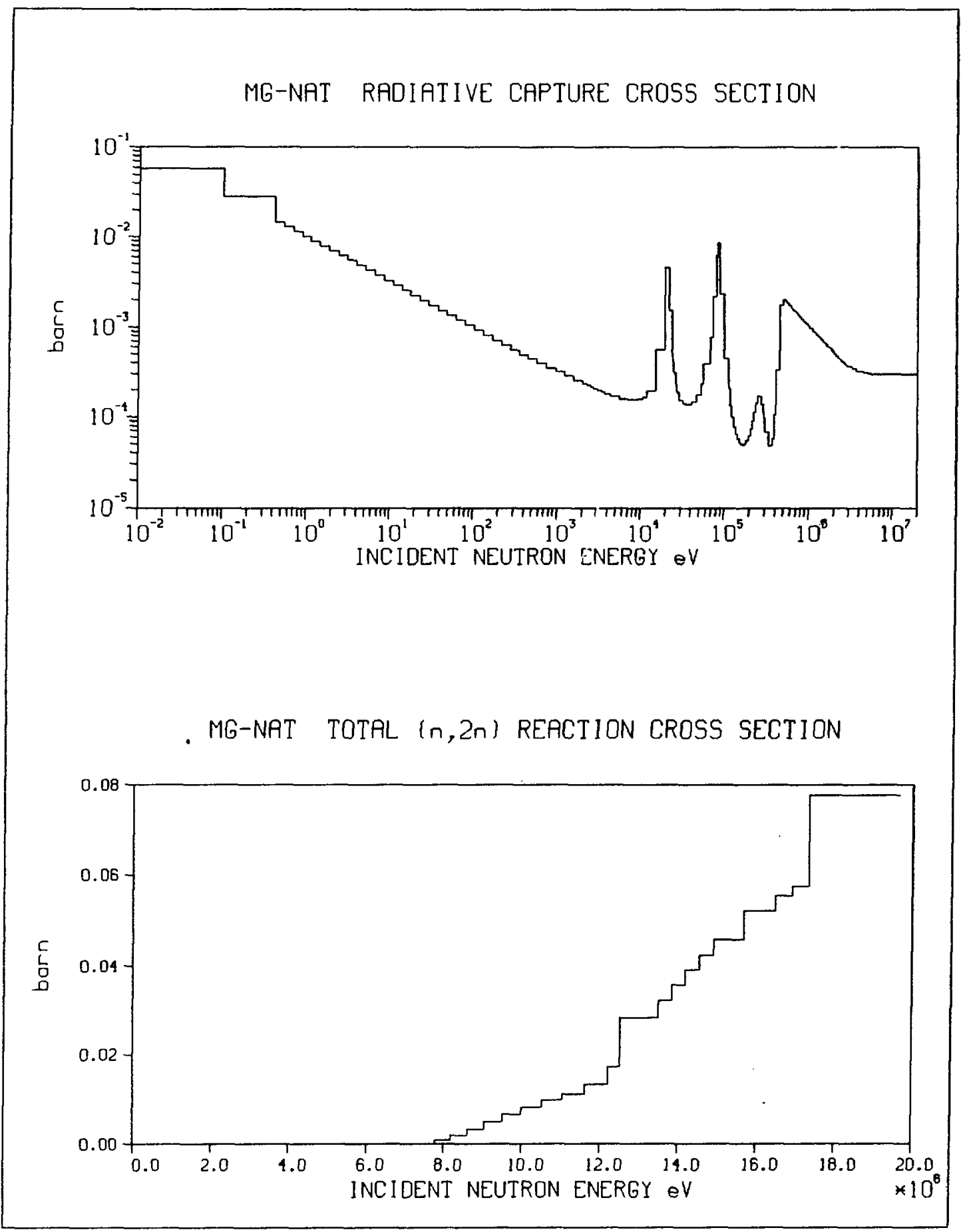




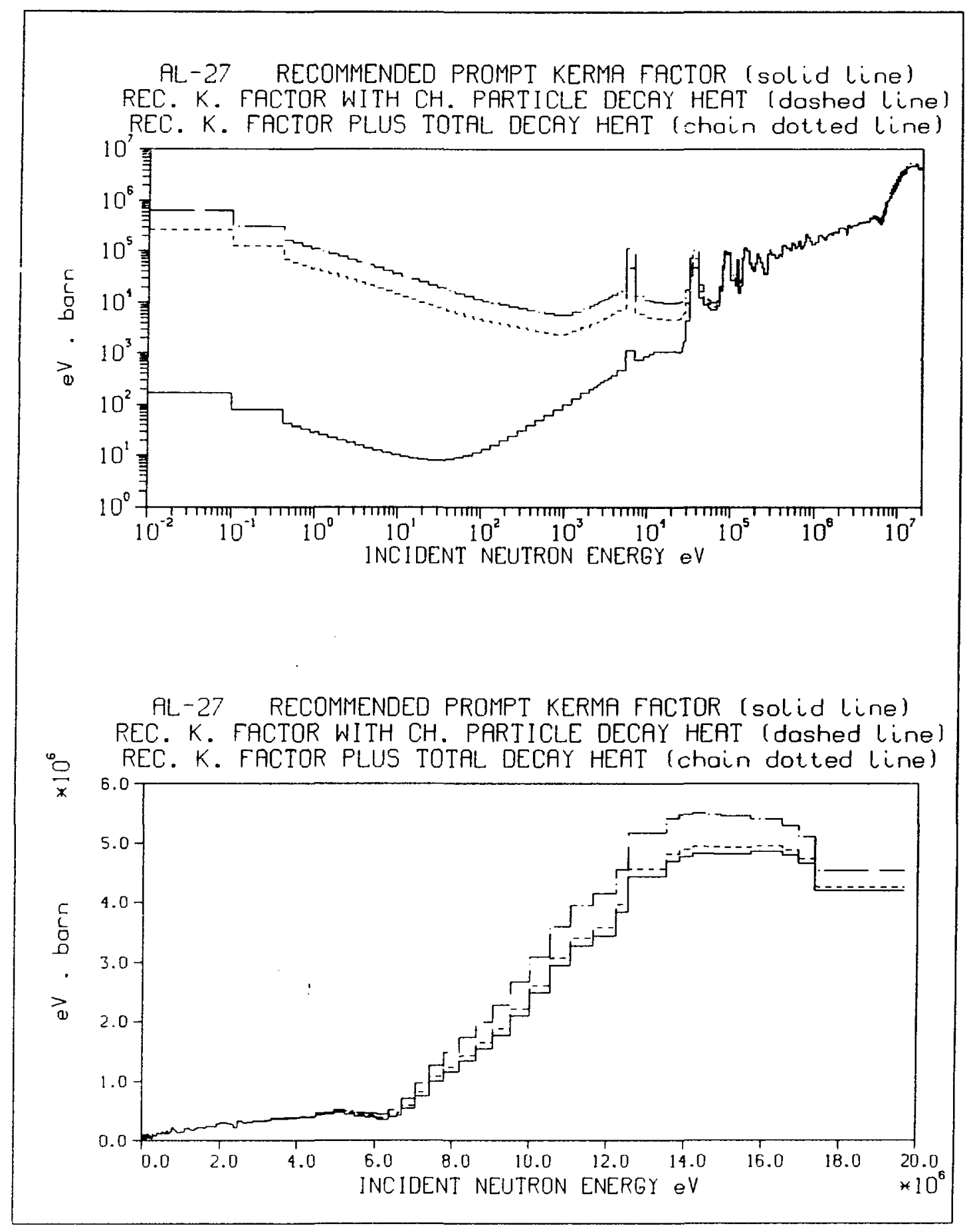




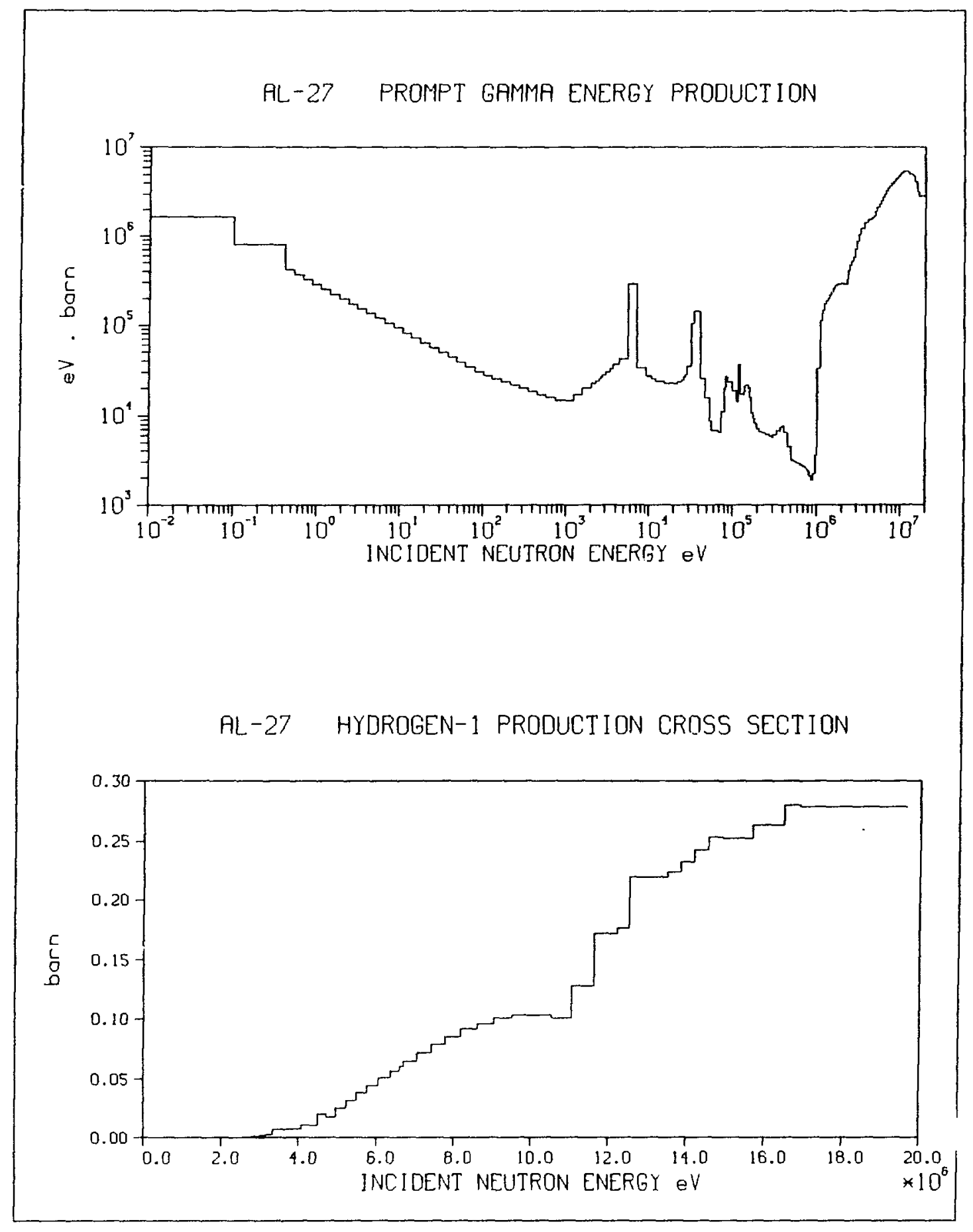




\section{AL-27 DEUTERIUM PRODUCTION CROSS SECTION}

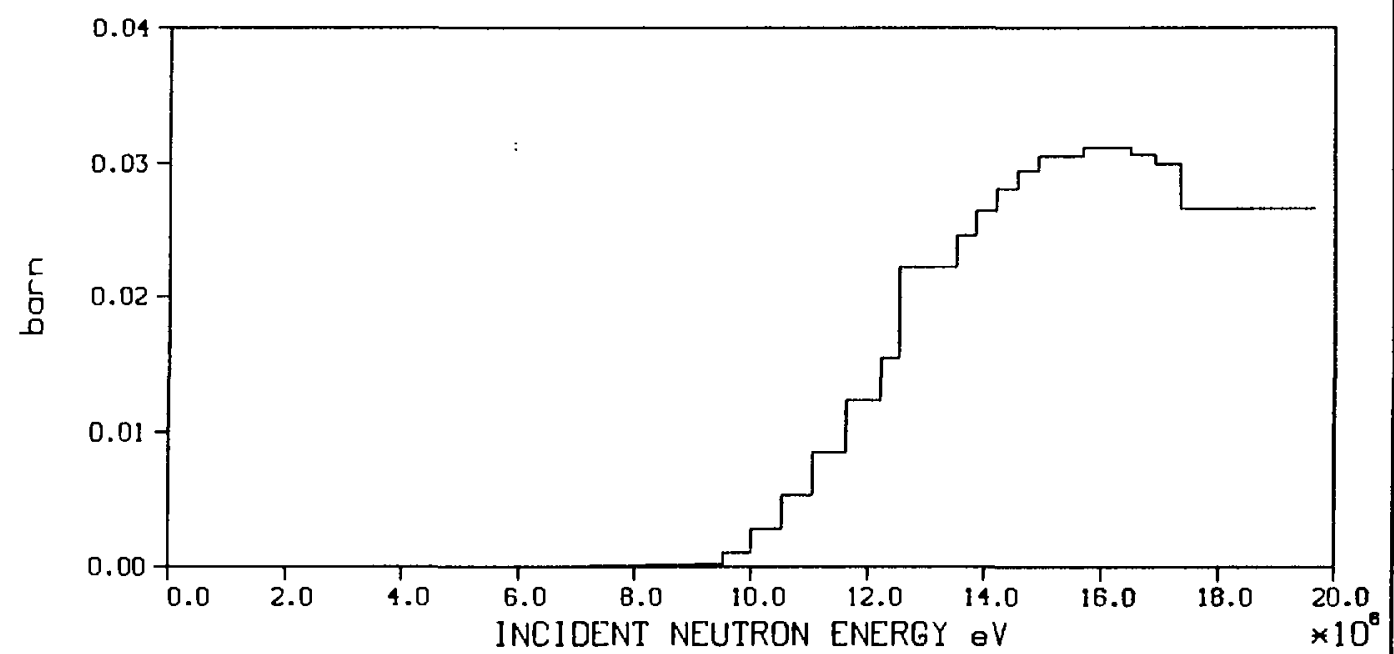

RL-27 TRITIUM PRODUCTION CROSS SECTION

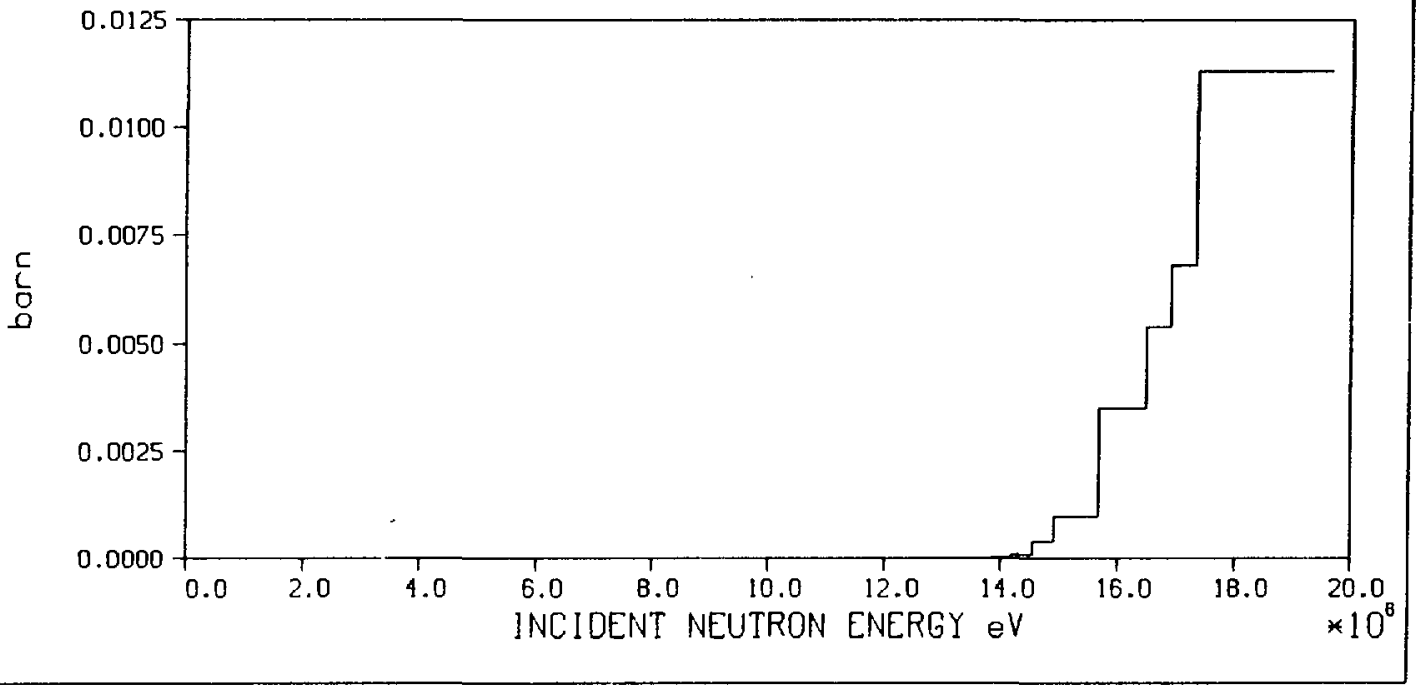




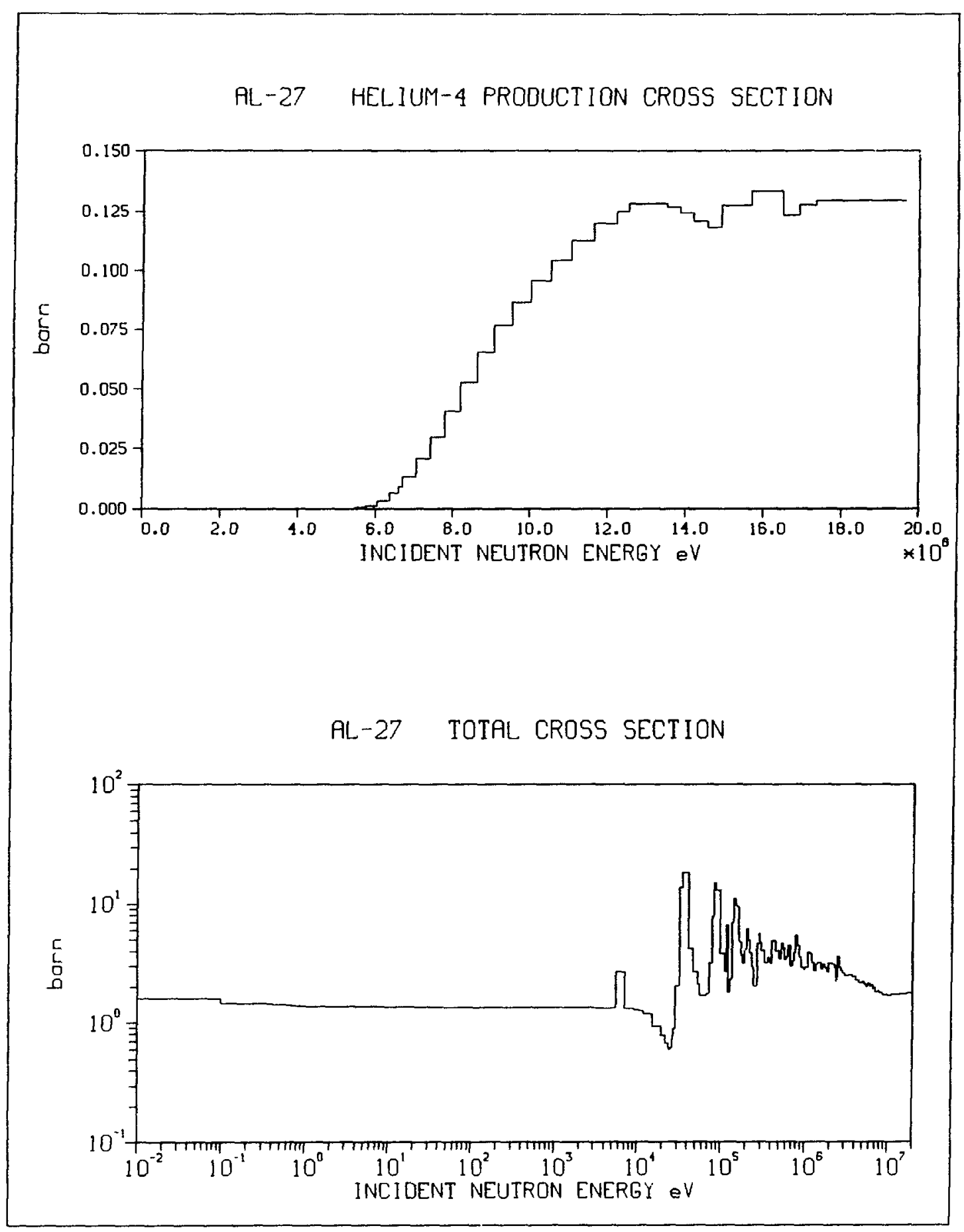




\section{AL-27 ELASTIC CROSS SECTION}

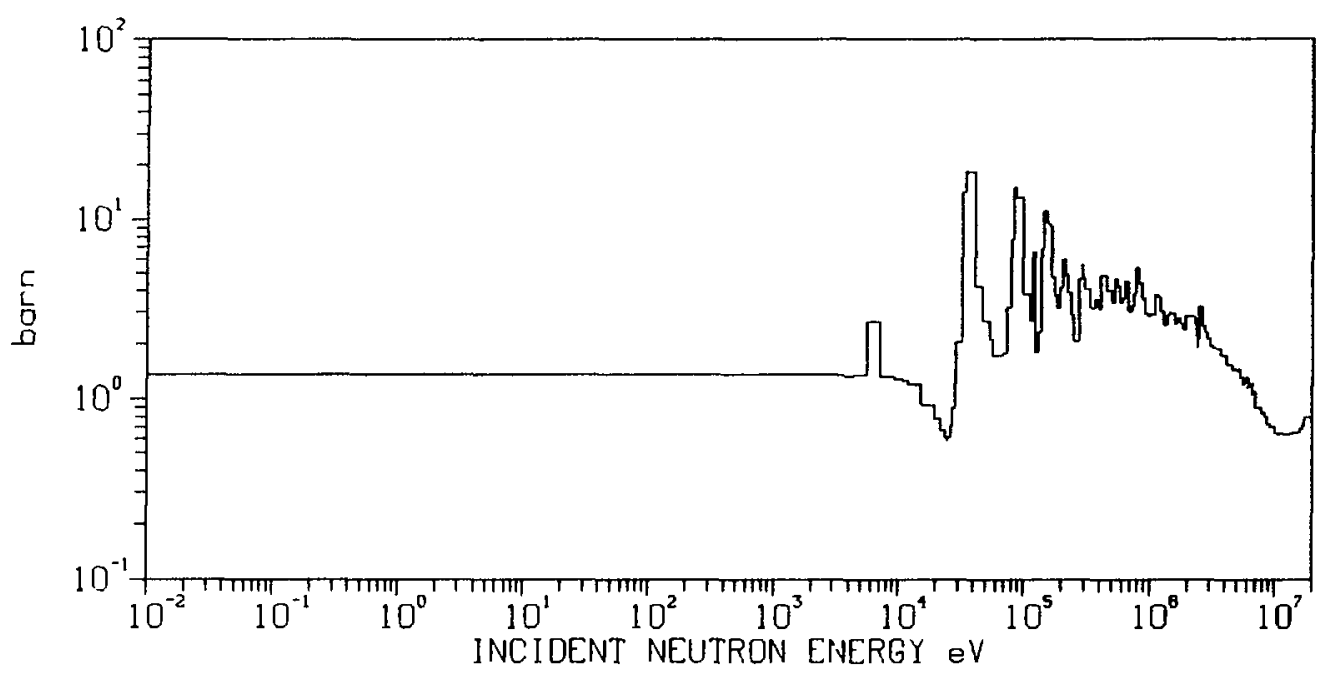

AL-27 TOTAL INELASTIC CROSS SECTION

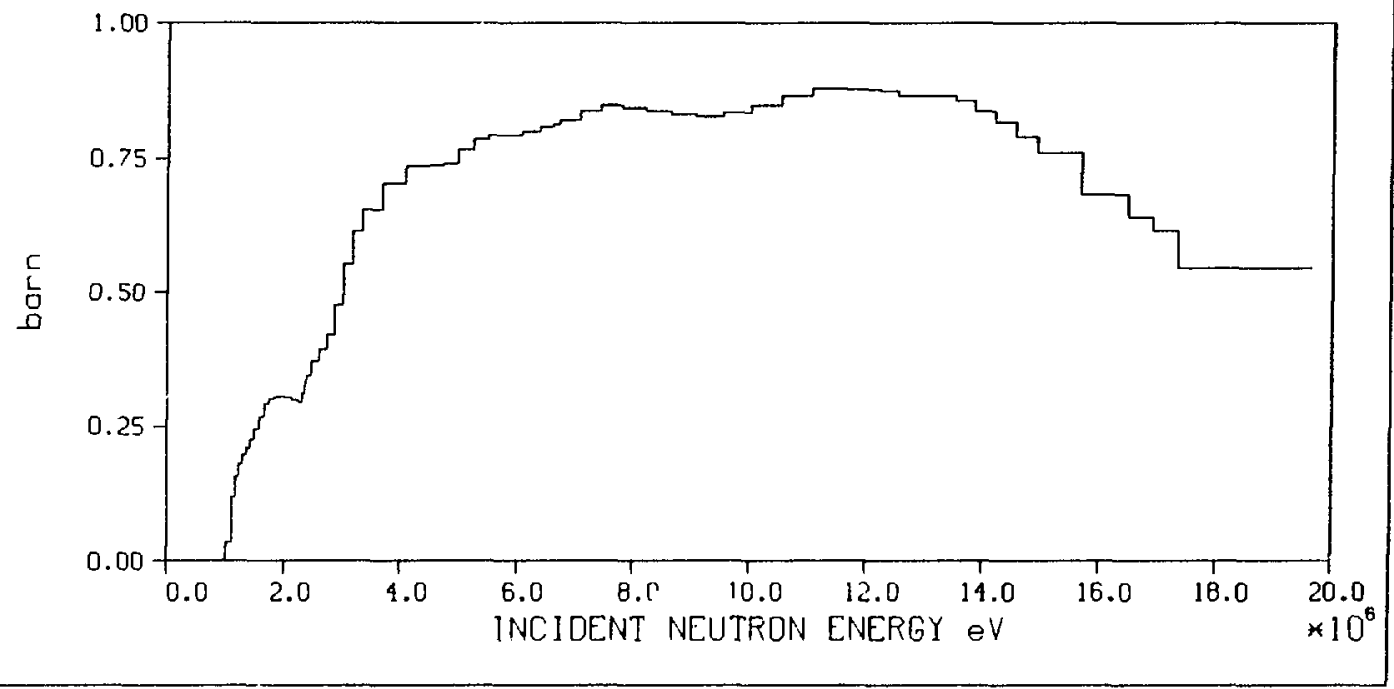




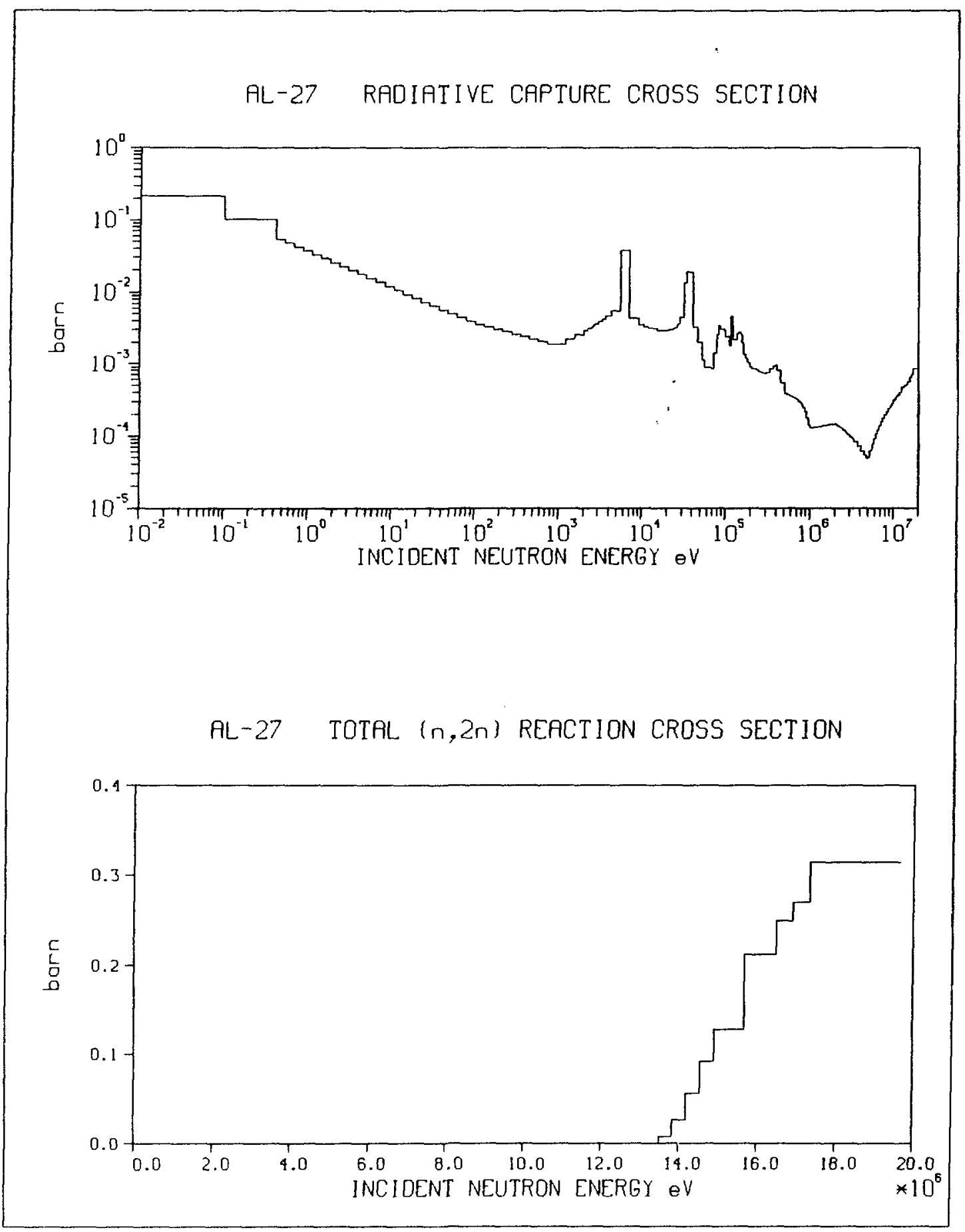




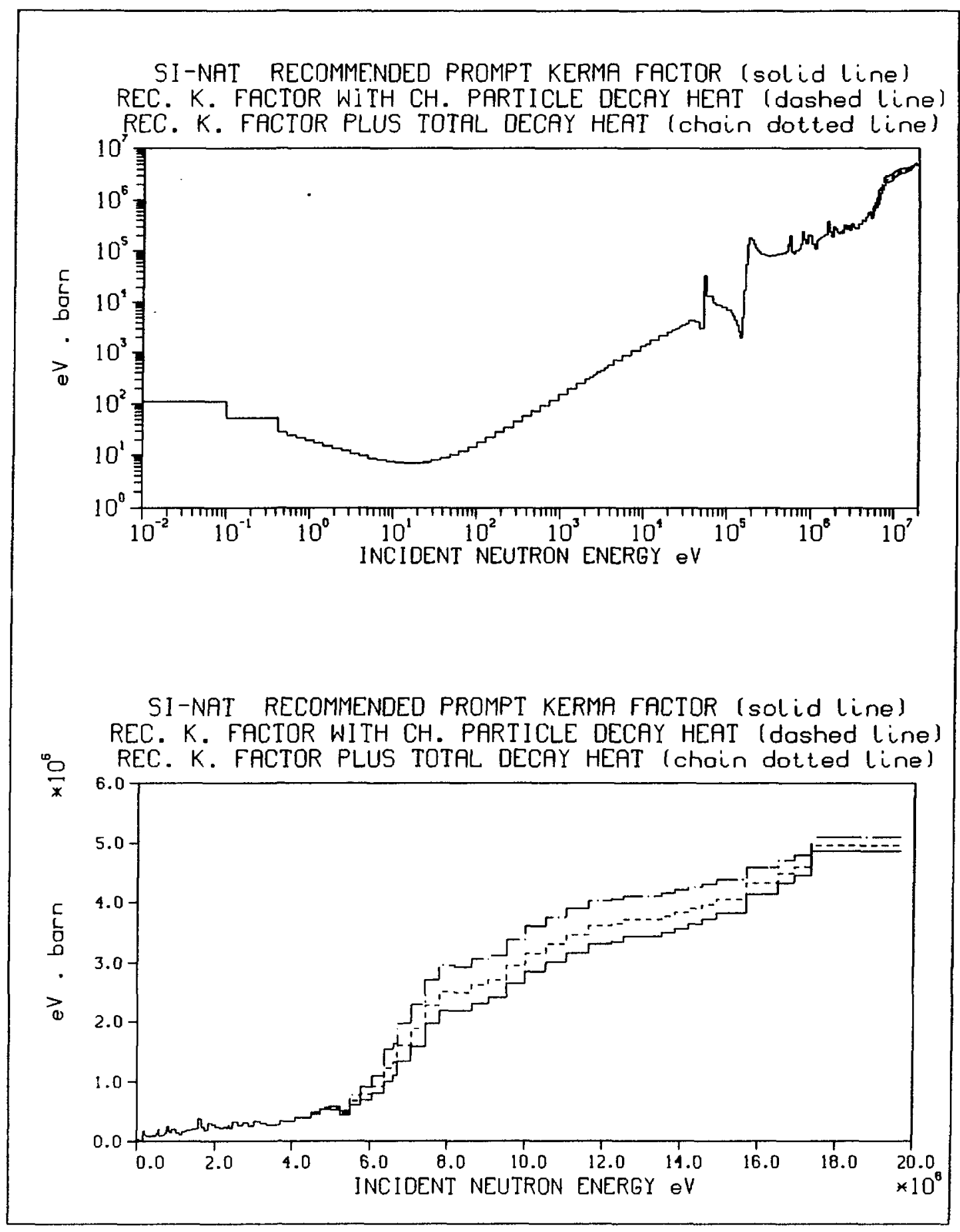




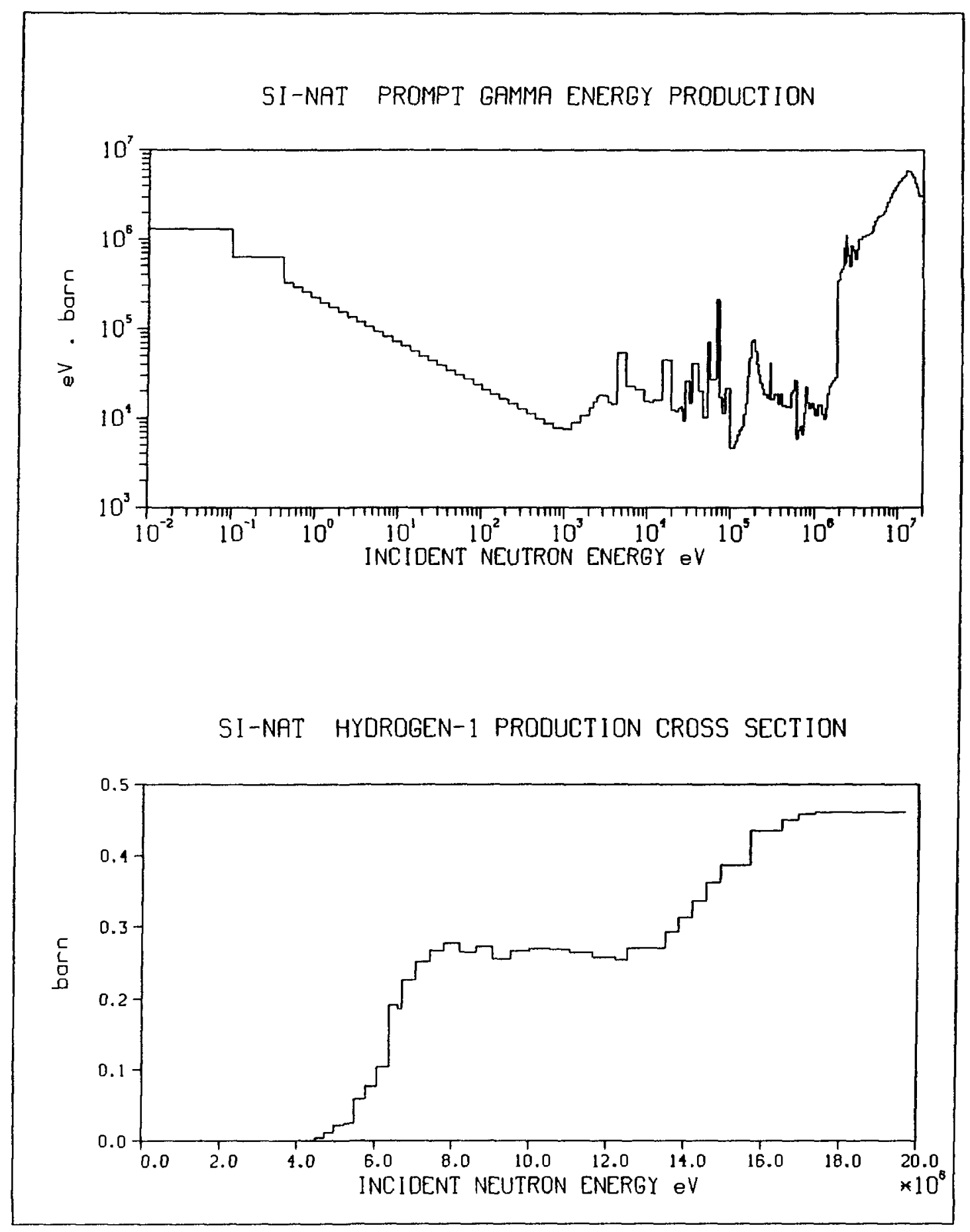




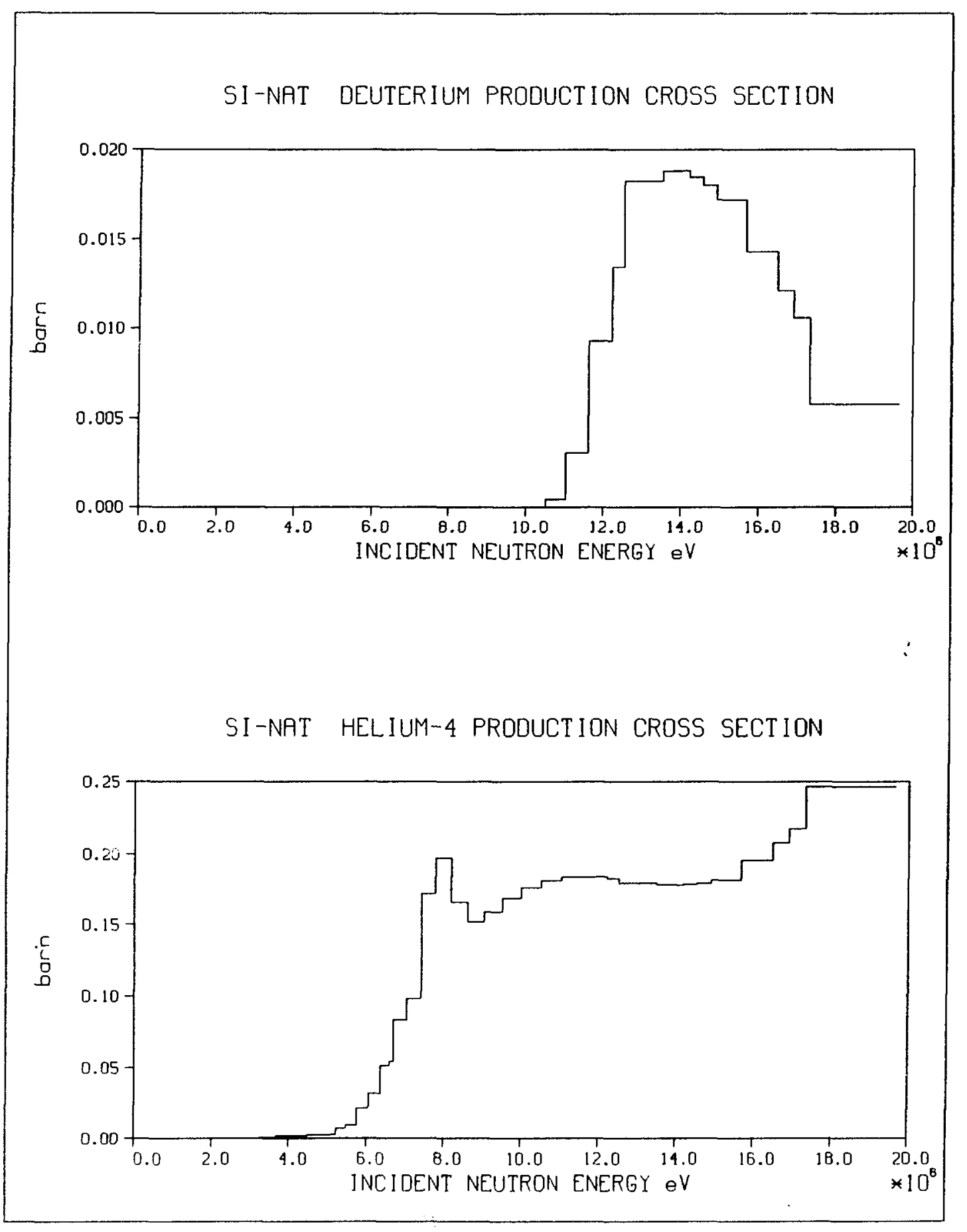


SI-NAT TOTAL CROSS SECTION

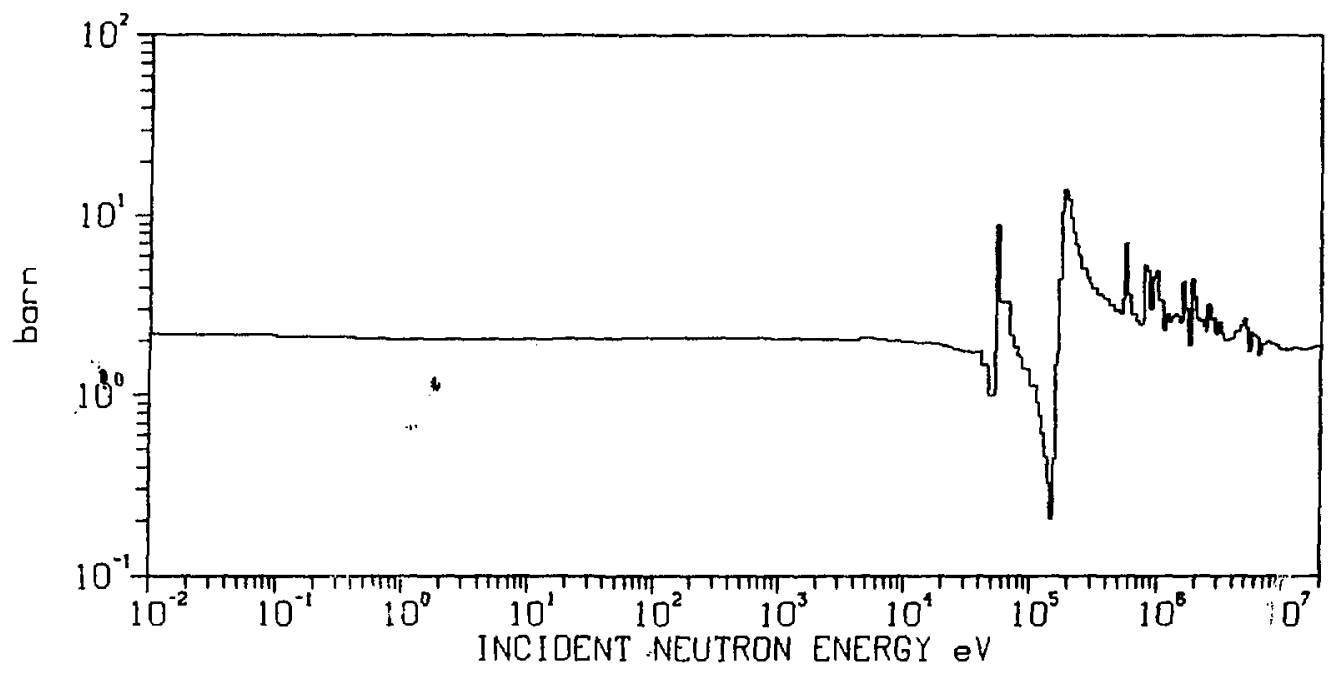

SI-NAT ELASTIC CROSS SECTION

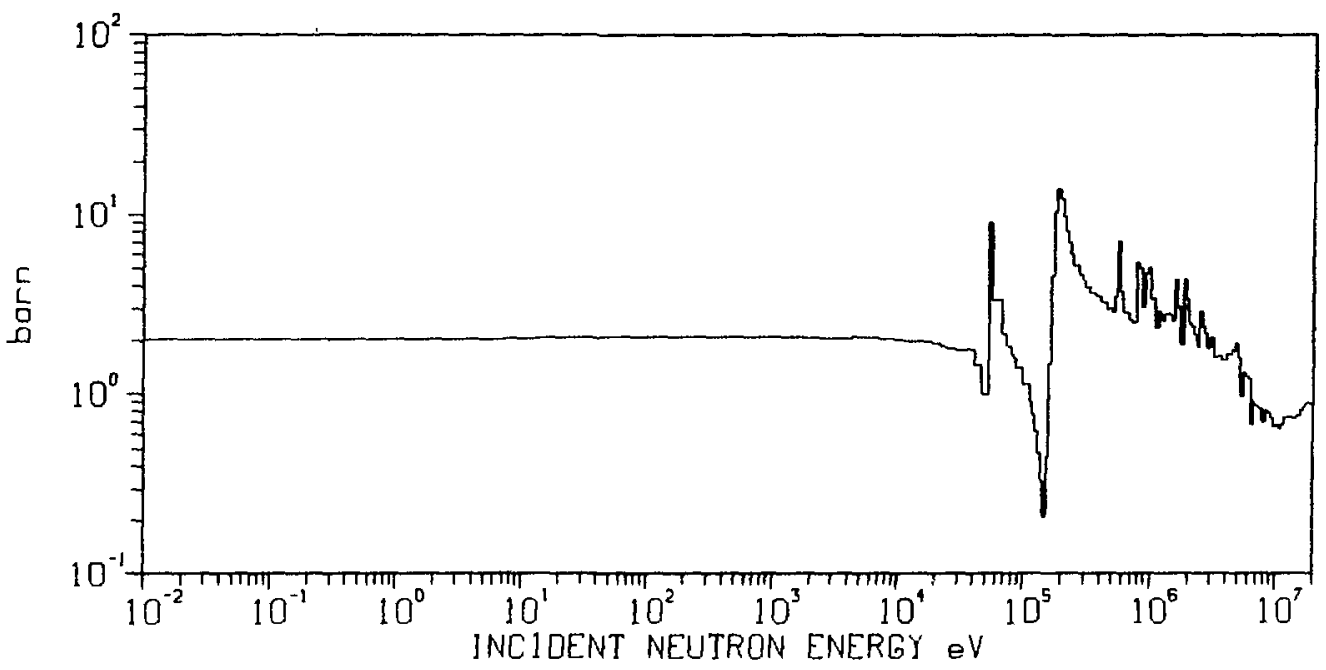




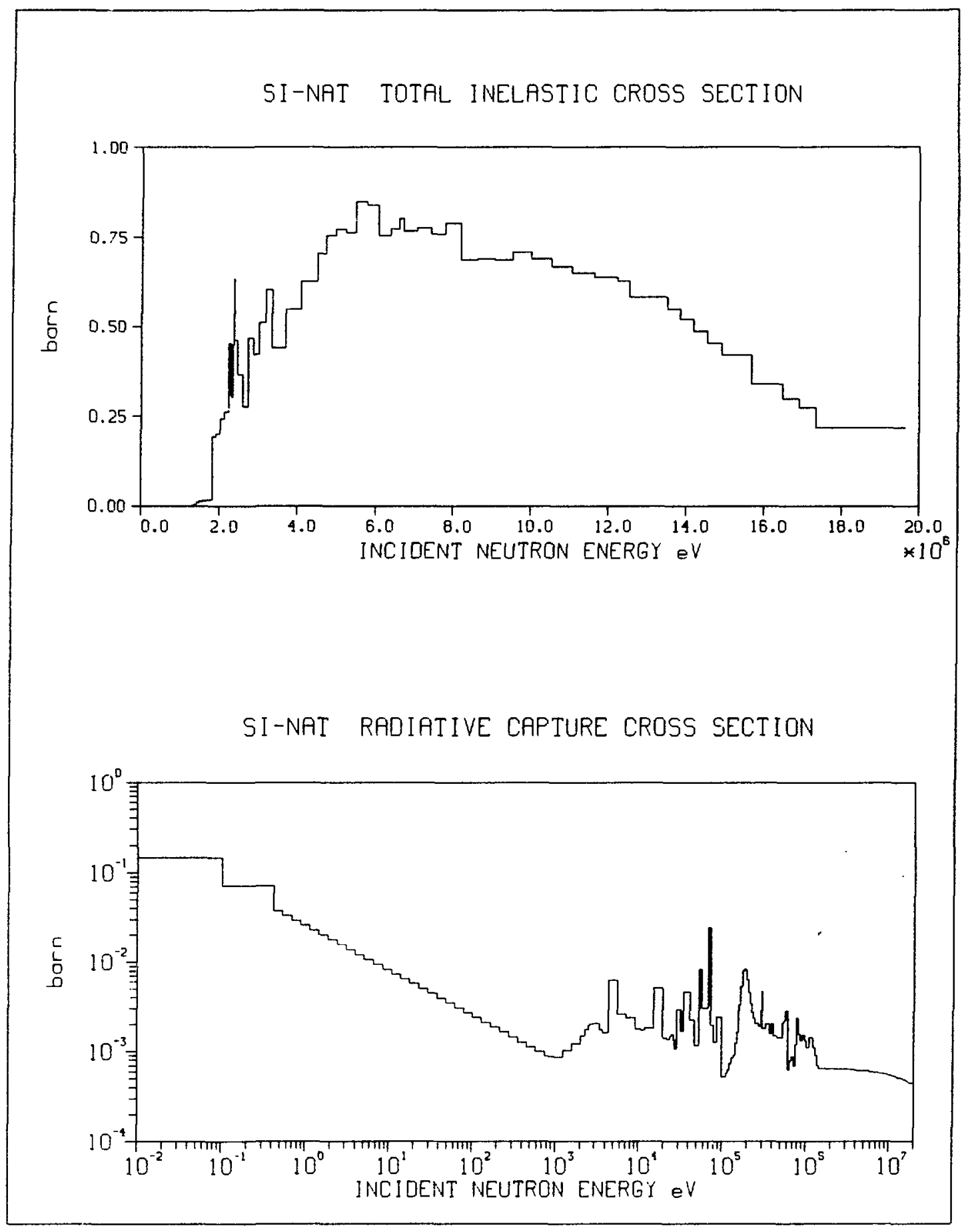




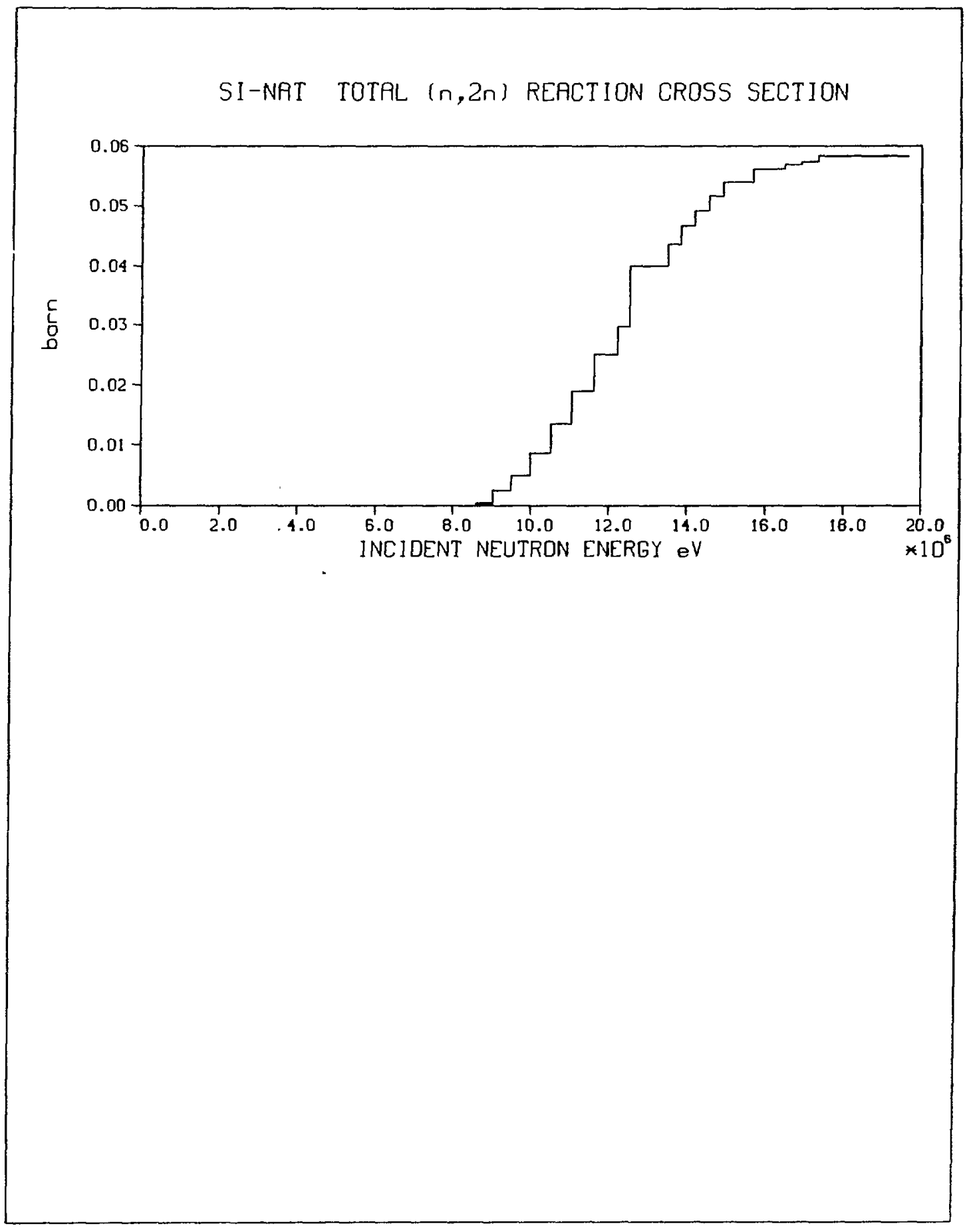




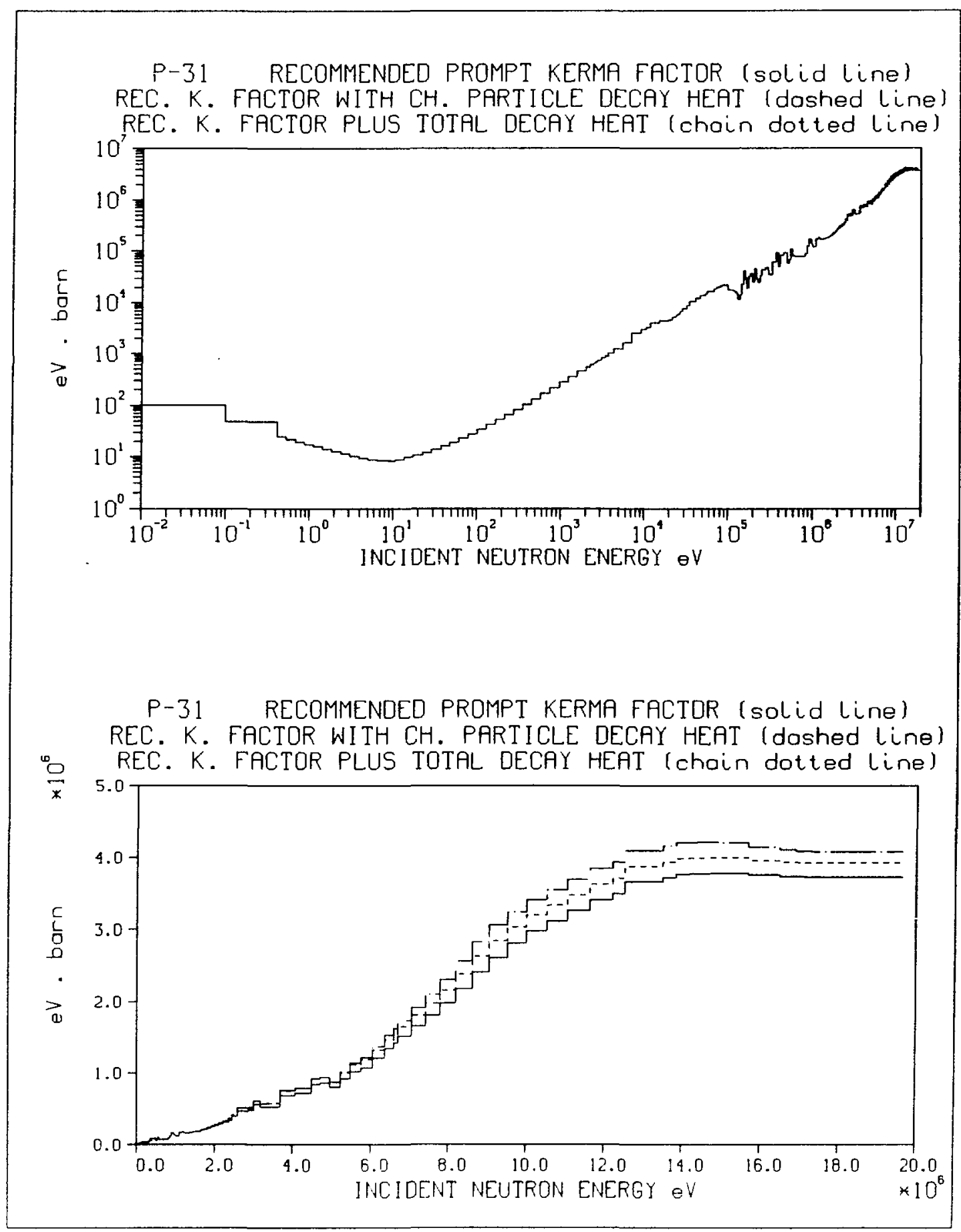




\section{P-31 PROMPT GAMMA ENERGY PRODUCTION}

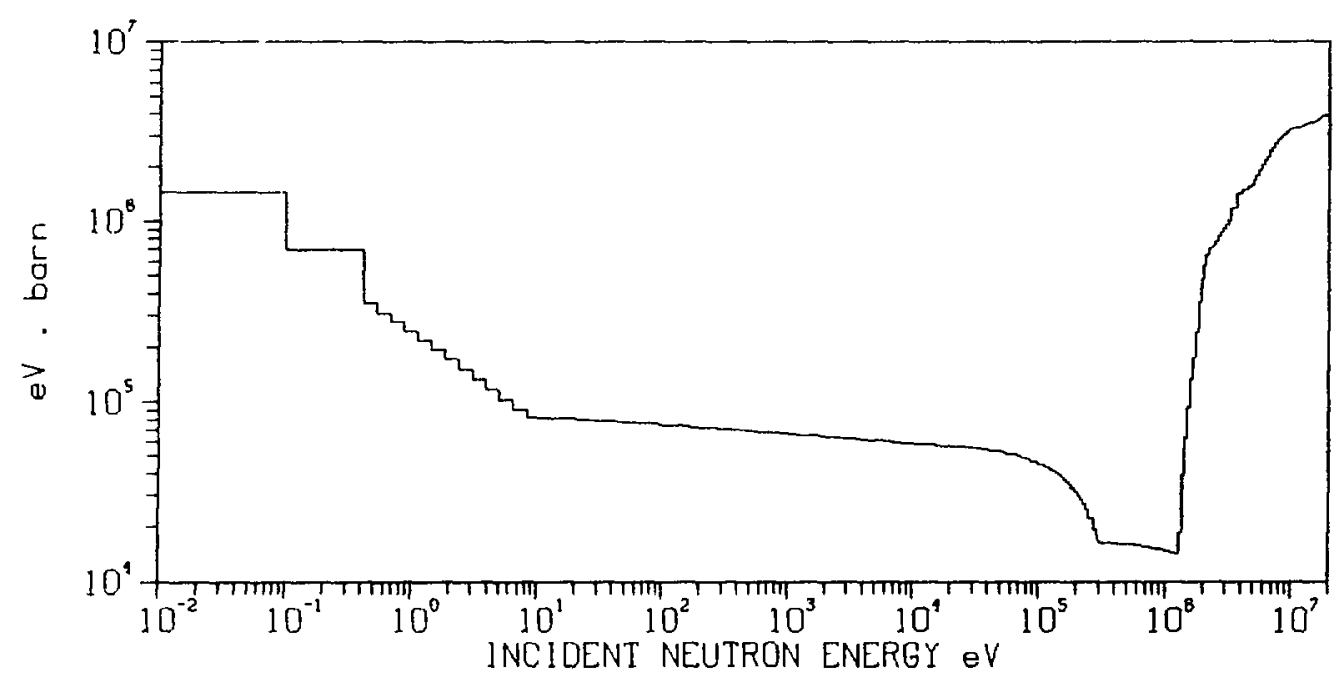

P-31 HYDROGEN-1 PRODUCTION CROSS SECTION

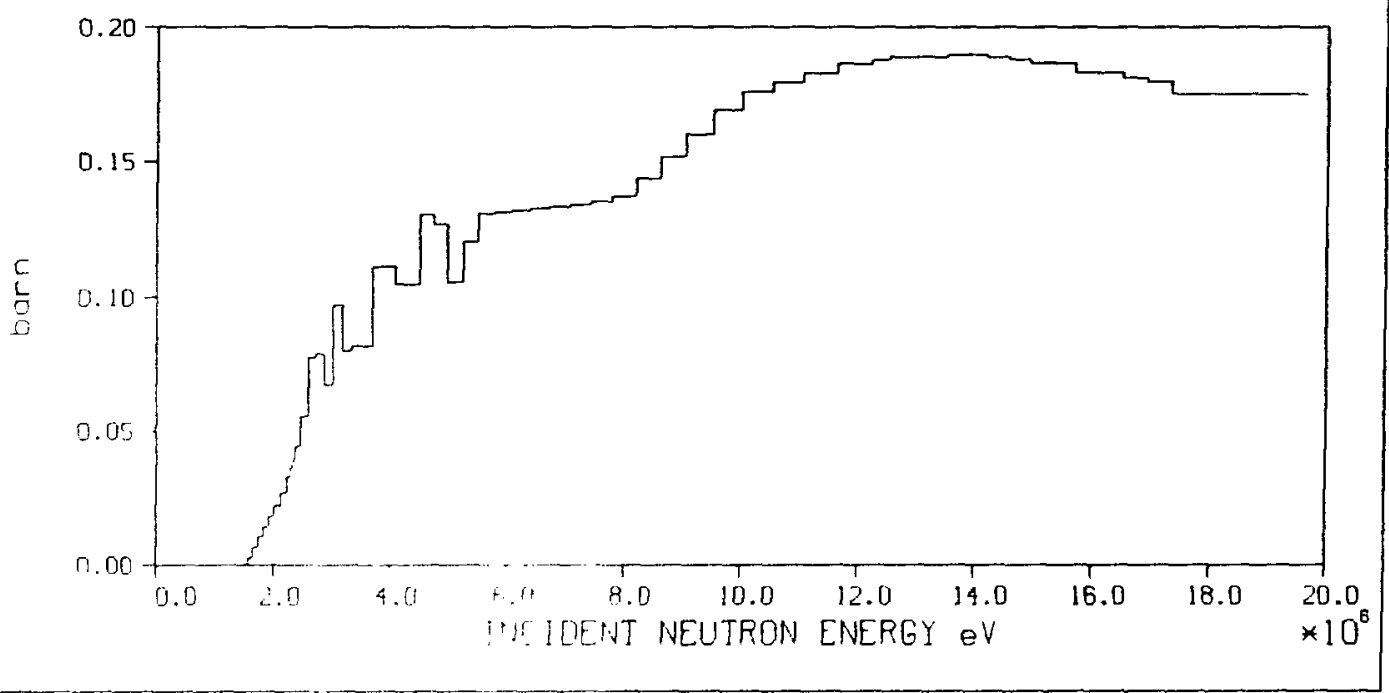




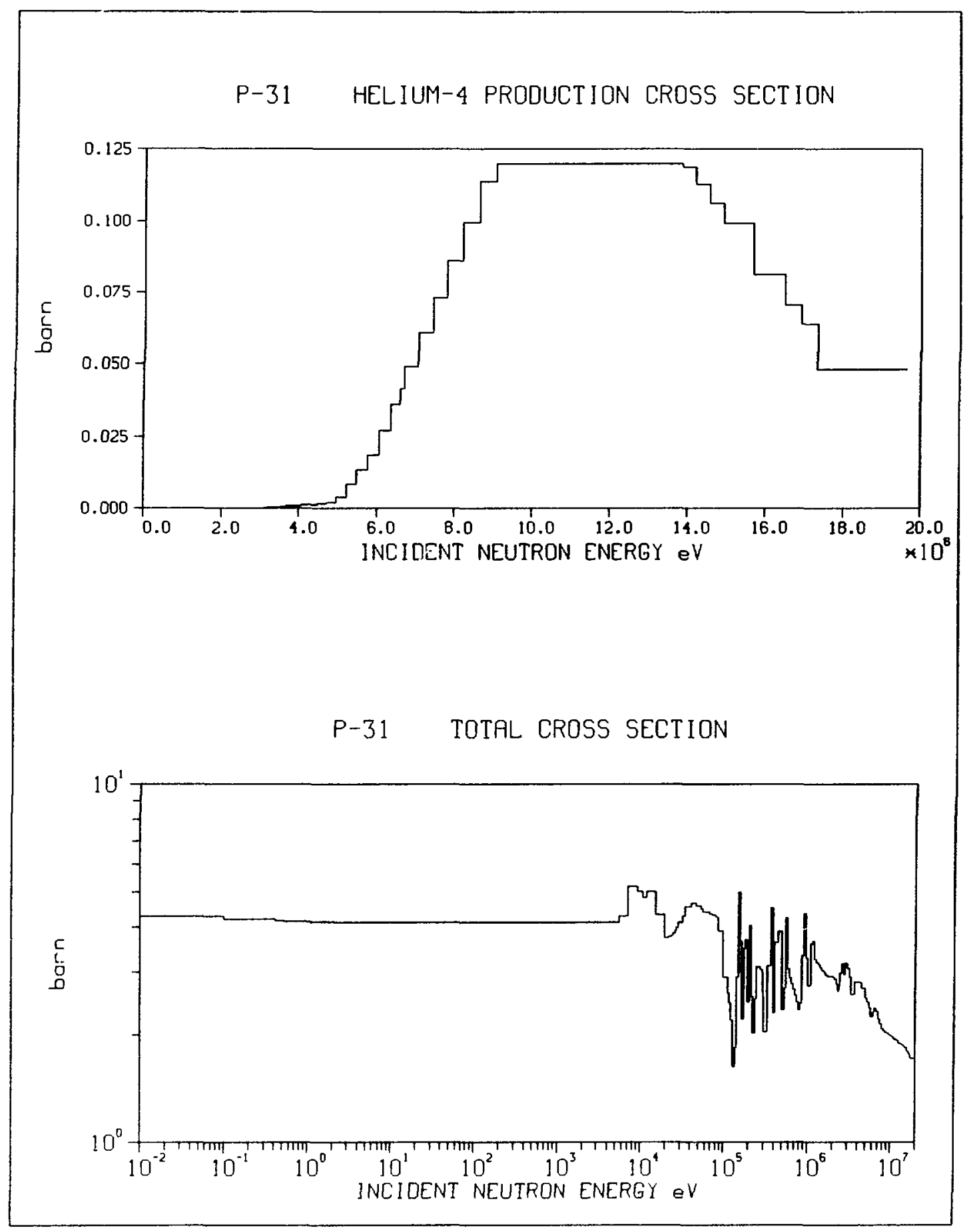




\section{$P-31$ ELASTIC CROSS SECTION}

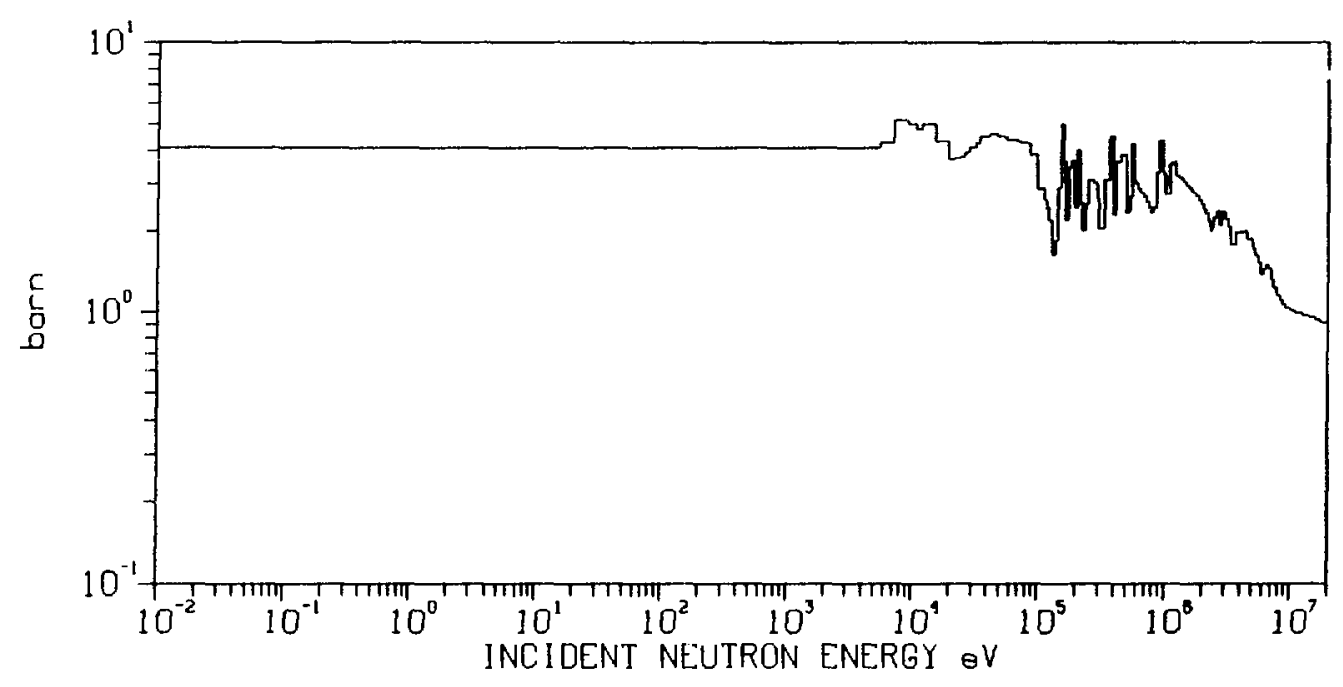

P-31 TOTAL INELASTIC CROSS SECTION

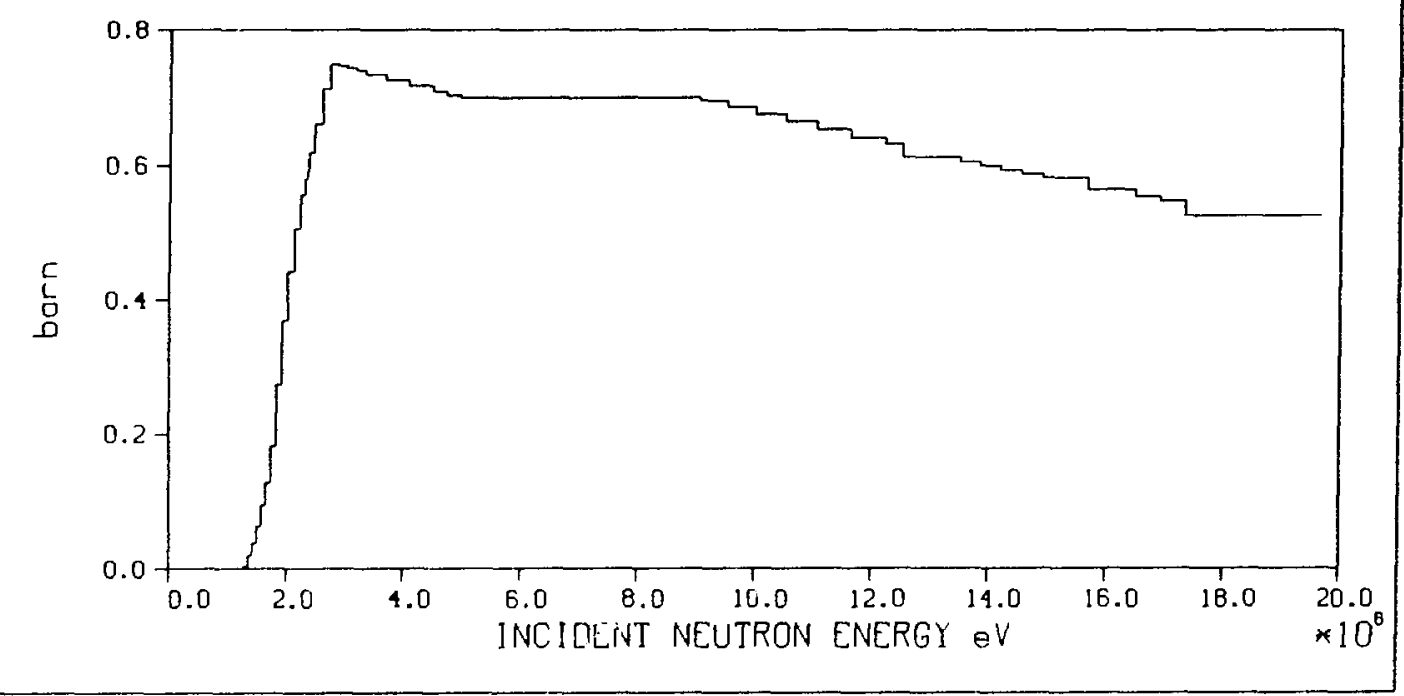




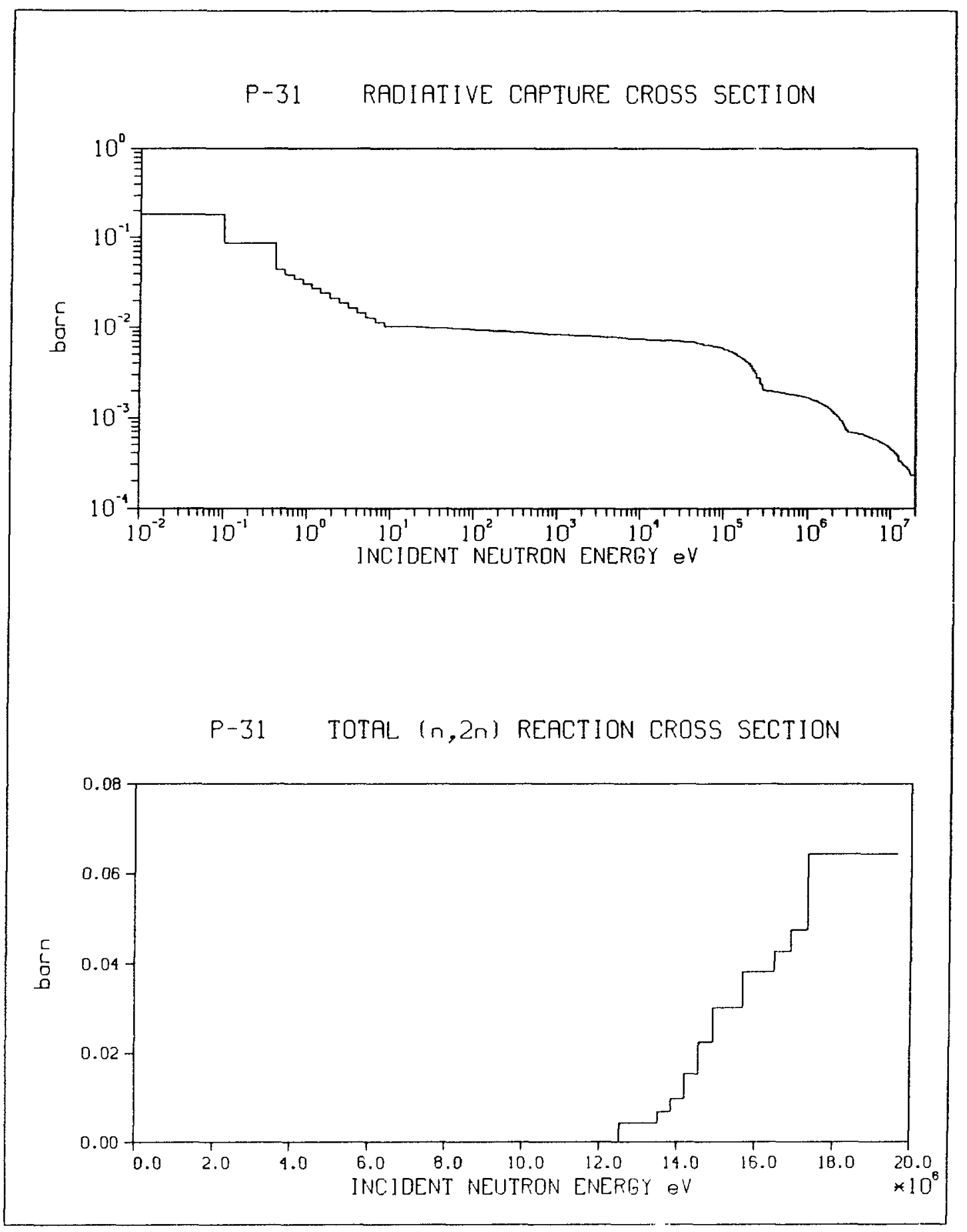




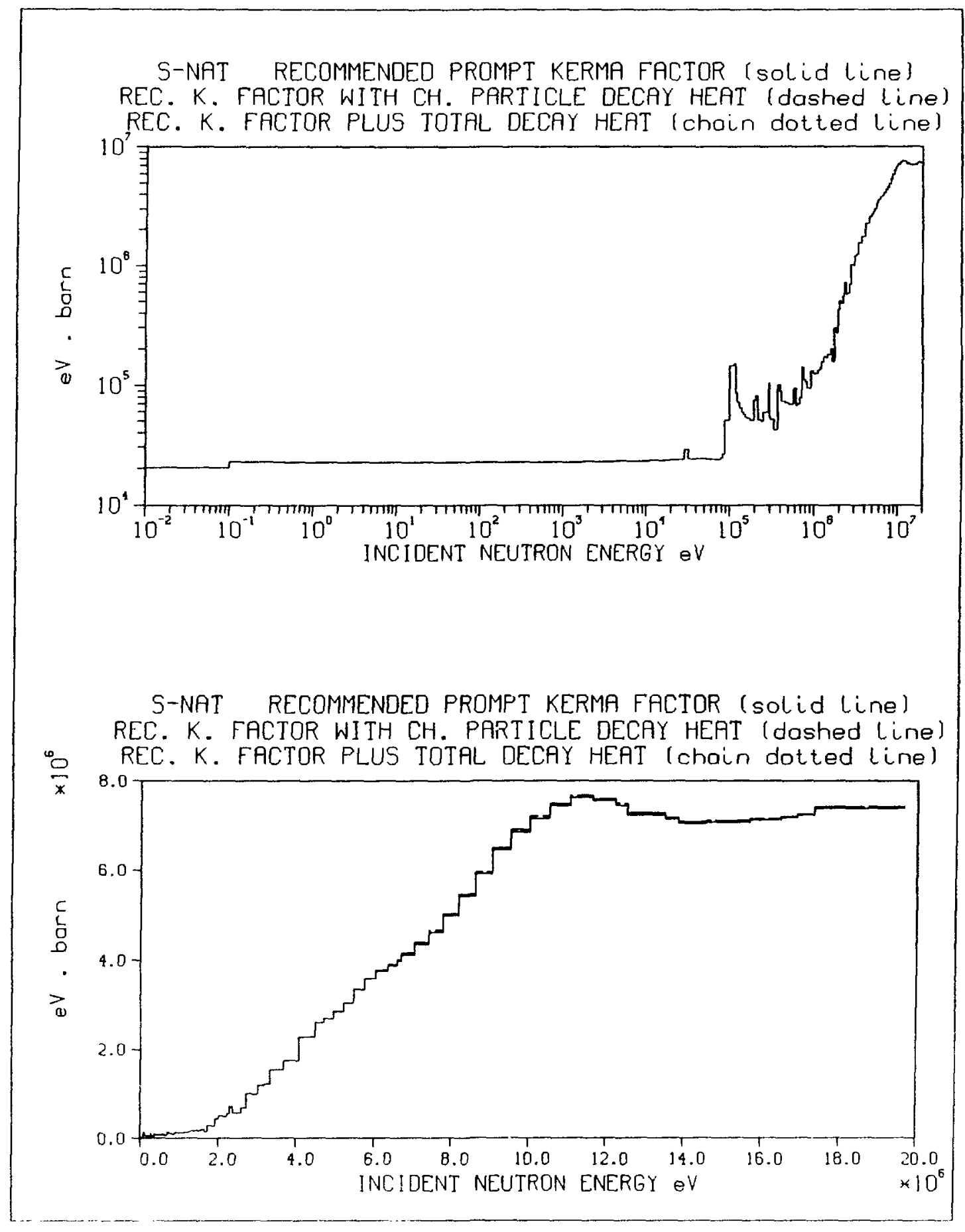




\section{S-NAT PROMPT GAMMA ENERGY PRODUCTION}

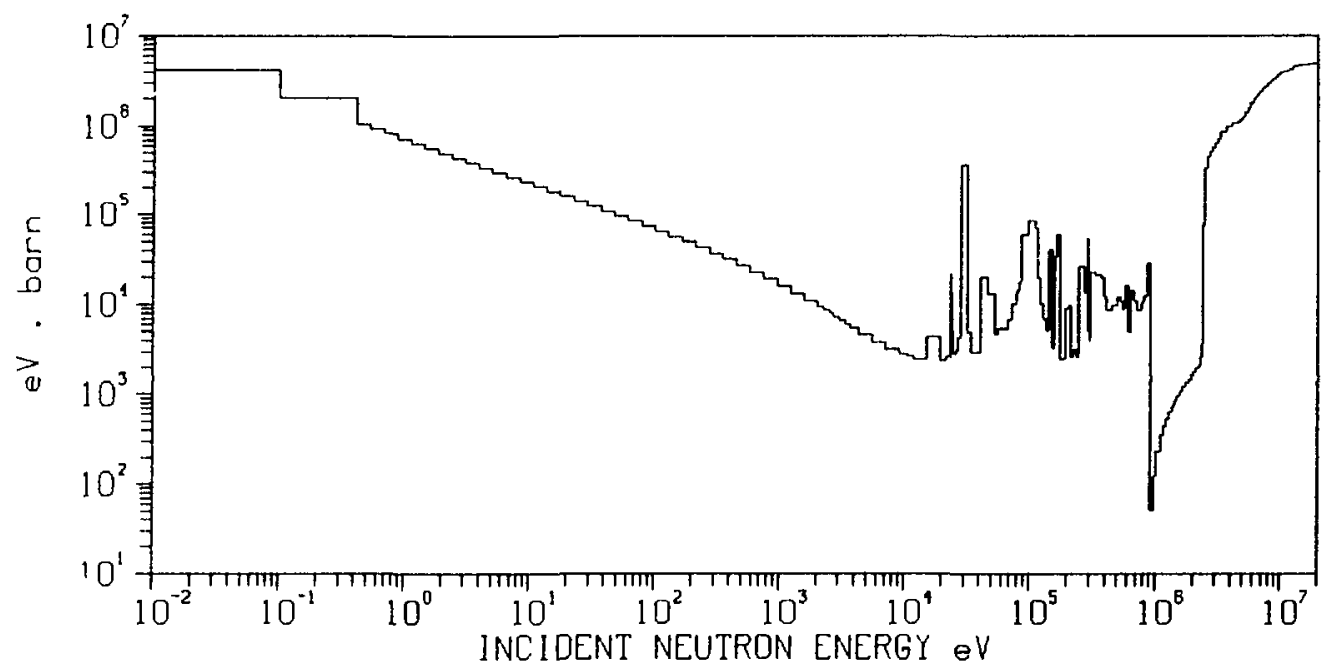

S-NAT HYDROGEN-1 PRODUCTION CROSS SECTION

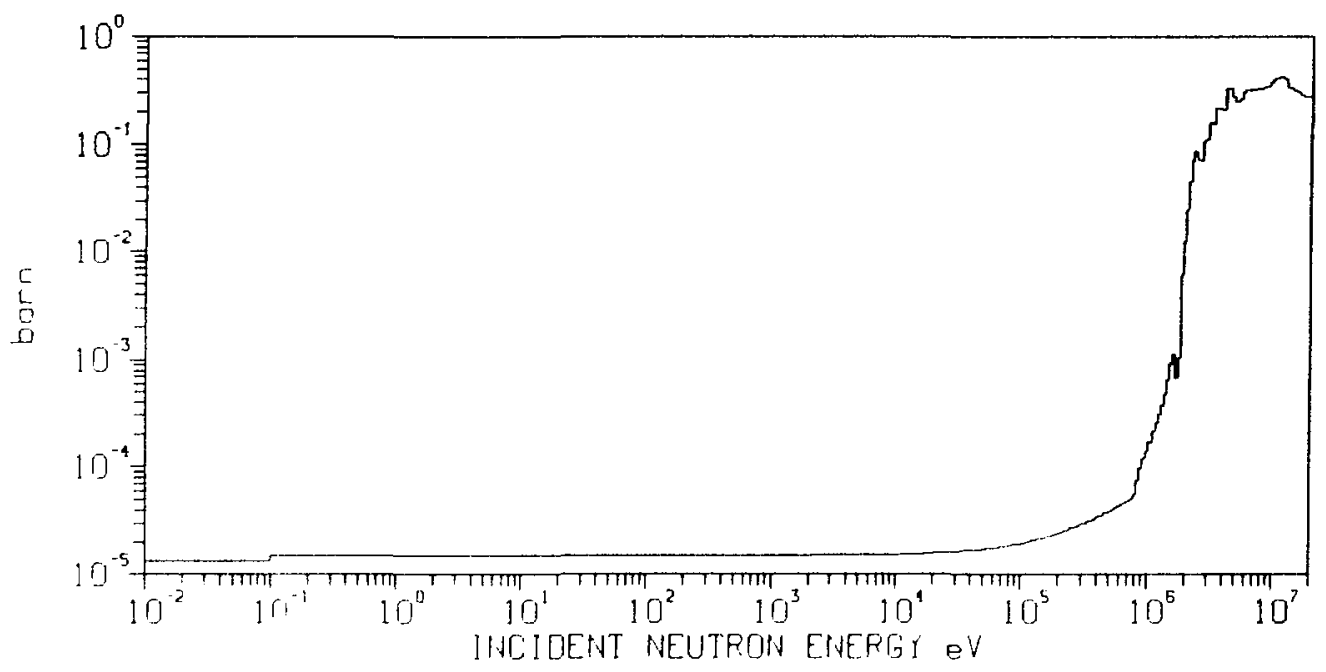




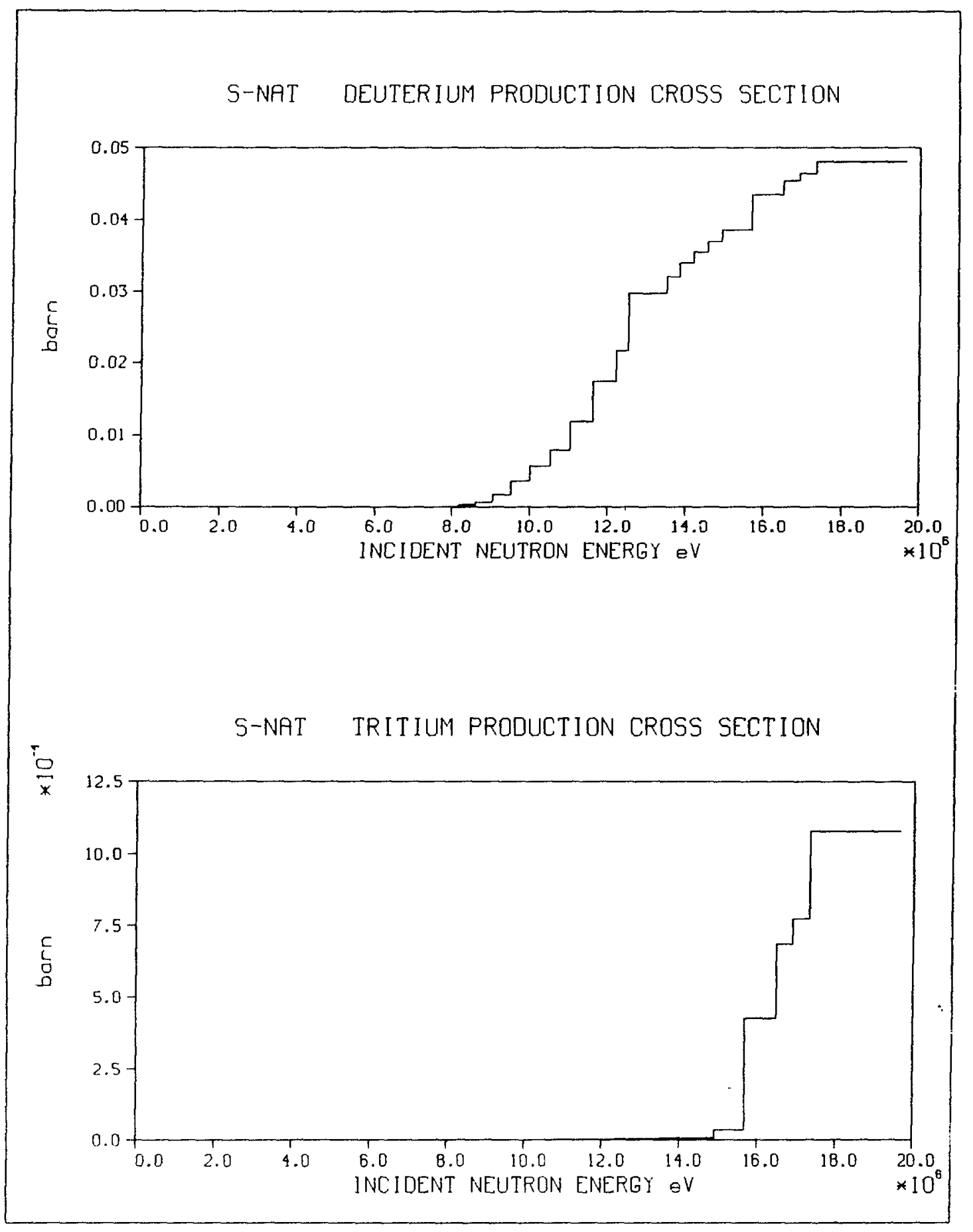




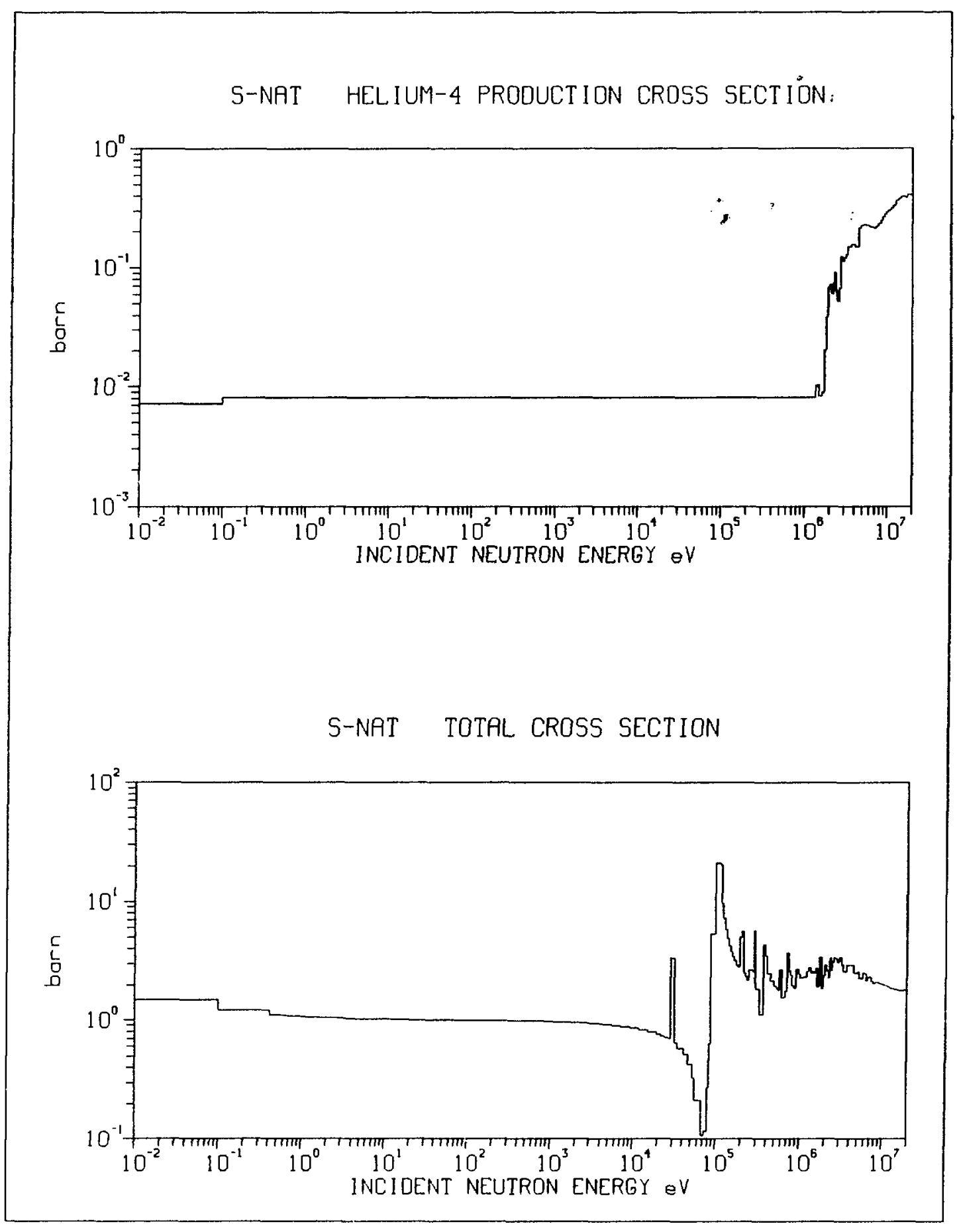




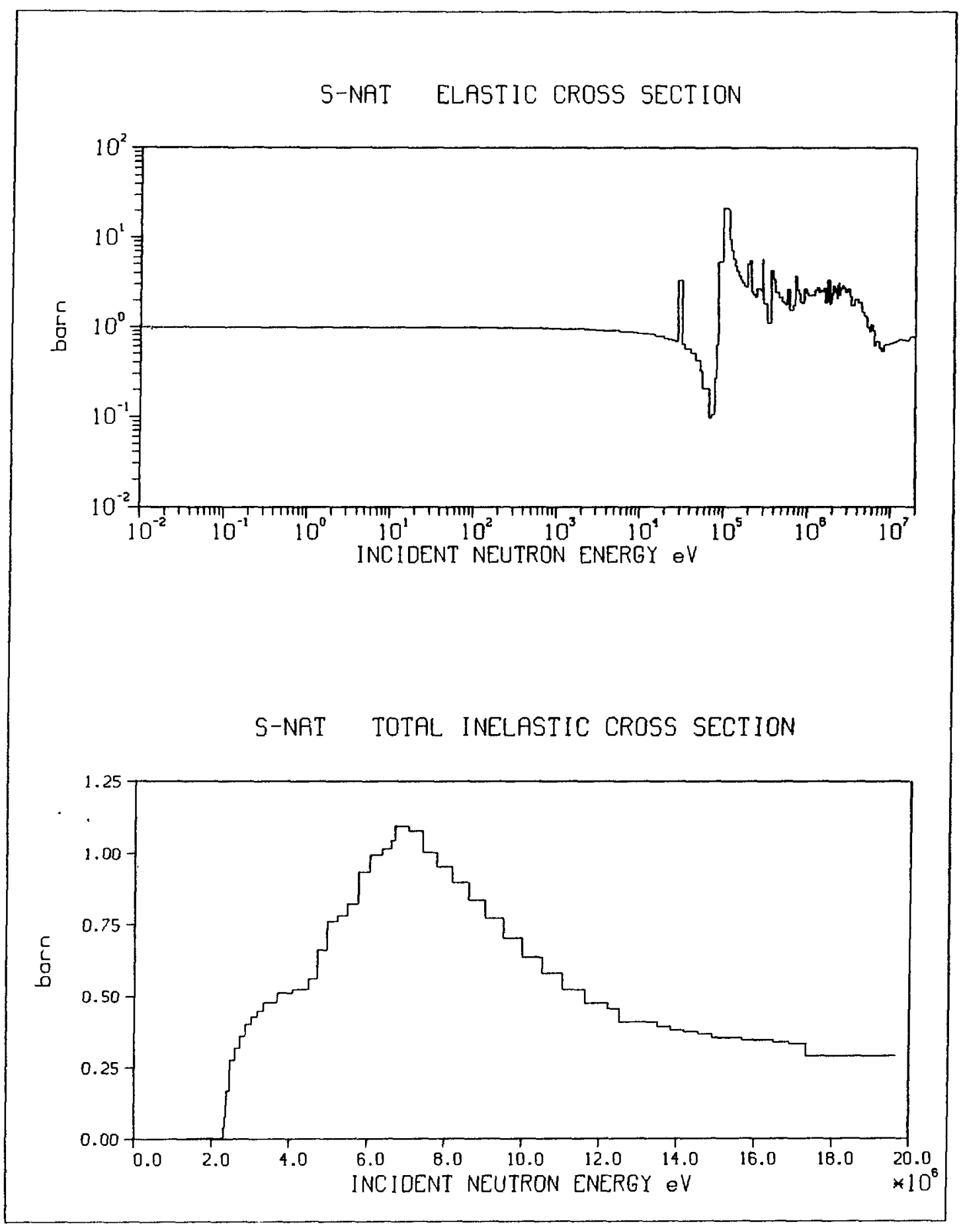


S-NAT RADIATIVE CAPTURE CROSS SECTION

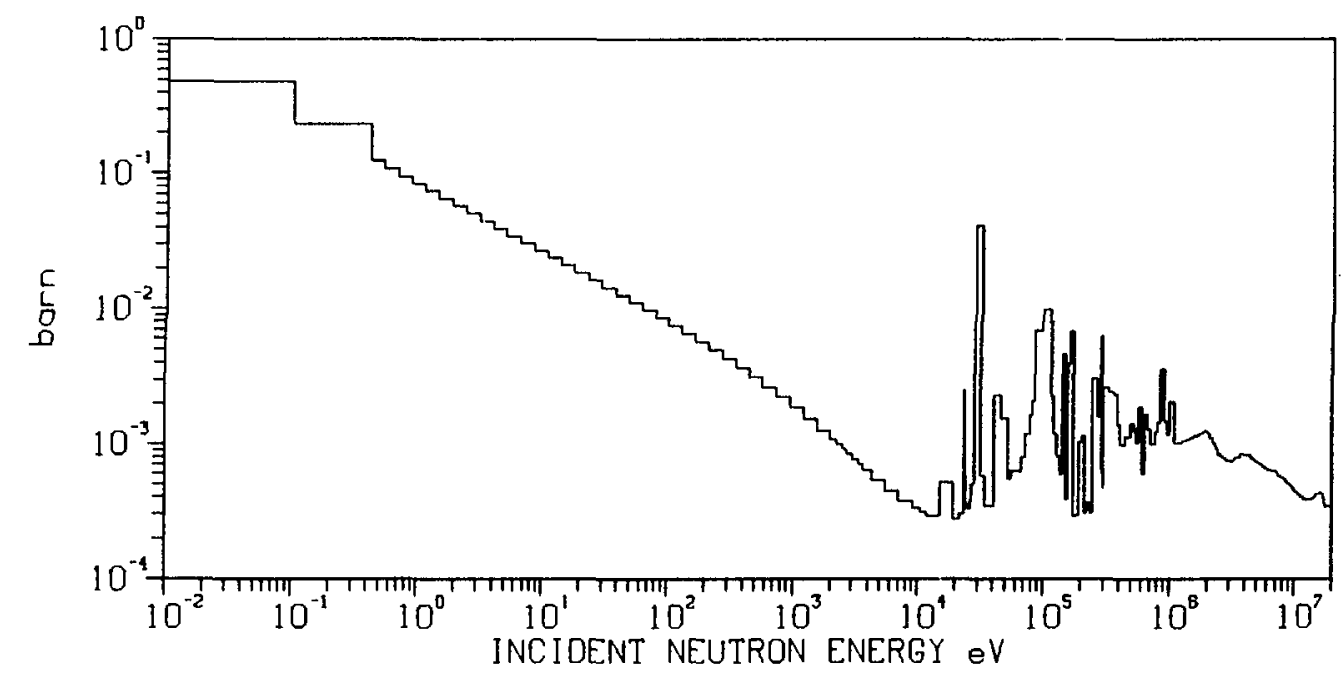

S-NAT TOTAL $(n, 2 n)$ REACTION CROSS SECTION

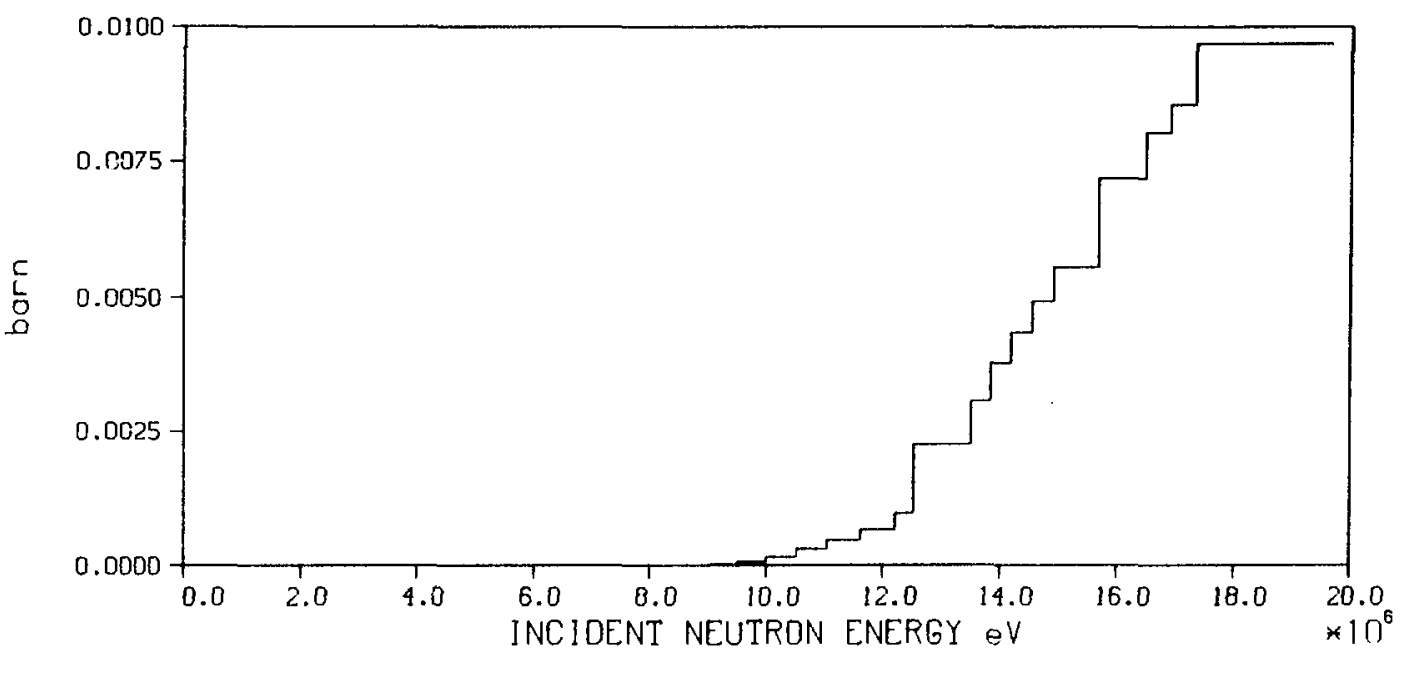




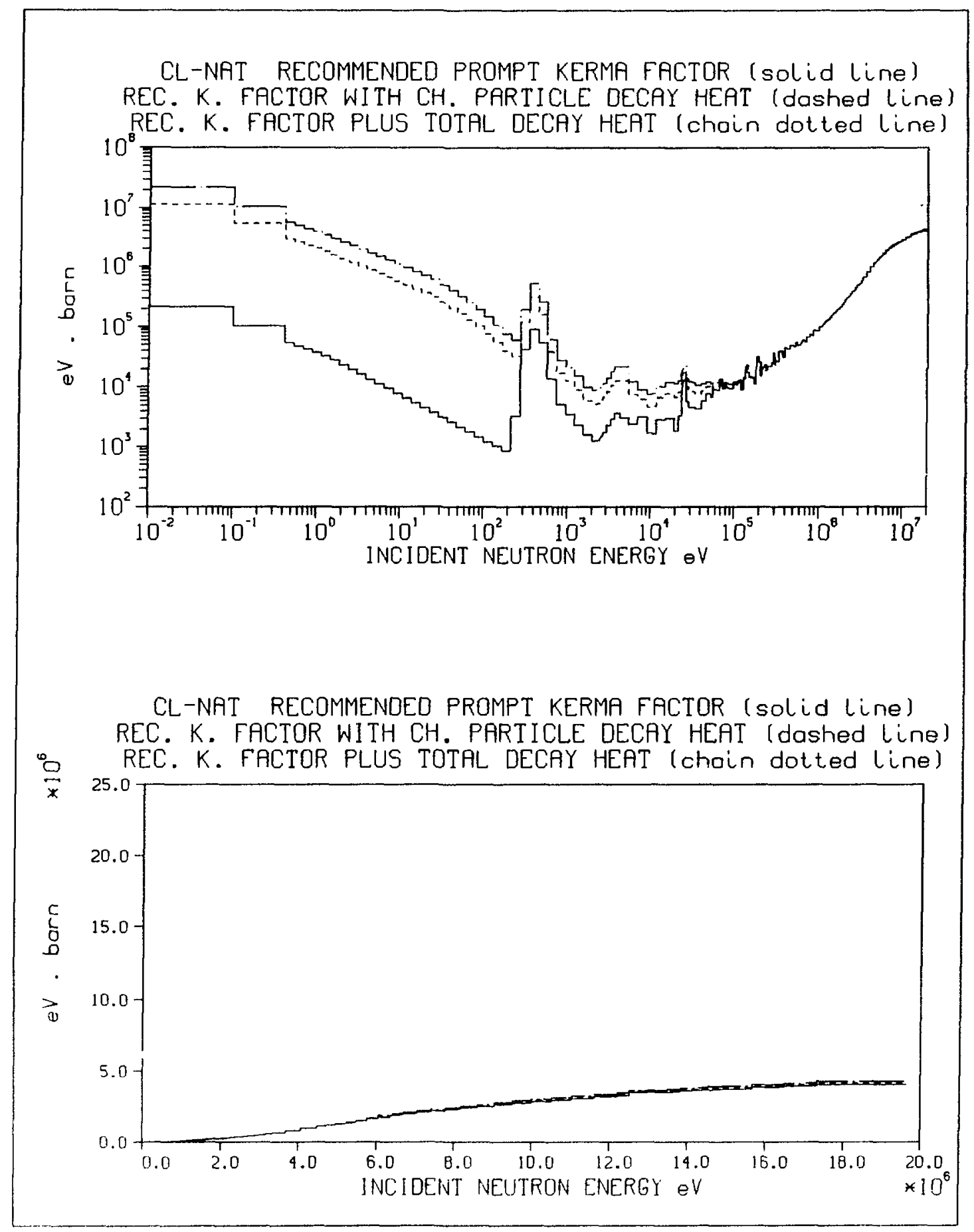




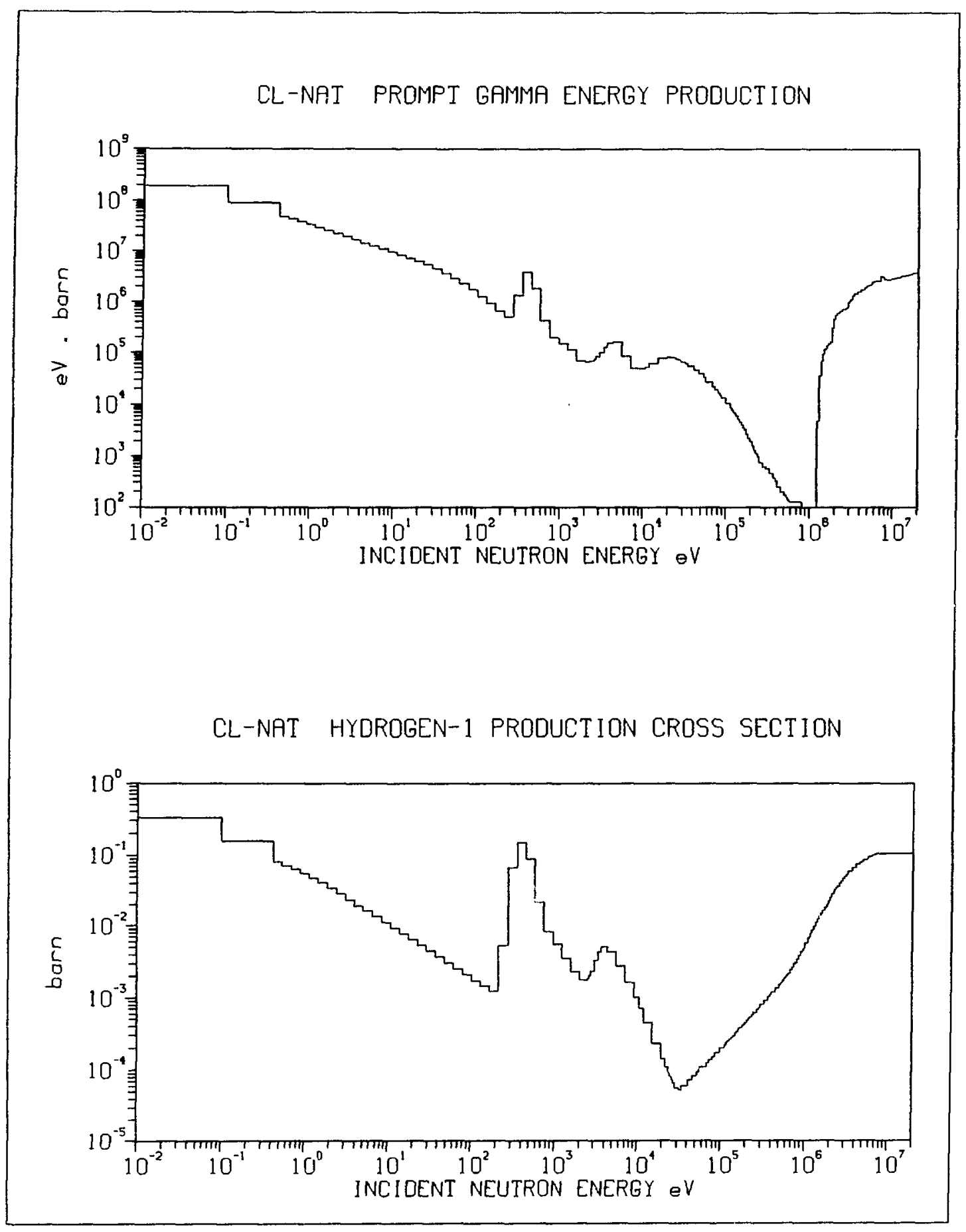




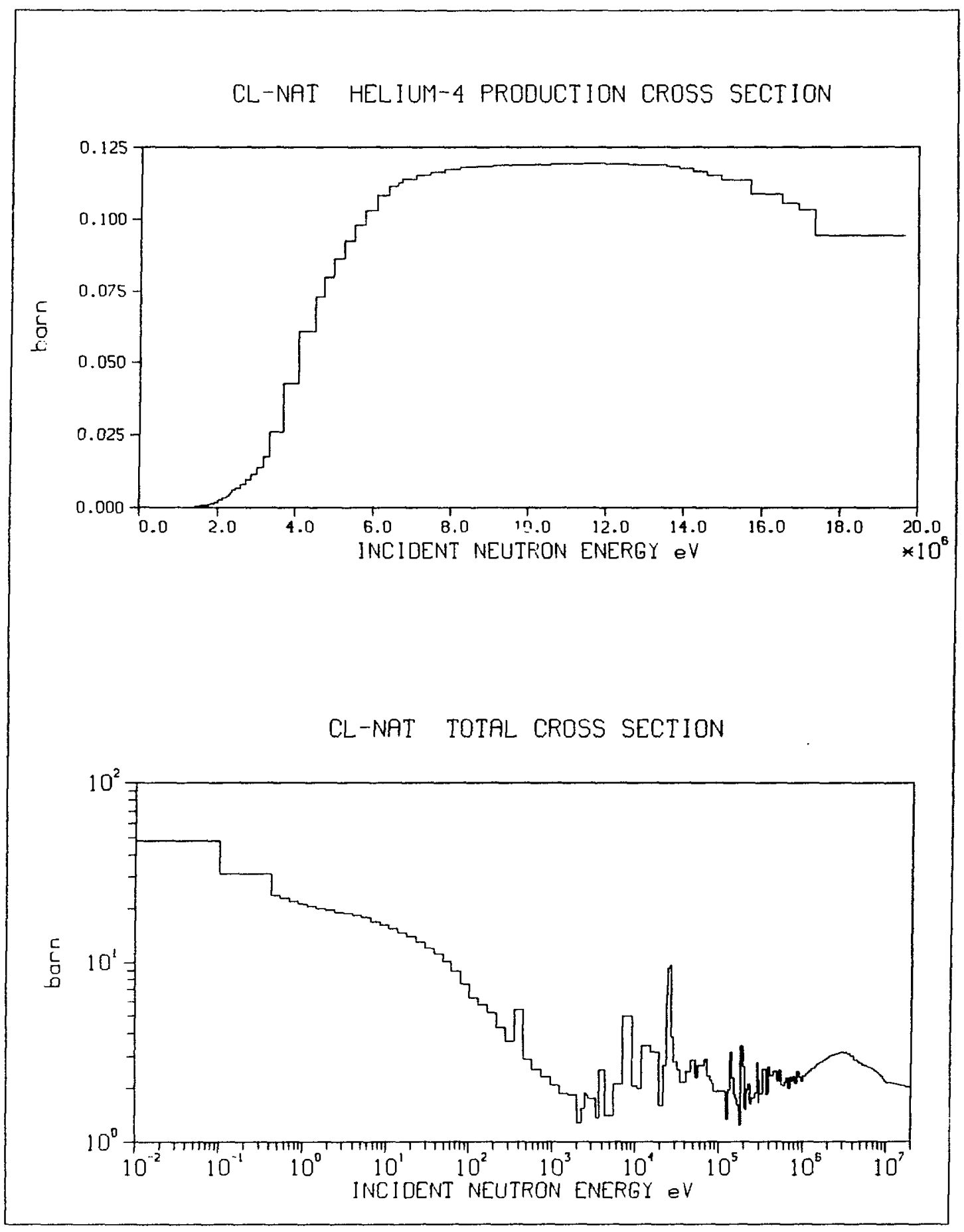


CL-NAT ELASTIC CROSS SECTION

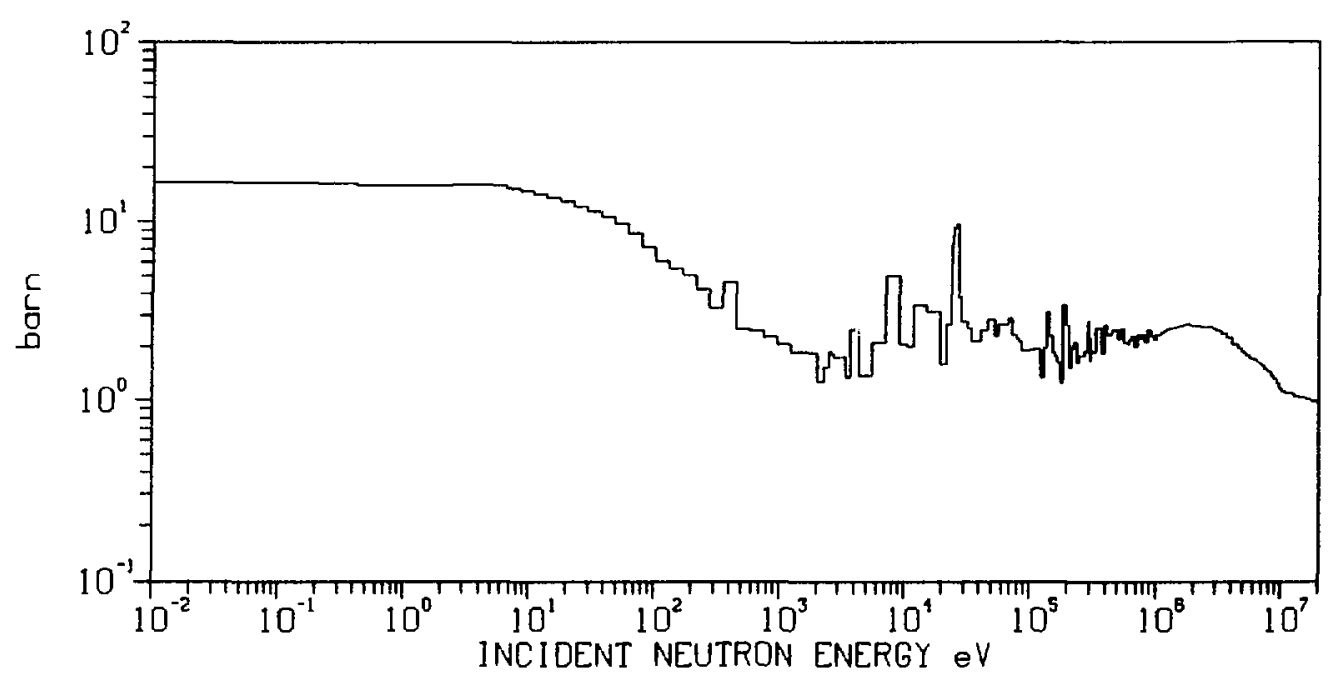

CL-NAT TOTAL INELASTIC CROSS SECTION

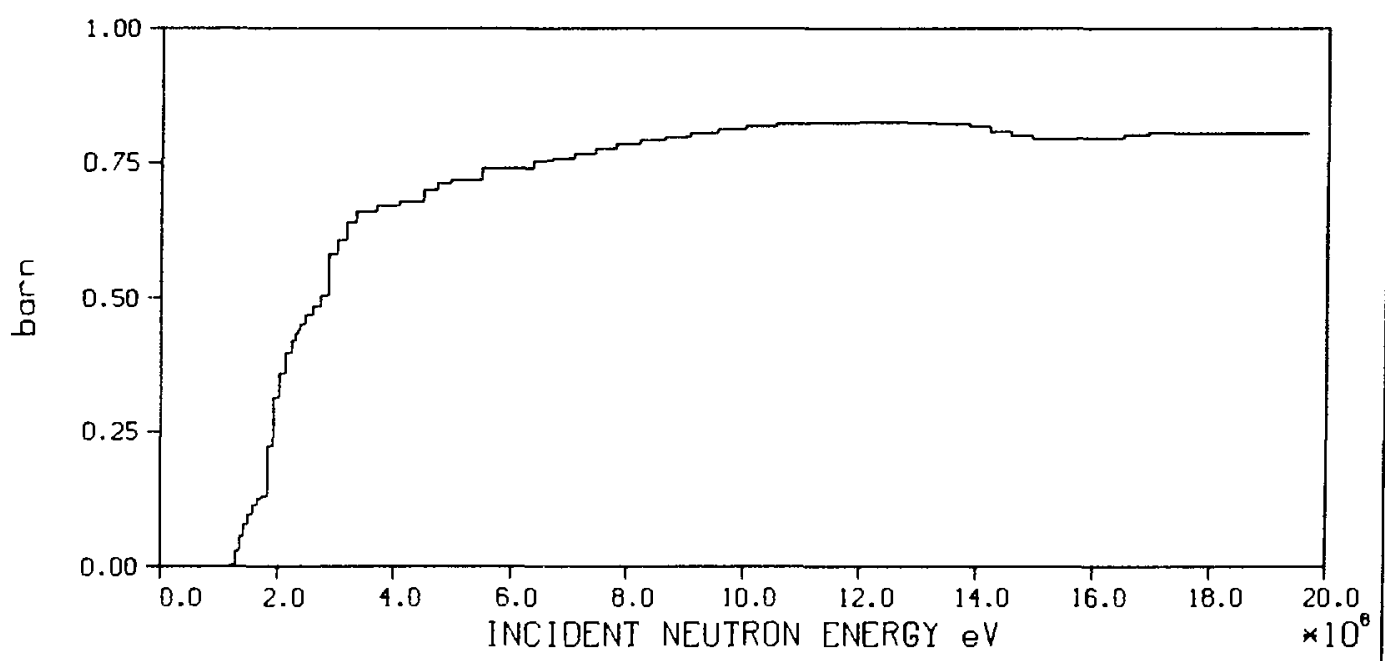




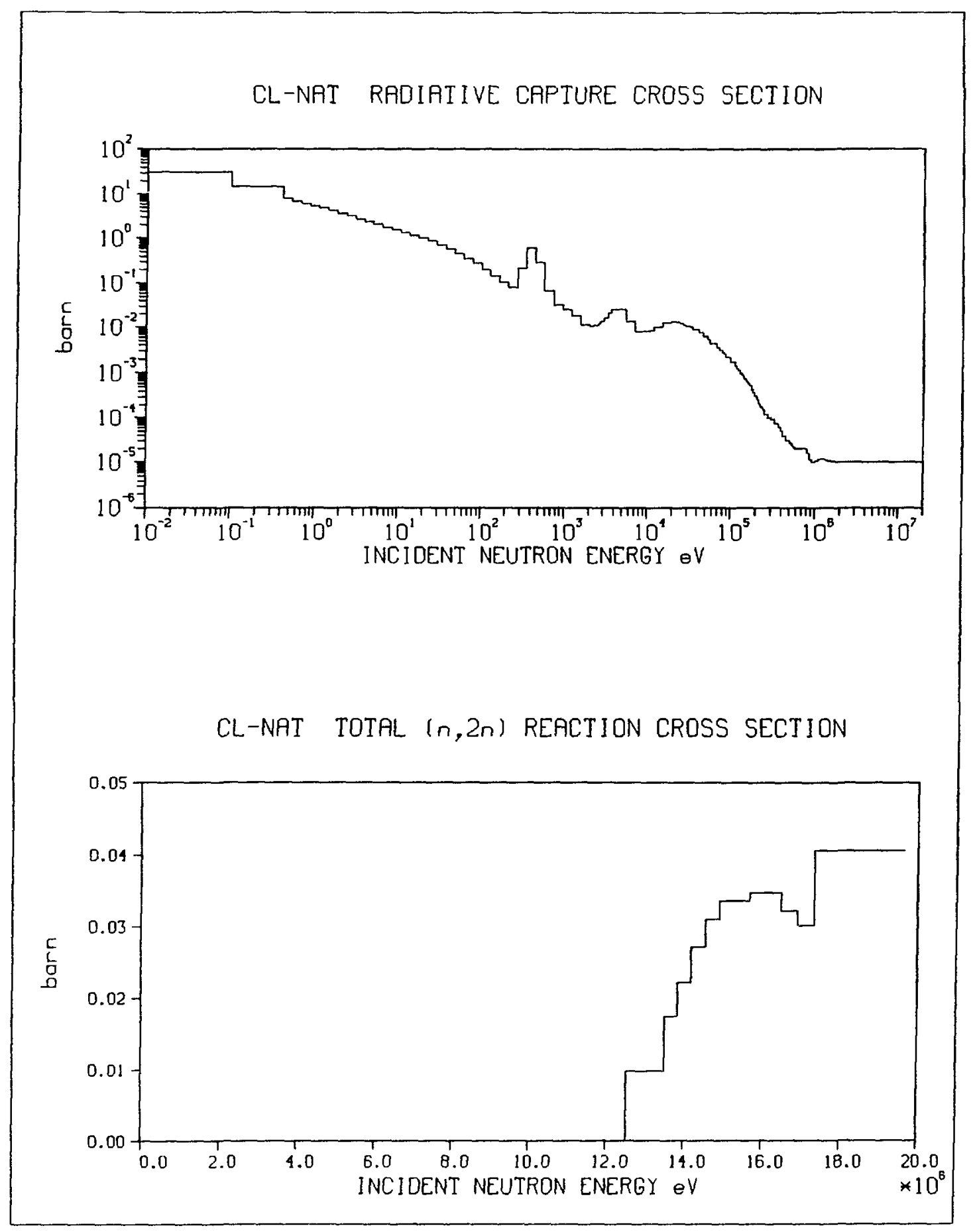




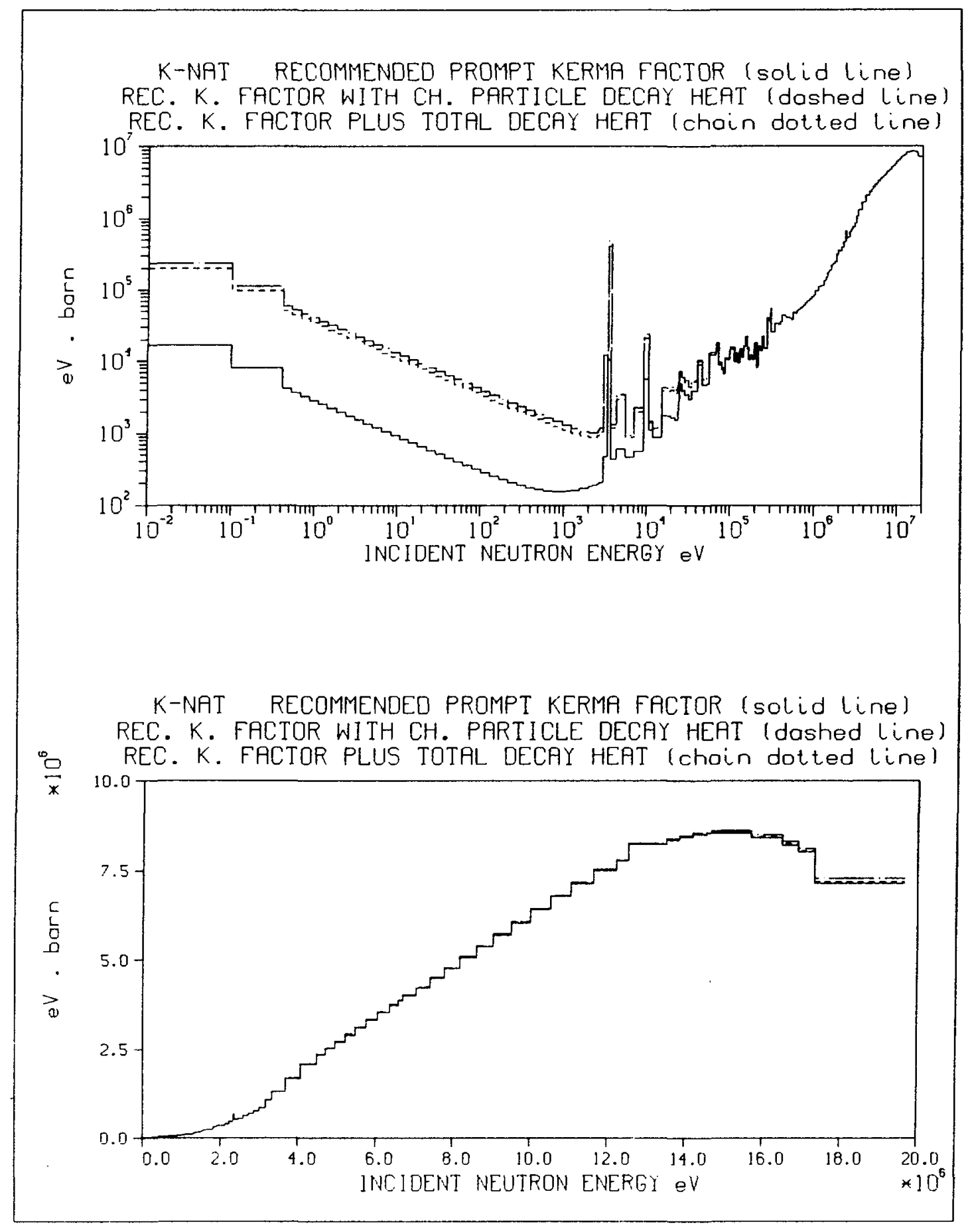




\section{K-NAT PROMPT GRMMA ENERGY PRODUCTION}

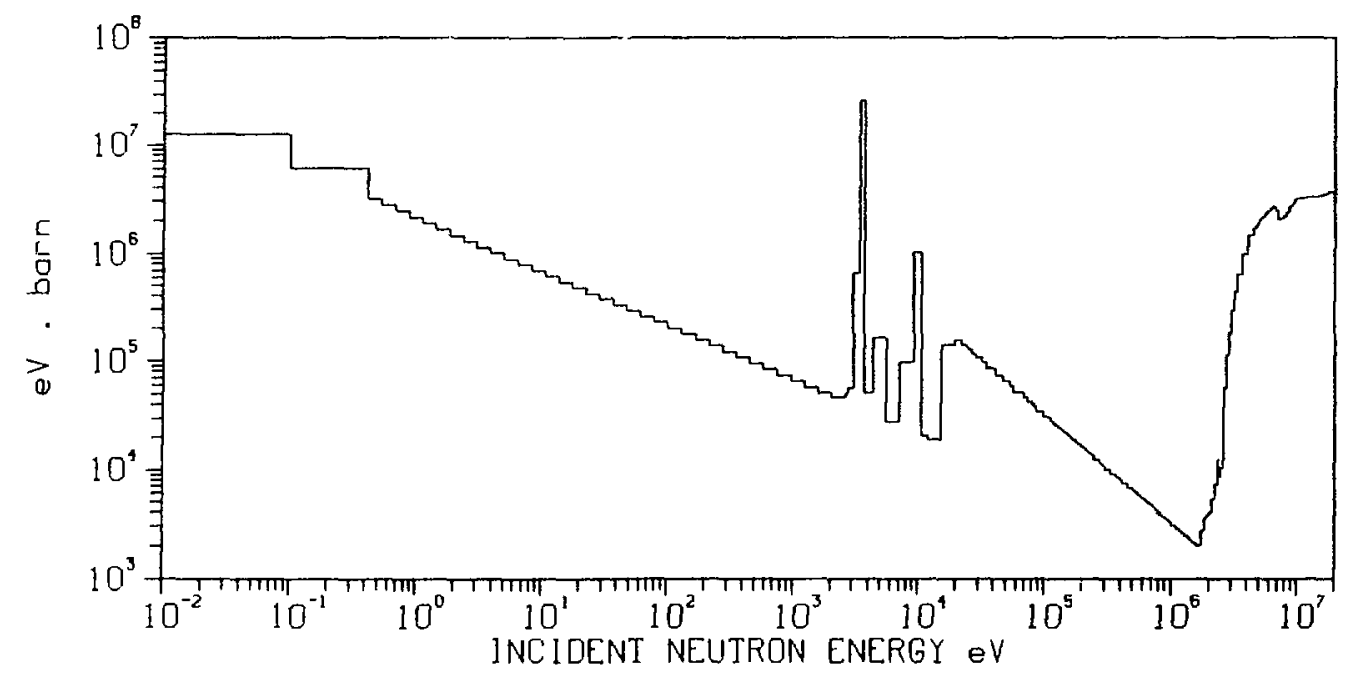

K-NAT HYOROGEN-1 PRODUCTION CROSS SECTION

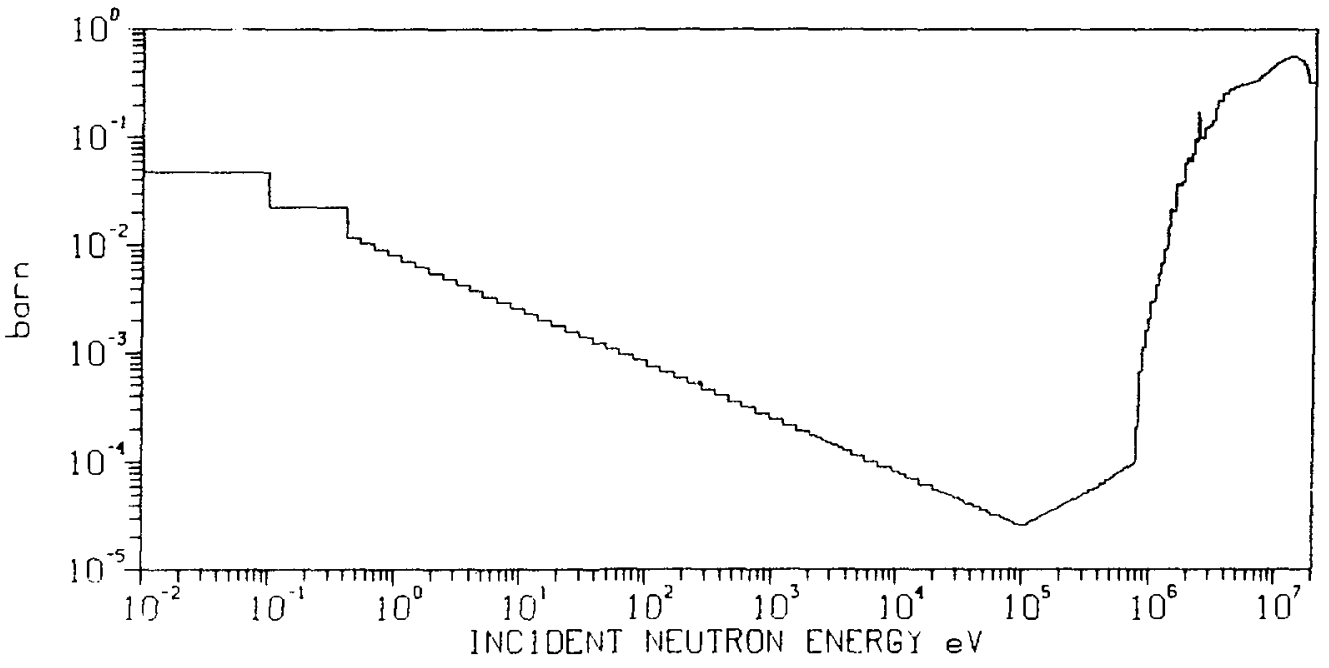




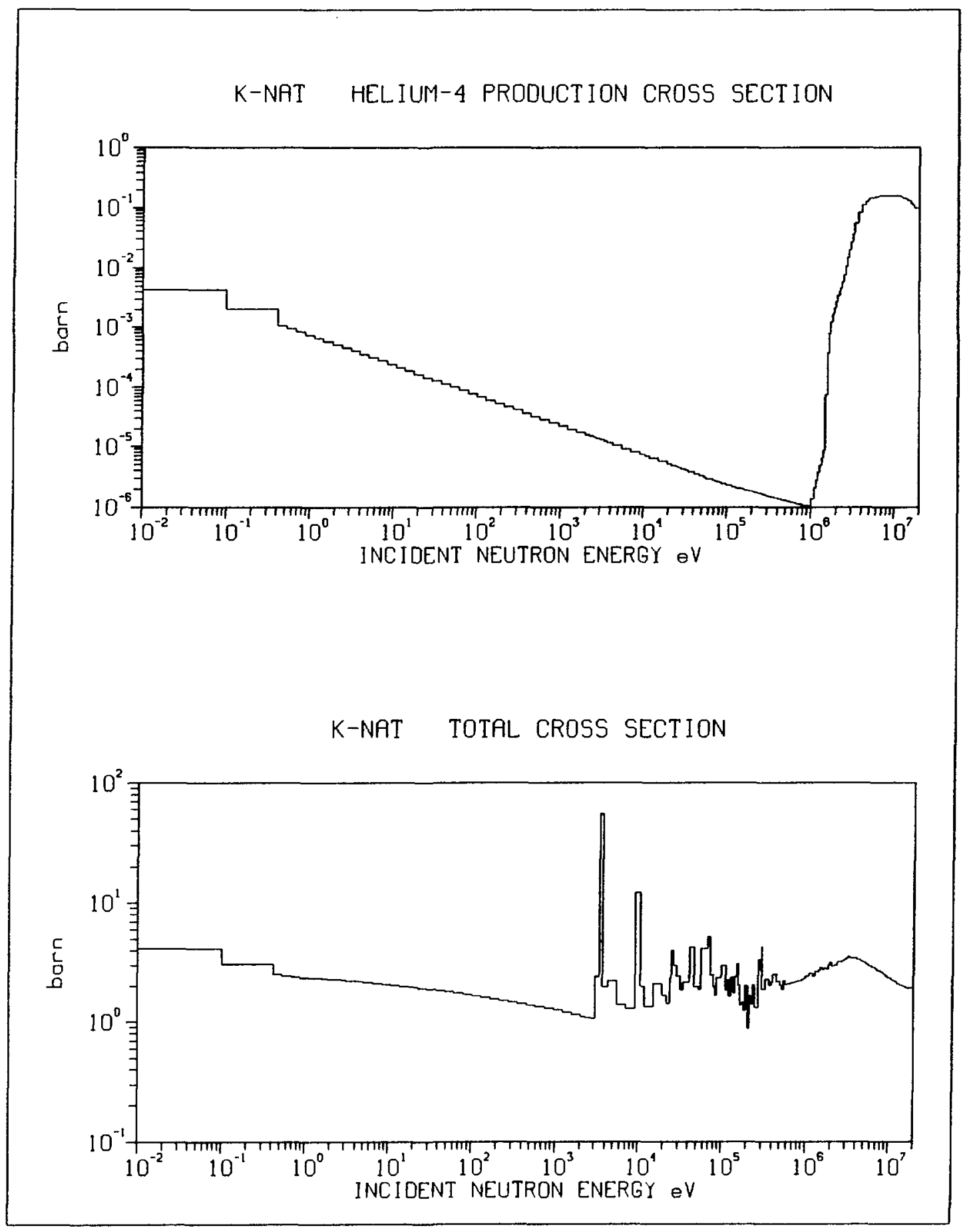




\section{K-NAT ELASTIC CROSS SECTION}

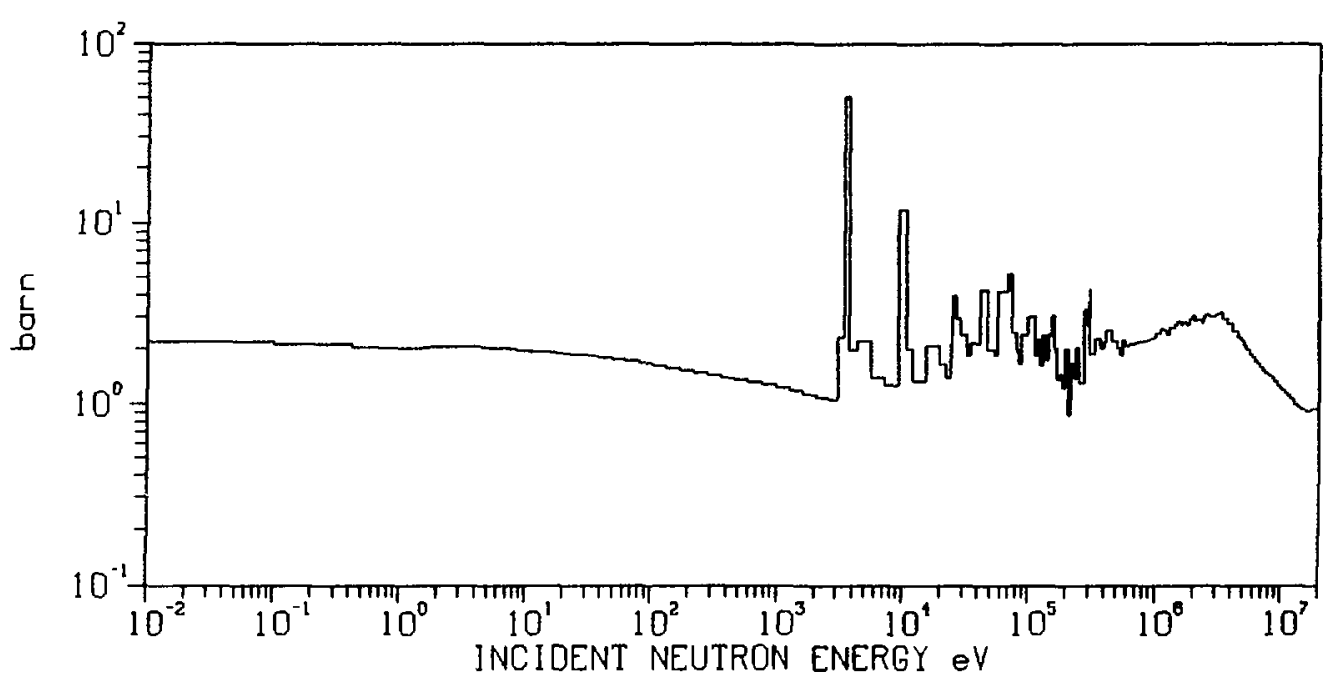

K-NAT TOTAL INELASTIC CROSS SECTION

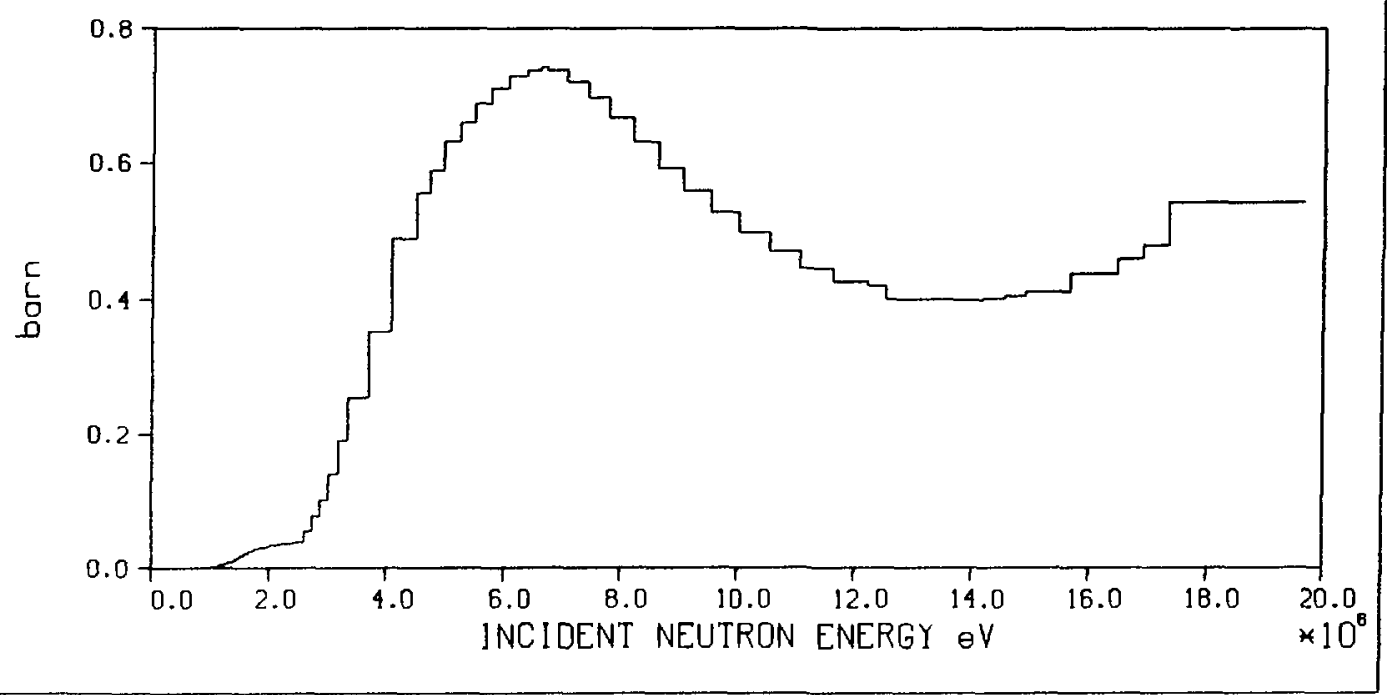




\section{K-NAT RADIATIVE CAPTURE CROSS SECTION}

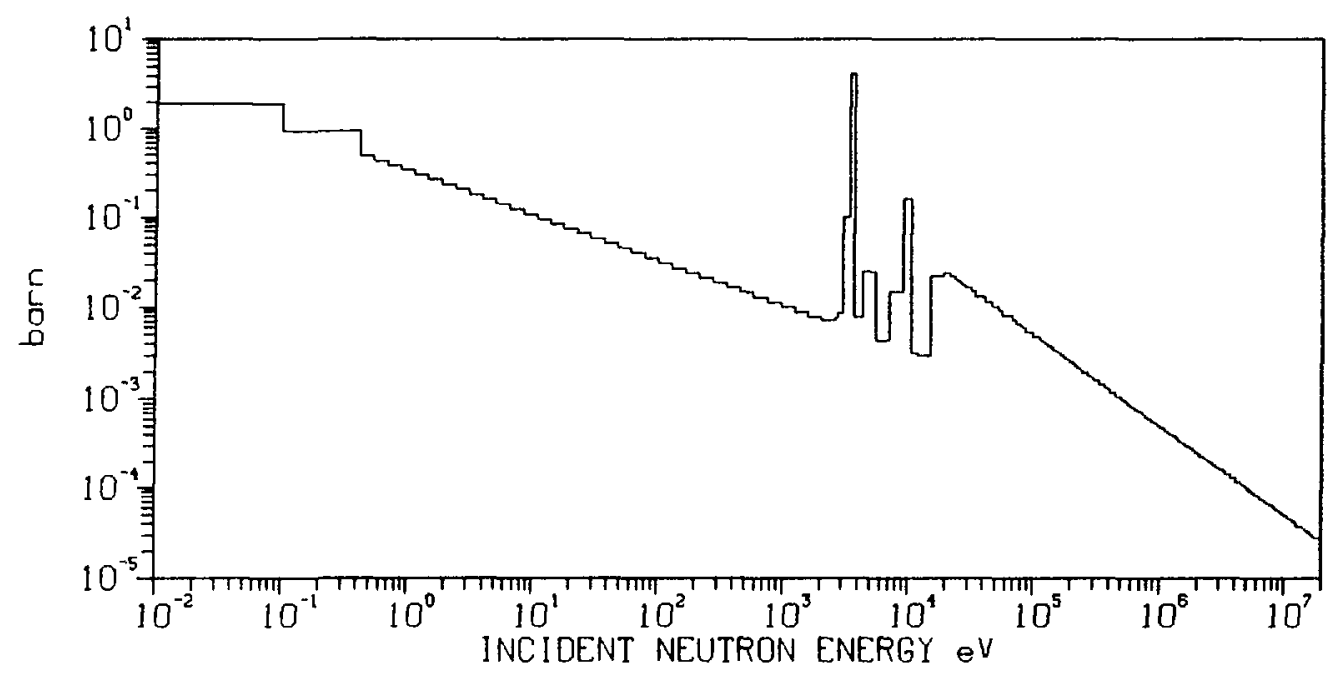

K-NAT TOTAL $(n, 2 n)$ REACTION CROSS SECTION

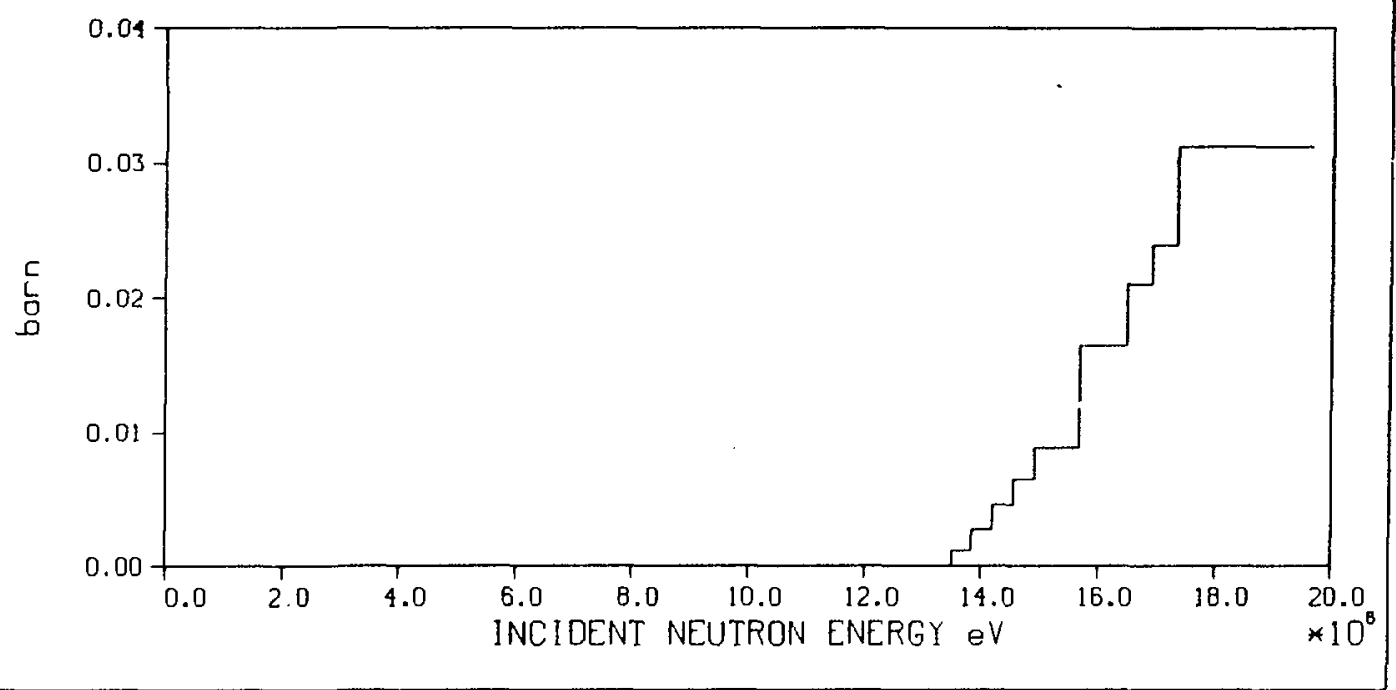


CA-NAT RECOMMENDED PROMPT KERMA FACTOR (solid Line)

REC. K. FACTOR WITH CH. PARTICLE DECAY HEAT (dashed line)

REC. K. FACTOR PLUS TOTAL DECAY HEAT (choin dotted line)

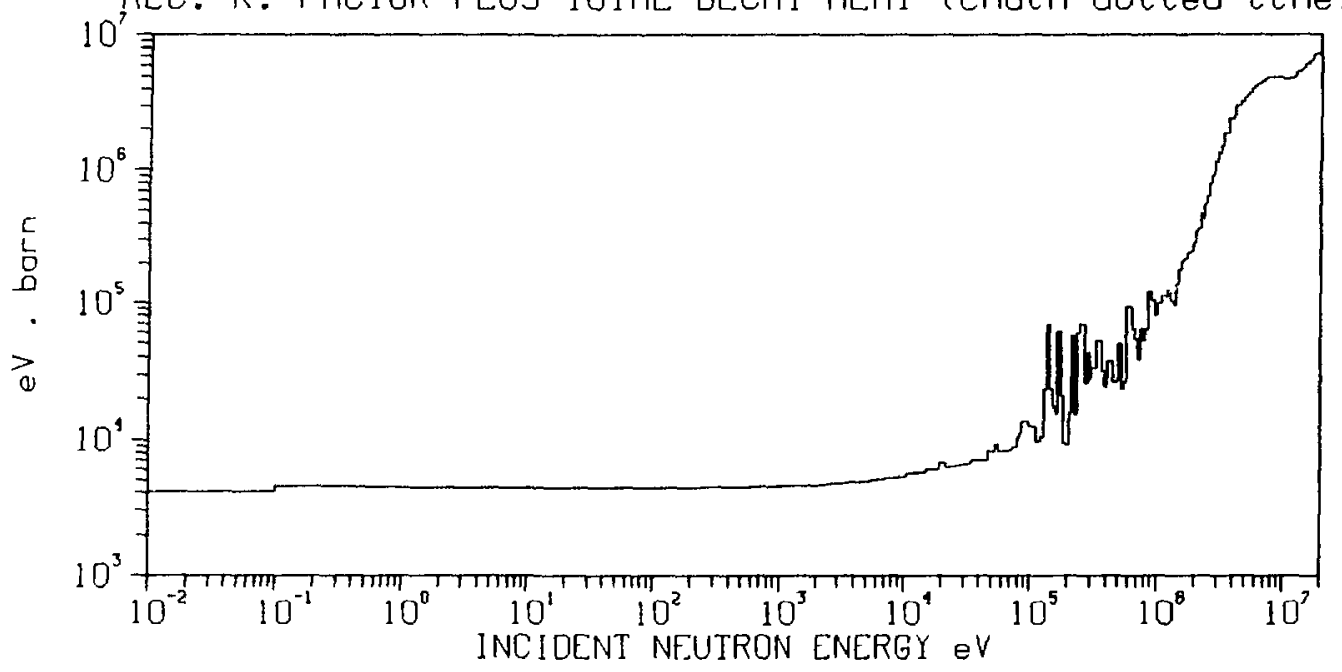

CA-NAT RECOMMENDED PROMPT KERMA FACTOR (solid line) REC. K. FACTOR WITH CH. PARTICLE DECAY HEAT (dashed line) $\because$ REC. K. FACTOR PLUS TOTAL DECAY HEAT (chain dotted line)

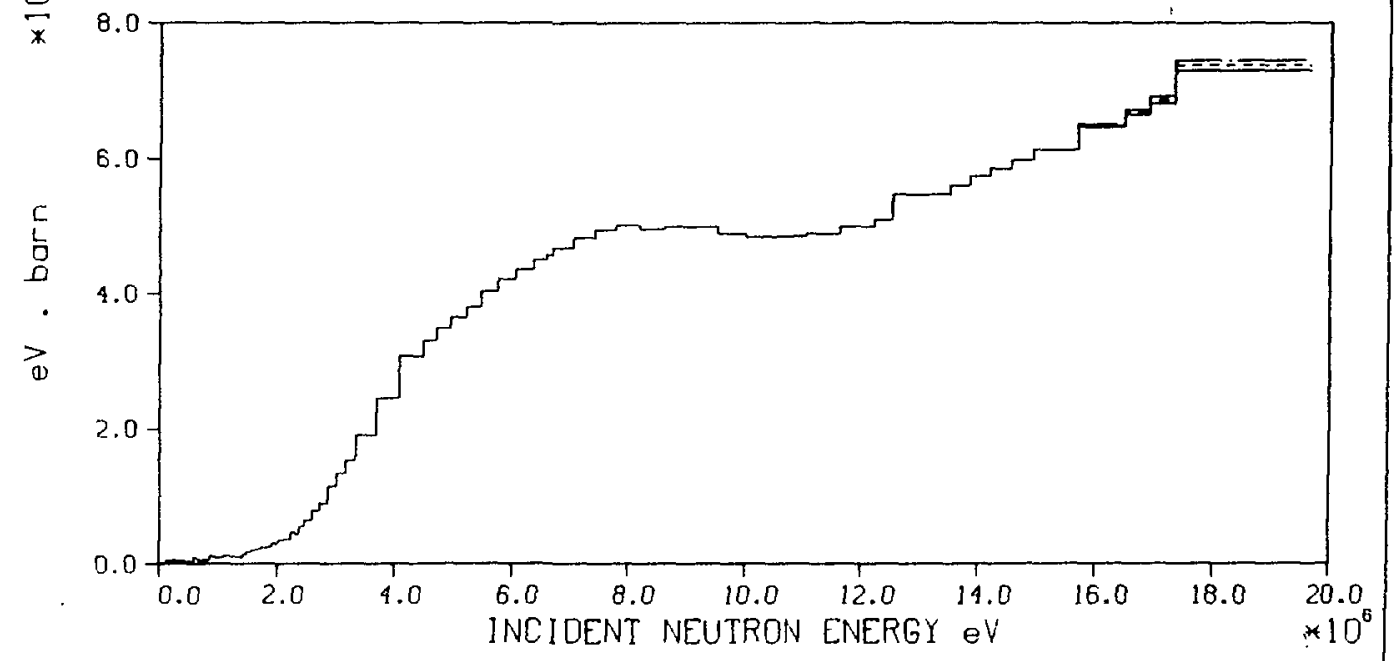




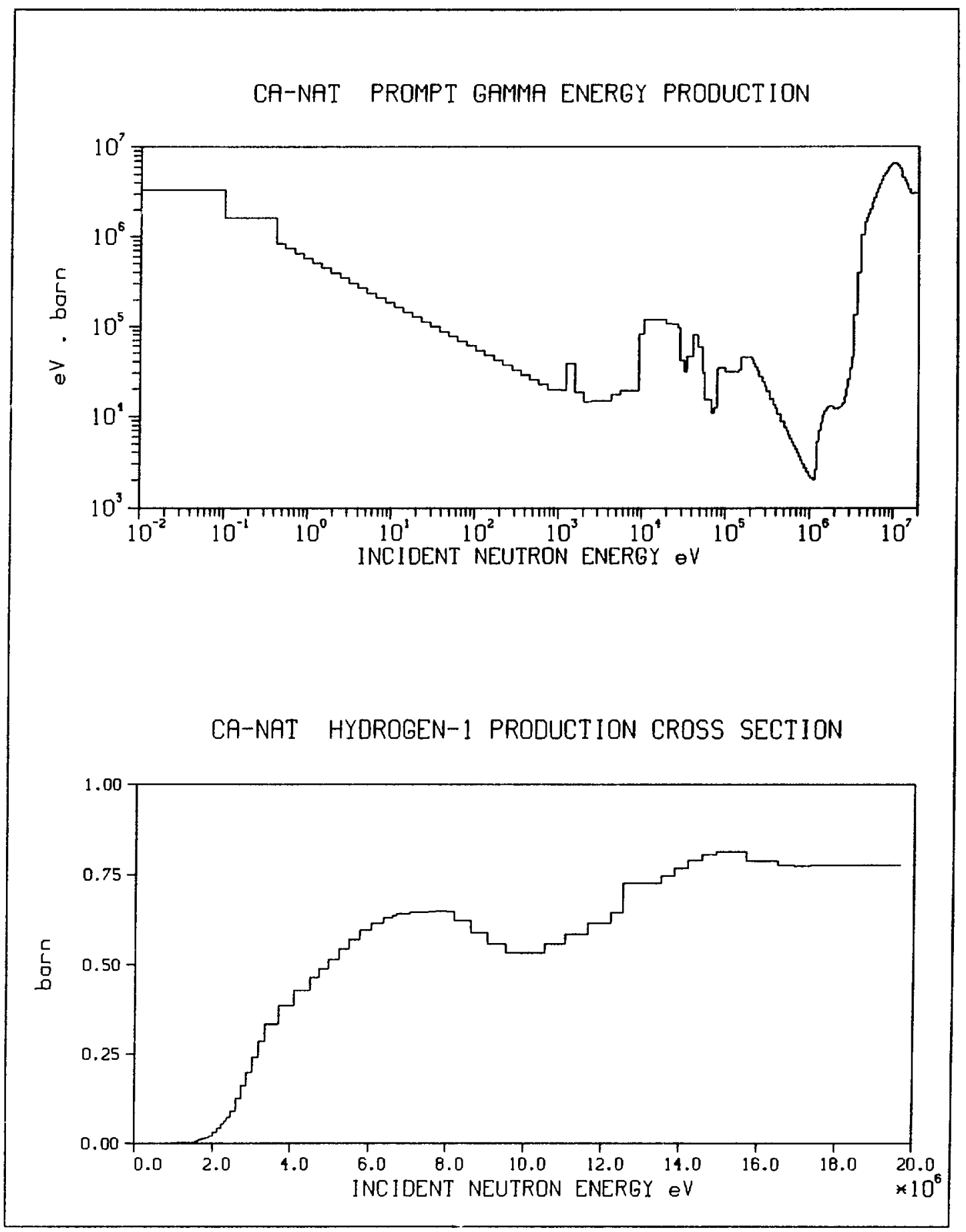




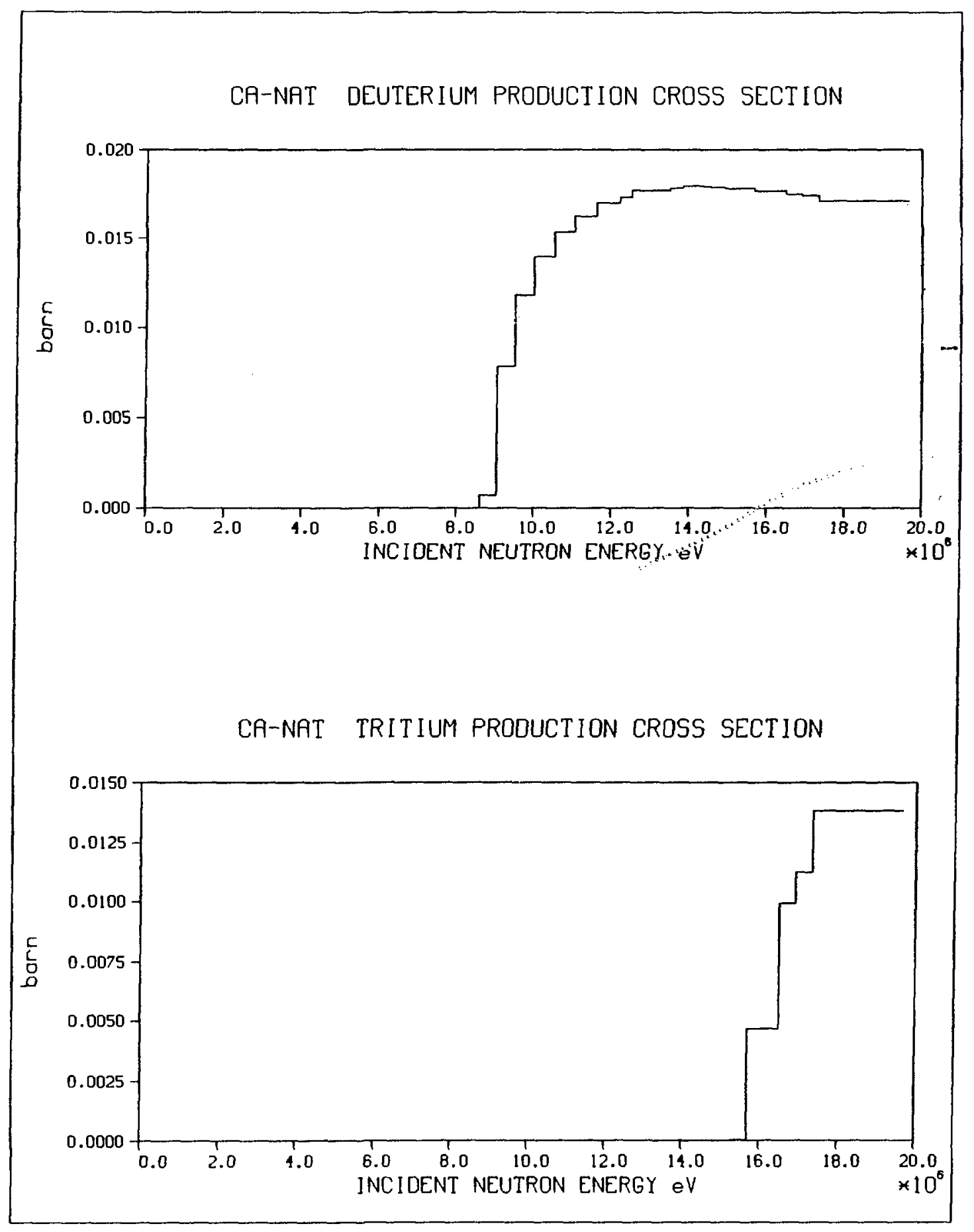




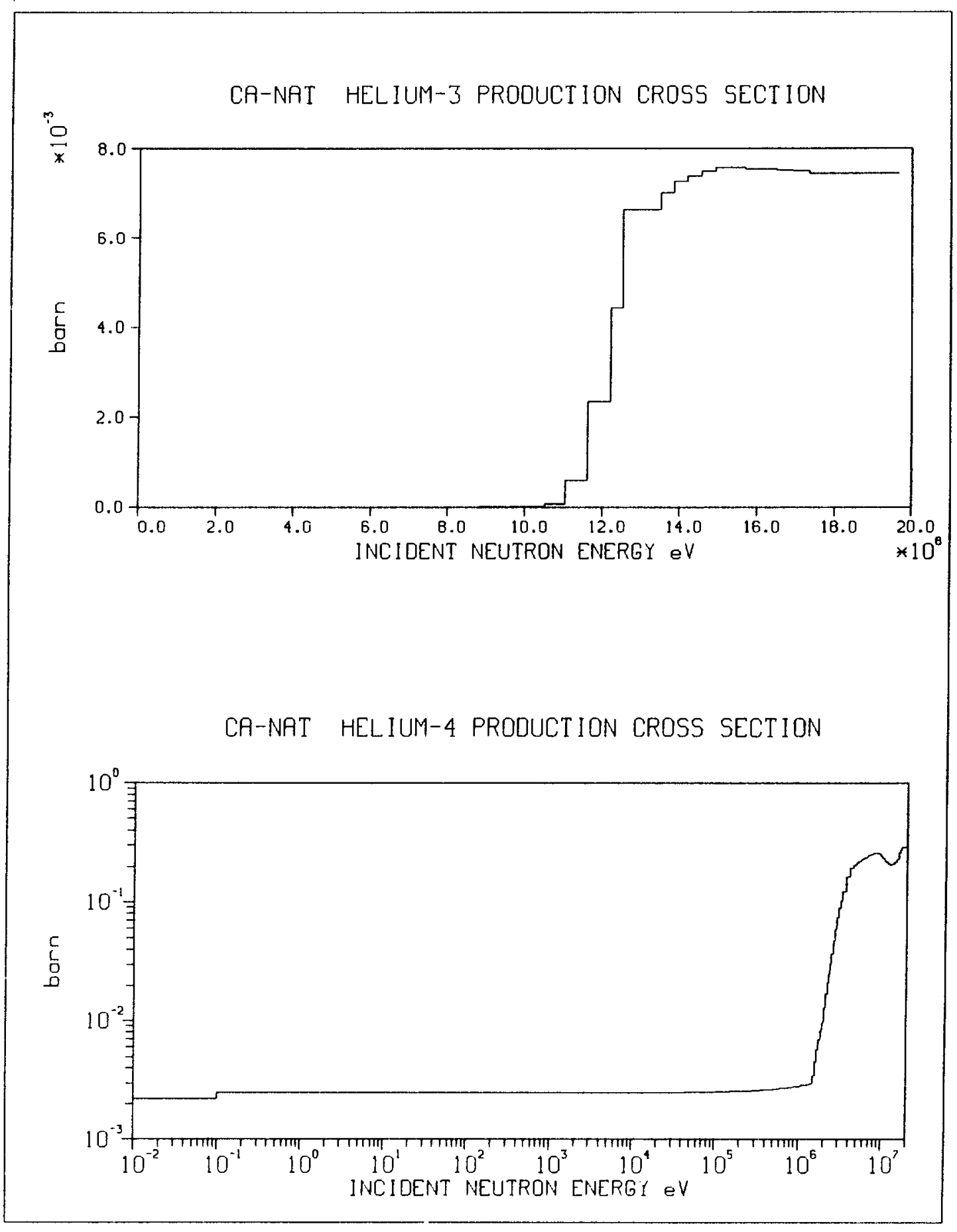




\section{CA-NAT TOTAL CROSS SECTION}

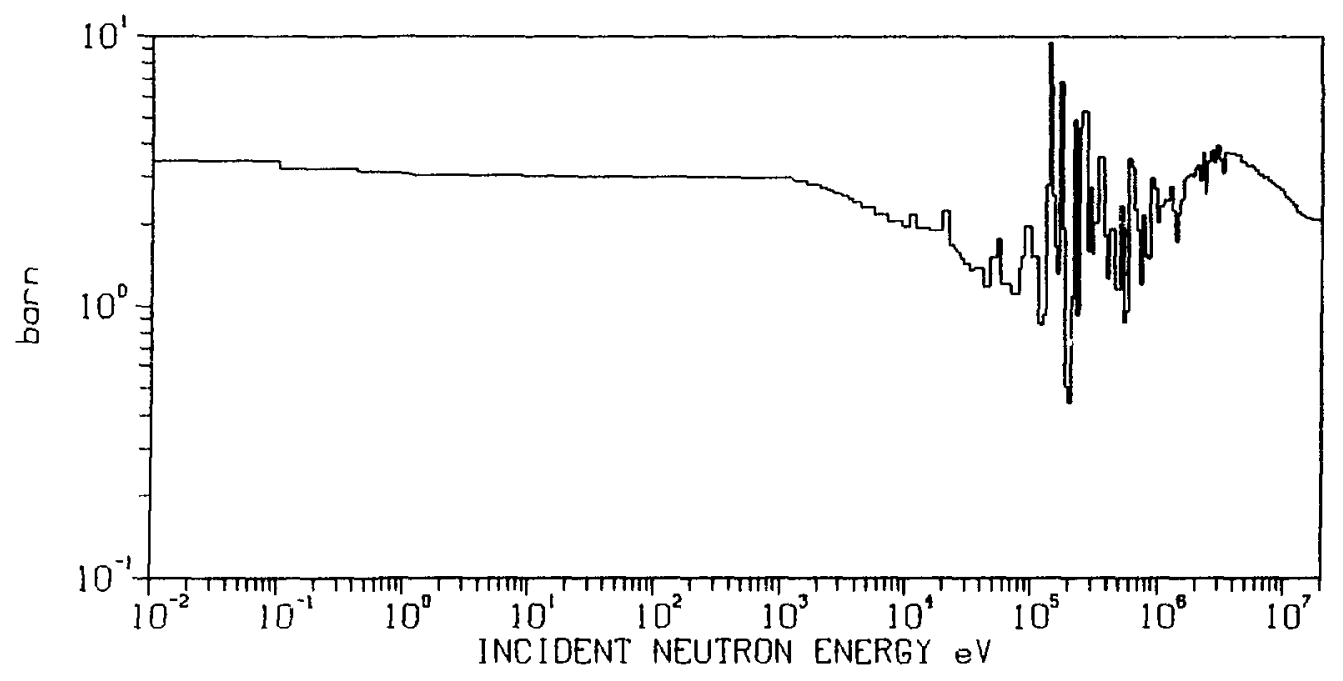

\section{CA-NAT ELASTIC CROSS SECTION}

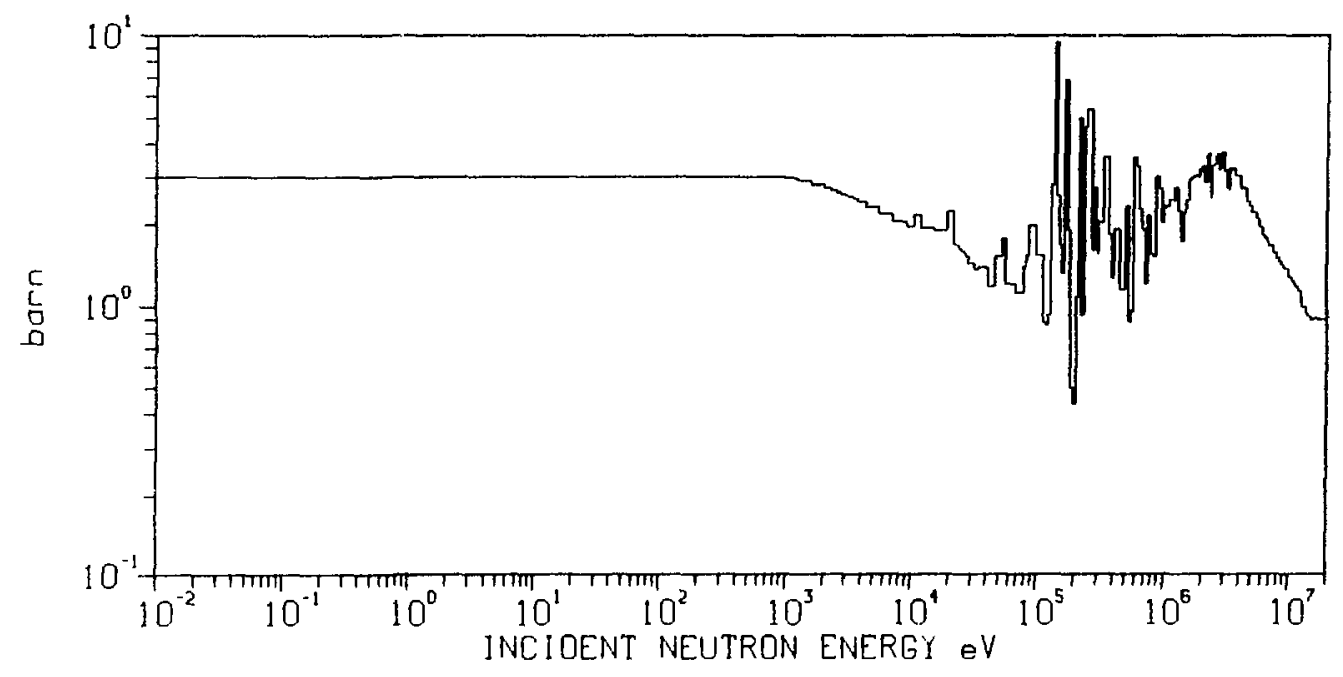




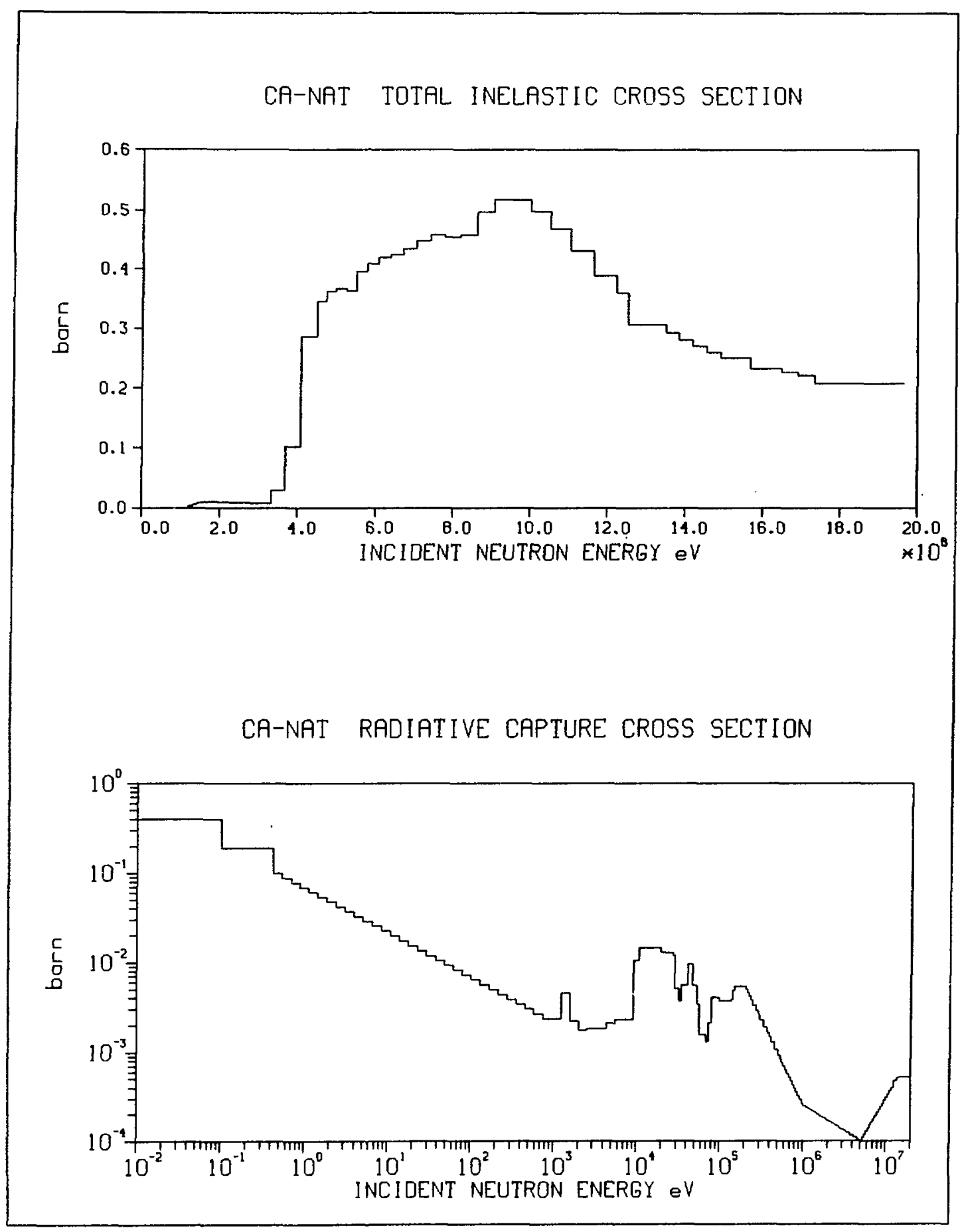




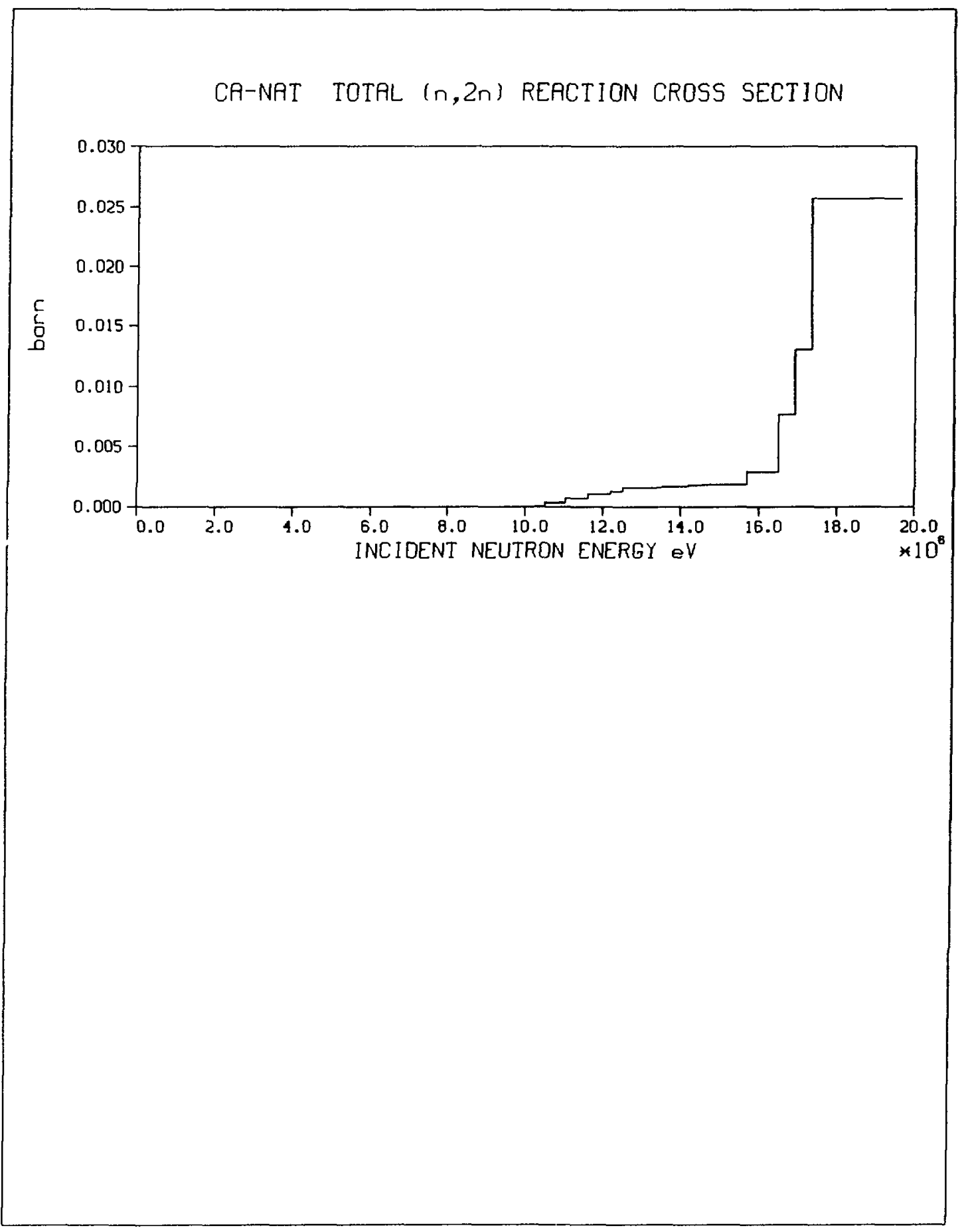




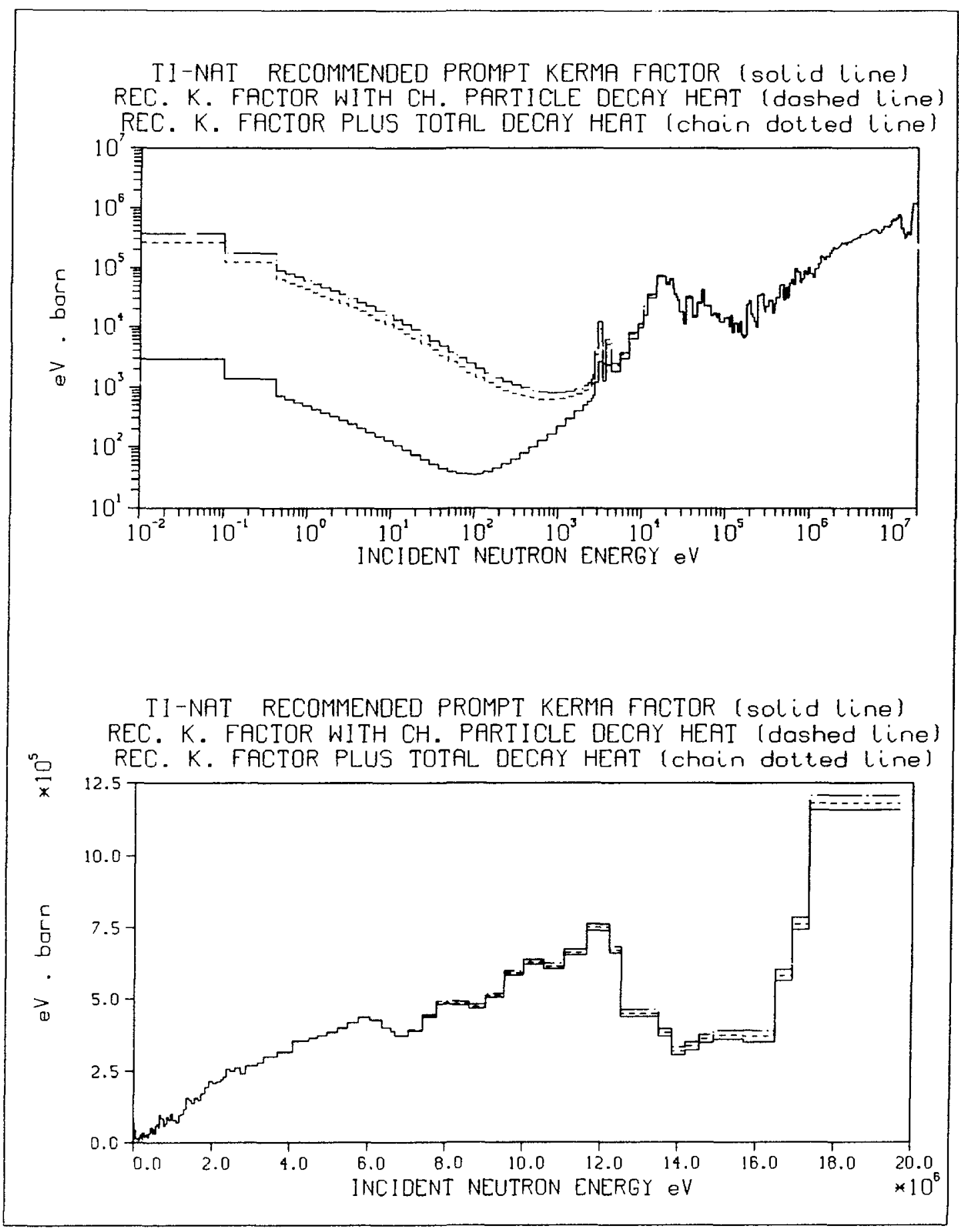




\section{TI-NAT PROMPT GAMMA ENERGY PRODUCTION}

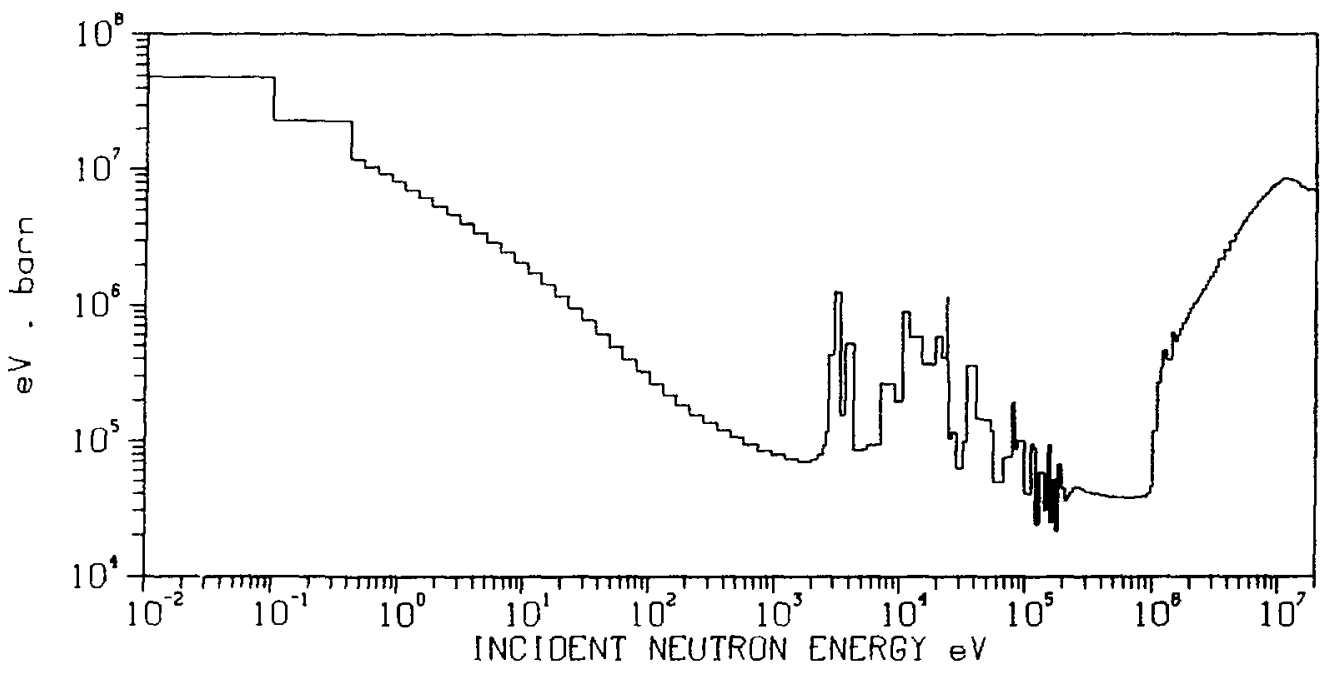

II-NAT HYDROGEN-1 PRODUCTION CROSS SECTION

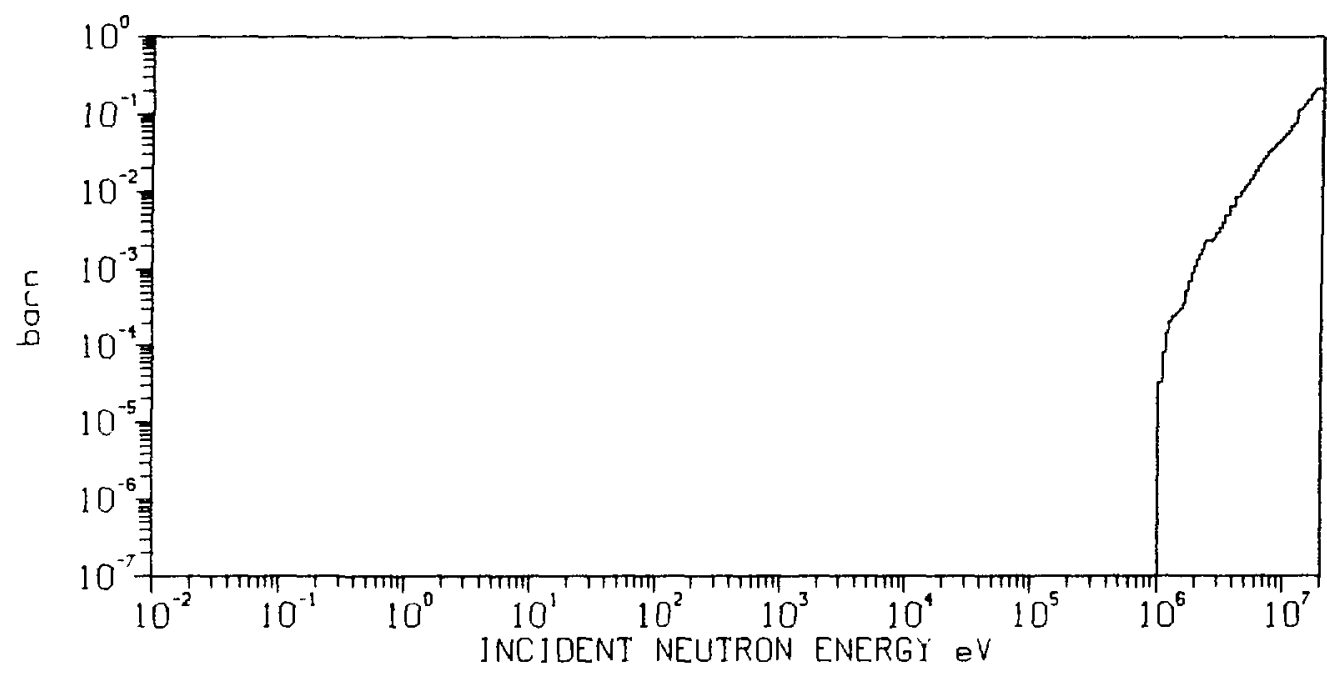




\section{TI-NAT OEUTERIUM PRODUCTION CROSS SECTION}

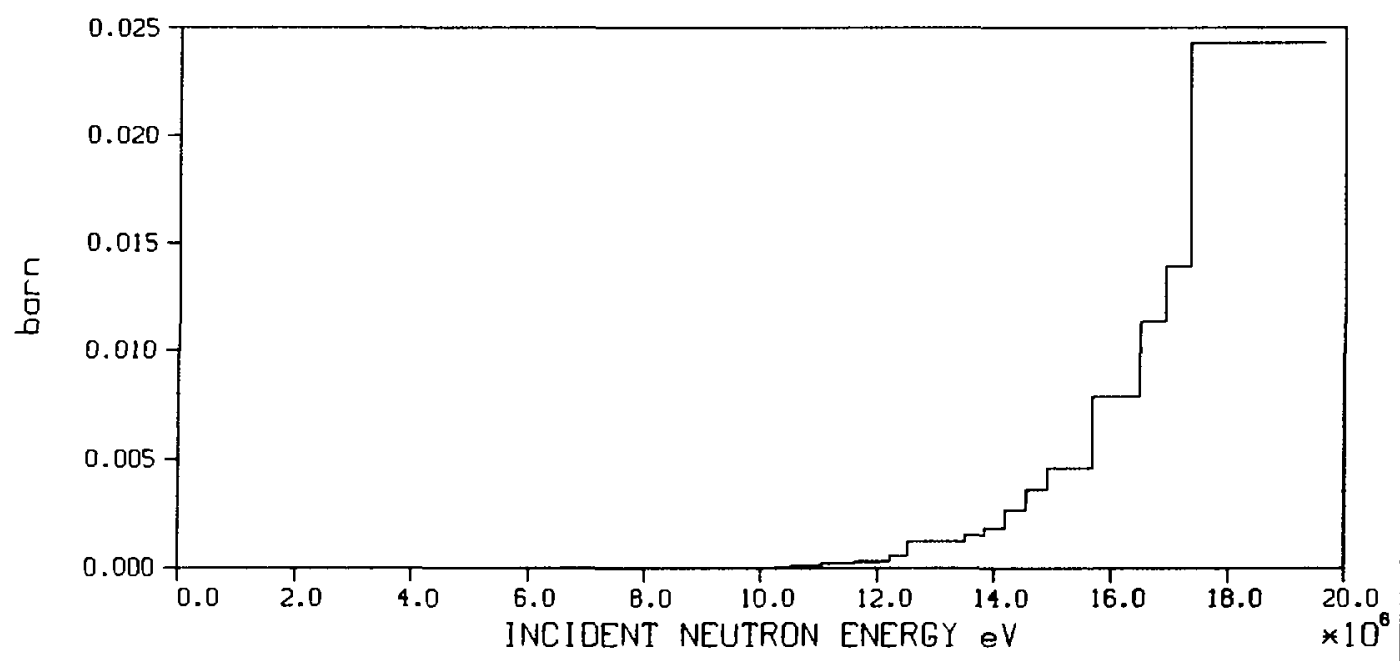

TI-NAT TRITIUM PRODUCTION CROSS SECTION

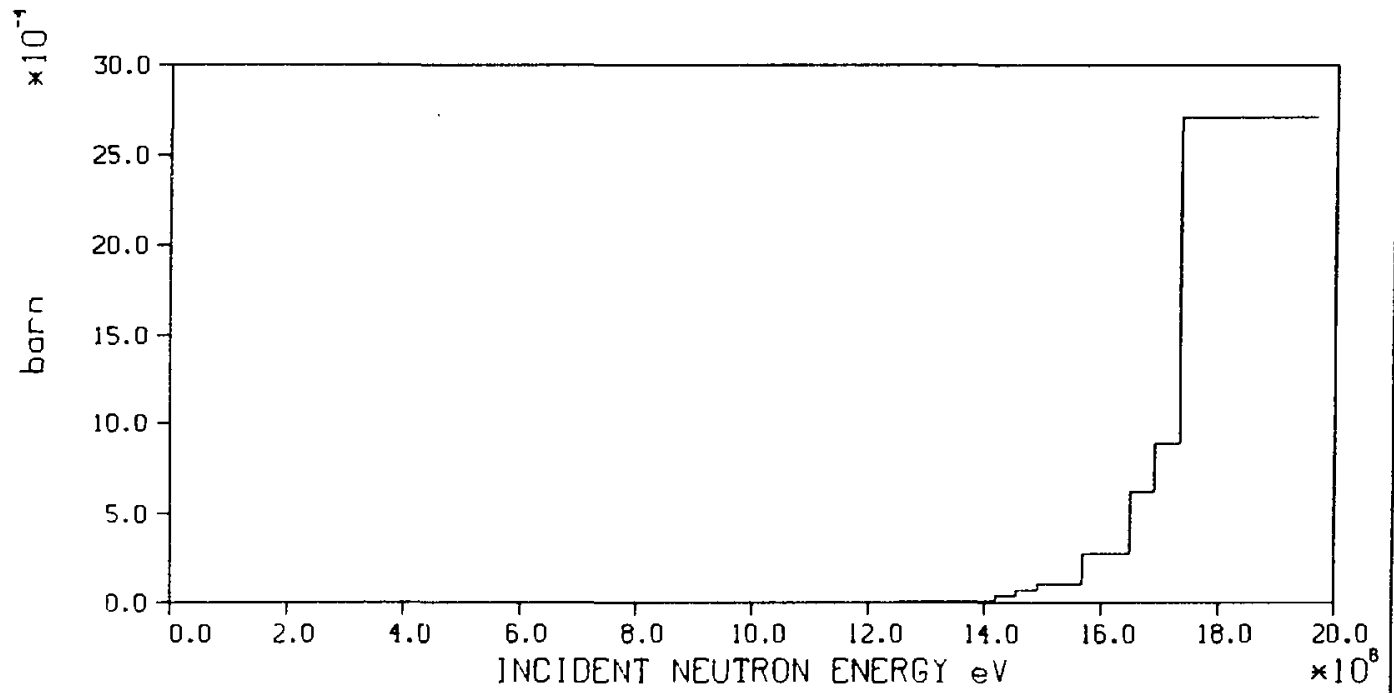




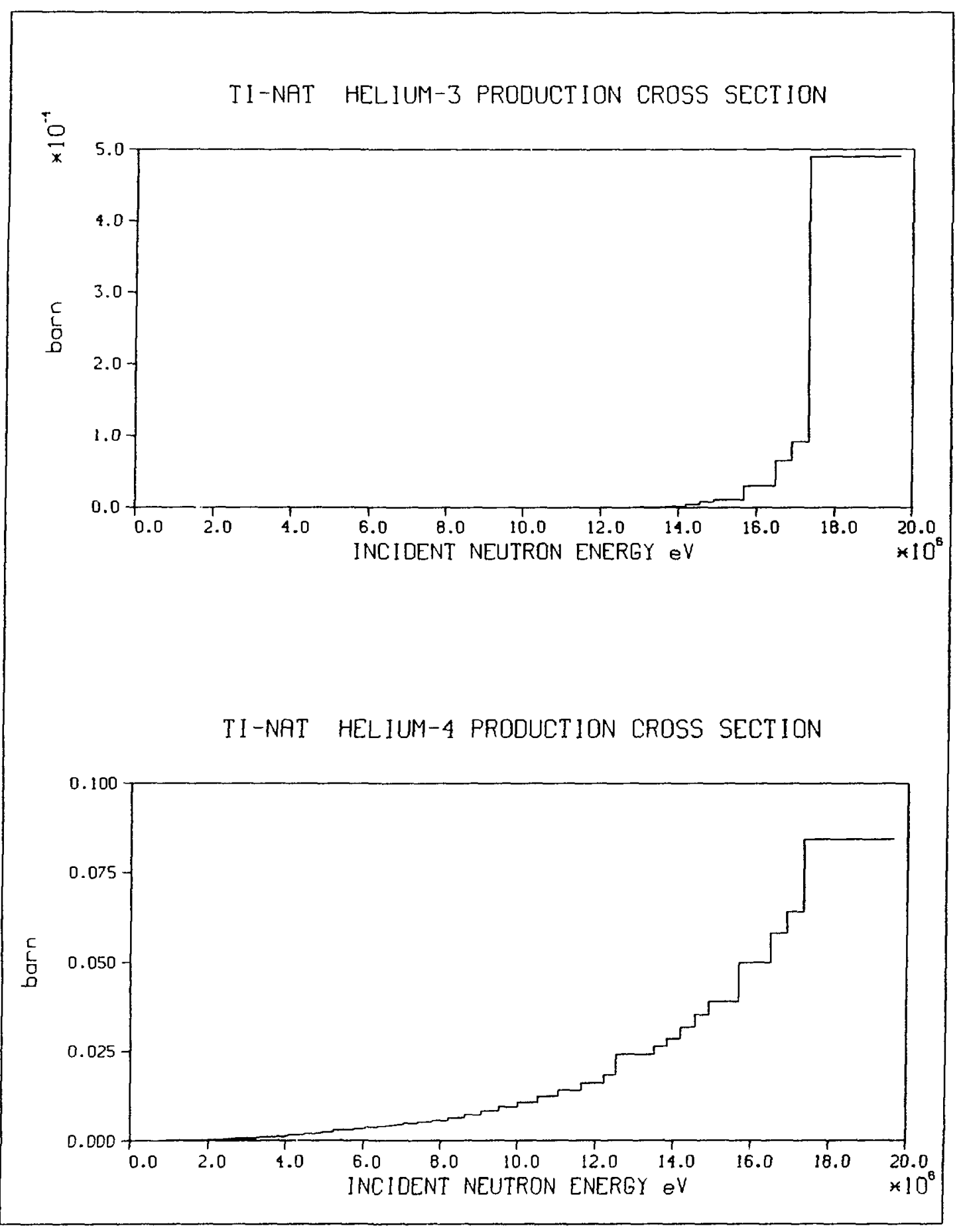




\section{II-NAT TOTAL CROSS SECTION}

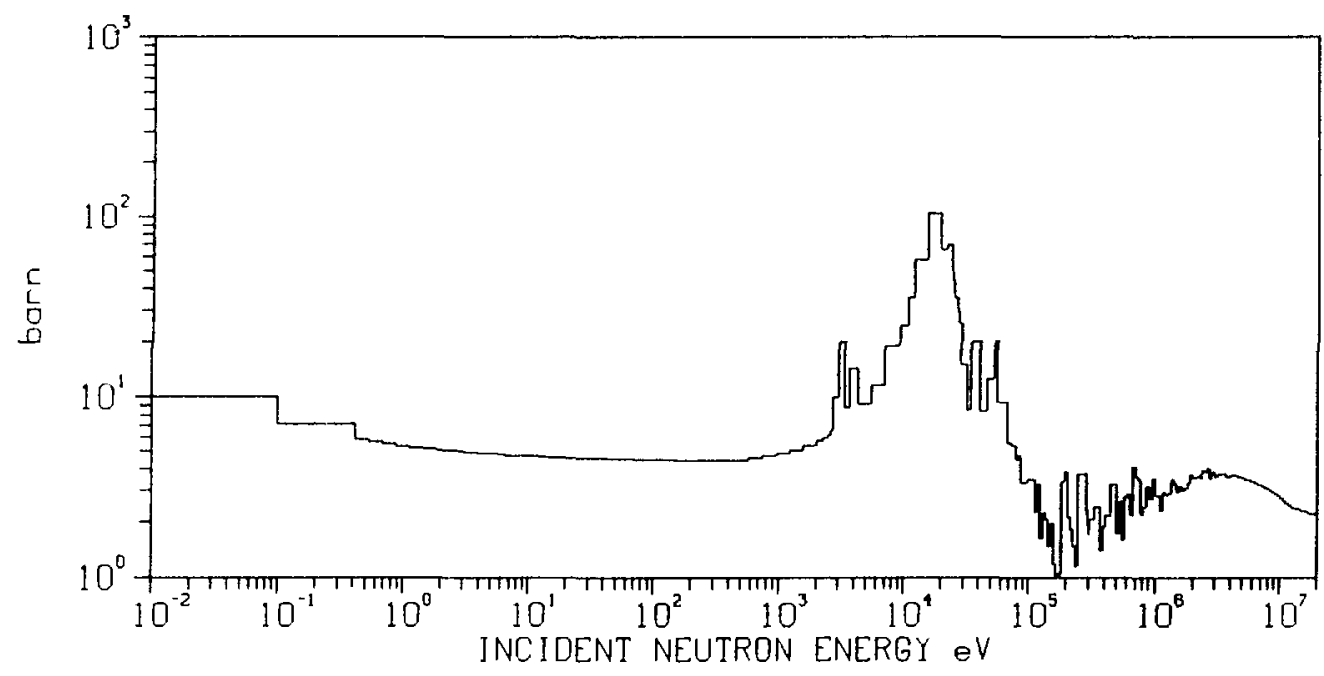

TI-NAT ELASTIC CROSS SECTION

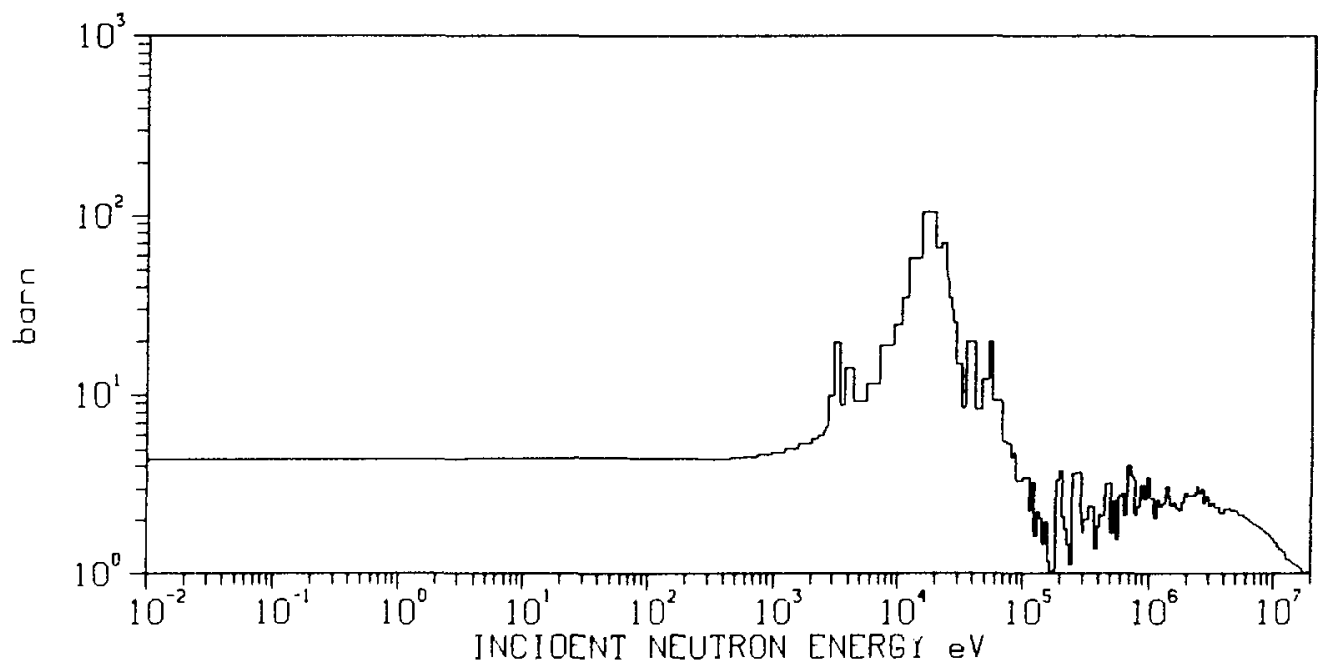




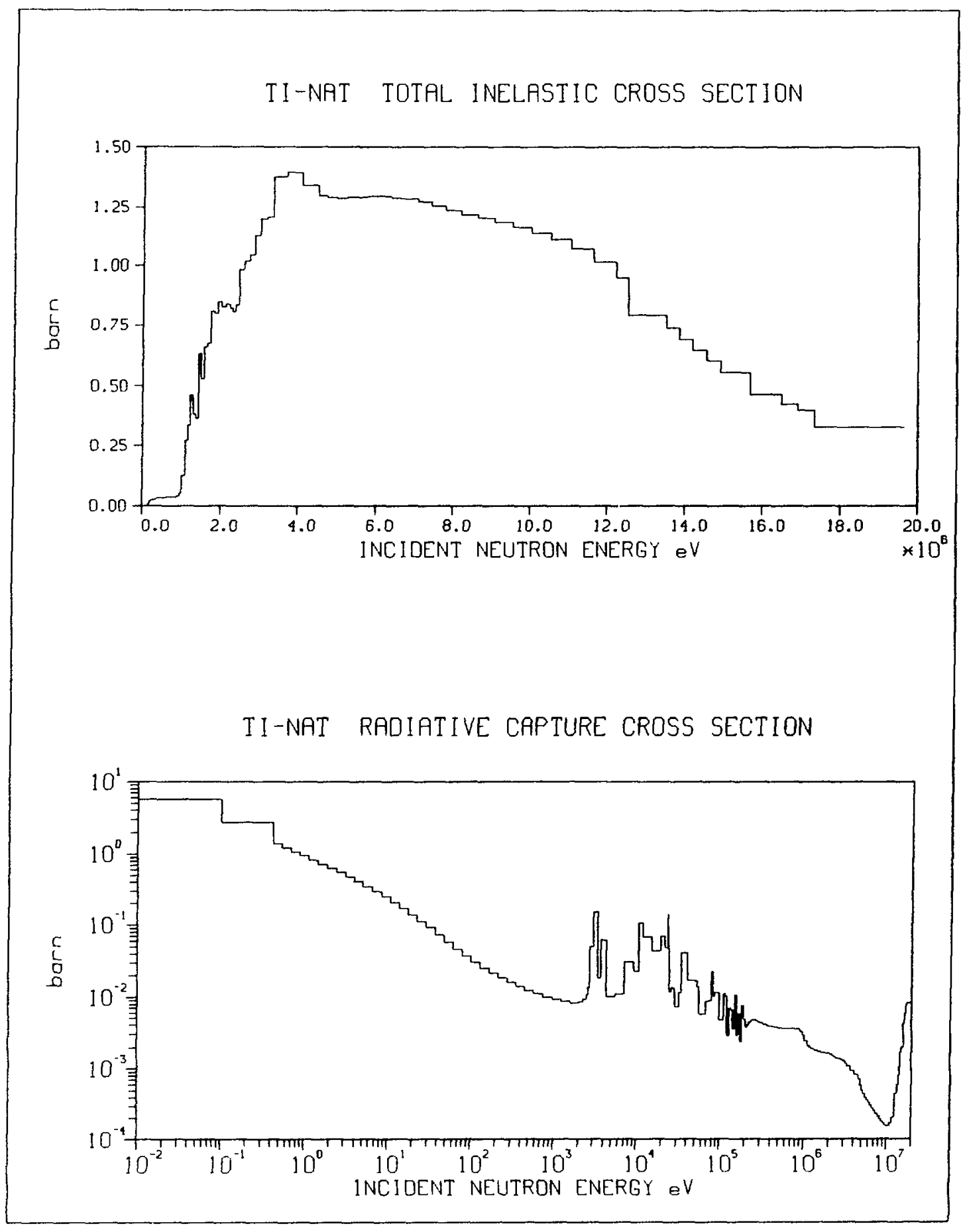




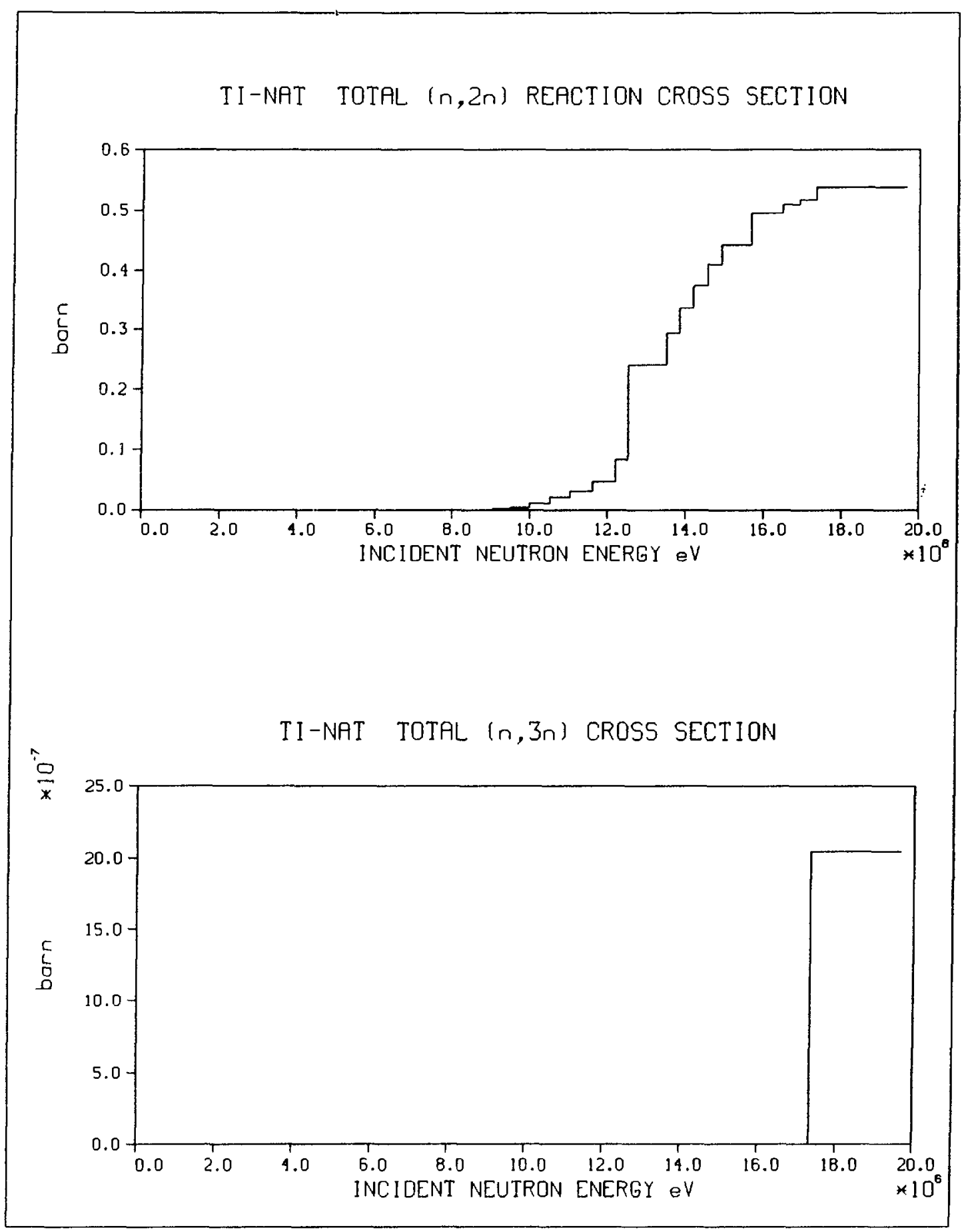




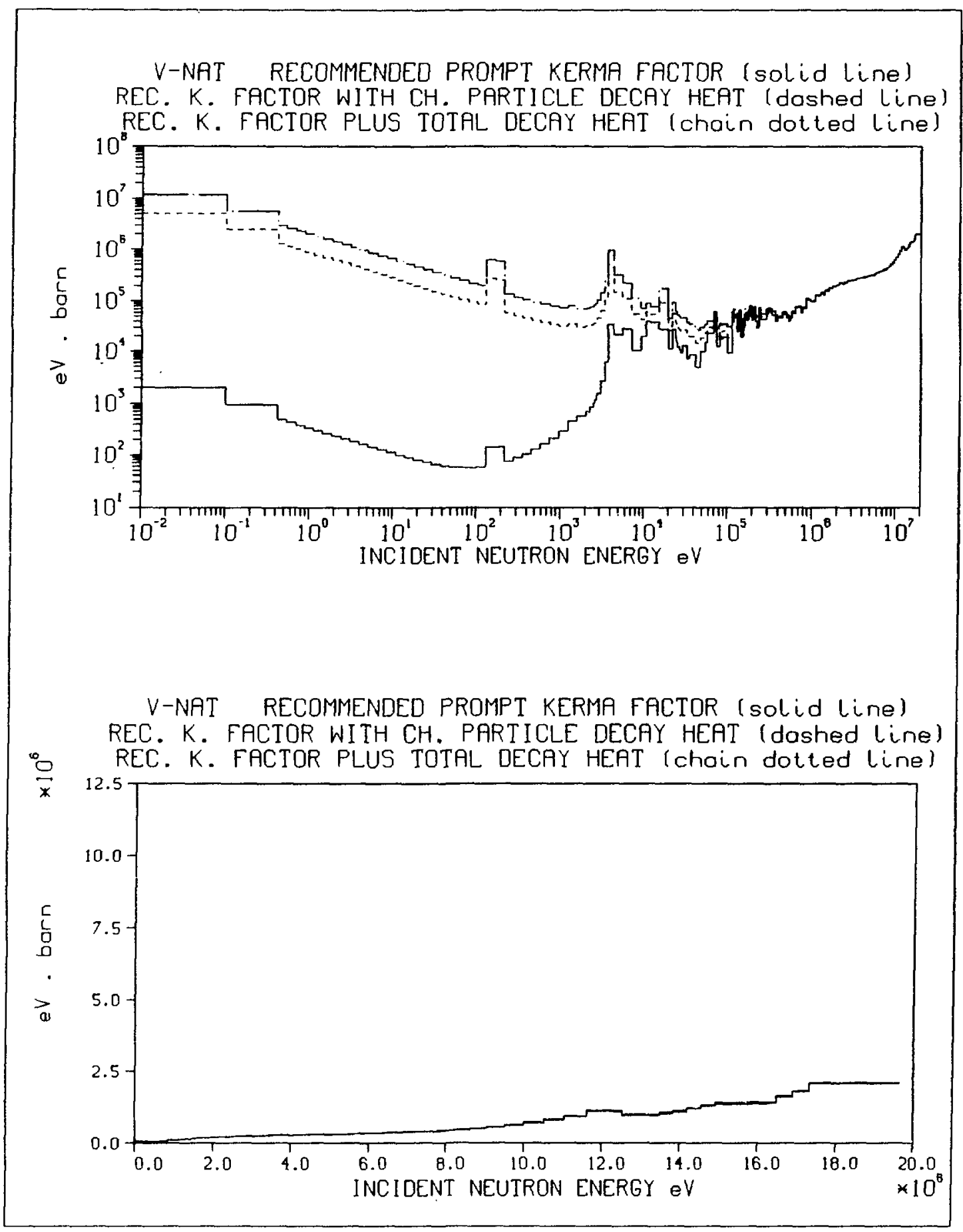




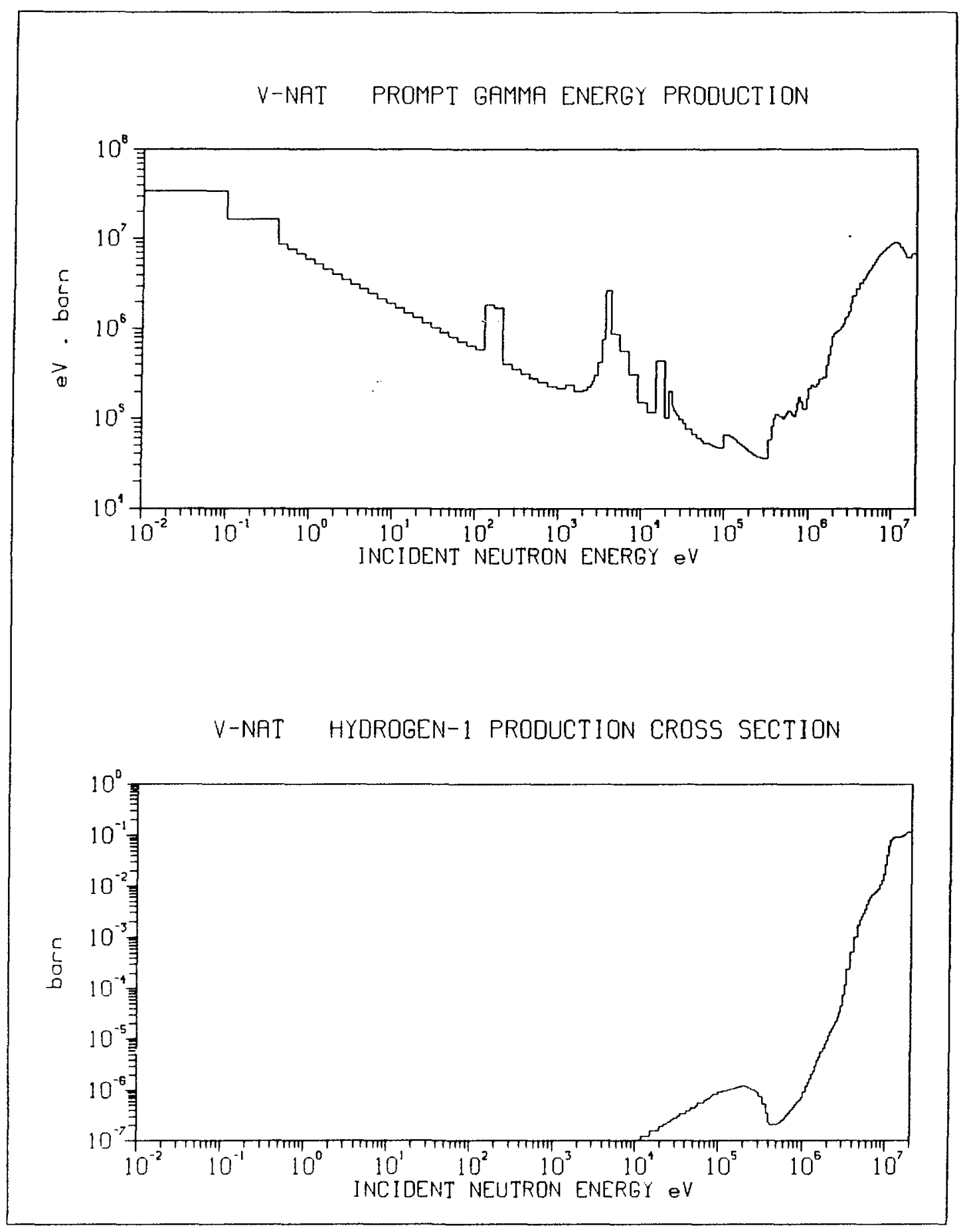




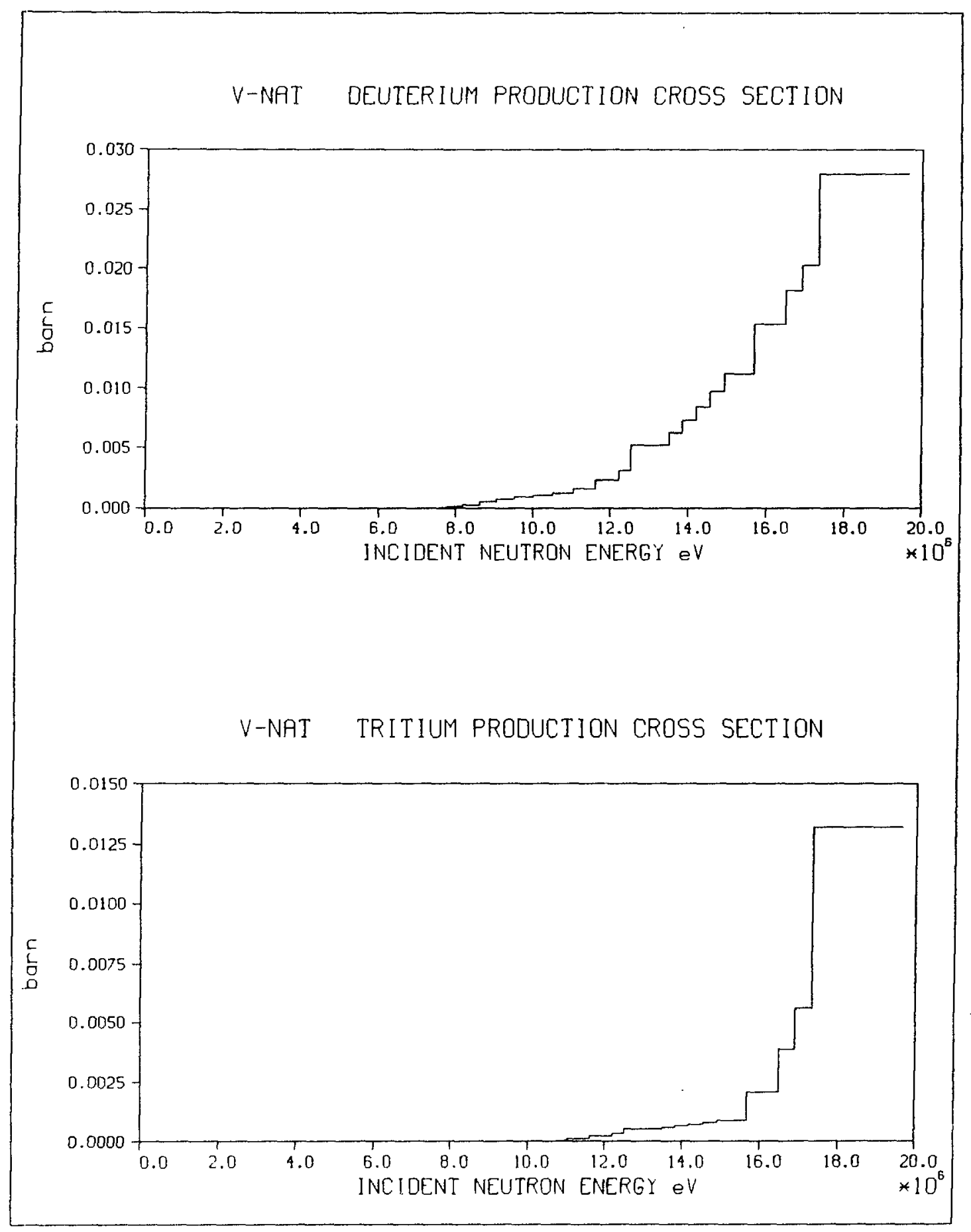




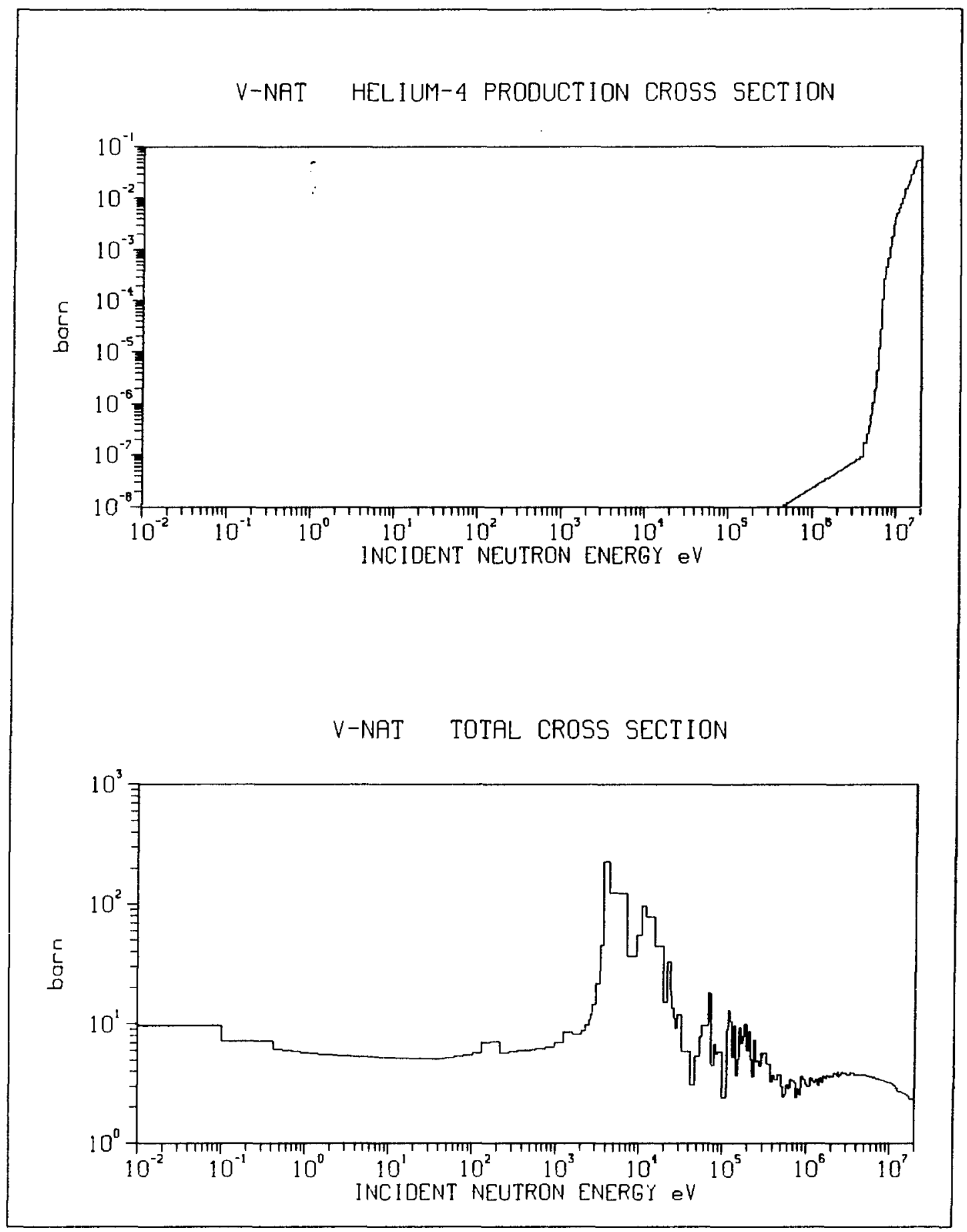




\section{V-NAT ELASTIC CROSS SECTION}

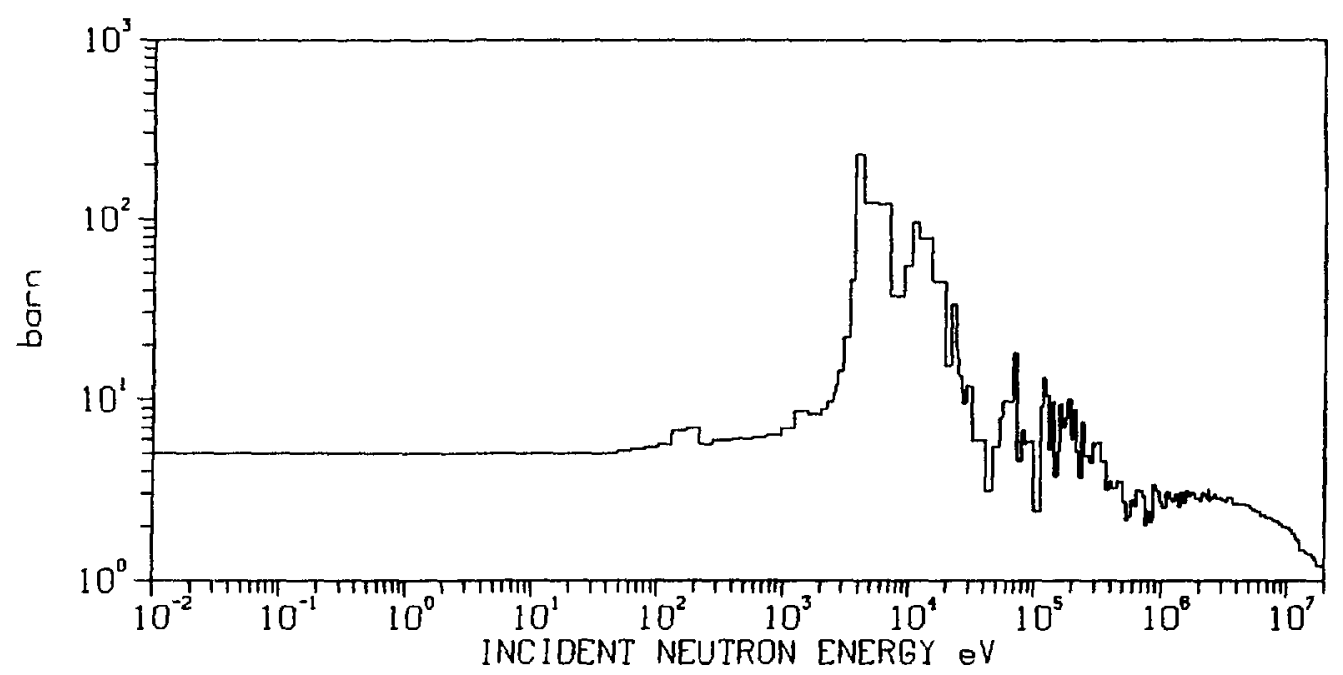

V-NAT TOTAL INELASTIC CROSS SECTION

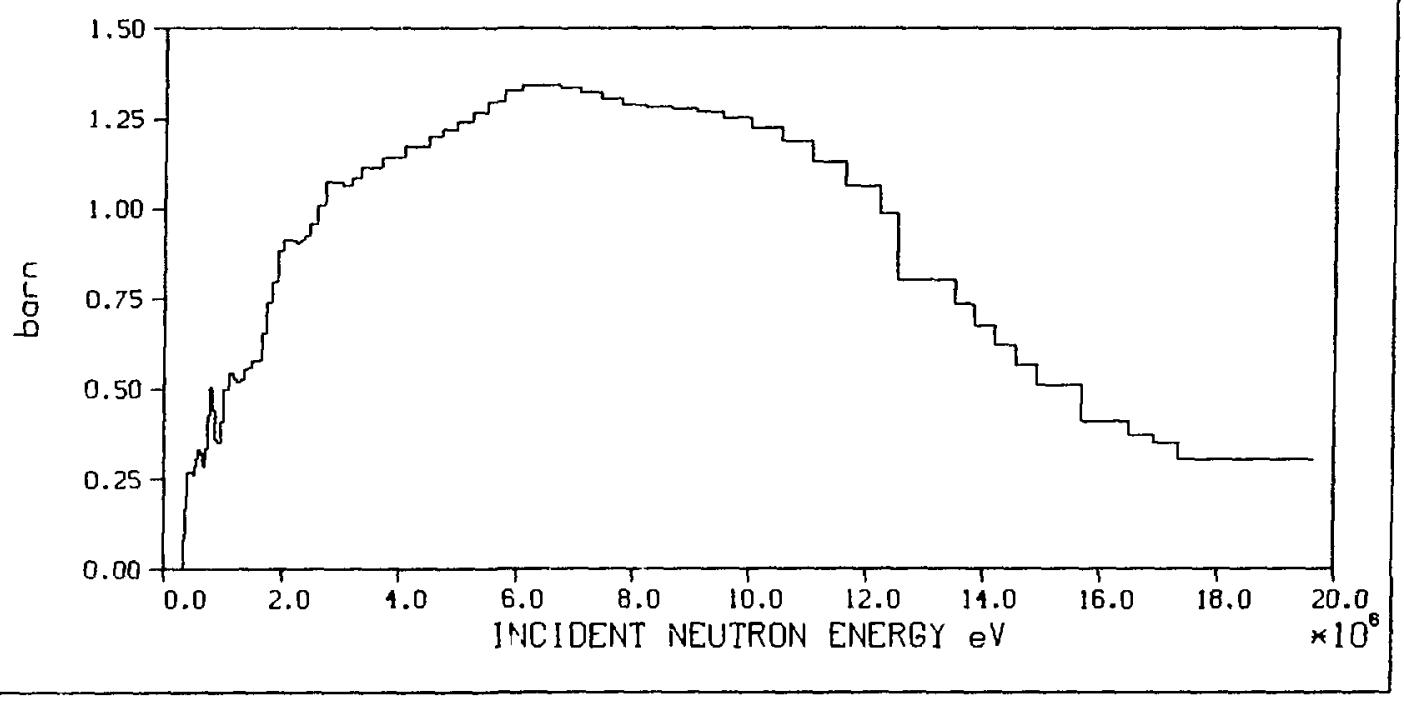




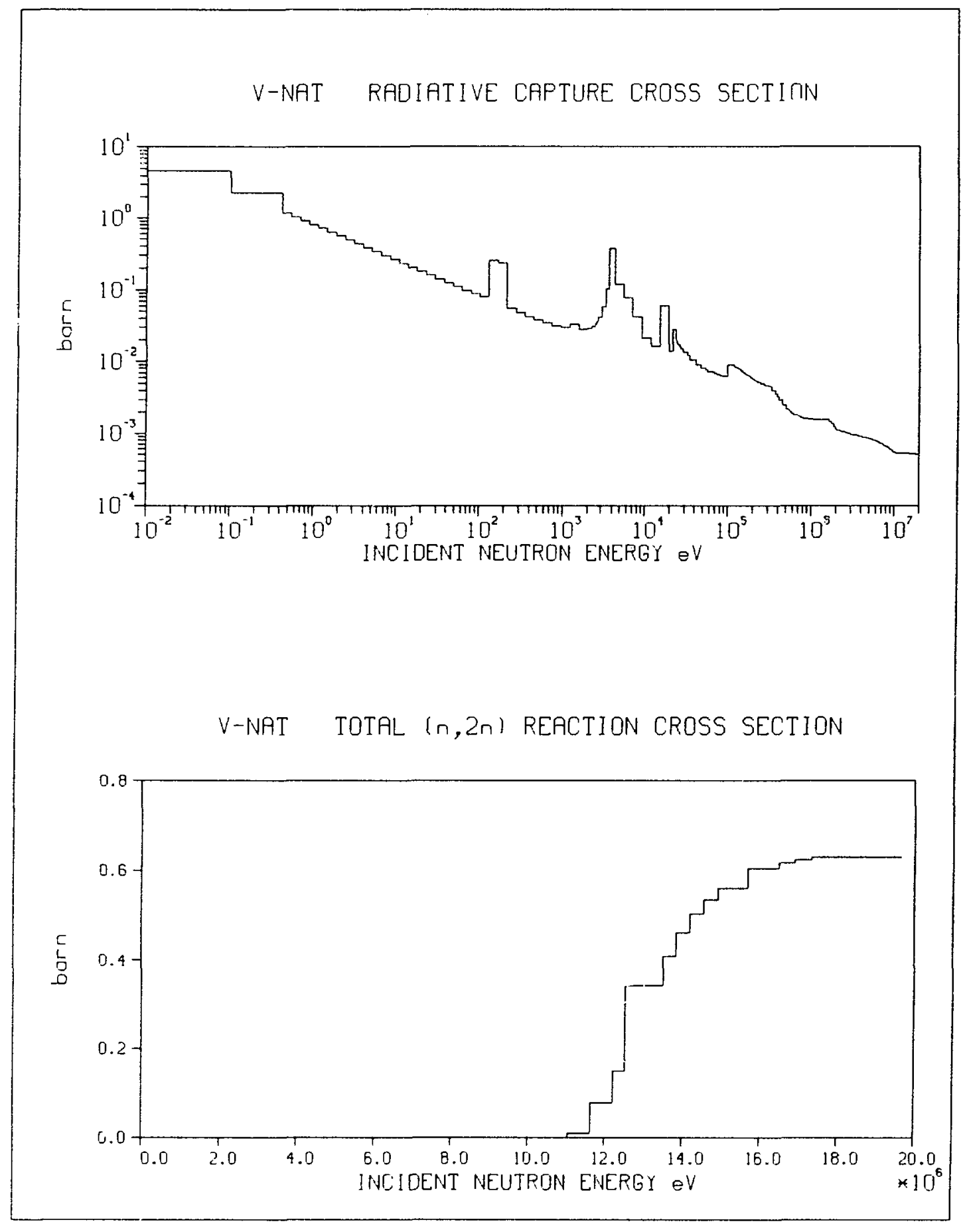




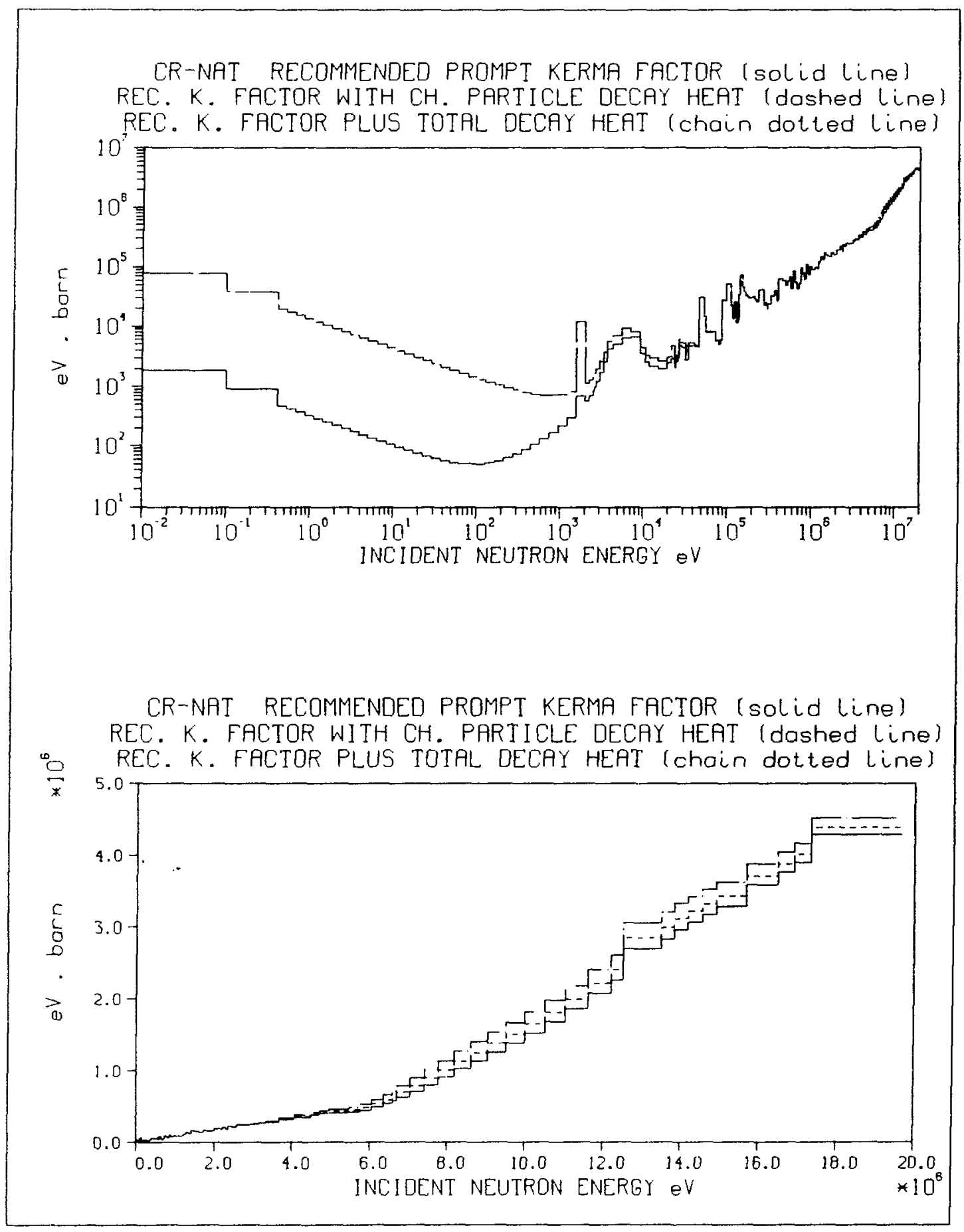




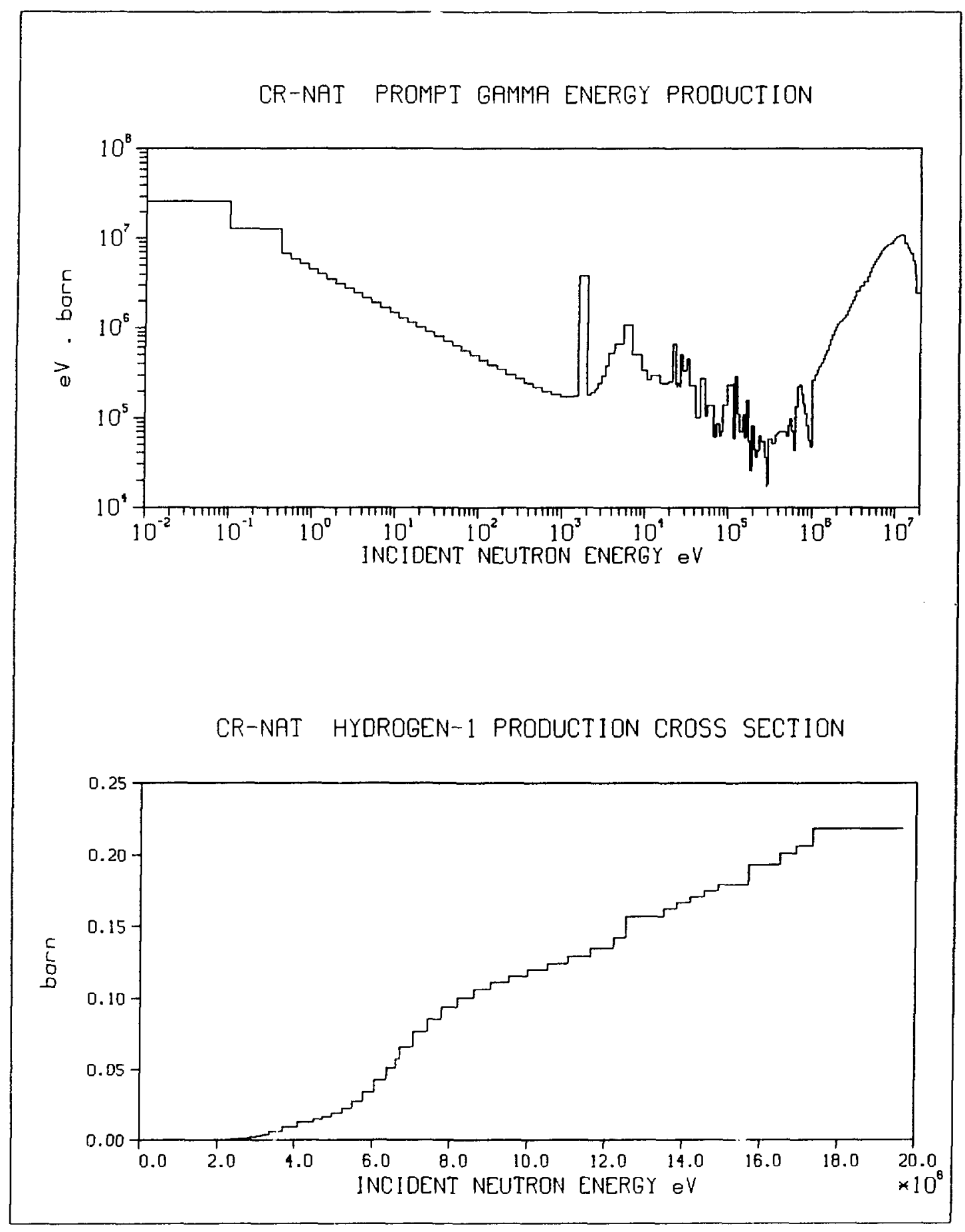




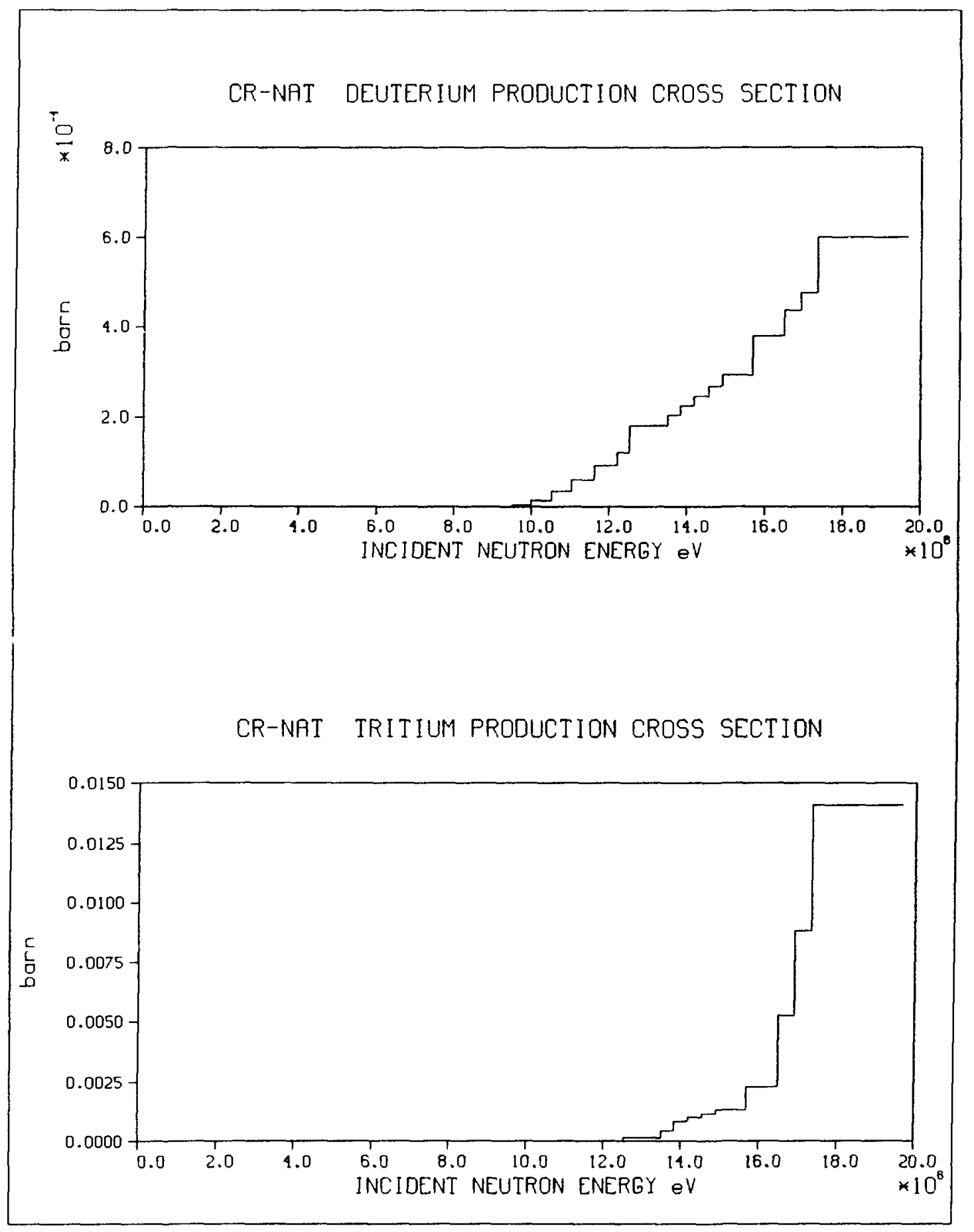




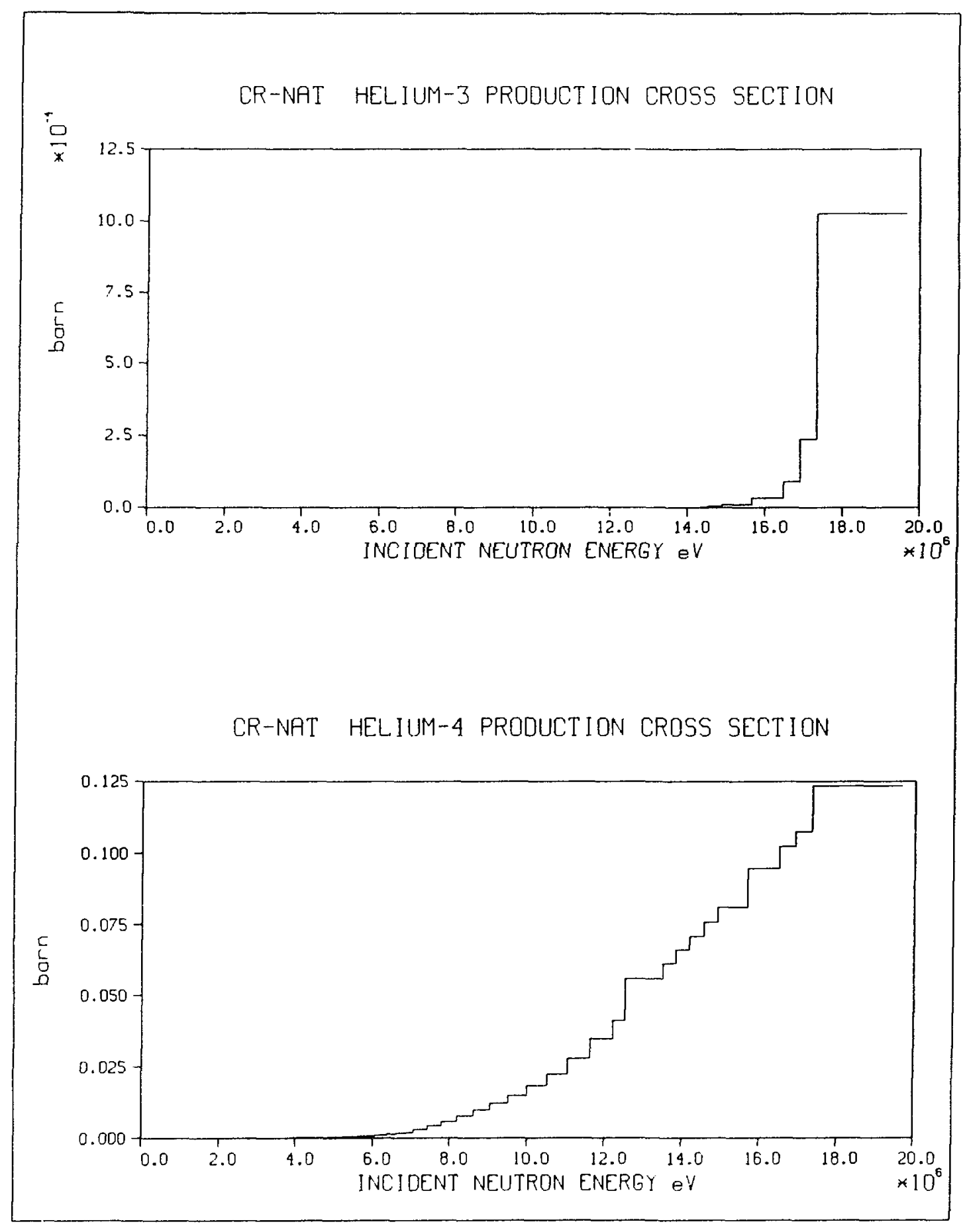




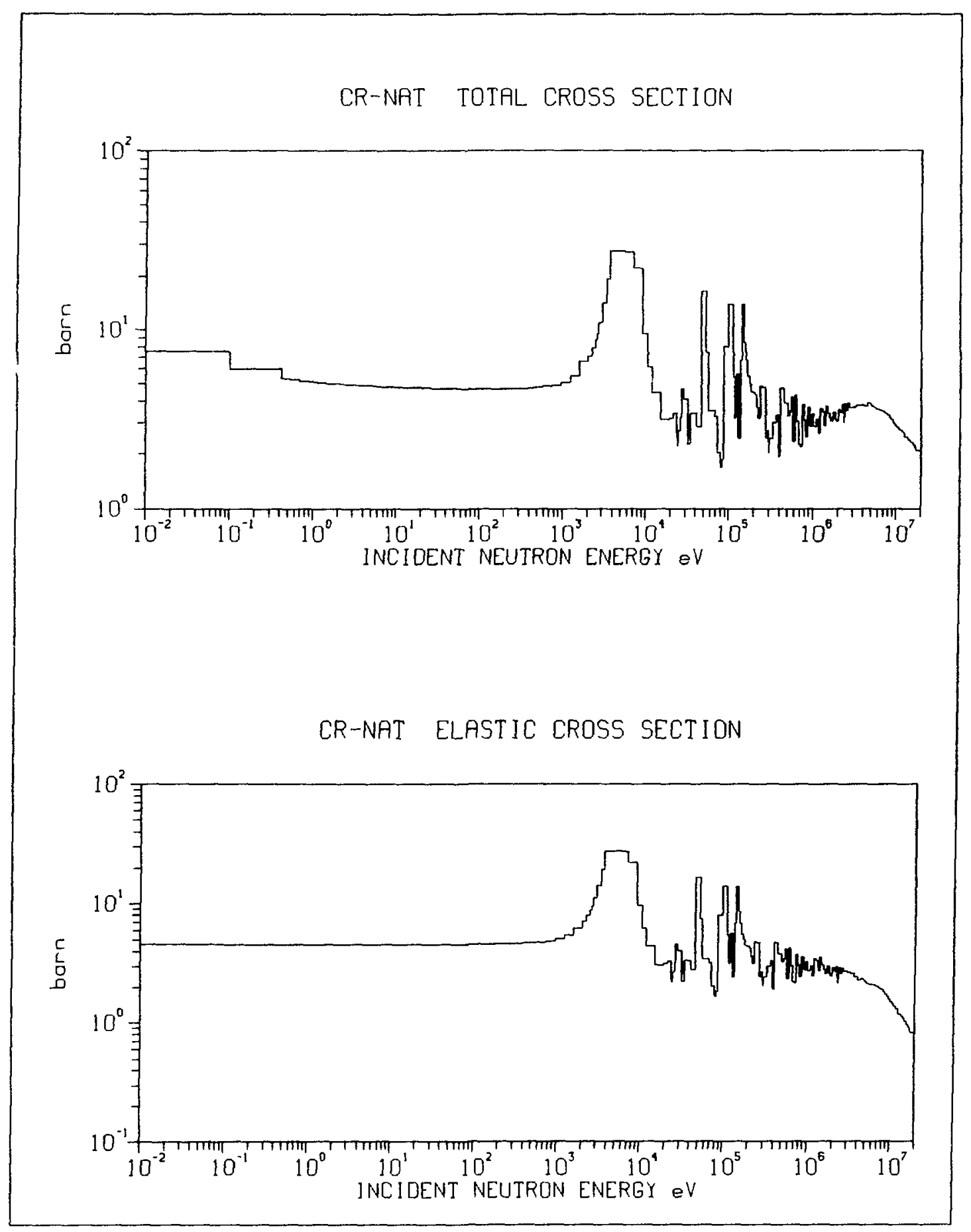




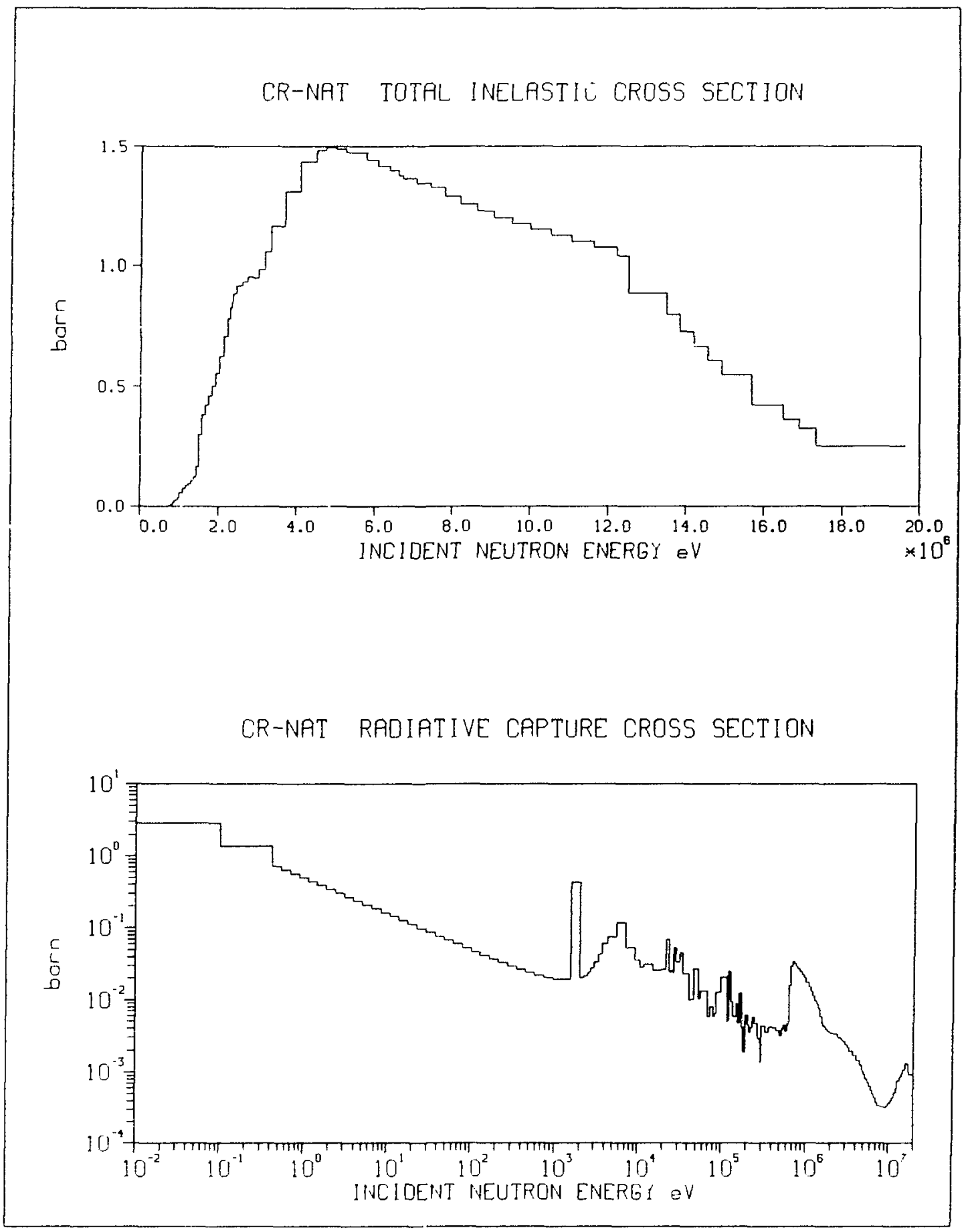




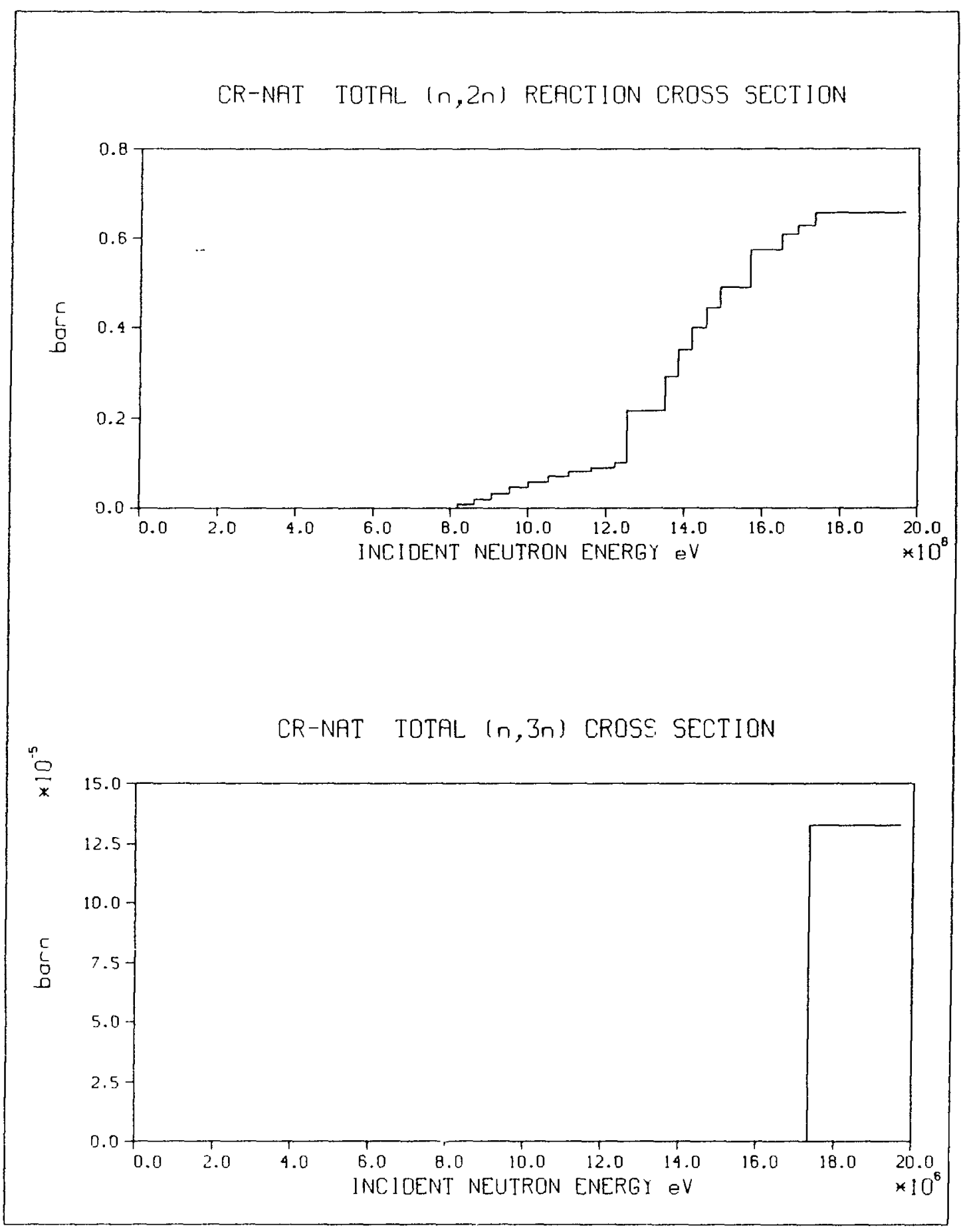




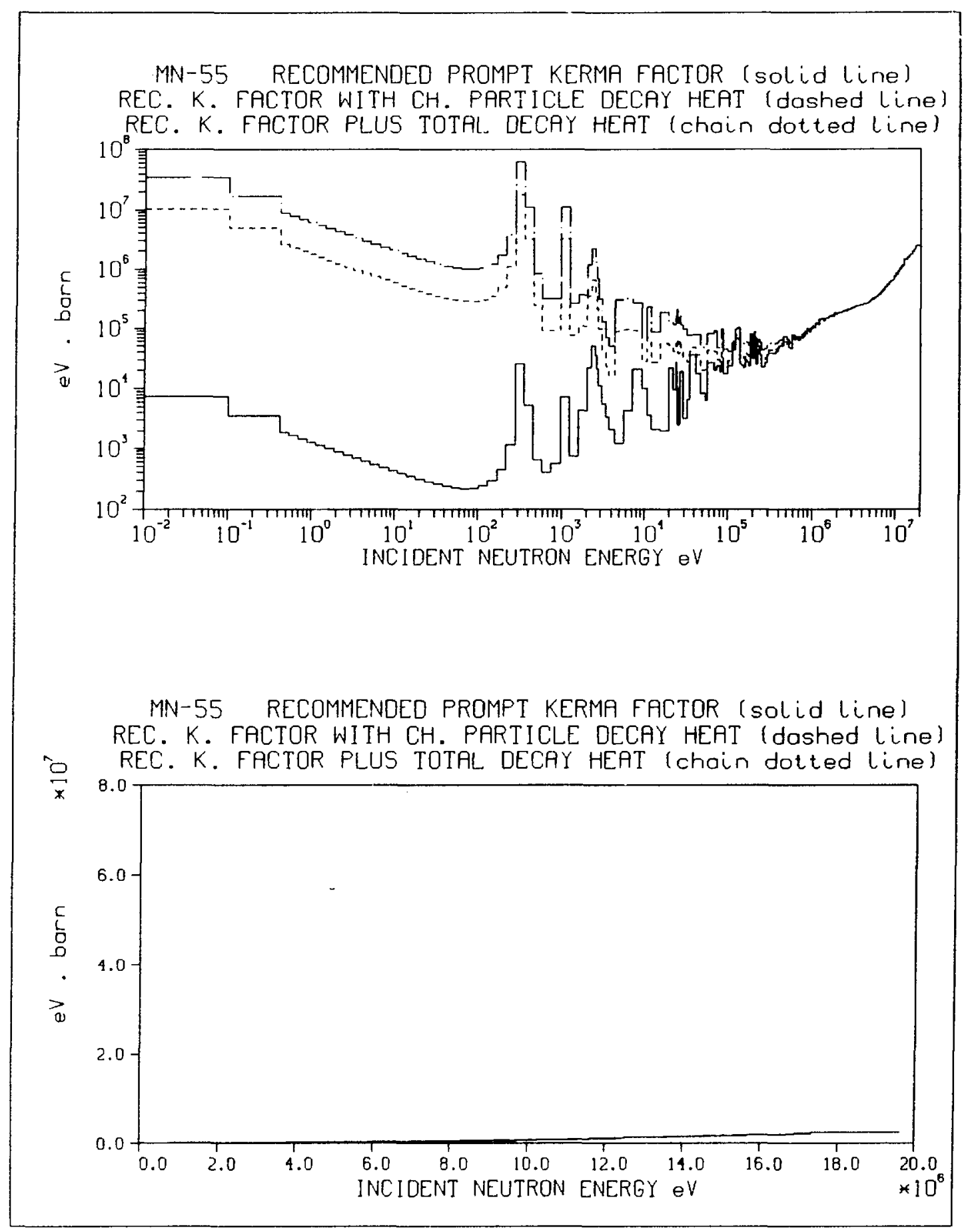




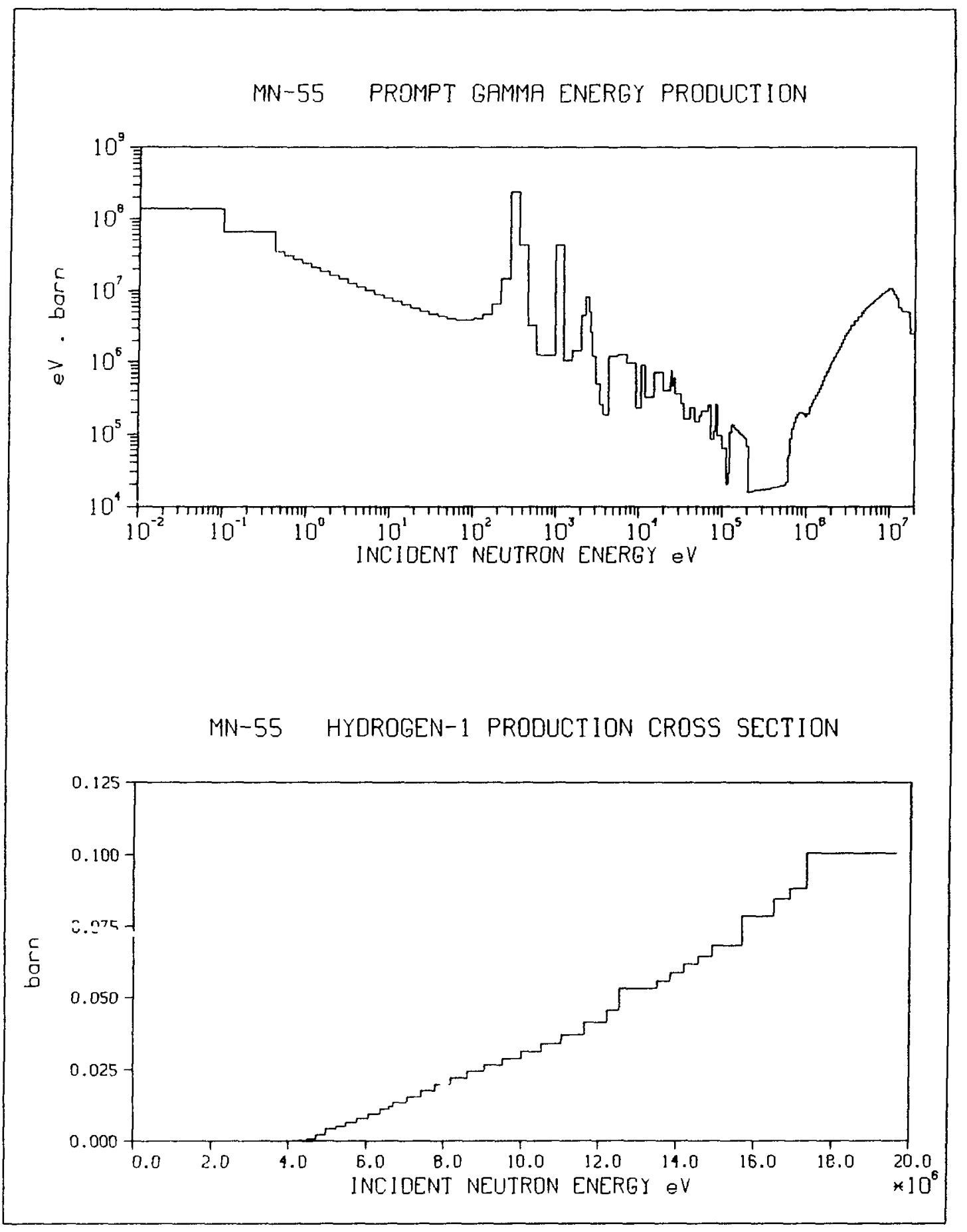




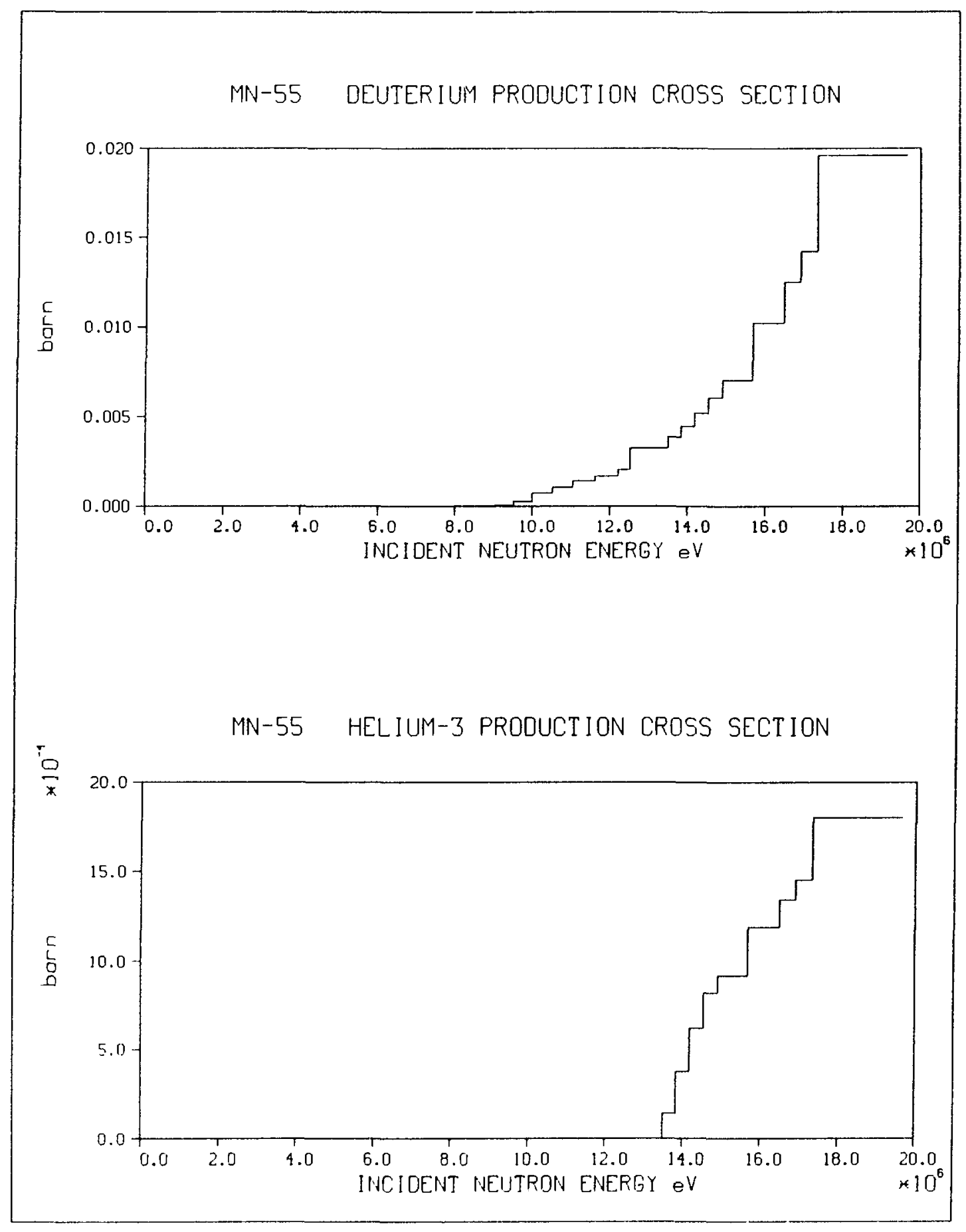




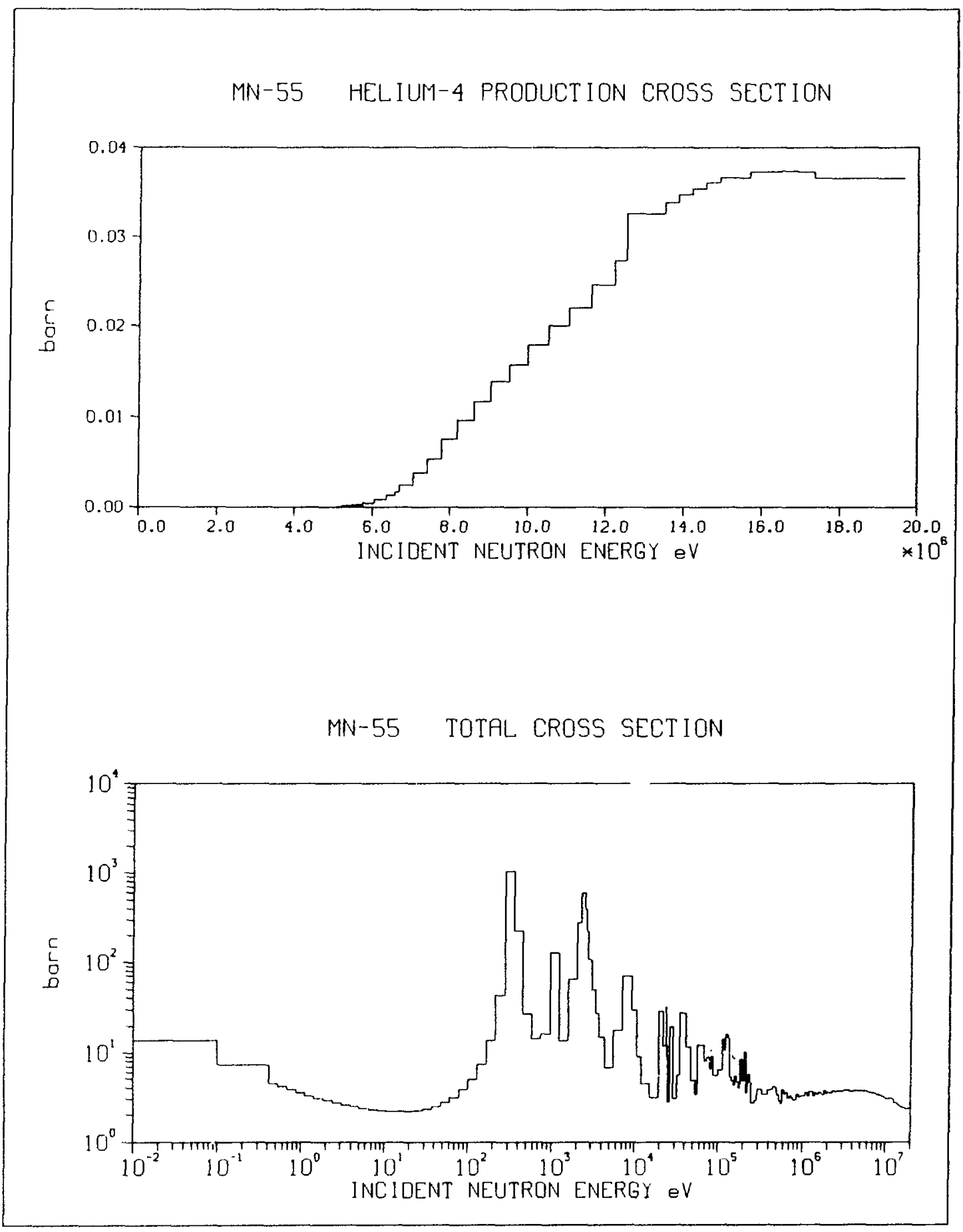


MN-55 ELASTIC CROSS SECTION

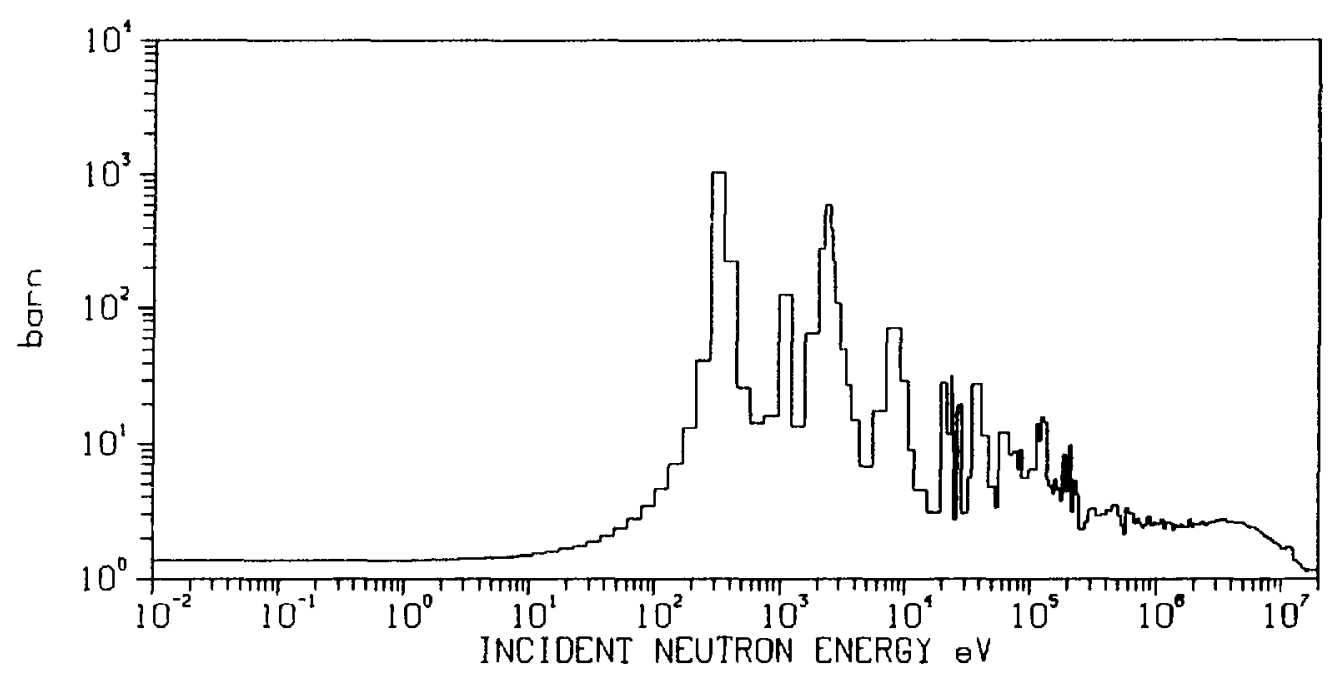

MN-55 TOTAL INELASTIC CROSS SECTION

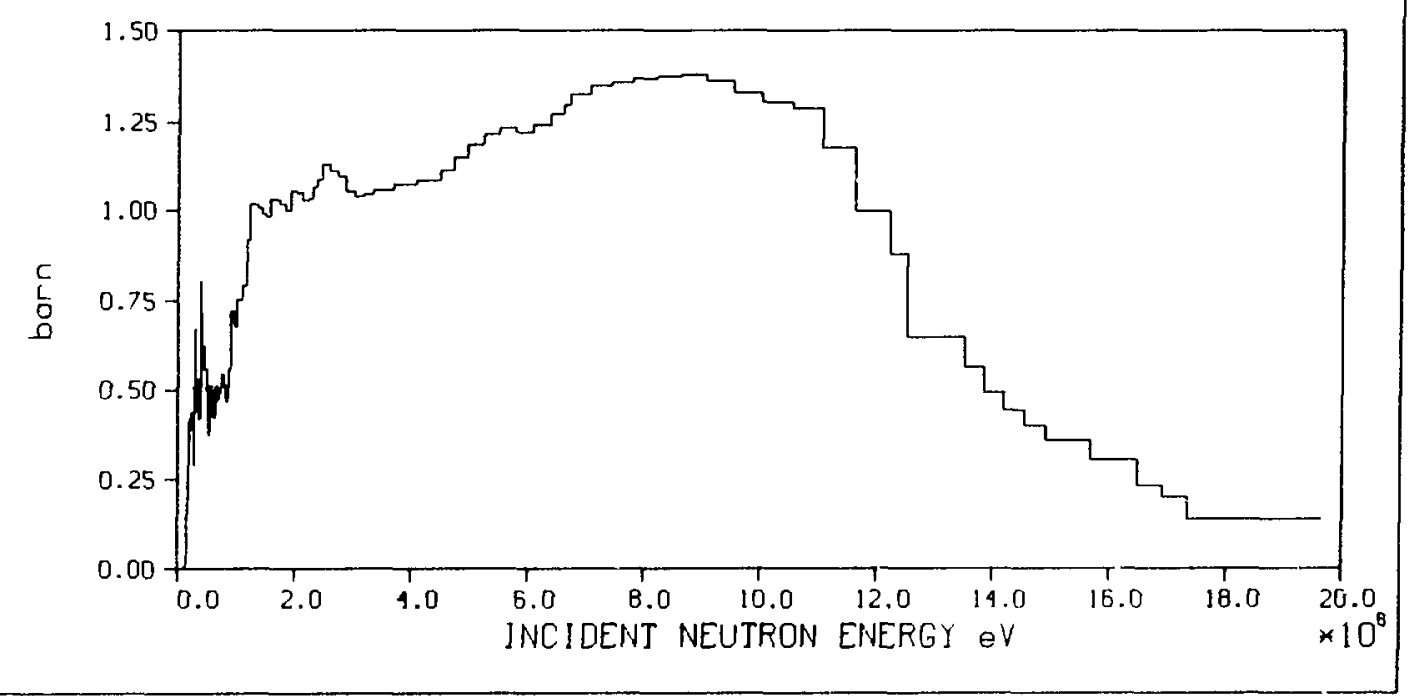


MN-55 RADIATIVE CAPTURE CROSS SECTION

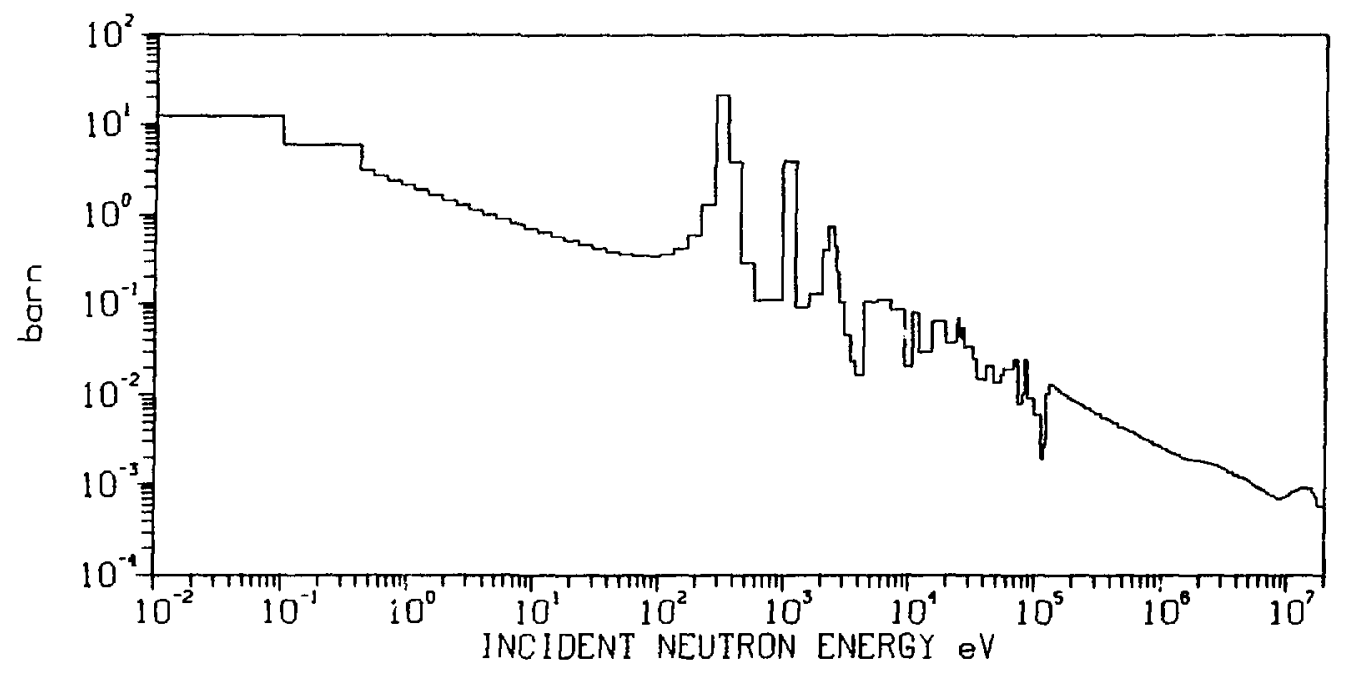

MN-55 TOTAL $(n, 2 n)$ REACTION CROSS SECTION

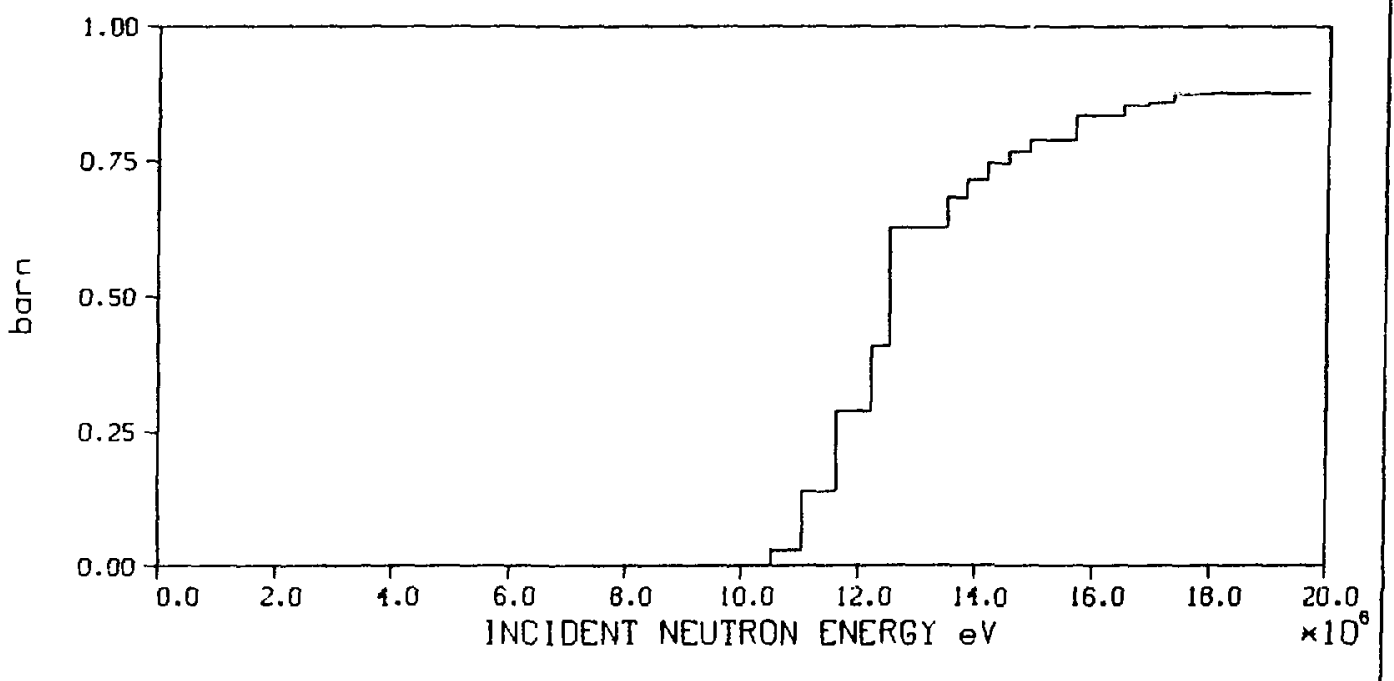




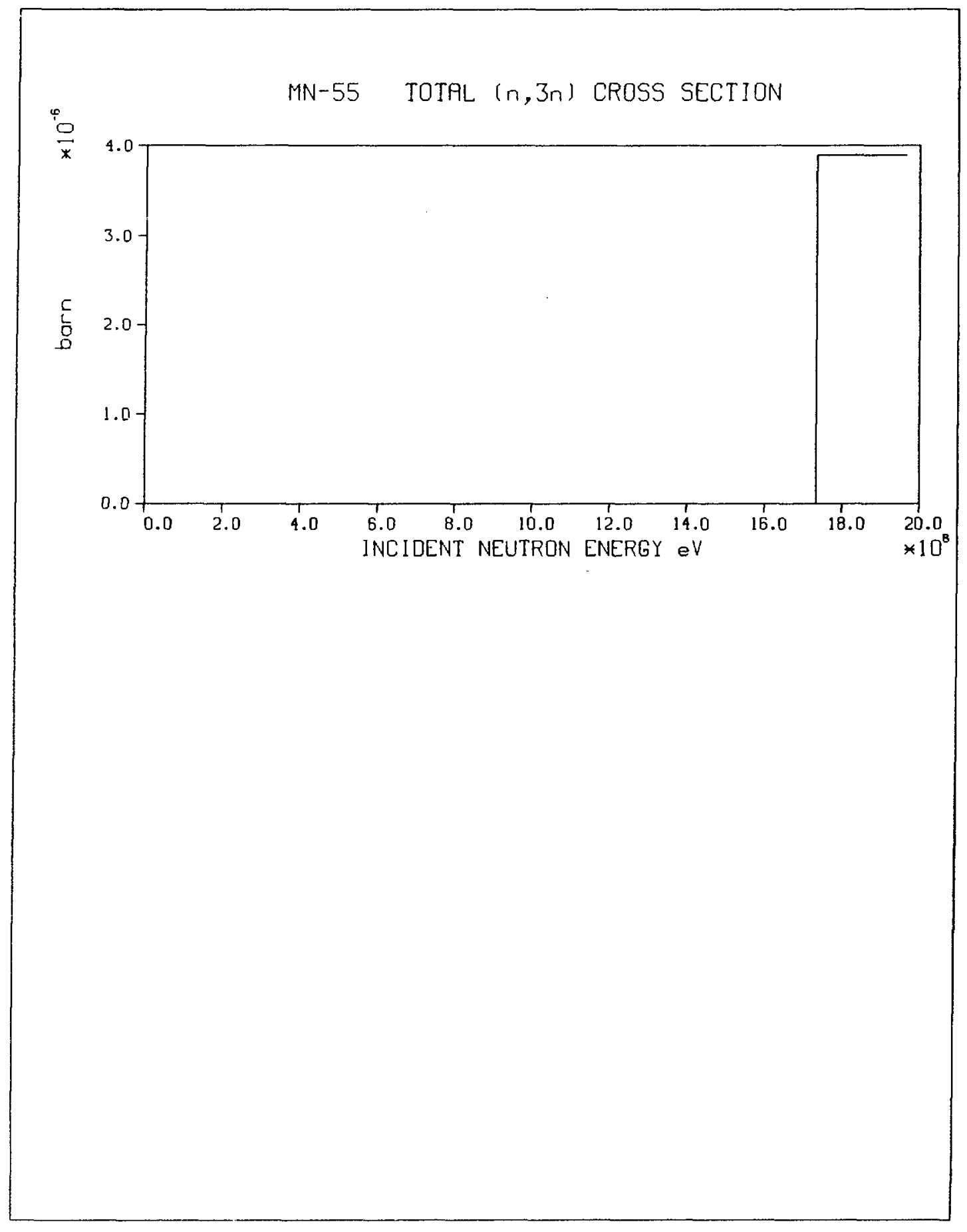




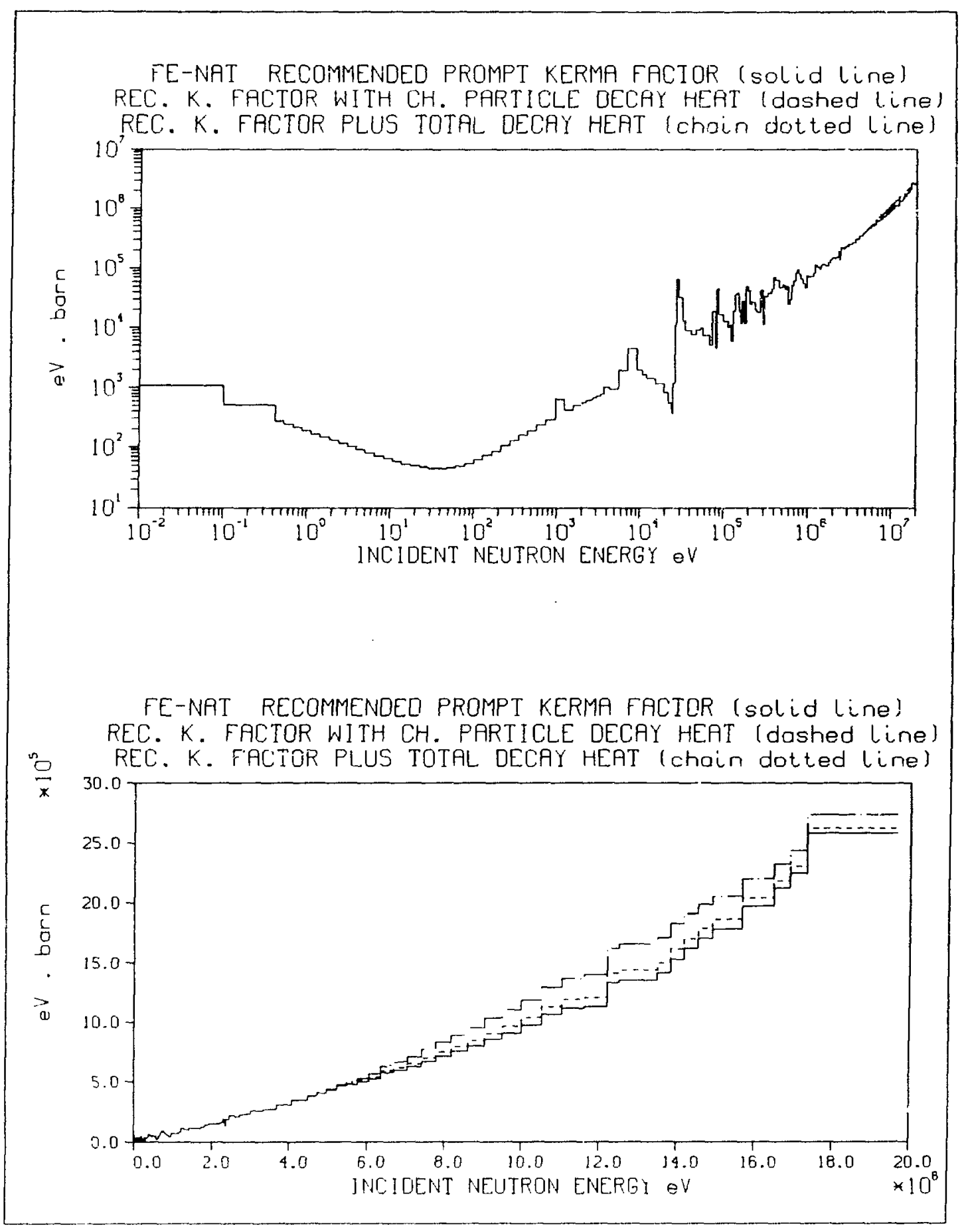




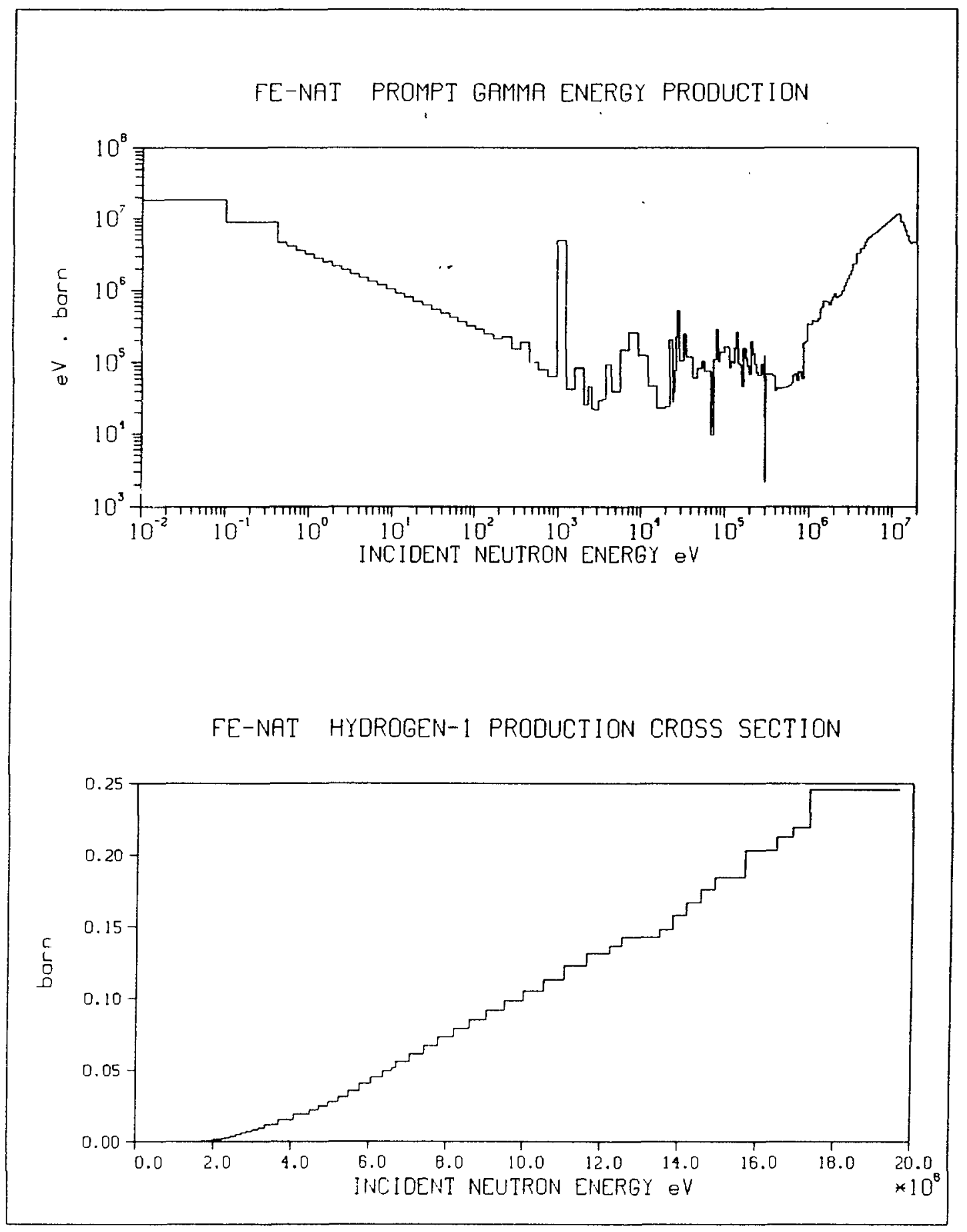




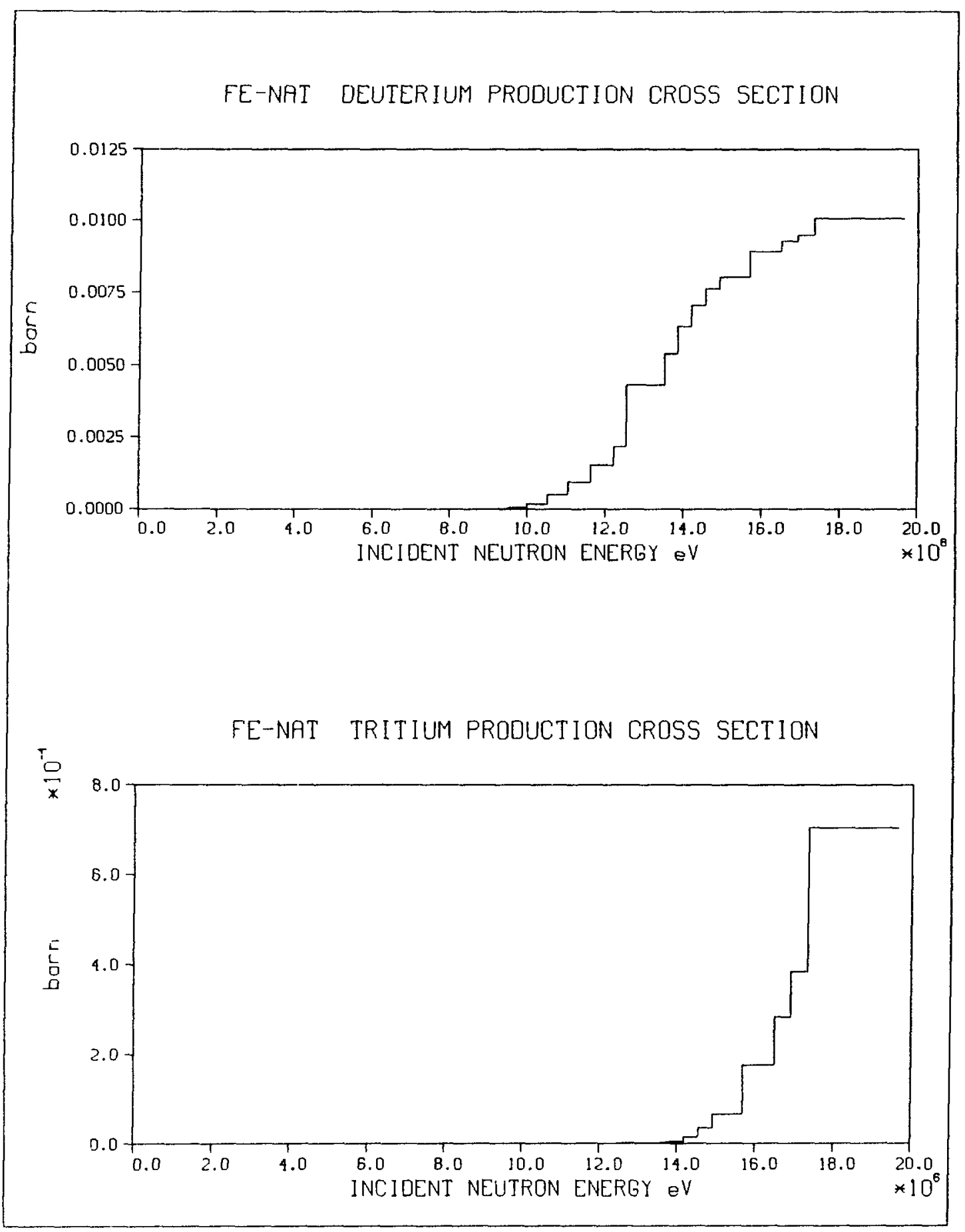




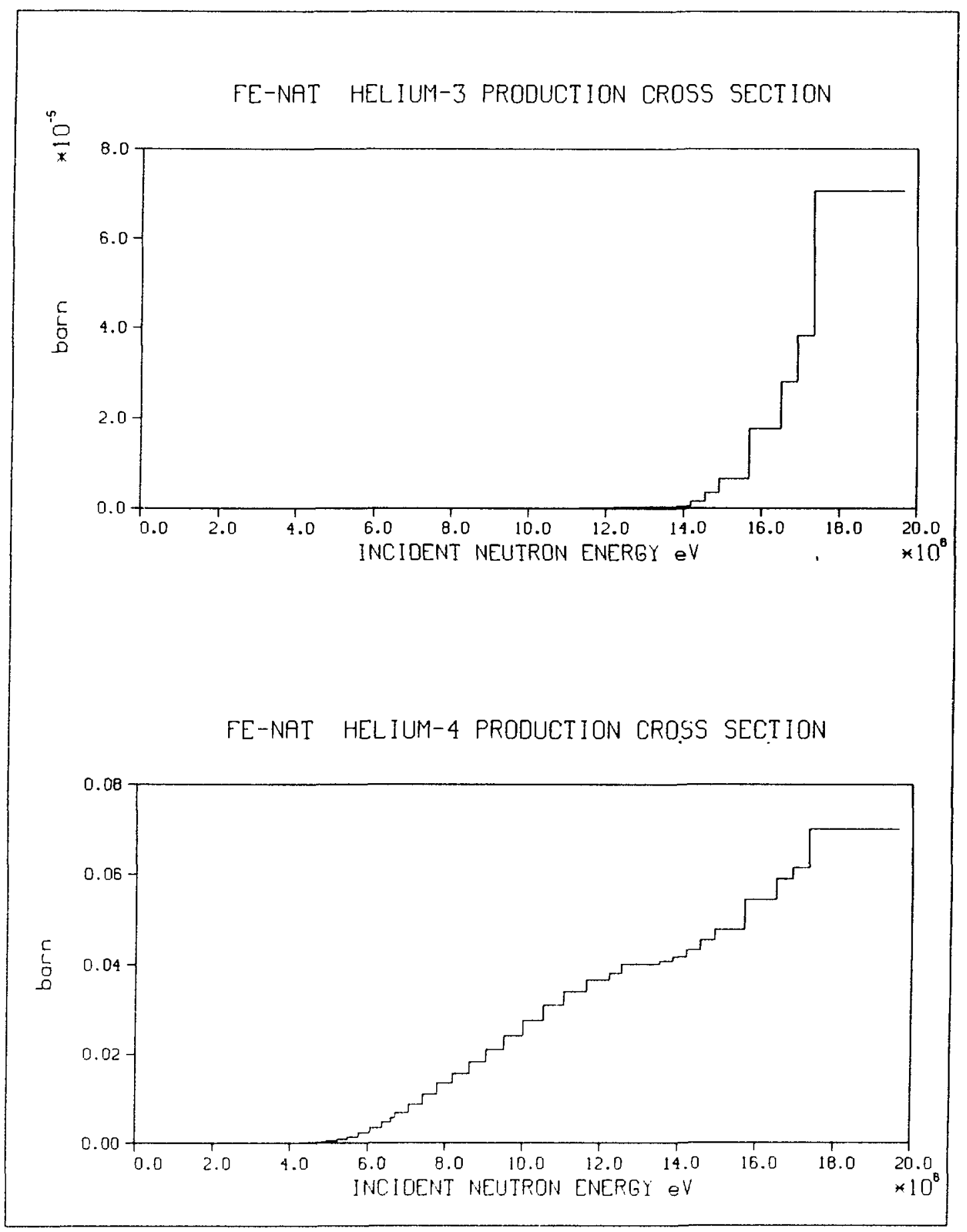




\section{FE-NAT TOTAL CROSS SECTION}

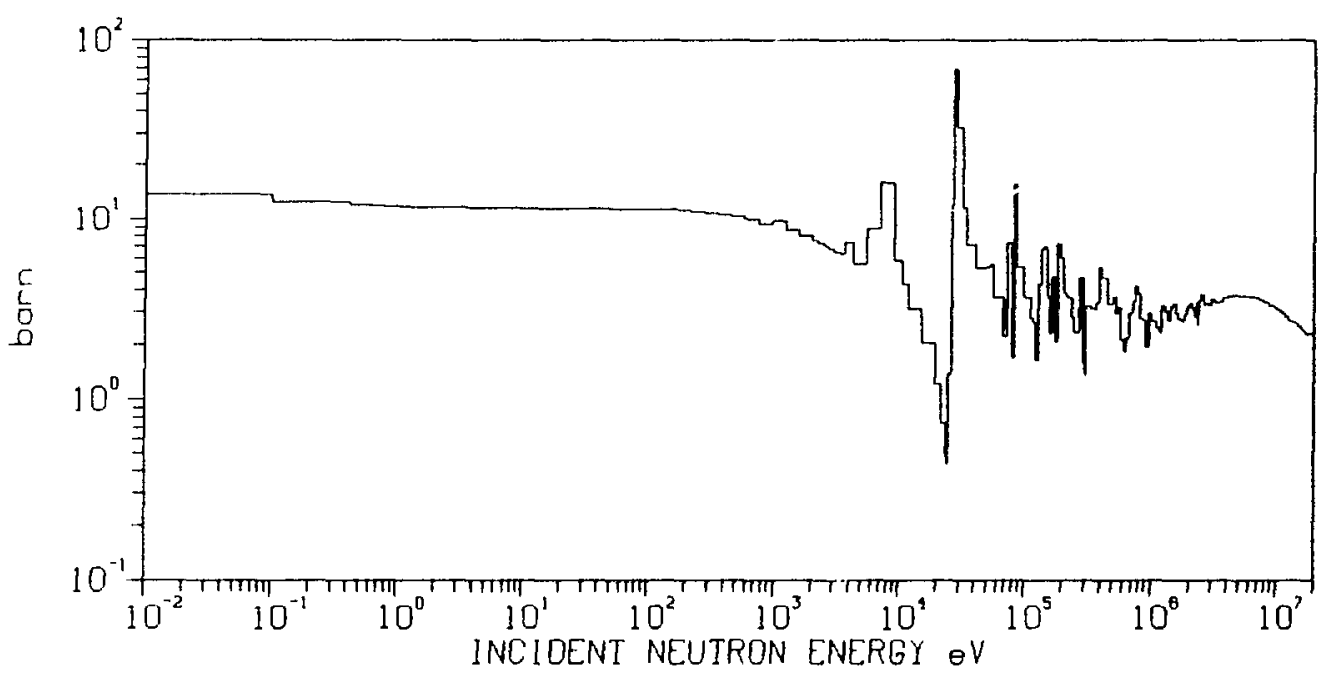

FE-NAT ELASTIC CROSS SECTION

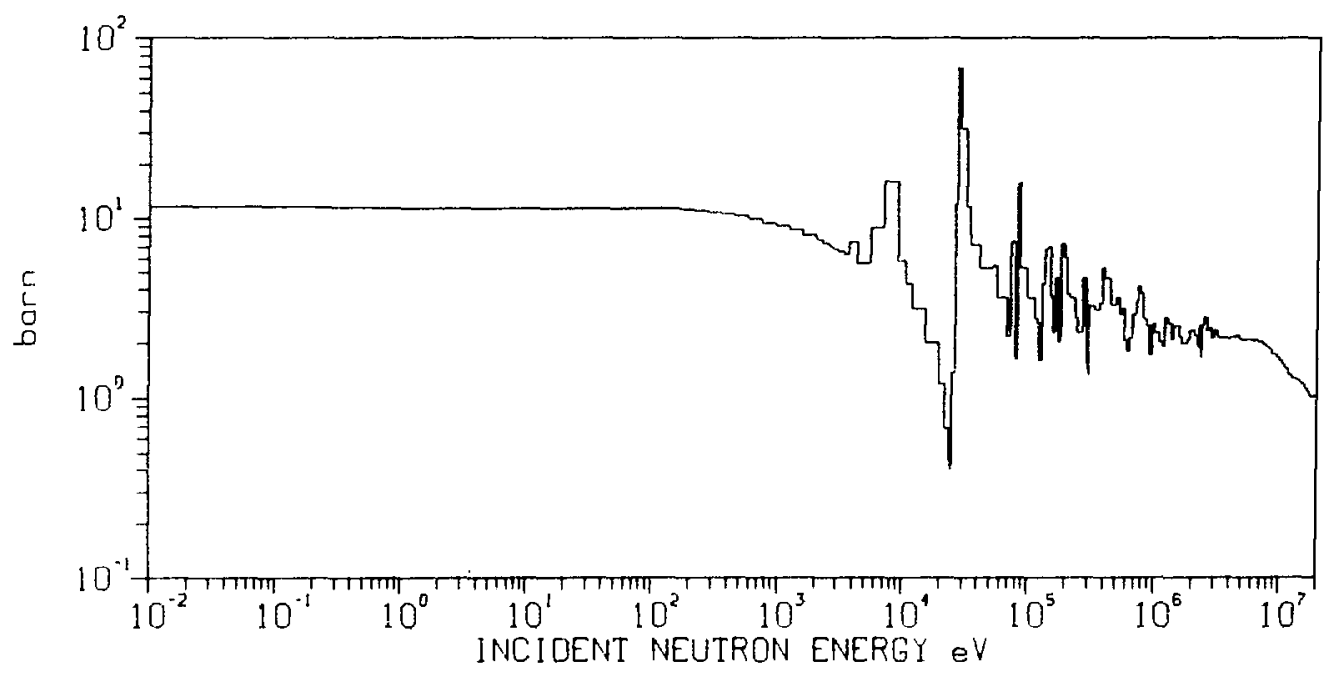


FE-NAT TOTHL INELASTIC CROSS SECTION

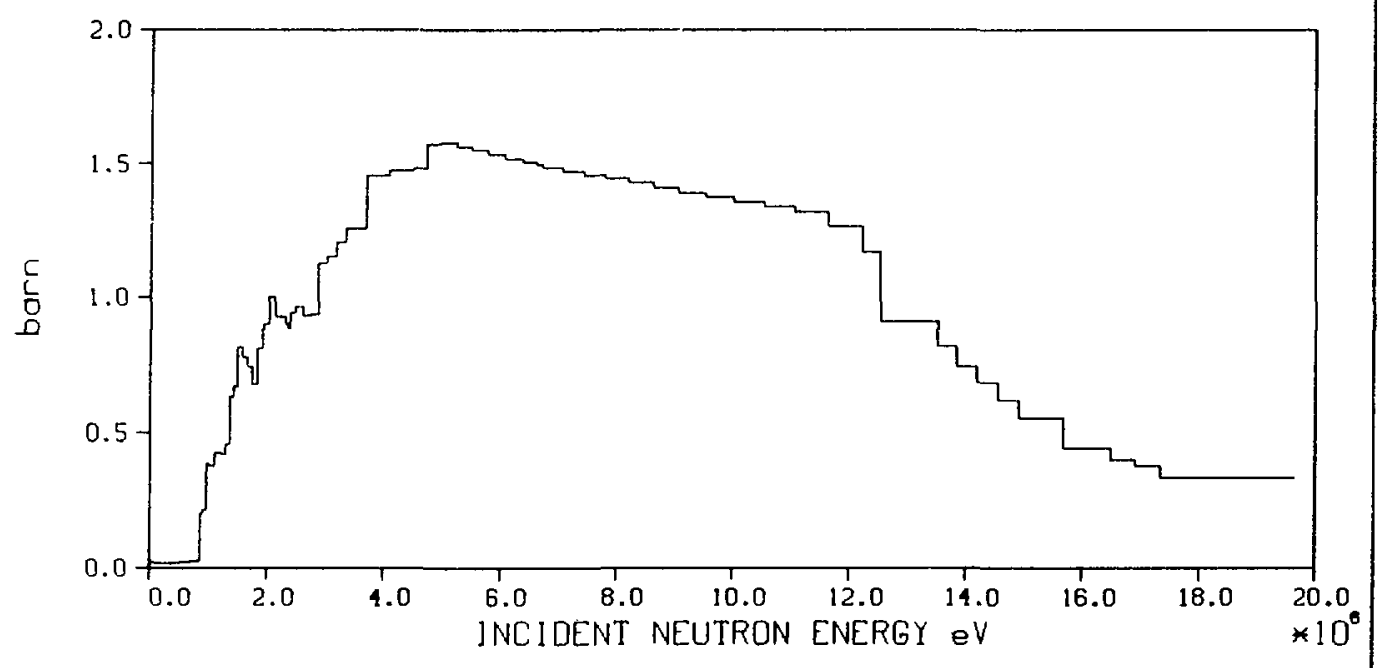

FE-NAT RADIATIVE CAPTURE CROSS SECTION

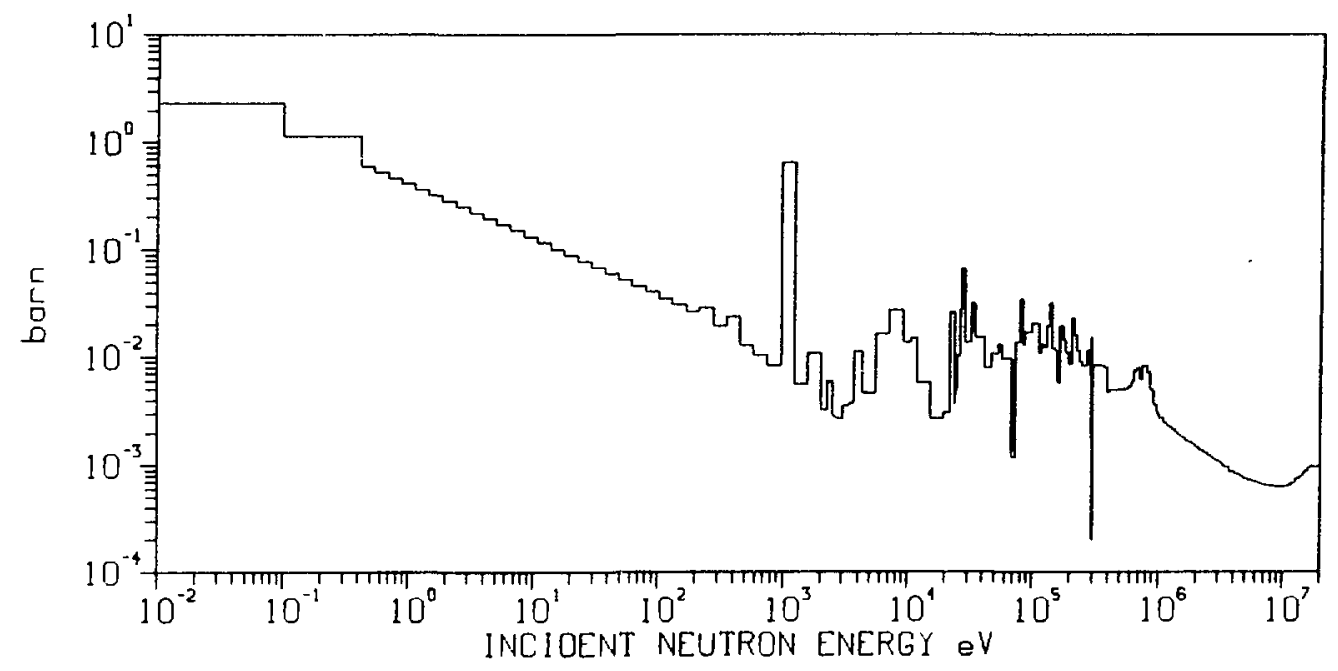




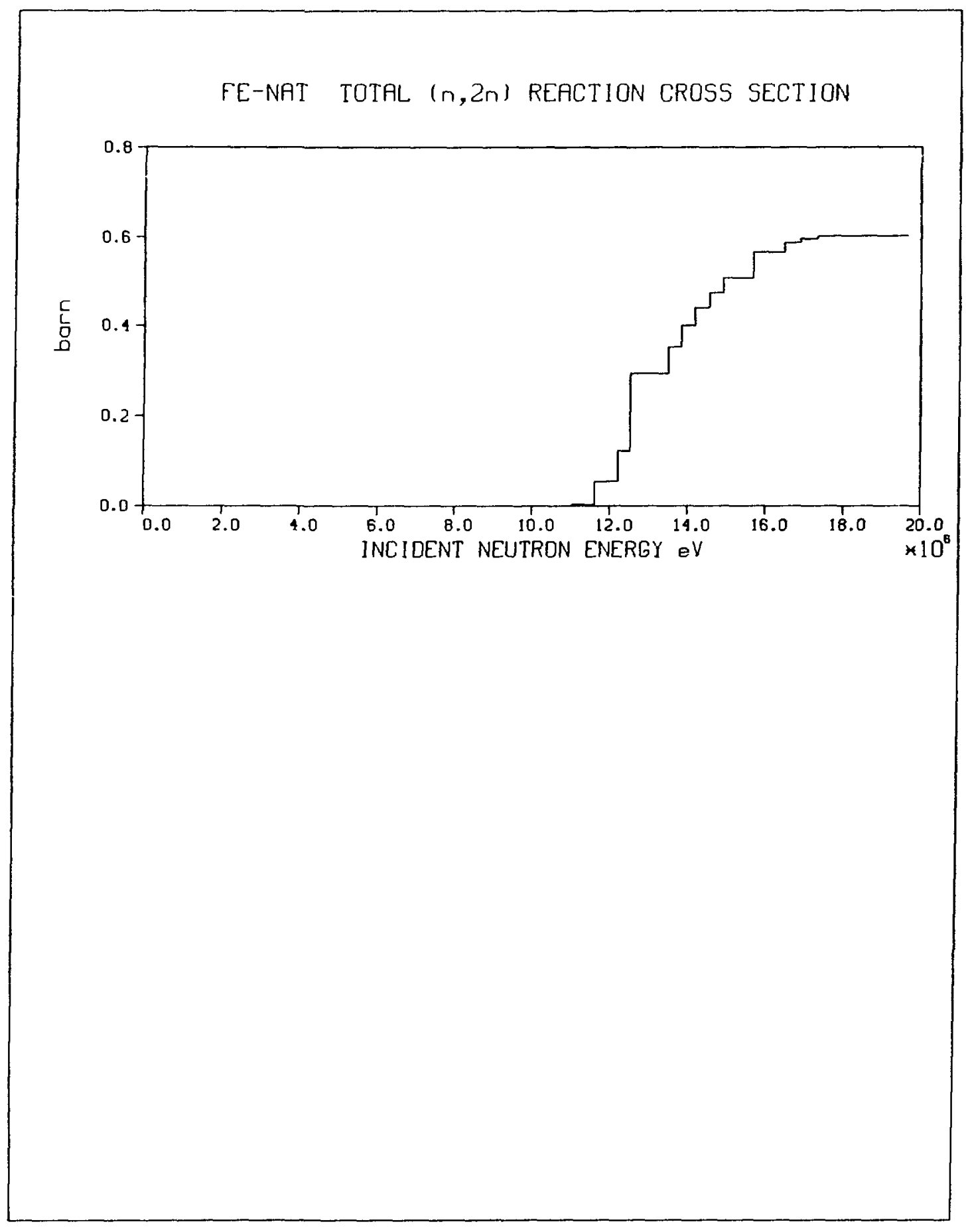




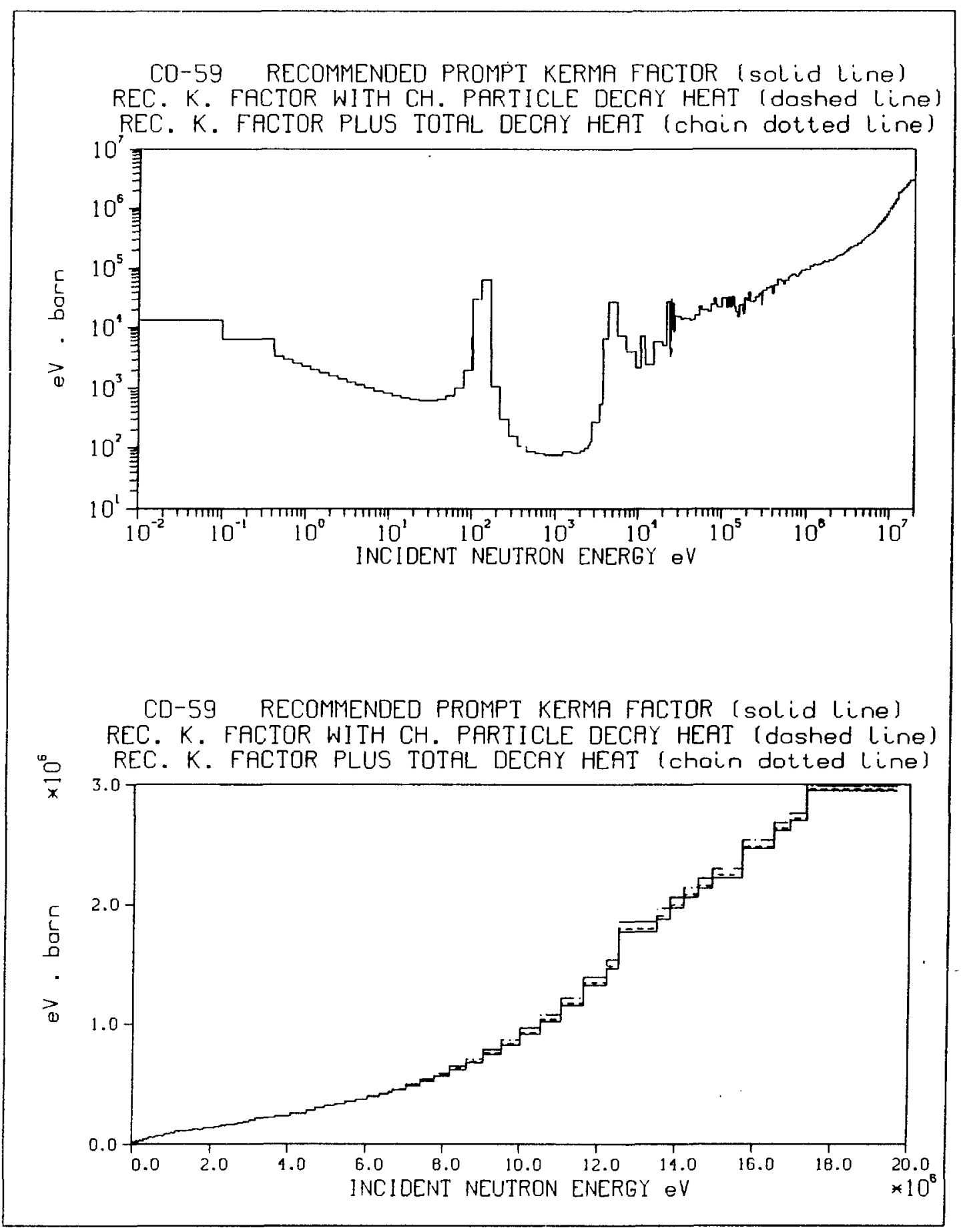




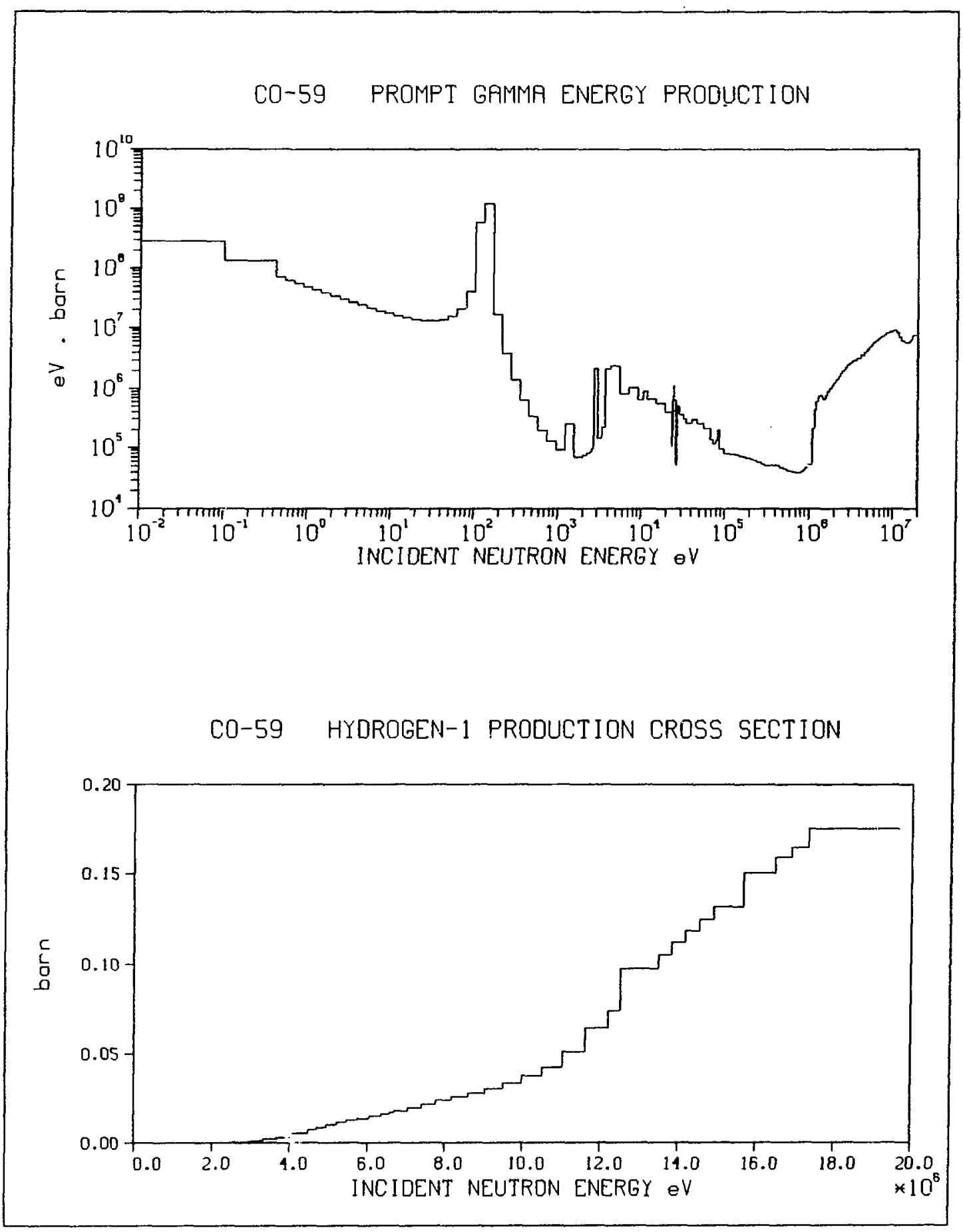




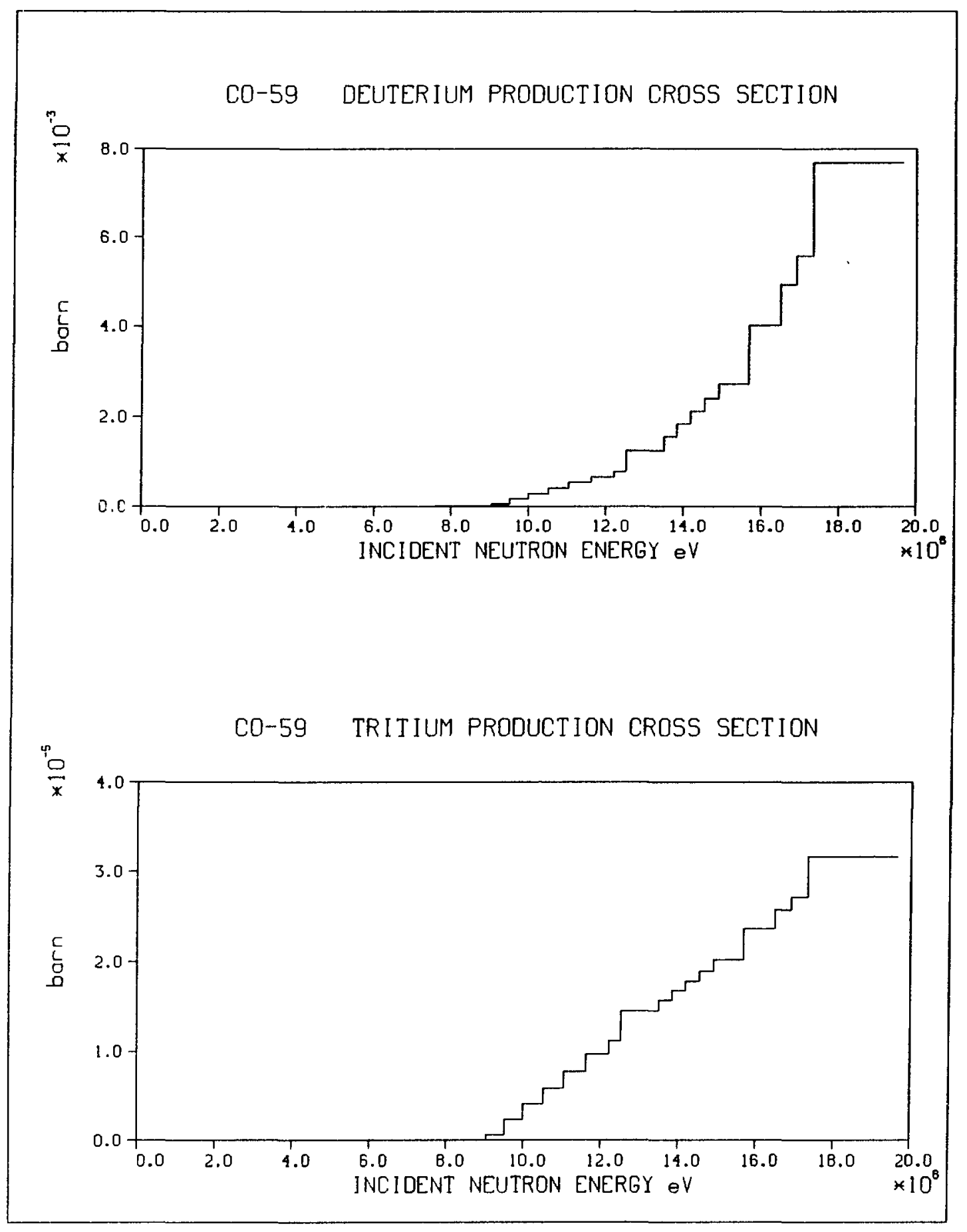




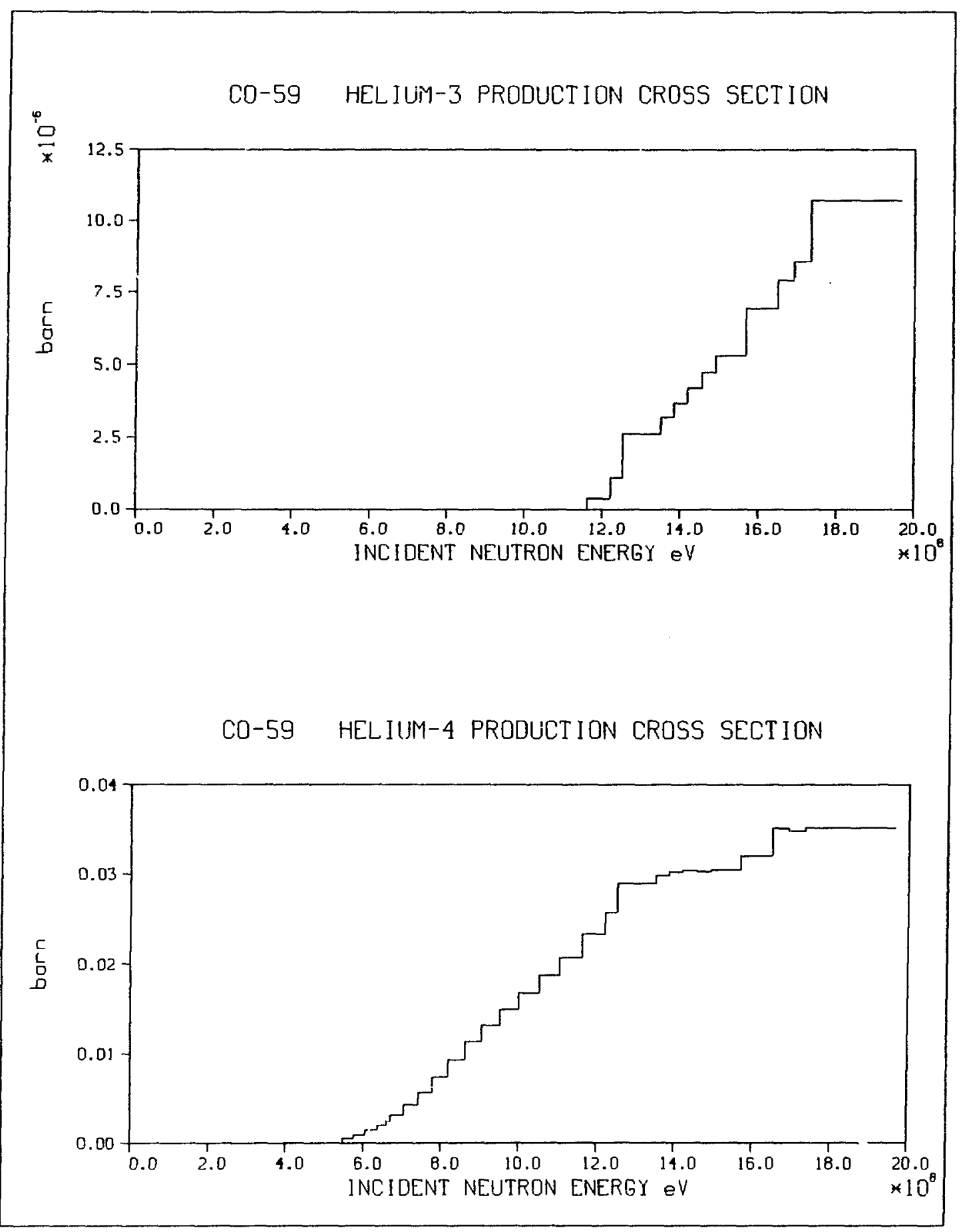


CO-59 TOTAL CROSS SECTION

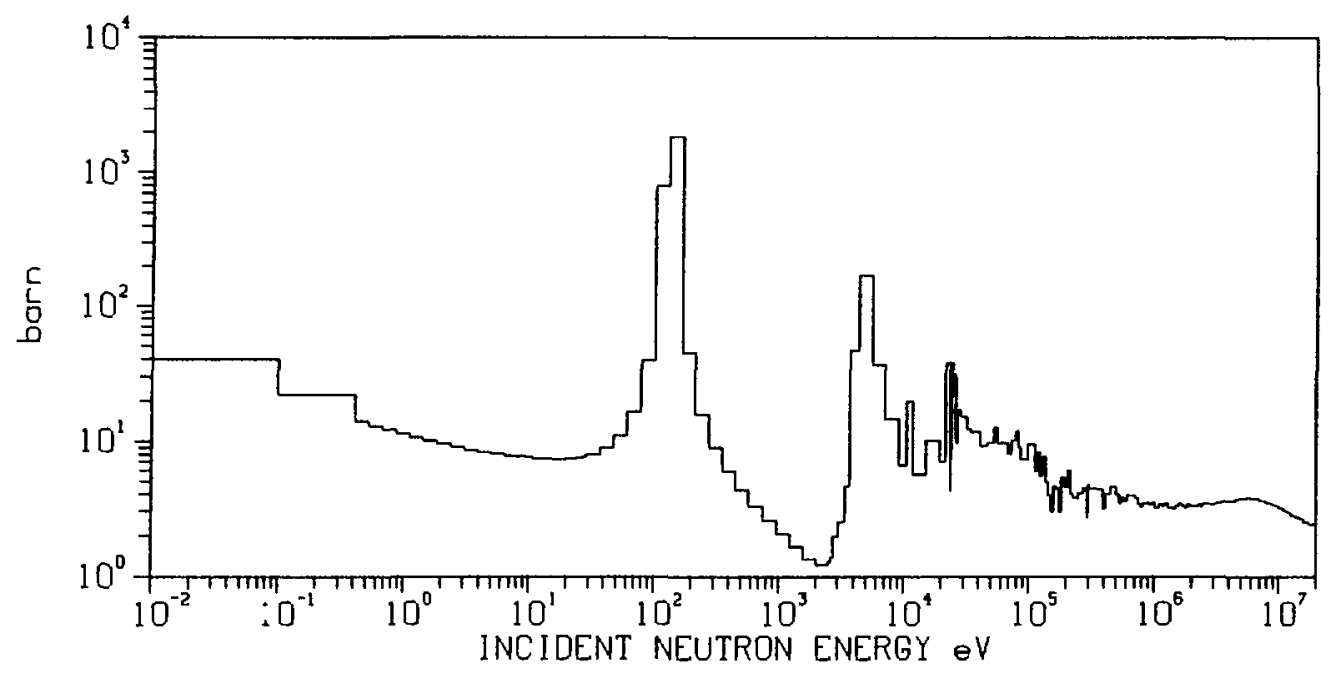

CO-59 ELASTIC CROSS SECTION

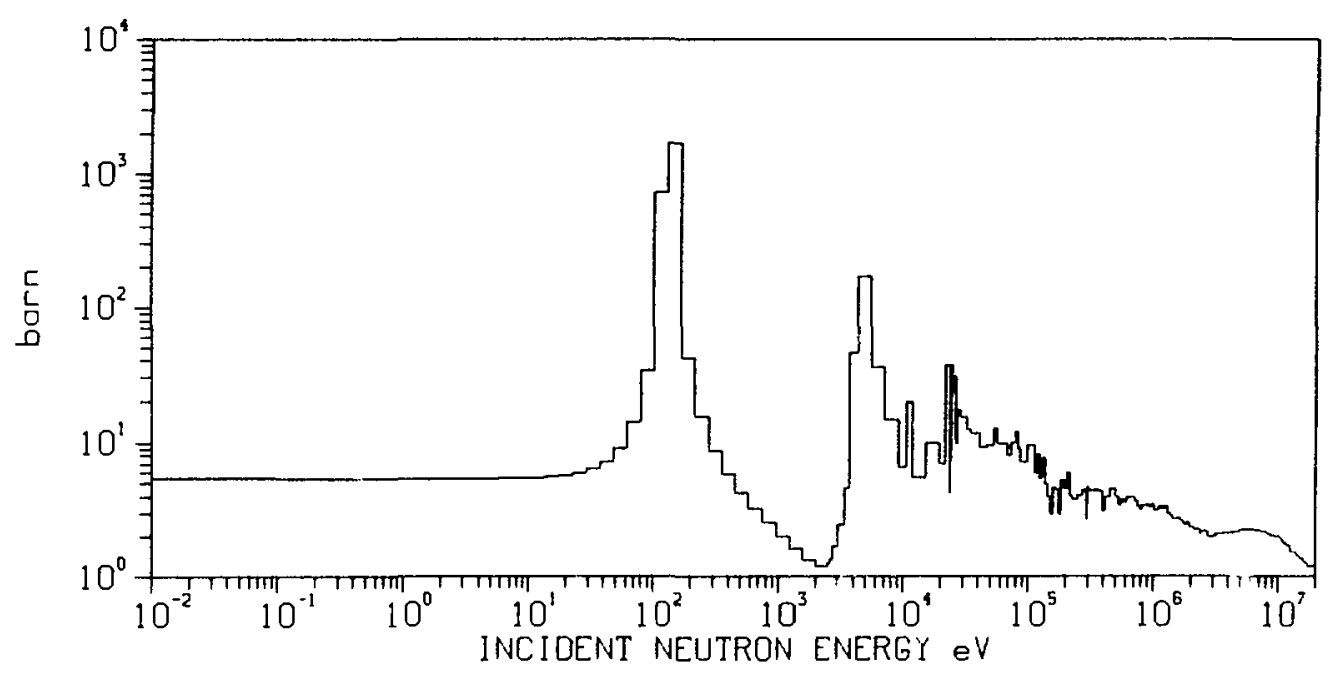




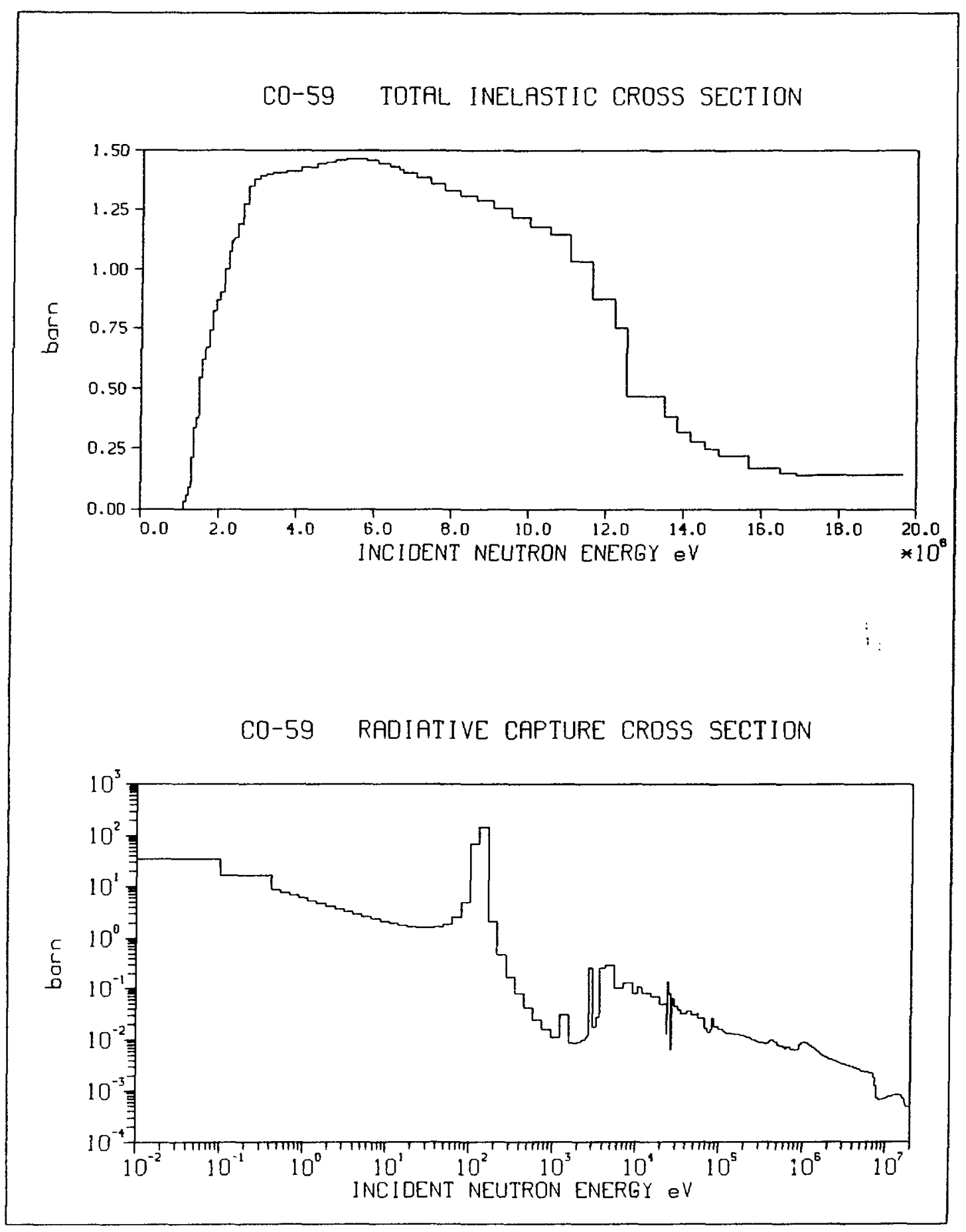




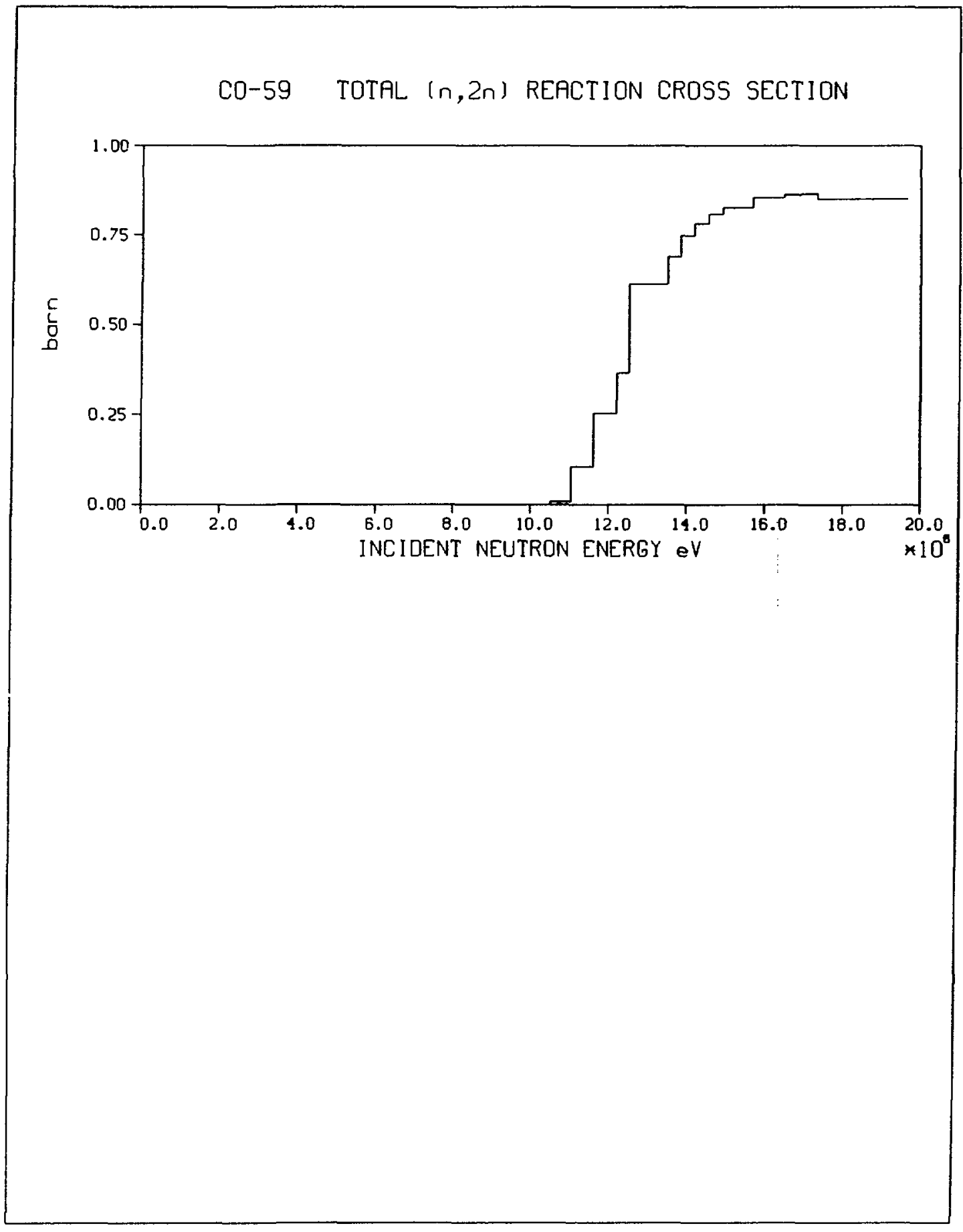




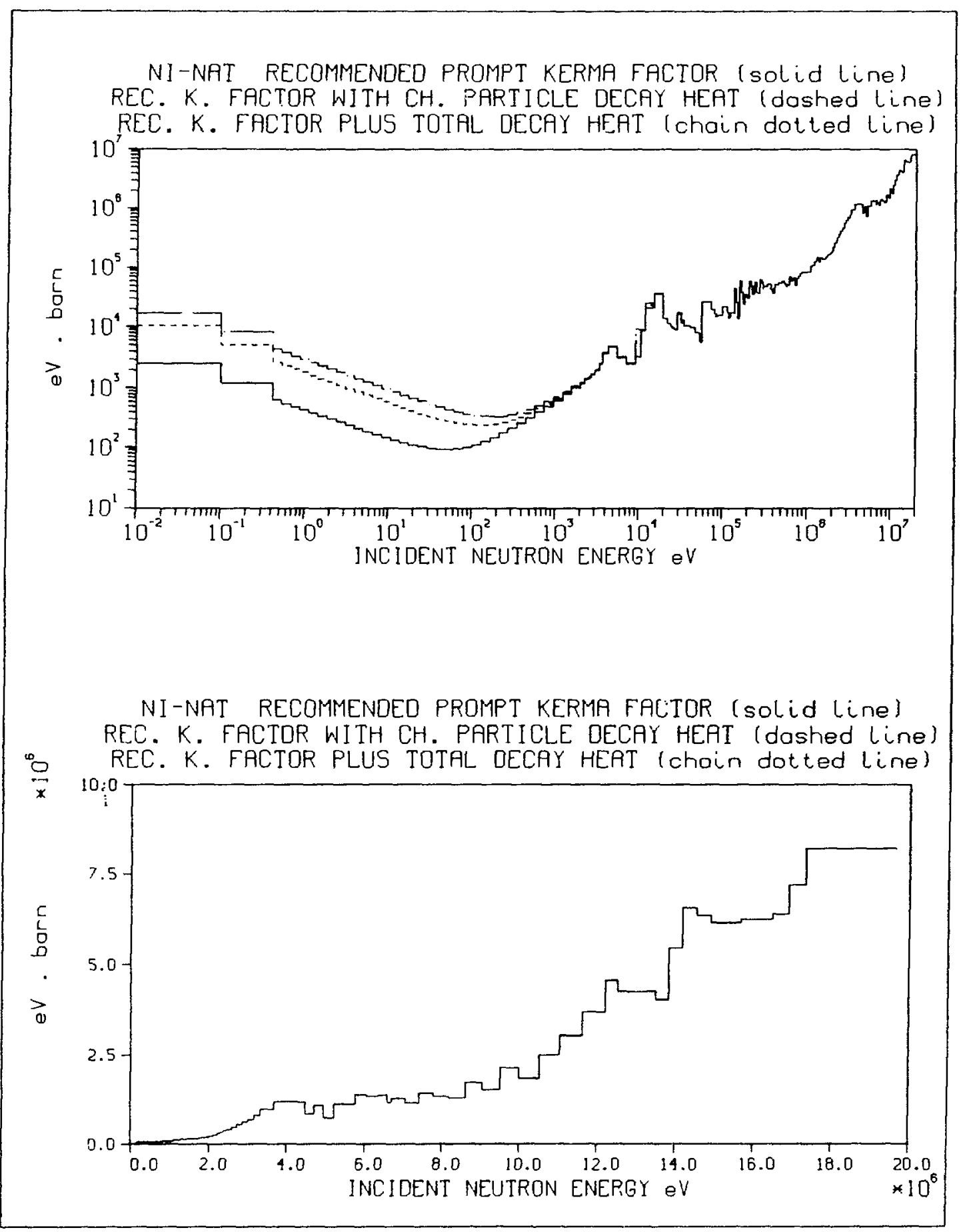




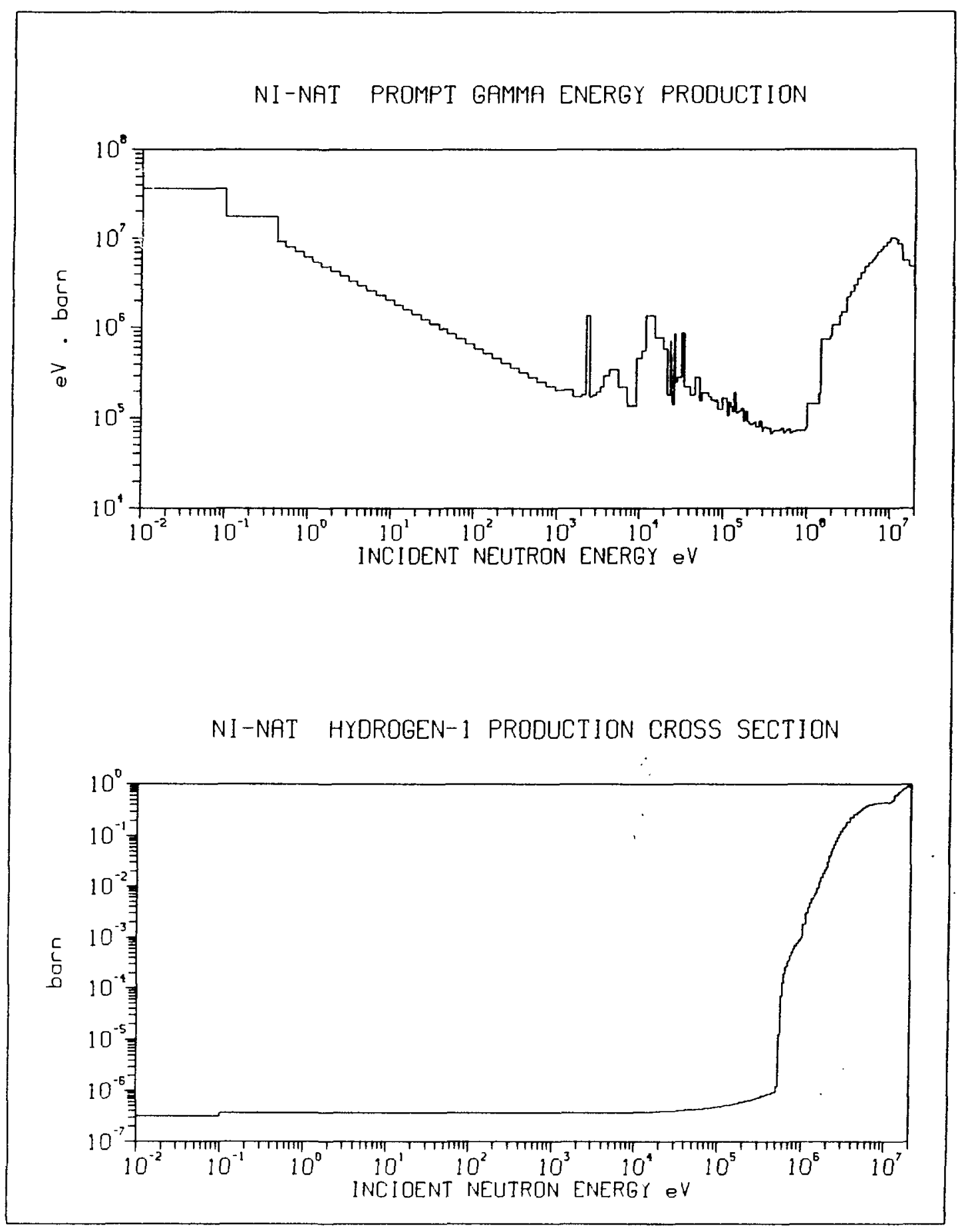




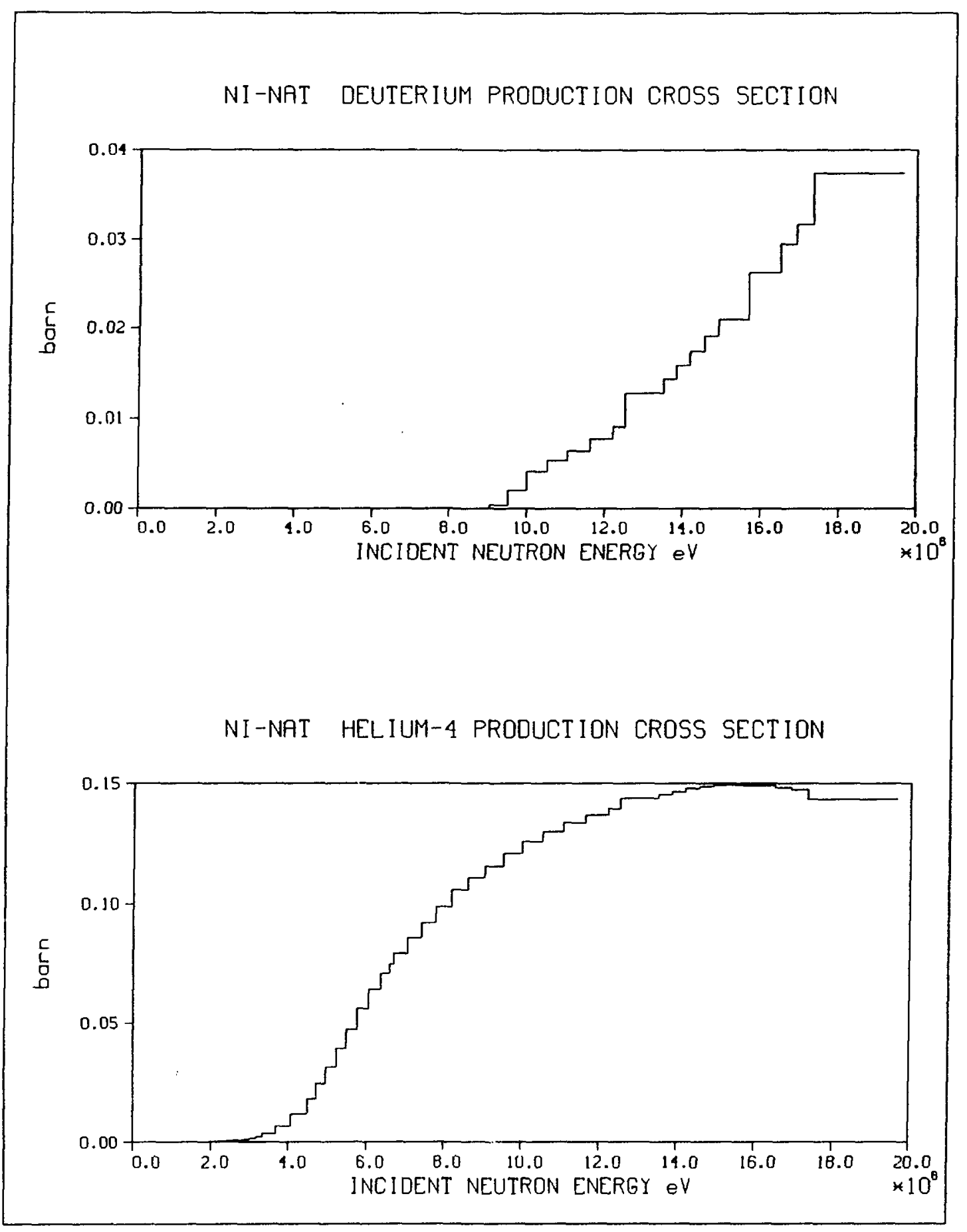




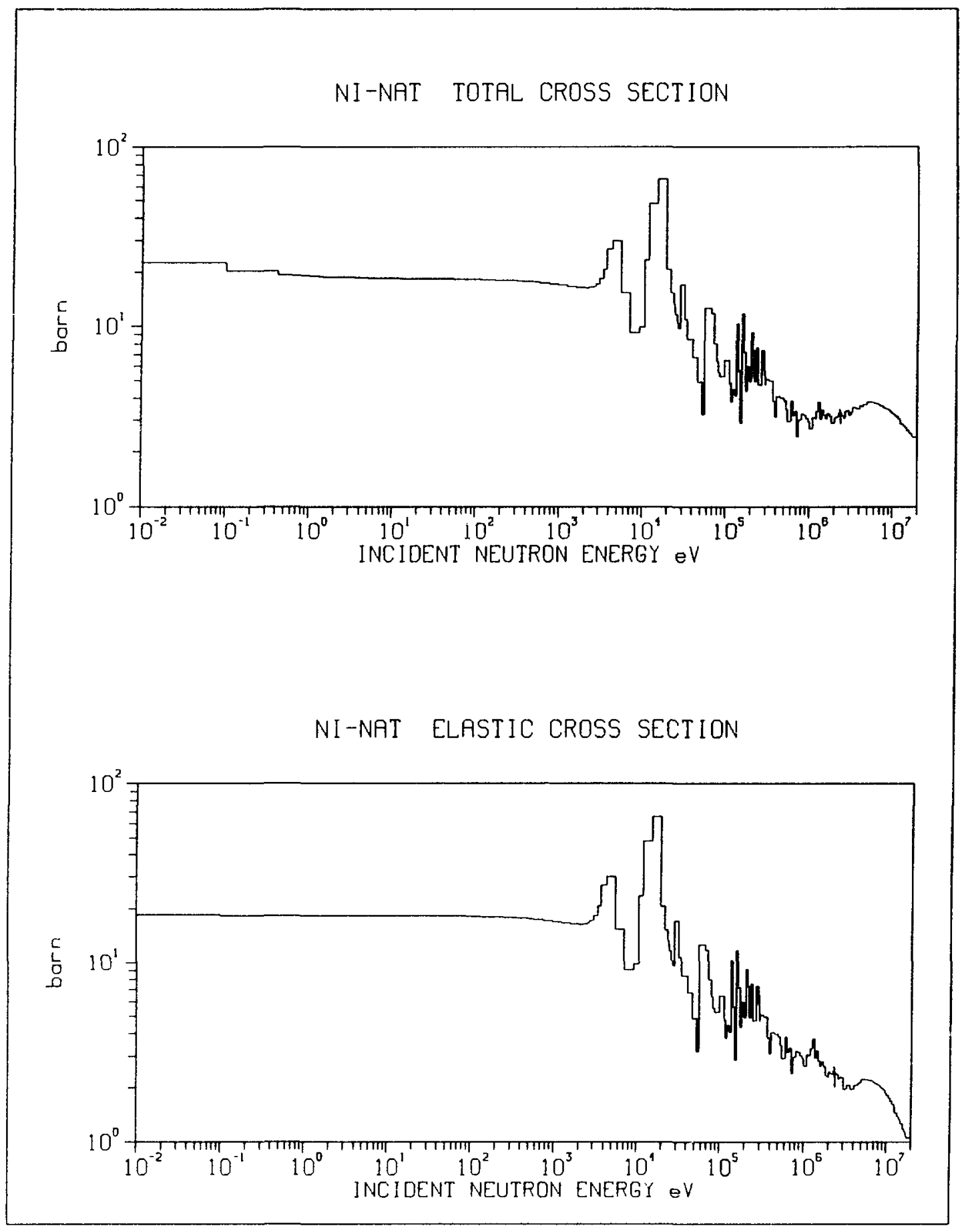




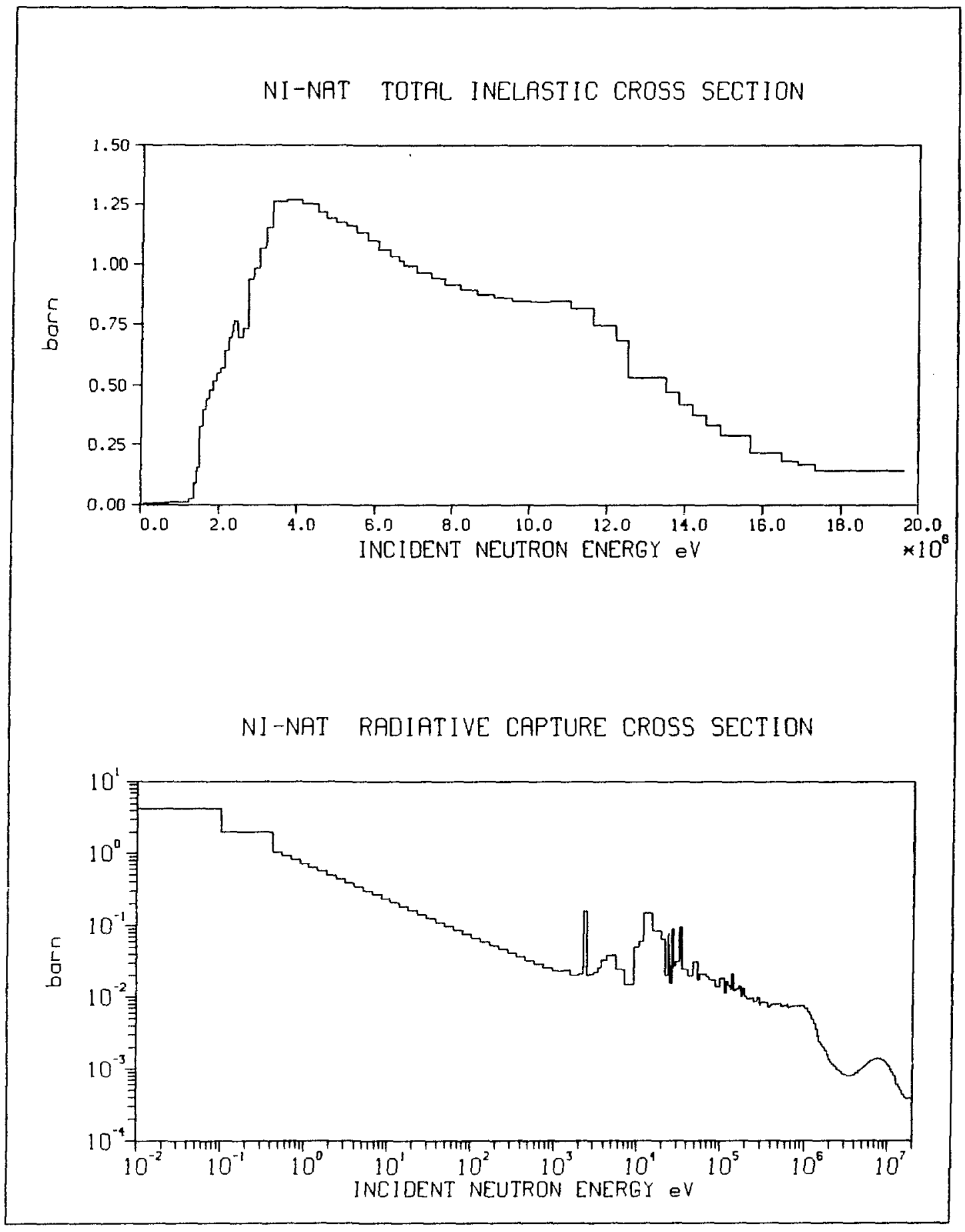




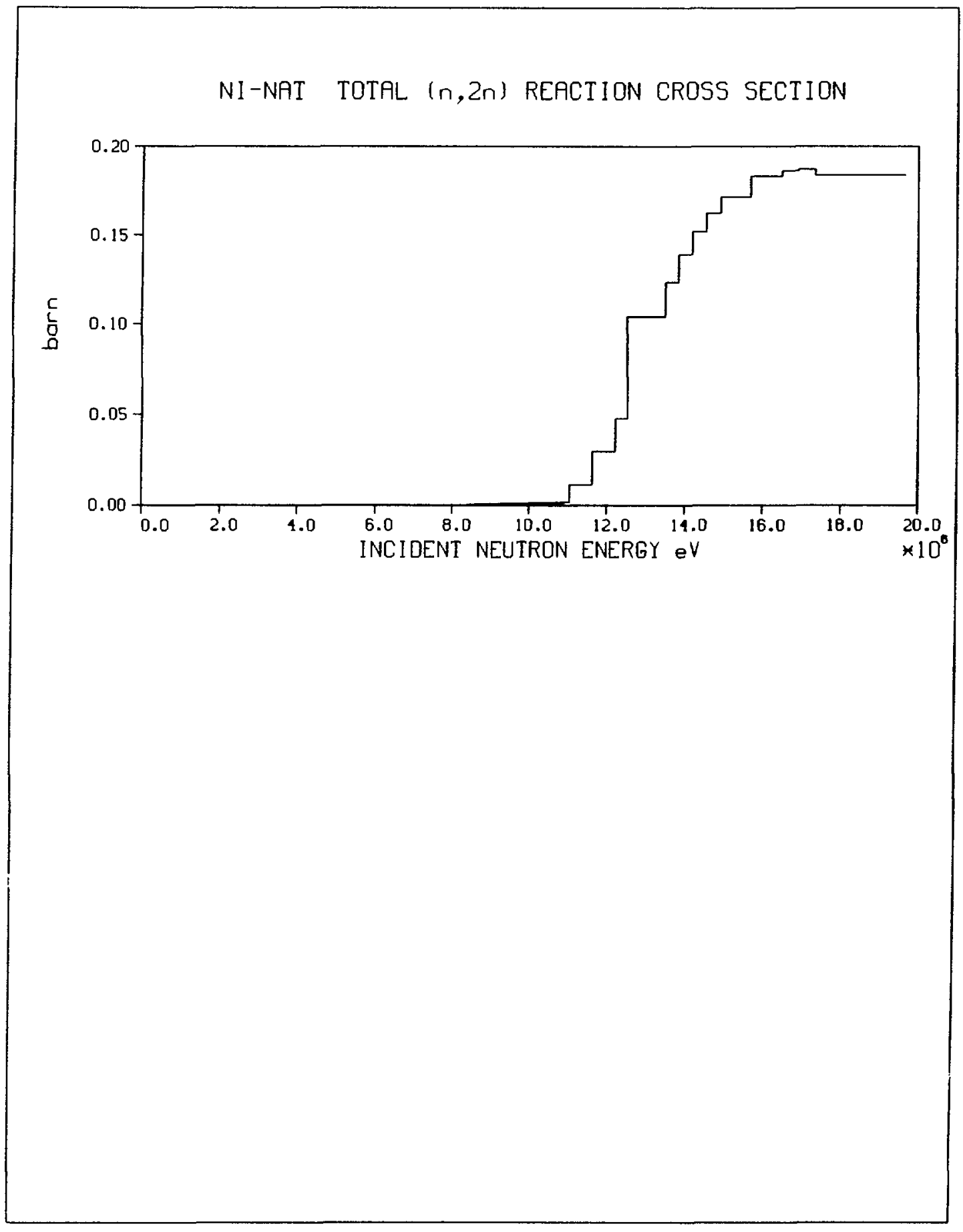




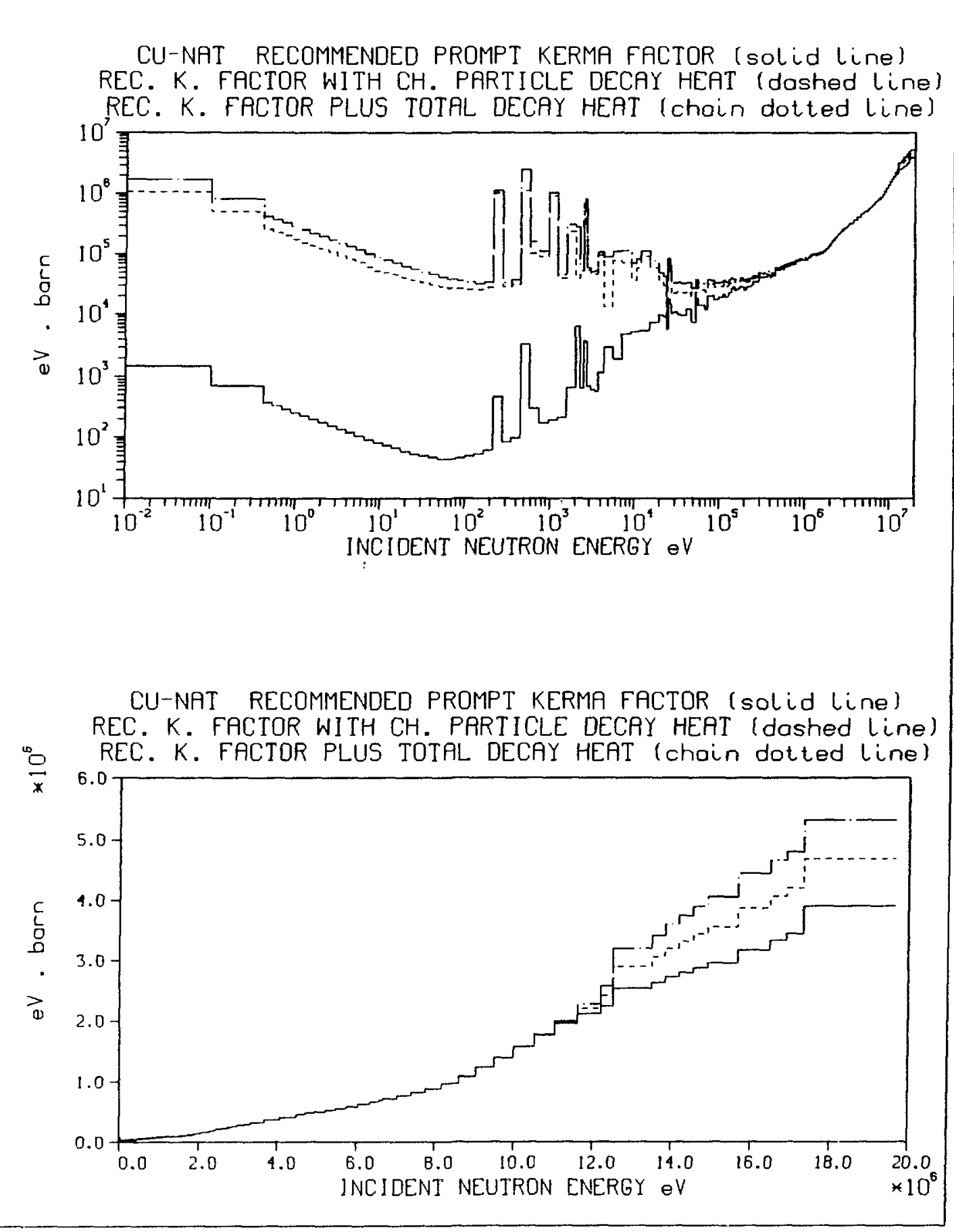




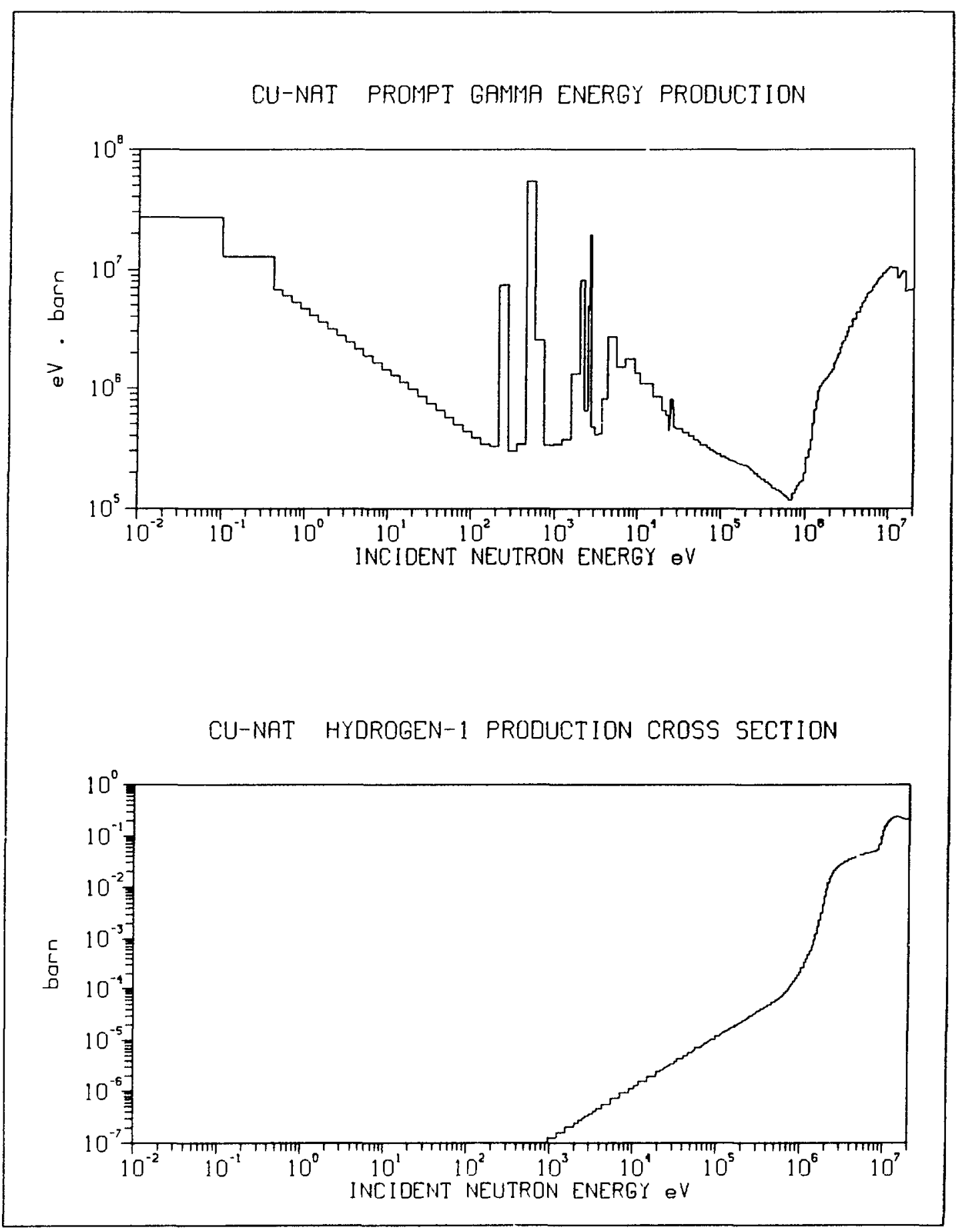




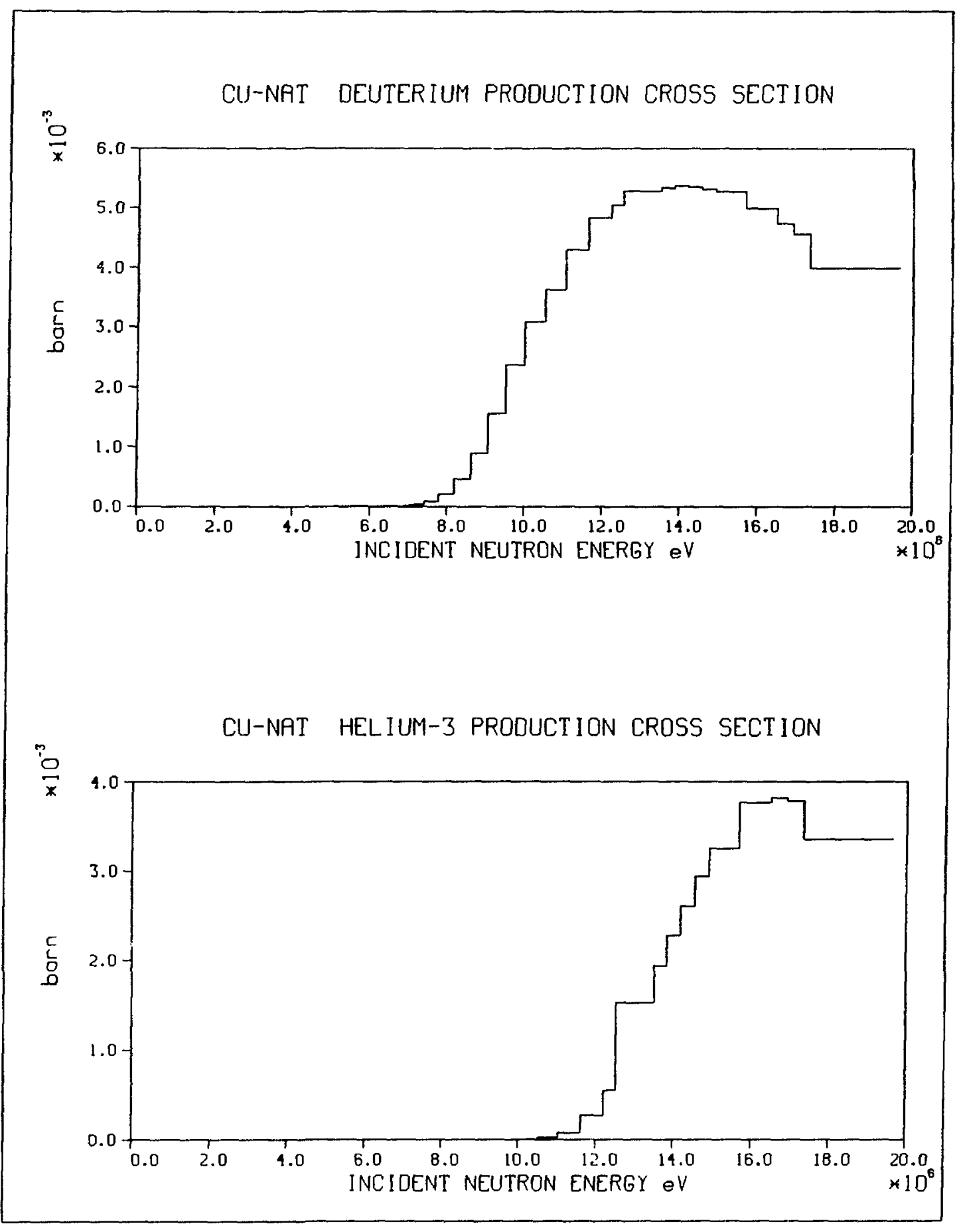




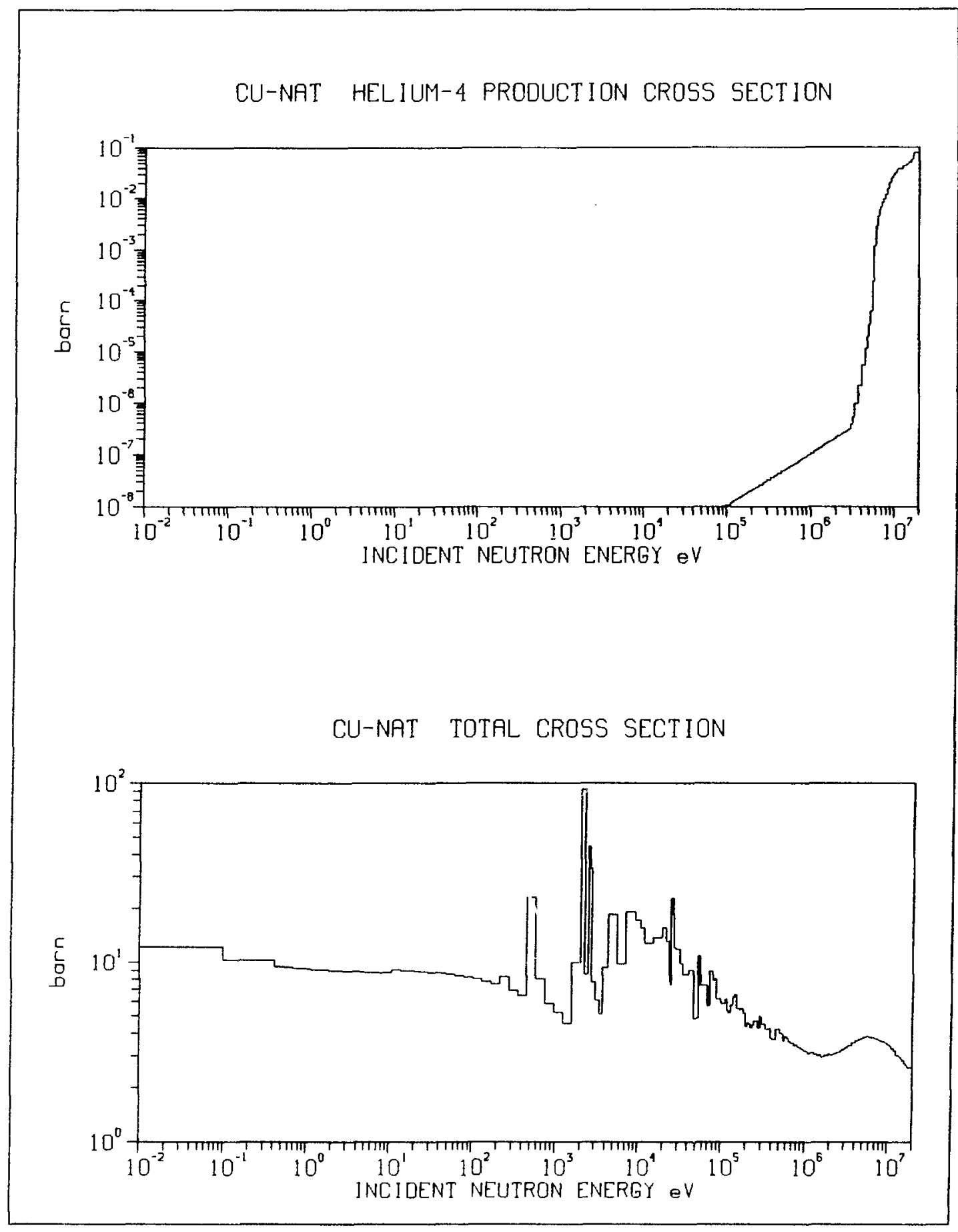




\section{CU-NAT ELASTIC CROSS SECTION}

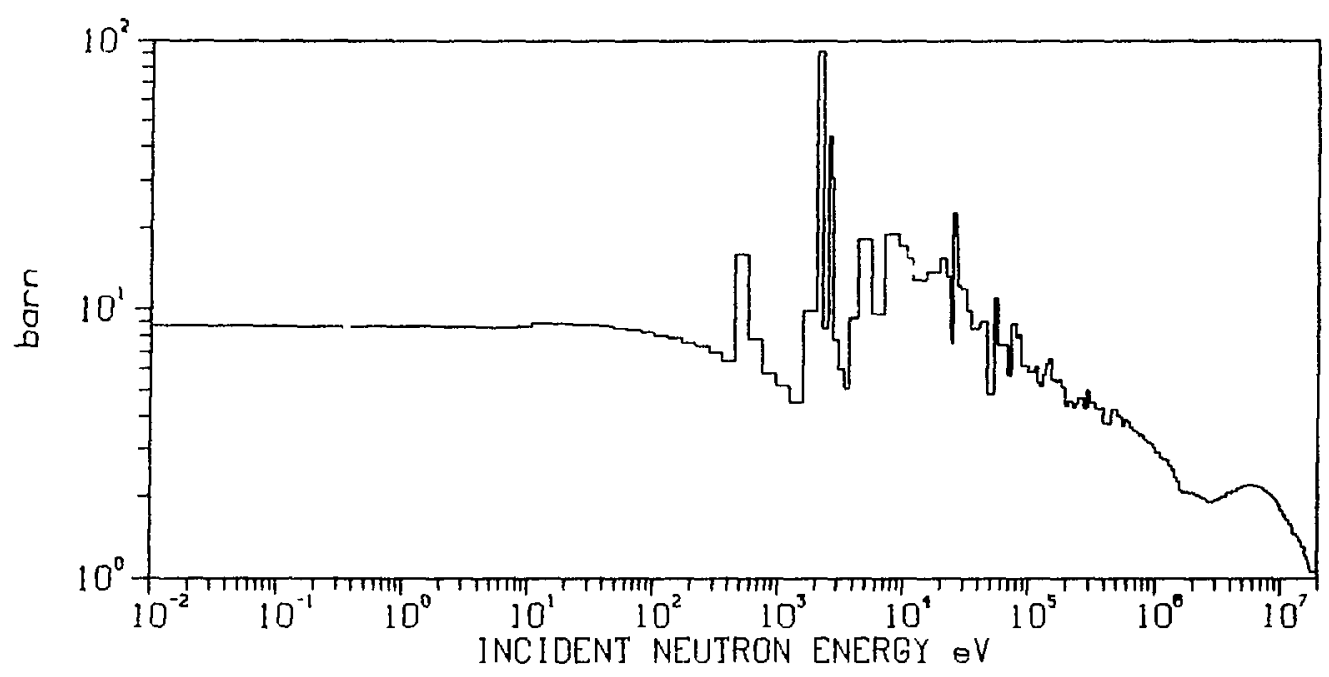

CUNAT TOTAL INELASTIC CROSS SECTION

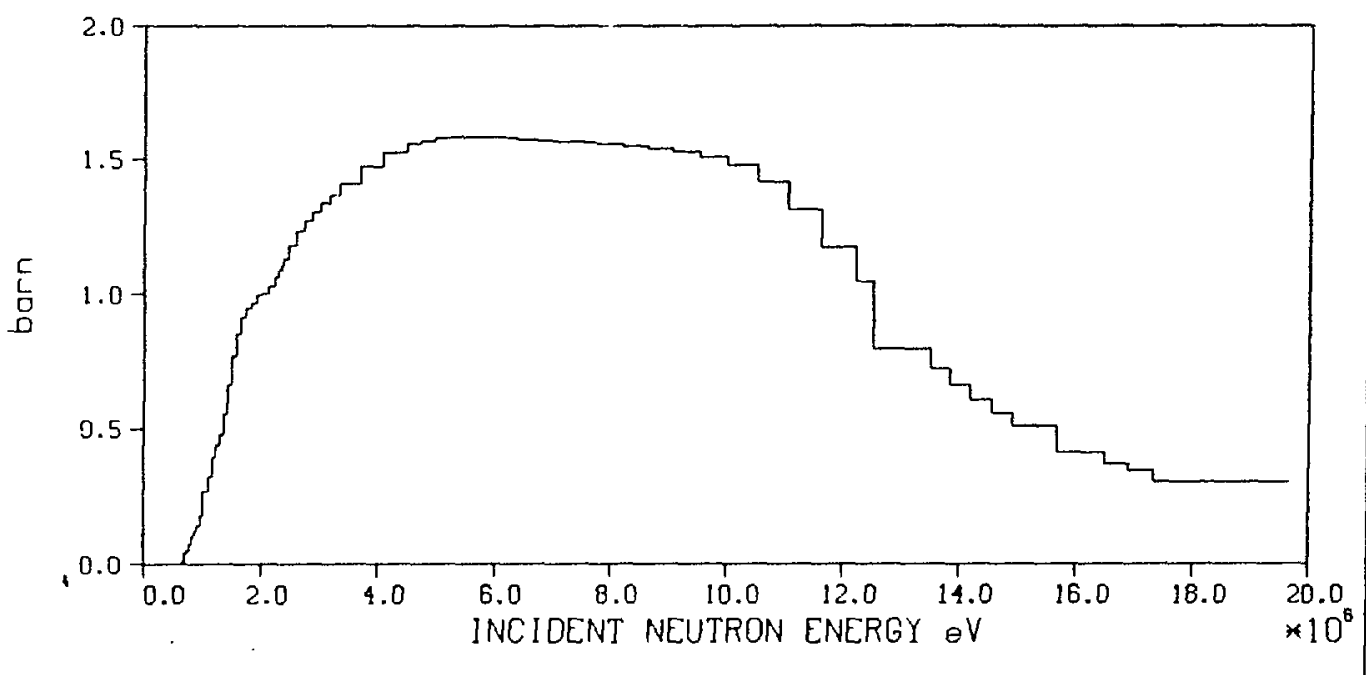




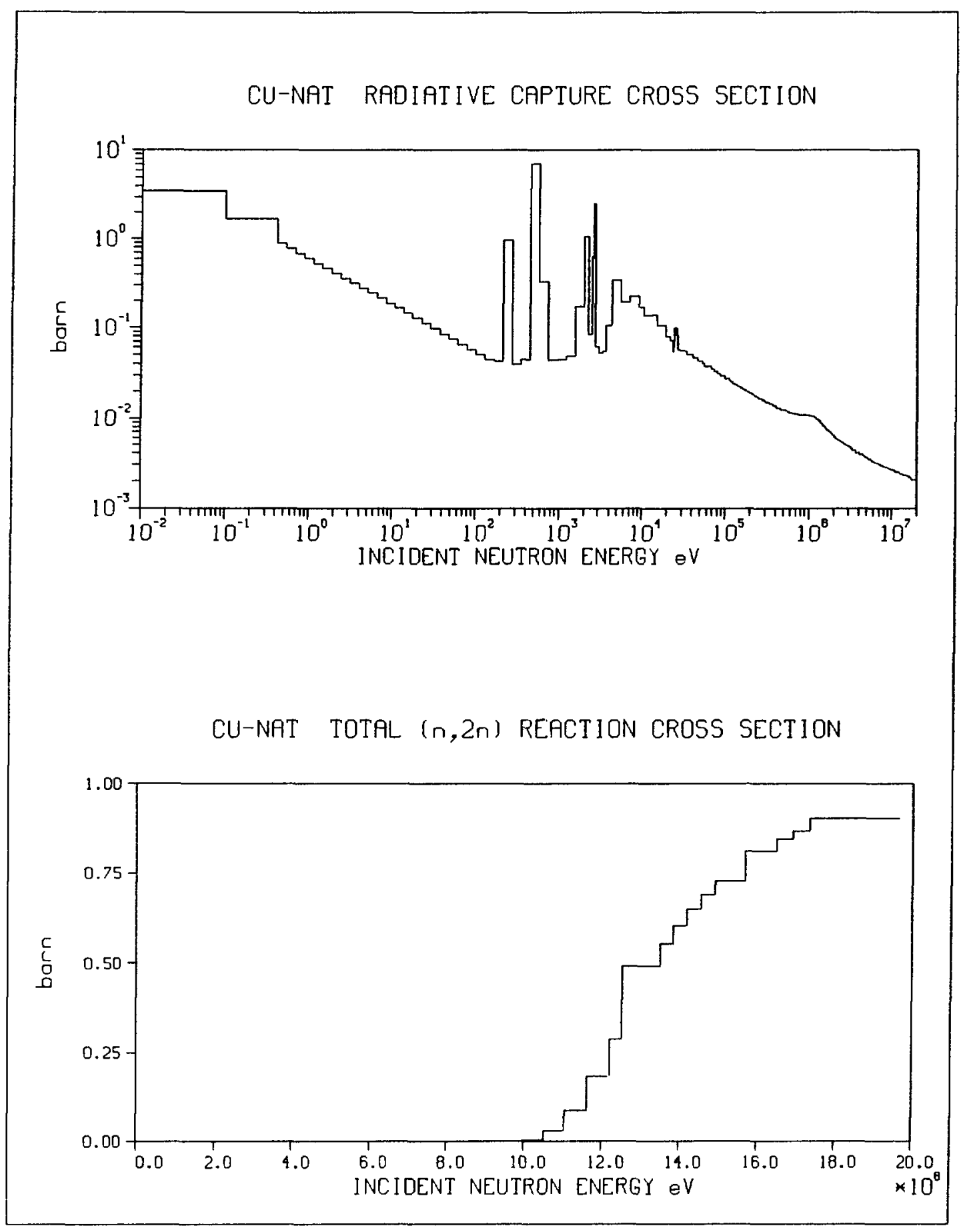




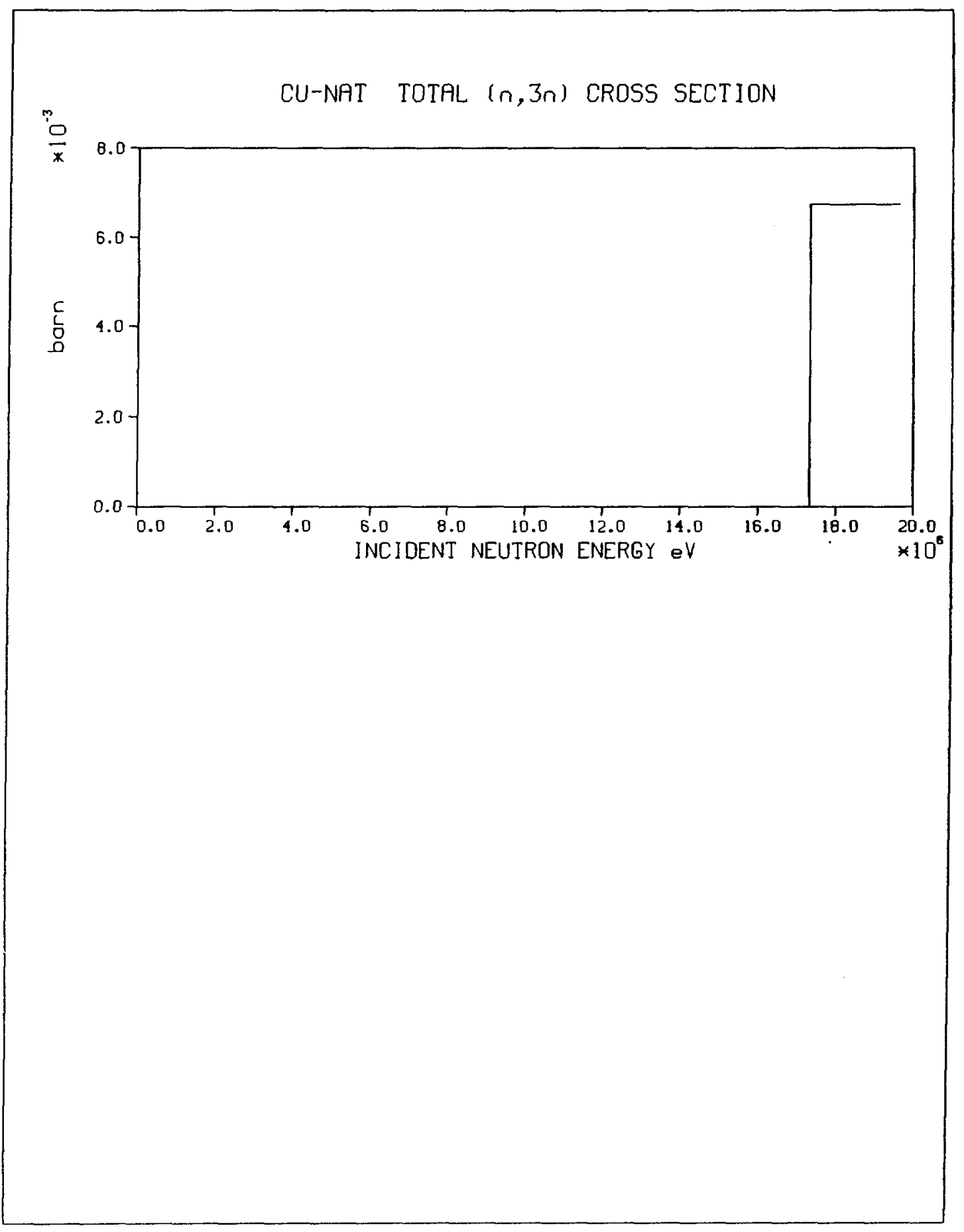




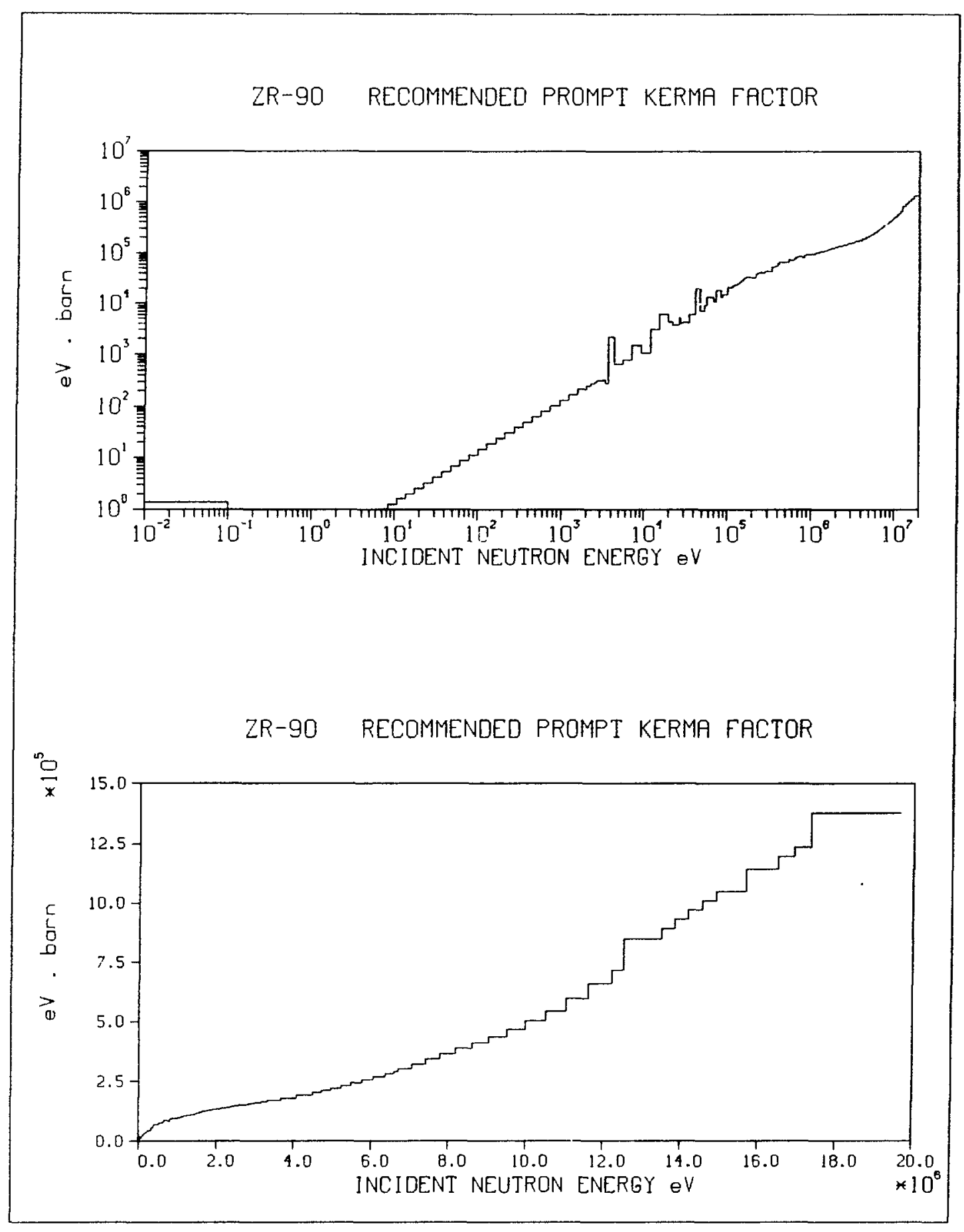


ZR-90 PROMPT GAMMA ENERGY PRODUCTION

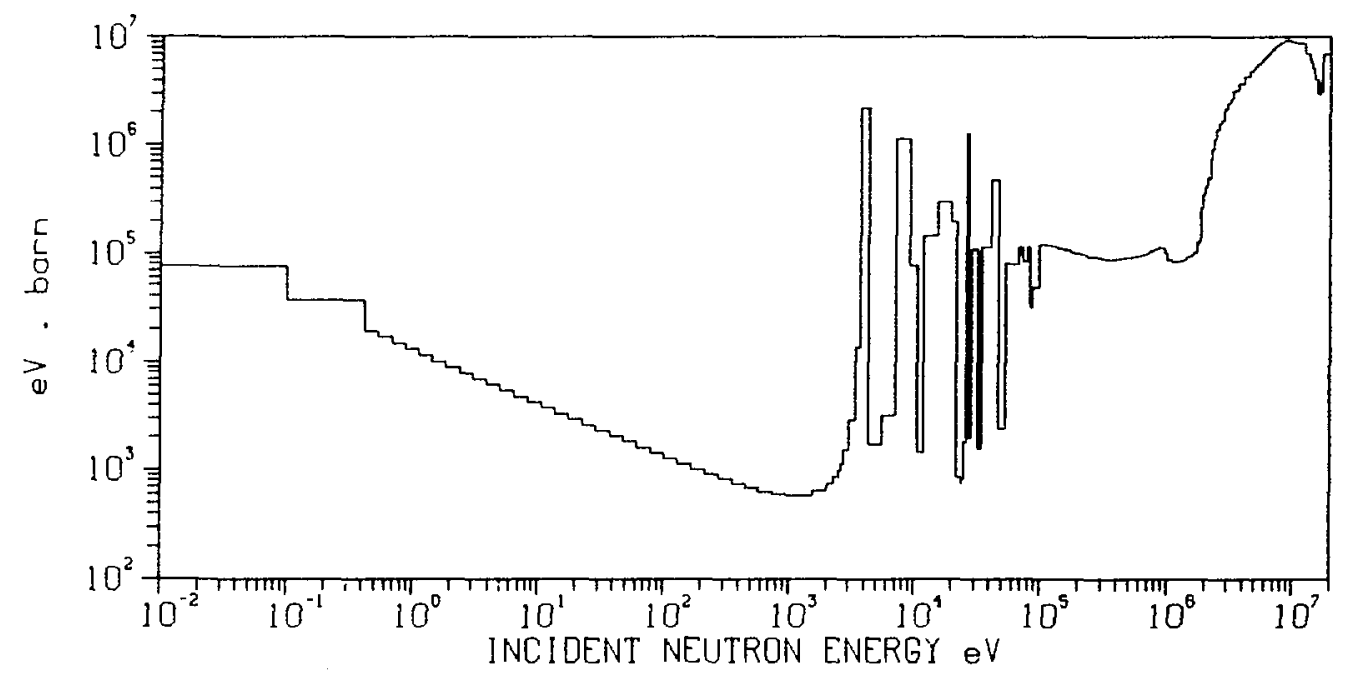

ZR-90 HYOROGEN-1 PRODUCTION CROSS SECTION

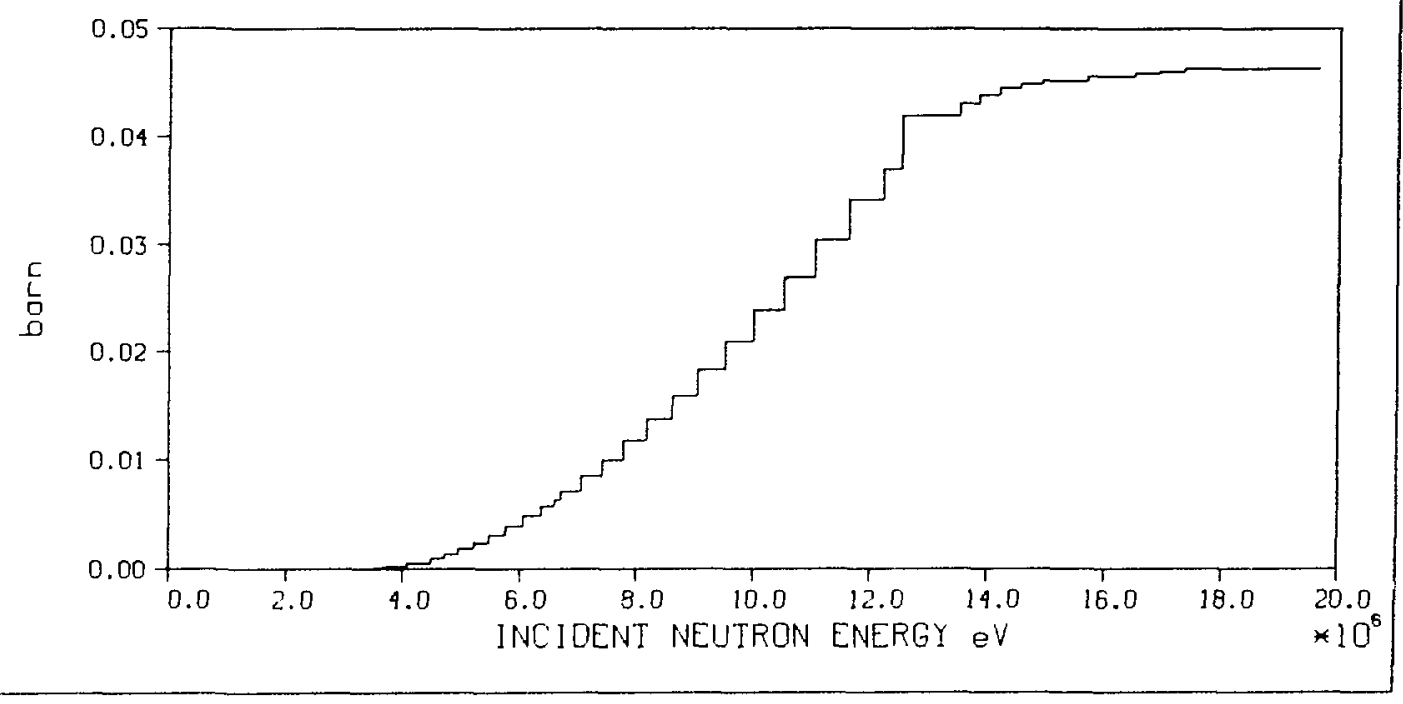




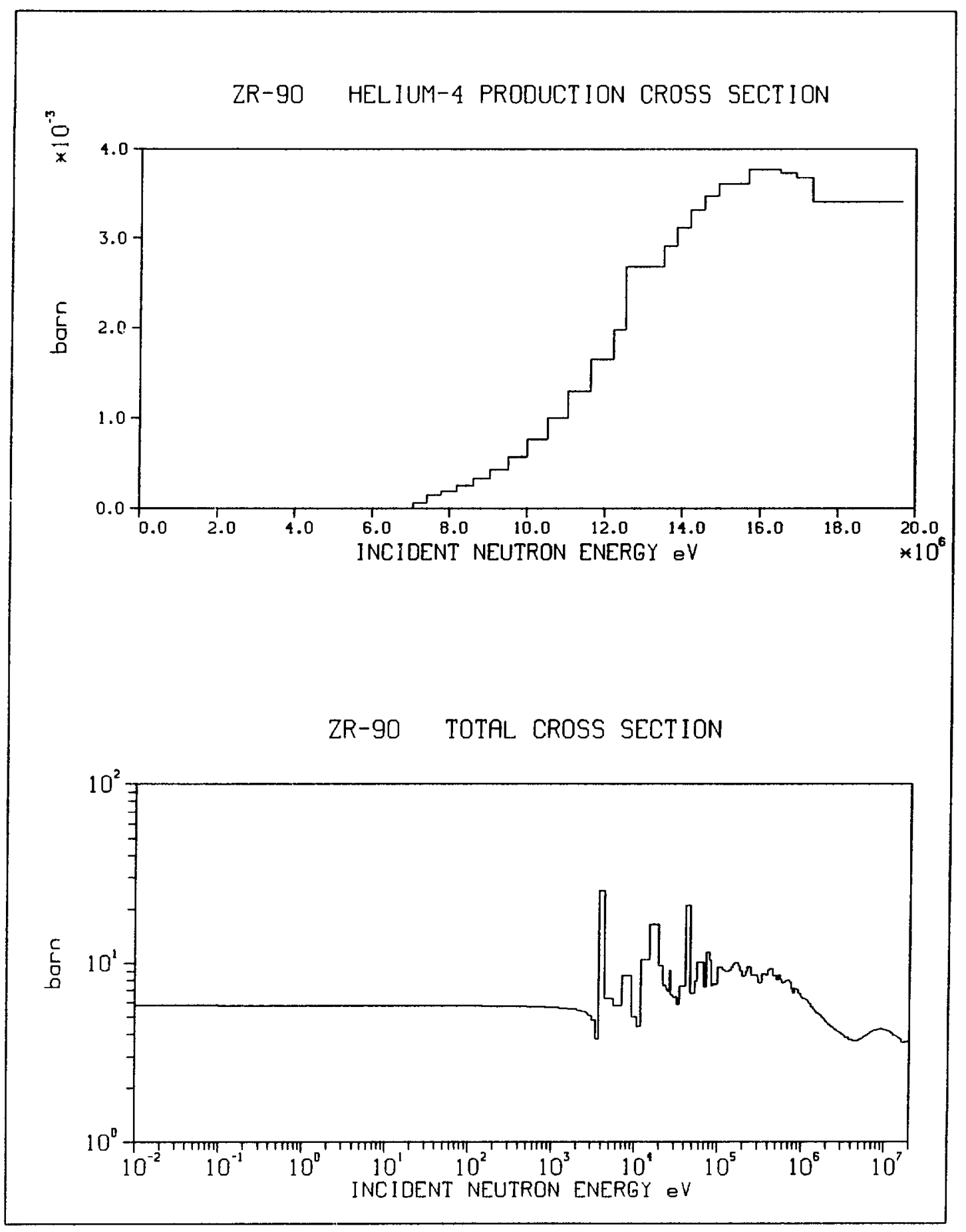




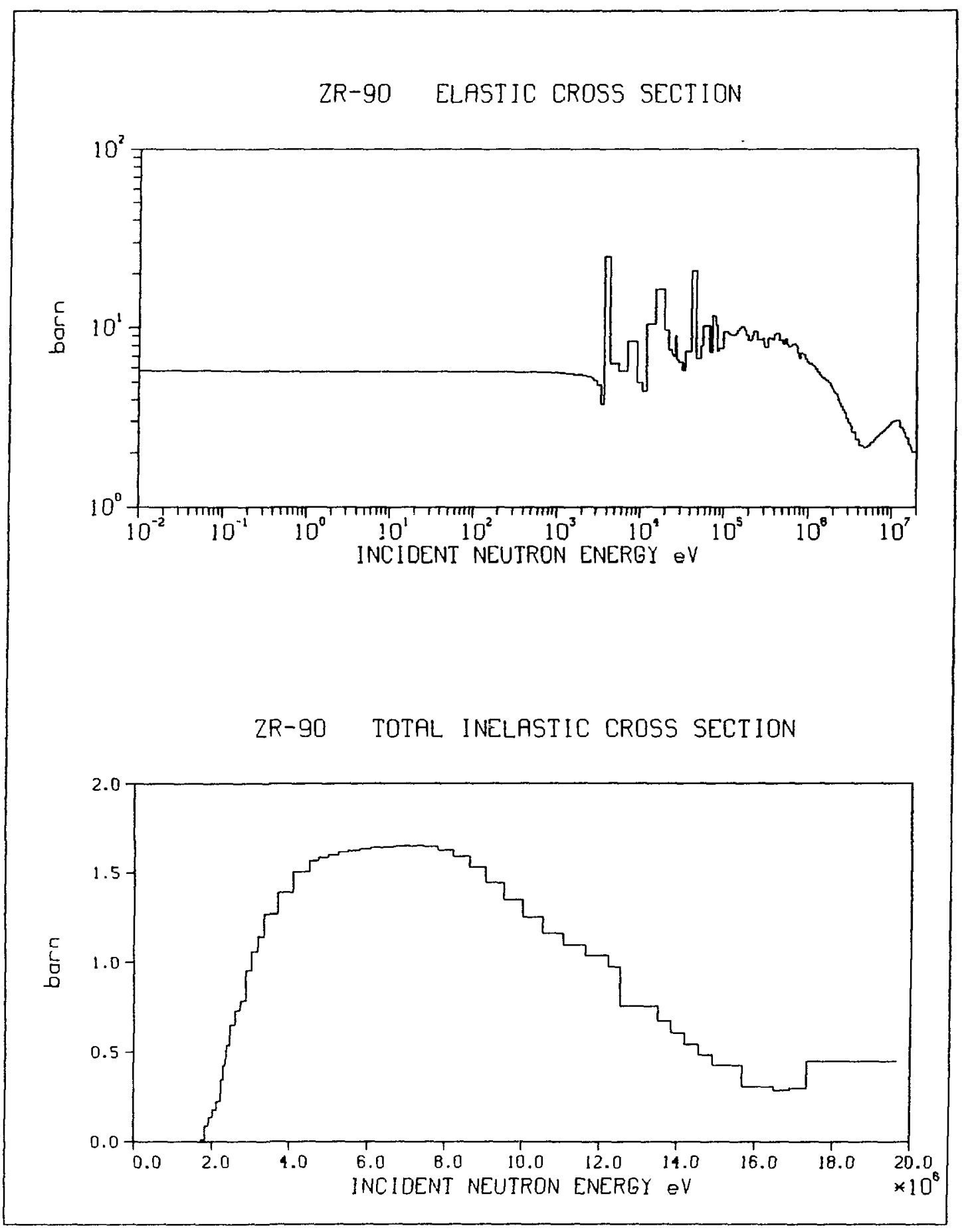


ZR-90 RADIATIVE CAPTURE CROSS SECTION

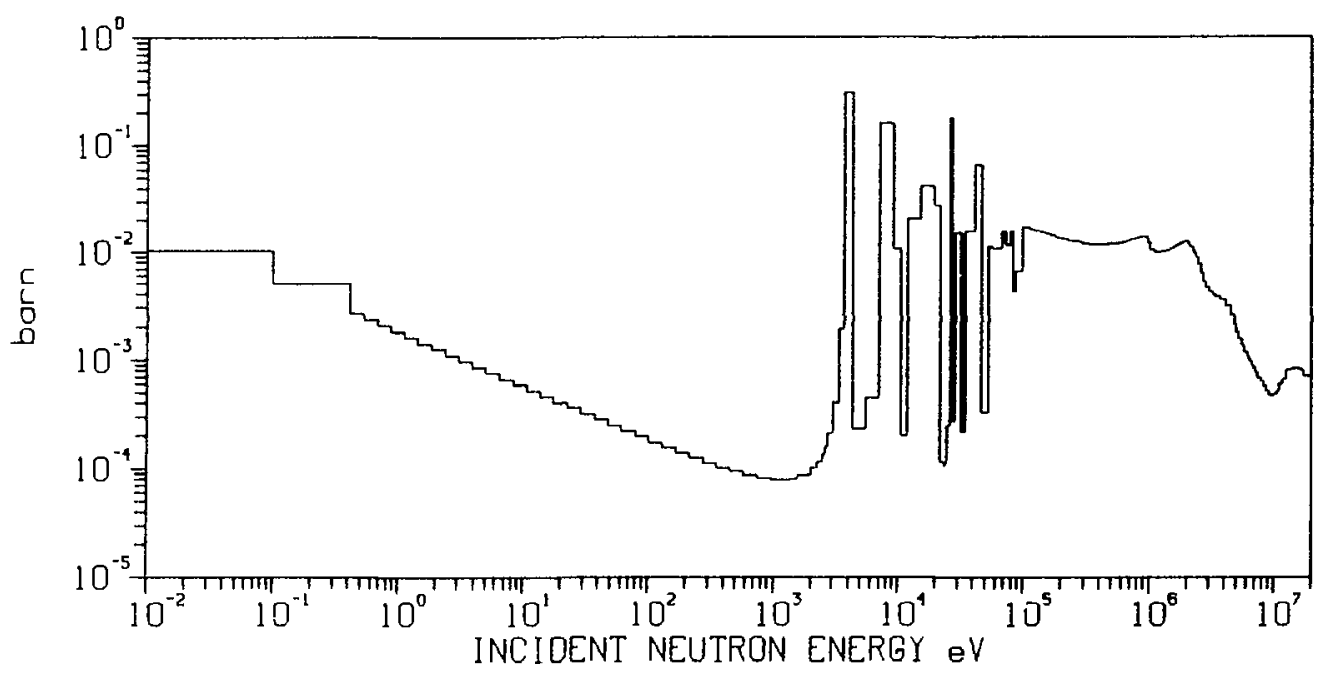

ZR-90 TOTAL $(n, 2 n)$ REACTION CROSS SECTION

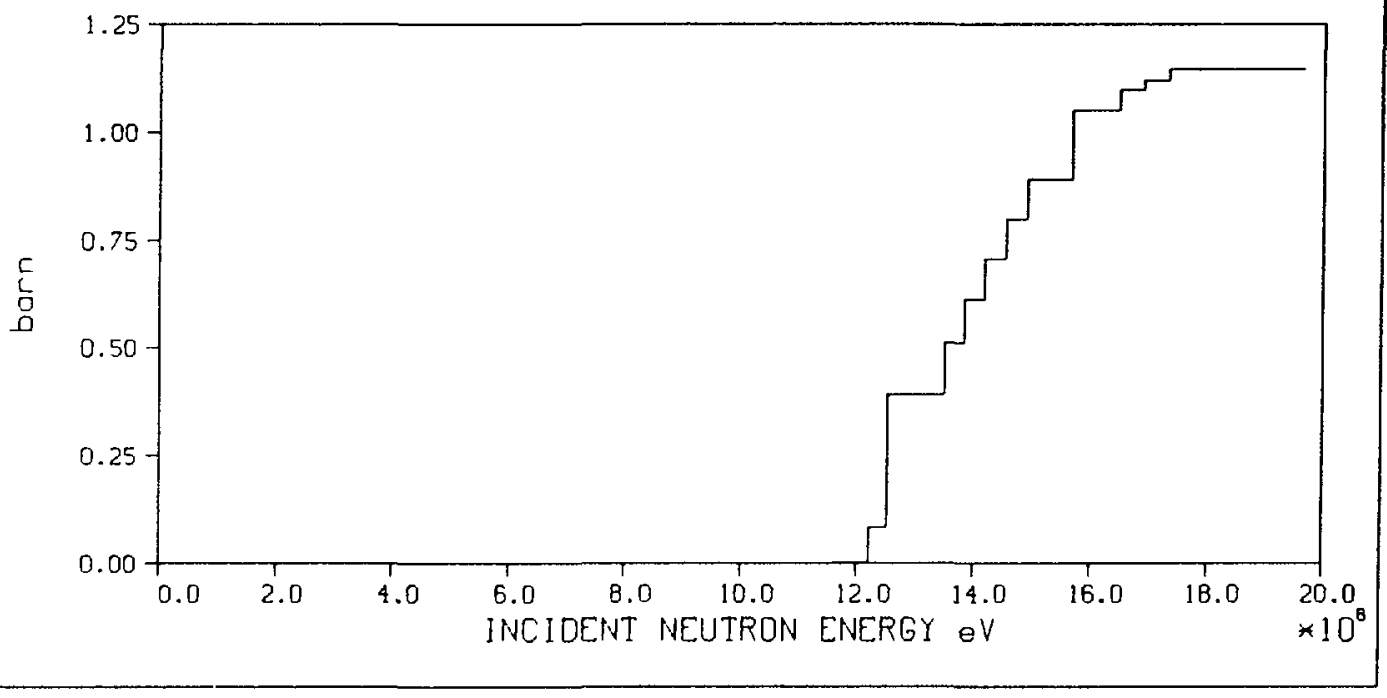




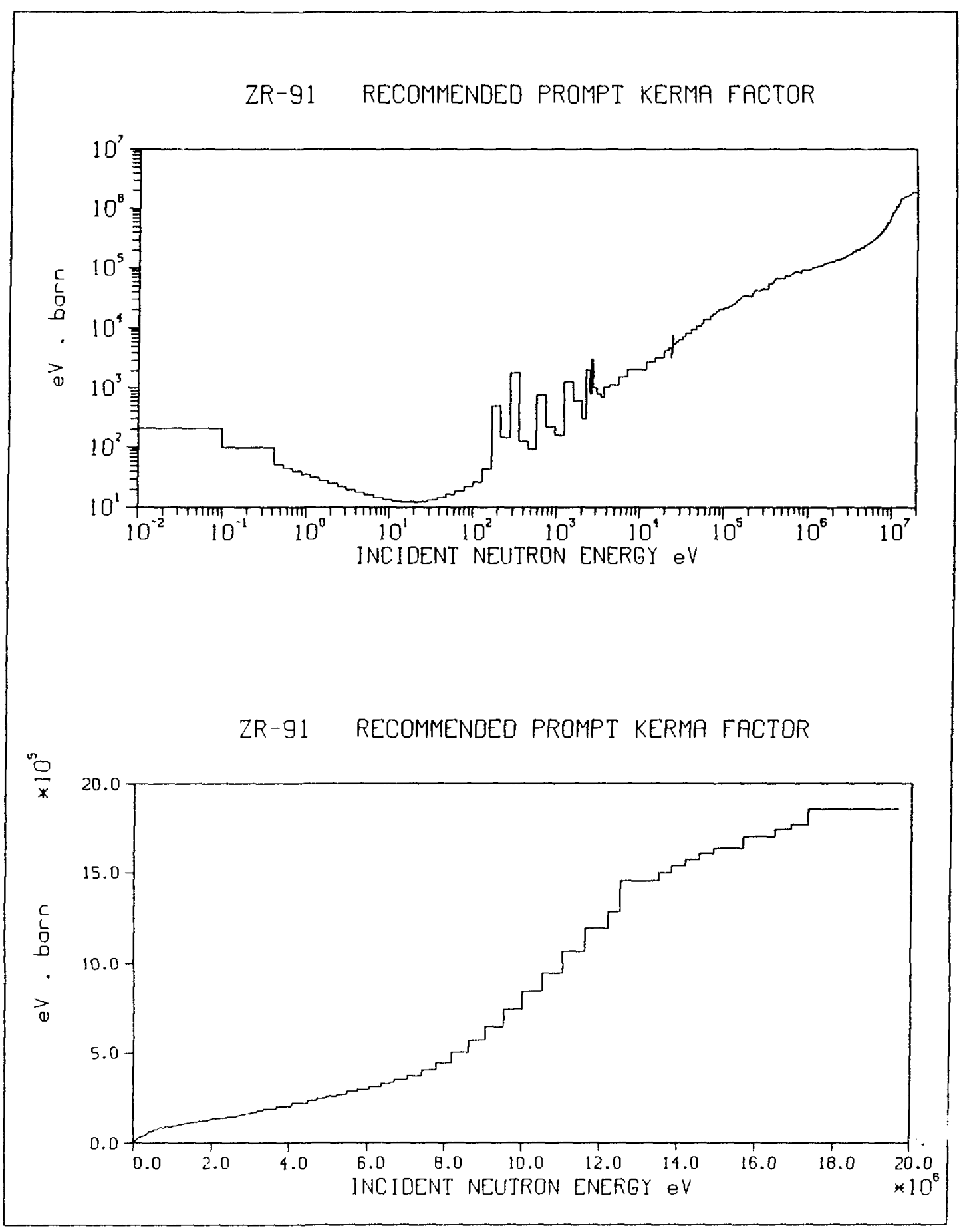




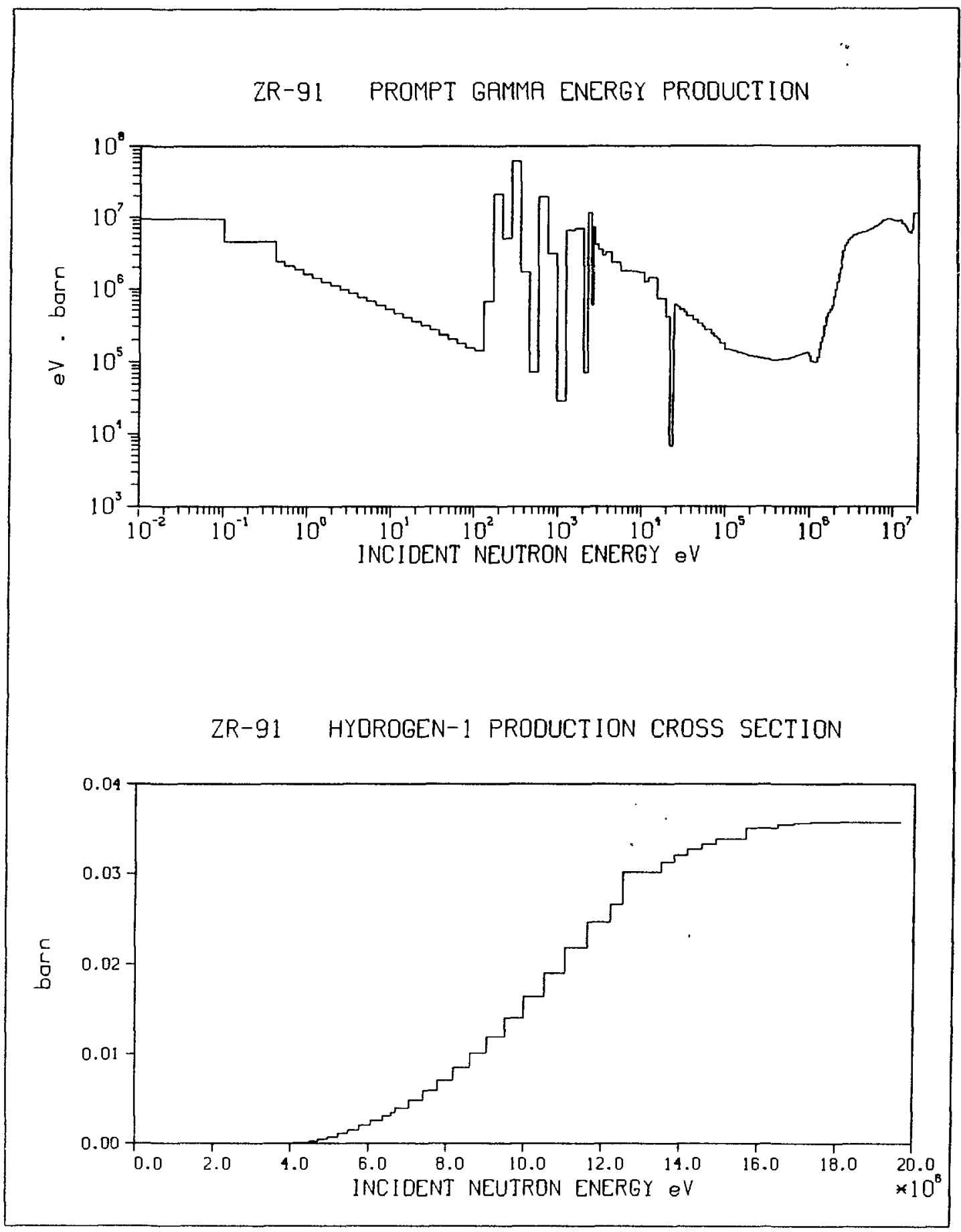




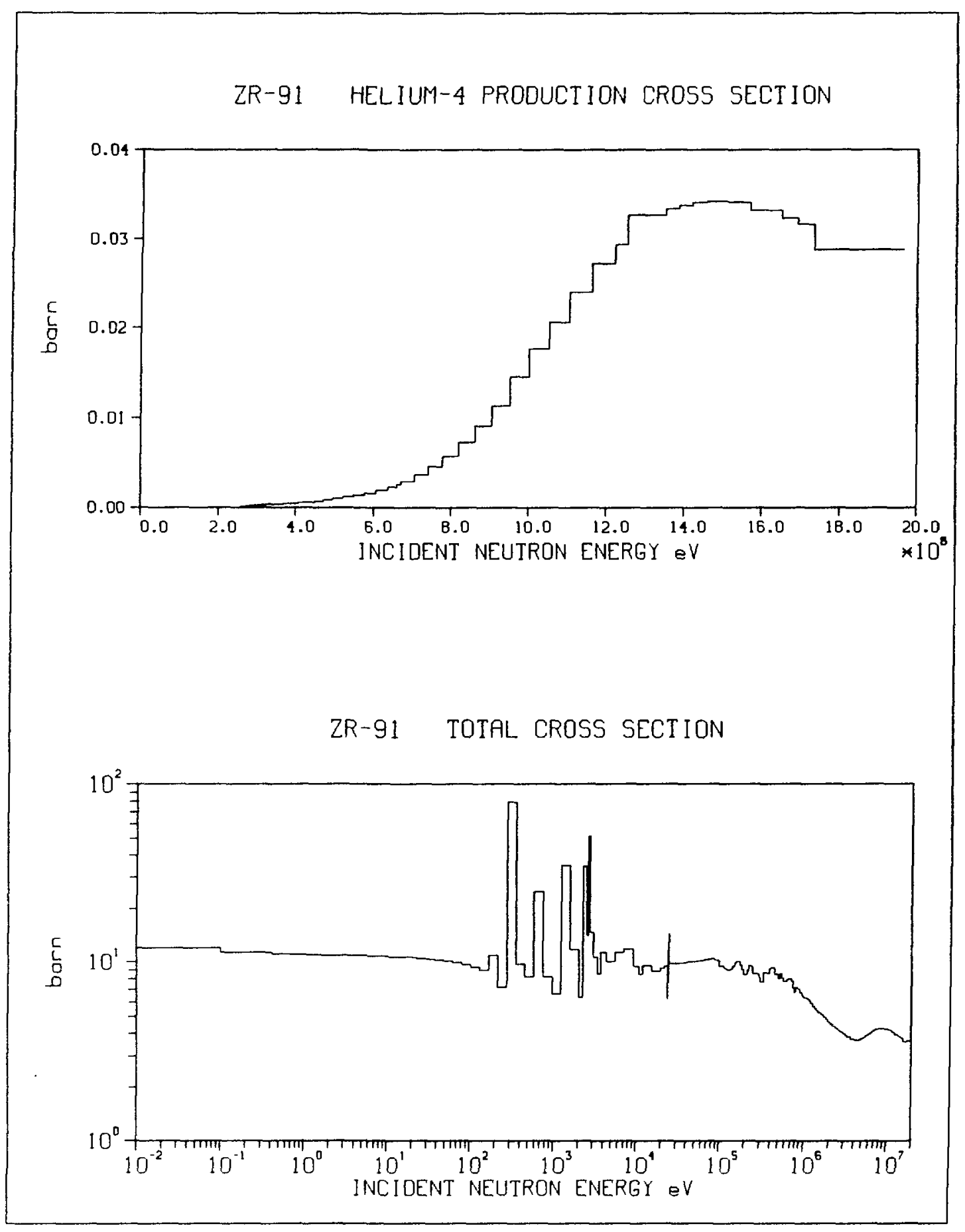




\section{ZR-91 ELASTIC CROSS SECTION}

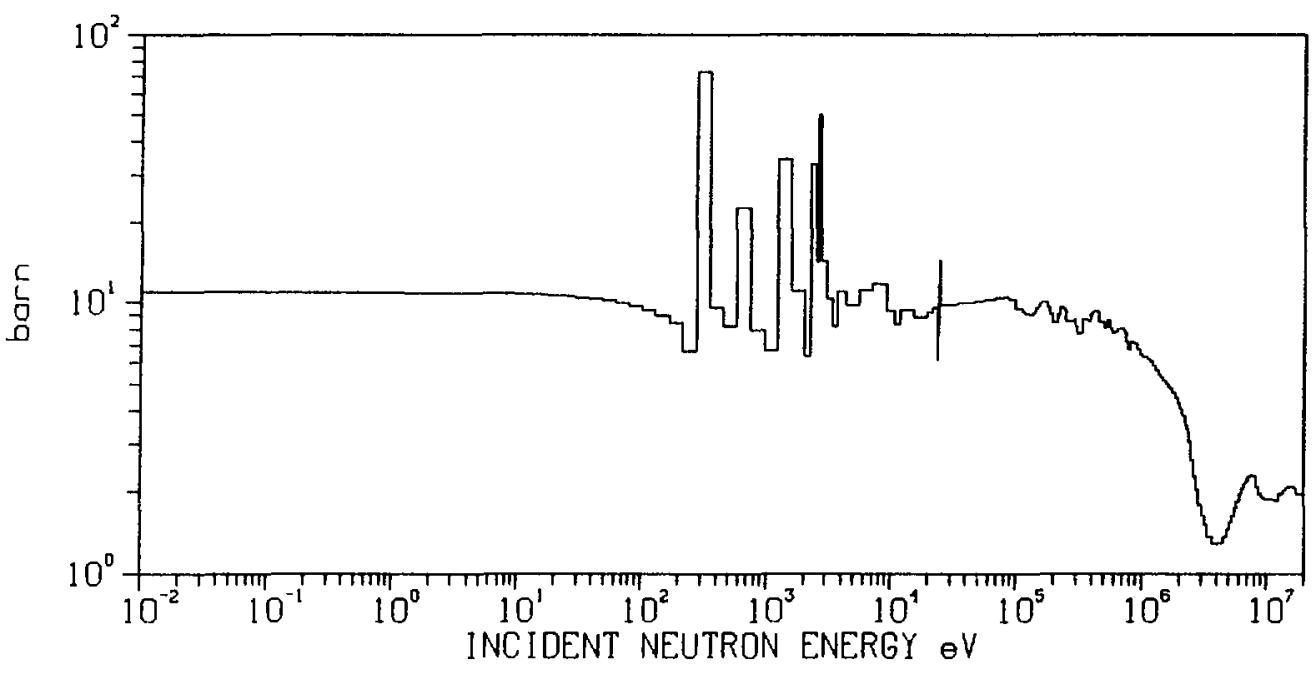

$\therefore \quad$ ZR-91 TOTAL INELASTIC CROSS SECTION

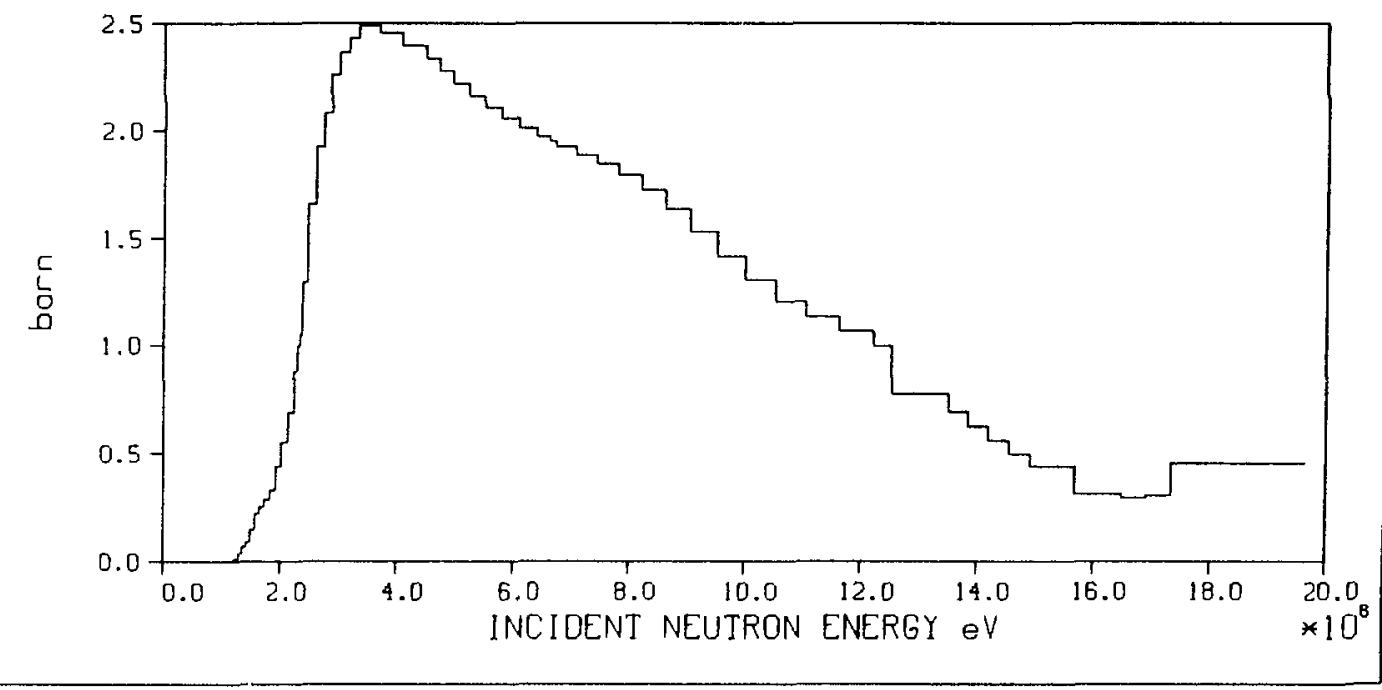




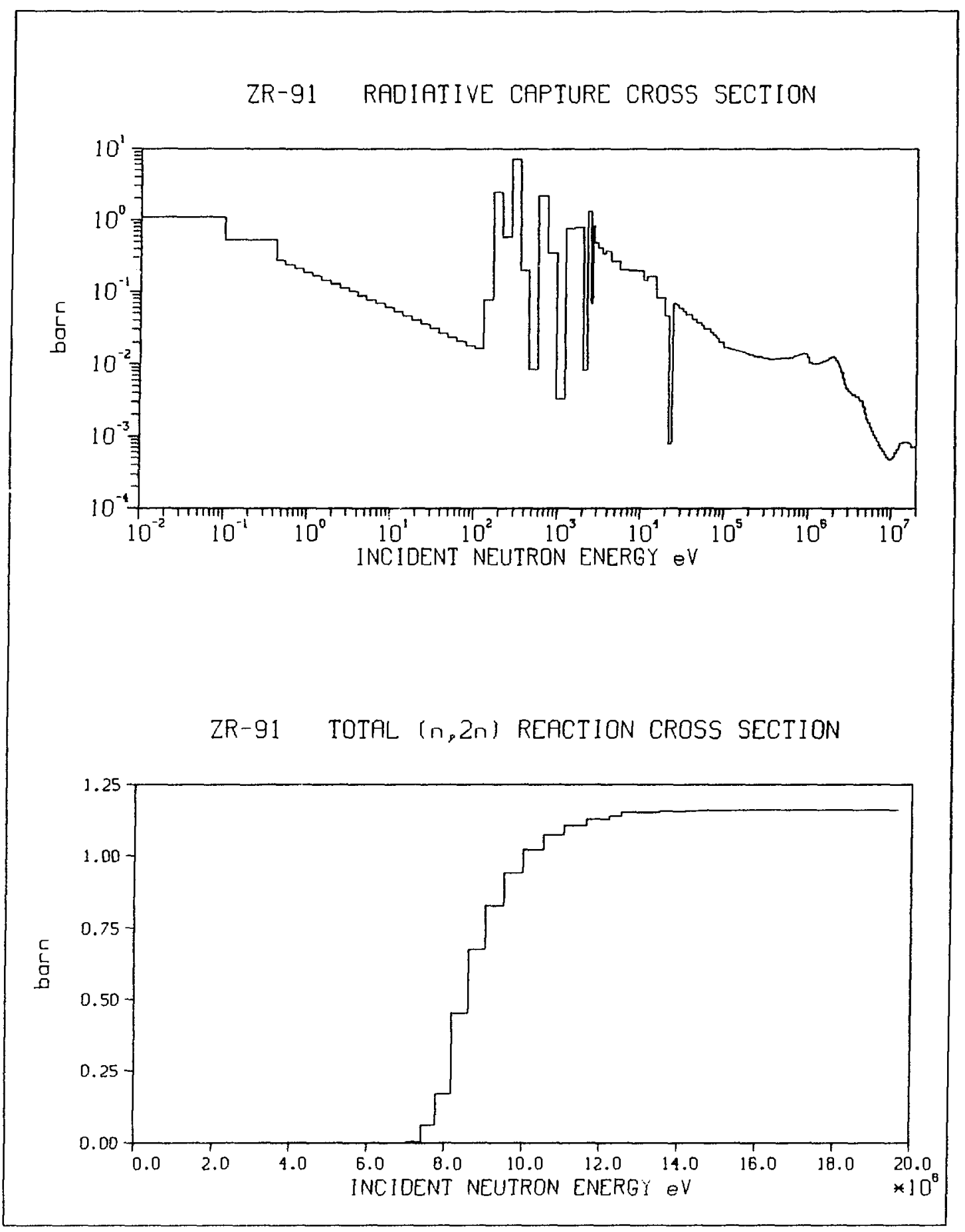


ZR-92 RECOMMENDED PROMPT KERMA FACTOR (solid line) REC. K. FACTOR WITH CH. PARTICLE DECAY HEAT (dashed line)

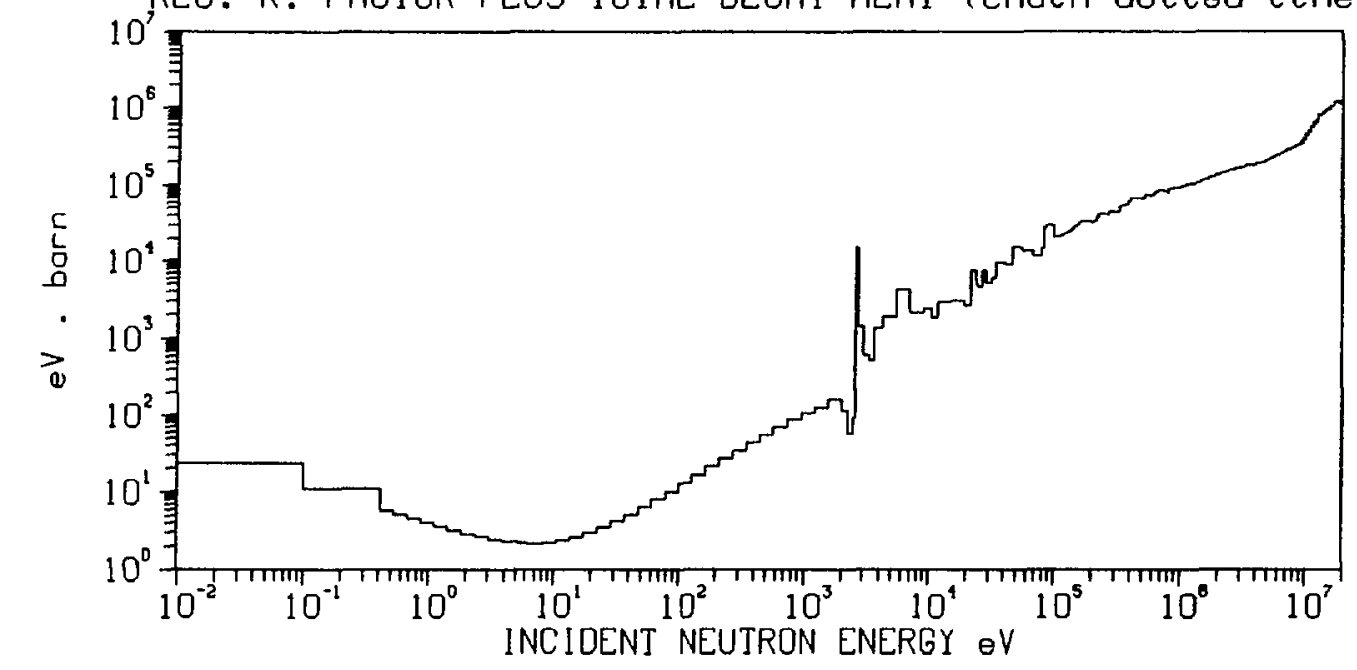

ZR-92 RECOMMENDED PROMPT KERMA FACTOR (solid line) REC. K. FACTOR WITH CH. PARTICLE DECAY HEAT (dashed line) "O REC. K. FACTOR PLUS TOTAL DECAY HEAT (chain dotted line)

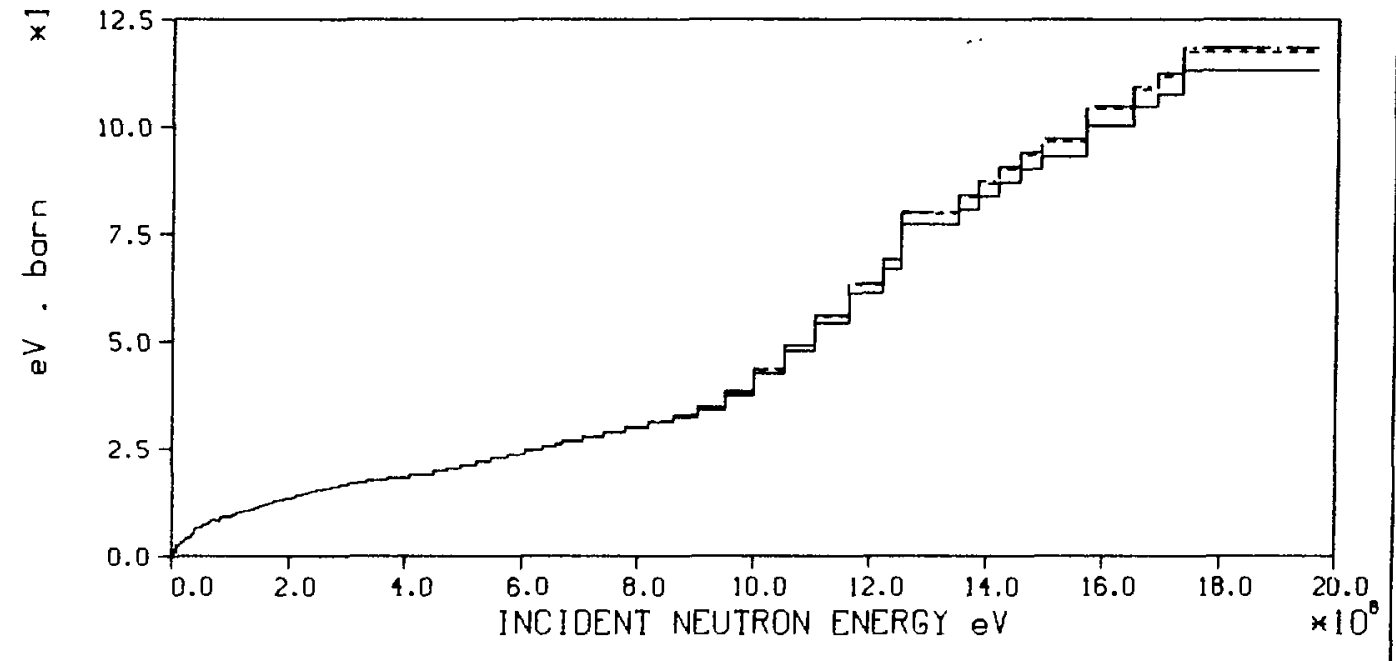




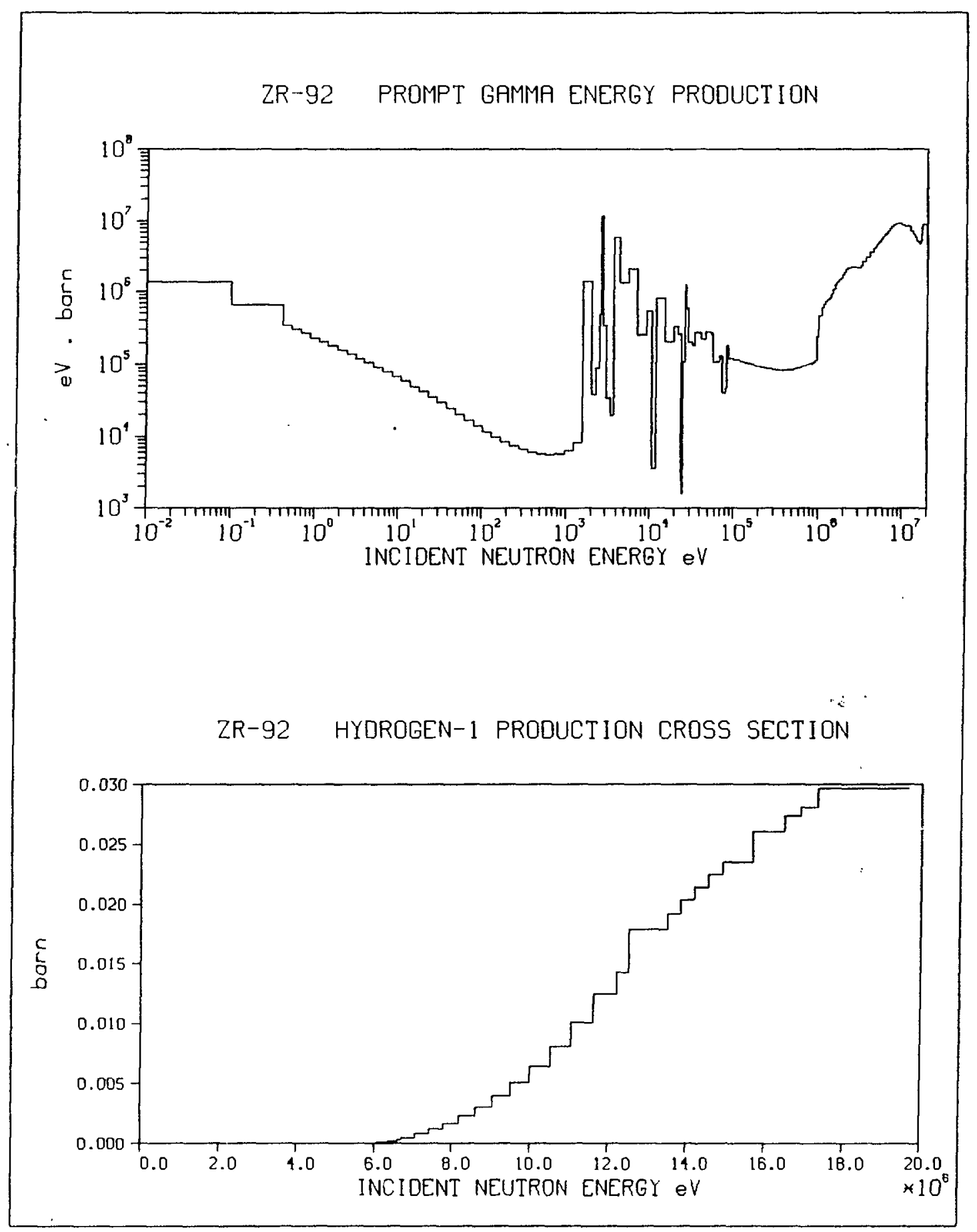




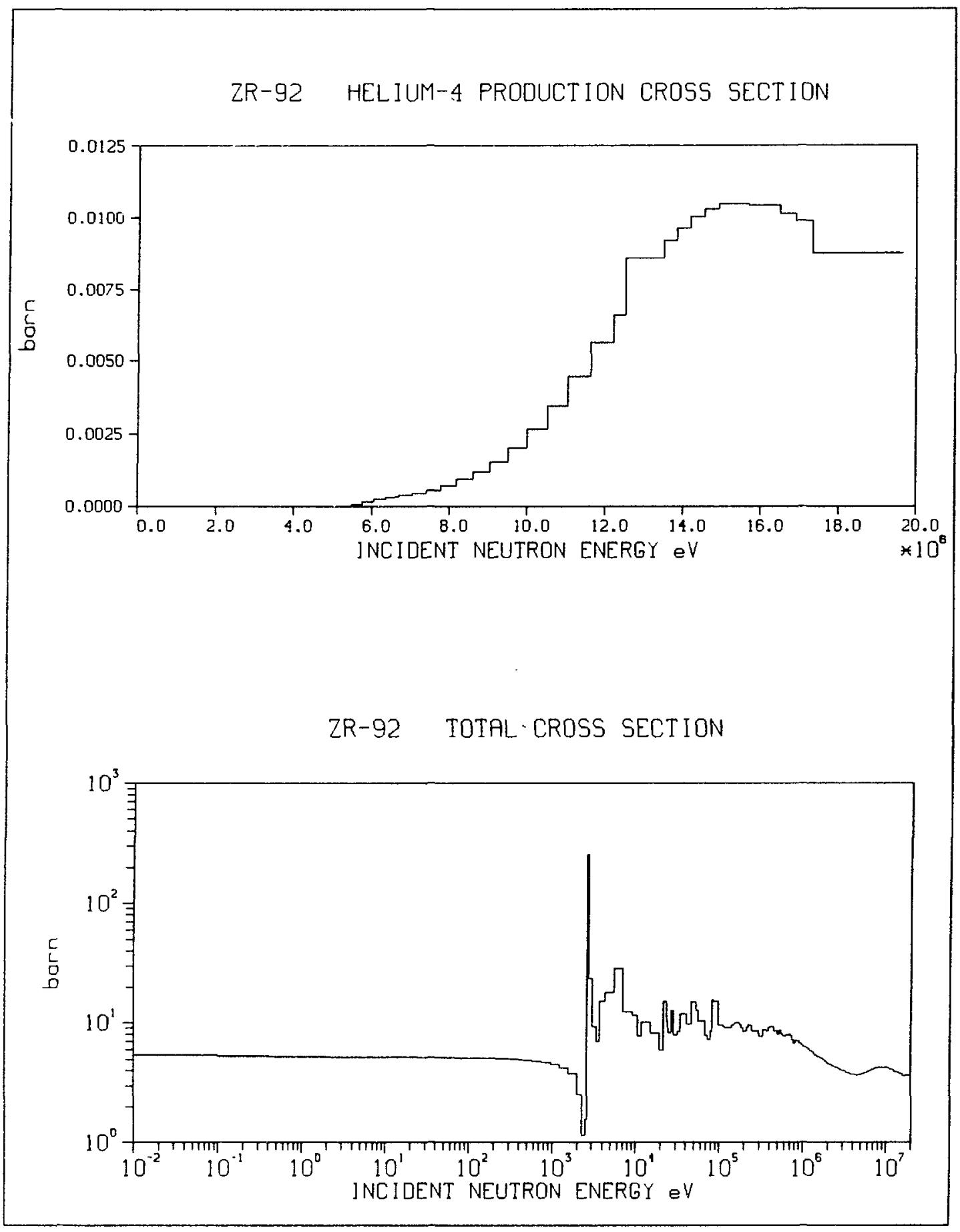


ZR-92 ELASTIC CROSS SECTION

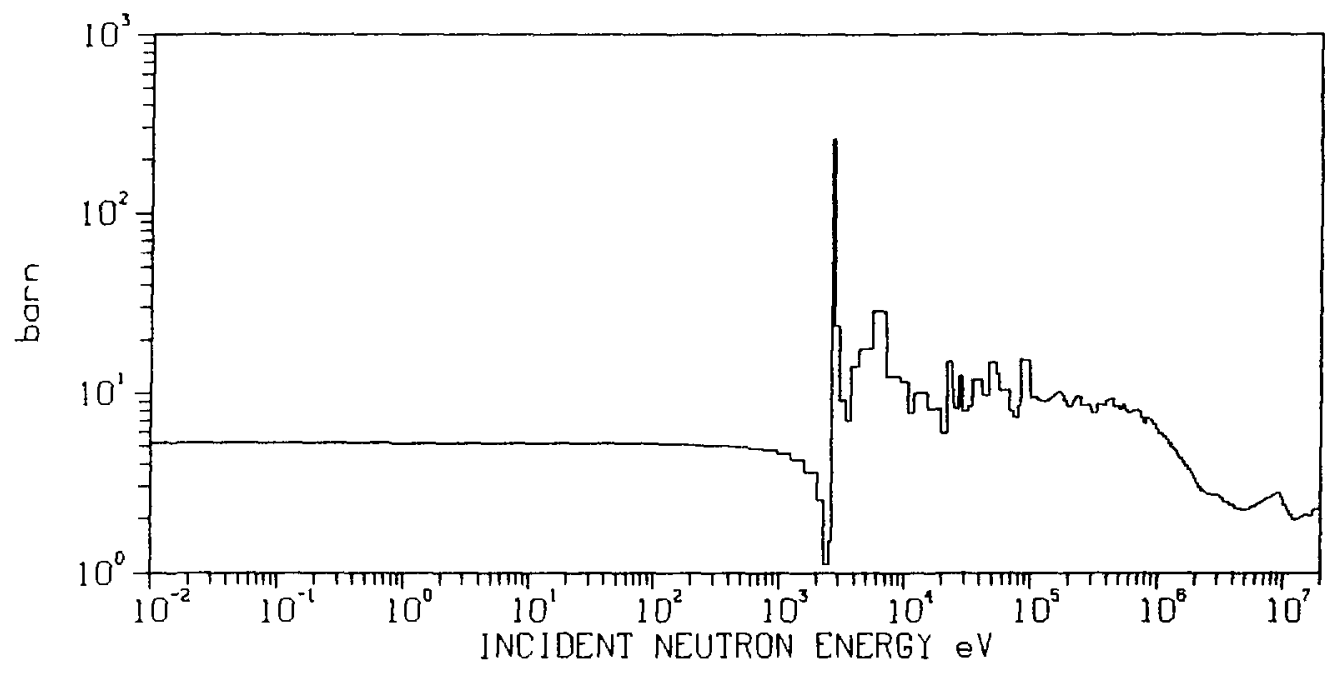

ZR-92 TOTAL INELASTIC CROSS SECTION

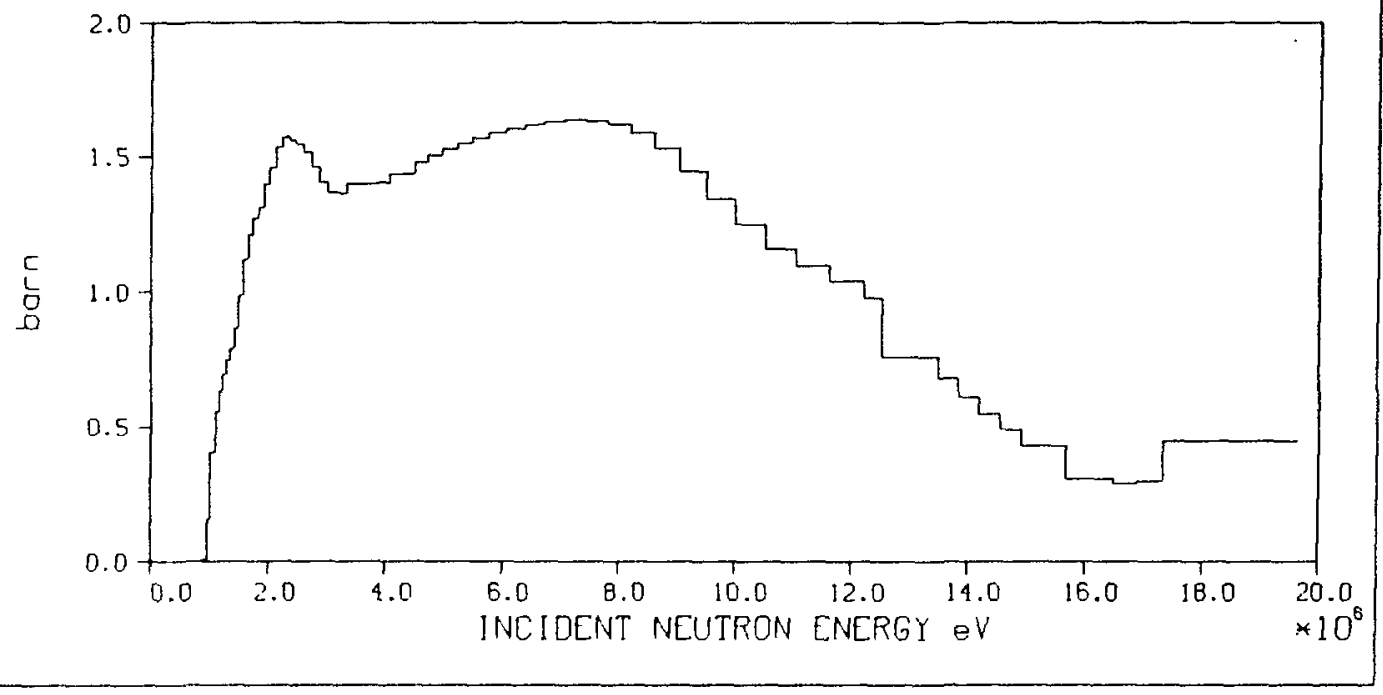




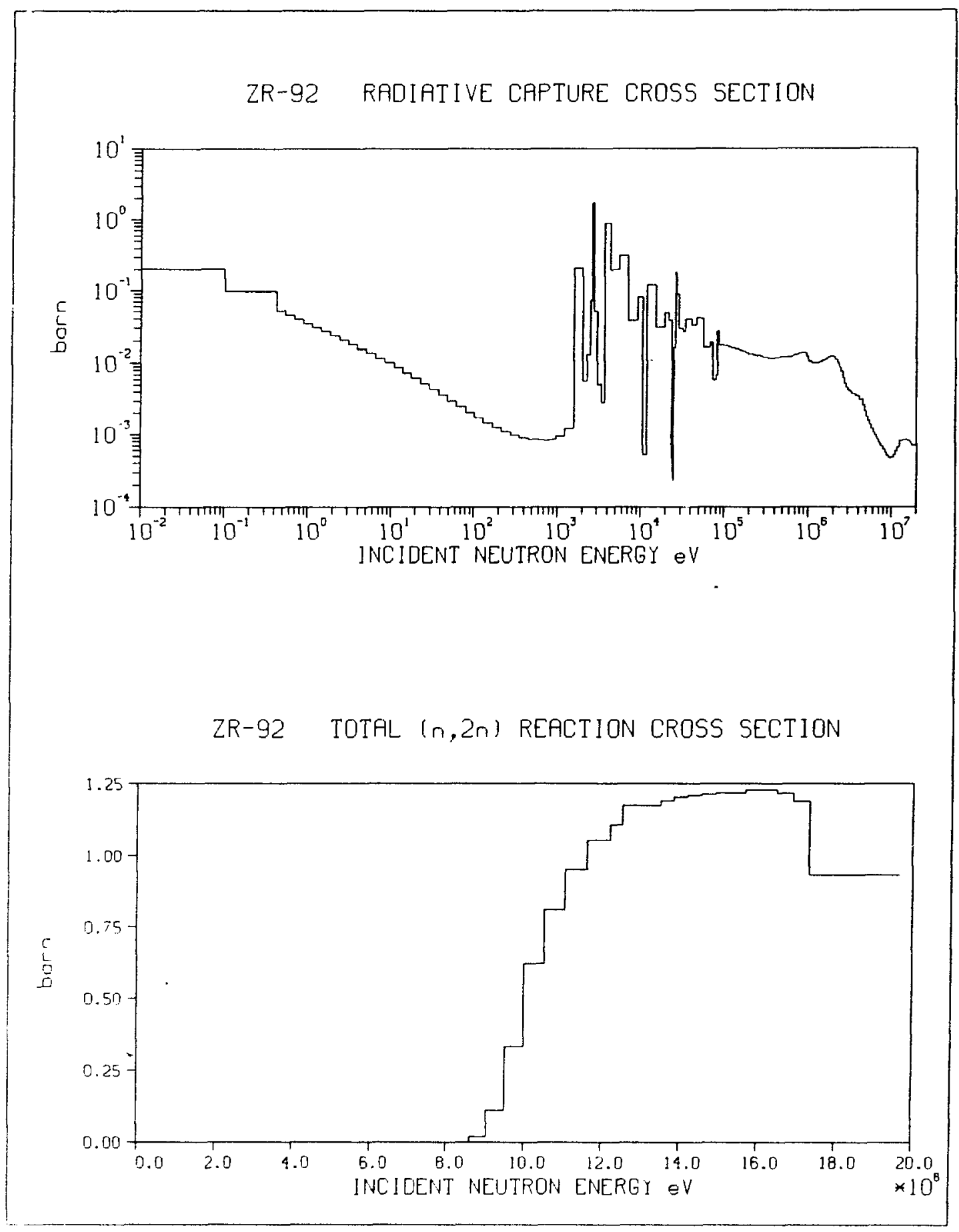




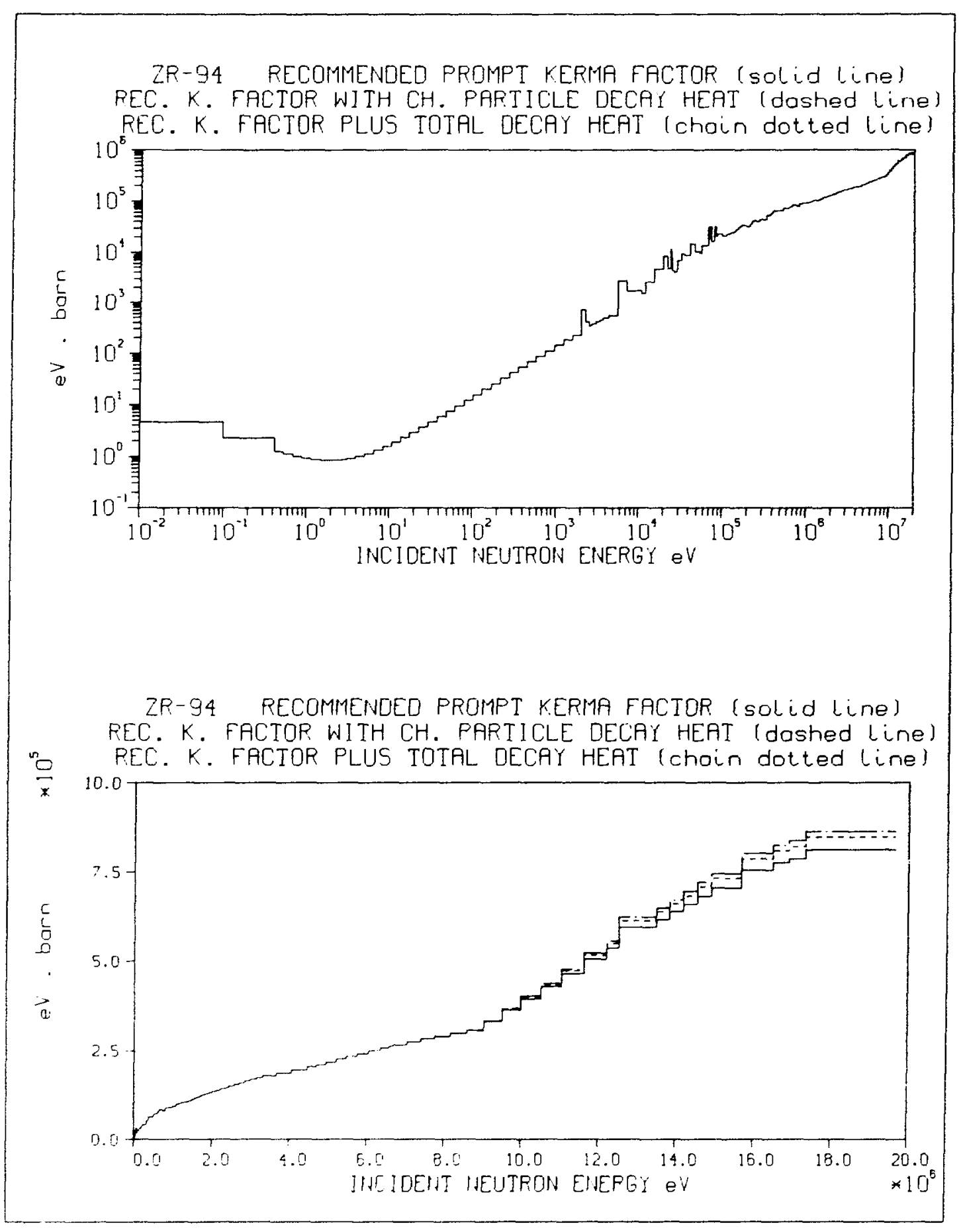




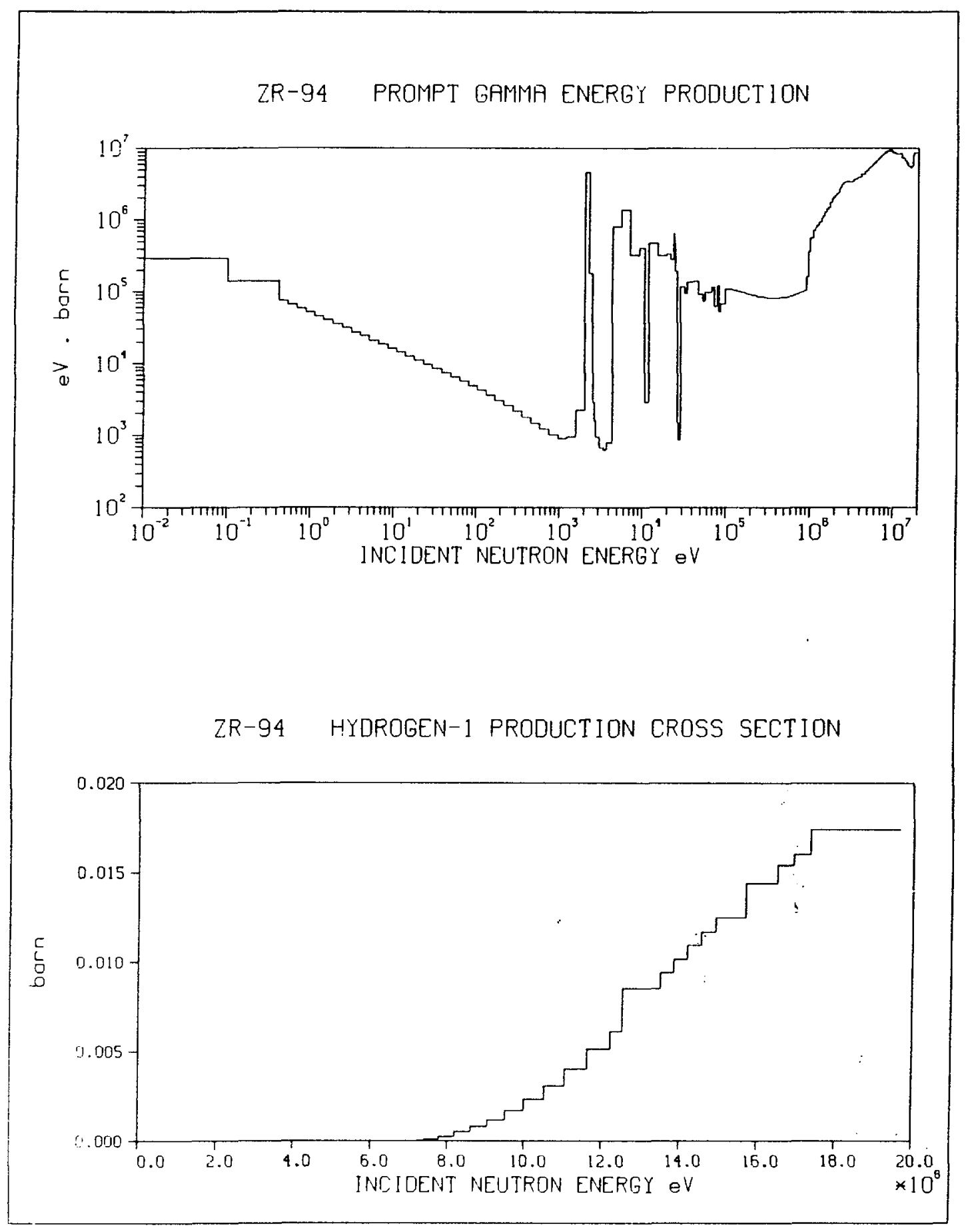




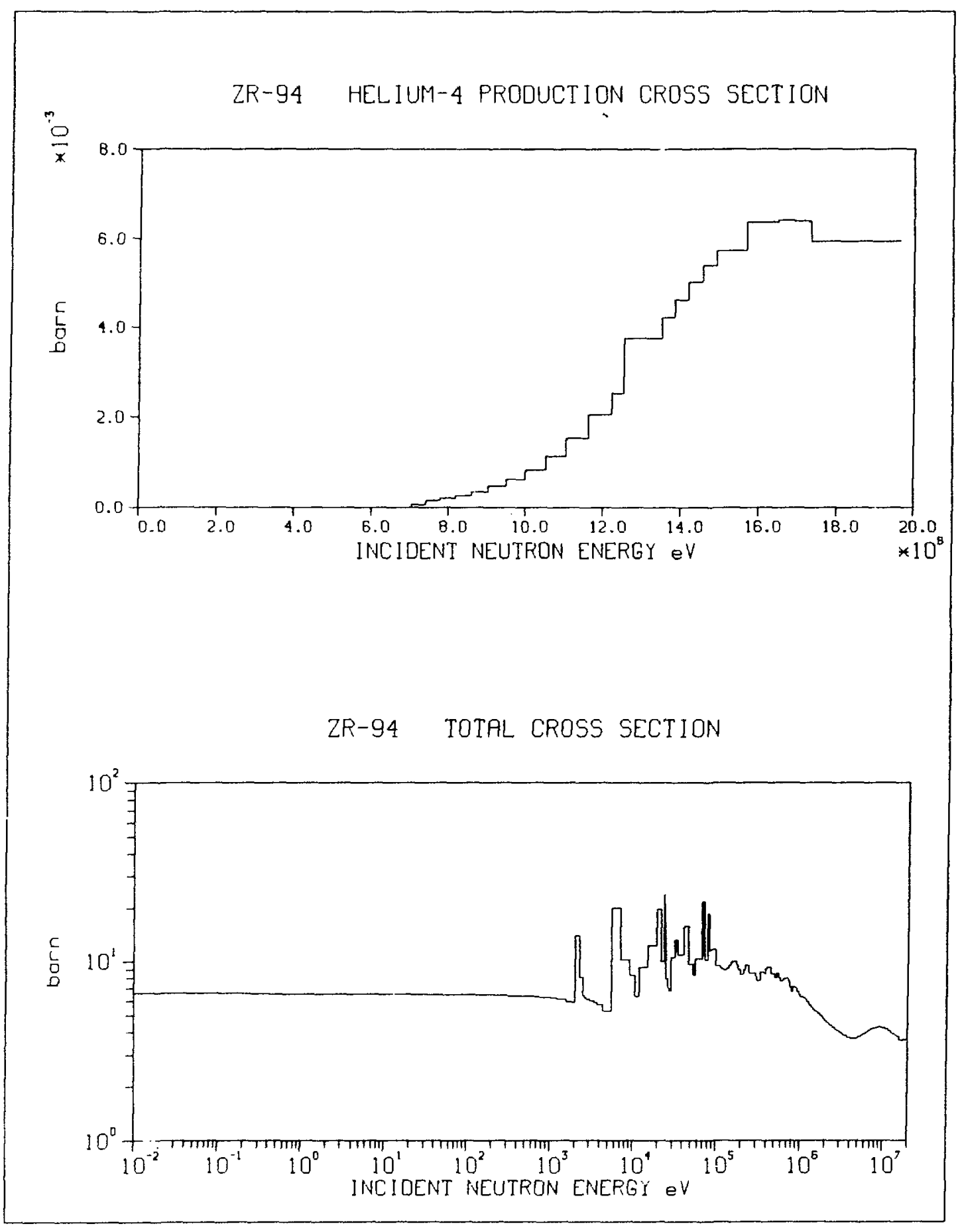




\section{ZR-94 ELASTIC CROSS SECTION}

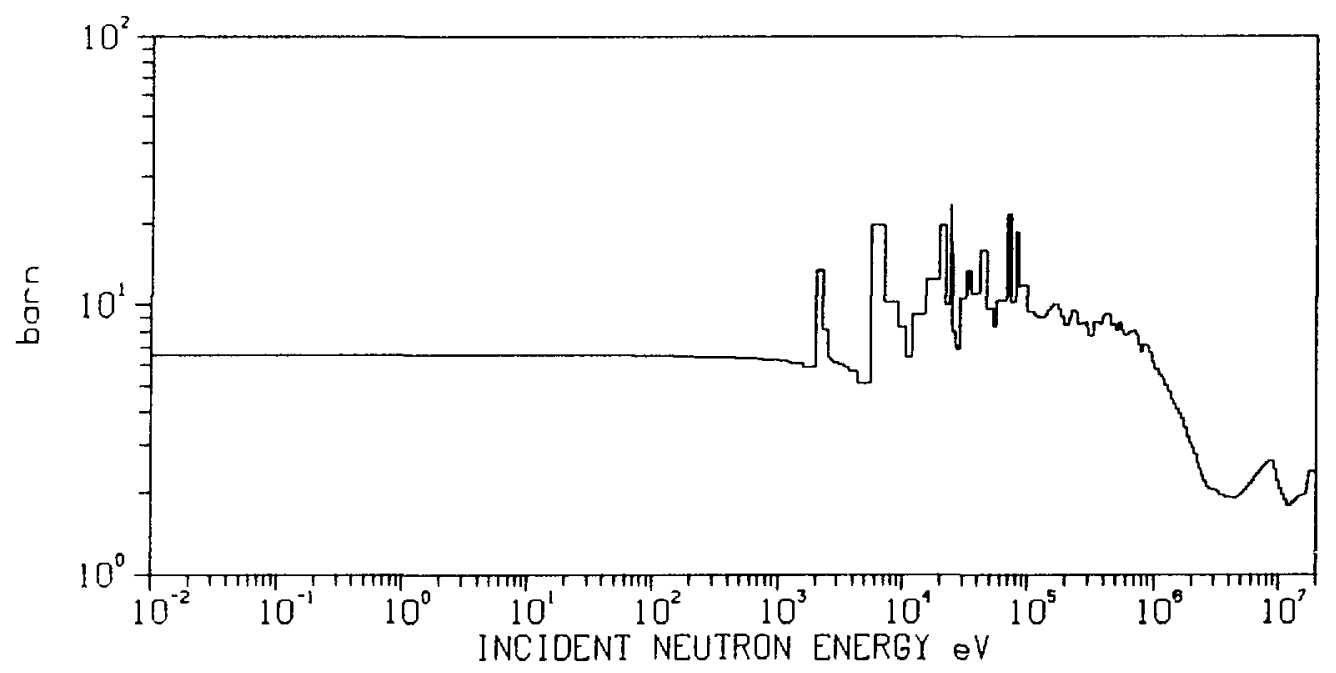

ZR-94 TOTAL INELASTIC CROSS SECTION

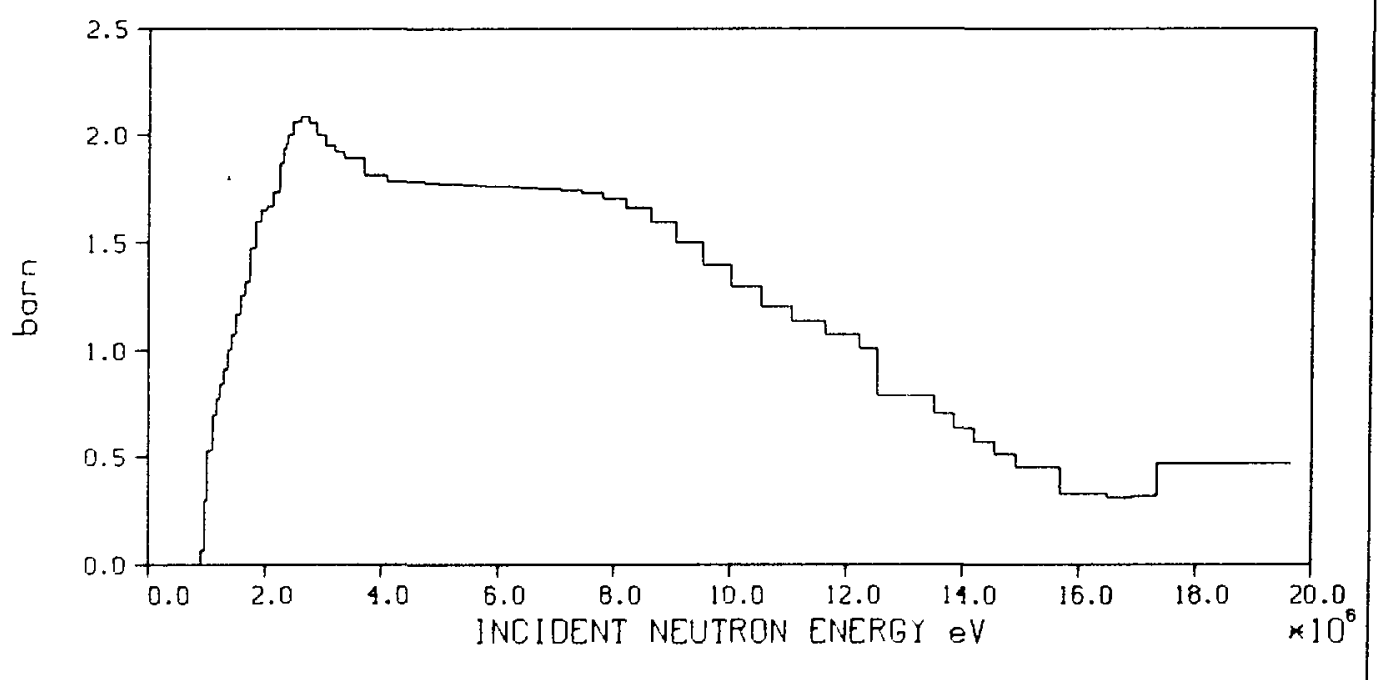


ZR-94 RADIATIVE CAPTURE CROSS SECTION

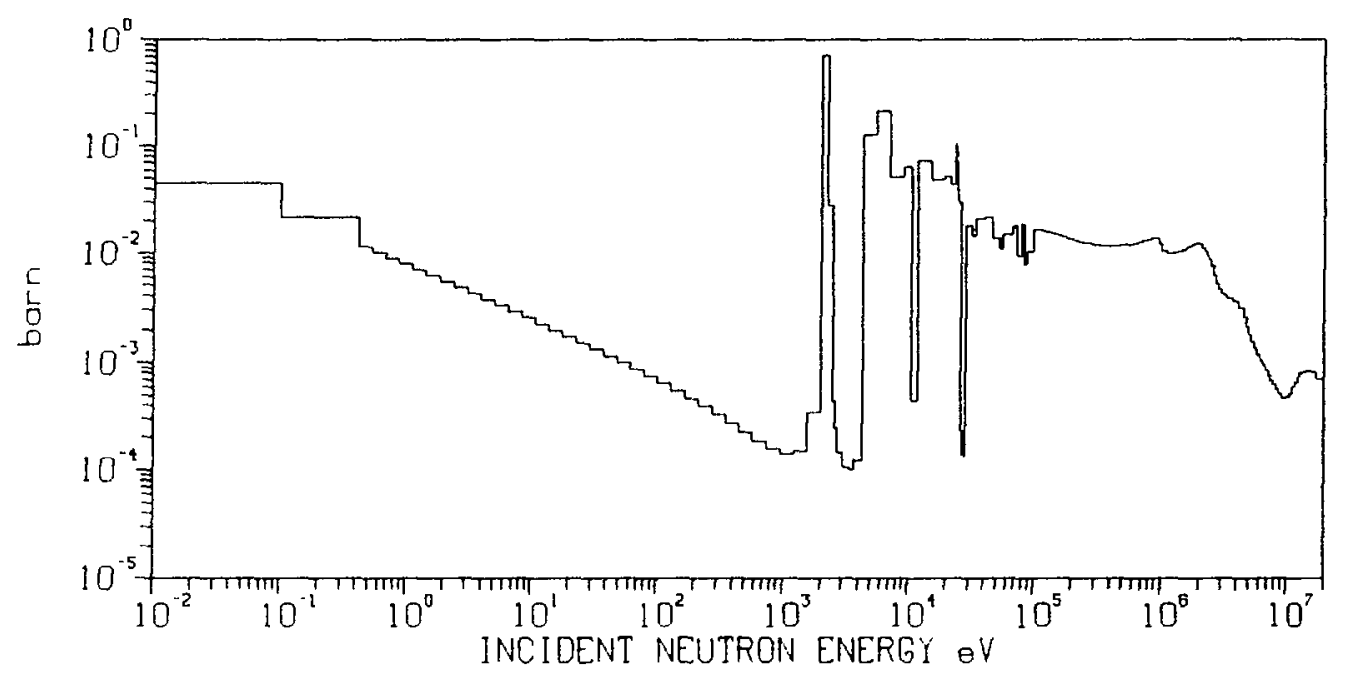

ZR-94 TOTAL $(n, 2 n)$ REACTION CROSS SECTION

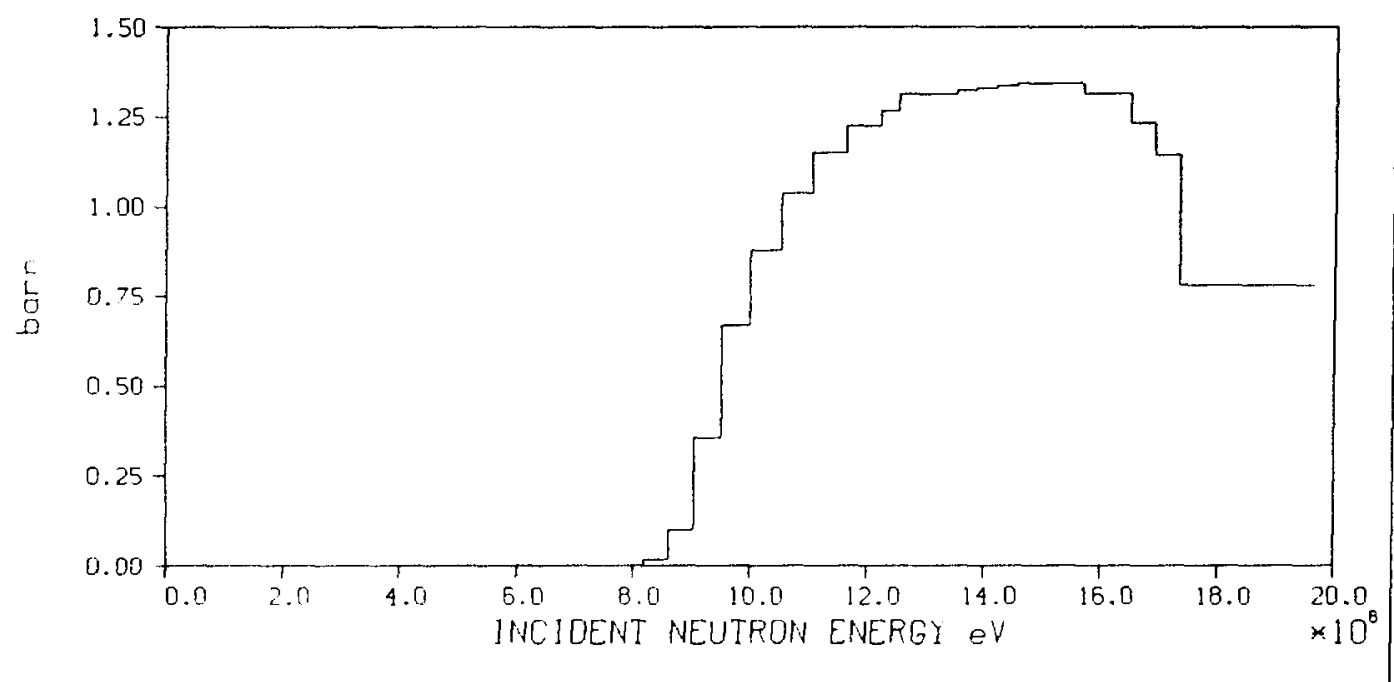




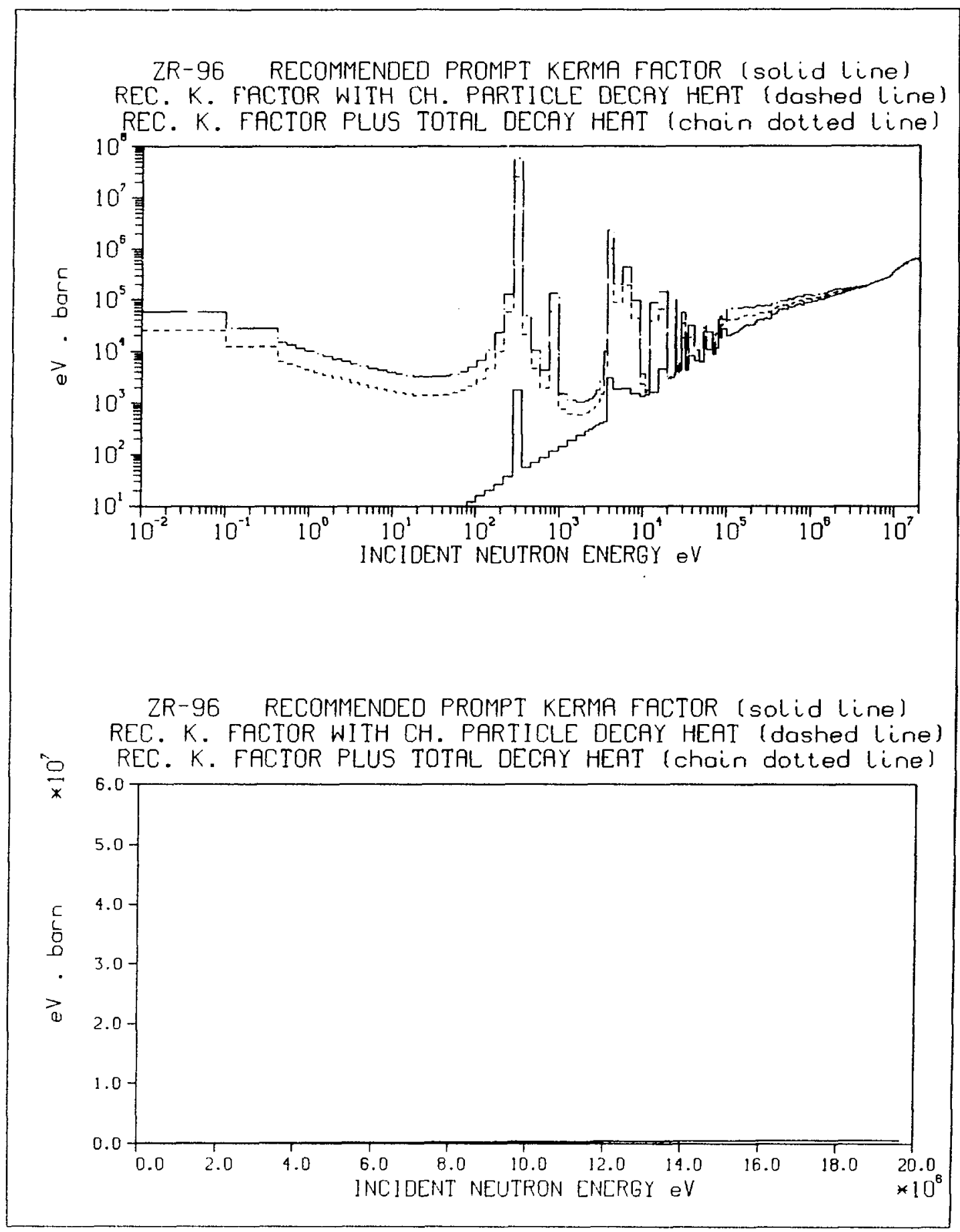




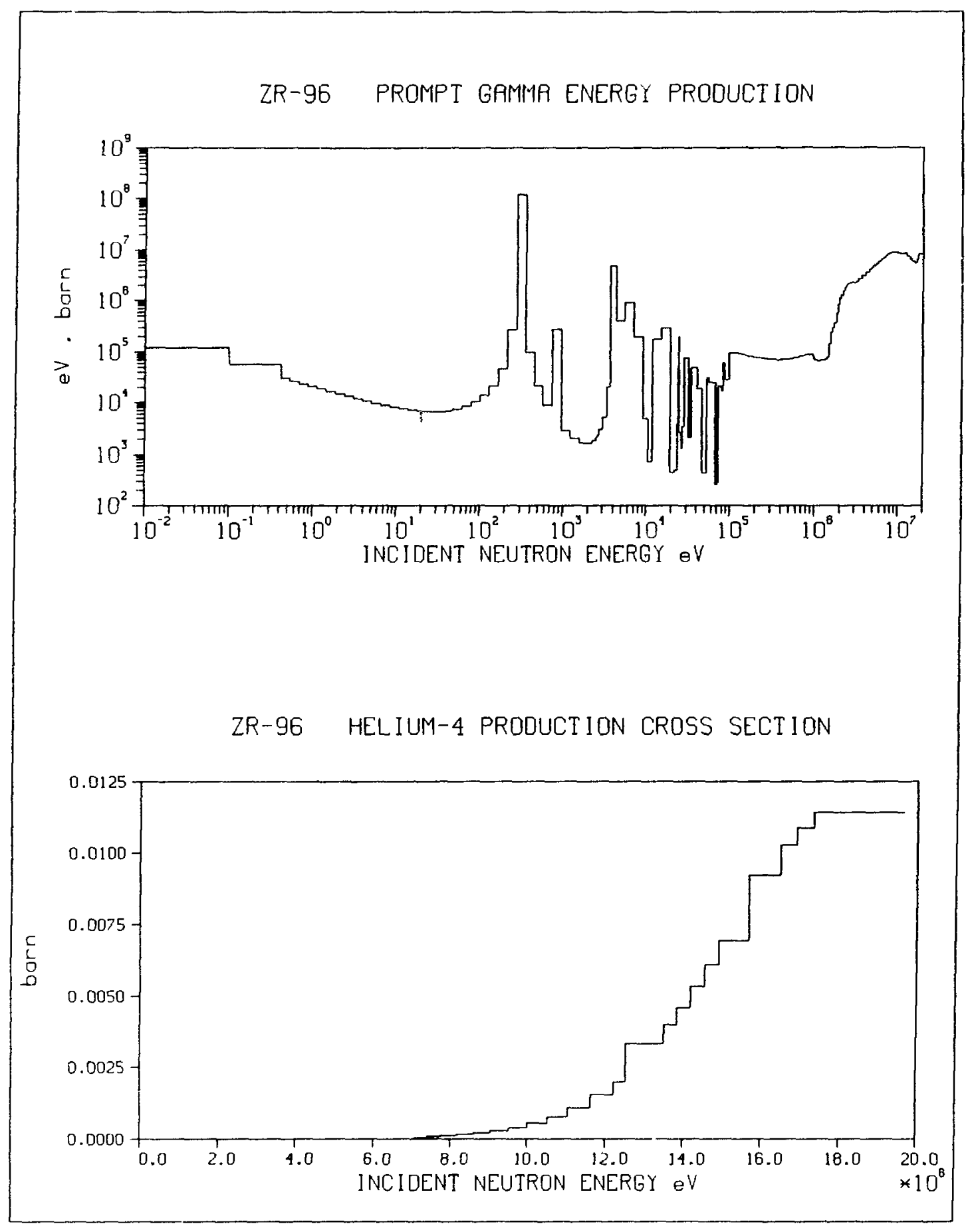




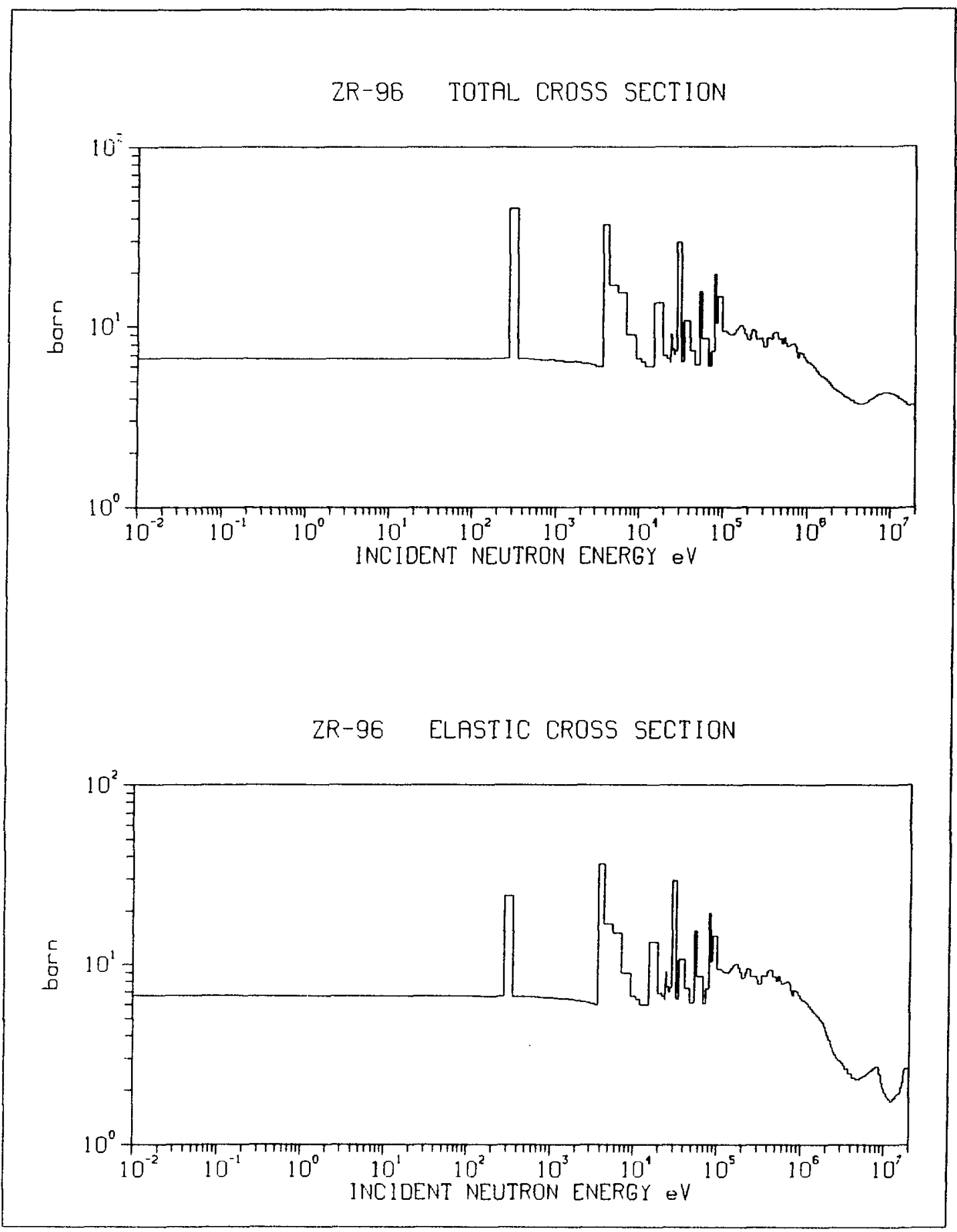




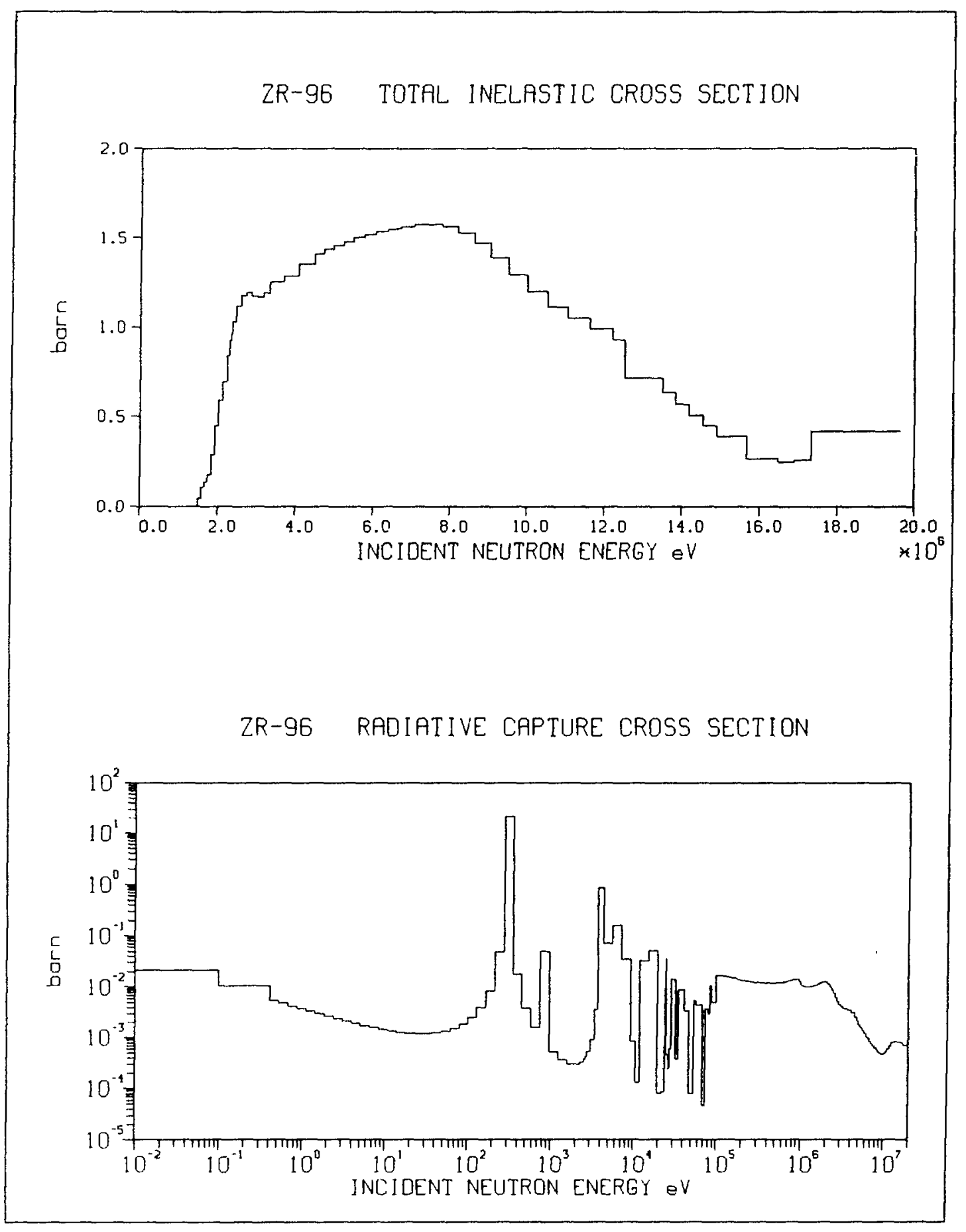




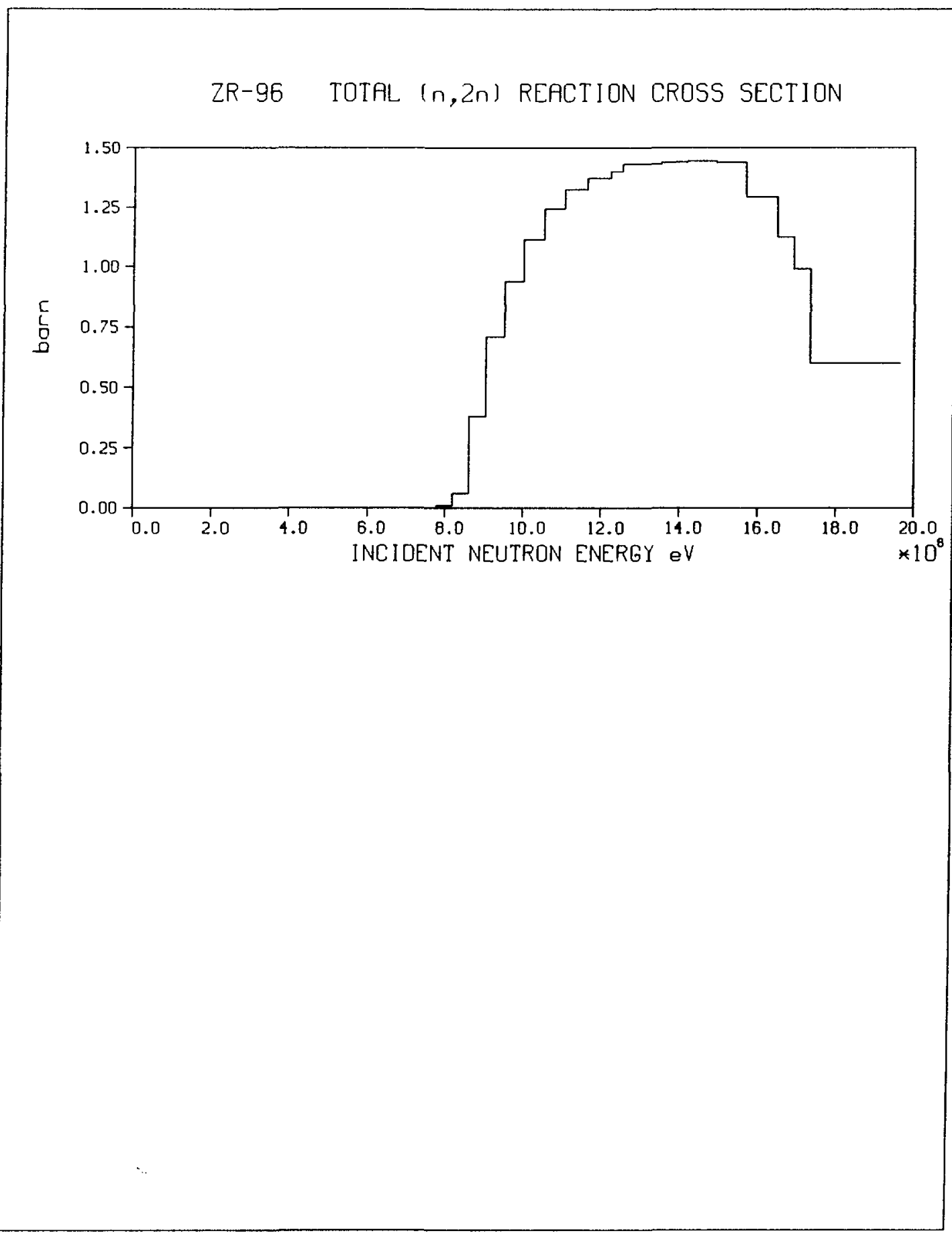




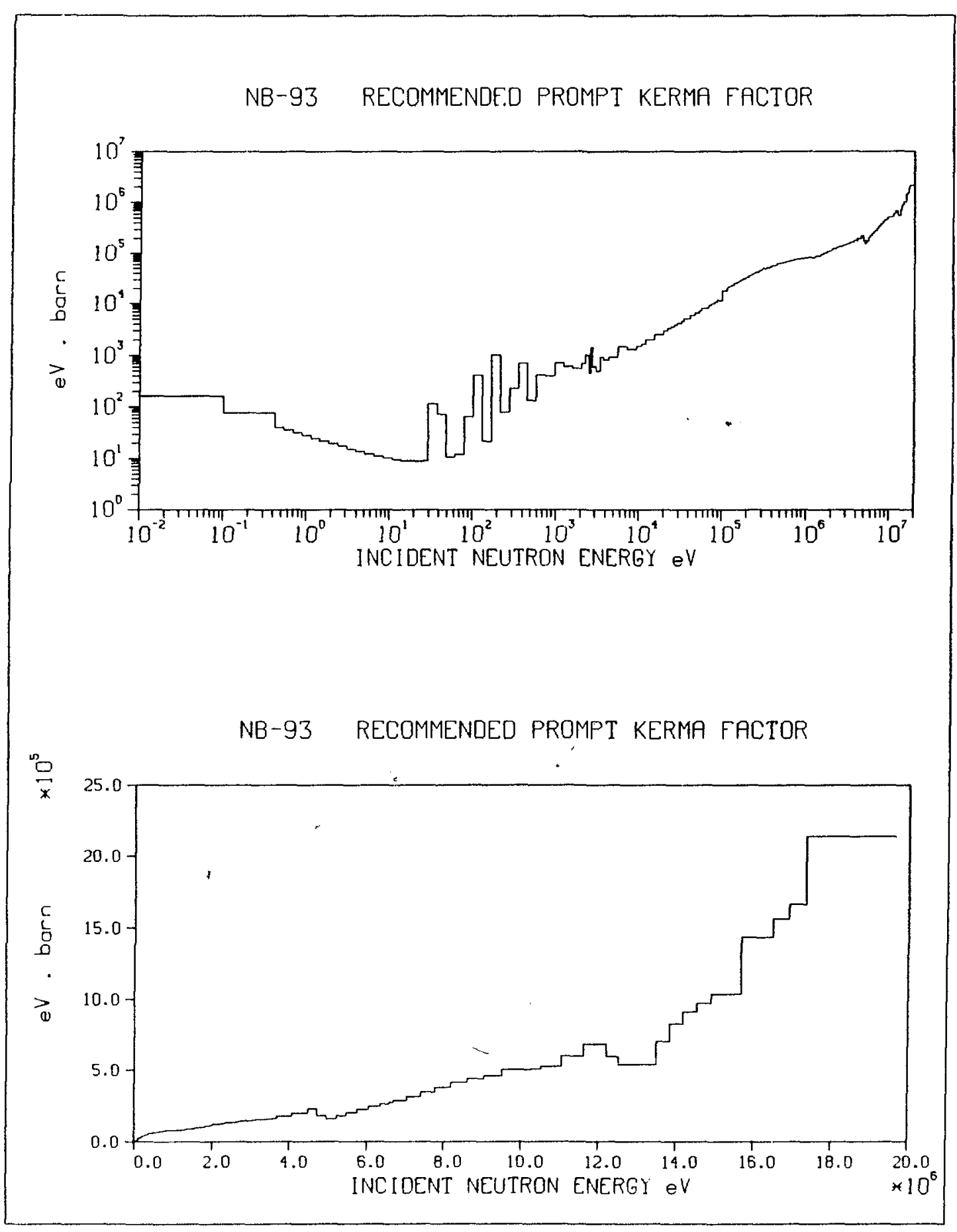




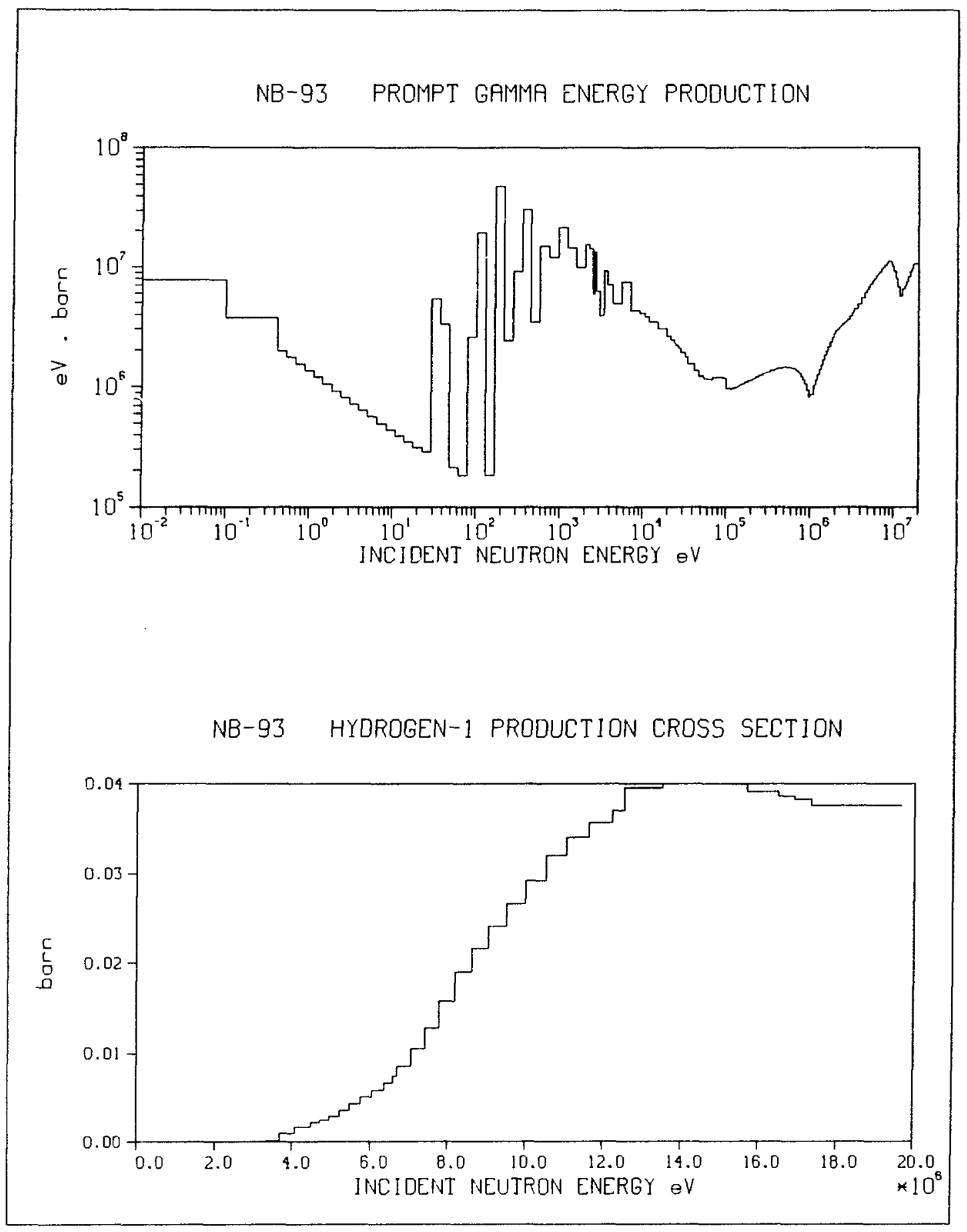




\section{NB-93 PROMPT GAIMHA EIERG PRODUCTION}

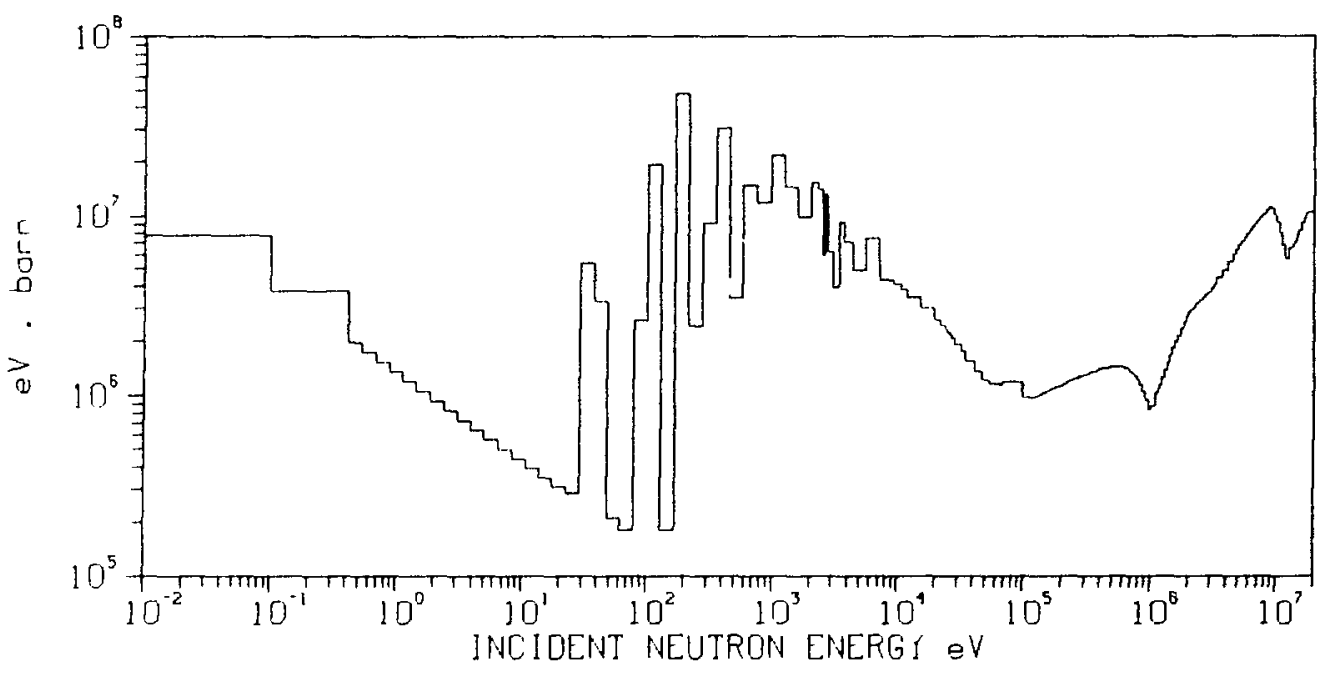

1H-93 HYOROGEN-1 PRODUCTION CROSS SECTION

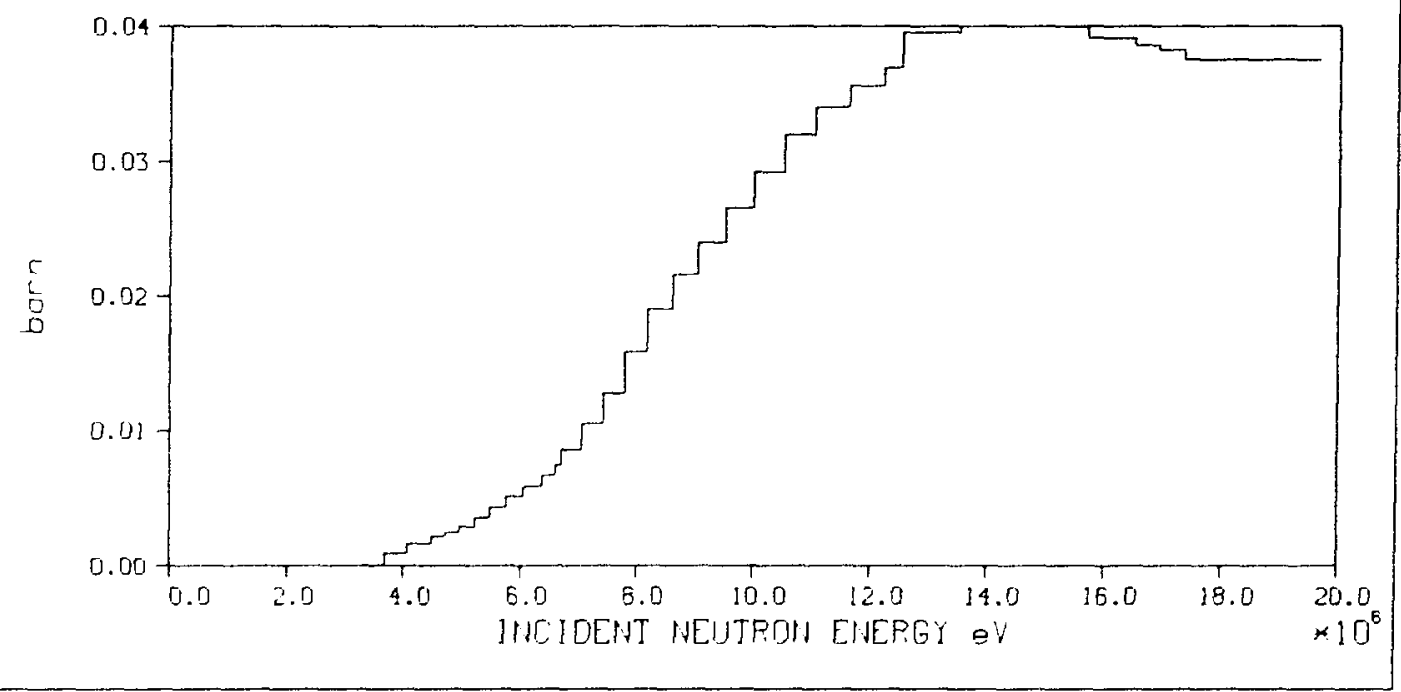




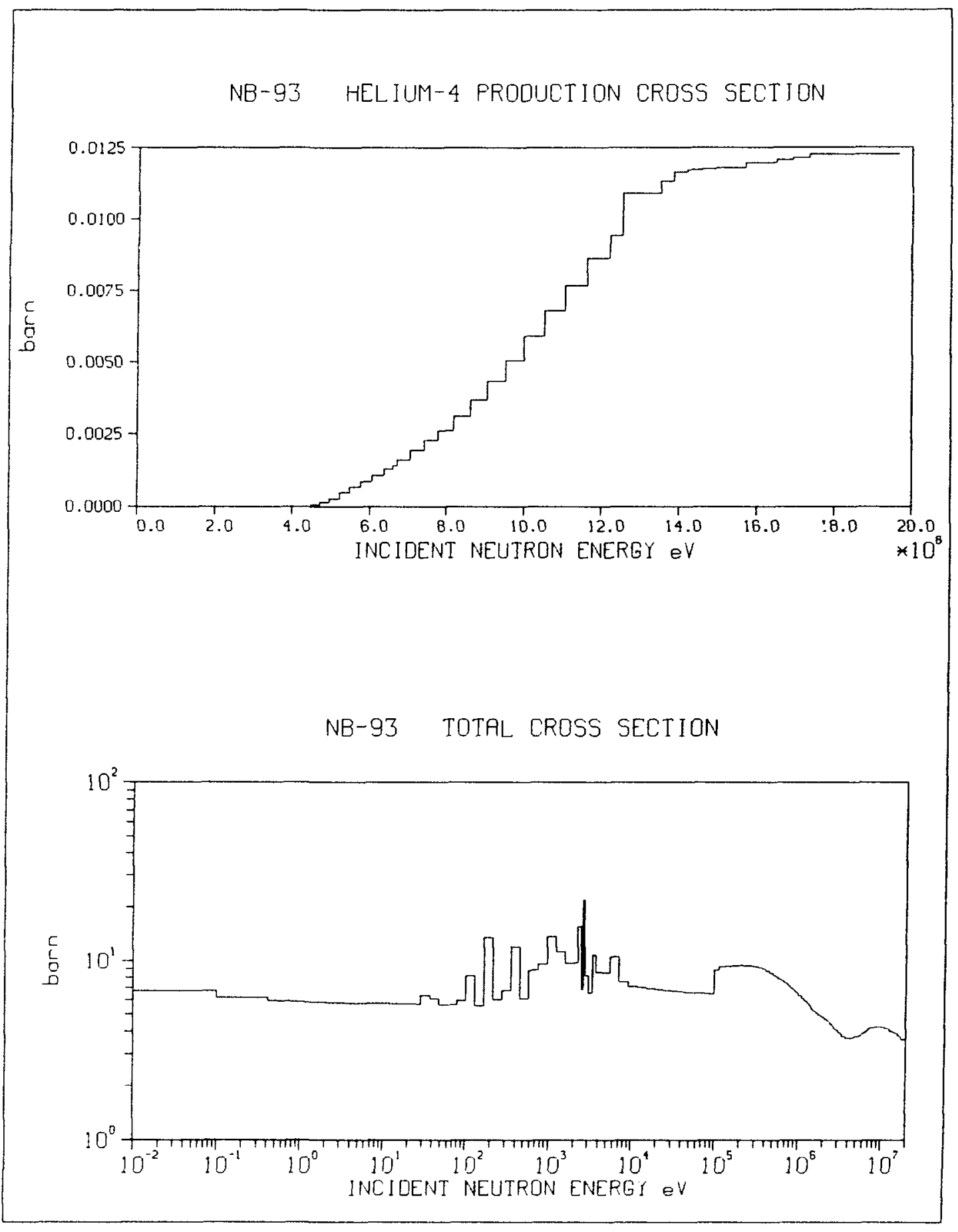




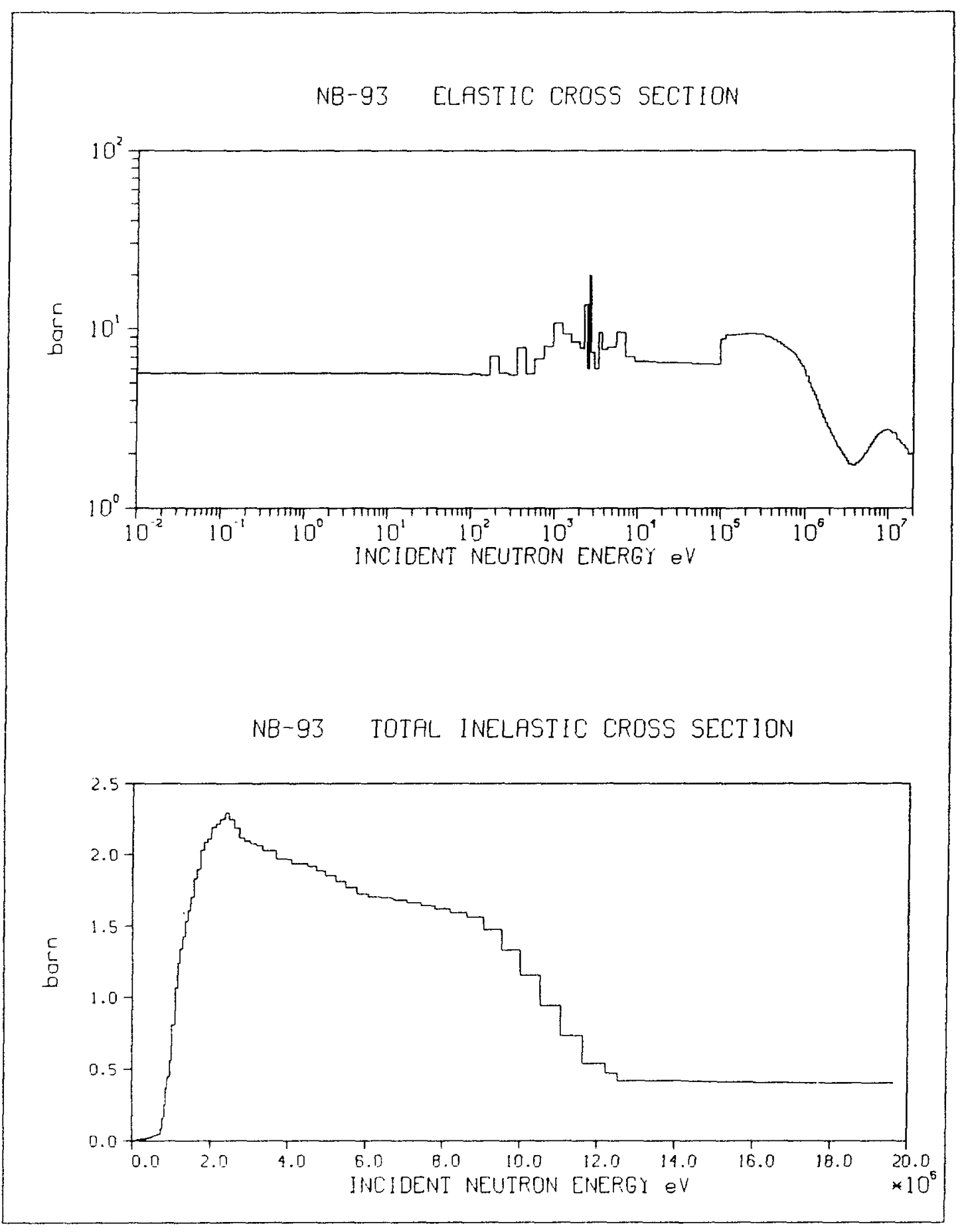




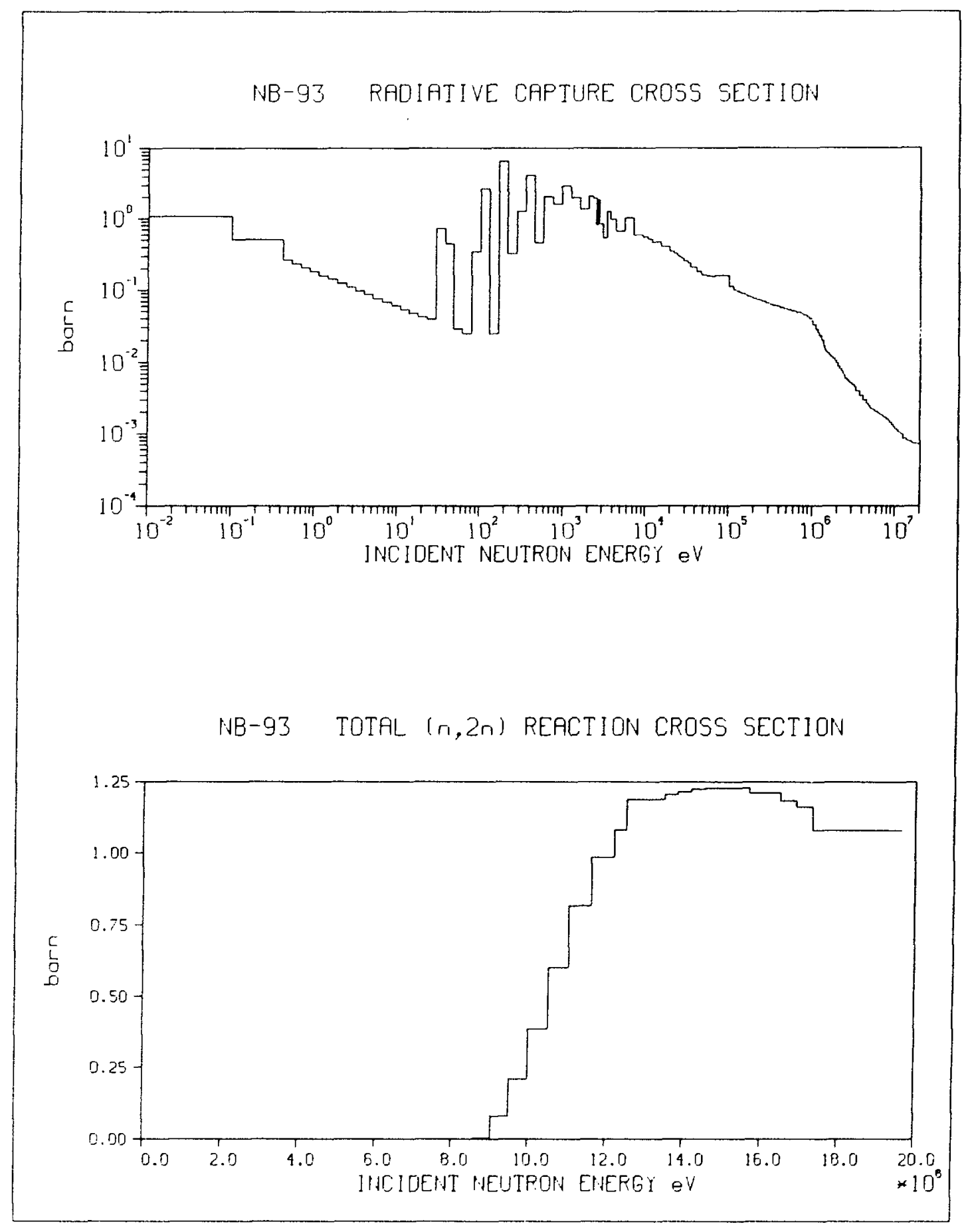


NB-93 TOTAL $(n, 3 n)$ CROSS SECTION

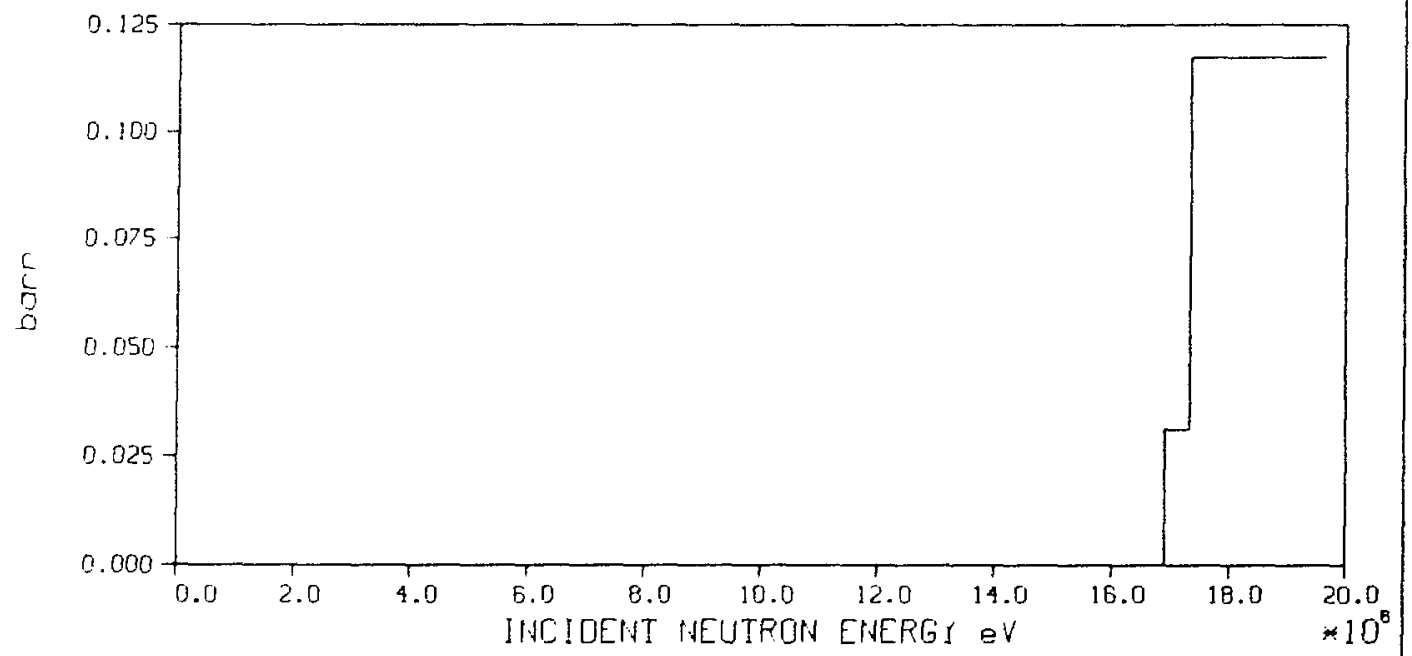


MO-NAT RECOMMENDED PROMPT KERMA FACTOR (solid line) REC. K. FACTOR WITH CH. PARTICLE DECAY HEAT (dashed line)

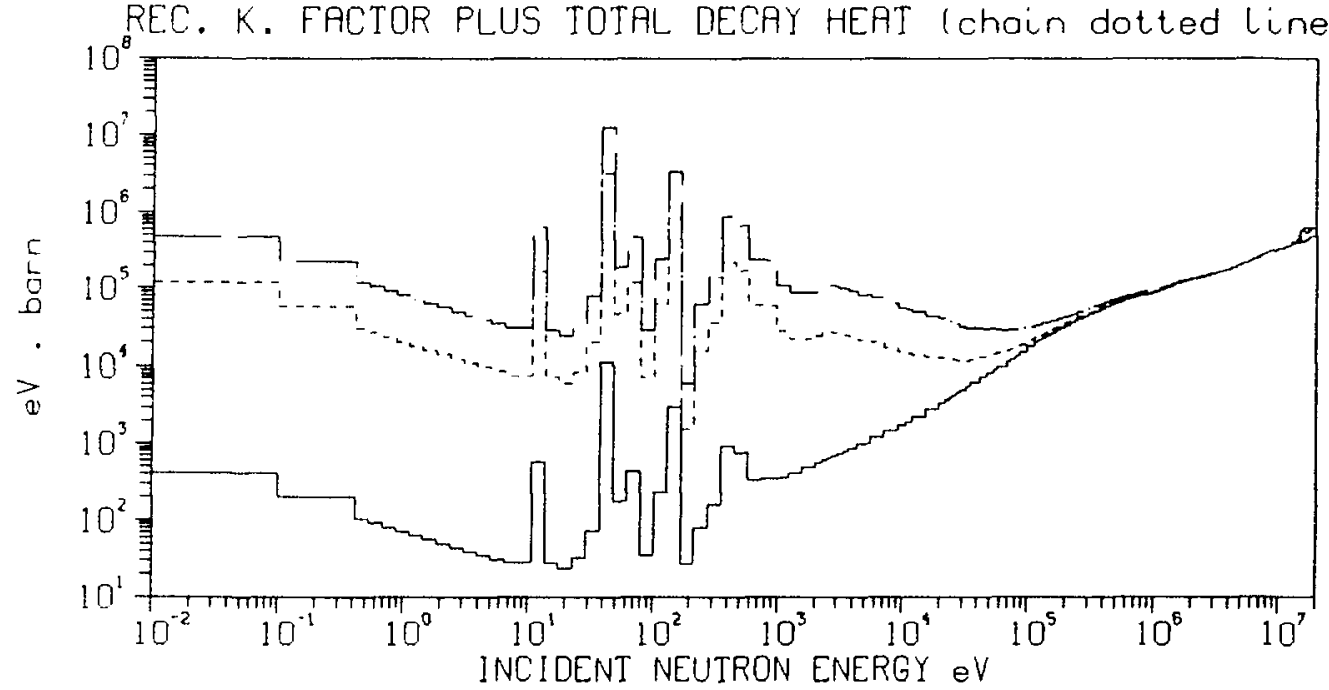

MO-NAT RECOMMENDED PROMPT KERMA FACTOR (solid line) REC. K. FACTOR WITH CH. PARTICLE DECAY HEAT (dashed line) $\because$ REC. K. FACTOR PLUS TOTAL. DECAY HEAT (chain dotted line)

$\vec{x}$

*

*

E 5.0

12.5

$10.0-$

7.5

a

$2.5-$

INIIDENT IUEUTRON ENERGY 


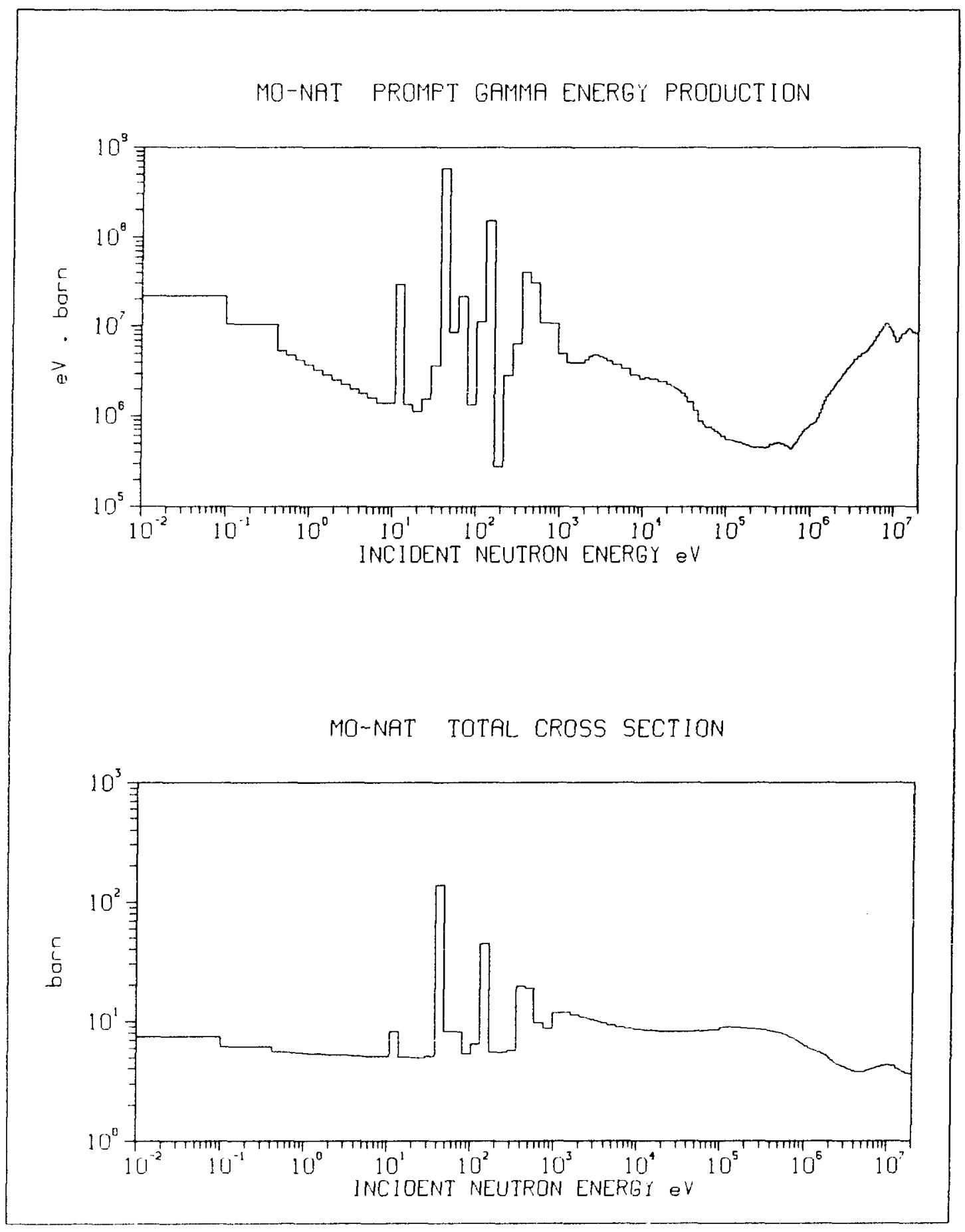




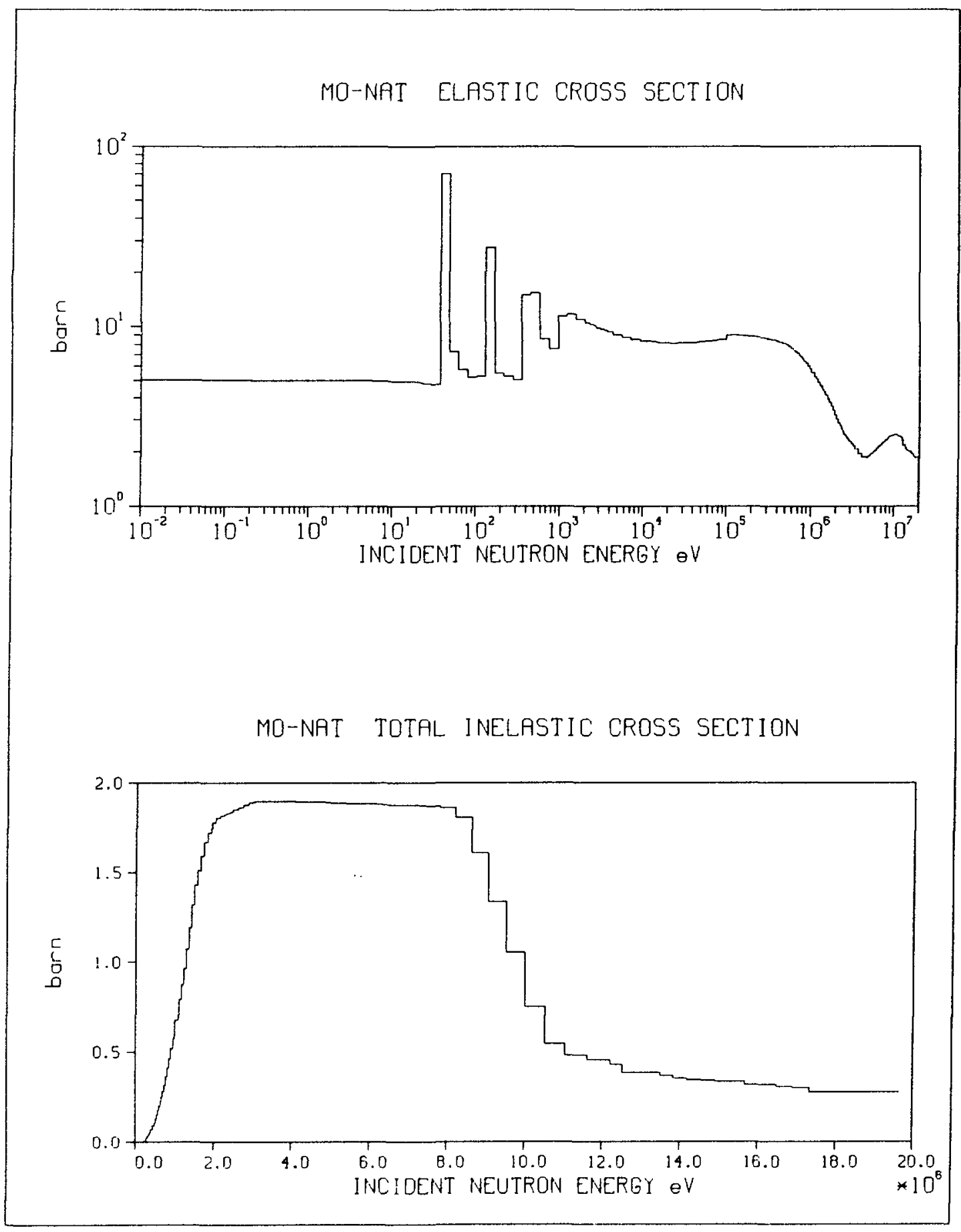


MO-NAT RADIATIVE CAPTURE CROSS SECTION

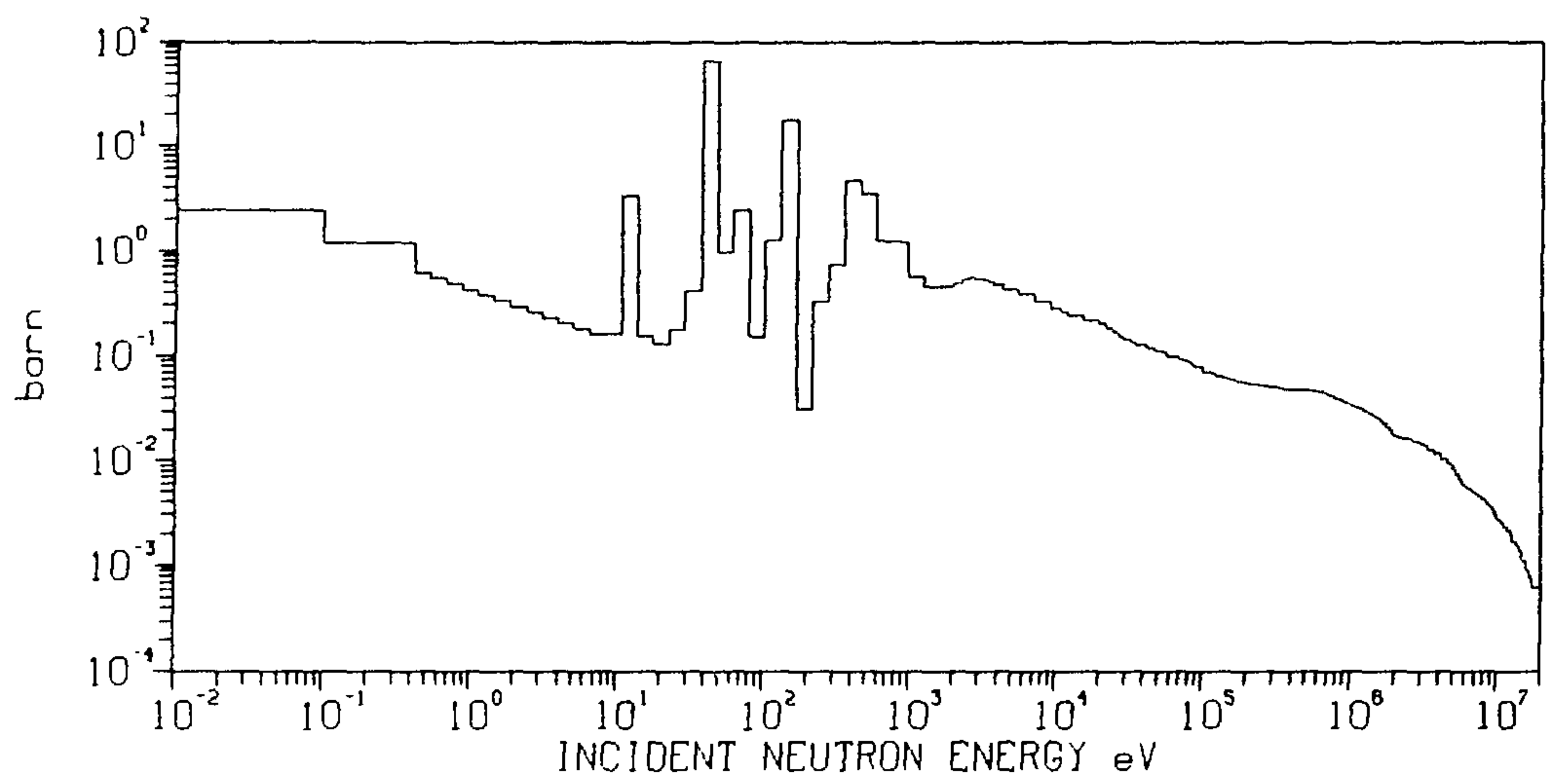

MO-NAT TOTAL $(n, 2 n)$ REACTION CROSS SECTION

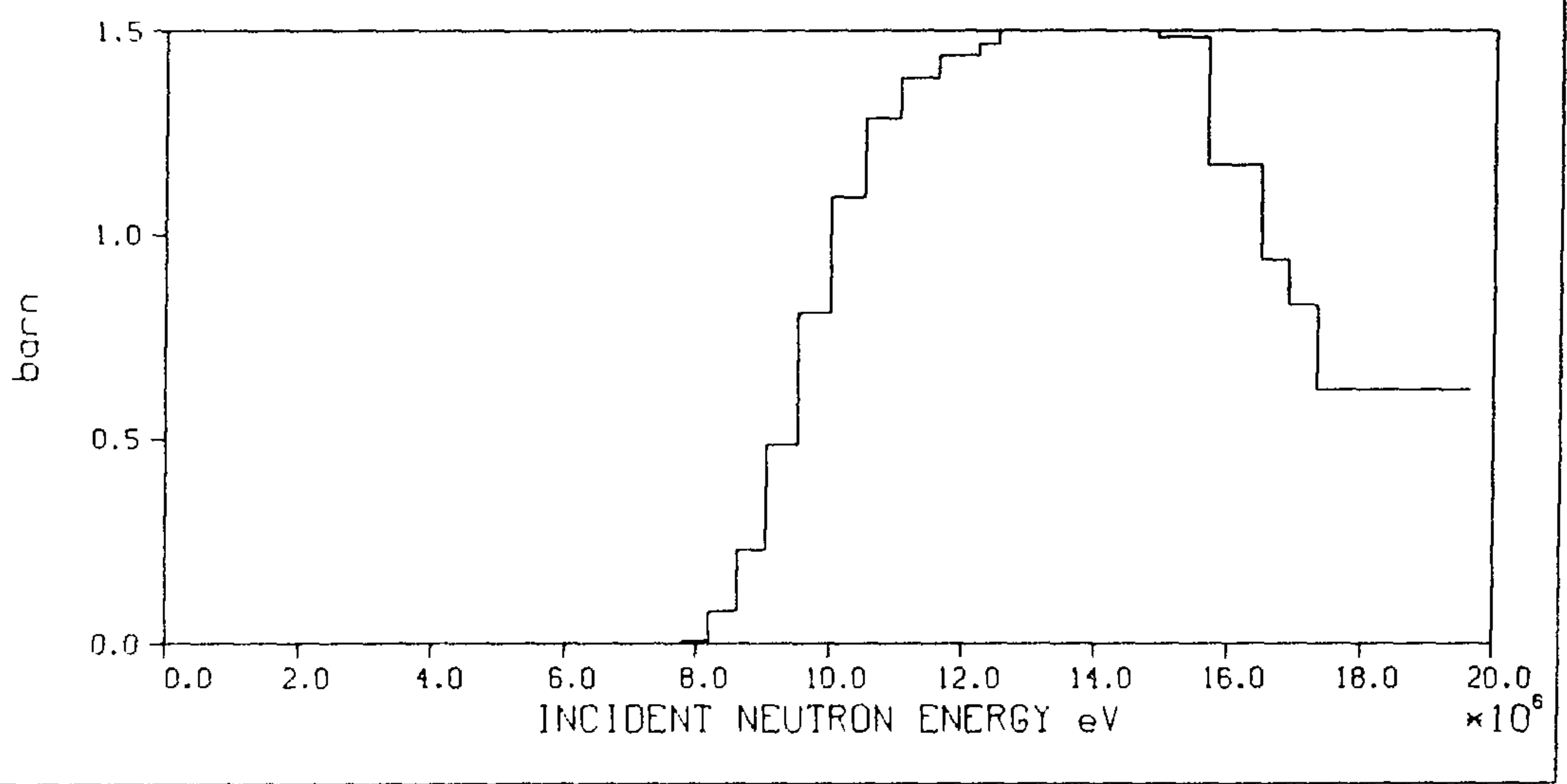


MO-NAT TOTAL $(n, 3 n)$ CROSS SECTION

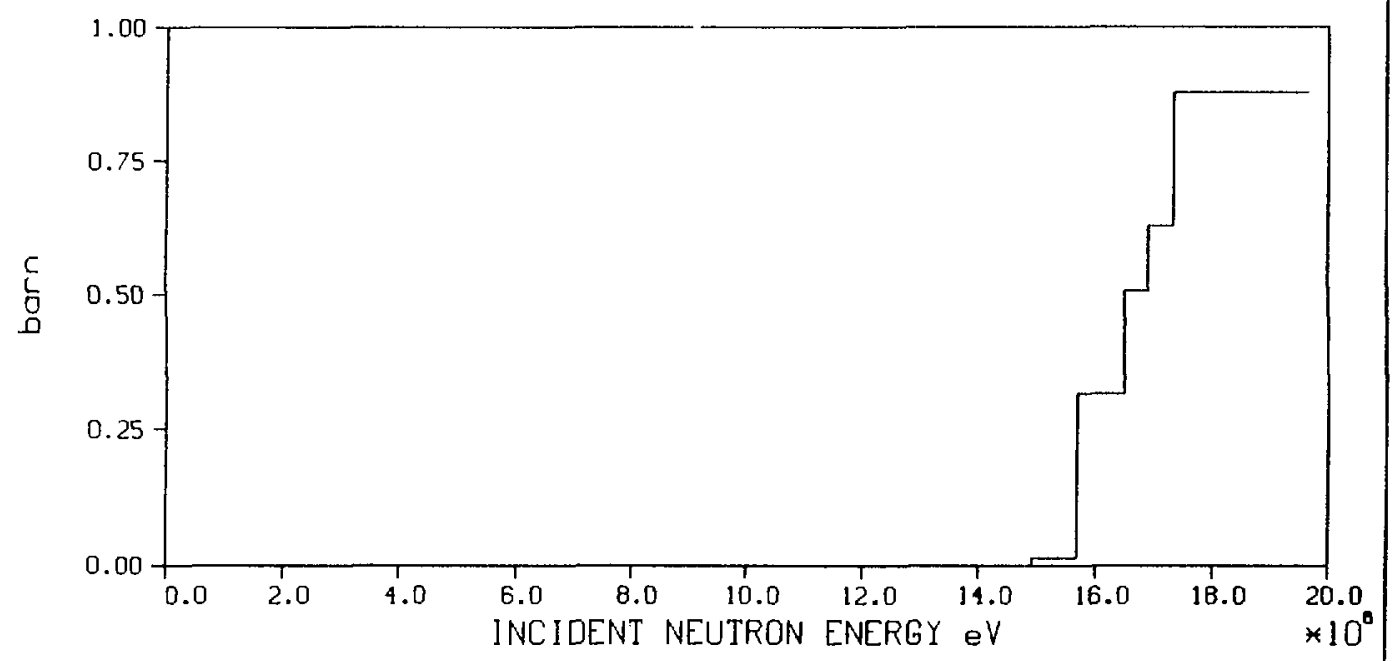




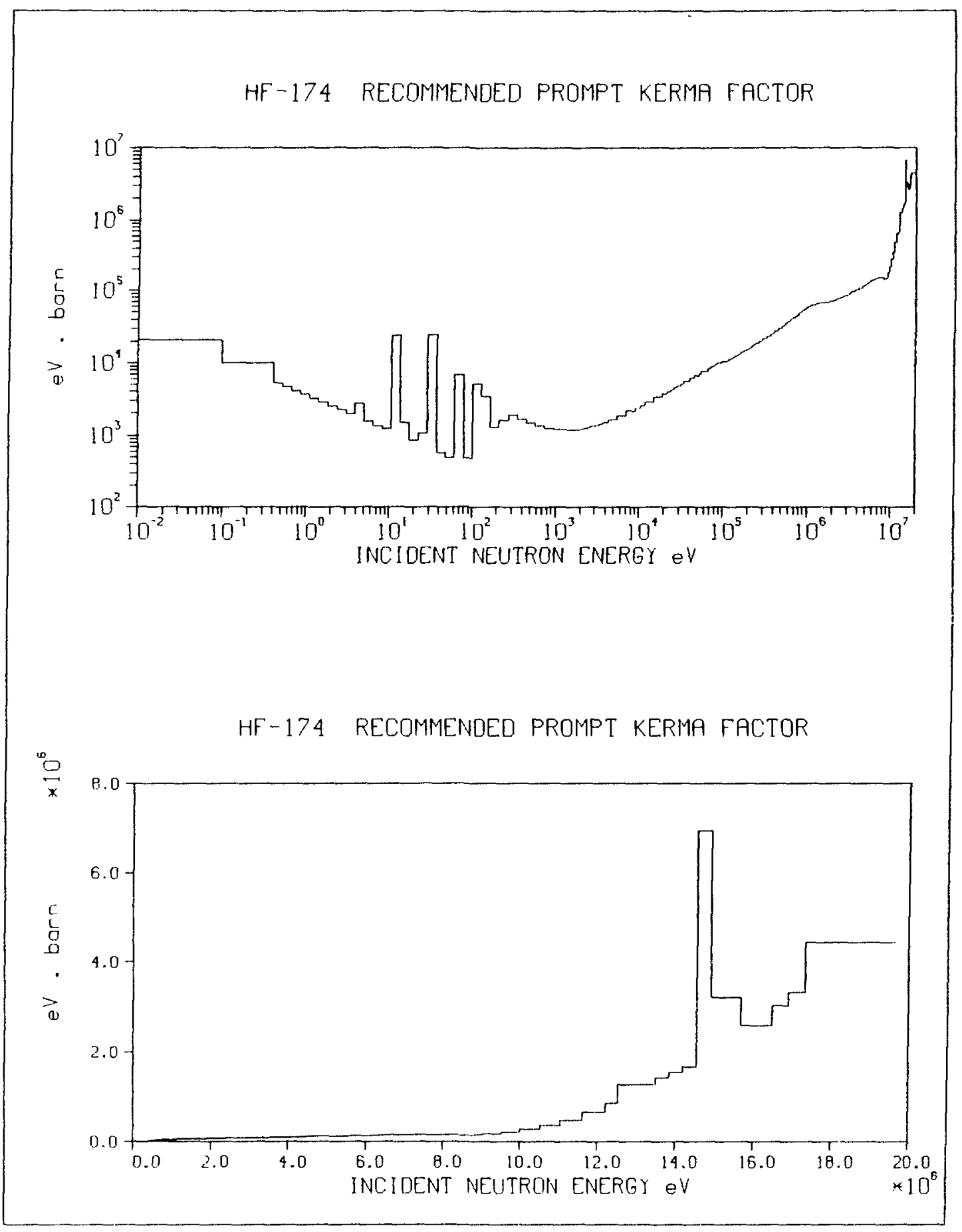




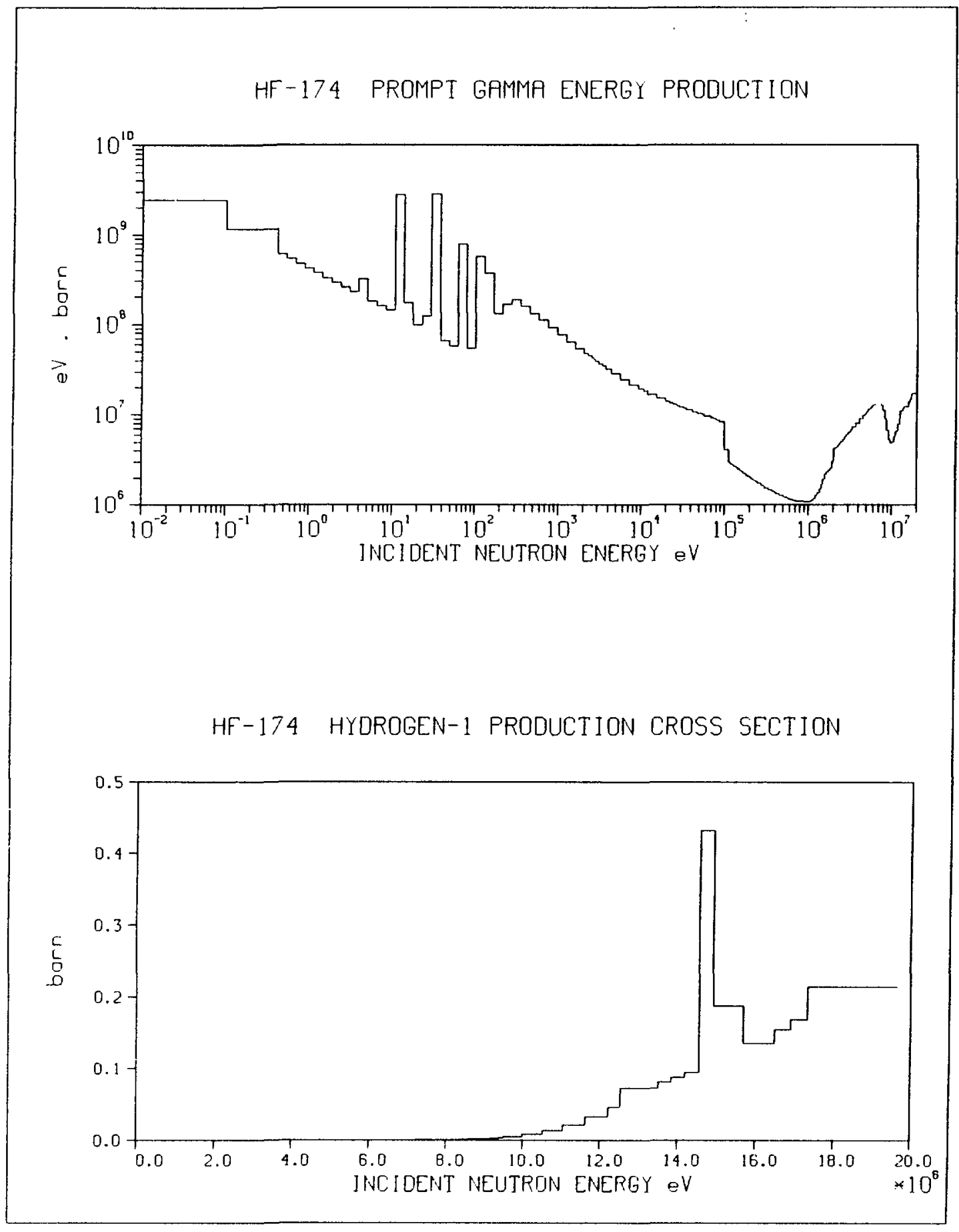




\section{HF-174 TOTAL CROSS SECTION}

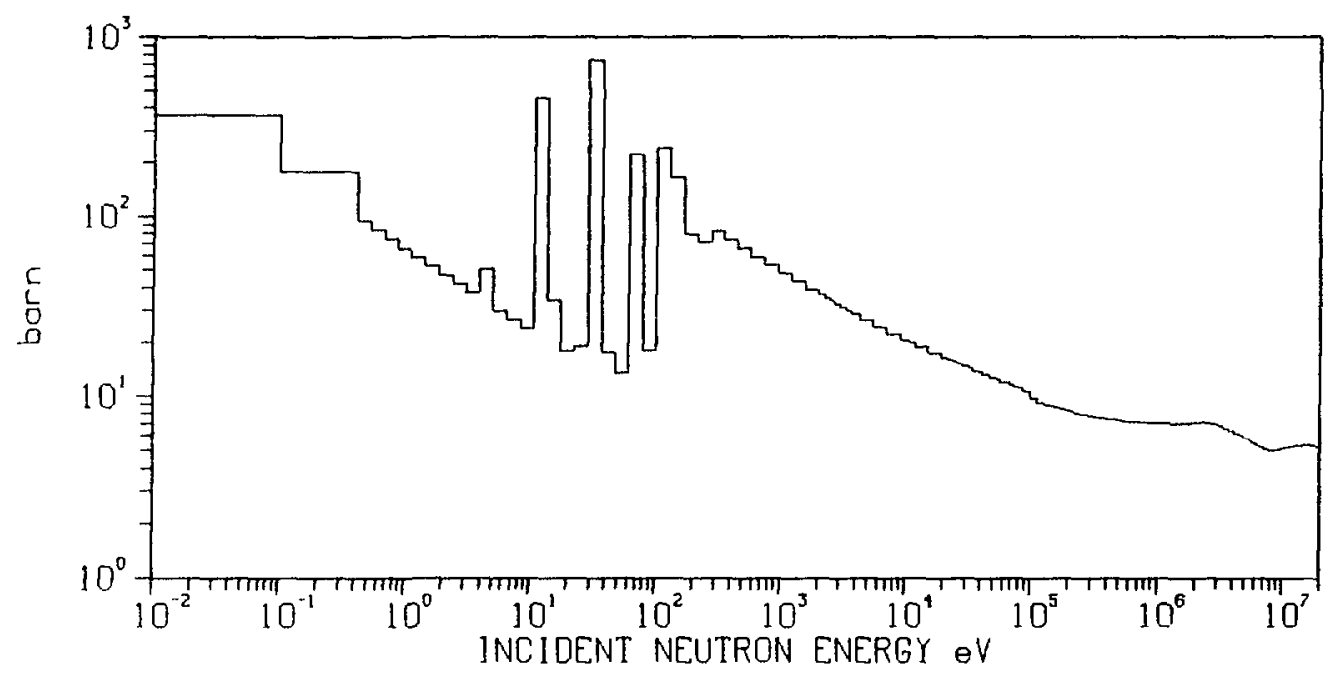

HF-174 ELASTIC CROSS SECTION

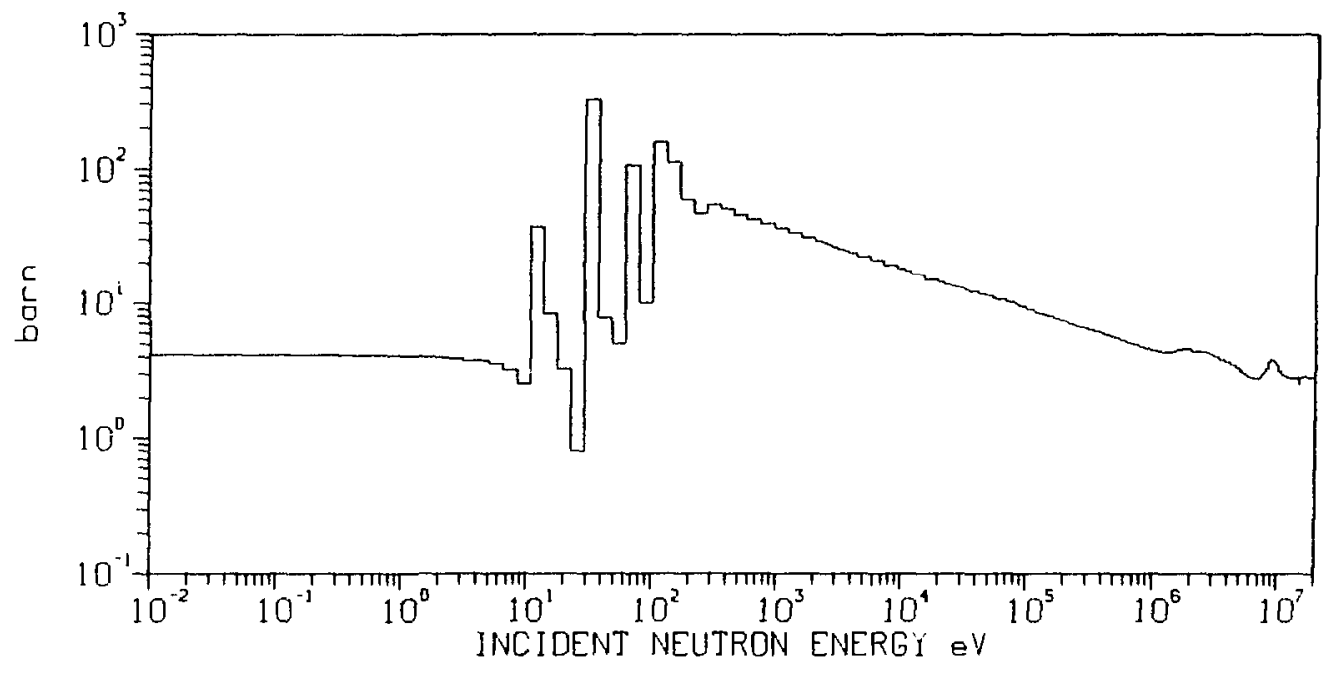




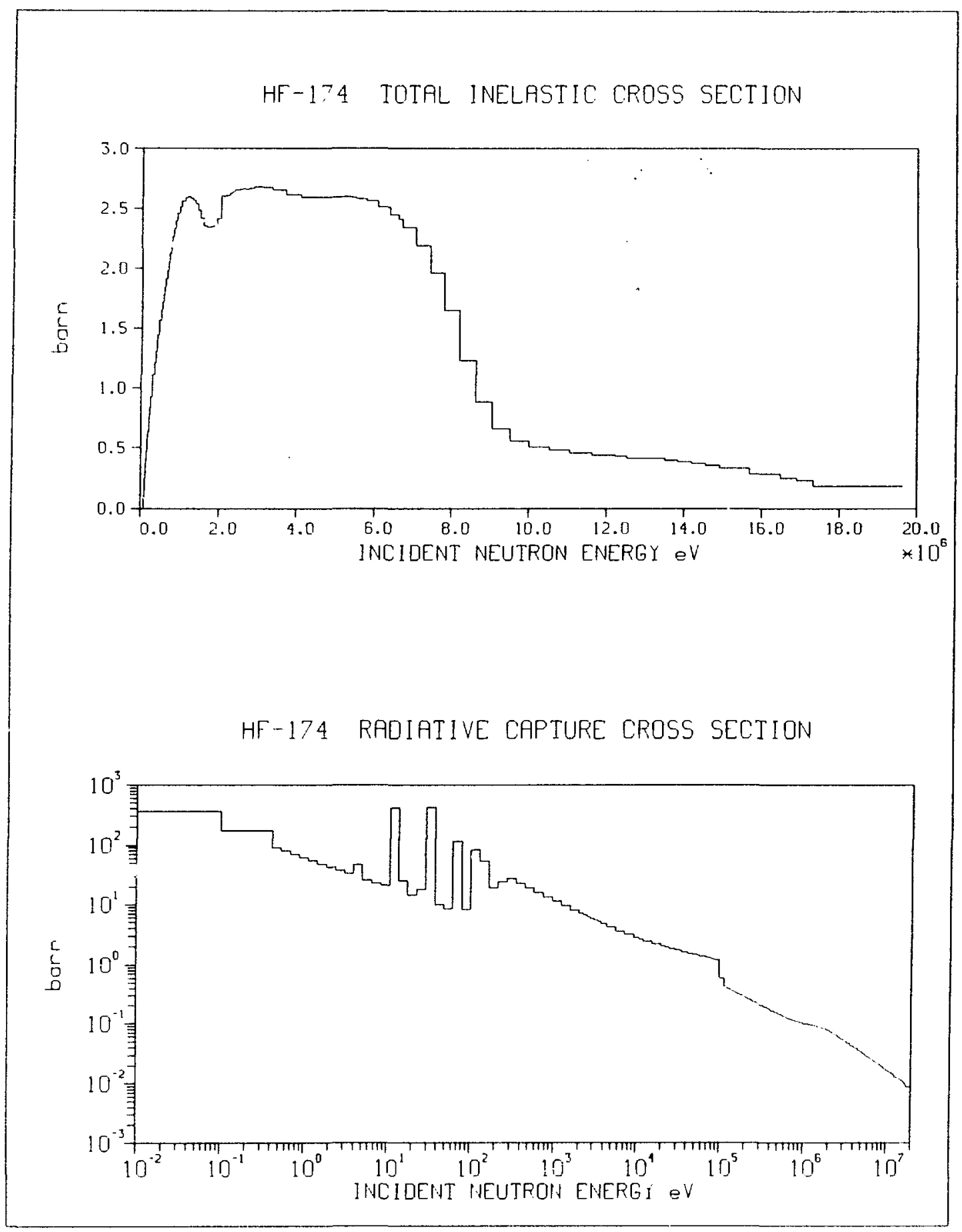




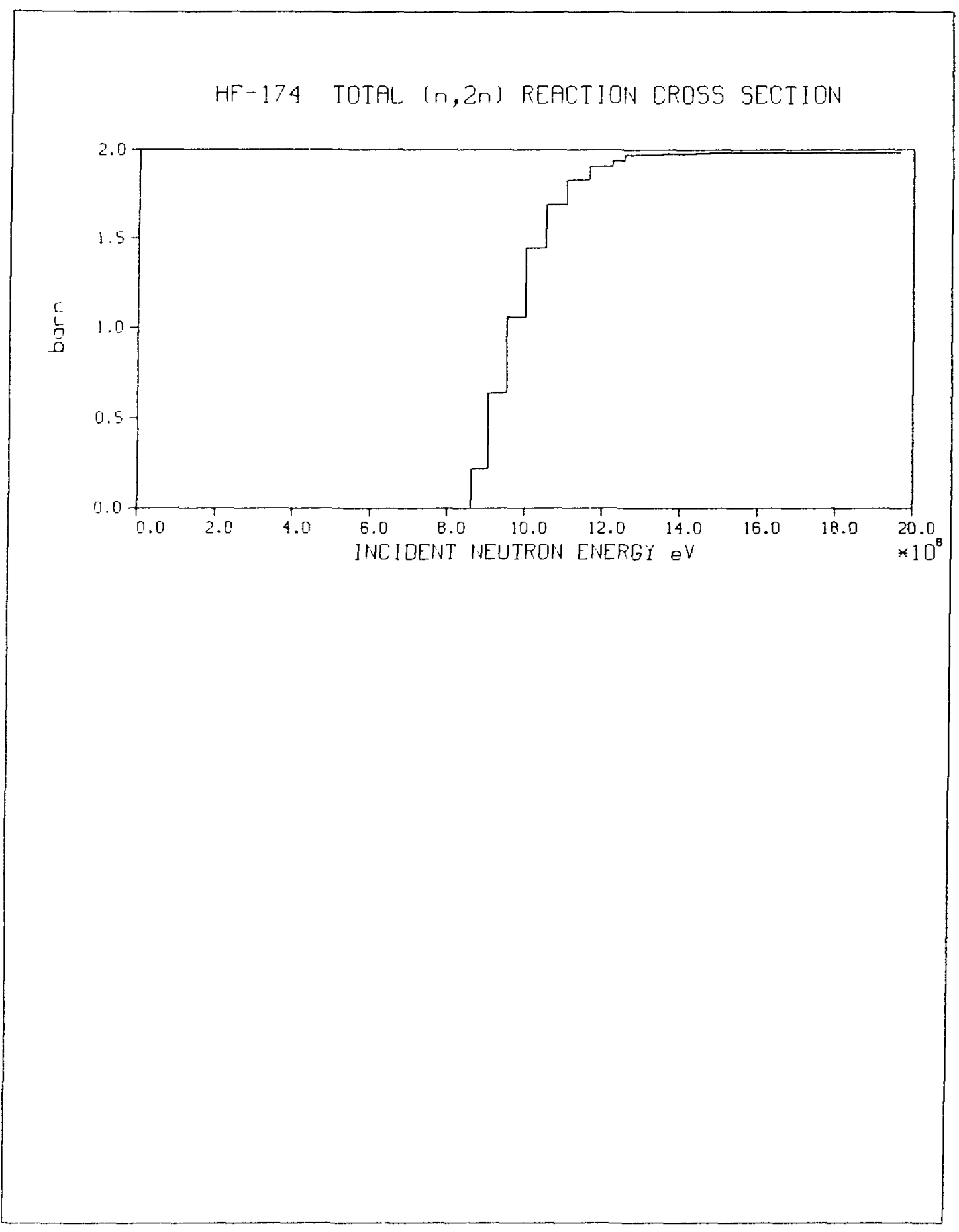




\section{HF-176 RECOMMENDED PROMPT KERMA FACTOR}

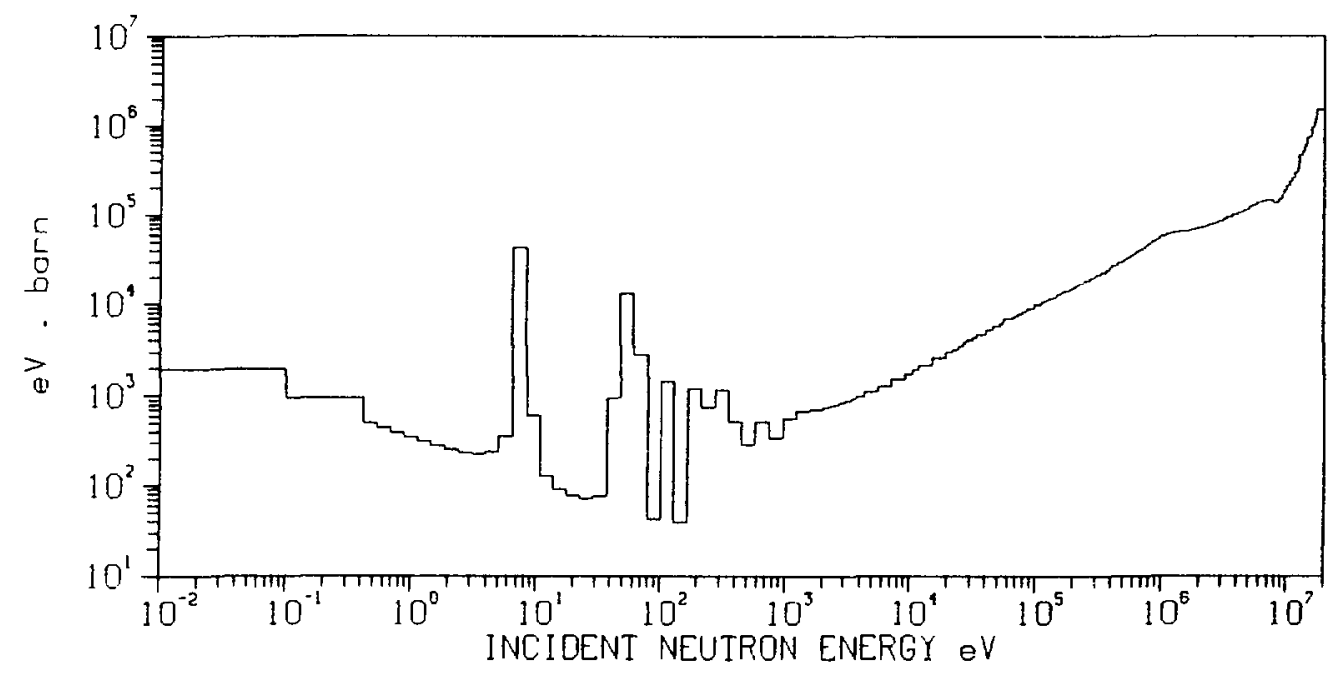

HF-176 RECOMMENDED PROMPT KERMA FACTOR

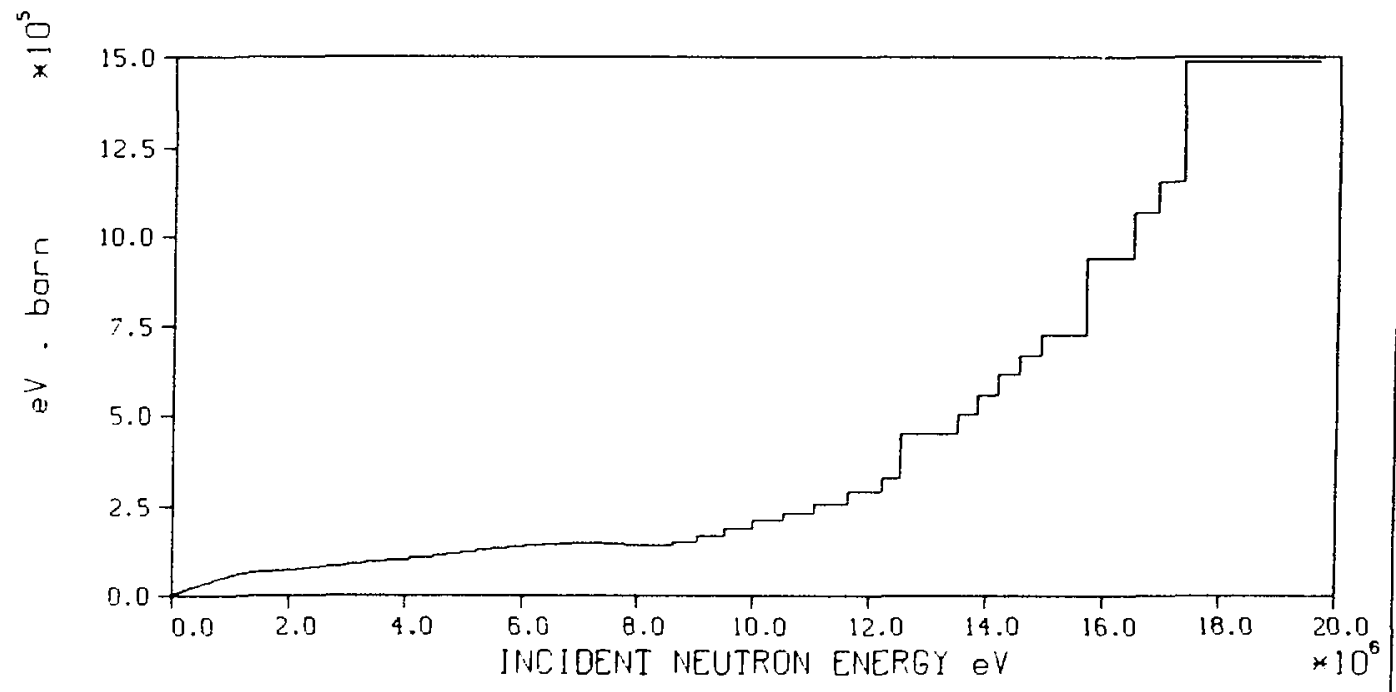




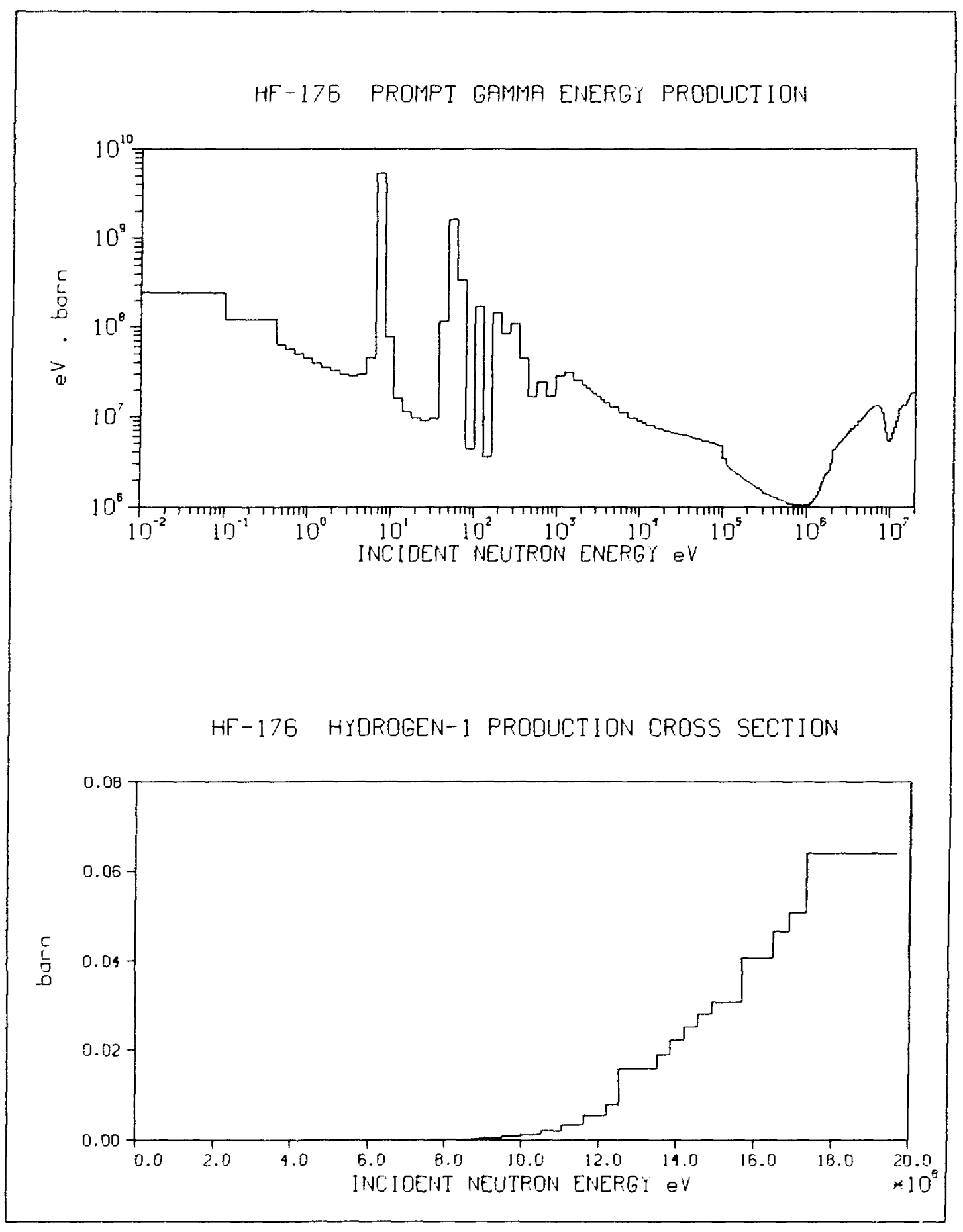




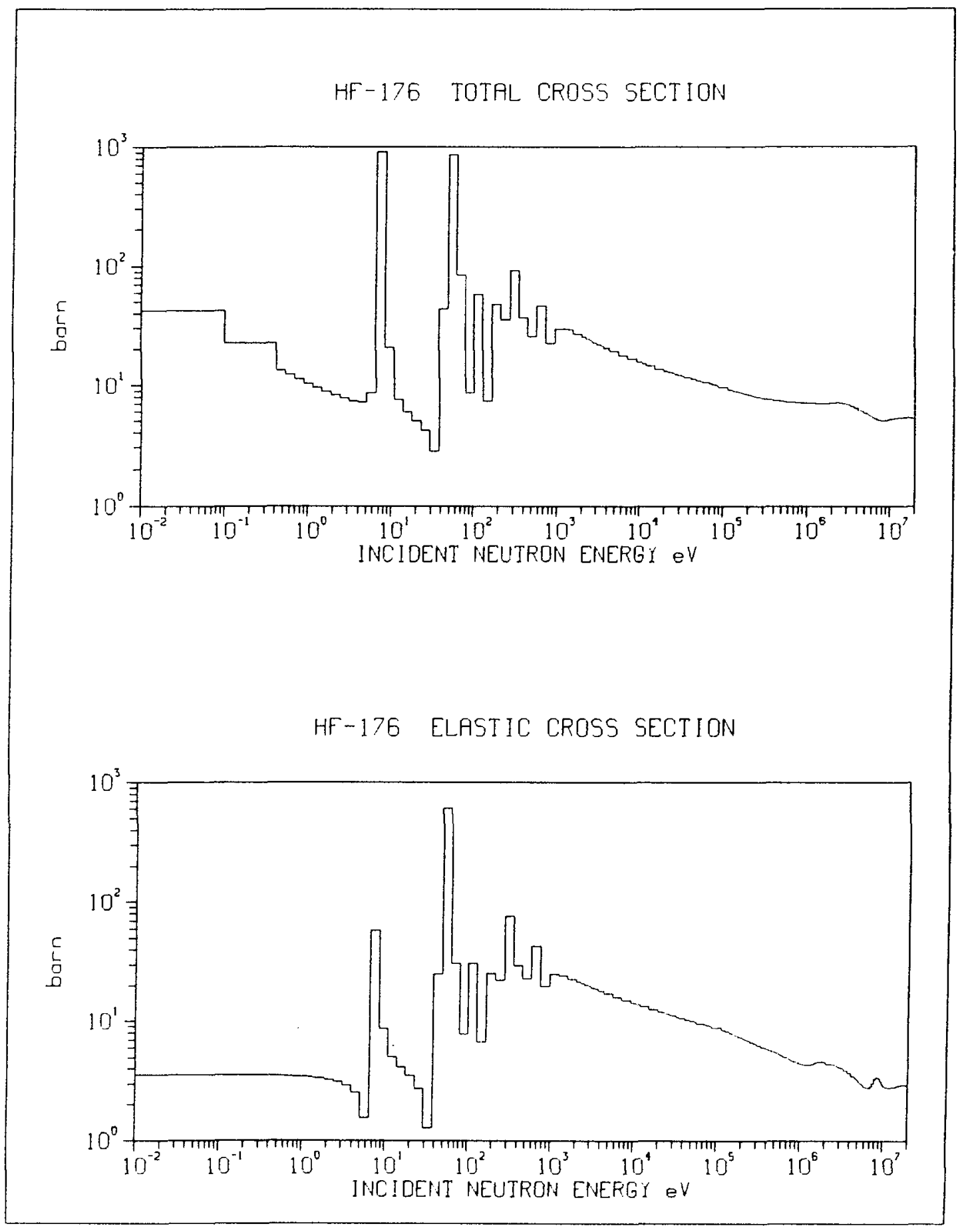




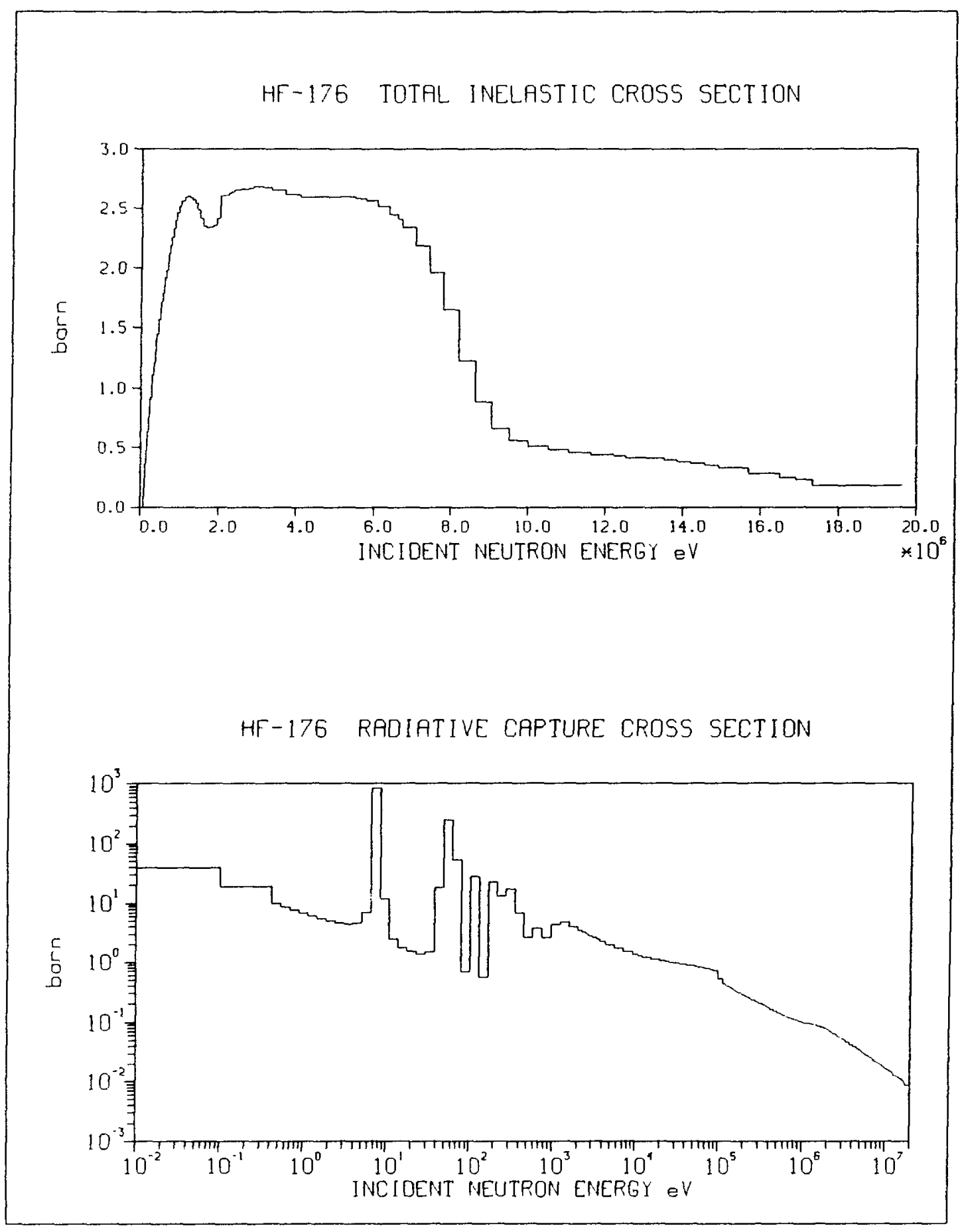




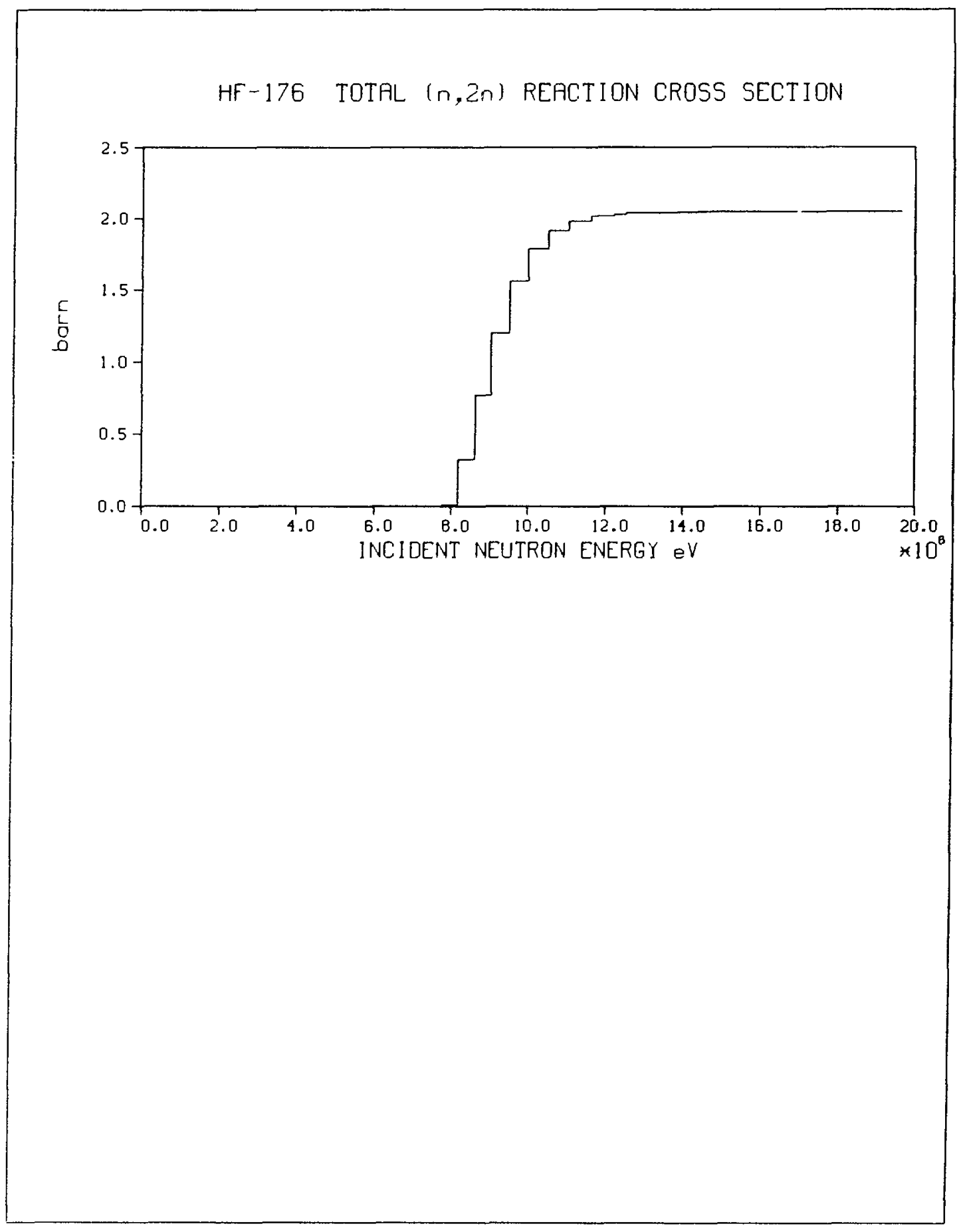




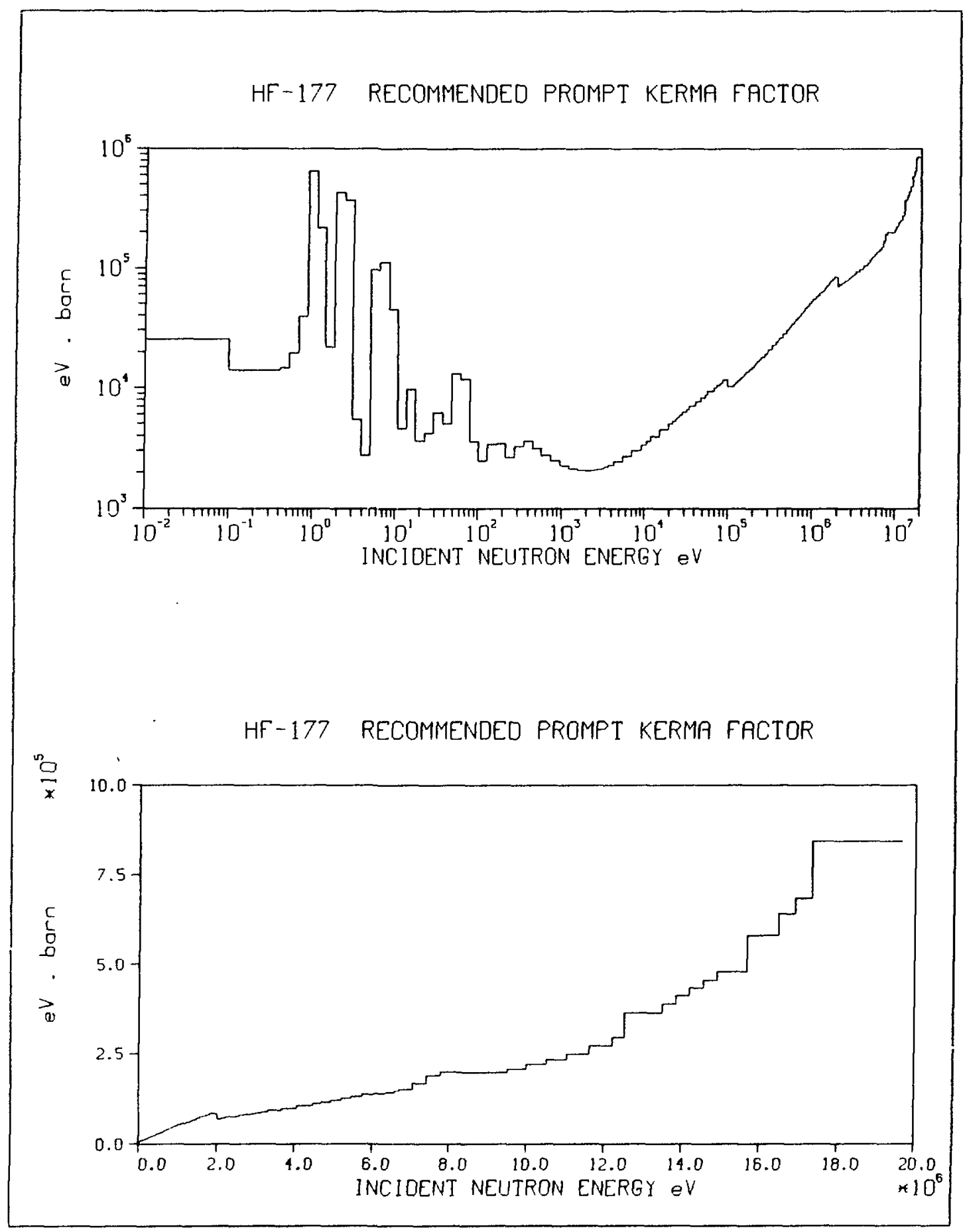


HF-177 PROMPT GAMMA ENERGY PRODUCTION

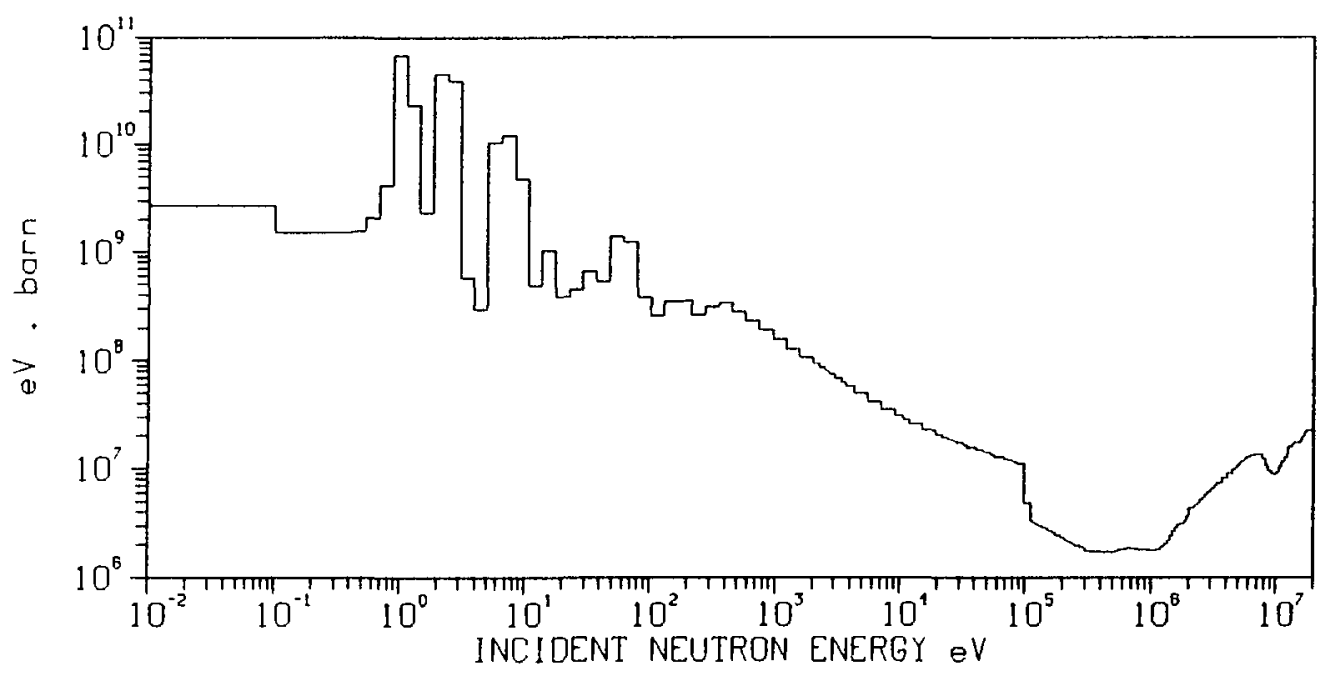

HF-177 HYOROGEN-1 PRODUCTION CROSS SECTION

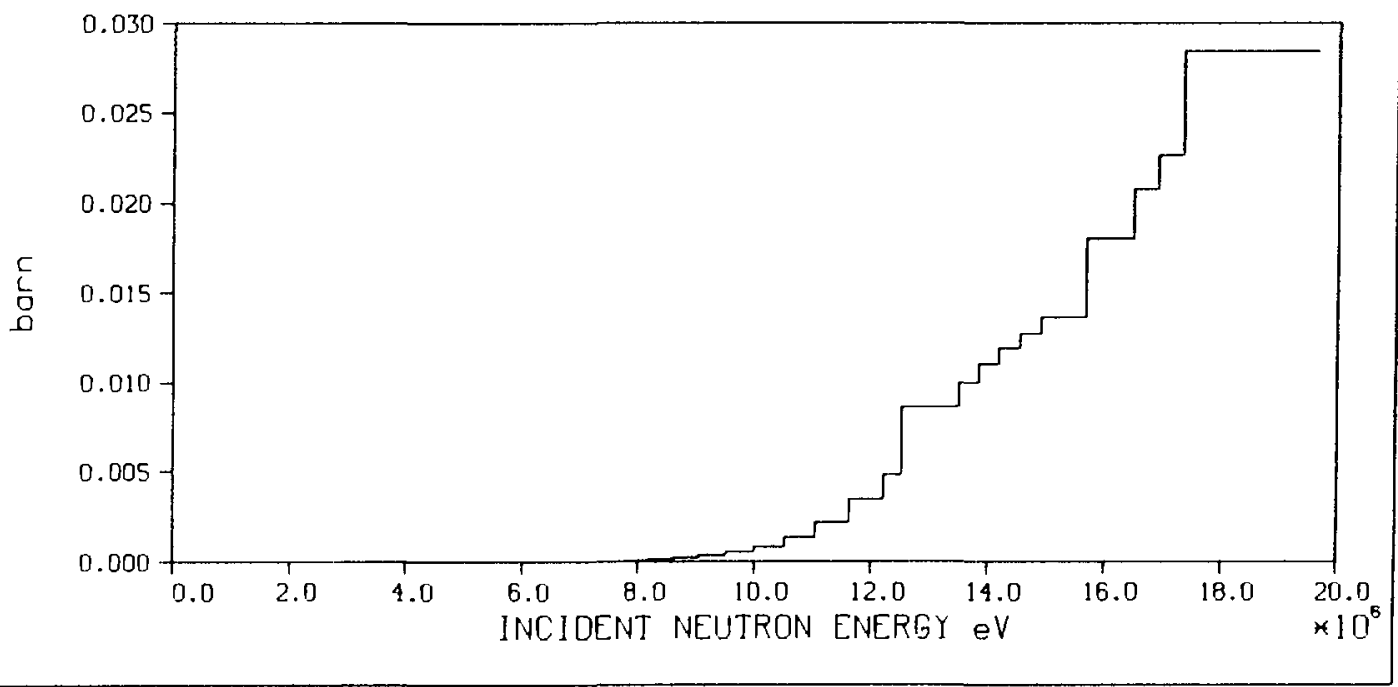


HF-177 TOTAL CROSS SECTION

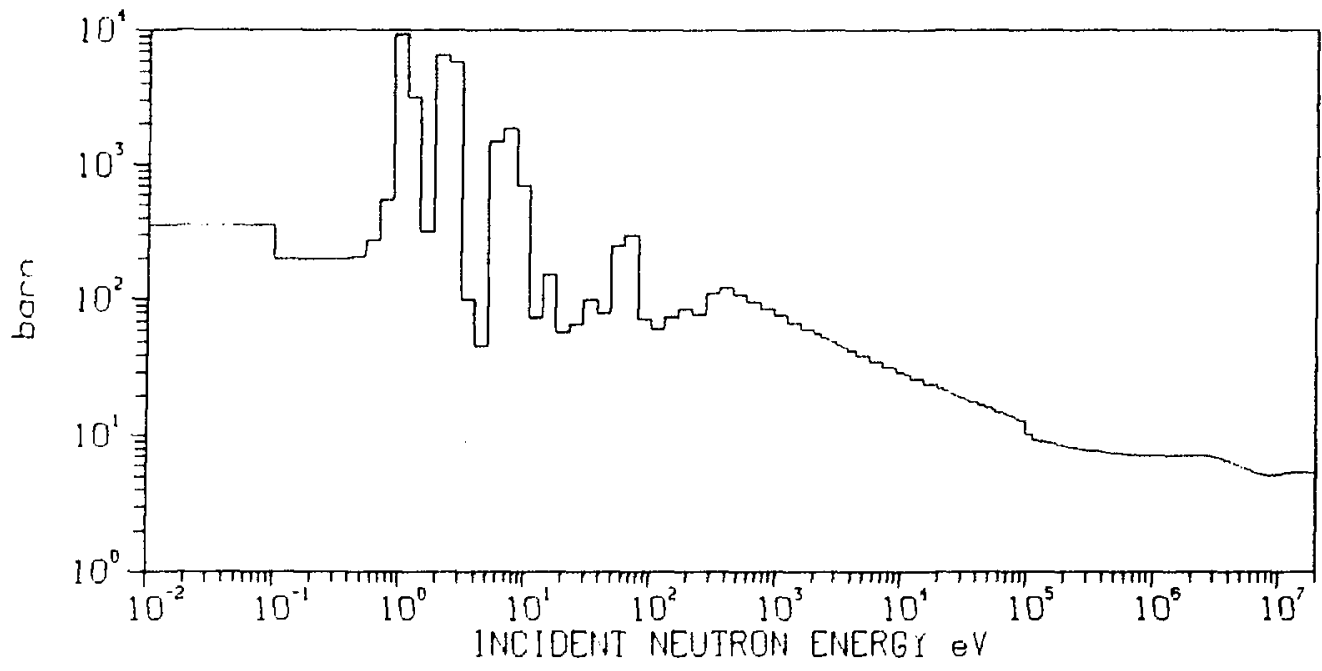

HF-177 ELASTIC CROSS SECTION

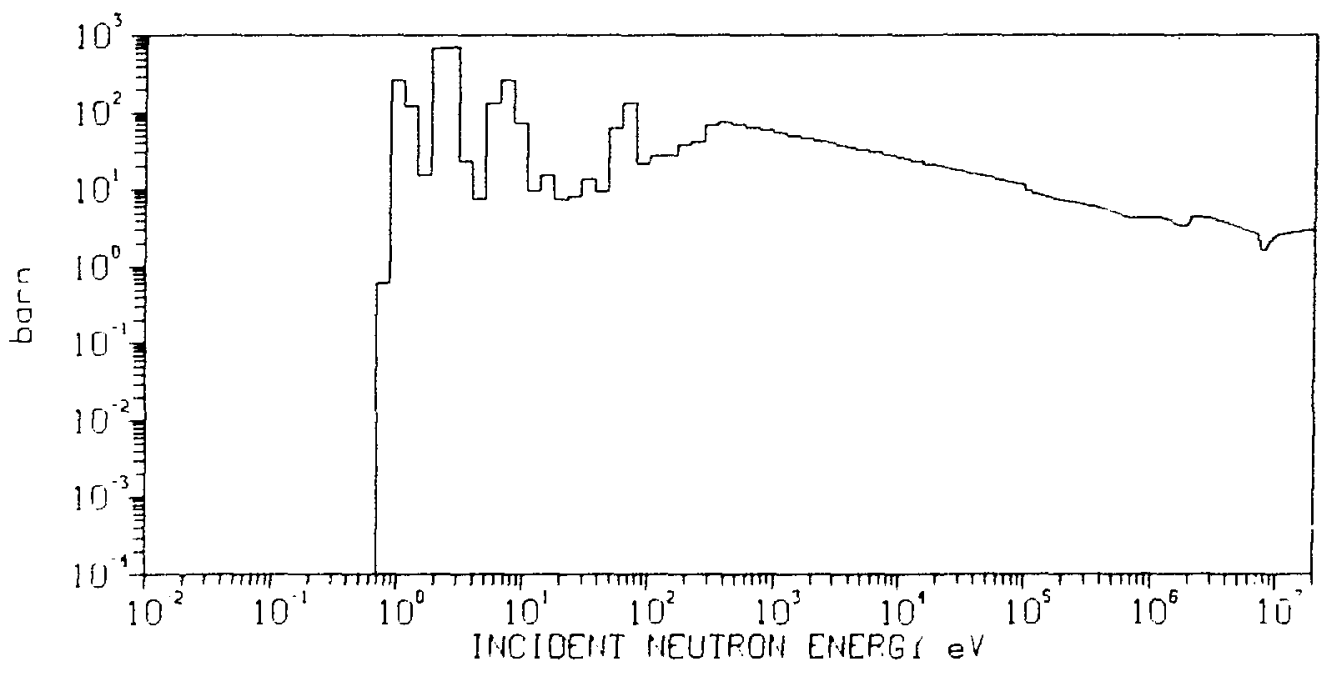




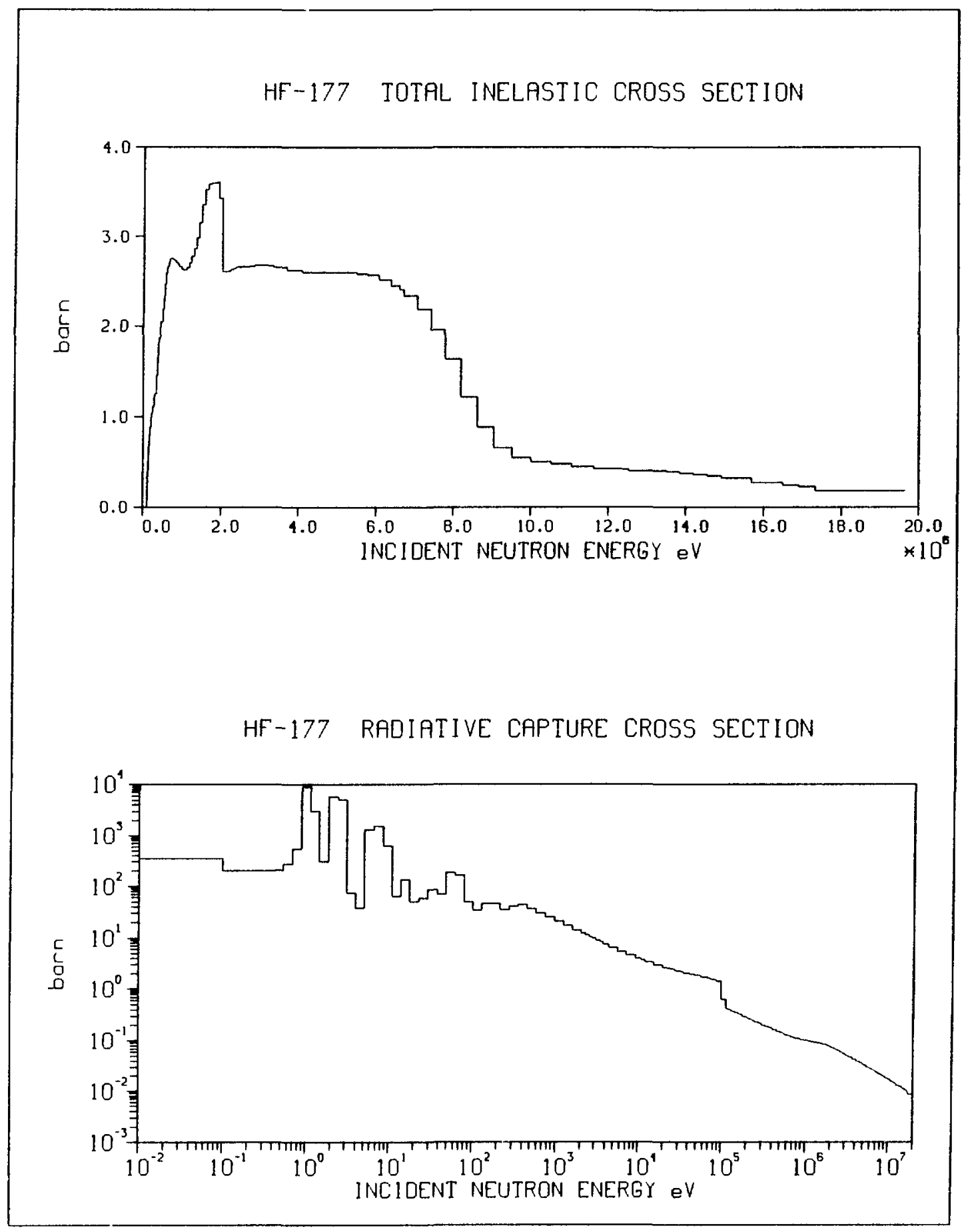




$$
\text { HF-177 TOTAL }(n, 2 n) \text { REACTION CROSS SECTION. }
$$

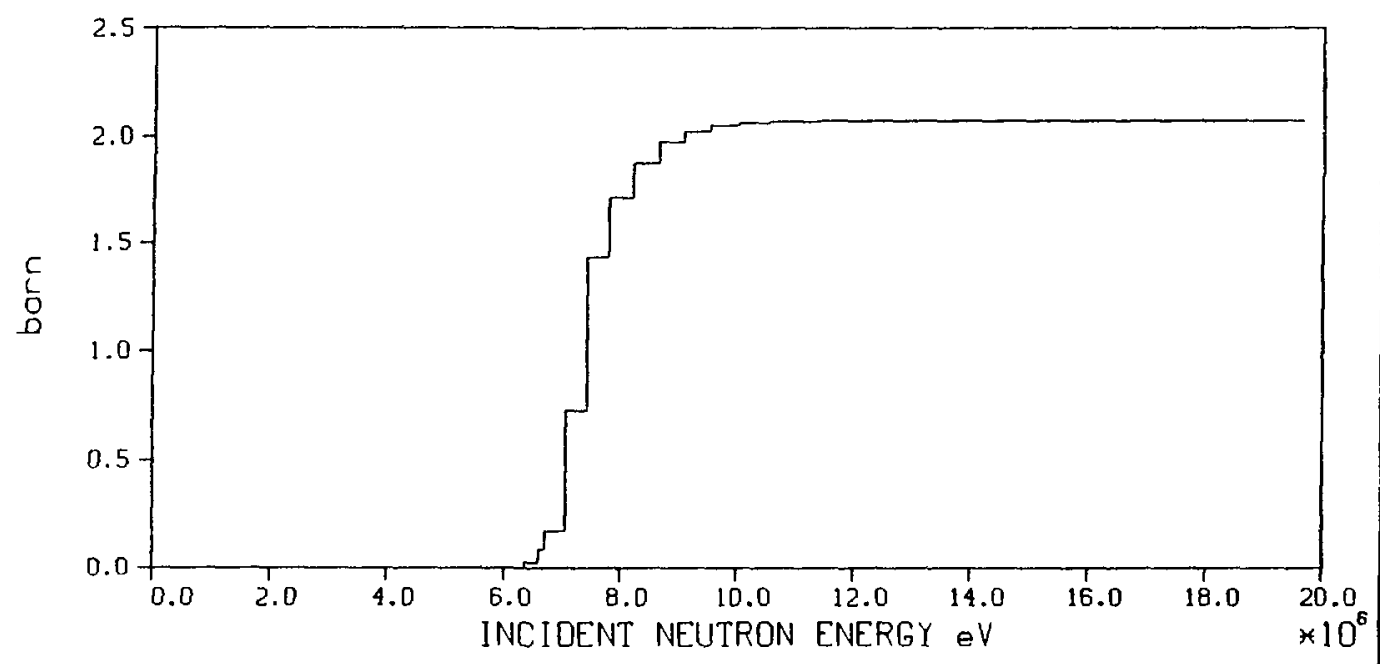




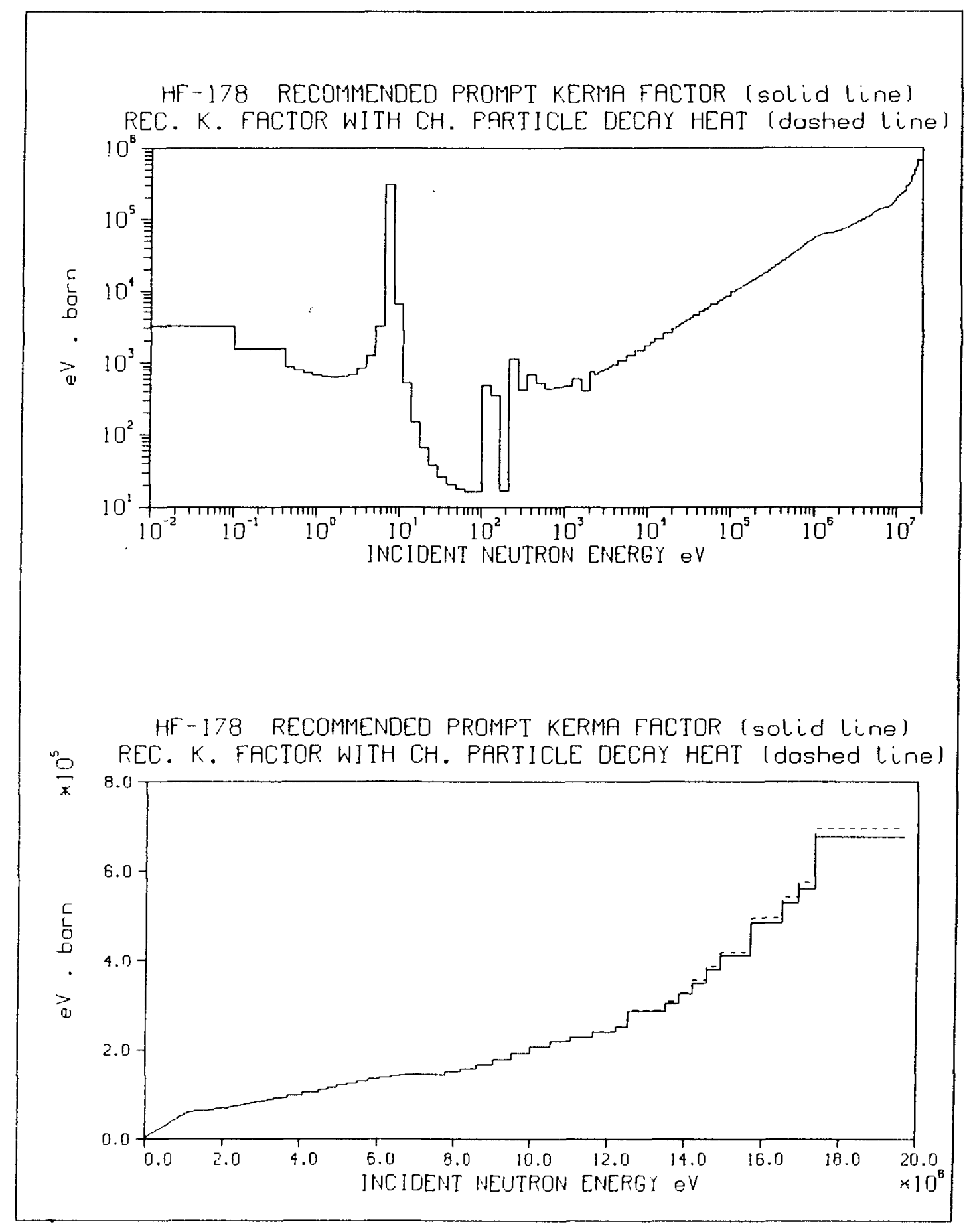




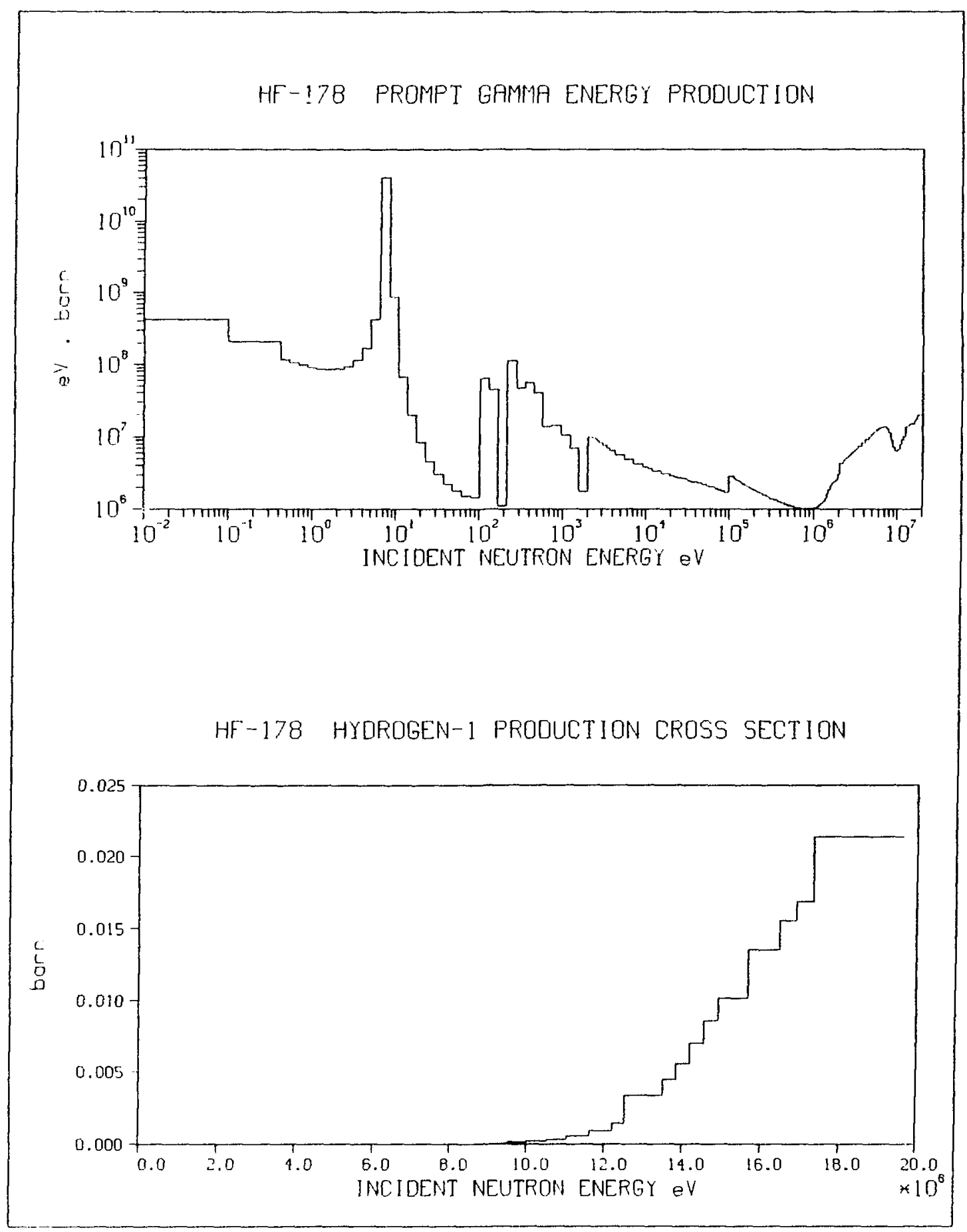




\section{HF-178 TOTAL CROSS SECTION}

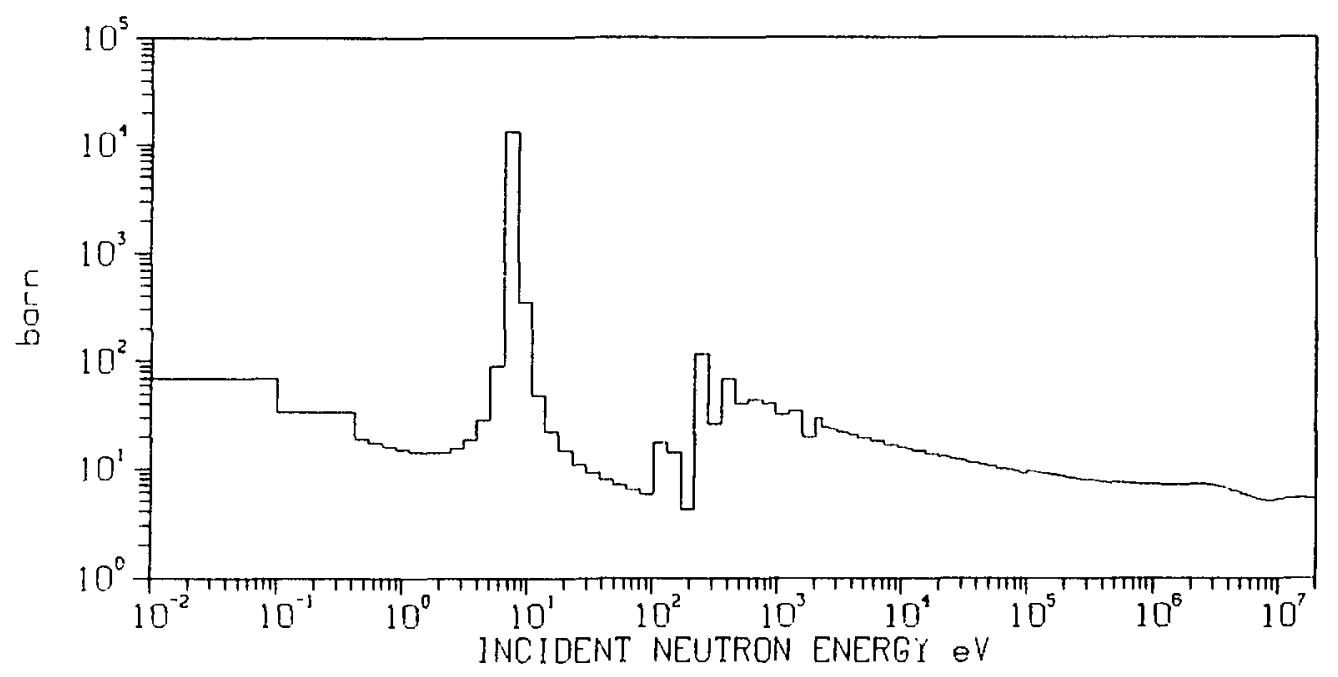

HF-178 ELASTIC CROSS SECTION

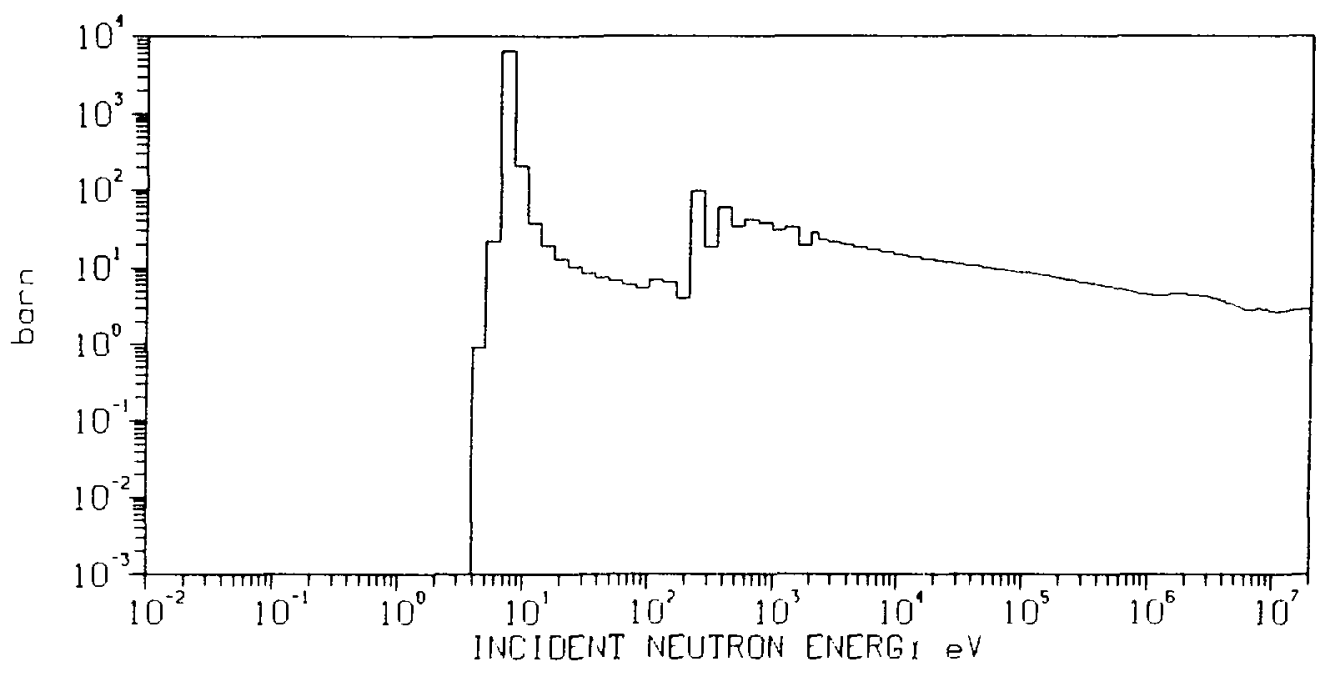




\section{HF-178 TOTAL INELASTIC CROSS SECTION}

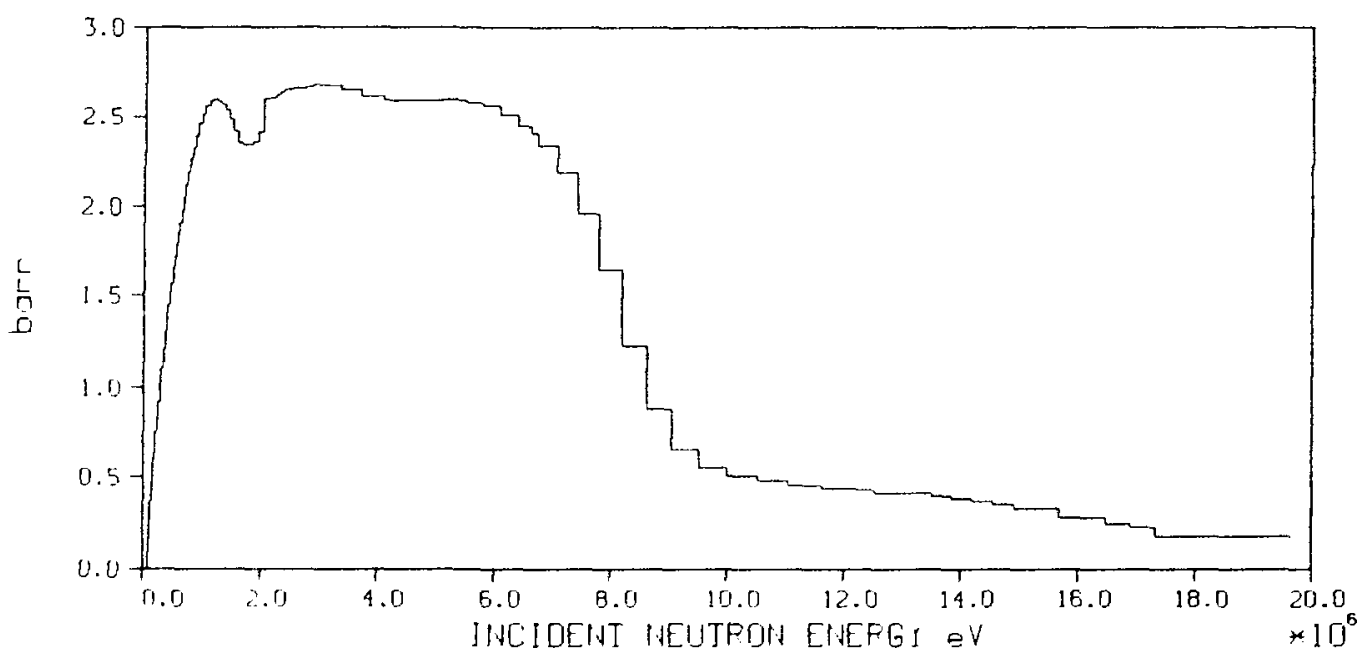

HF-178 RAOIATIVE CAPTURE CROSS SECTION

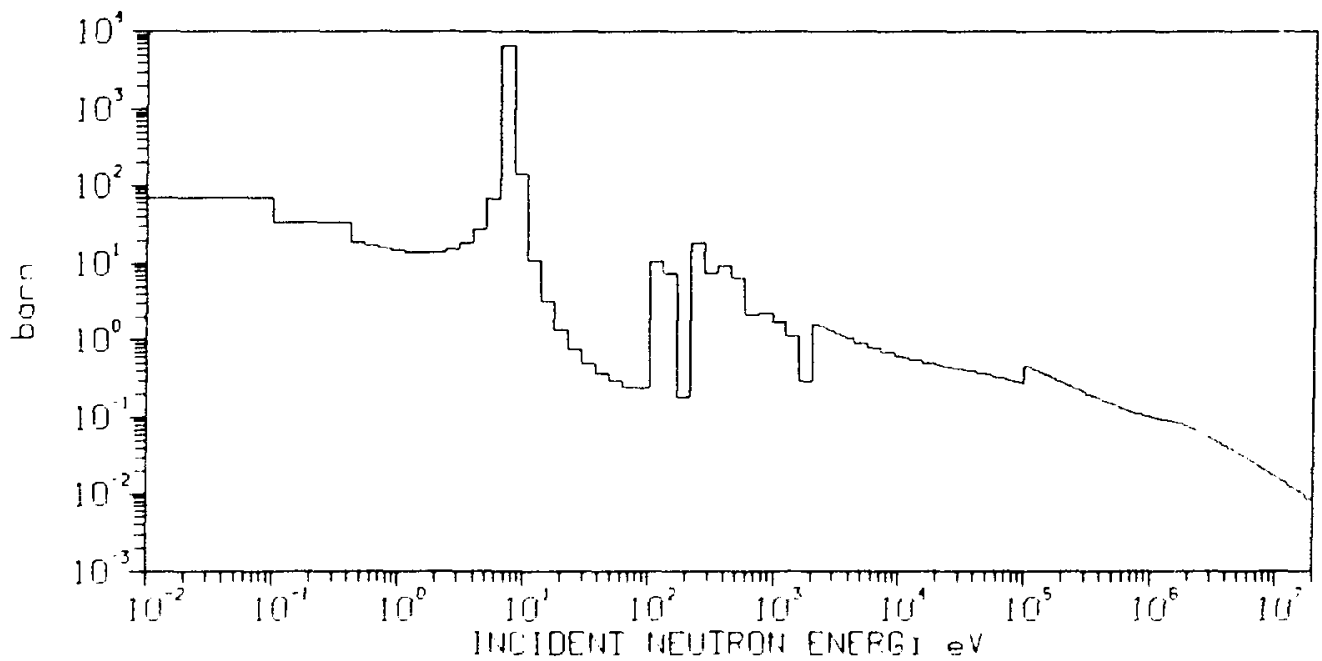




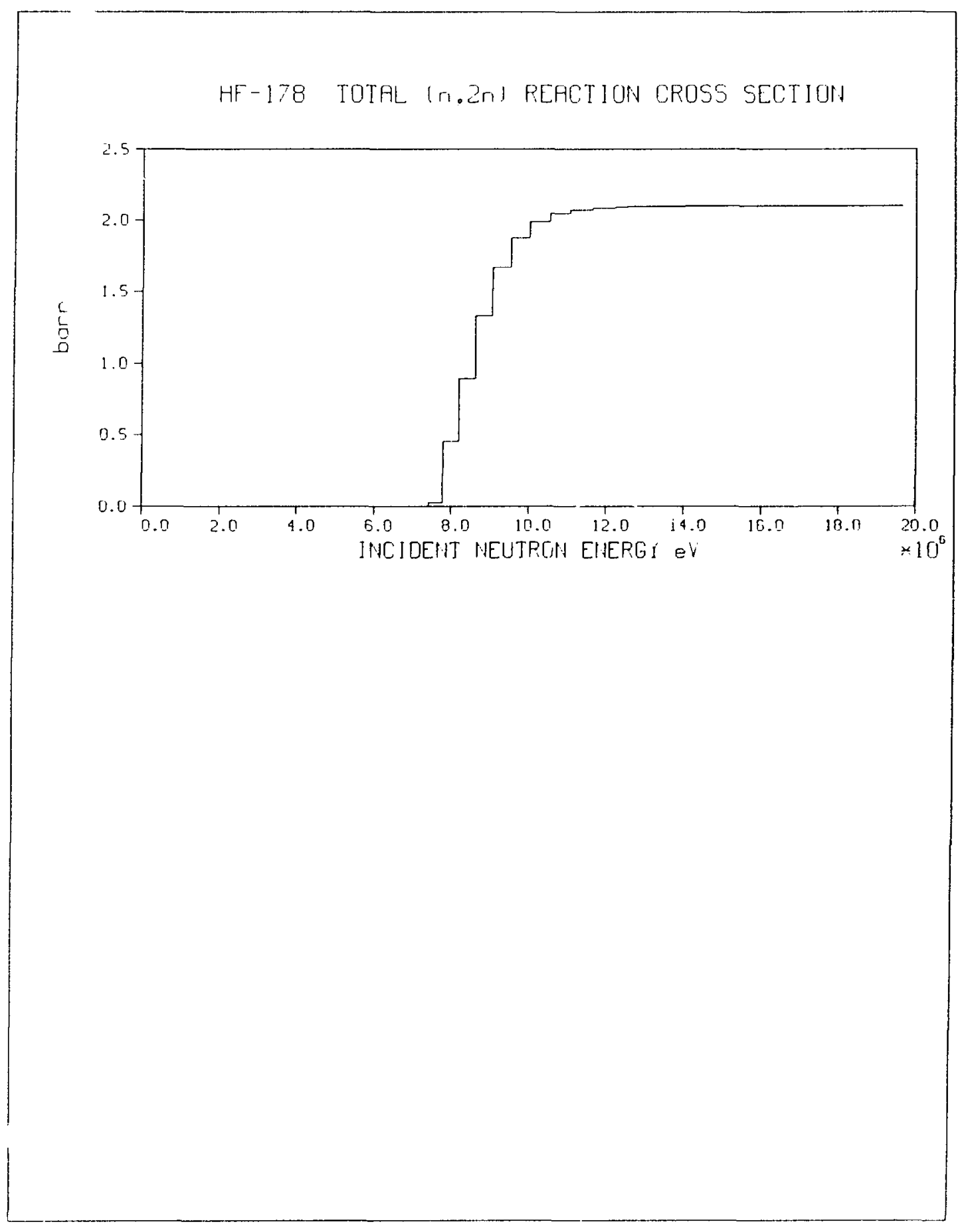




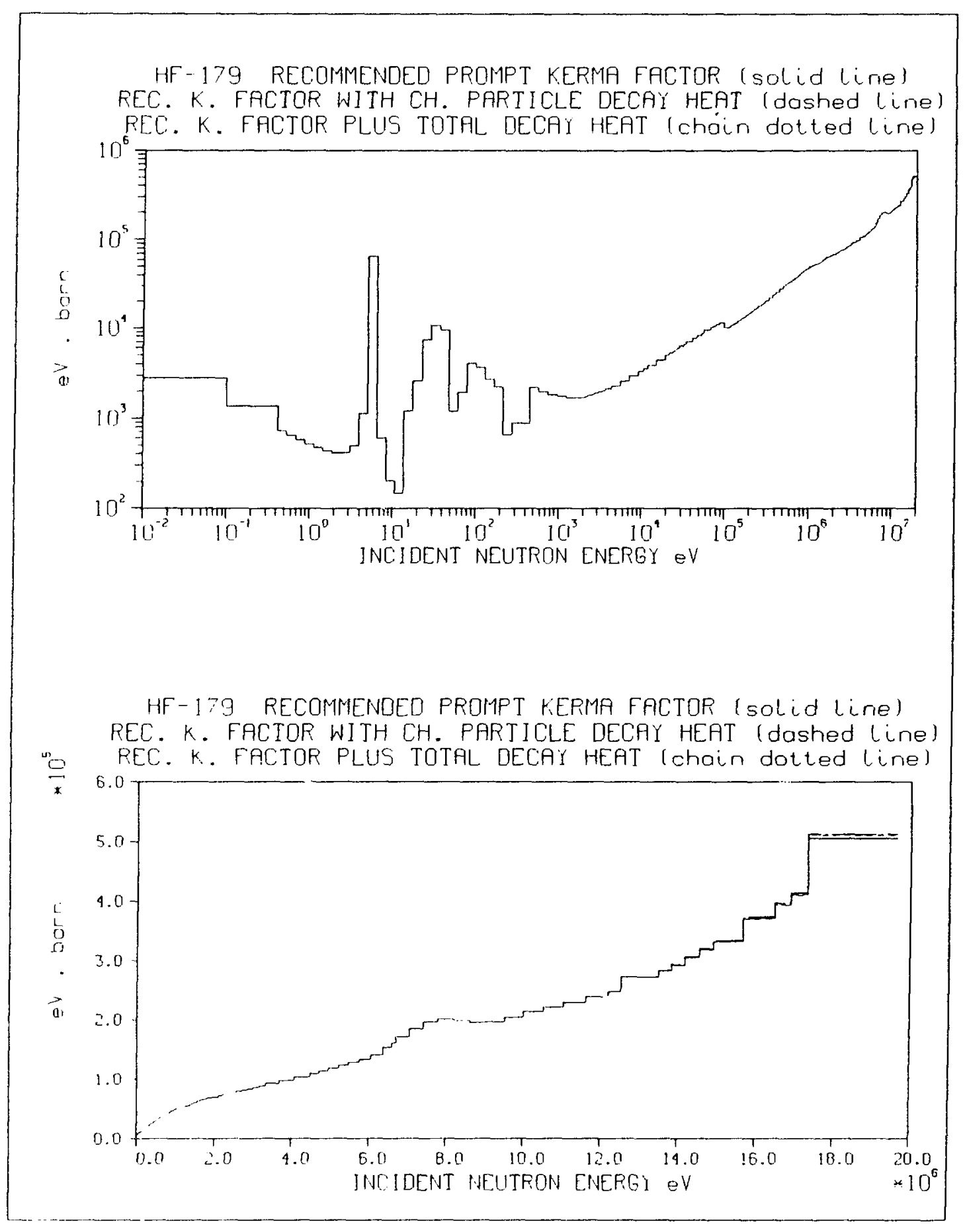




\section{HF-179 PROMPT GRMMA ENERGY PRODUCTION}

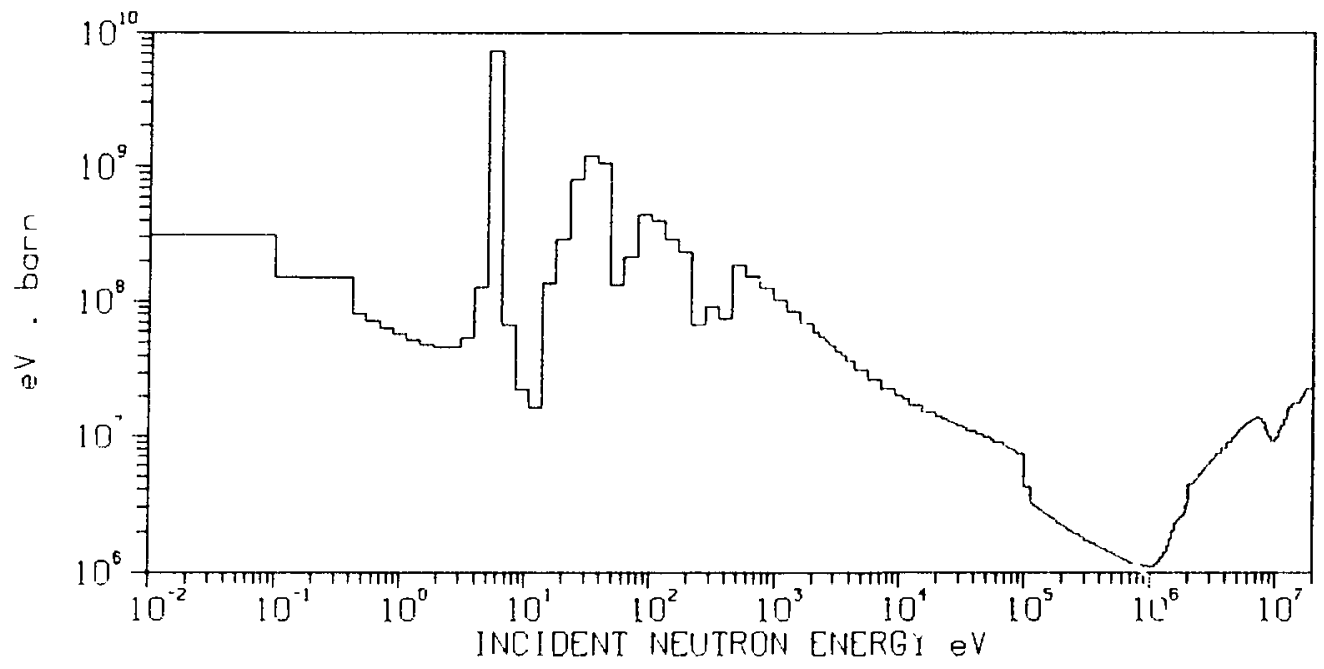

HF-179 HYDROGEN-1 PRODUCTION CROSS SECTION

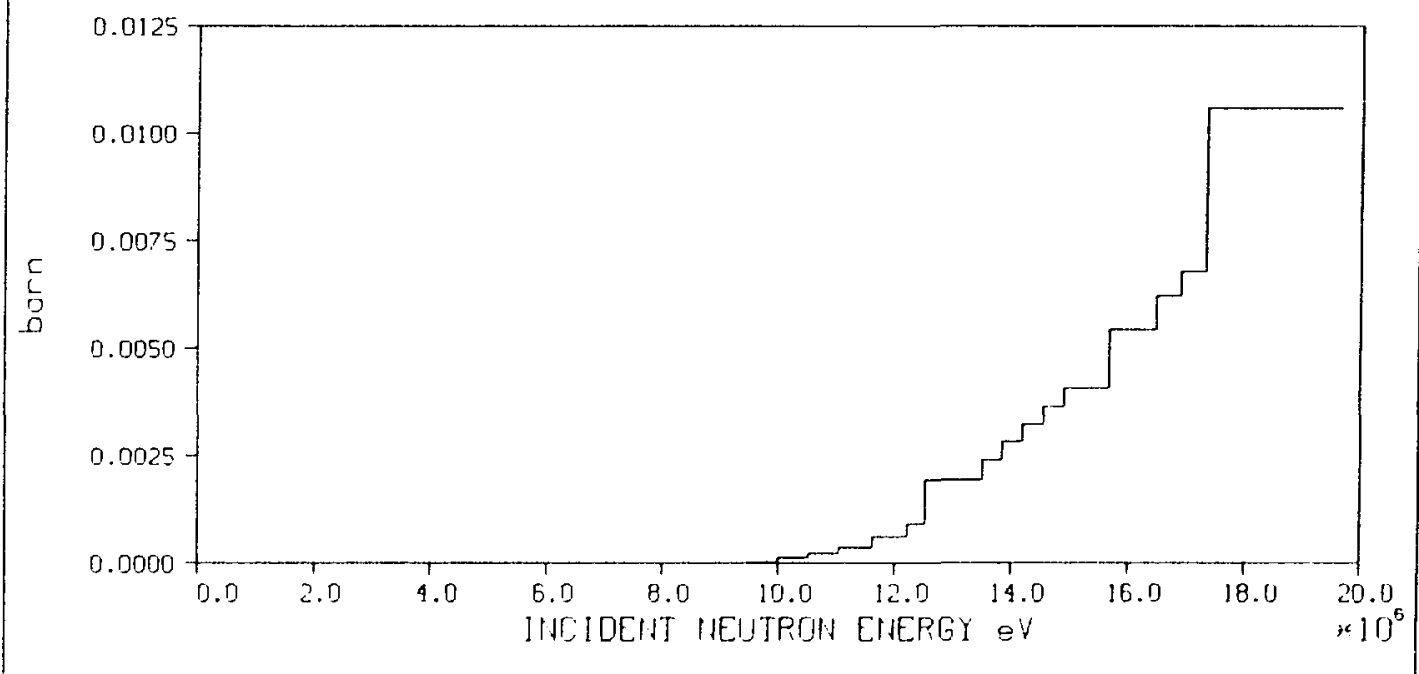




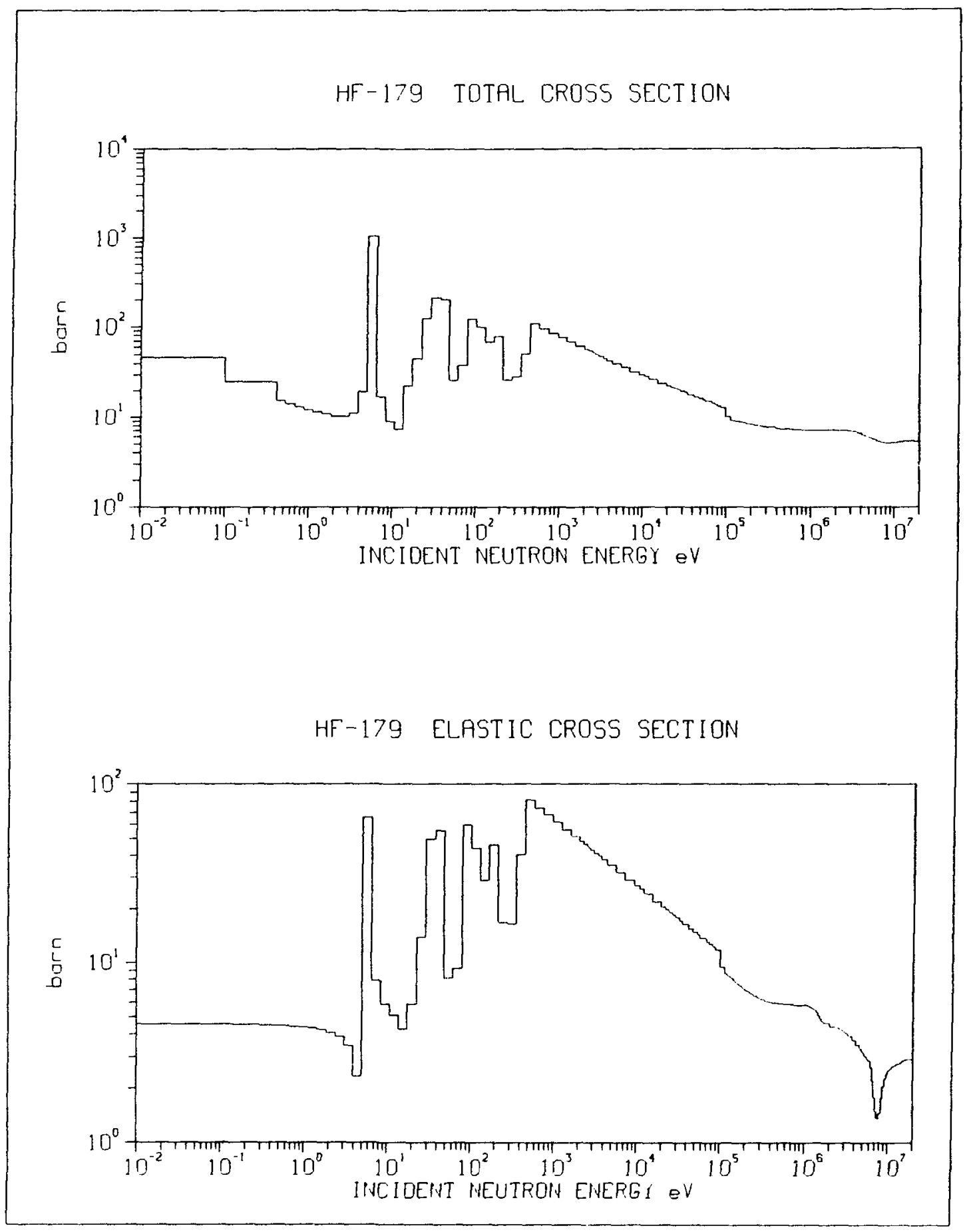


HF-179 TDTAL INELASTIC CROSS SECTION

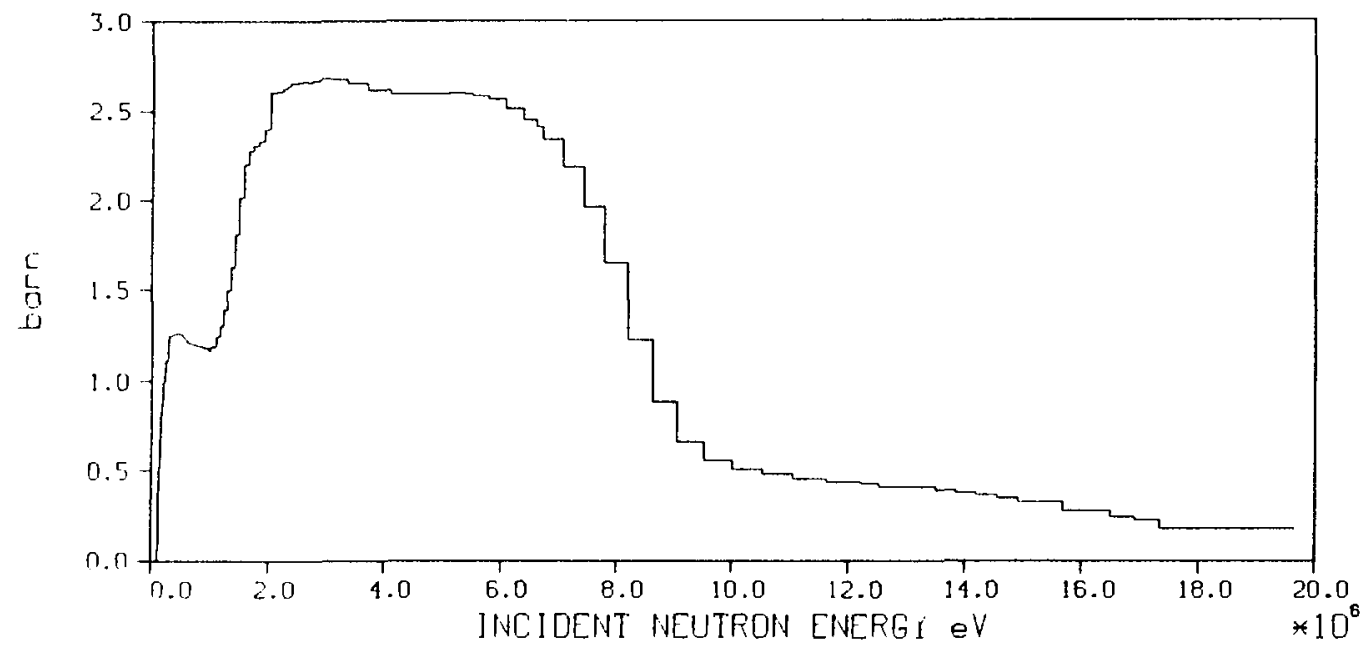

HF-179 RADIATIVE CAPTURE CROSS SECTION

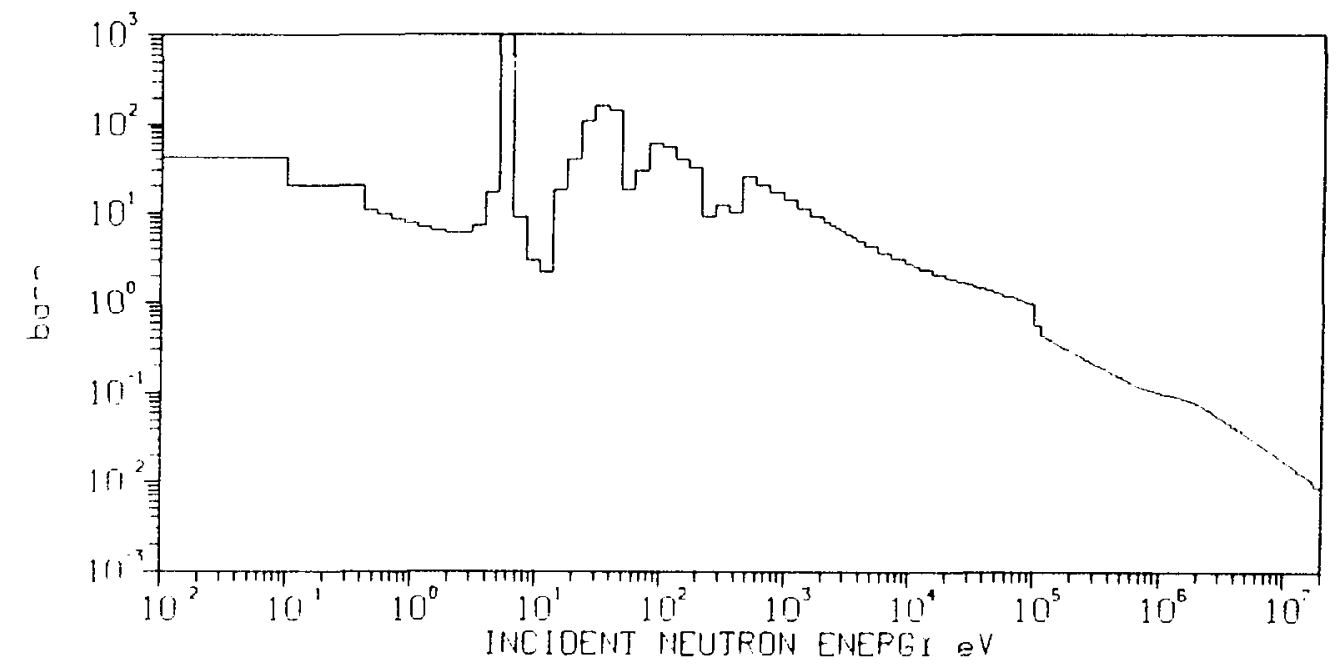


HF-179 TOTAL $(n, 2 n)$ REACTION CPOSS SECTION

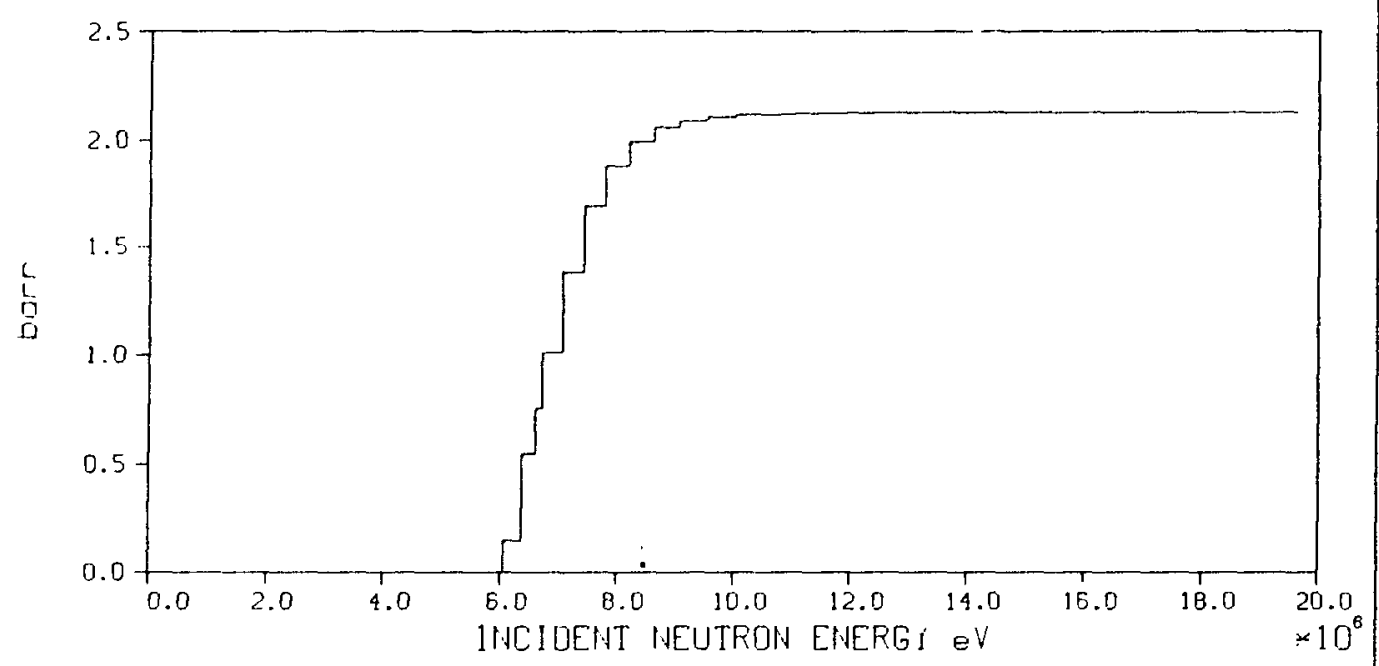


HF-180 RECOMMENDED PROMPT KERMA FACTOR (solid line) REC. K. FACTOR WITH CH. PAFTICLE [IECAI HEAT Idashed Iine) REC. K. FACTOR PLUS TOTAL DECAI HEAT (chain dot ted line)

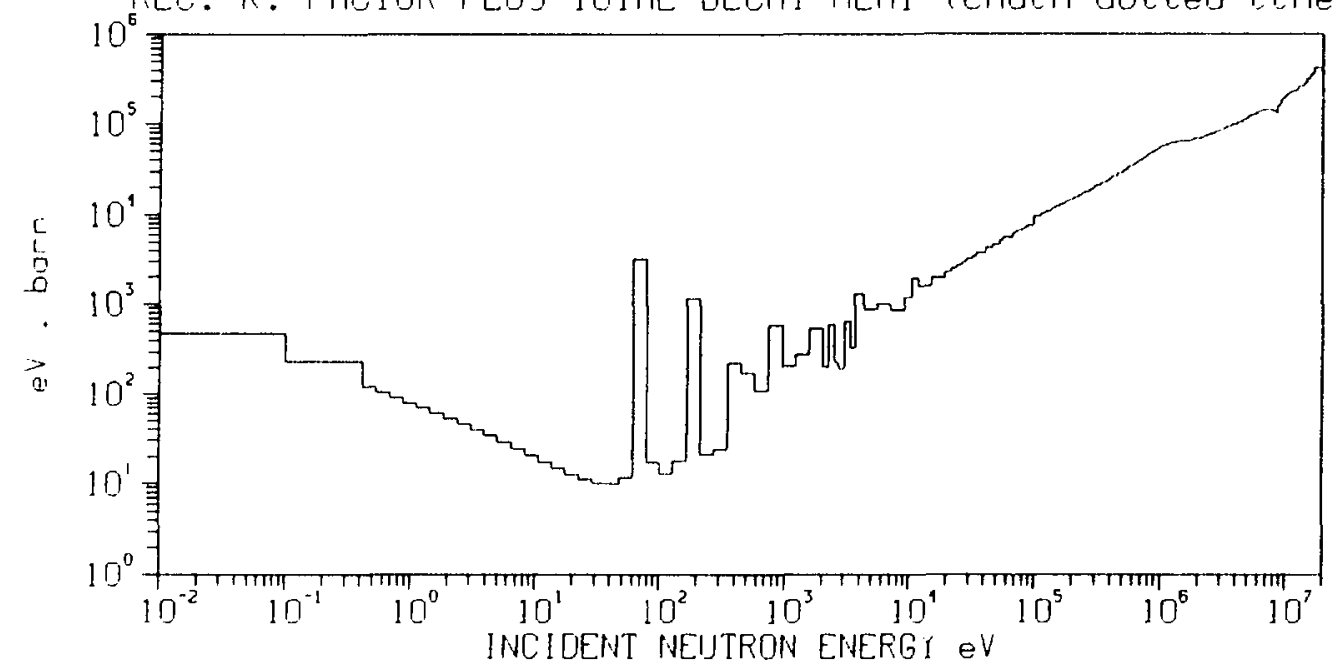

HF- 180 RECOMIENDED PRDIPT KERIA FACTOR (solid line) REC. K. FACTOR WITH CH. PARTICLE DECAI HEAT I doshed Line) $\because$ REC. $K$. FACTOR PLUS TOTAL DECAI HEAT (chain dotted lirie)

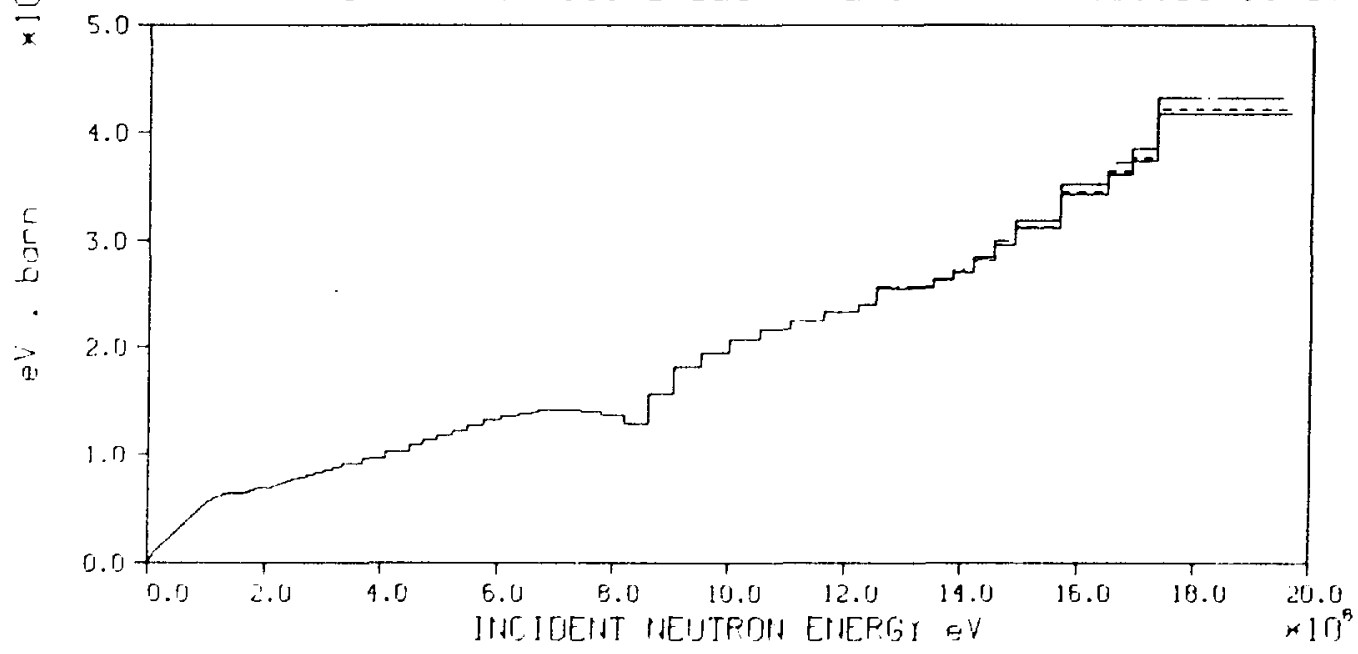


HF-180 PROMPT GAMMA ENERGI PRODUCTIDN

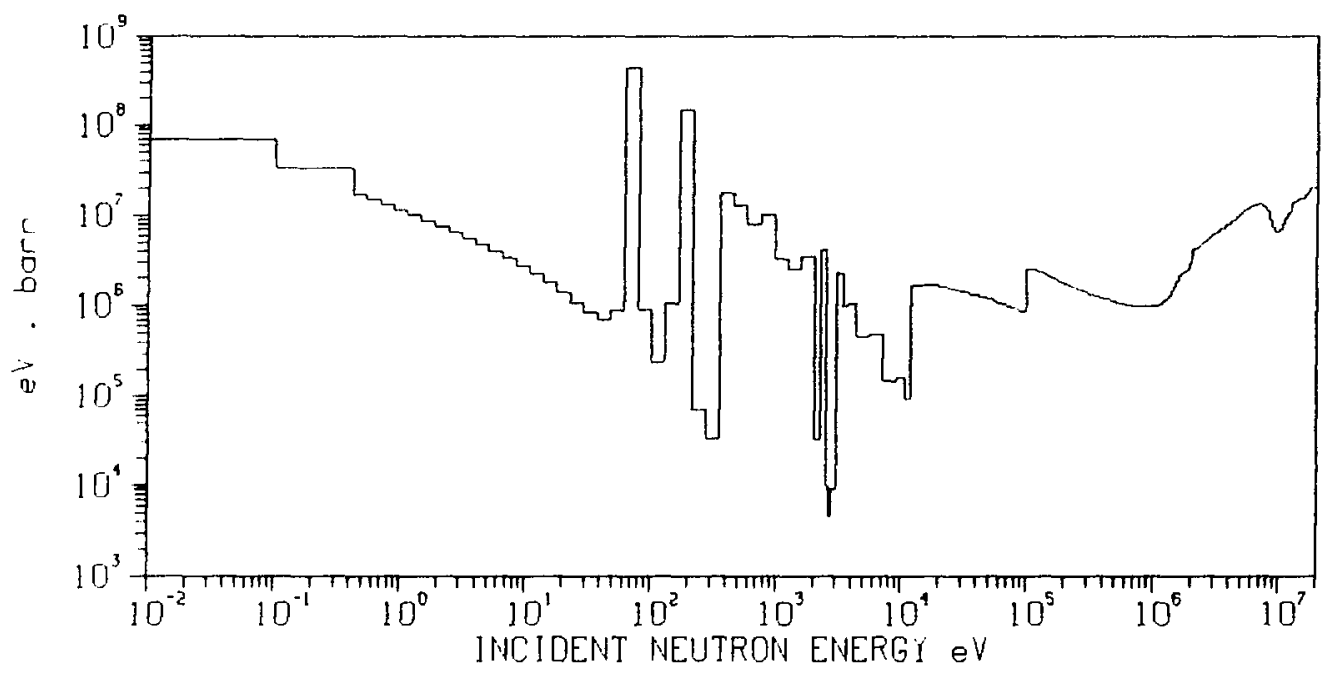

HF-180 HYOROGEN-1 PRODUCTION CROSS SECTION

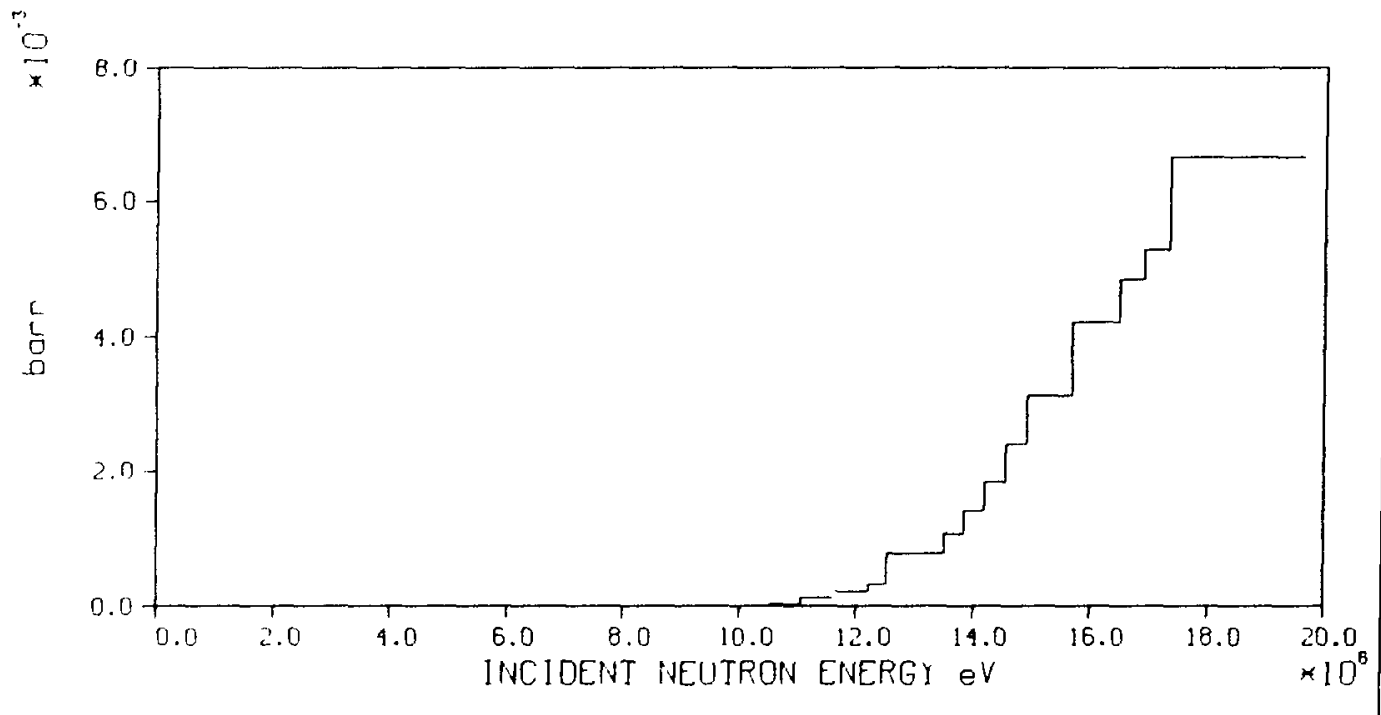


HF-180 TOTAL CROSS SECTION

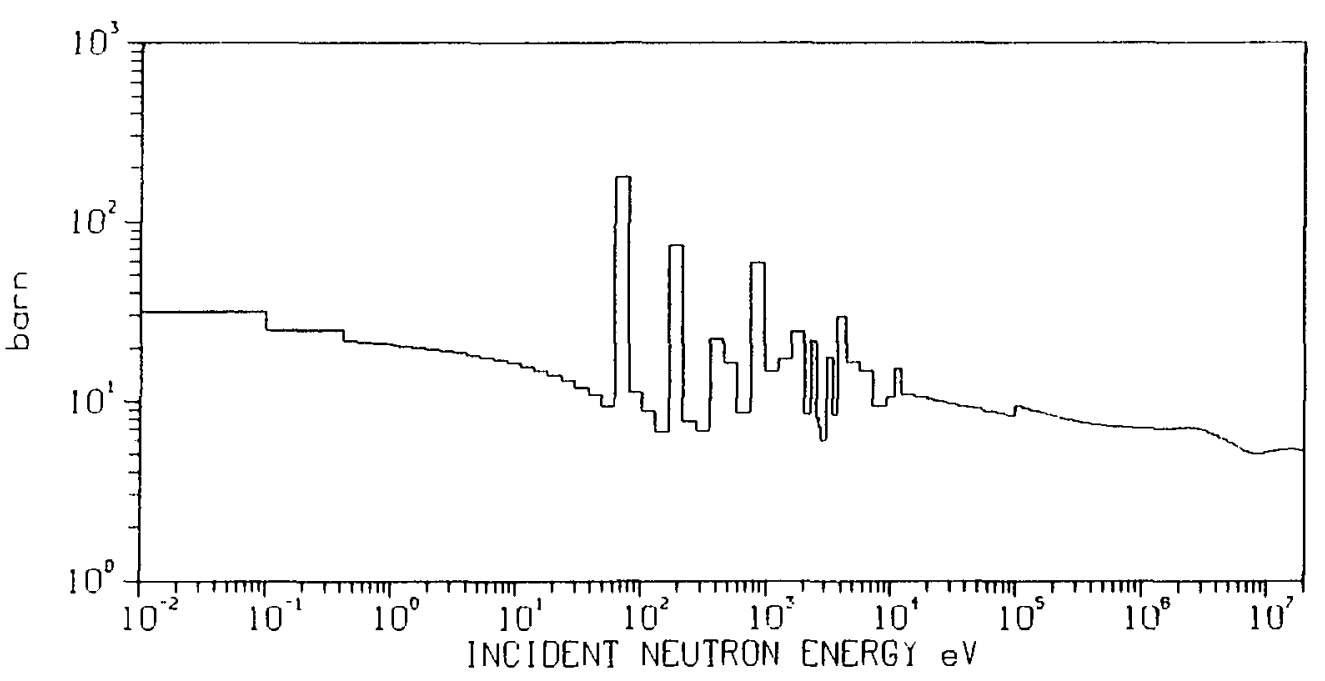

HF-180 ELASTIC CROSS SECTION

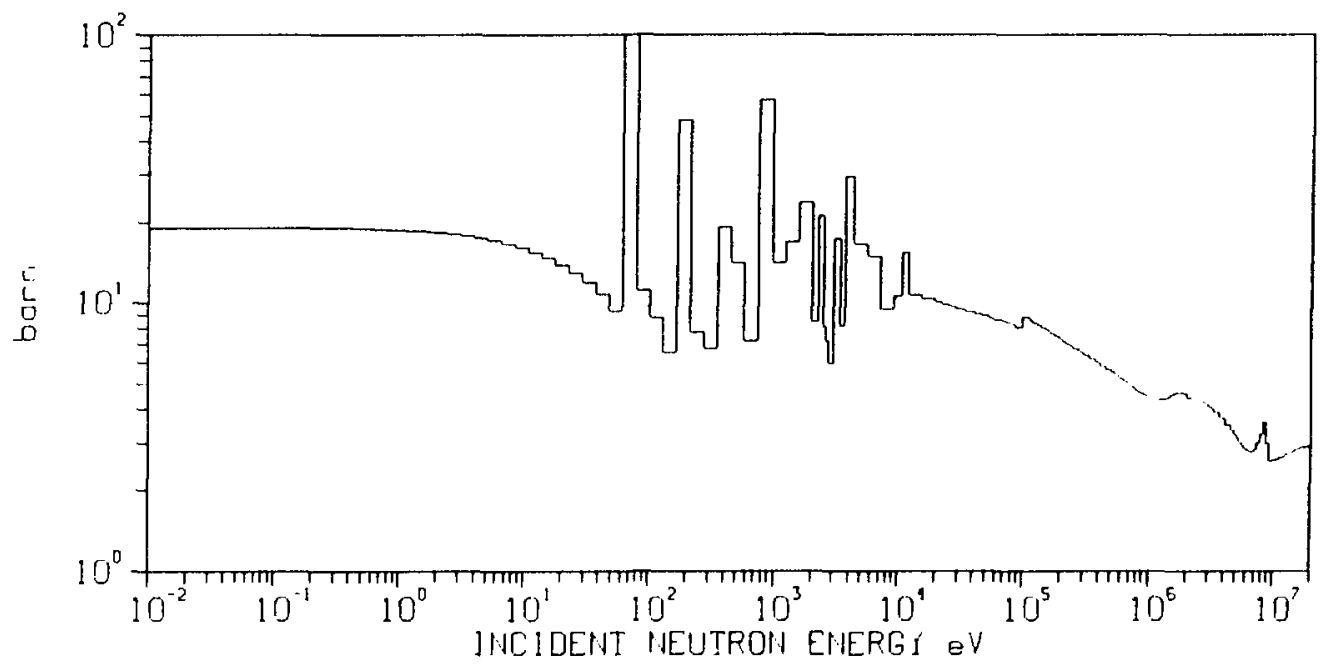




\section{HF-180 TOTAL INELASTIC CROSS SECTION}

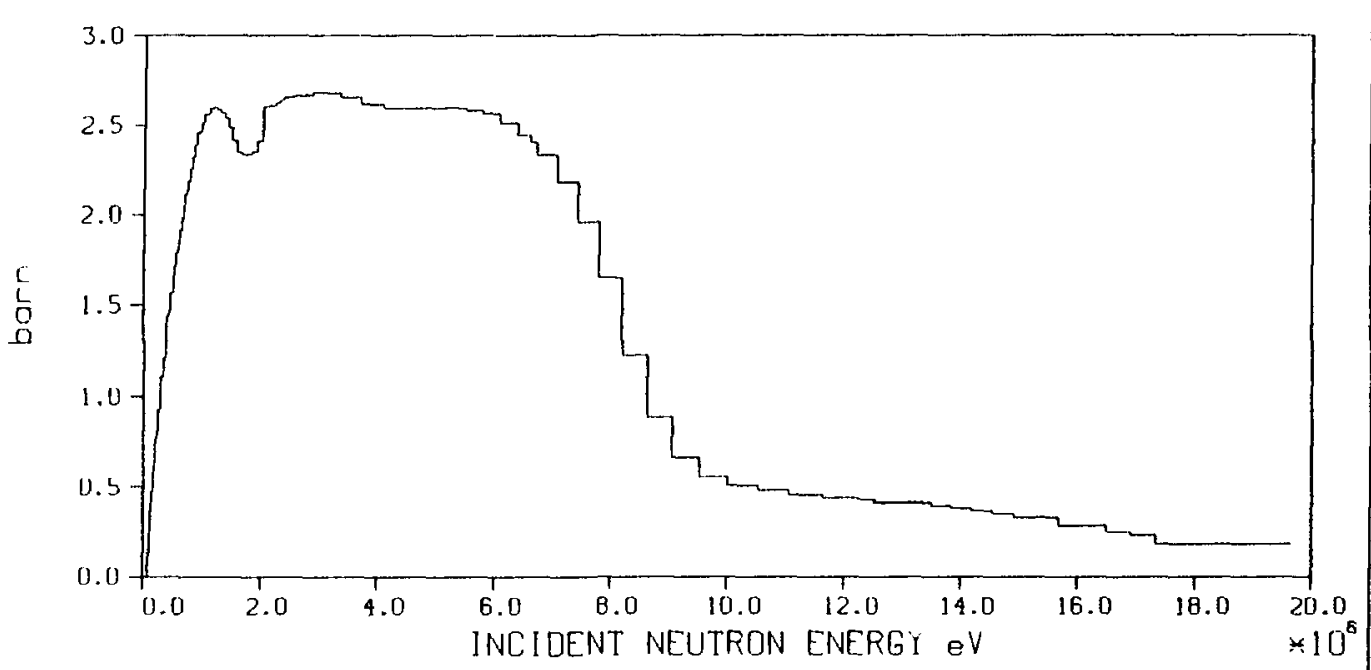

HF-180 RADIATIVE CAPTURE CROSS SECTION

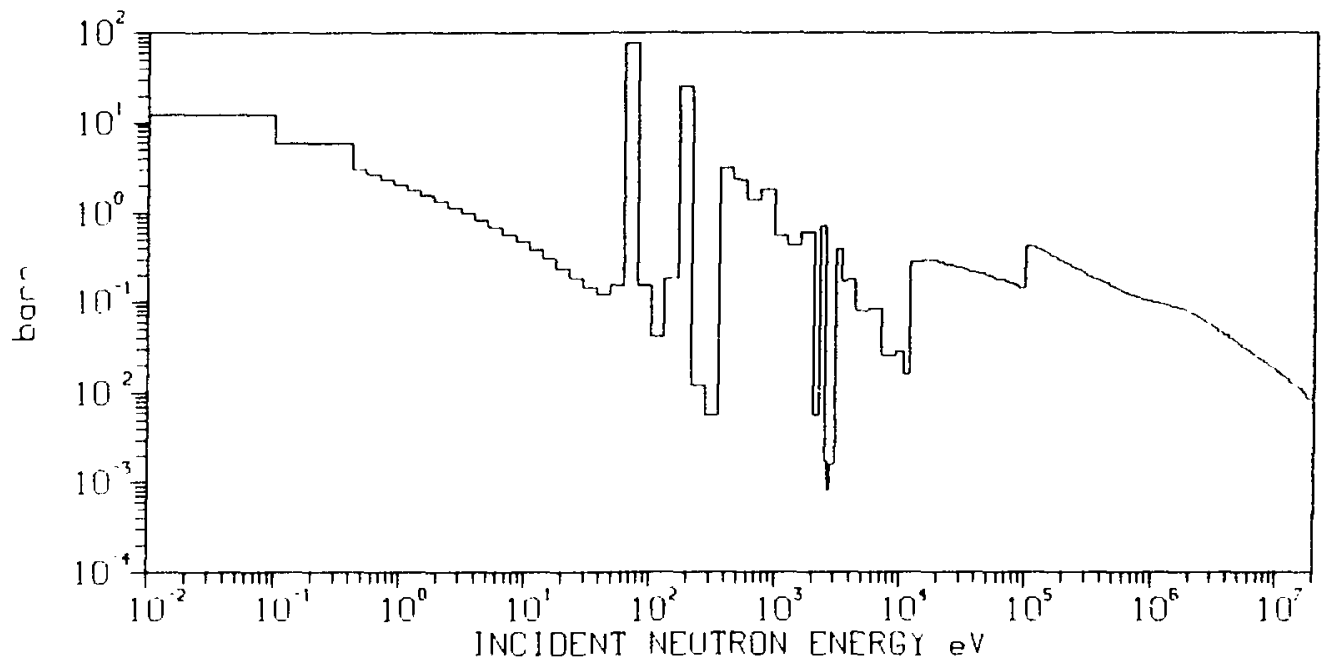


HF-180 TOTAL $(n, 2 n)$ REACTION CROSS SECTION

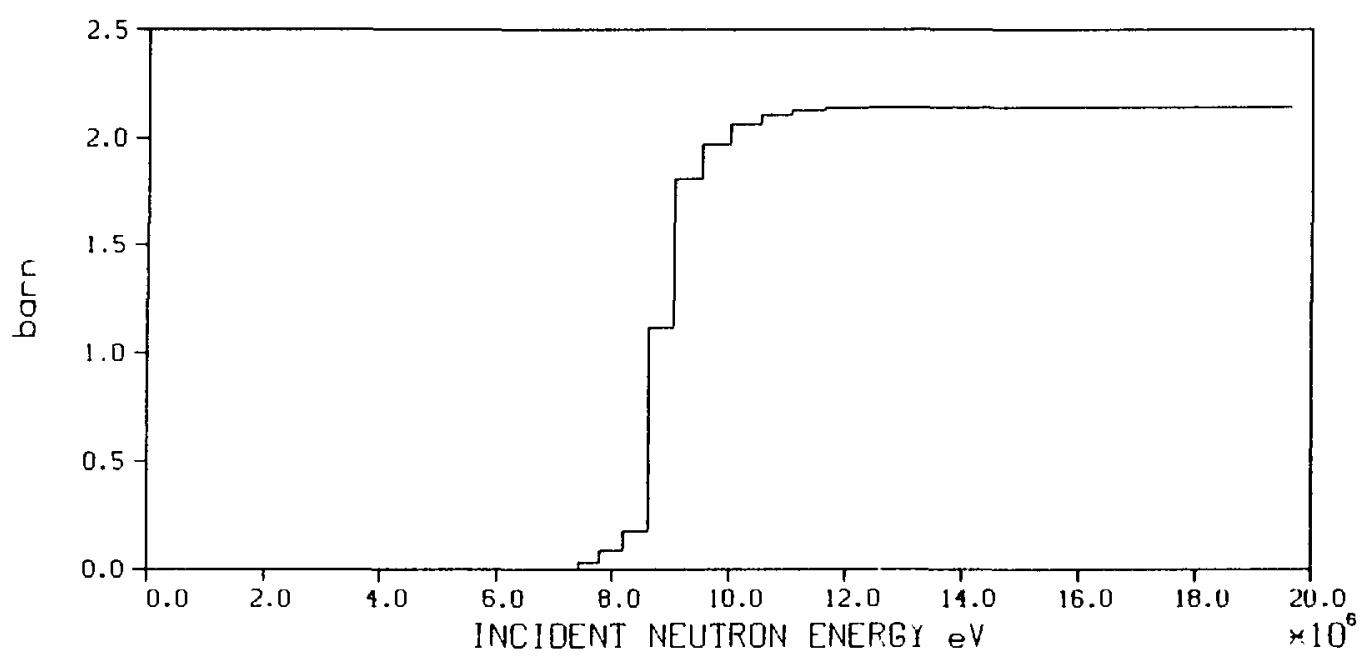




\section{TA-181 RECOMMENDED PROMPT KERMA FACTOR}

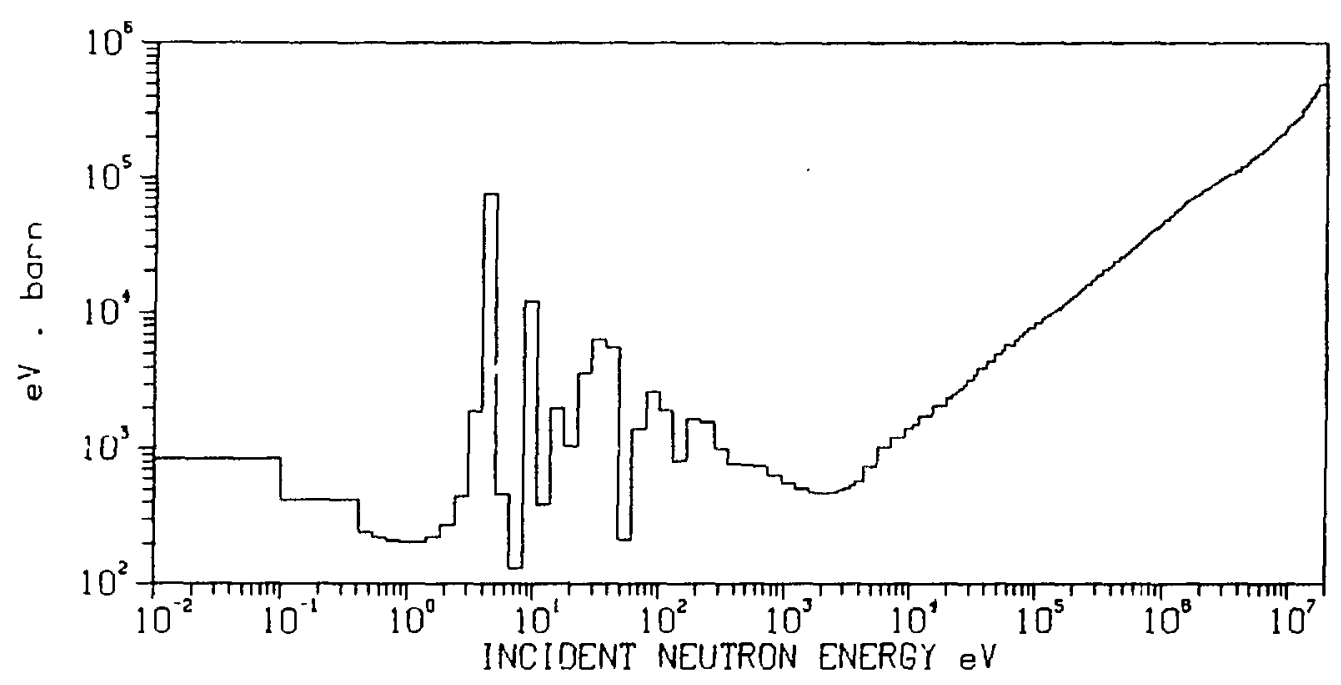

TA-181 RECOMMENDED PROMPT KERMA FACTOR

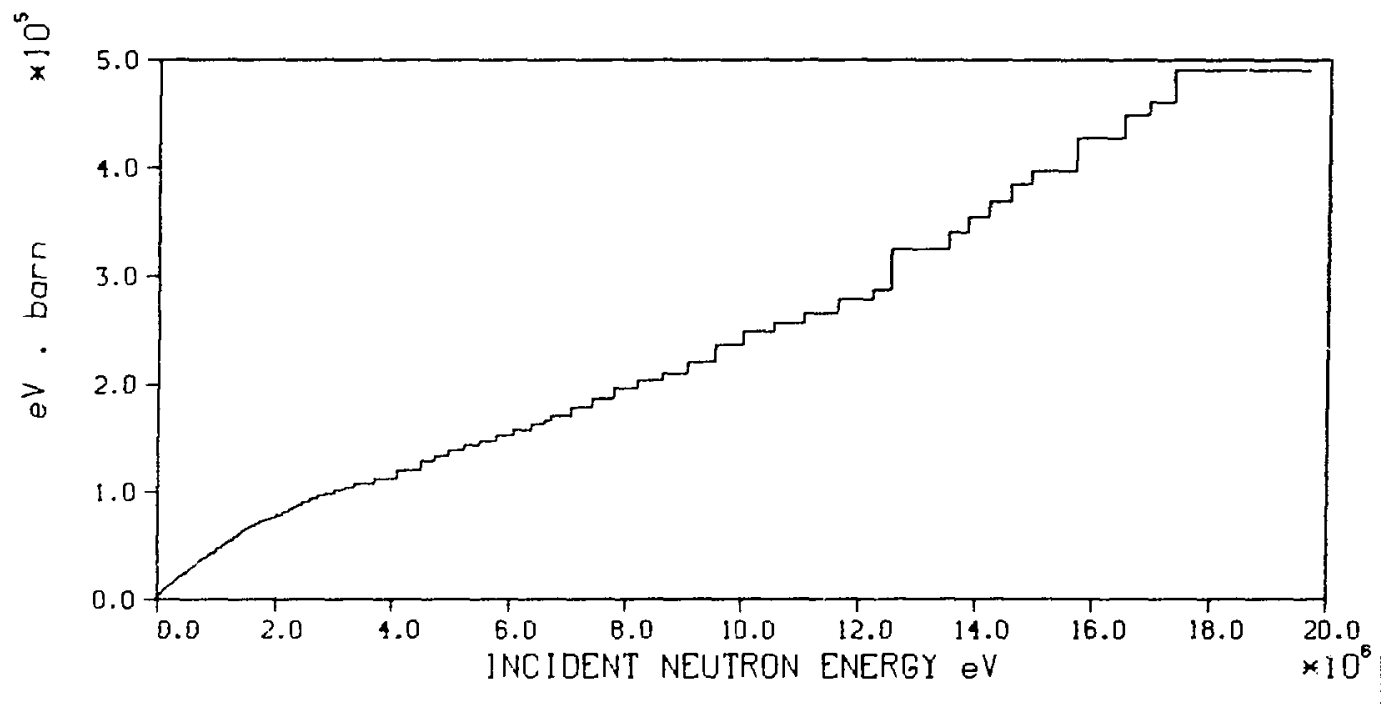




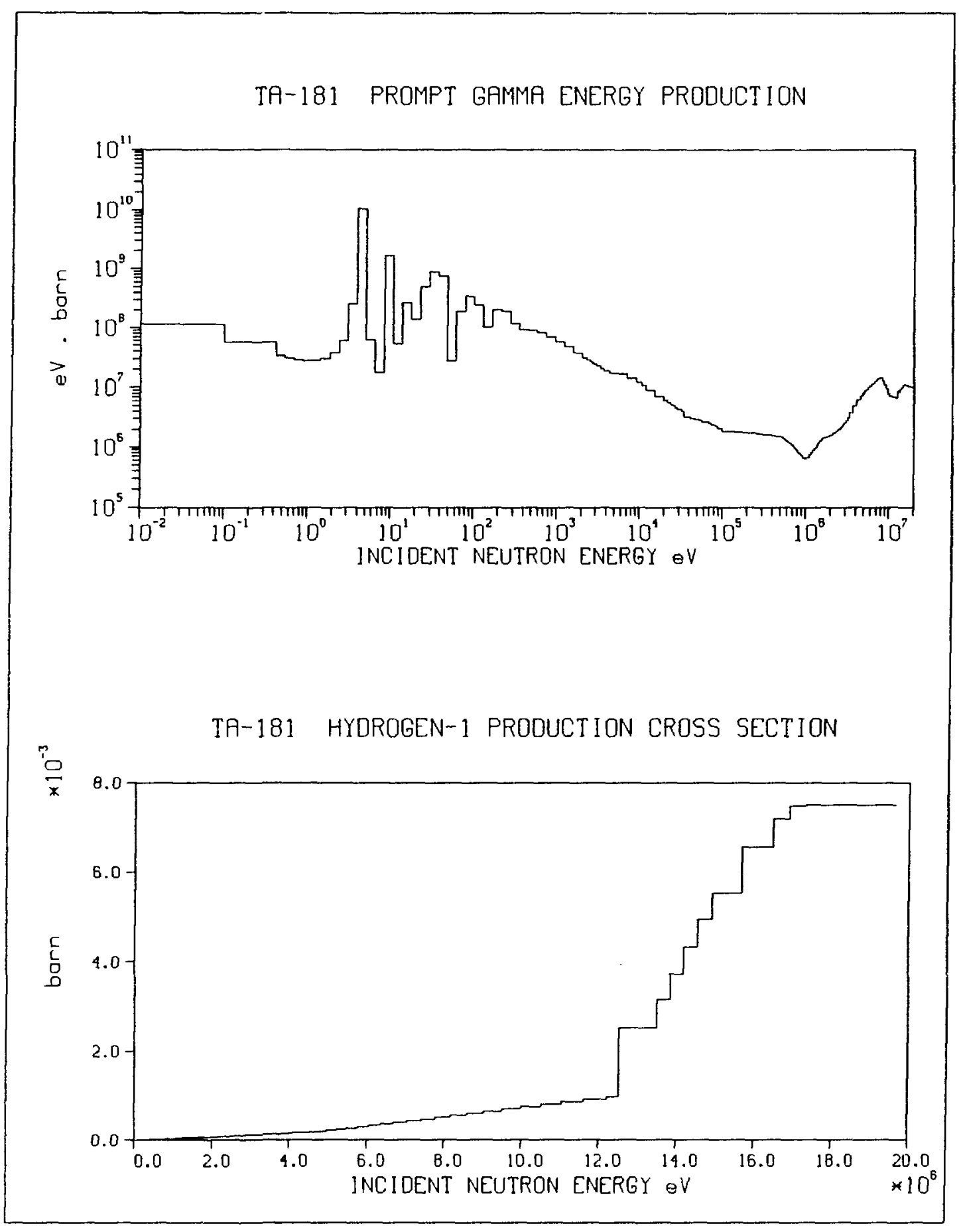


TA-181 TOTAL CROSS SECTION

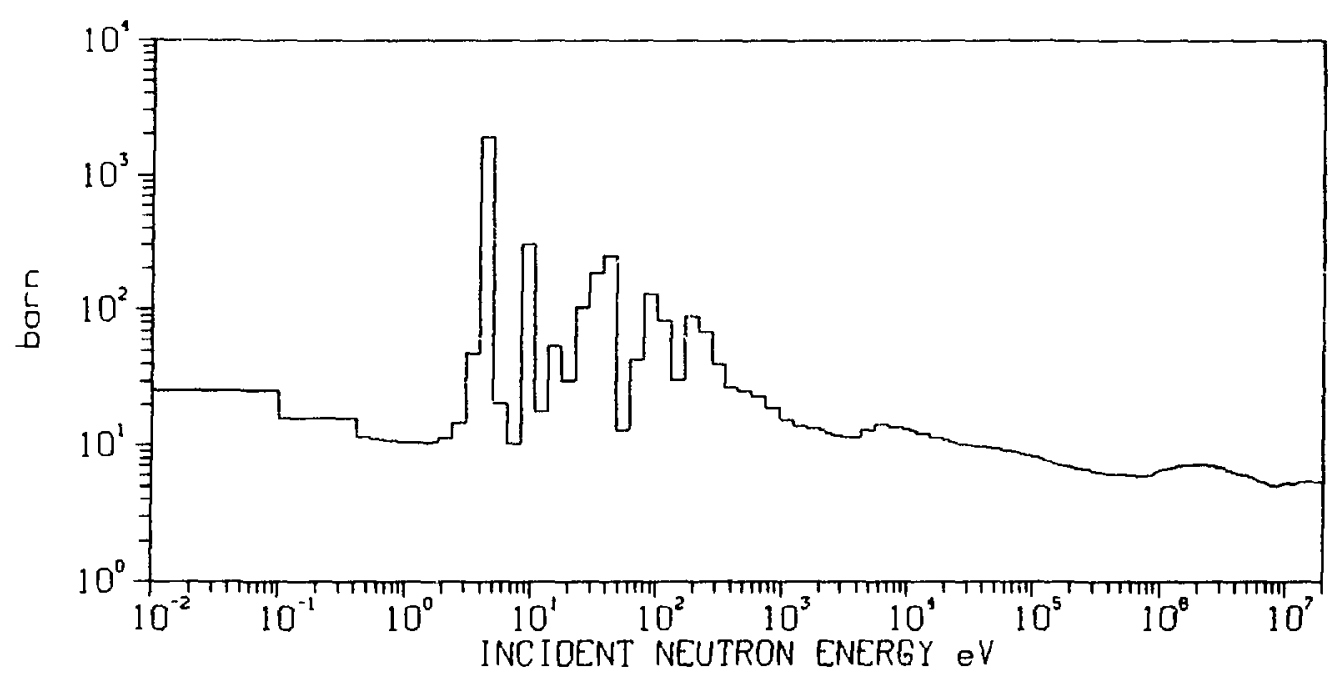

TA-181 ELASTIC CROSS SECTION

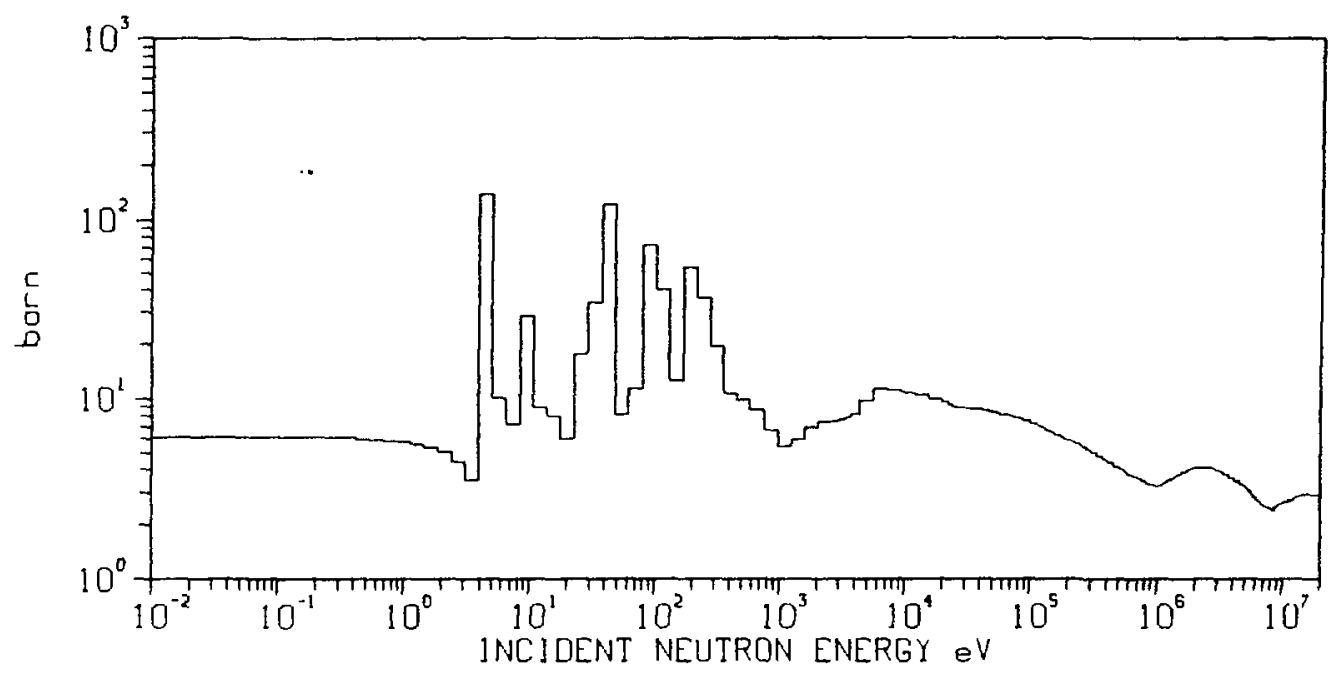


TA-181 TOTAL INELASTIC CROSS SECTION

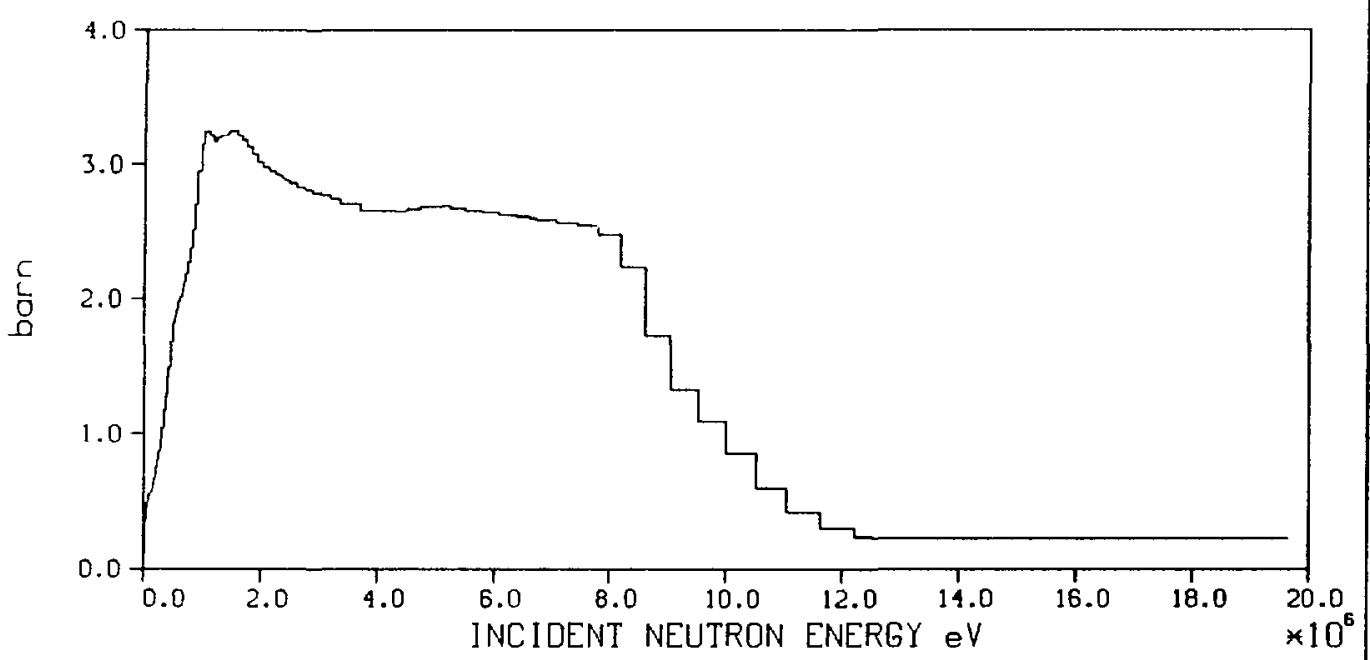

TA-181 RADIATIVE CAPTURE CROSS SECTION

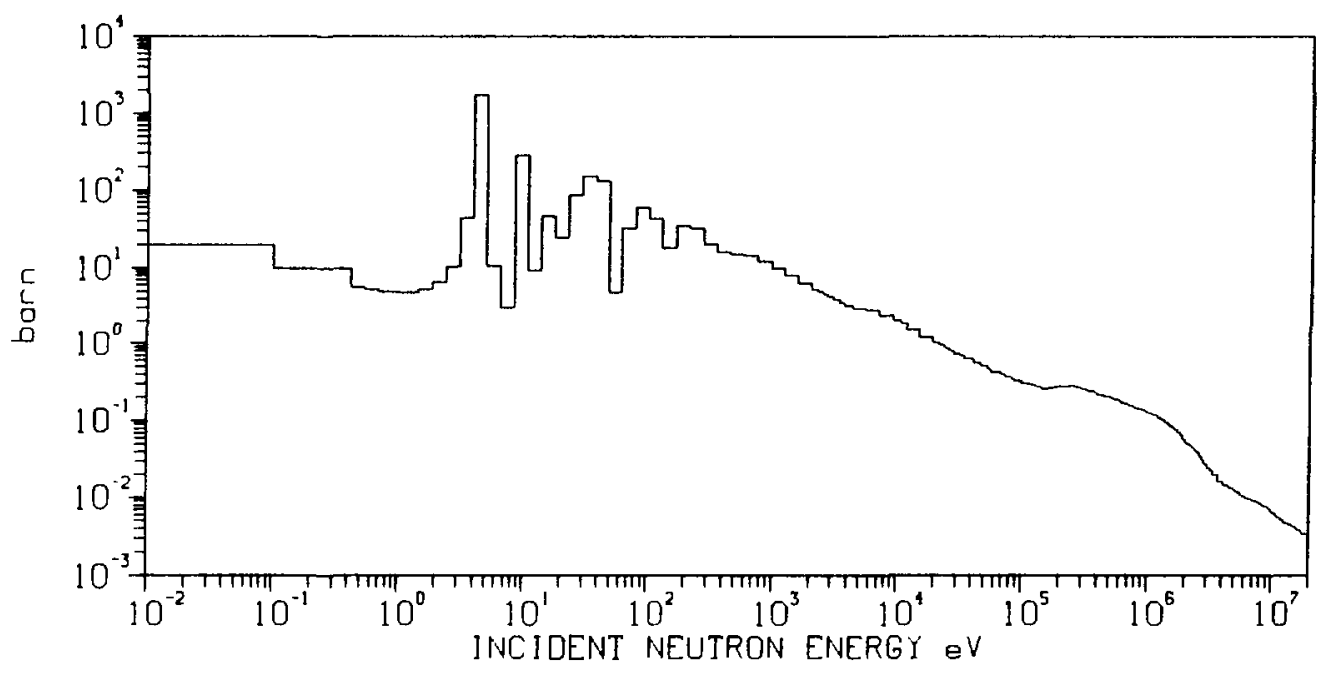




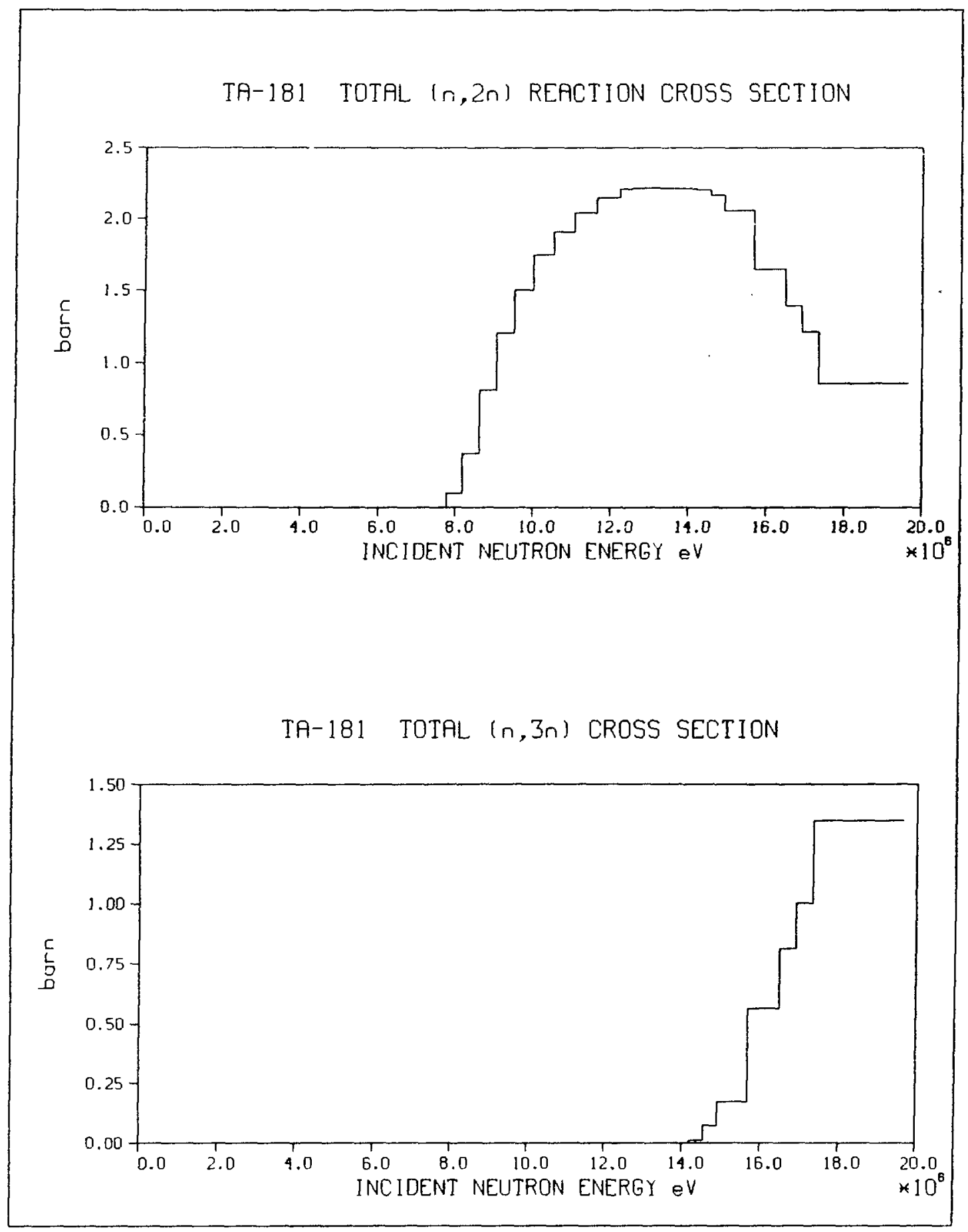


W-182 RECOMMENDEO PROMPT KERMA FACTOR

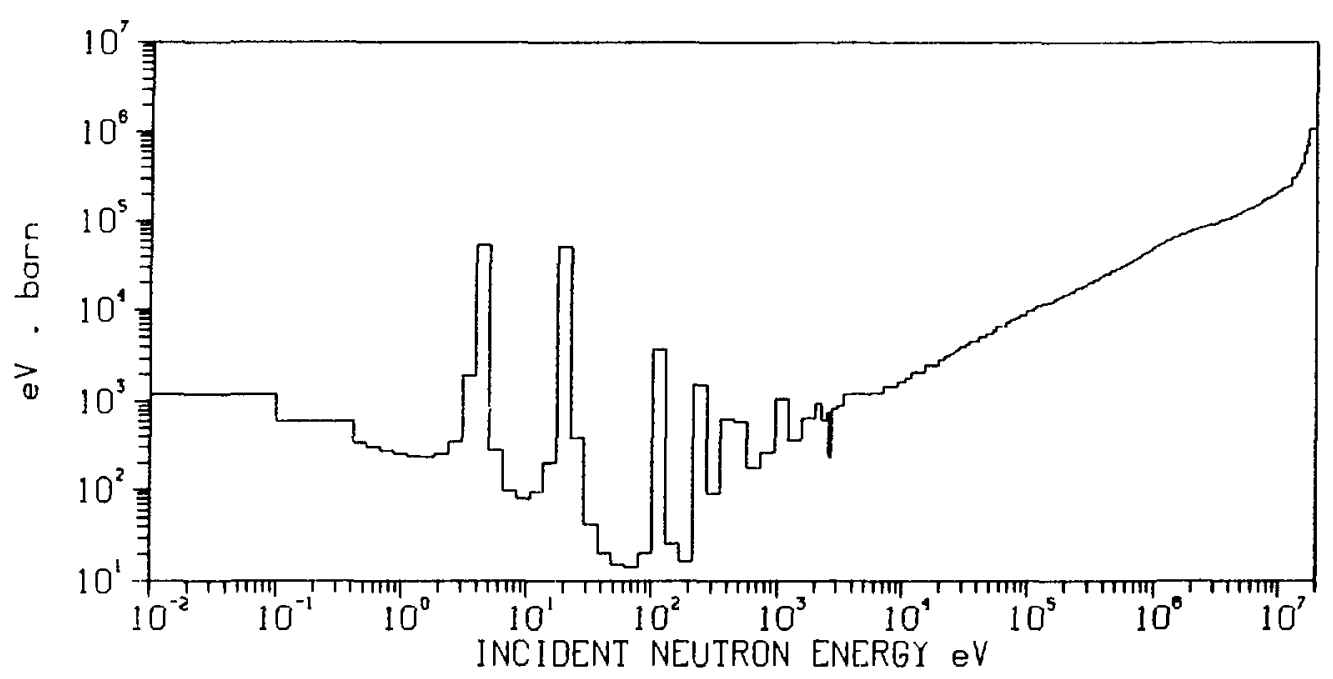

W-182 RECOMMENDED PROMPT KERMA FACTOR

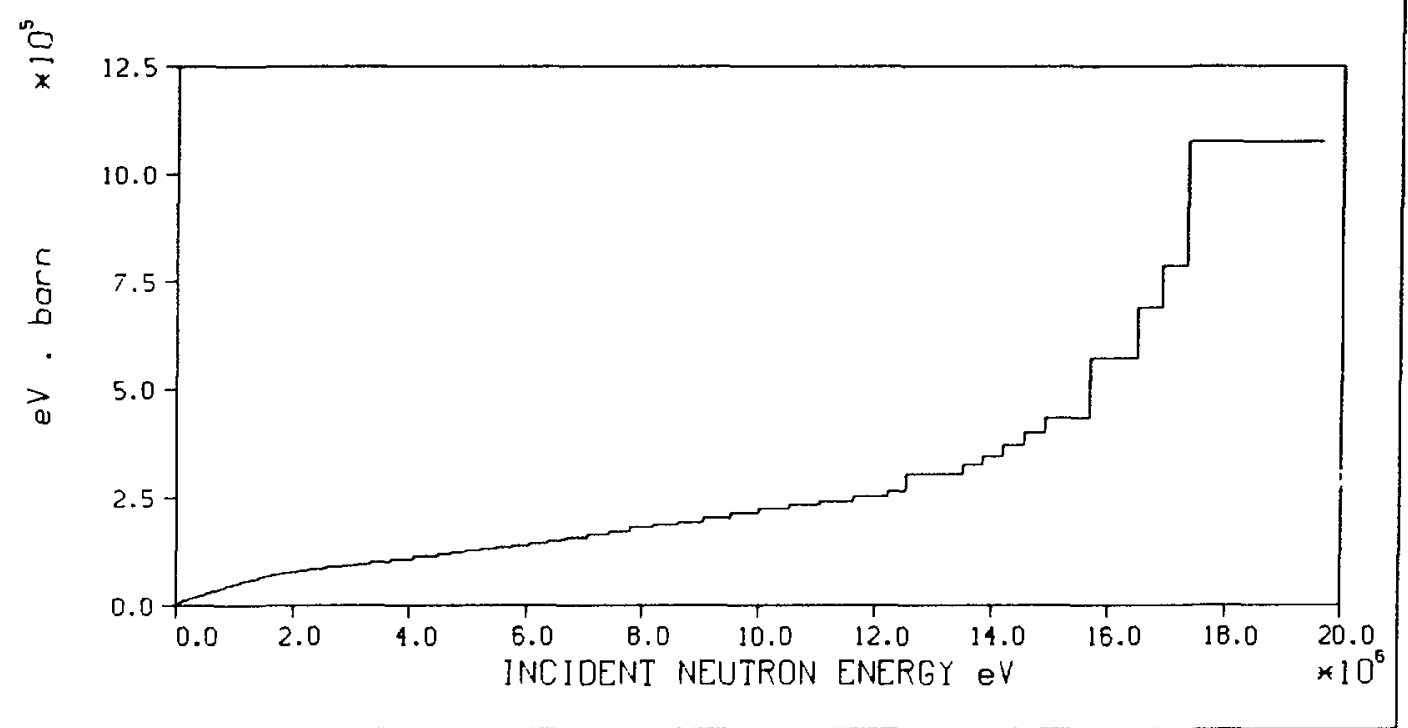


W-182 PROMPT GAMMA ENERGY PRODUCTION

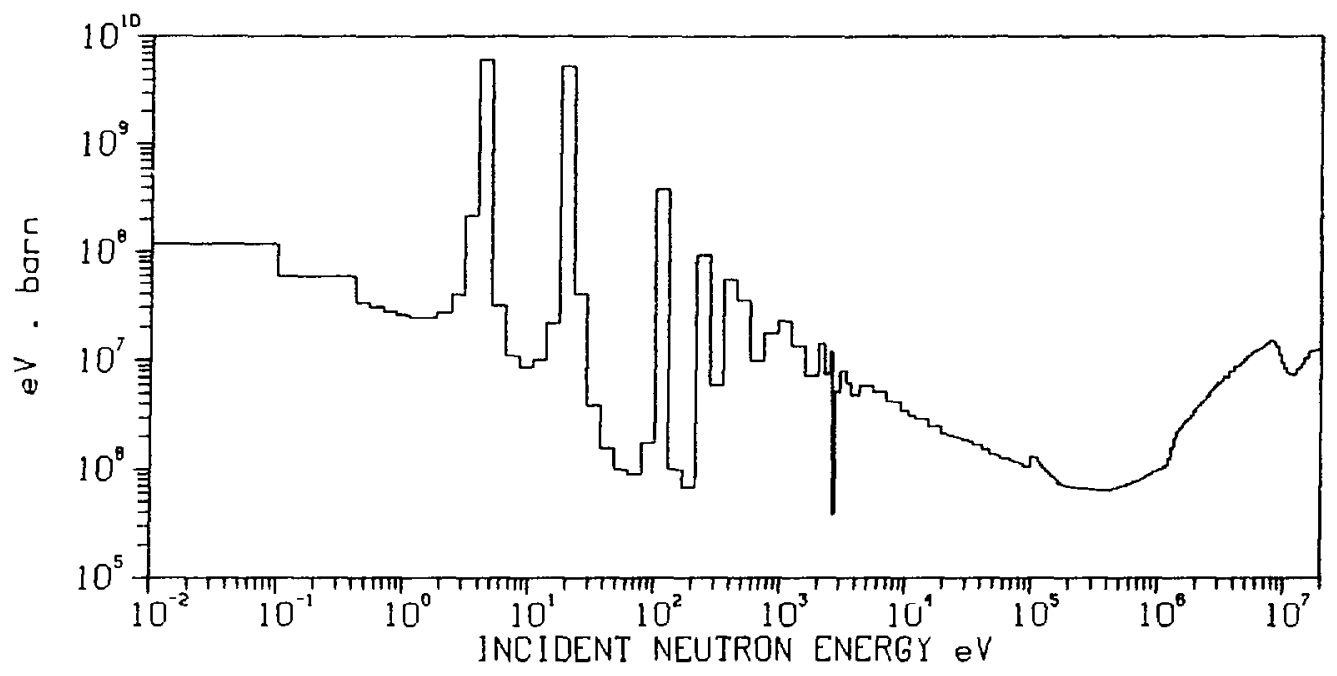

W-182 HYOROGEN-1 PRODUCTION CROSS SECTION

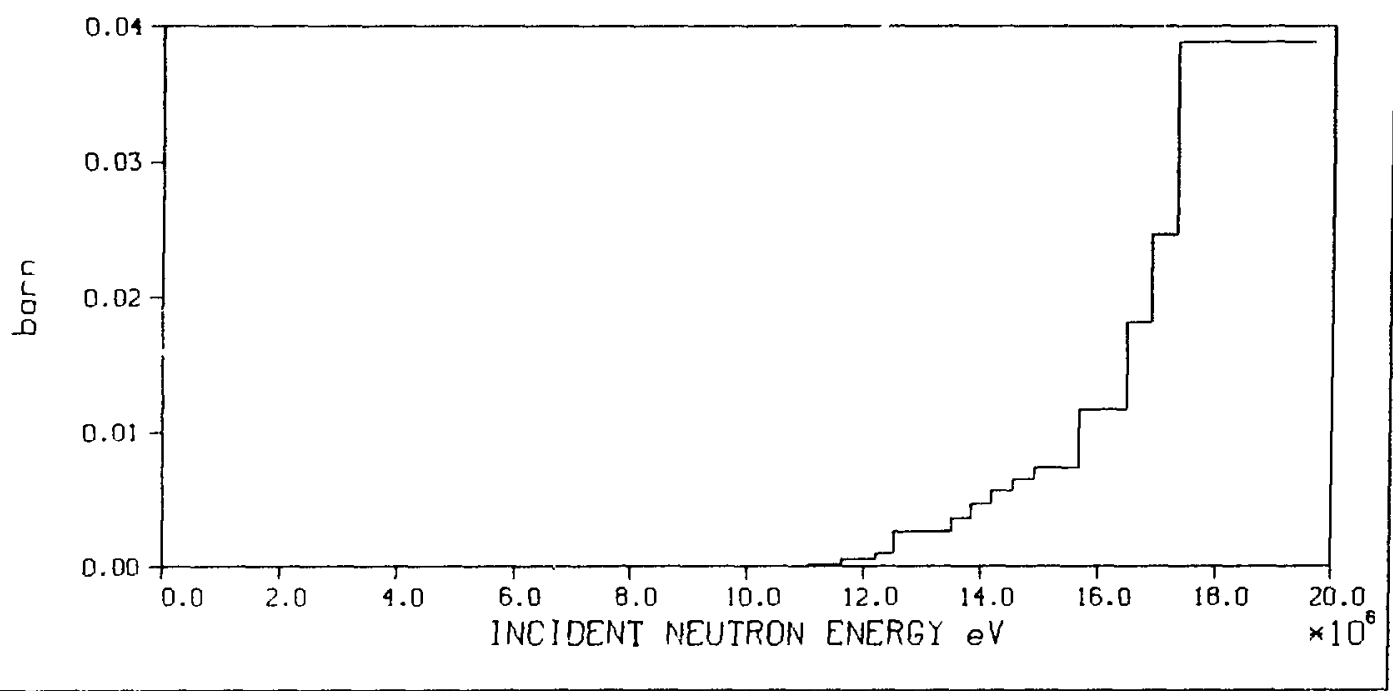




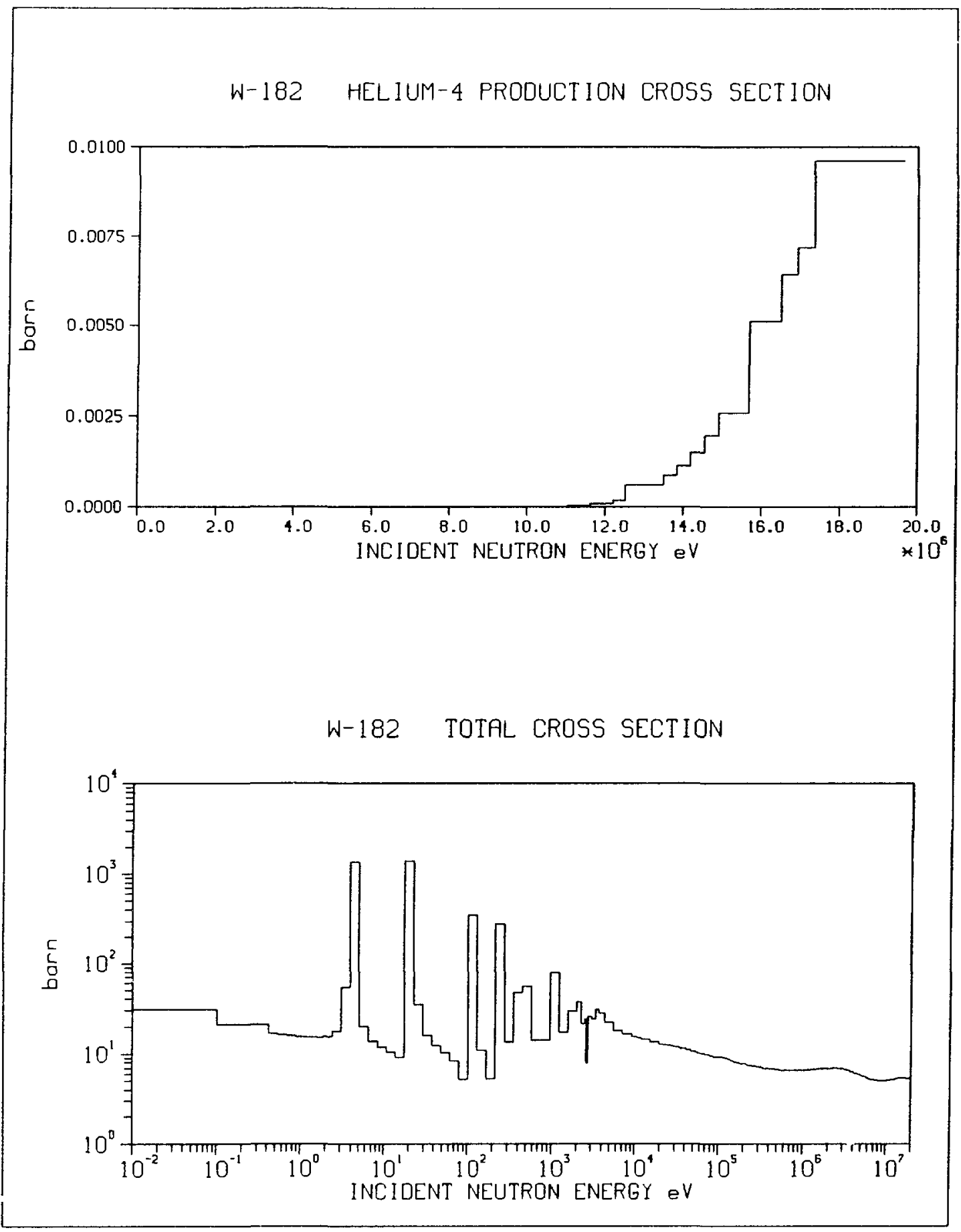




\section{W-182 ELASTIC CROSS SECTION}

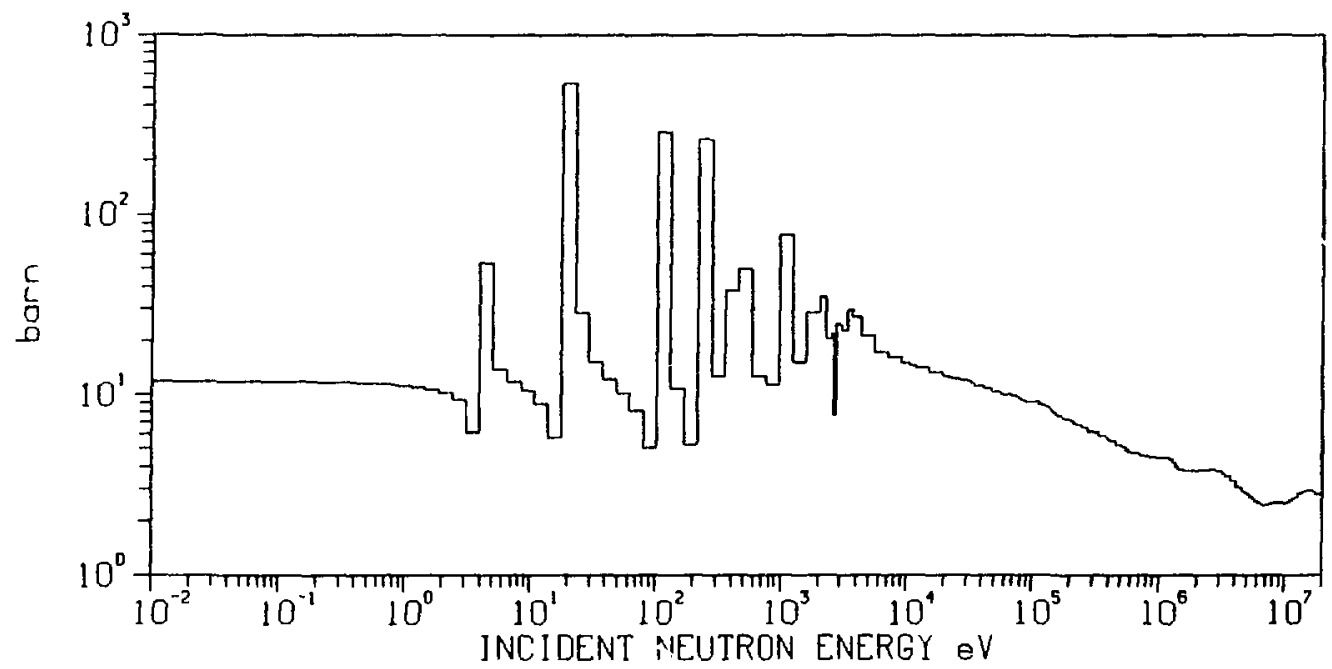

W-182 TOTAL INELASTIC CROSS SECTION

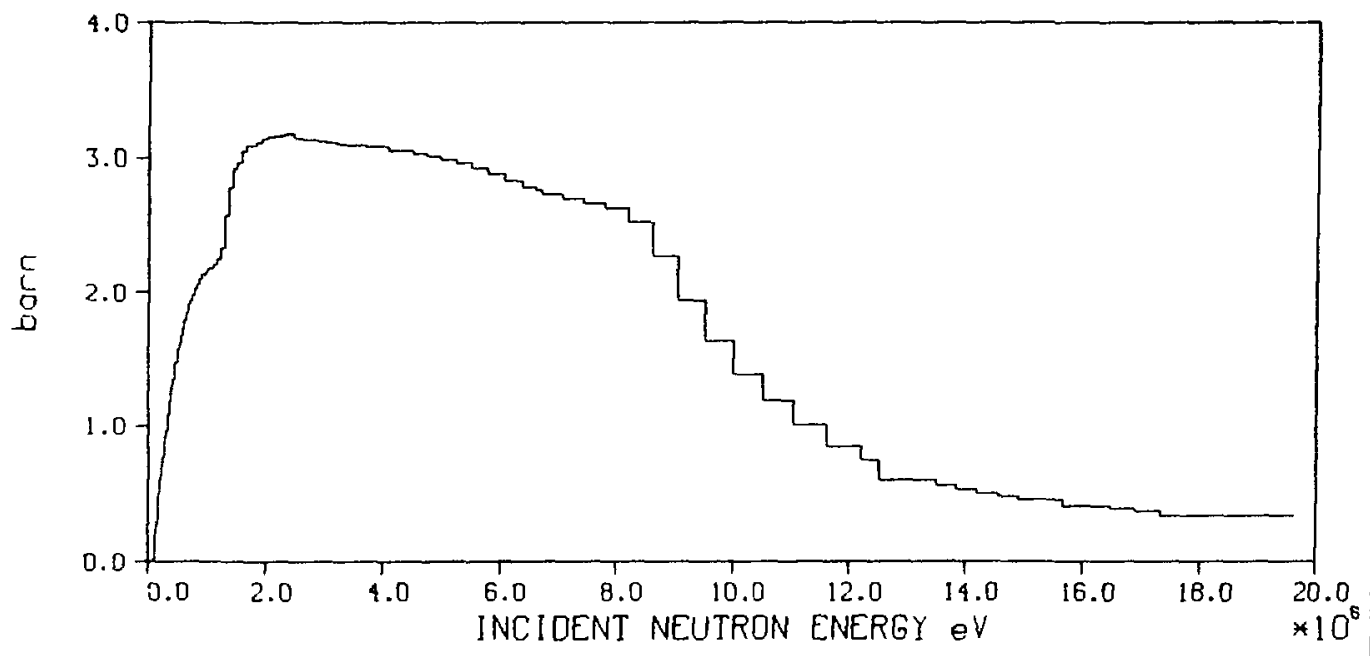


W-182 RAOIATIVE CAPTURE CROSS SECTION

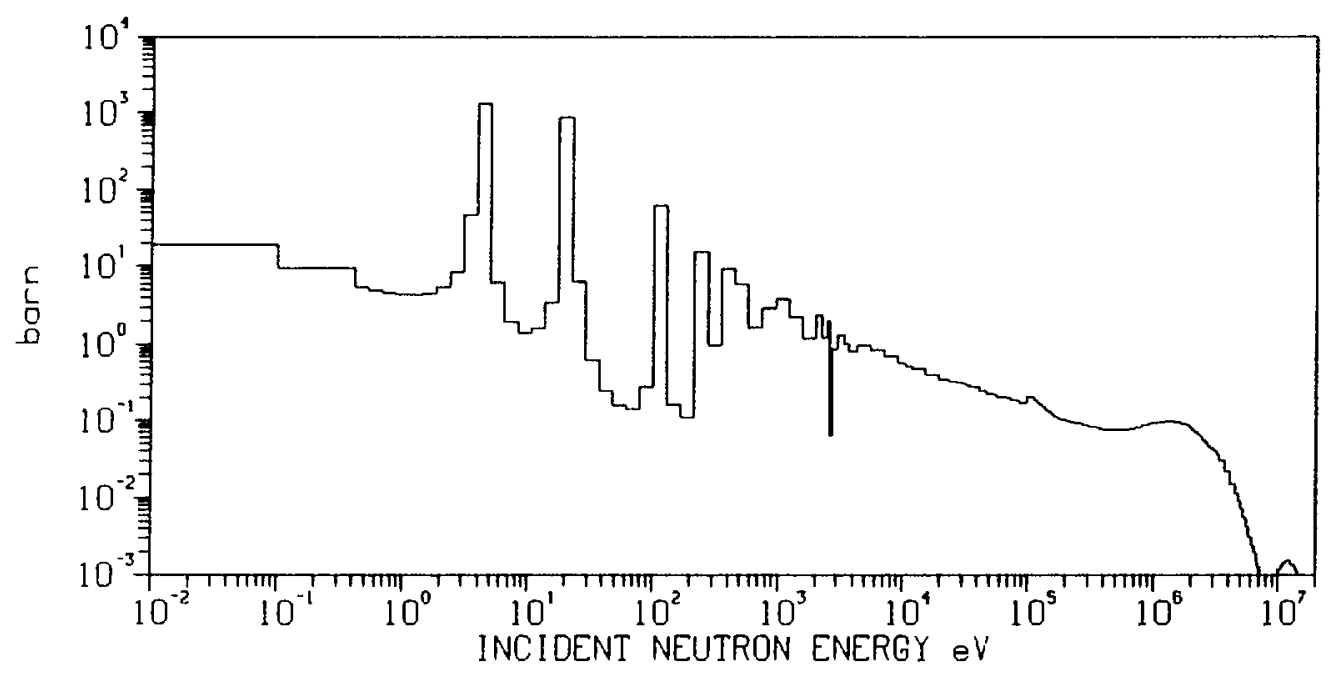

W-182 TOTAL $(n, 2 n)$ REACTION CROSS SECTION

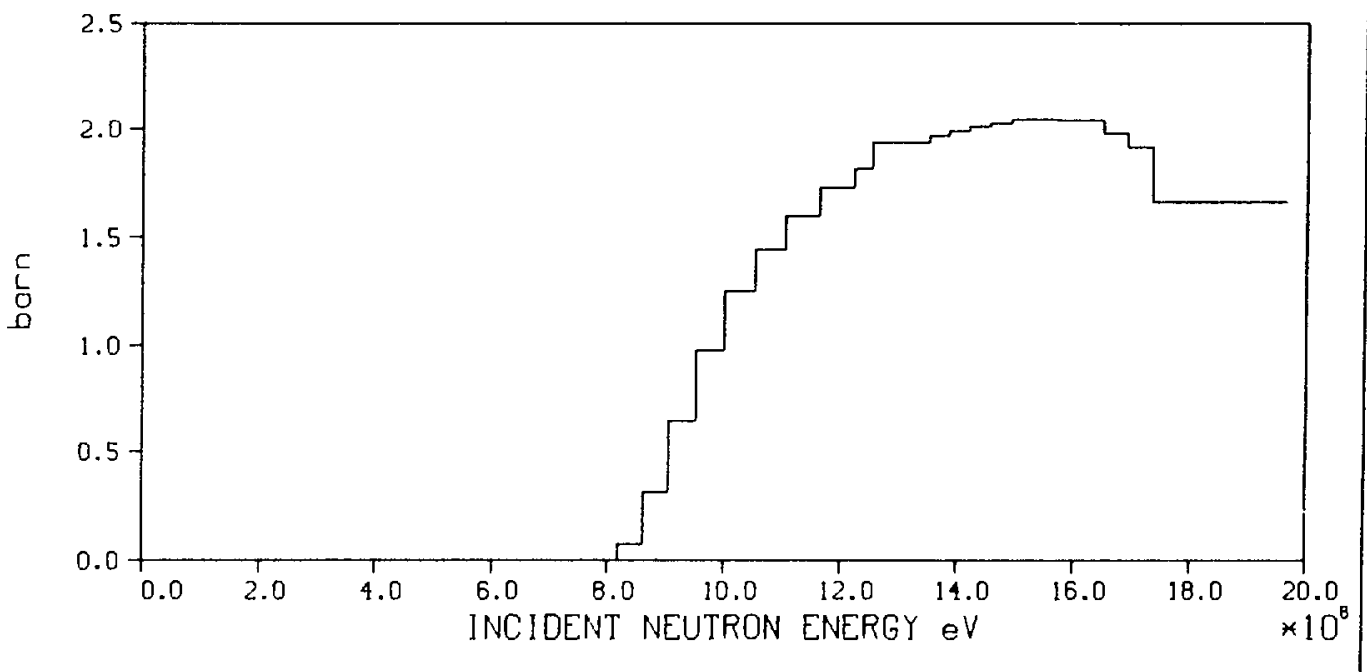




\section{w-182 TOTAL $(n, 3 n)$ CROSS SECTION}

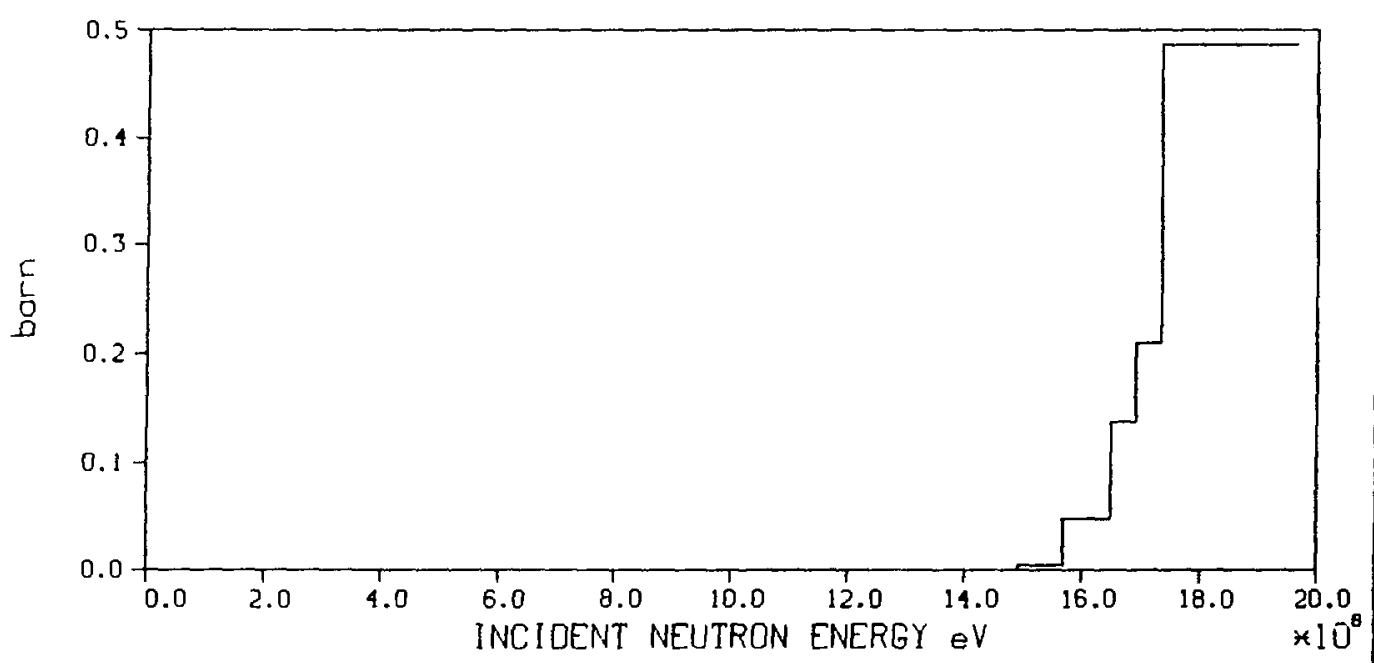




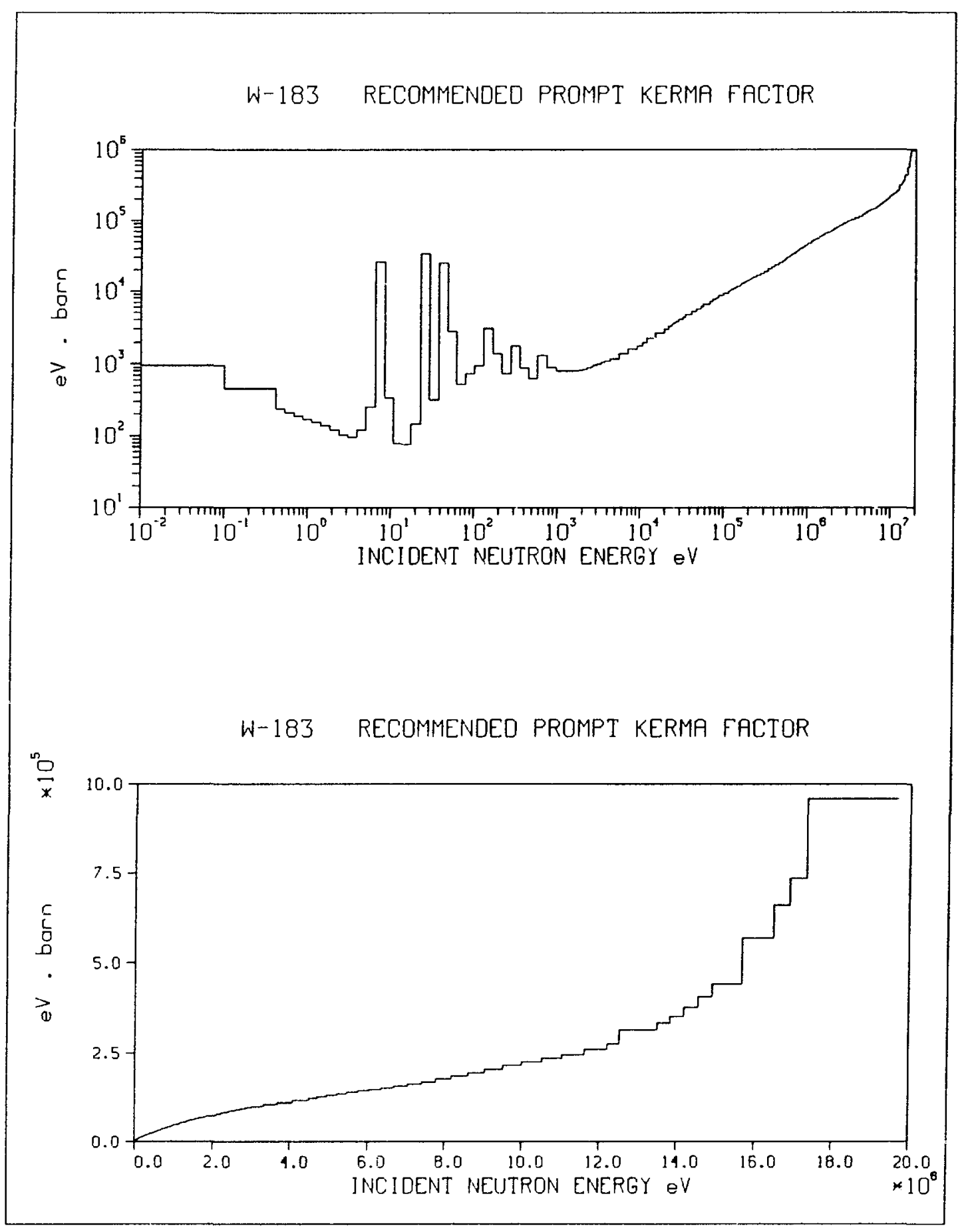




\section{W-183 PROMPT GAMMA ENERGI PRODUCTION}

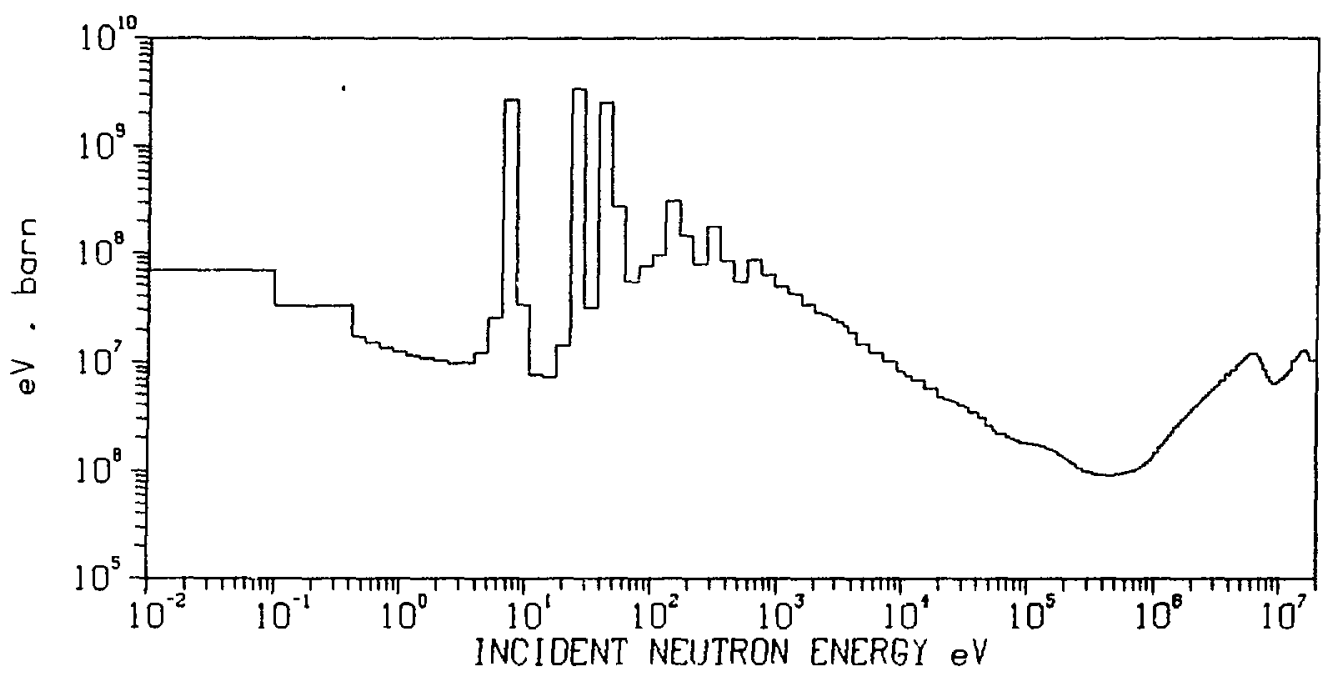

W-183 HYOROGEN-1 PRODUCTION CRQSS SECTION

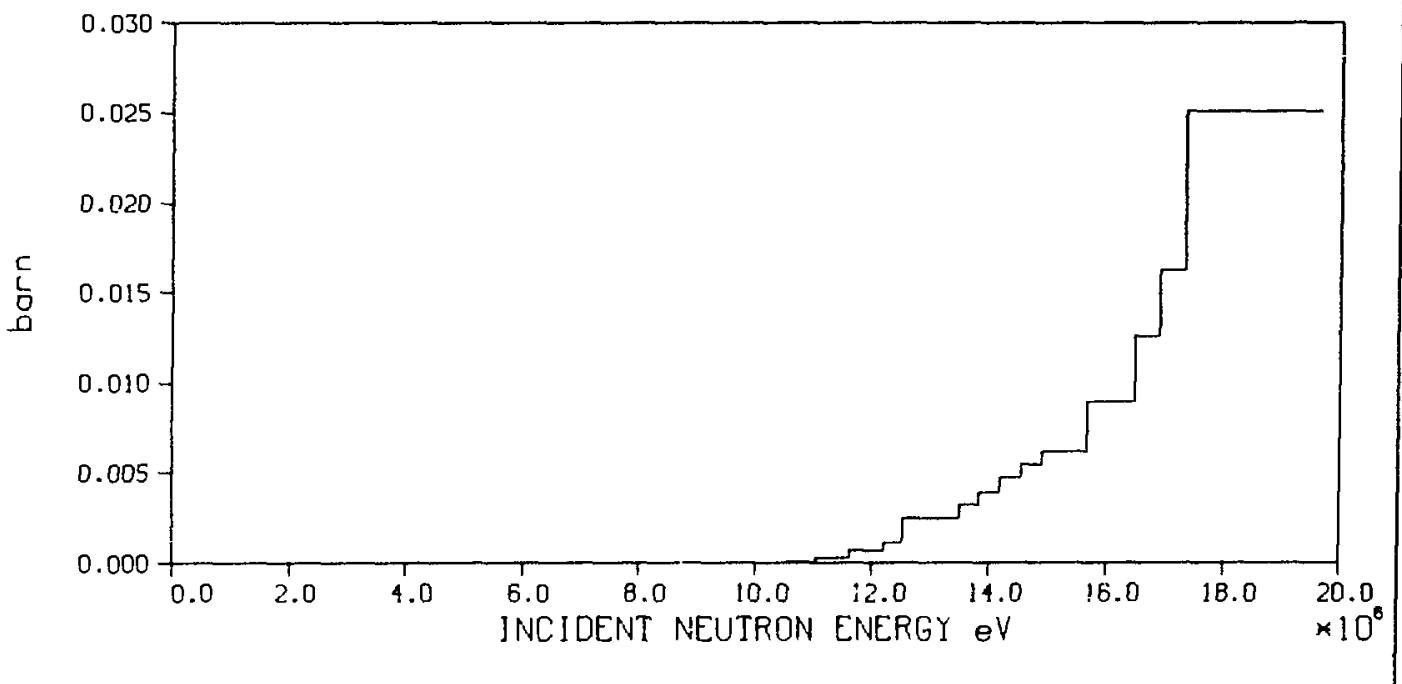




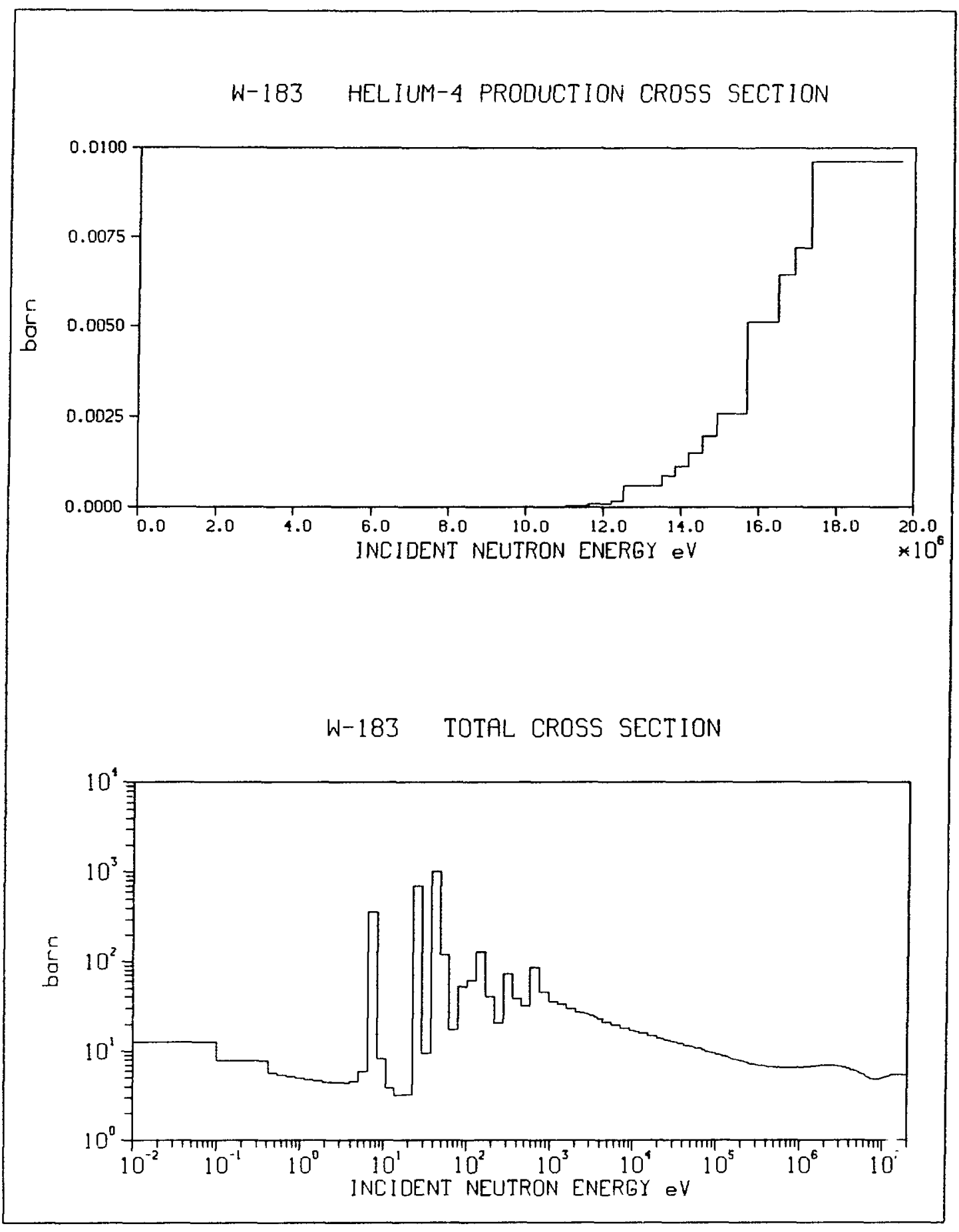




\section{W-183 ELASTIC CROSS SECTION}

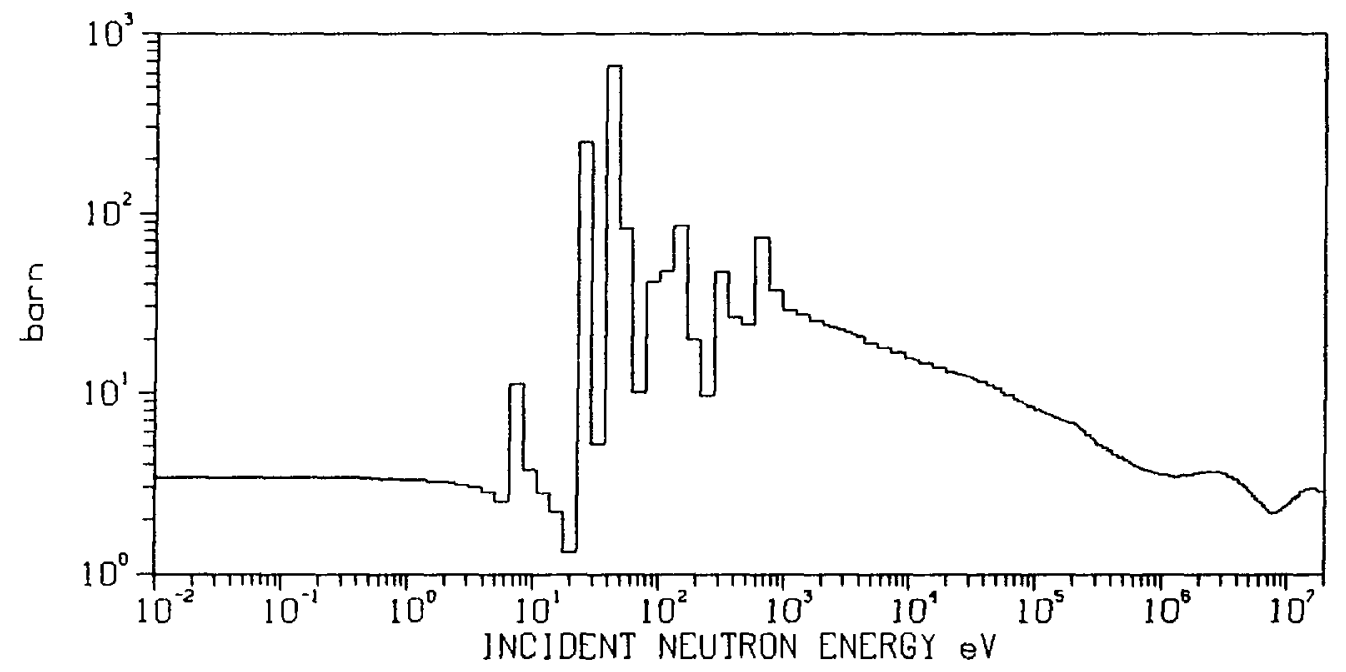

W-183 TOTAL INELASTIC CROSS SECTION

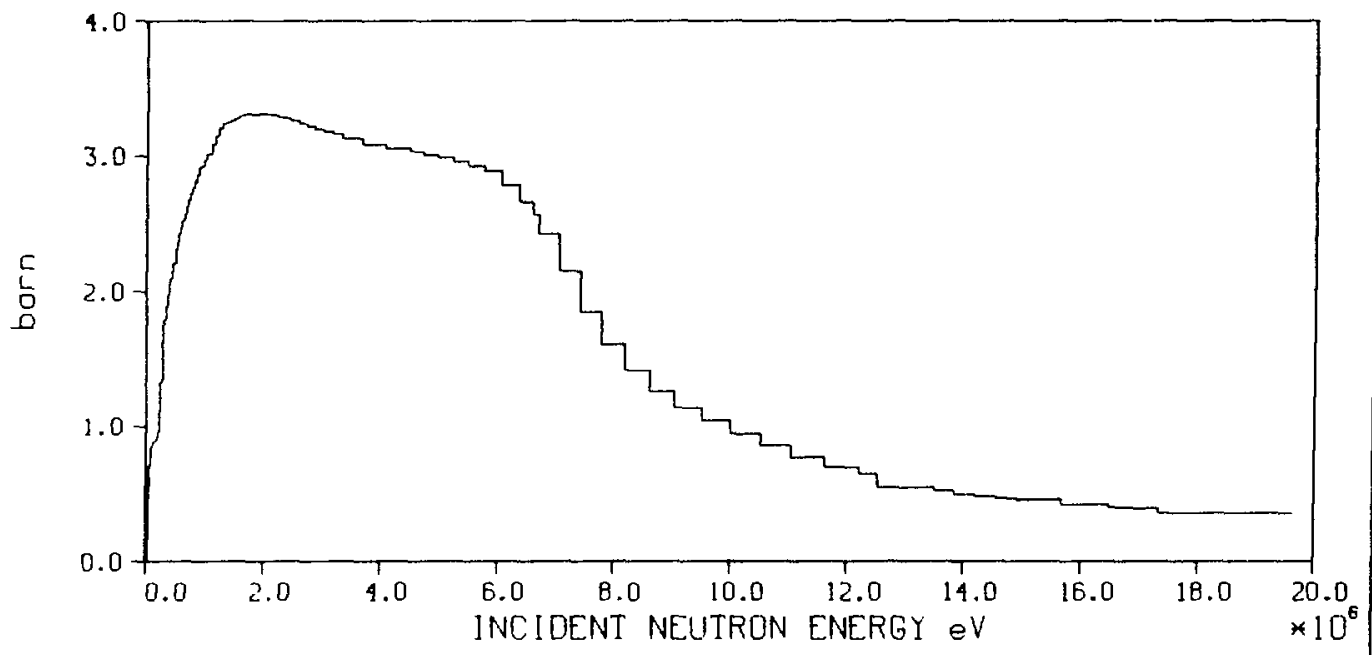




\section{W-183 RADIATIVE CAPTURE CROSS SECTION}

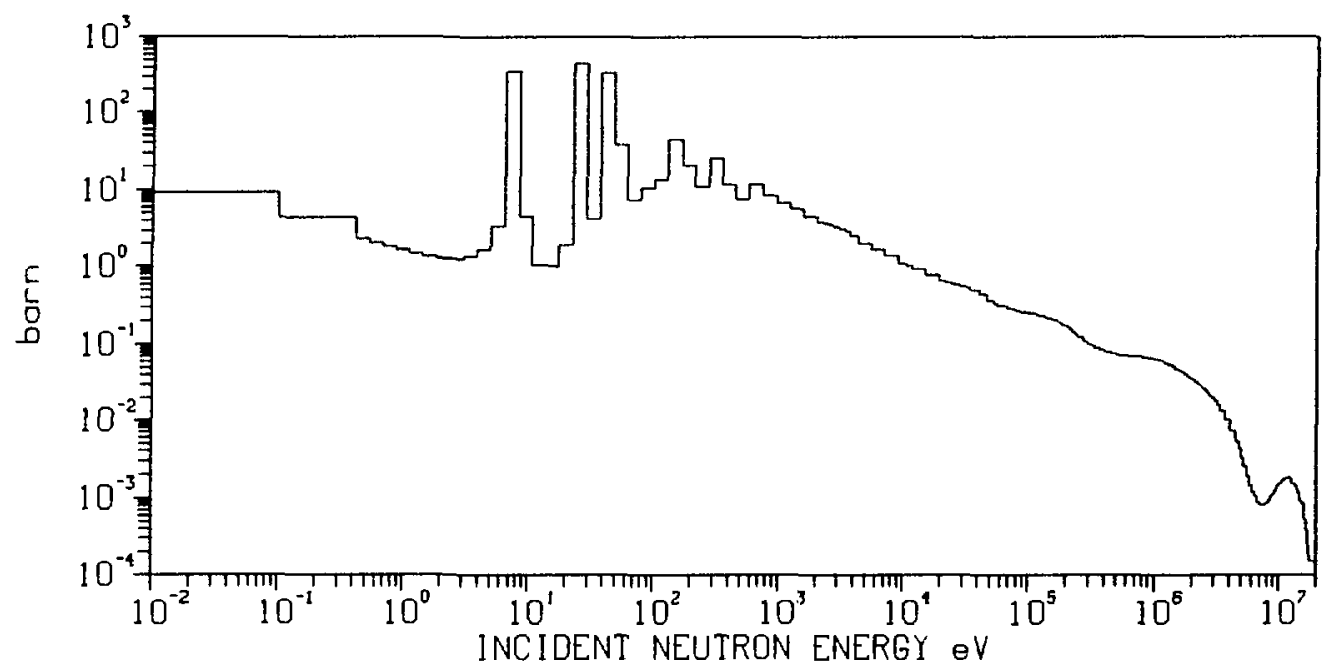

W-183 TOTAL $(n, 2 n)$ REACTION CROSS SECTION

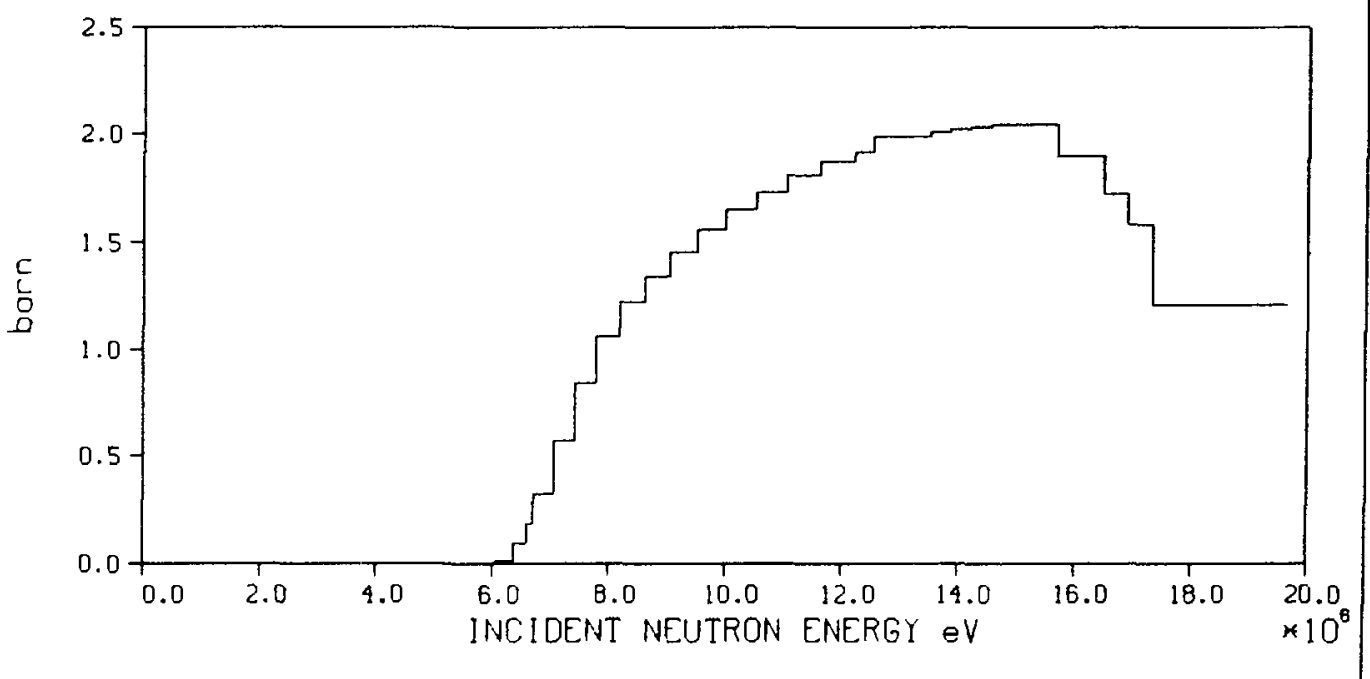


w-183 TOTAL $(n, 3 n)$ CROSS SECTION

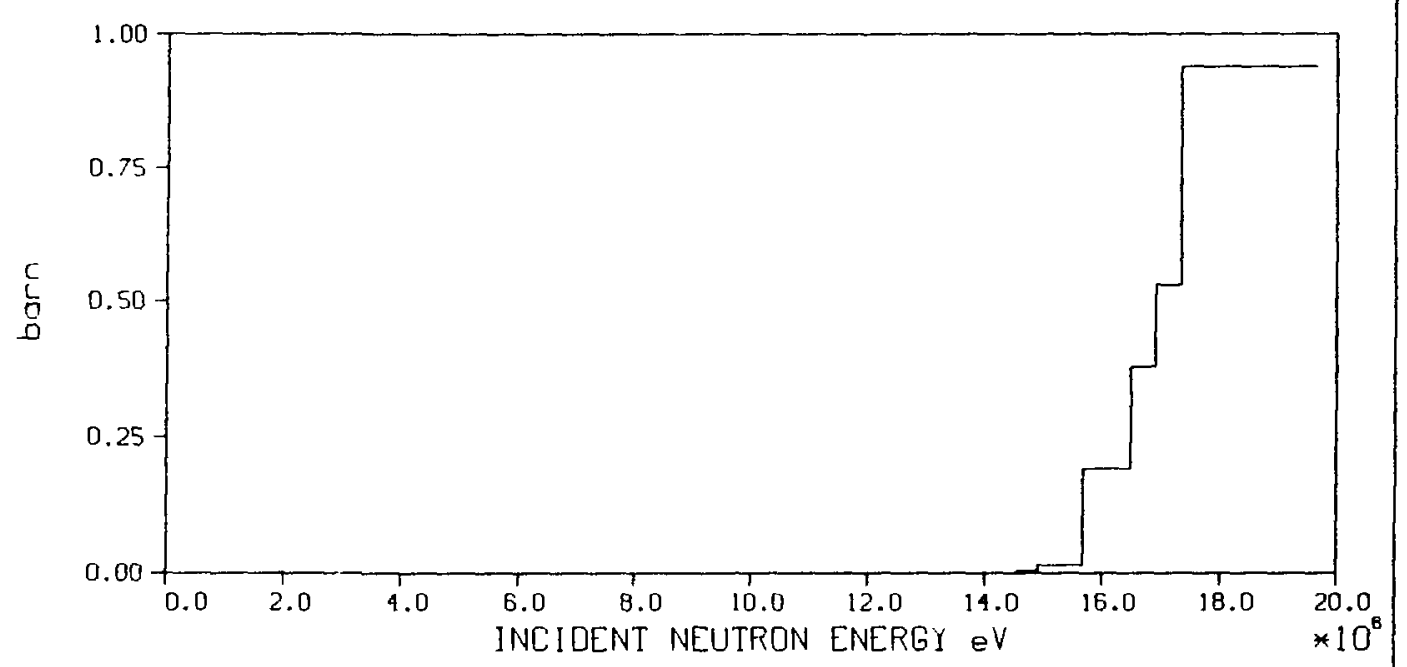


W-184 RECOMMENDED PROMPT KERMA FACTOR (solid line) REC. K. FACTOR WITH CH. PARTICLE DECAY HEAT (dashed line)

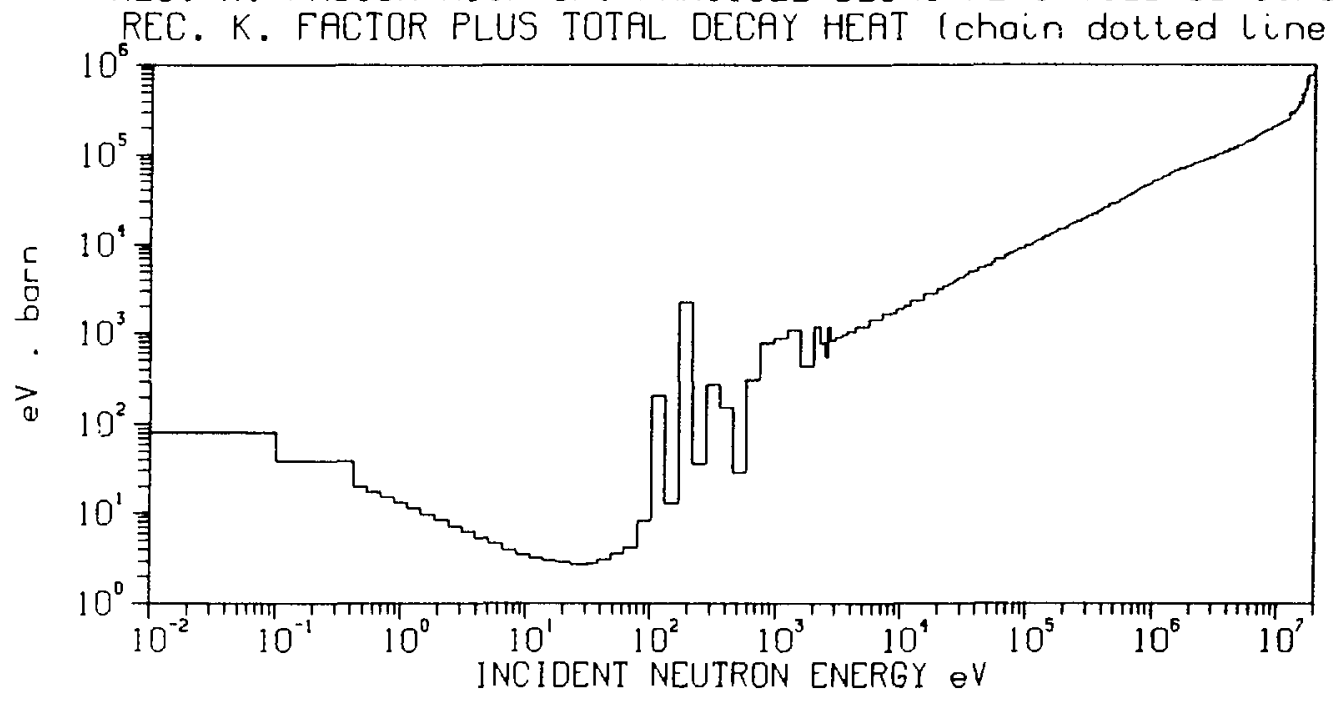

W-184 RECOMMENDED PROMPT KERMA FACTOR (solid line) REC. K. FACTOR WITH CH. PARTICLE DECAY HEAT (dashed line) "REC. K. FACTOR PLUS TOTAL DECAY HEAT (chain dotted line)

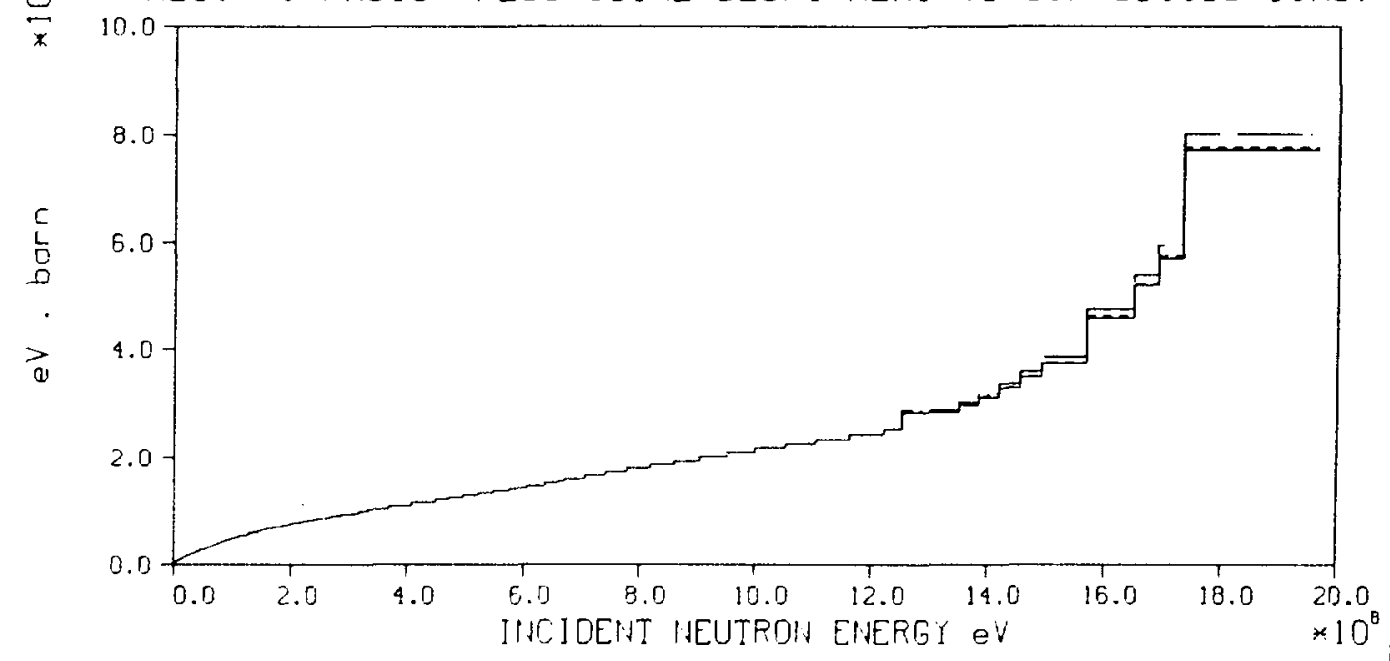




\section{W-184 PROMPT GAMMA ENERGY PRODUCTION}

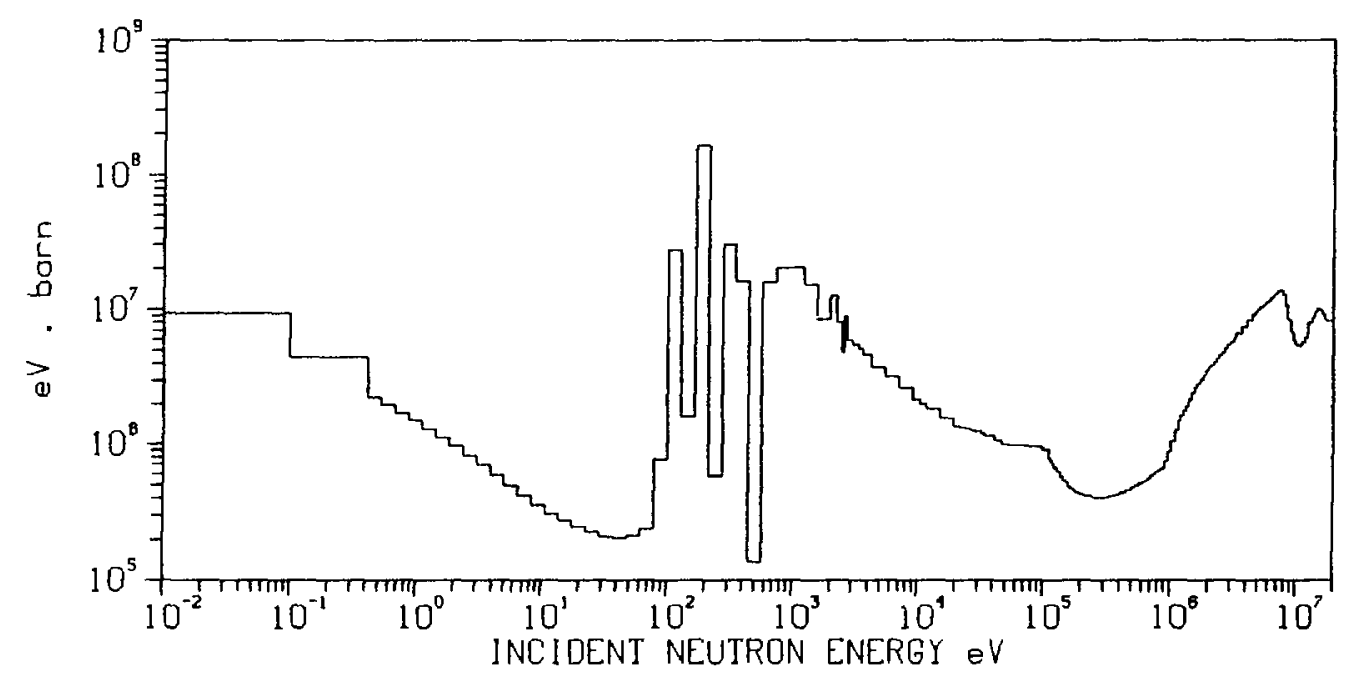

W-184 HYDROGEN-1 PRODUCTION CROSS SECTION

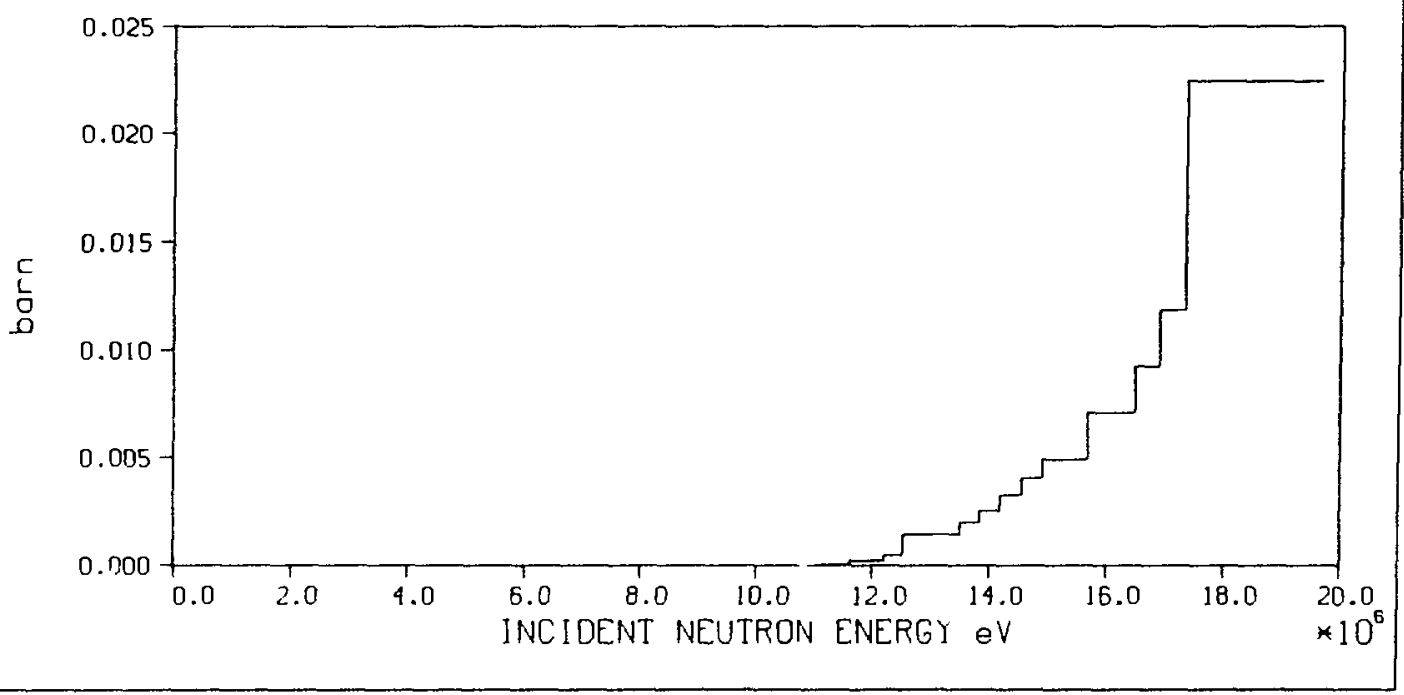




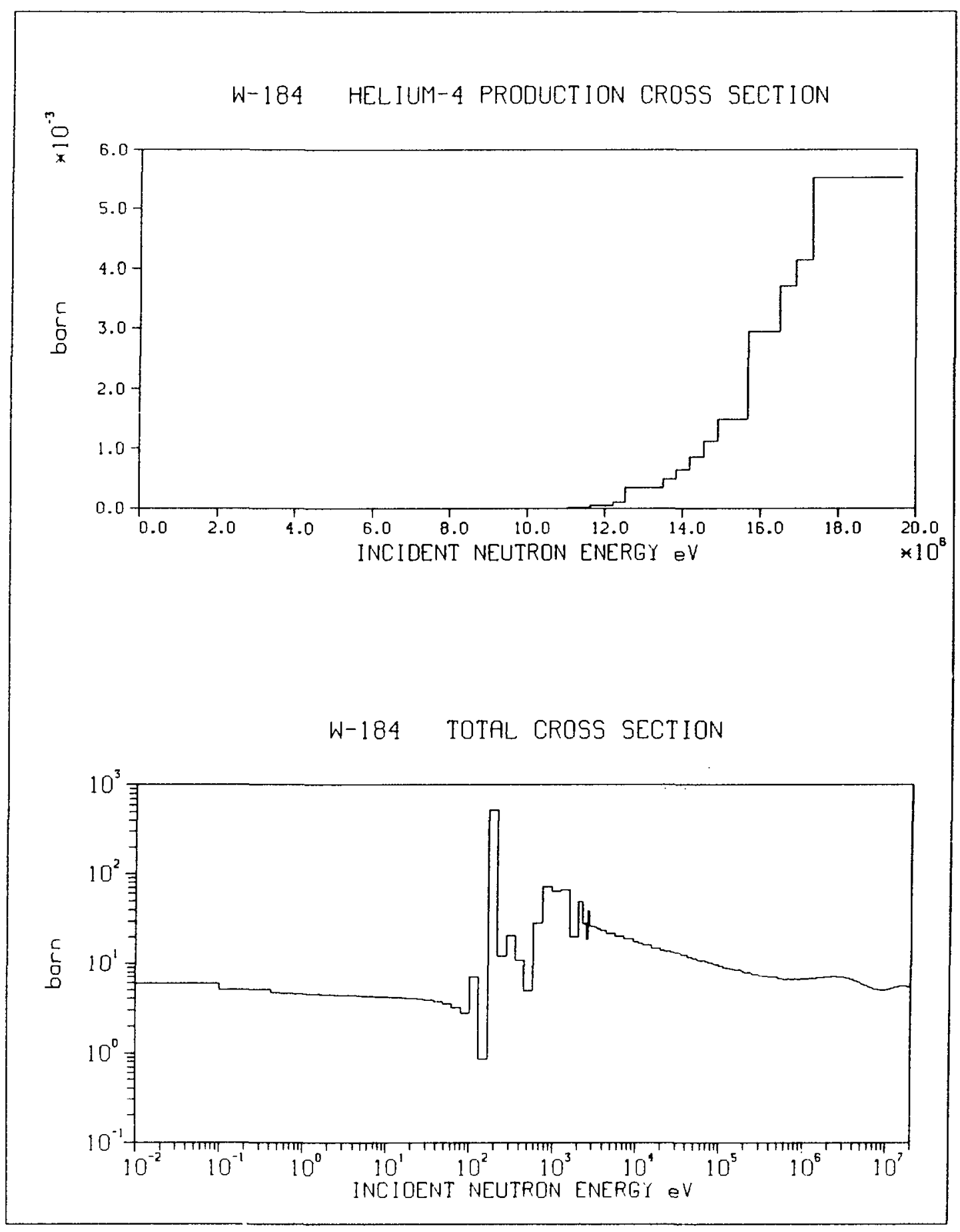




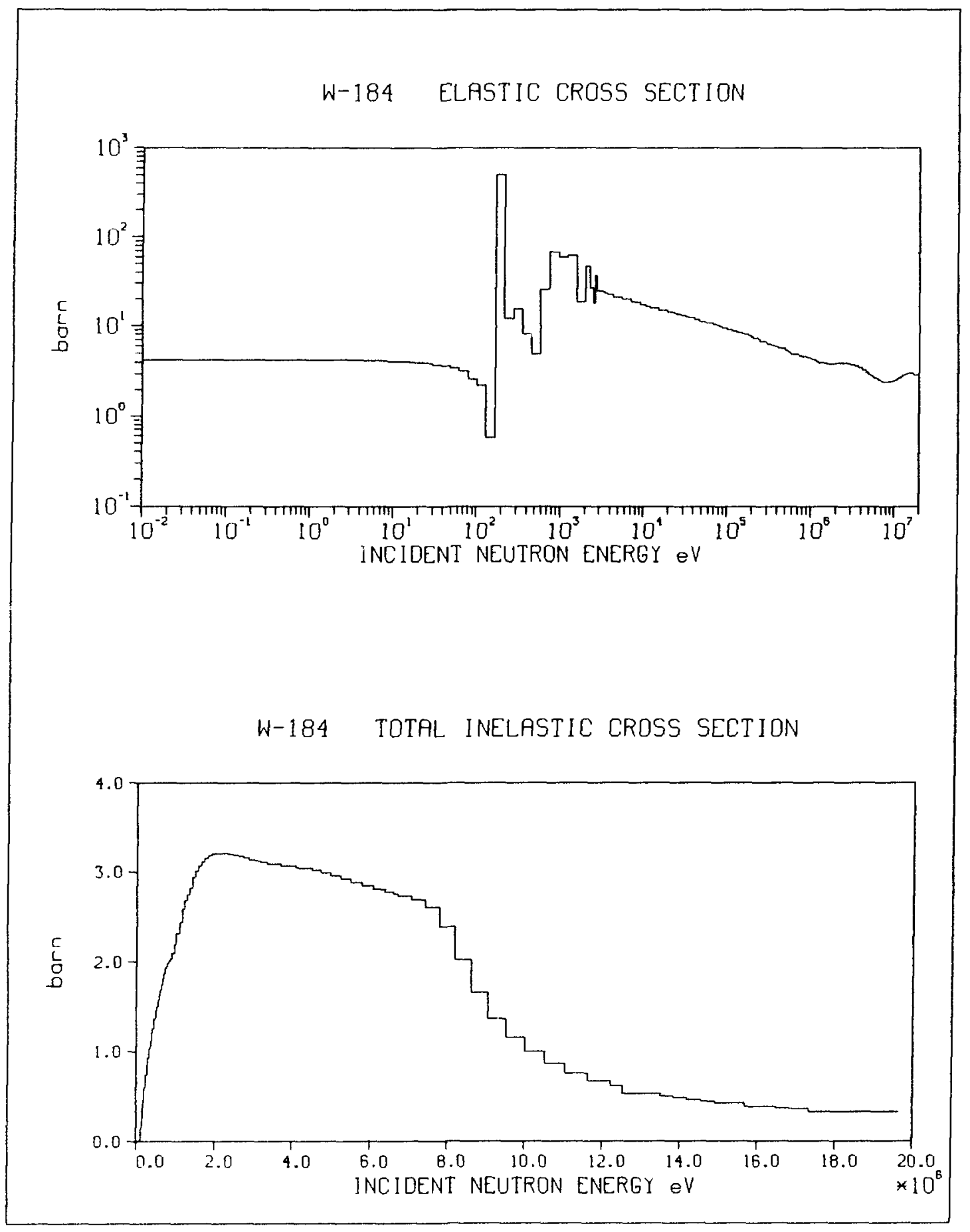




\section{W-184 RADIATIVE CAPTURE CROSS SECTION}

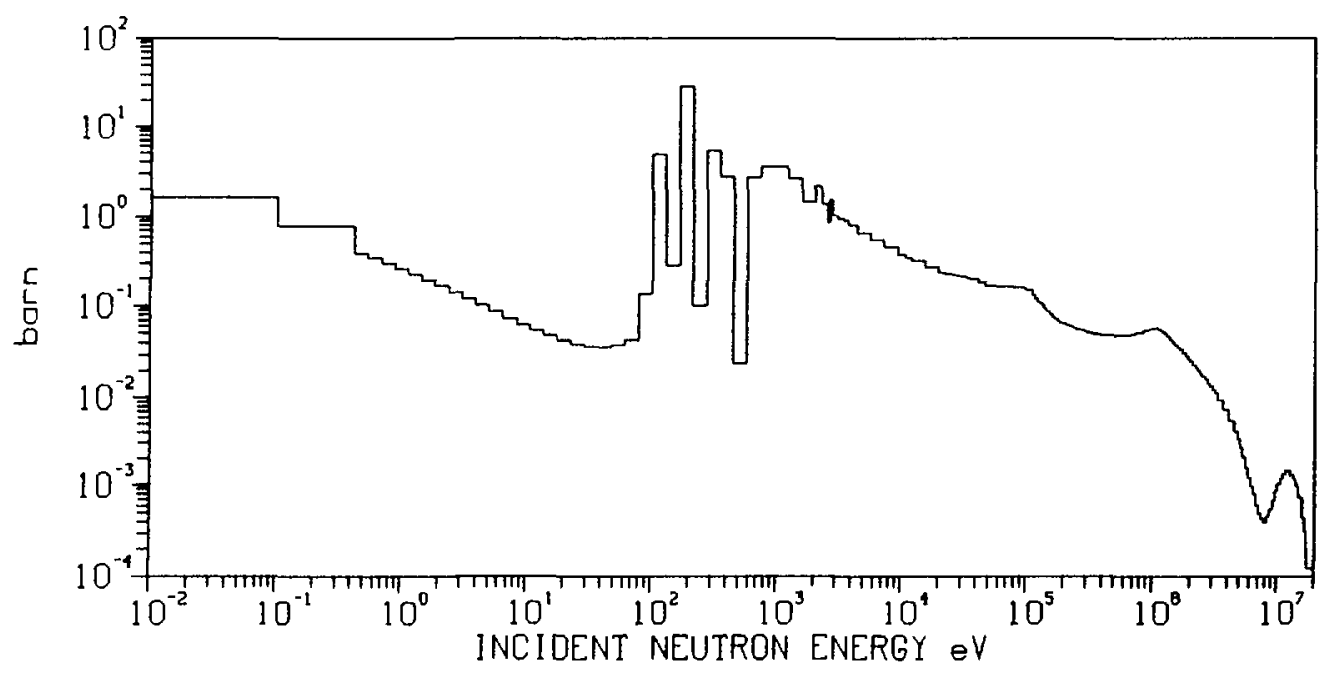

W-184 TOTAL $(n, 2 n)$ REACTION CROSS SECTION

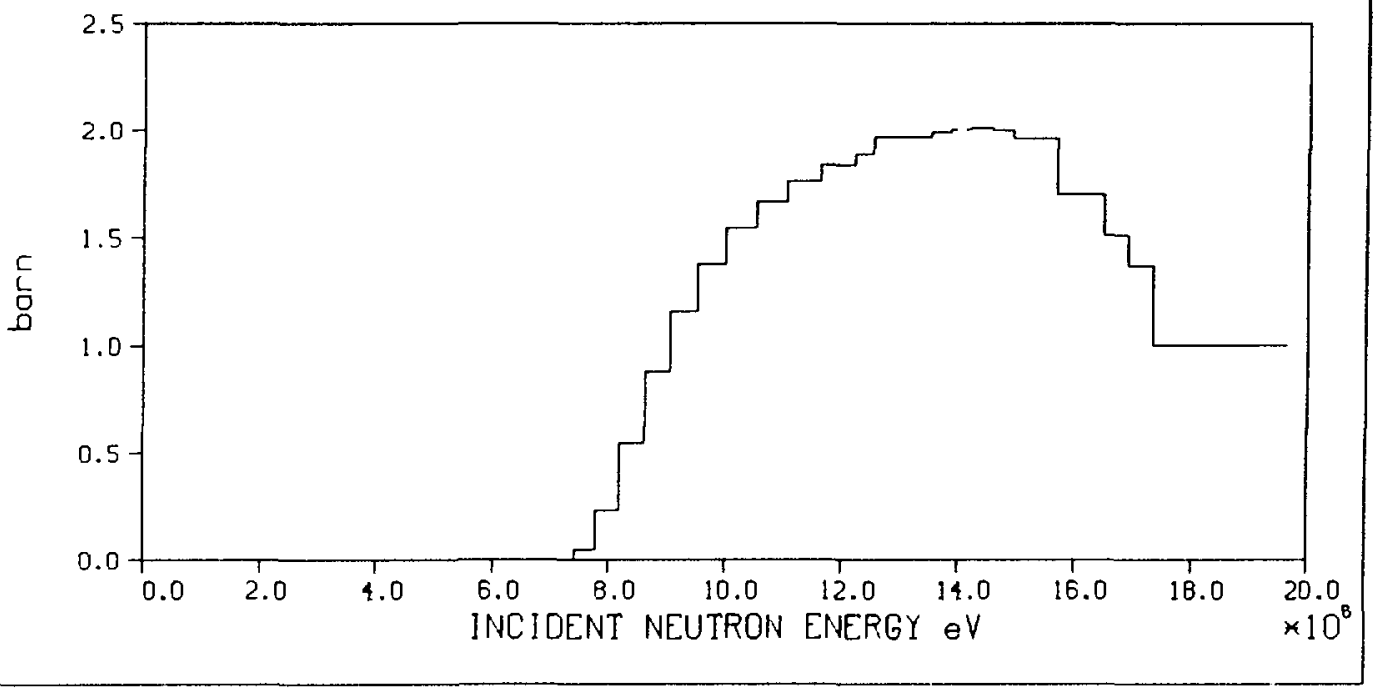




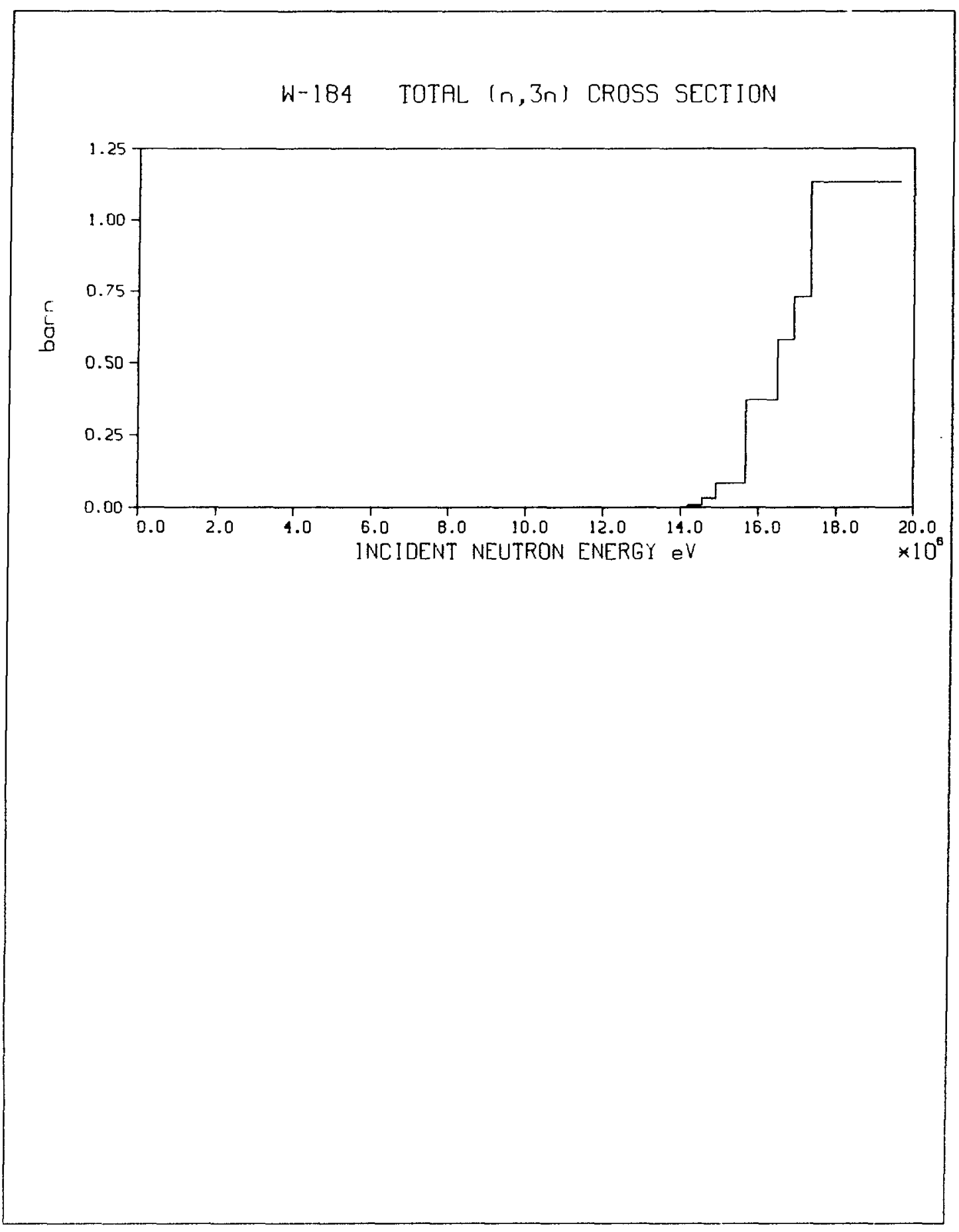




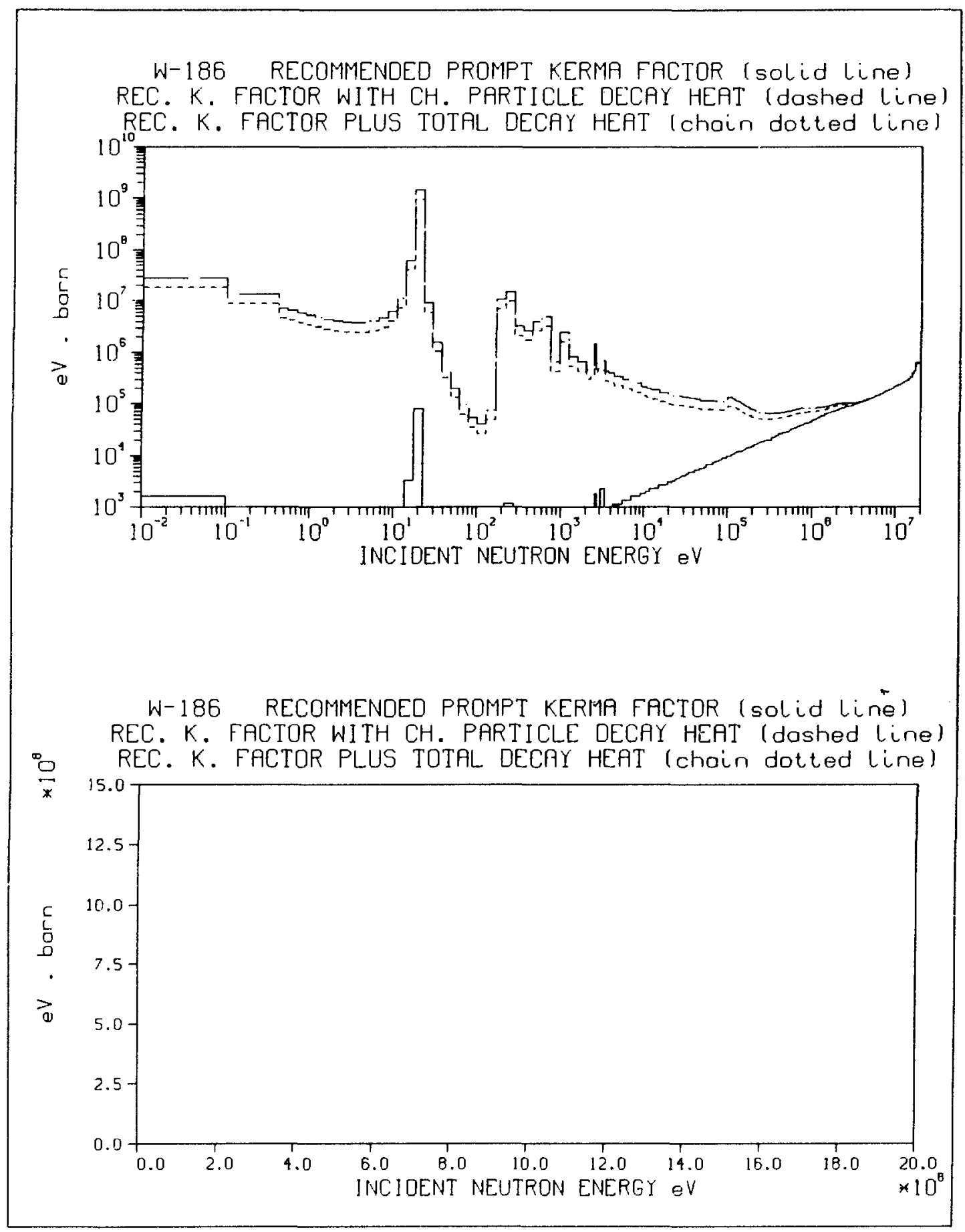


W-186 PROMPT GAMMA ENERGY PRODUCTION

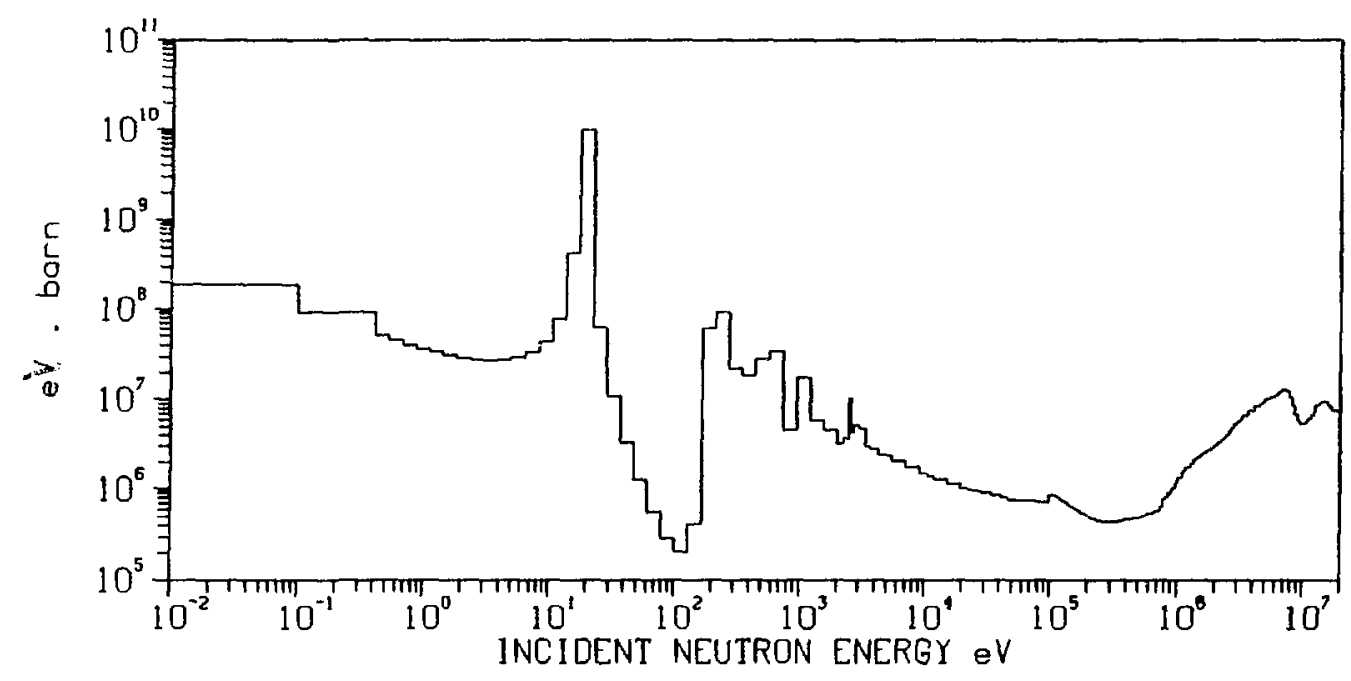

W-186 HYDROGEN-1 PRODUCTION CROSS SECTION

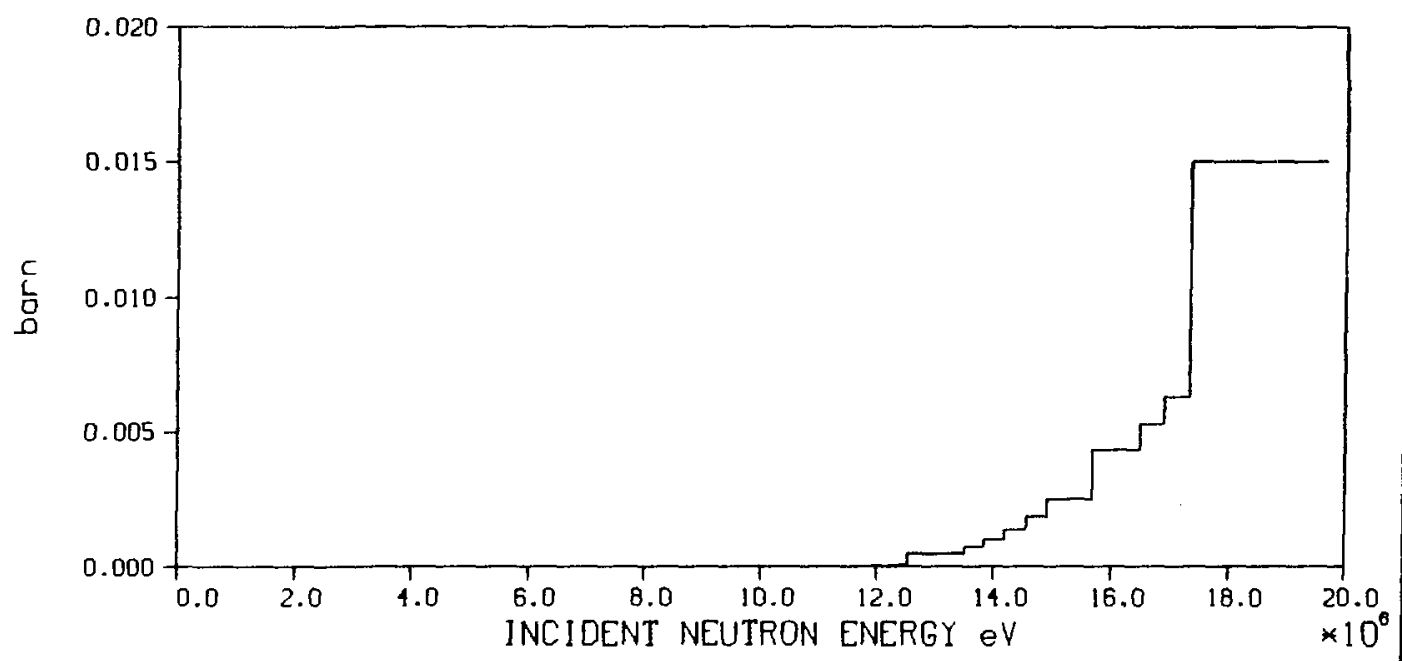




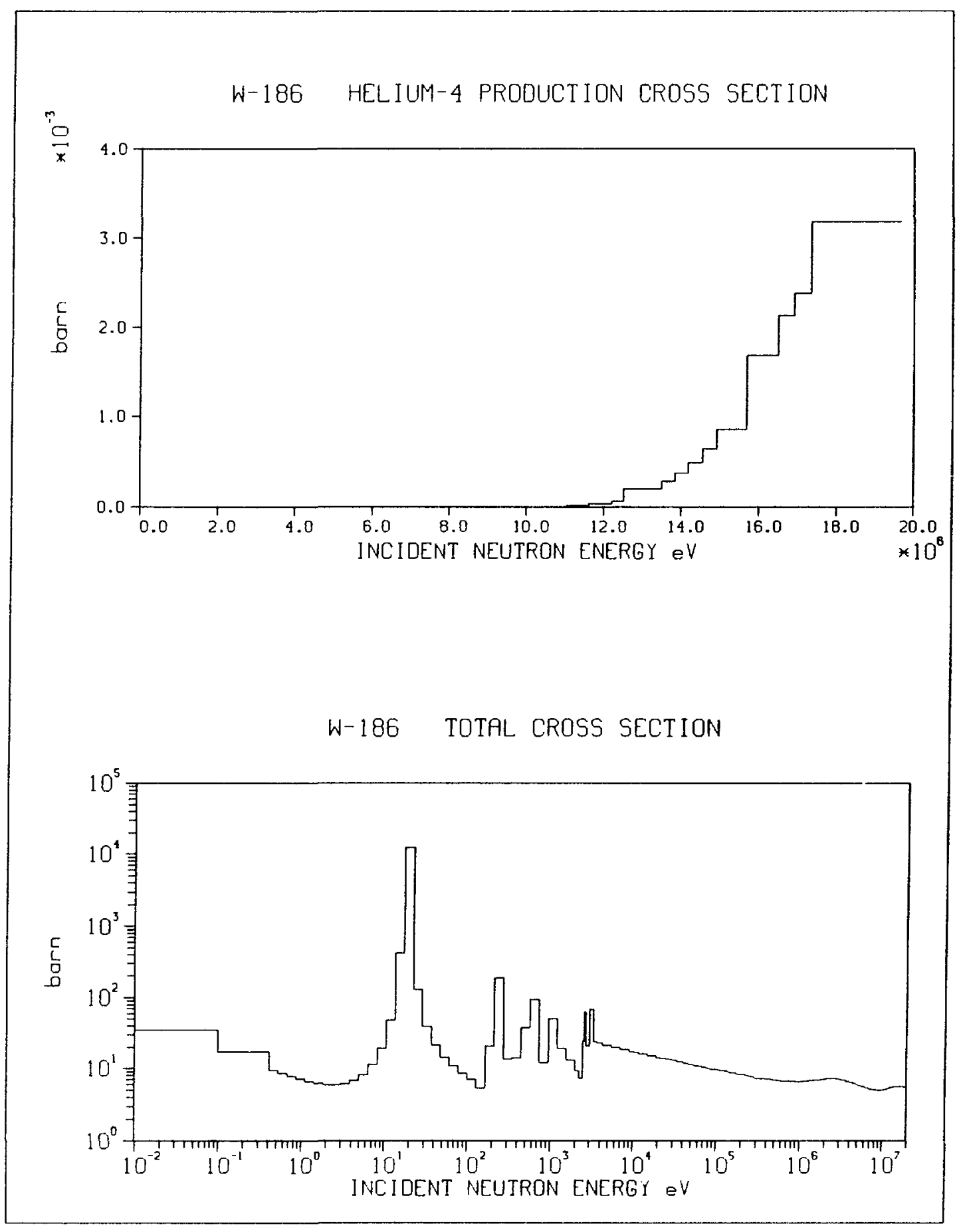




\section{W-186 ELASTIC CROSS SECTION}

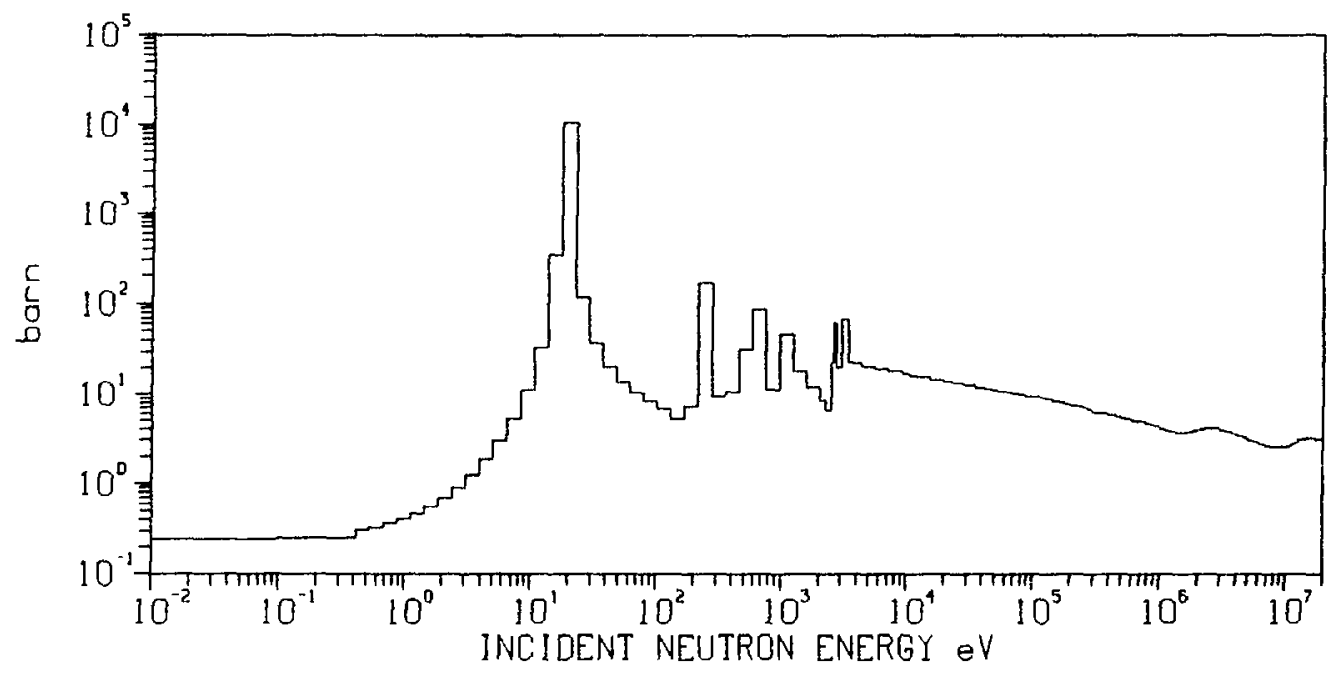

W-186 TOTAL INELASTIC CROSS SECTION

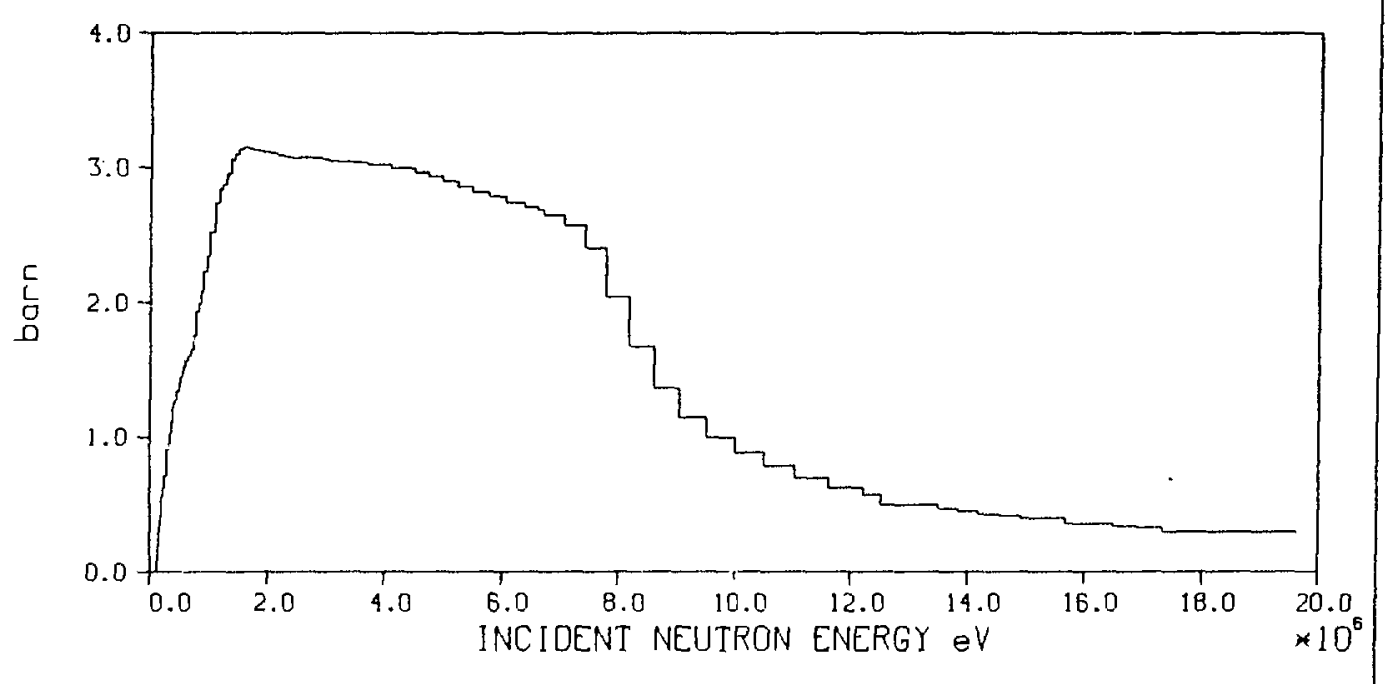




\section{W-186 RADIATIVE CAPTURE CROSS SECTION}

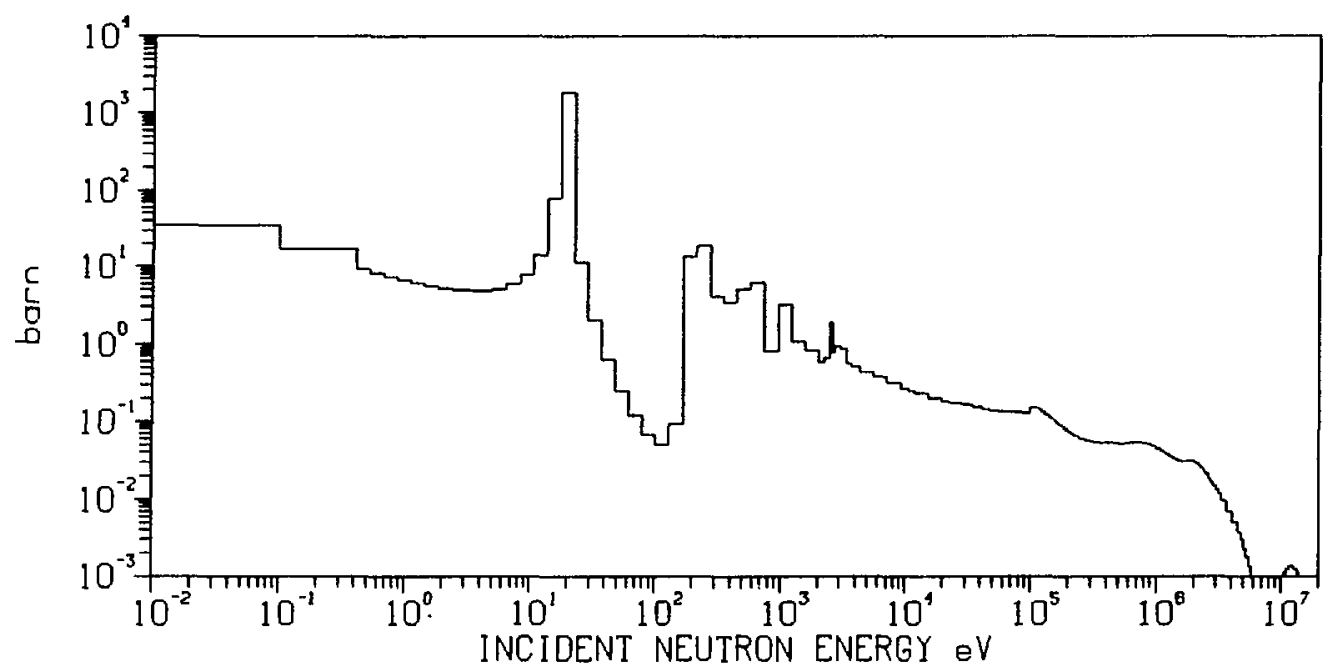

W-186 TOTAL $(n, 2 n)$ REACTION CROSS SECTION

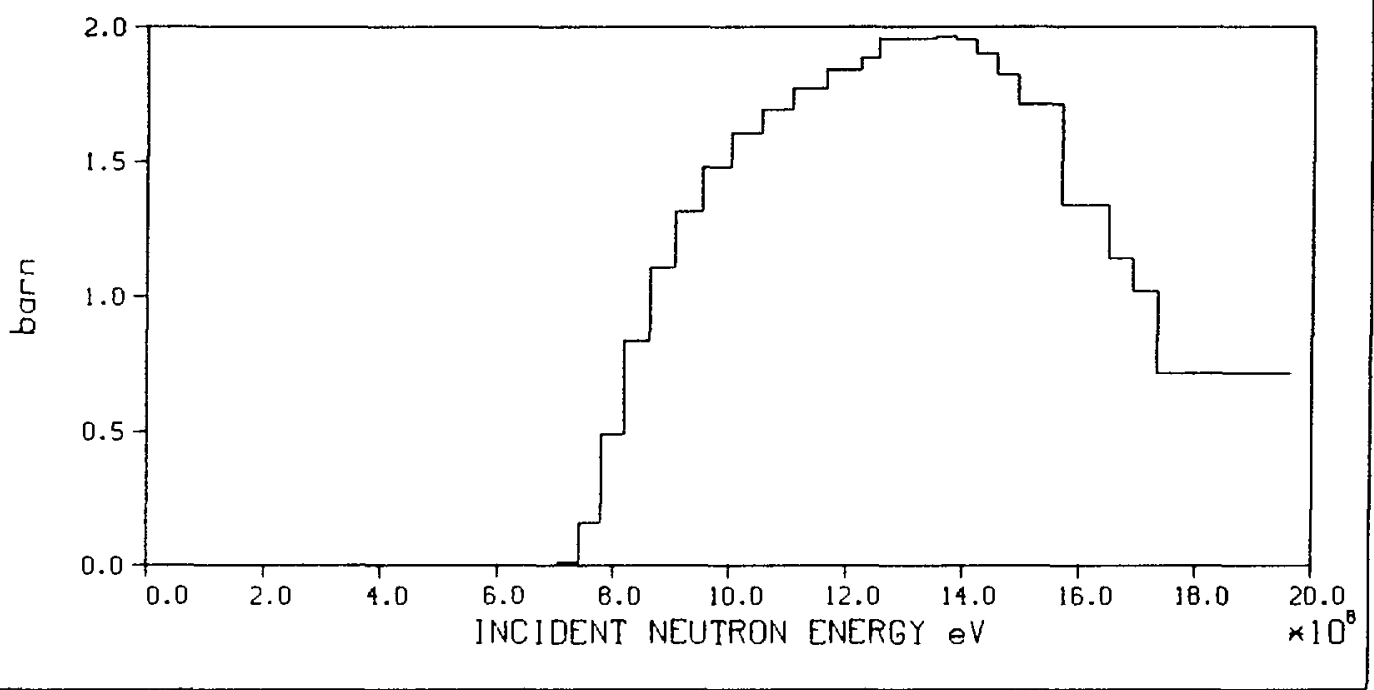


$w-186$ TOTAL $(n, 3 n)$ CROSS SECTION

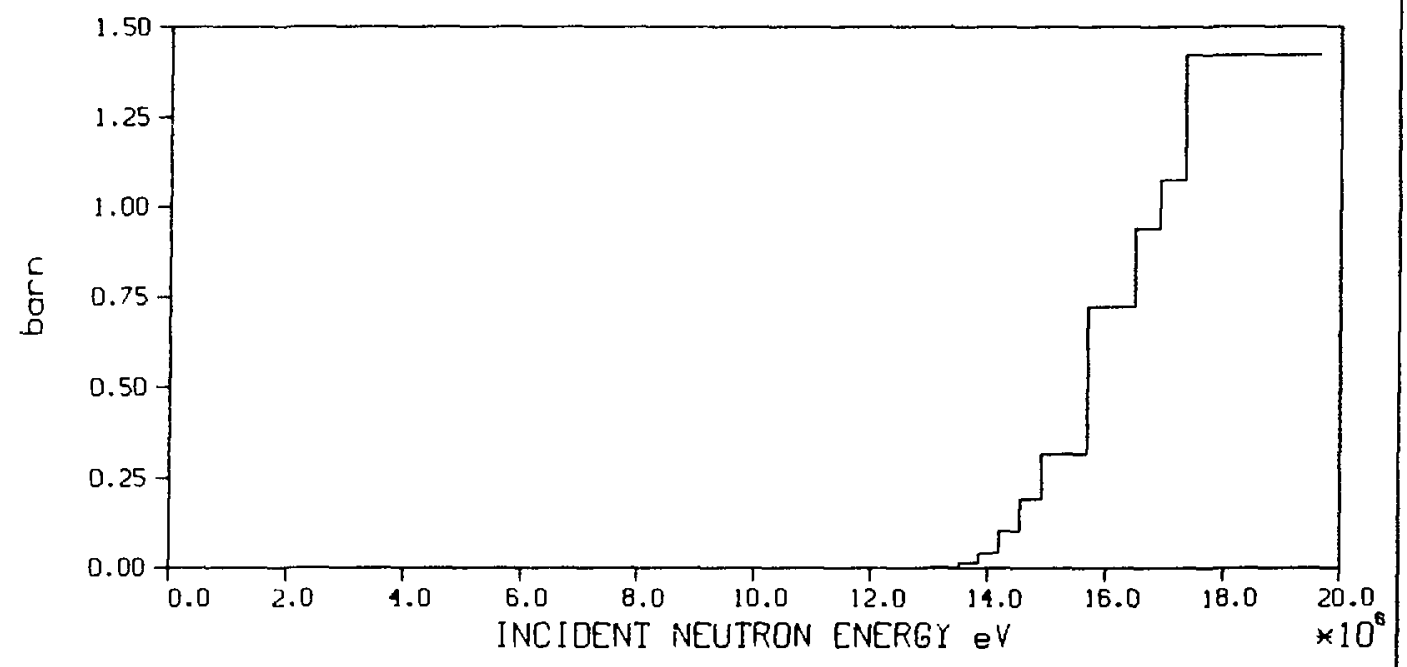


PB-NAT RECOMMENDED PROMPT KERMA FACTOR (solid line) REC. K. FACTOR WITH CH. PARTICLE DECAY HEAT (dashed line)

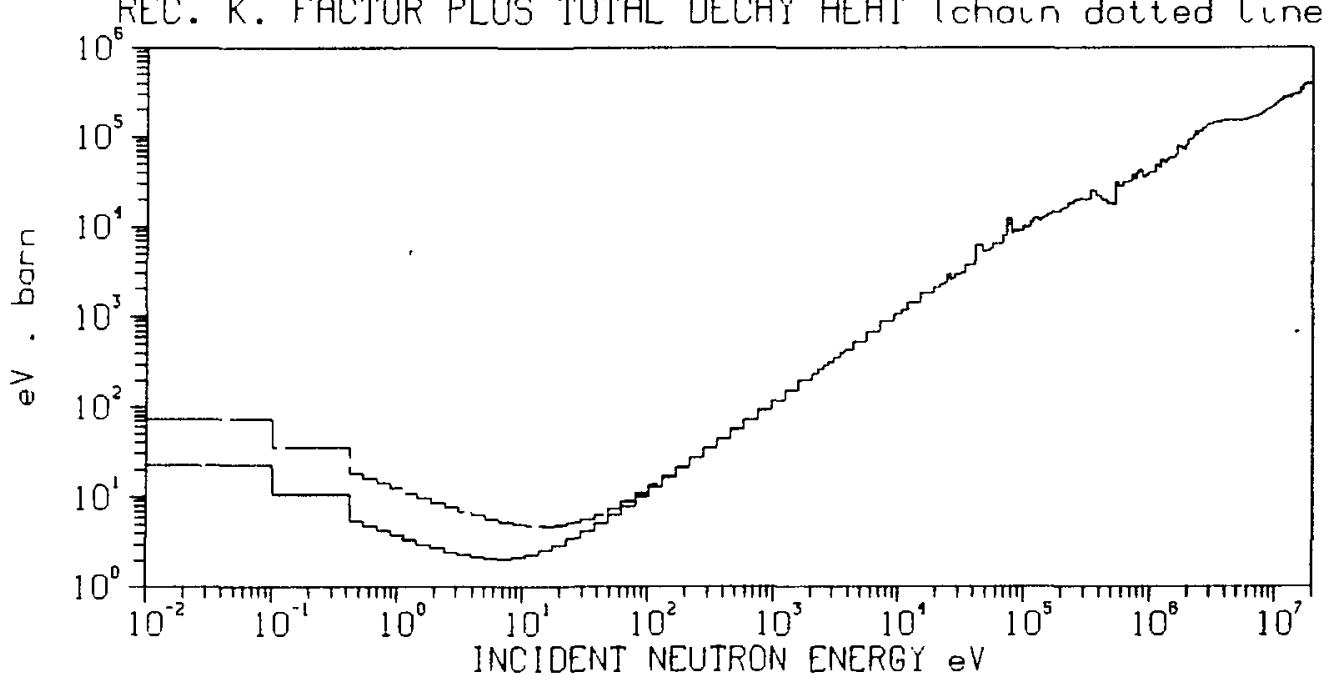

PB-NAT RECOMMENDED PROMPT KERMA FACTOR (solid line) REC. K. FACTOR WITH CH. PARTICLE DECAY HEAT (dashed line) ¿ REC. K. FACTOR PLUS TOTAL DECAY HEAT (chain dotted line)

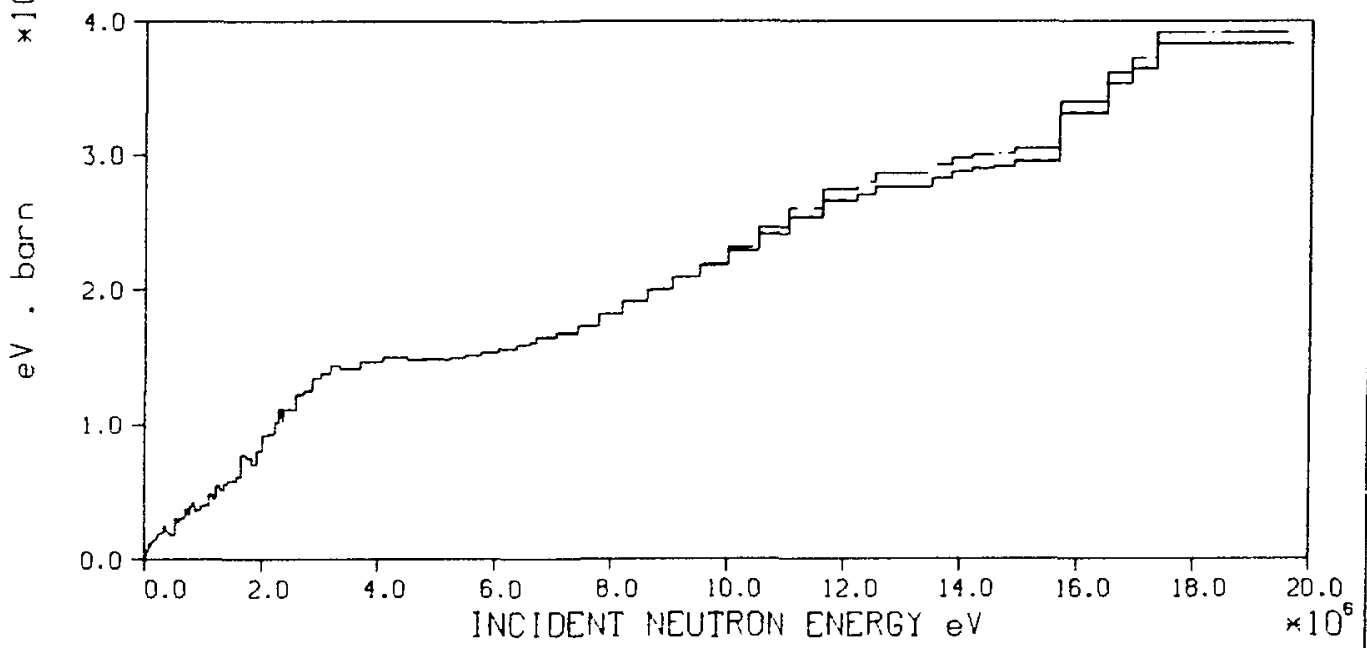




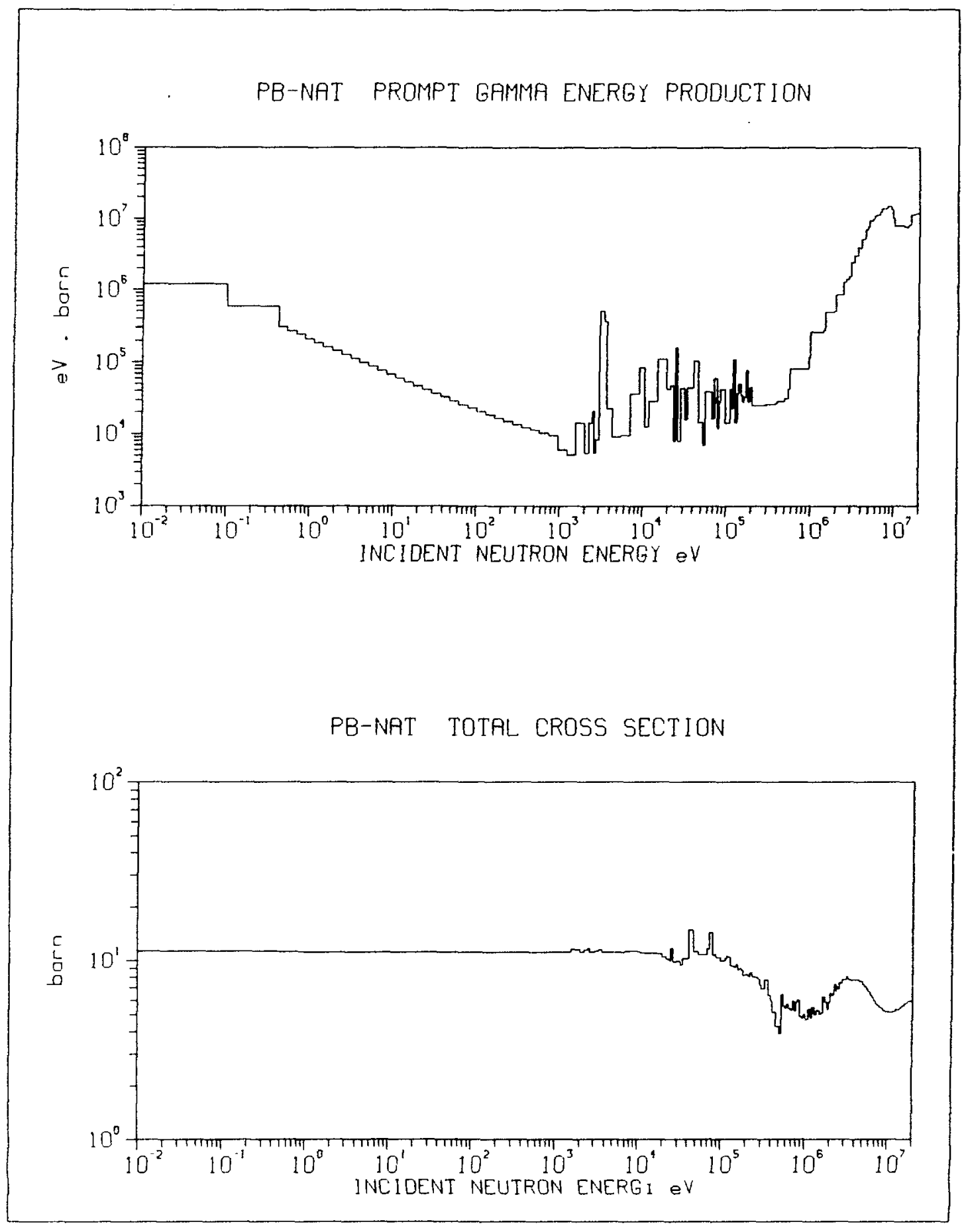


PB-NAT ELASTIC CROSS SECTION

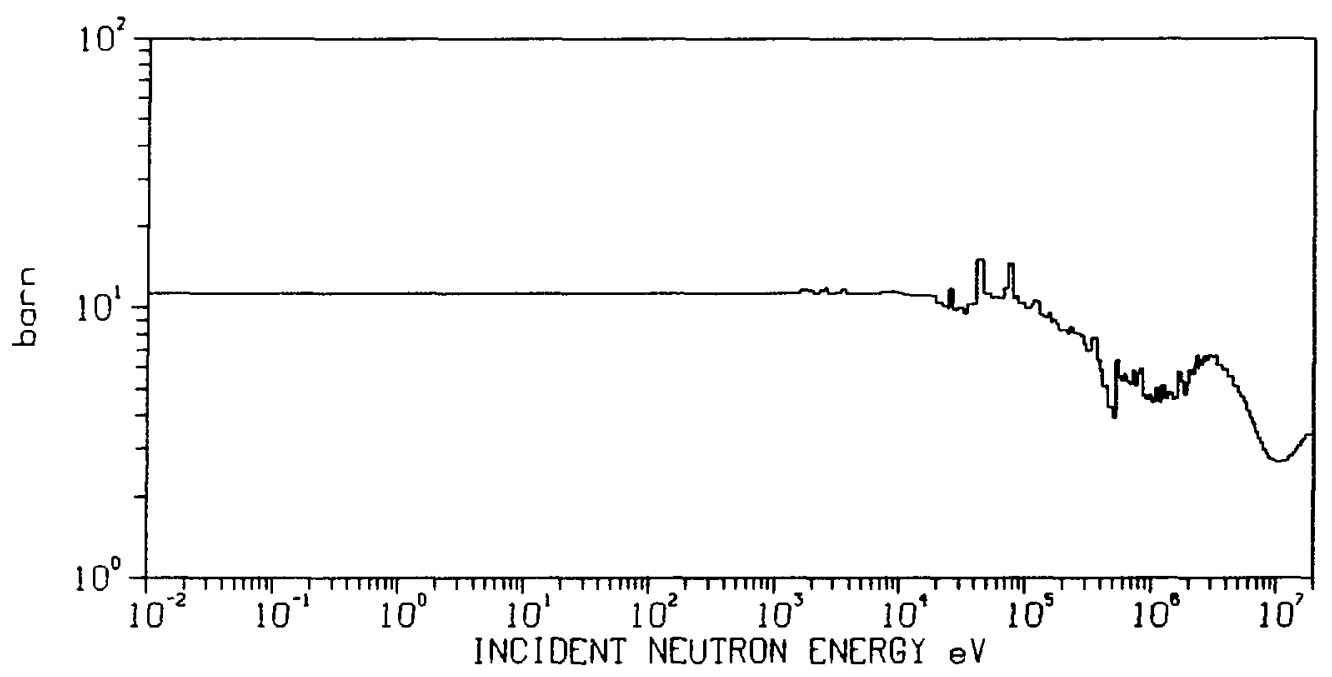

PB-NAT TOTAL INELASTIC CROSS SECTION

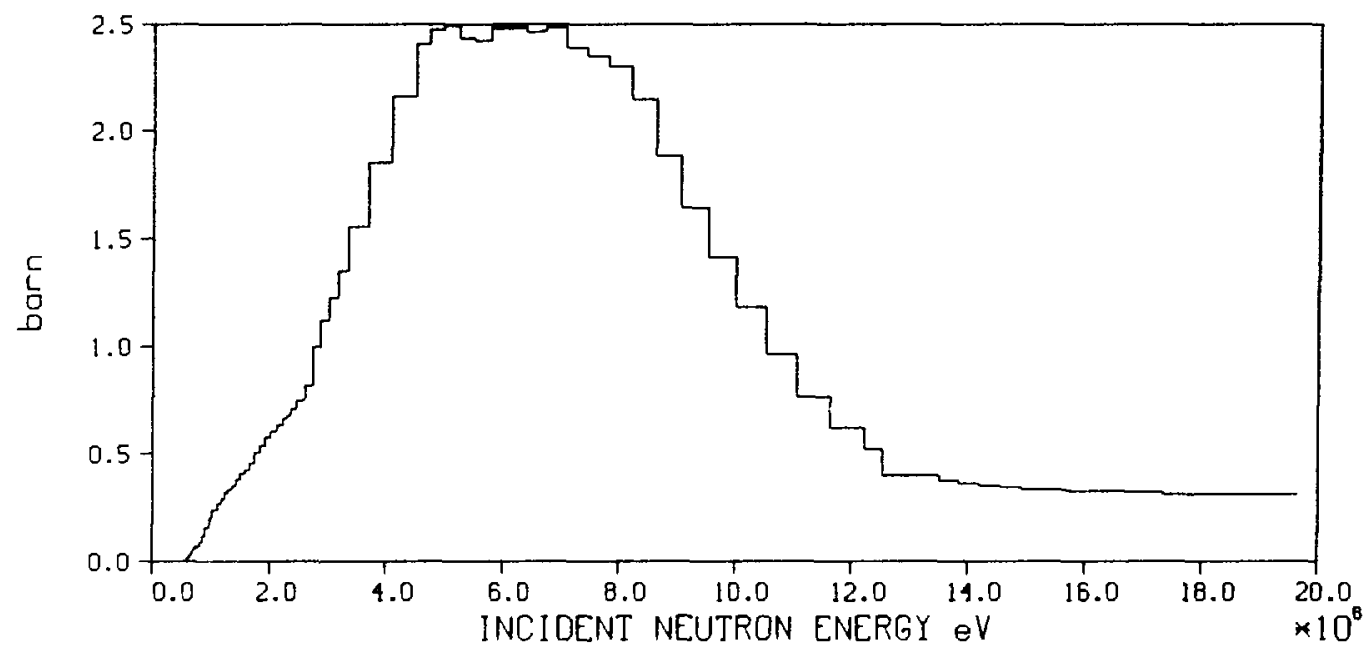


PB-NAT RADIATIVE CAPTURE CROSS SECTION

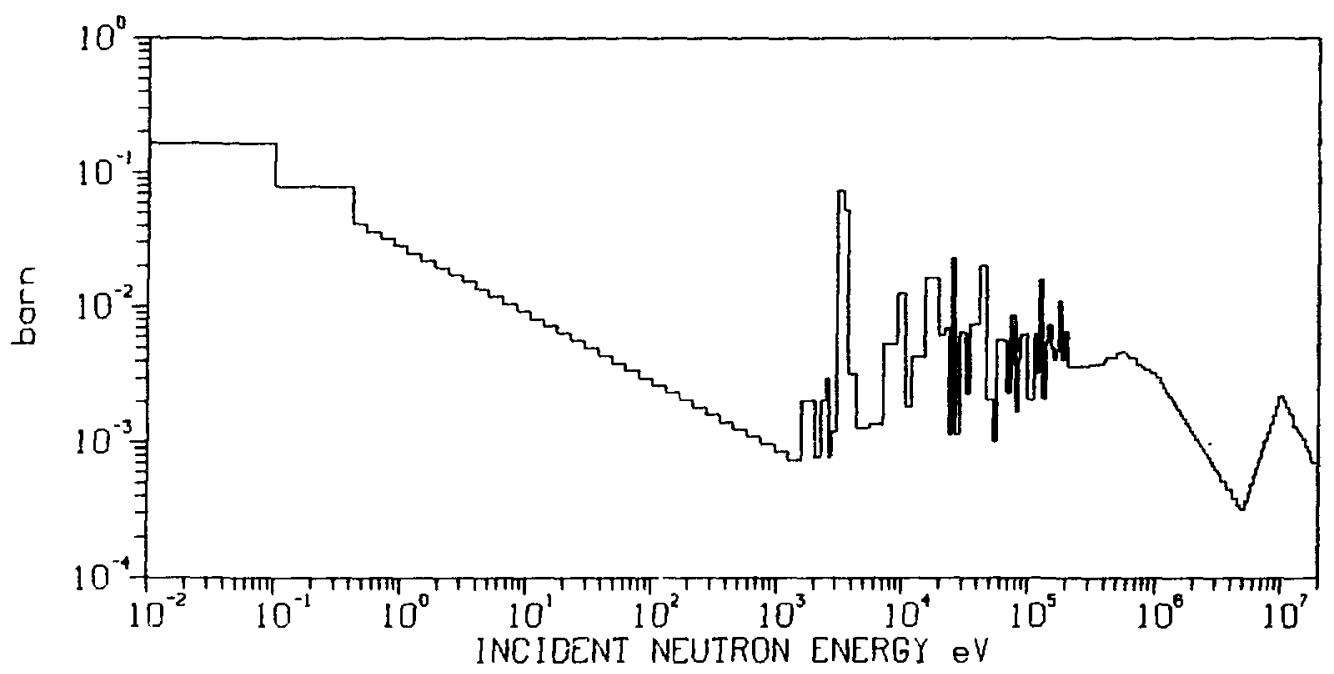

PB-NAT TOTAL $\{n, 2 n]$ REACTION CROSS SECTION

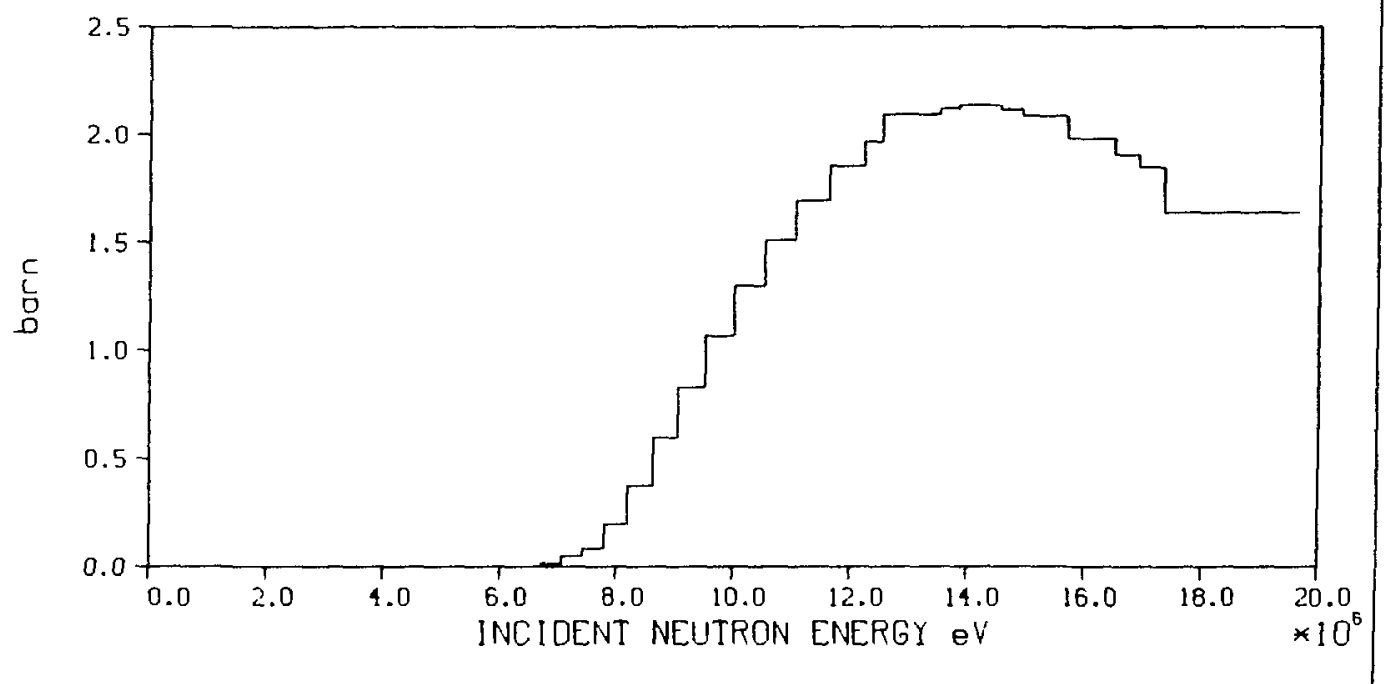


PB-NAT TOTAL $(n, 3 n)$ CROSS SECTION

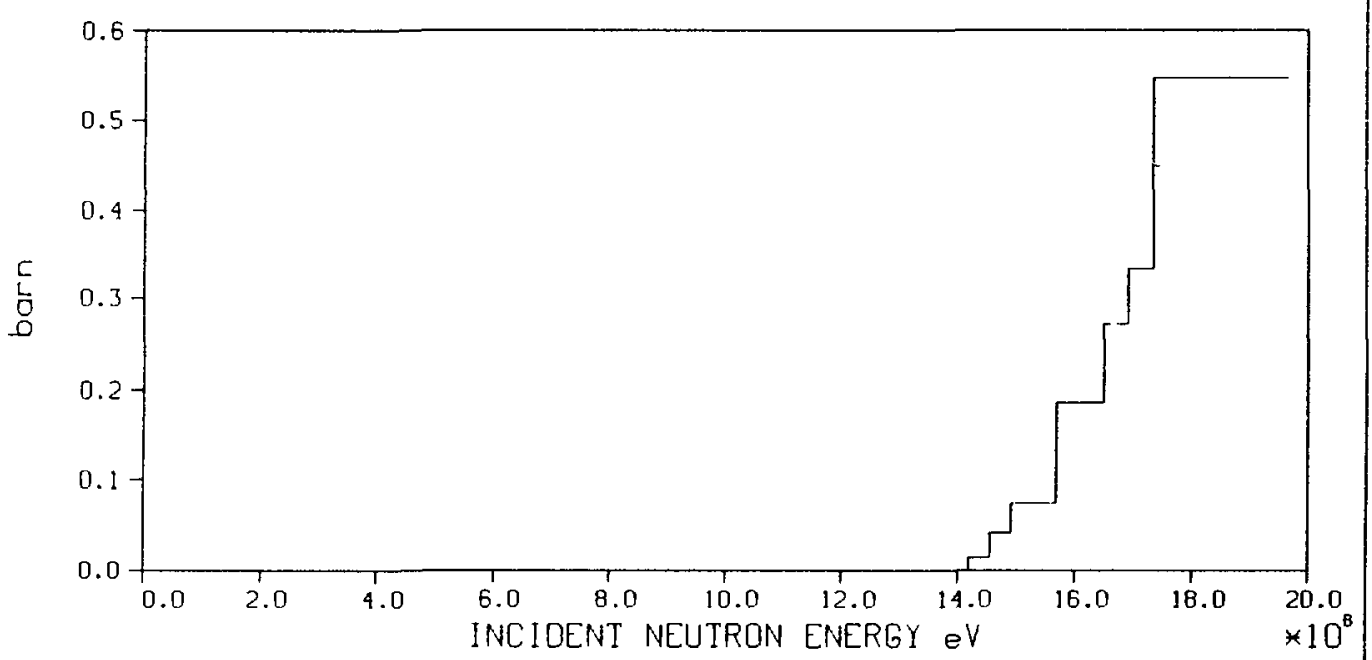




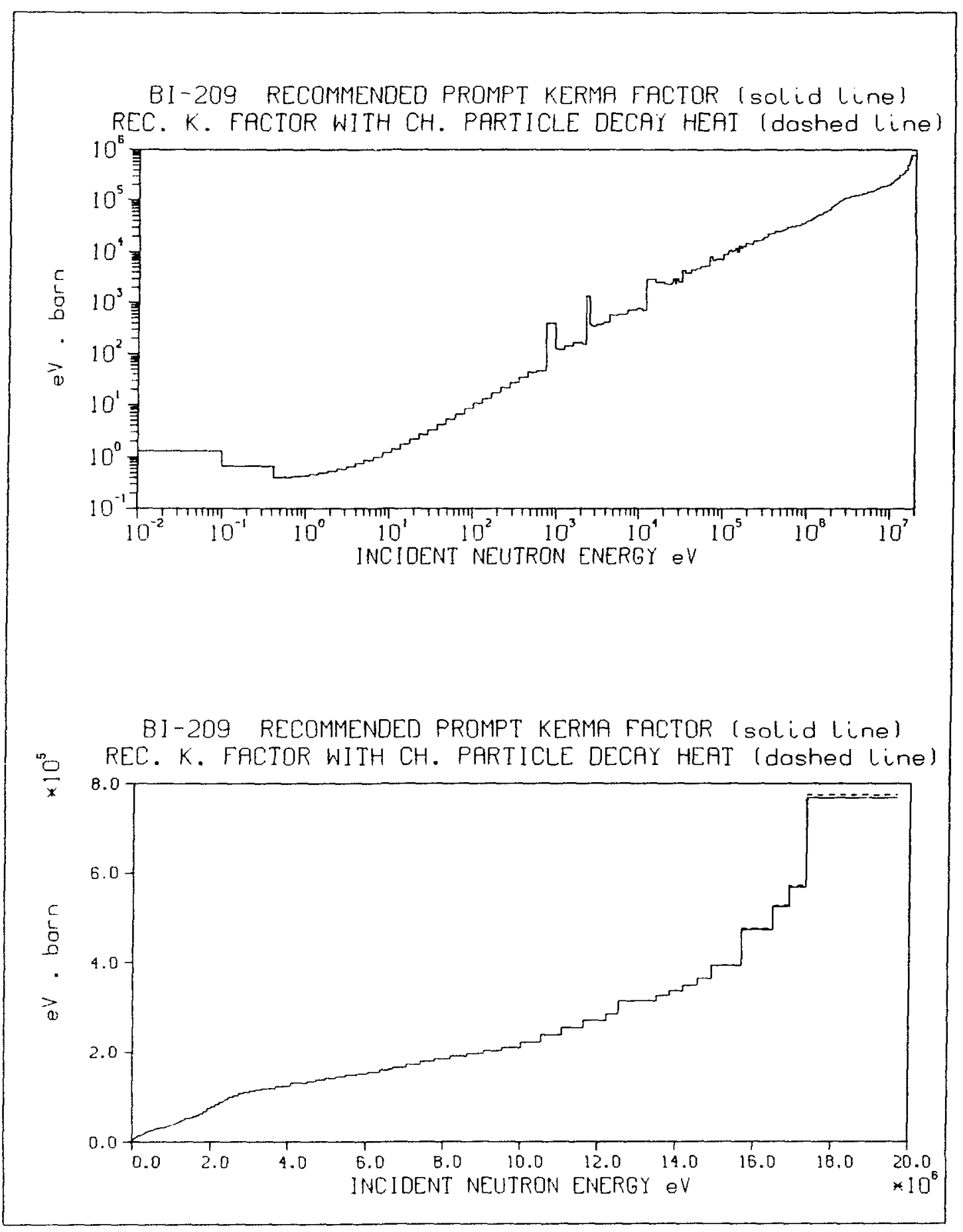




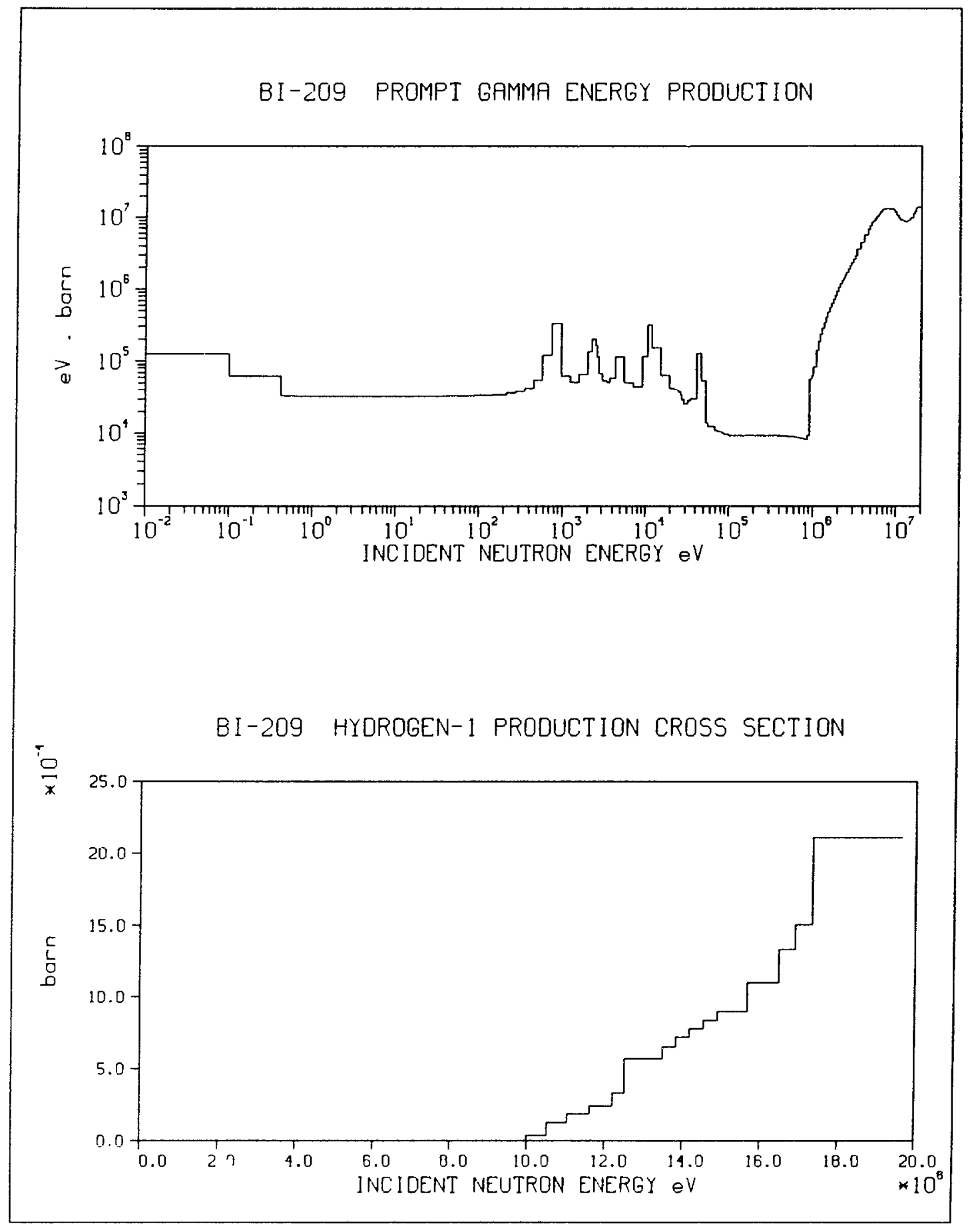




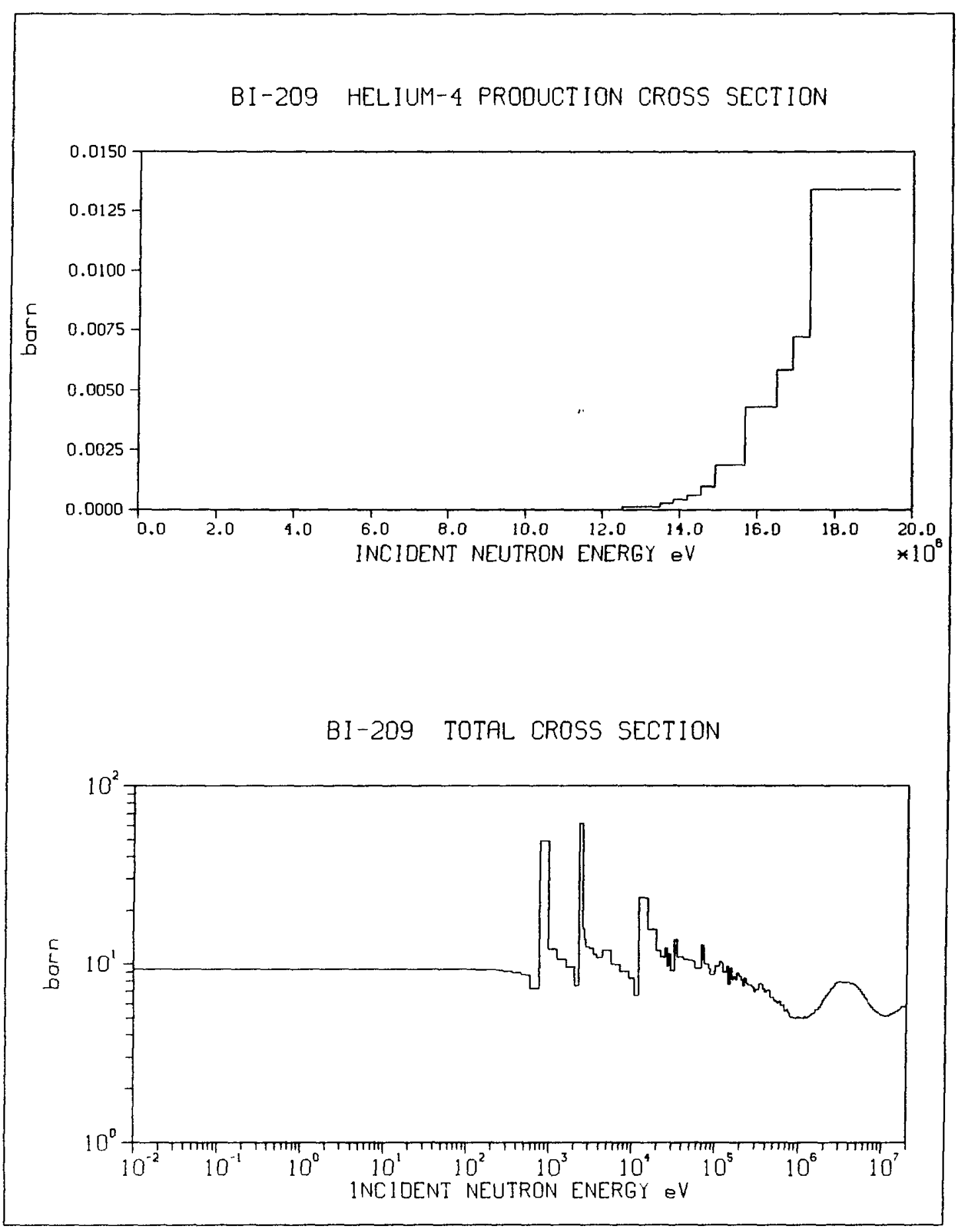




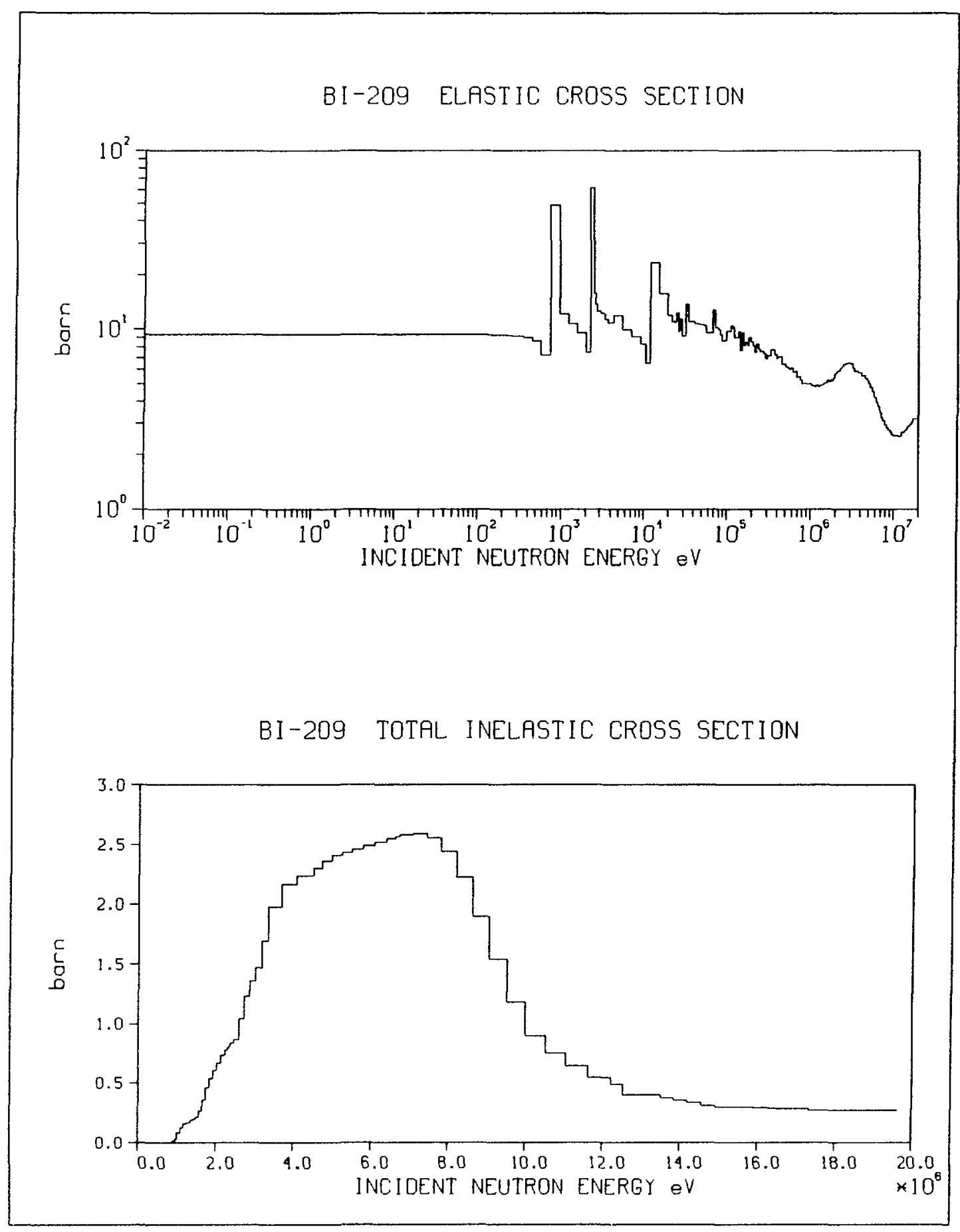




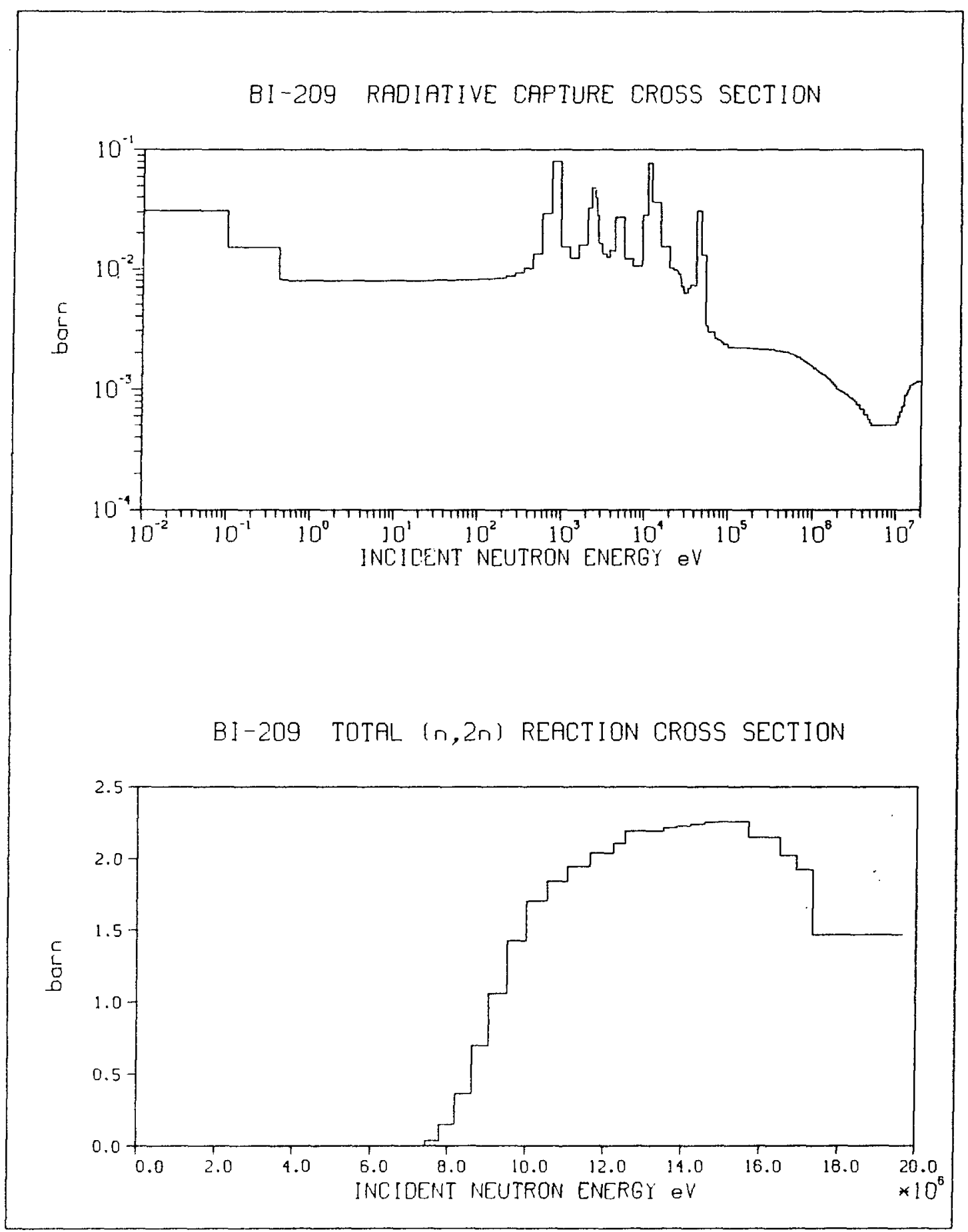




\section{BI-209 TOTAL $[n, 3 n]$ CROSS SECTION}

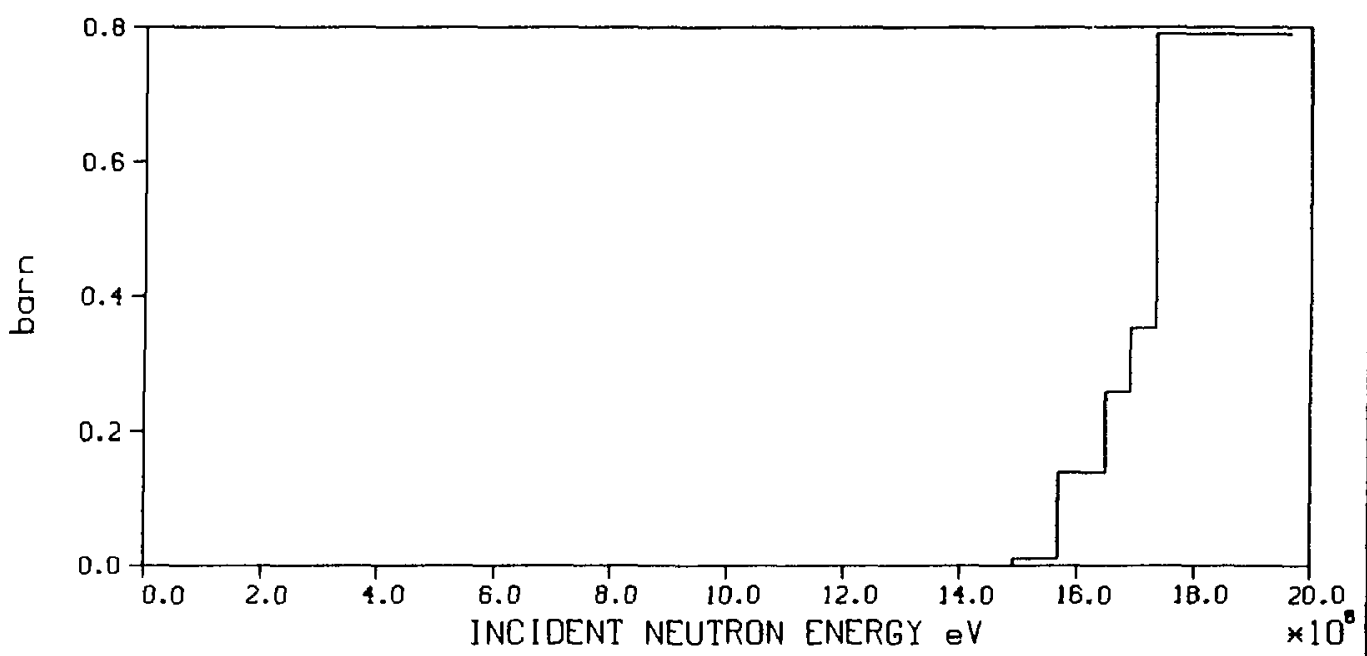


TH-232 RECOMMENDED PROMPT KERMA FACTOR (solid line) REC. K. FACTOR WITH CH. PARTICLE DECAY HEAT (dashed line) REC. K. FACTOR PLUS TOTAL DECAY HEAT (chain dotted line)

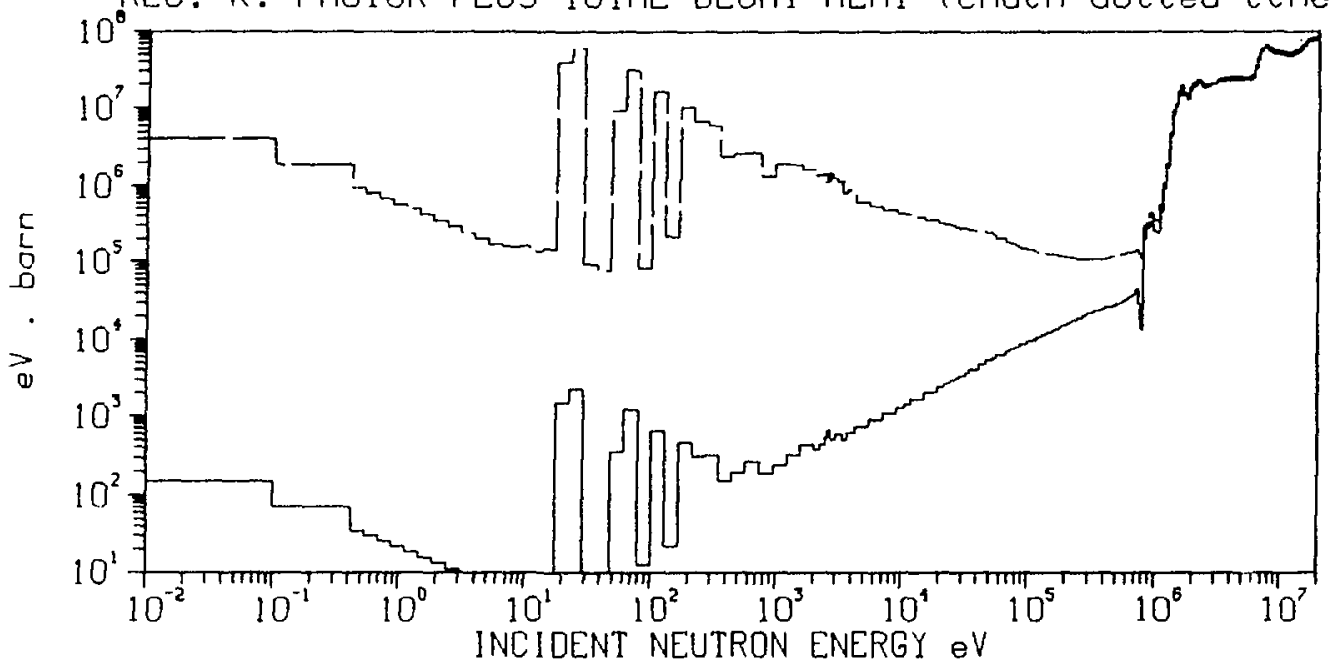

TH-232 RECOMMENDED PROMPT KERMA FACTOR (solid line) REC. K. FACTOR WITH CH. PARTICLE DECAY HEAT (dashed line) - REC. K. FACTOR PLUS TOTAL DECAY HEAT (chain dotted Line) ㄱ.

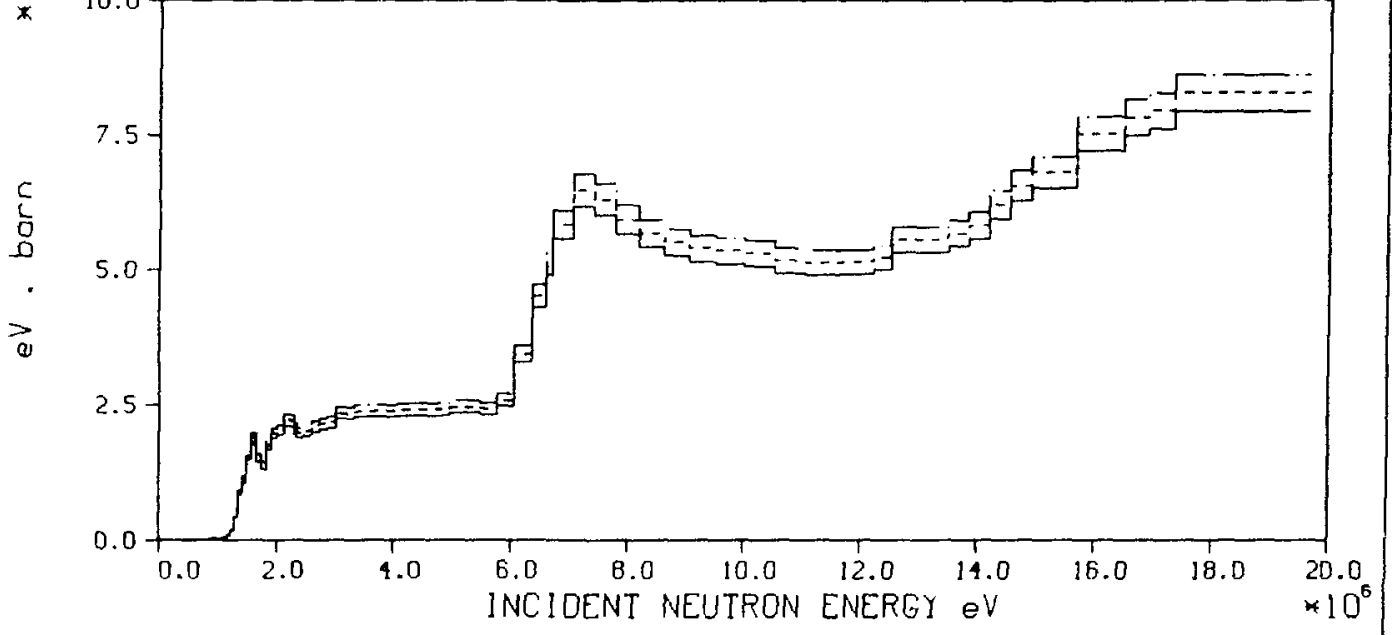


TH-232 PROMPT GAMMA ENERGY PRODUCTION

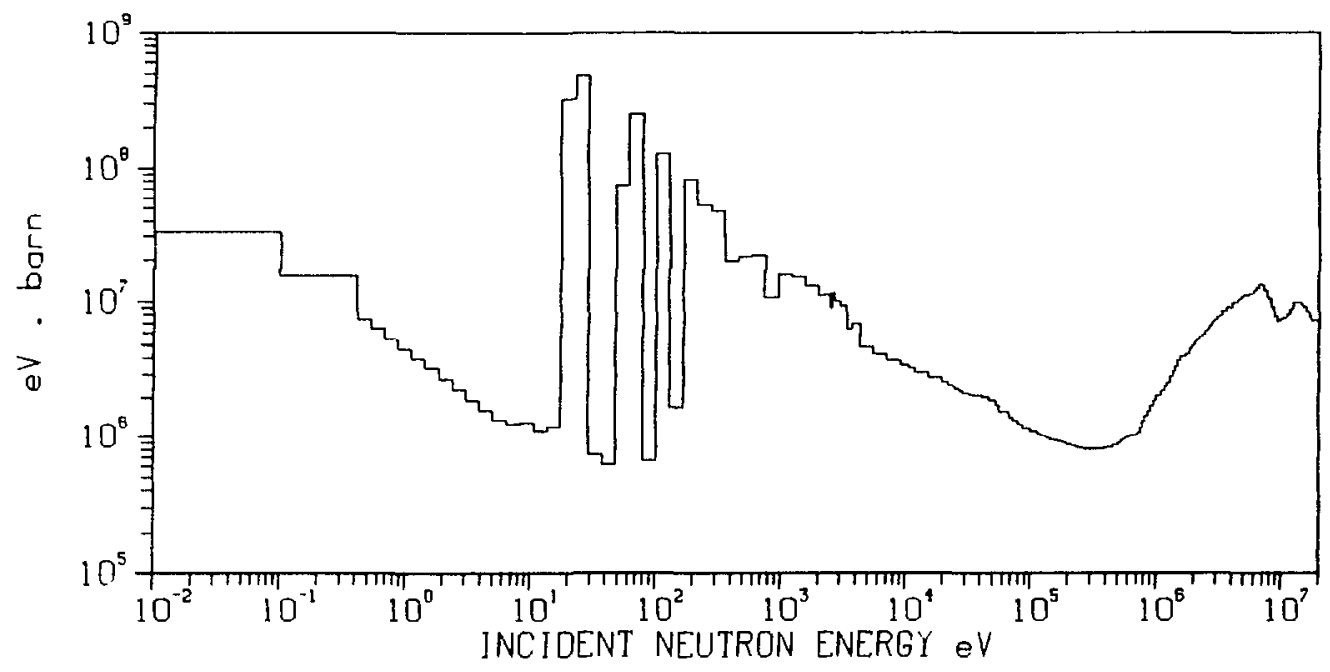

TH-232 TOTAL CROSS SECTION

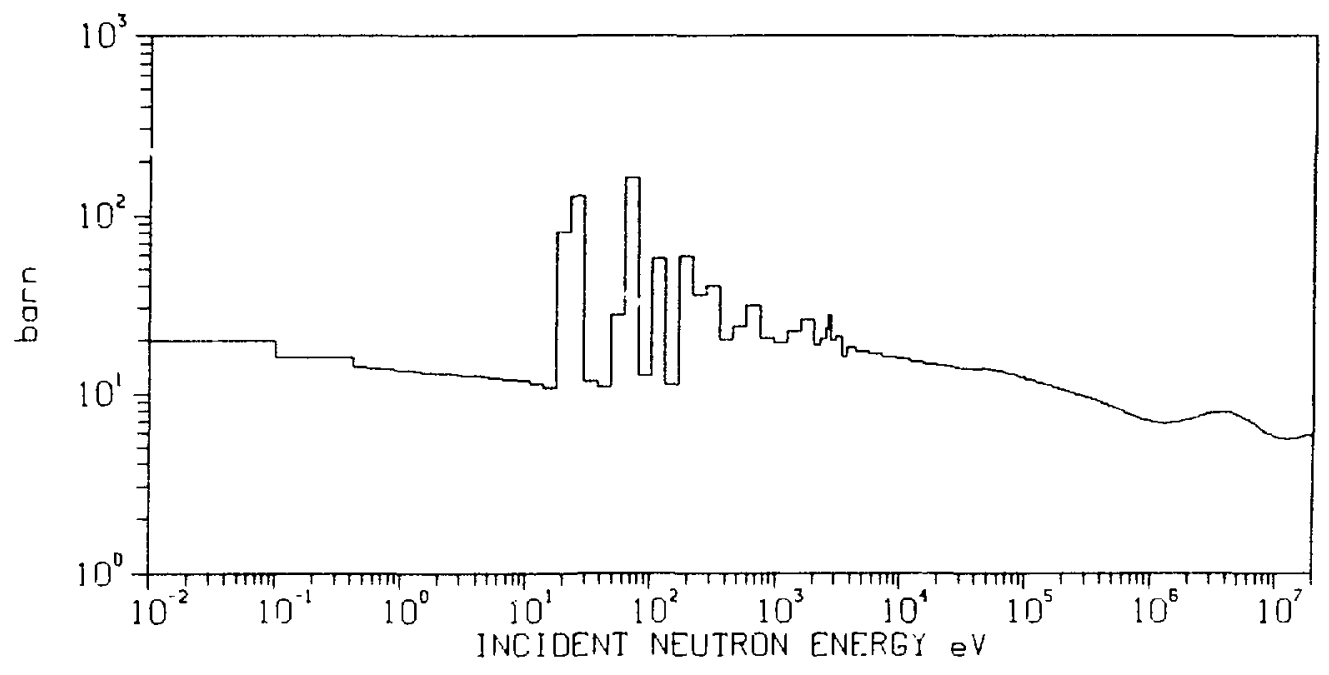




\section{TH-232 ELASTIC CROSS SECTION}

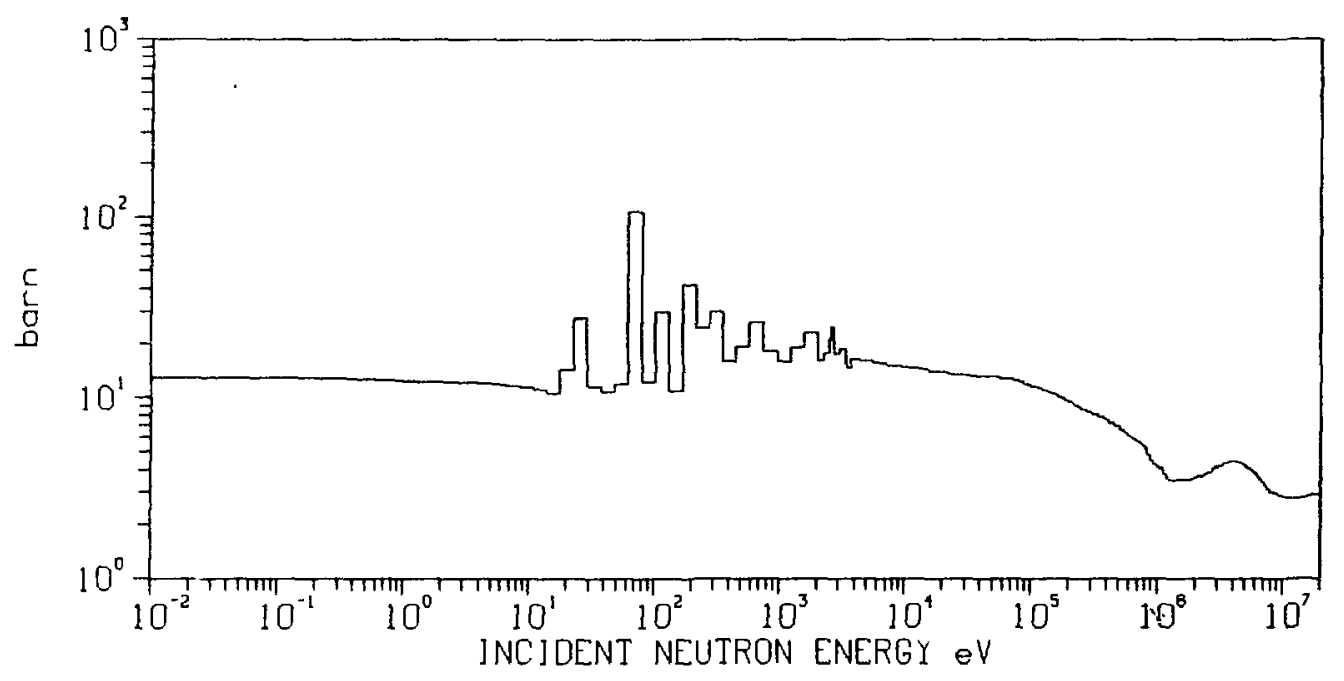

TH-232 TOTAL INELASTIC CROSS SECTION

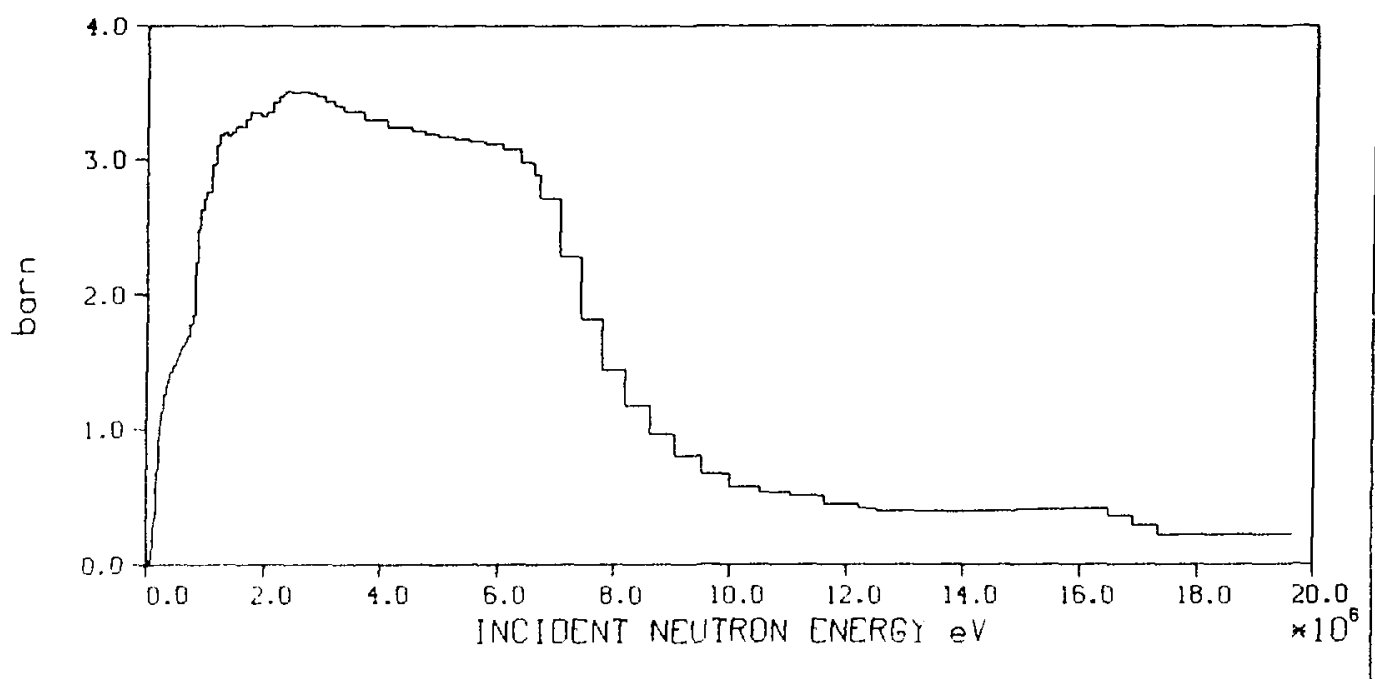


TH-232 RADIATIVE CAPTURE CROSS SECTION

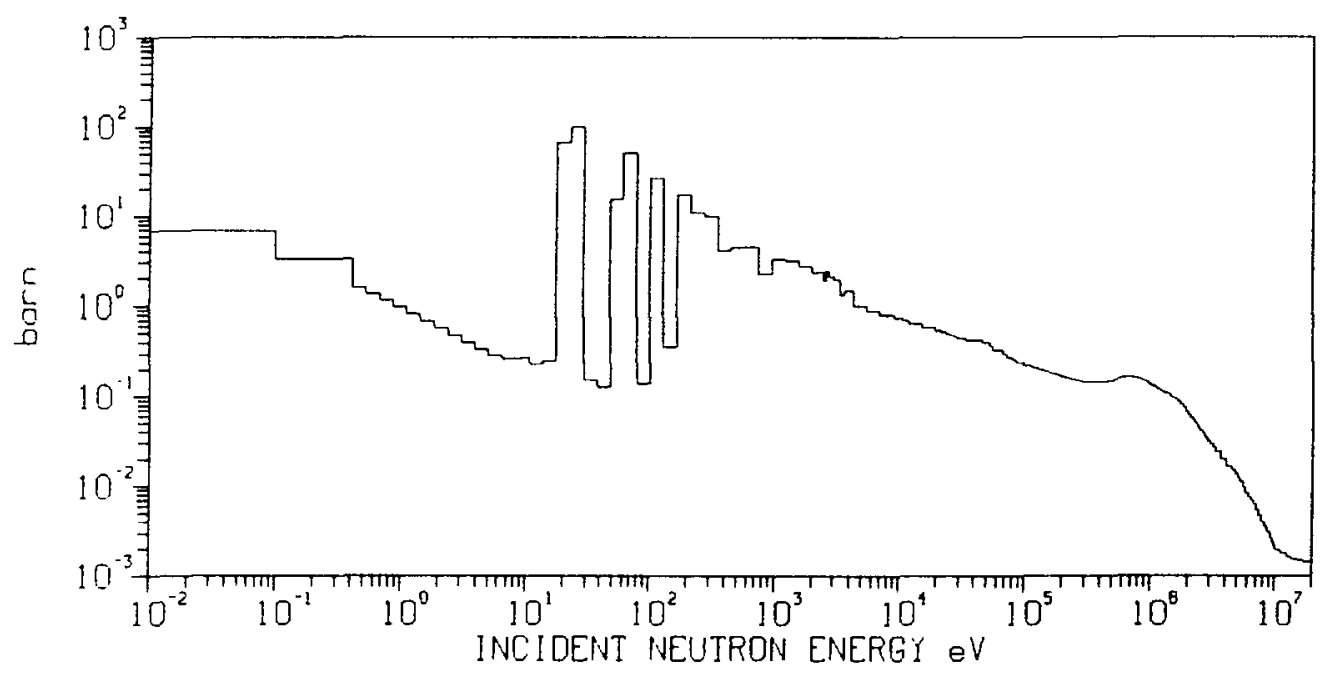

TH-232 TOTAL $(n, 2 n)$ REACTION CROSS SECTION

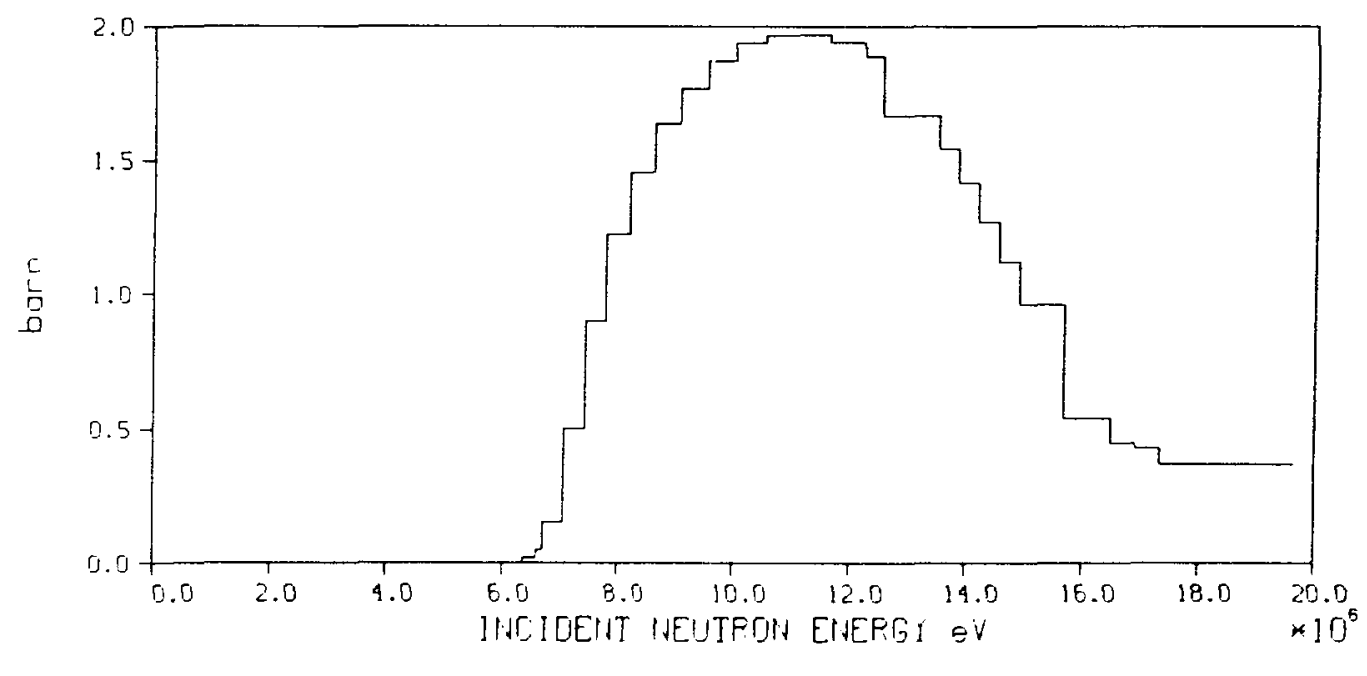


TH-232 TOTAL $(n, 3 n)$ CROSS SECTION

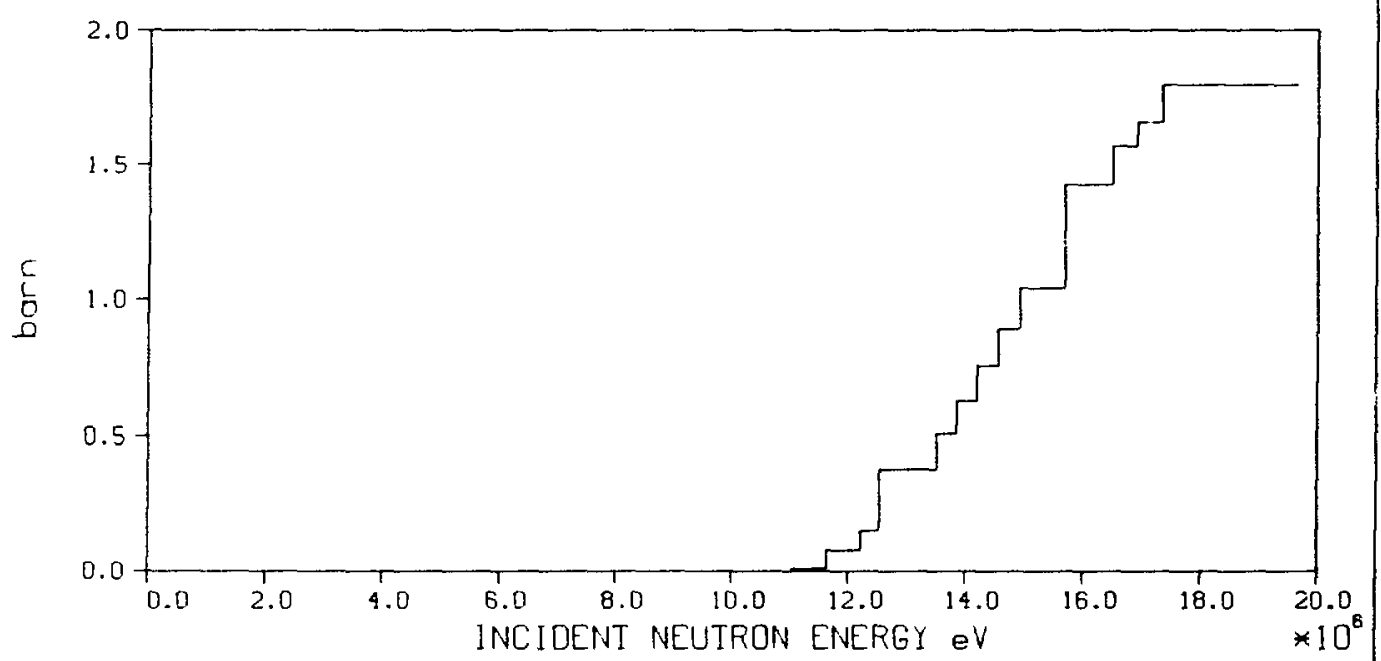

TH-232 NUMBER OF FISSION NEUTRONS * CROSS SECTION

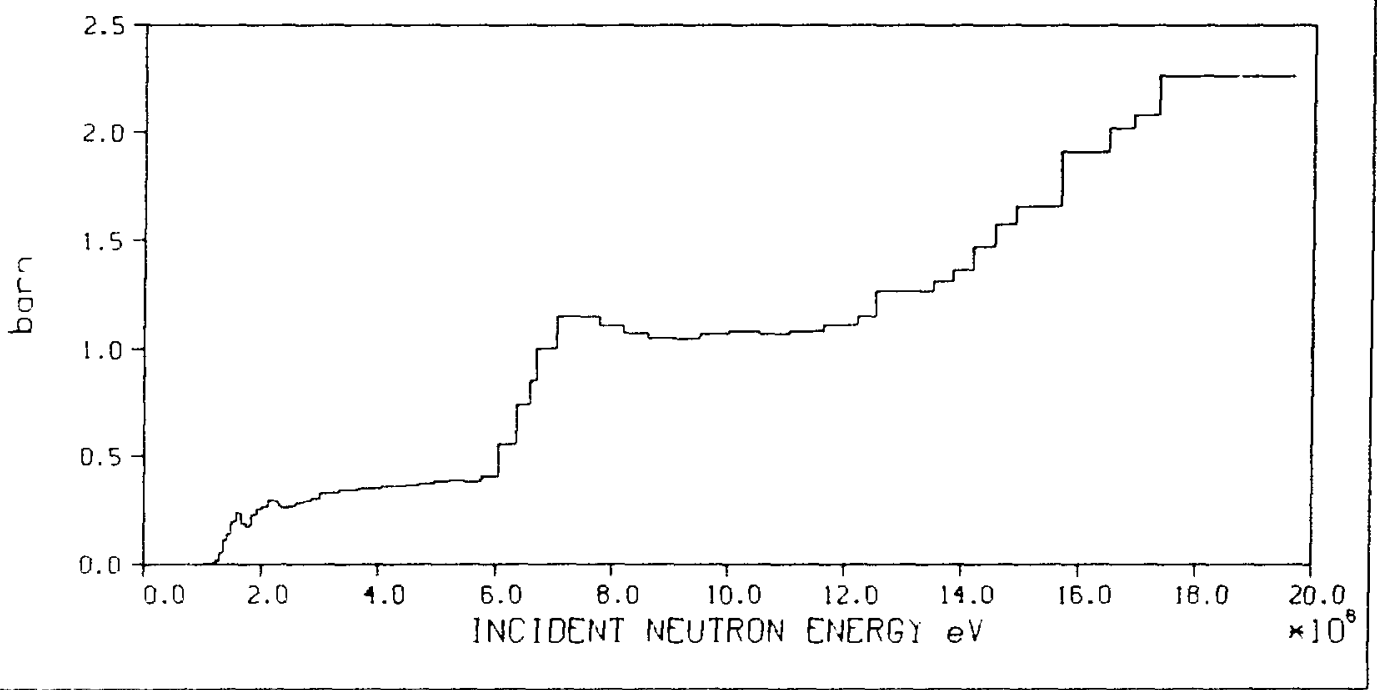


PA-233 RECOMMENDED PROMPT KERMA FACTOR (solid line)

REC. K. FACTOR WITH CH. PARTICLE DECAY HEAT (dashed line)

REC. K. FACTOR PLUS TOTAL DECAY HEAT (chain dotted line)

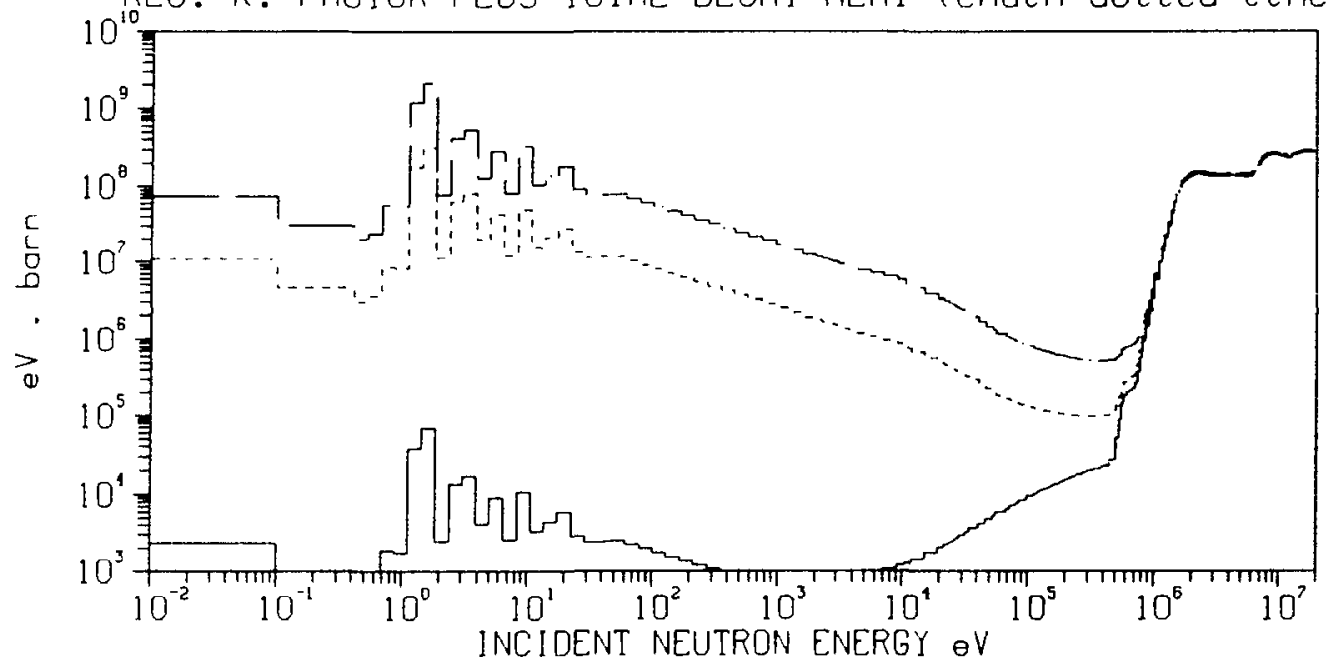

PA-233 RECOMMENDED PROMPT KERMA FACTOR (solid line) REC. K. FACTOR WITH CH. PARTICLE DECAY HEAT (dashed line) REC. K. FACTOR FLUS TOTAL DECAY HEAT (chain dotted line)

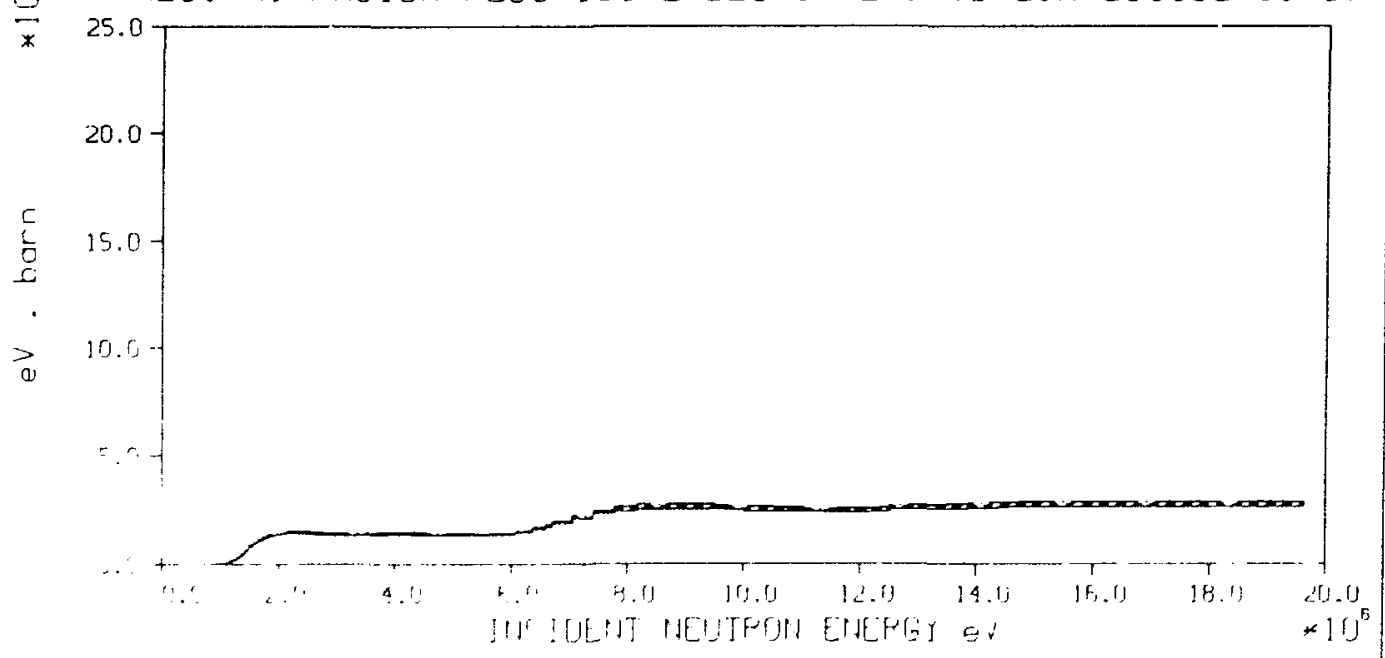


PR-233 TOTRL CROSS SECTION

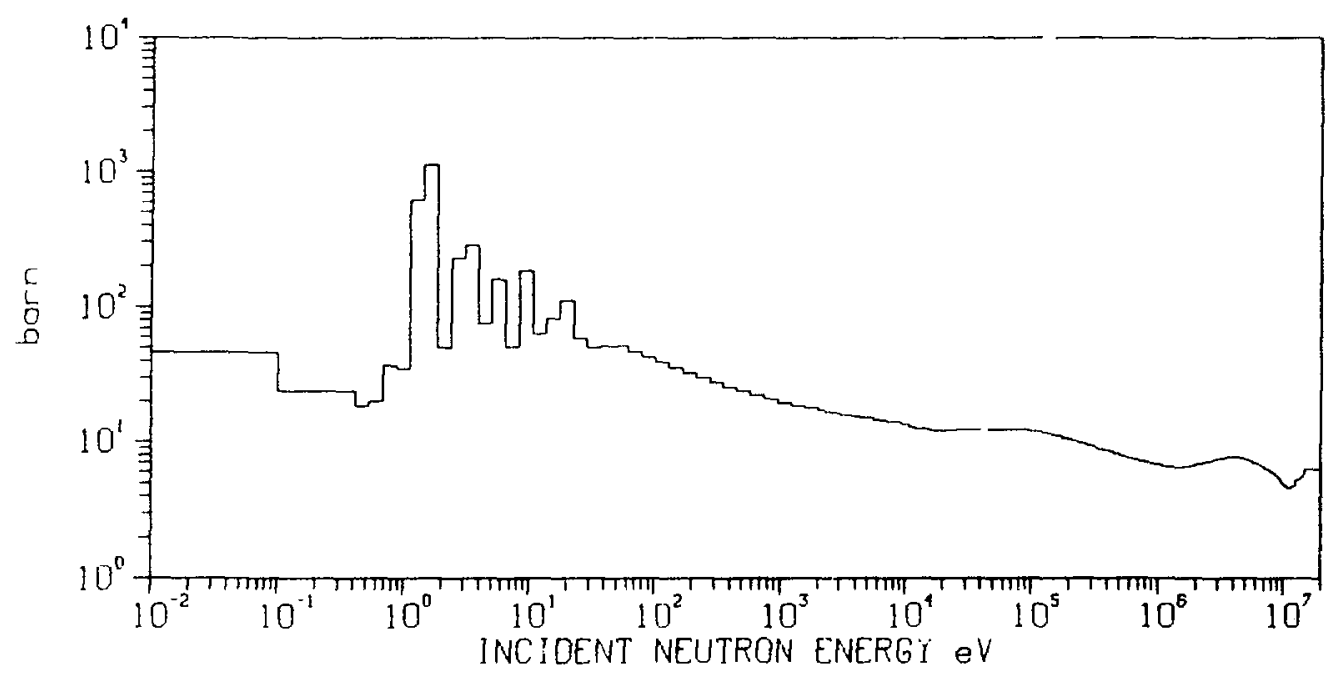

PA-233 ELASTIC CROSS SECTION

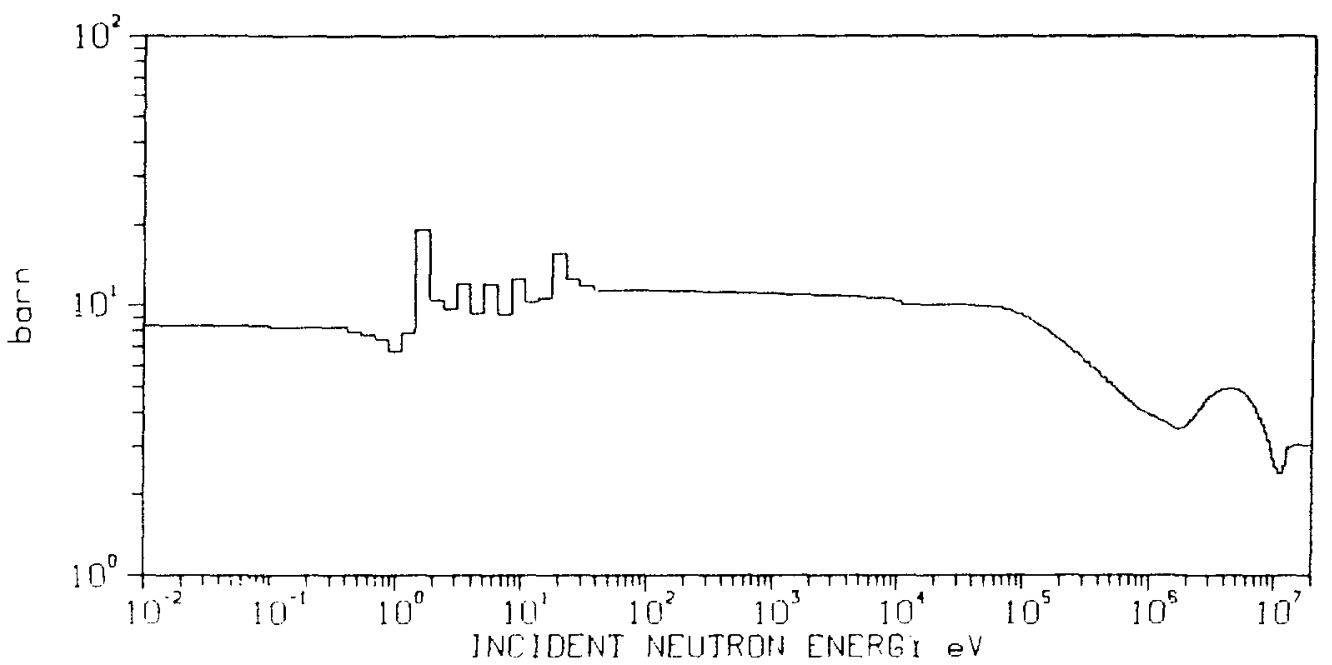


PA-233 TOTAL INELASTIC CROSS SECTION

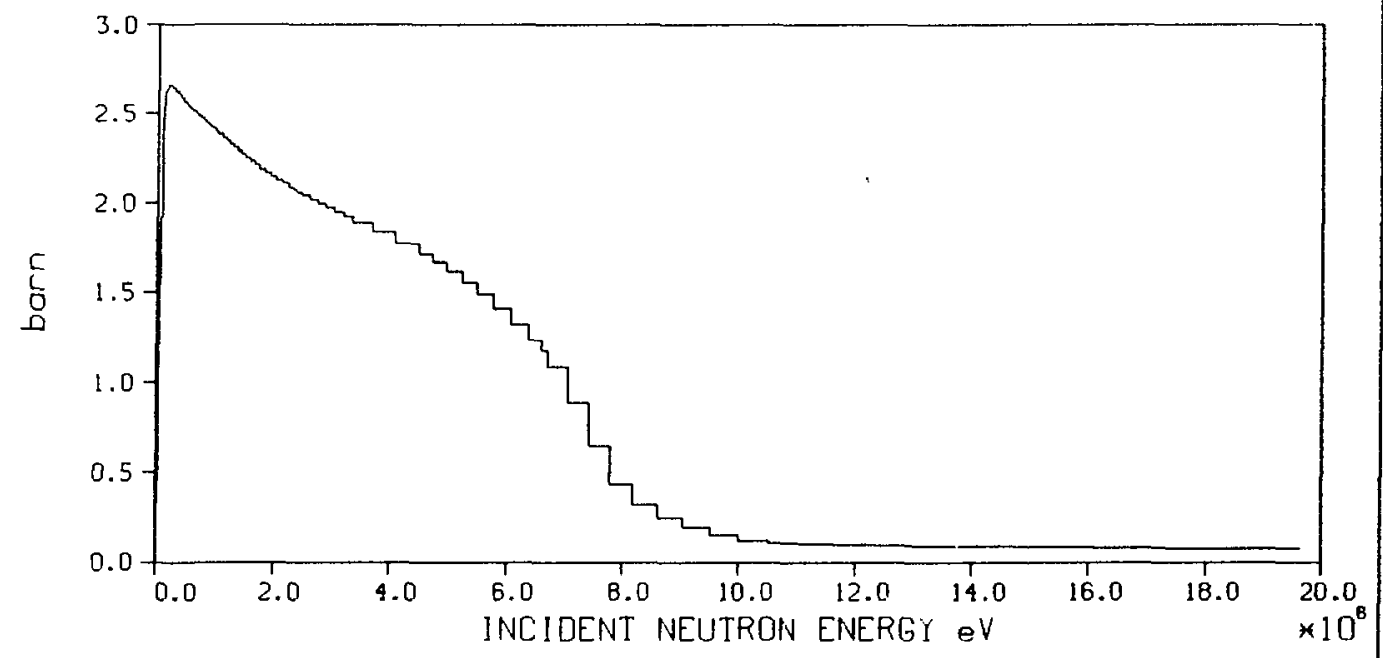

PA-233 RADIATIVE CAPTURE CROSS SECTION

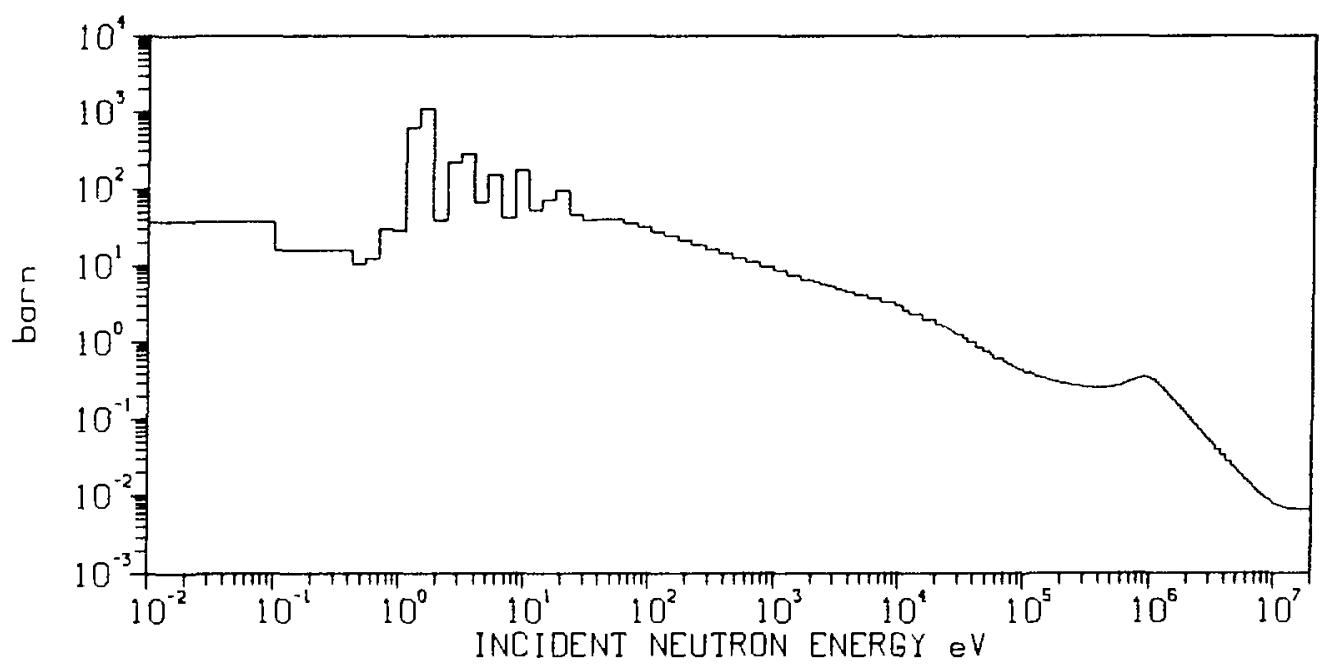


PA-233 TOTAL $(n, 2 n)$ REACTION CROSS SECTION

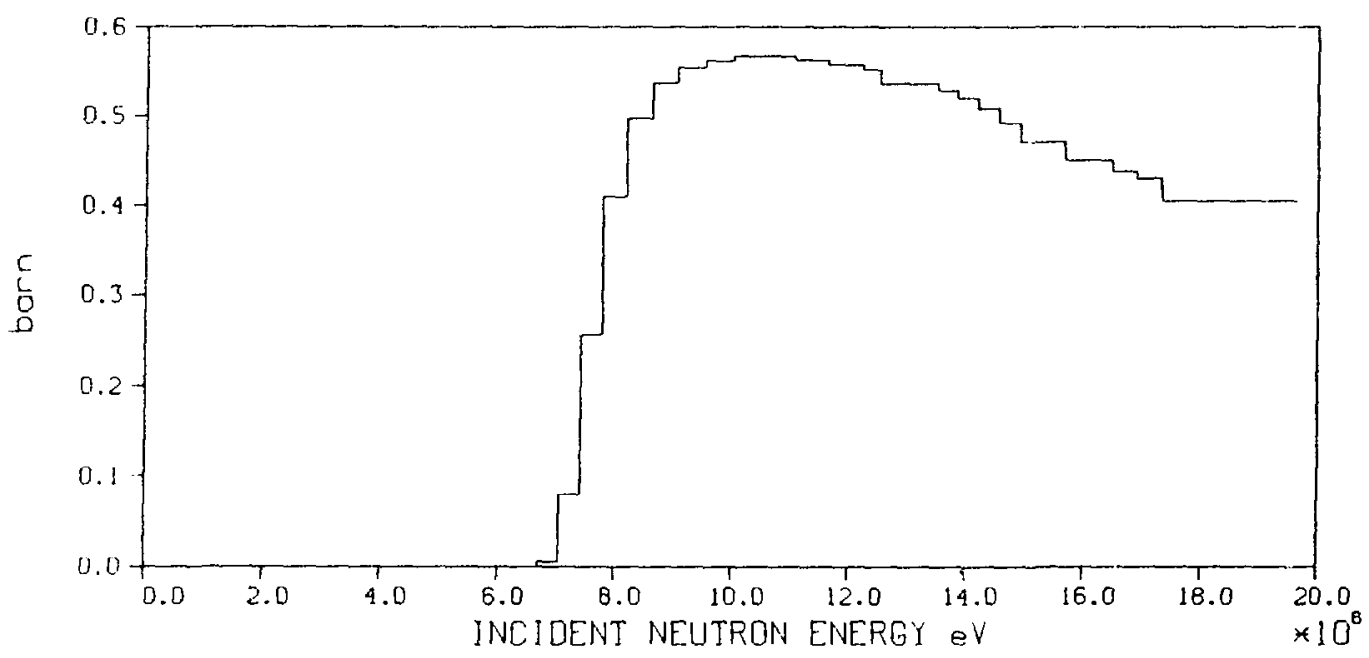

PA-233 TOTAL $(n, 3 n)$ CROSS SECTION

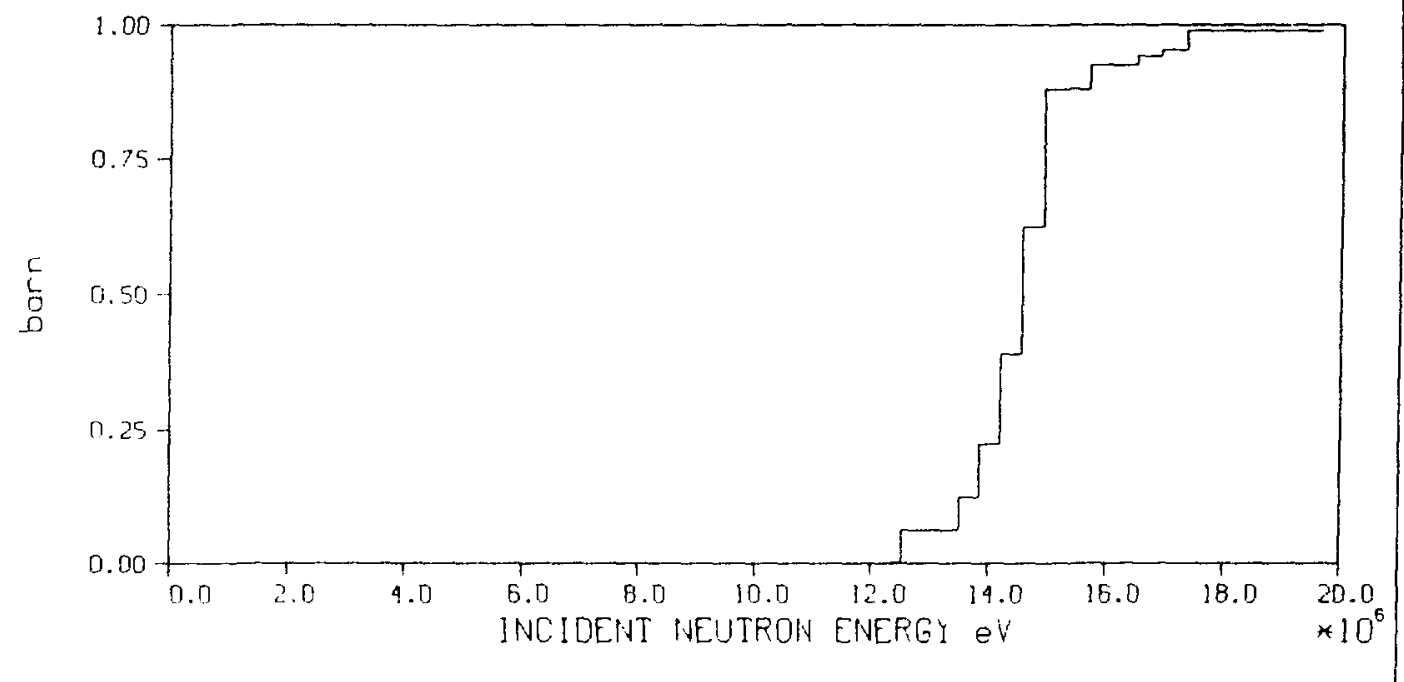




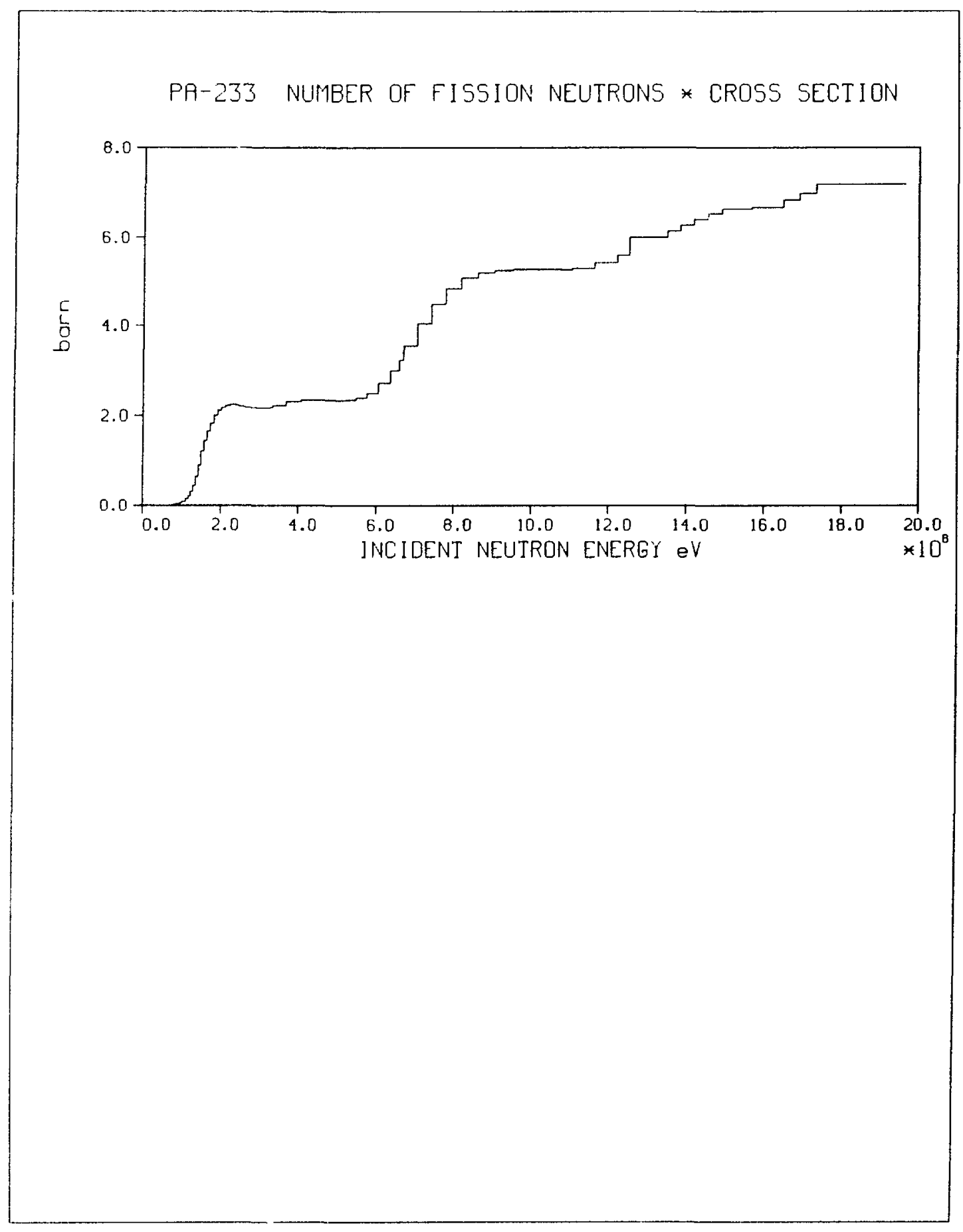




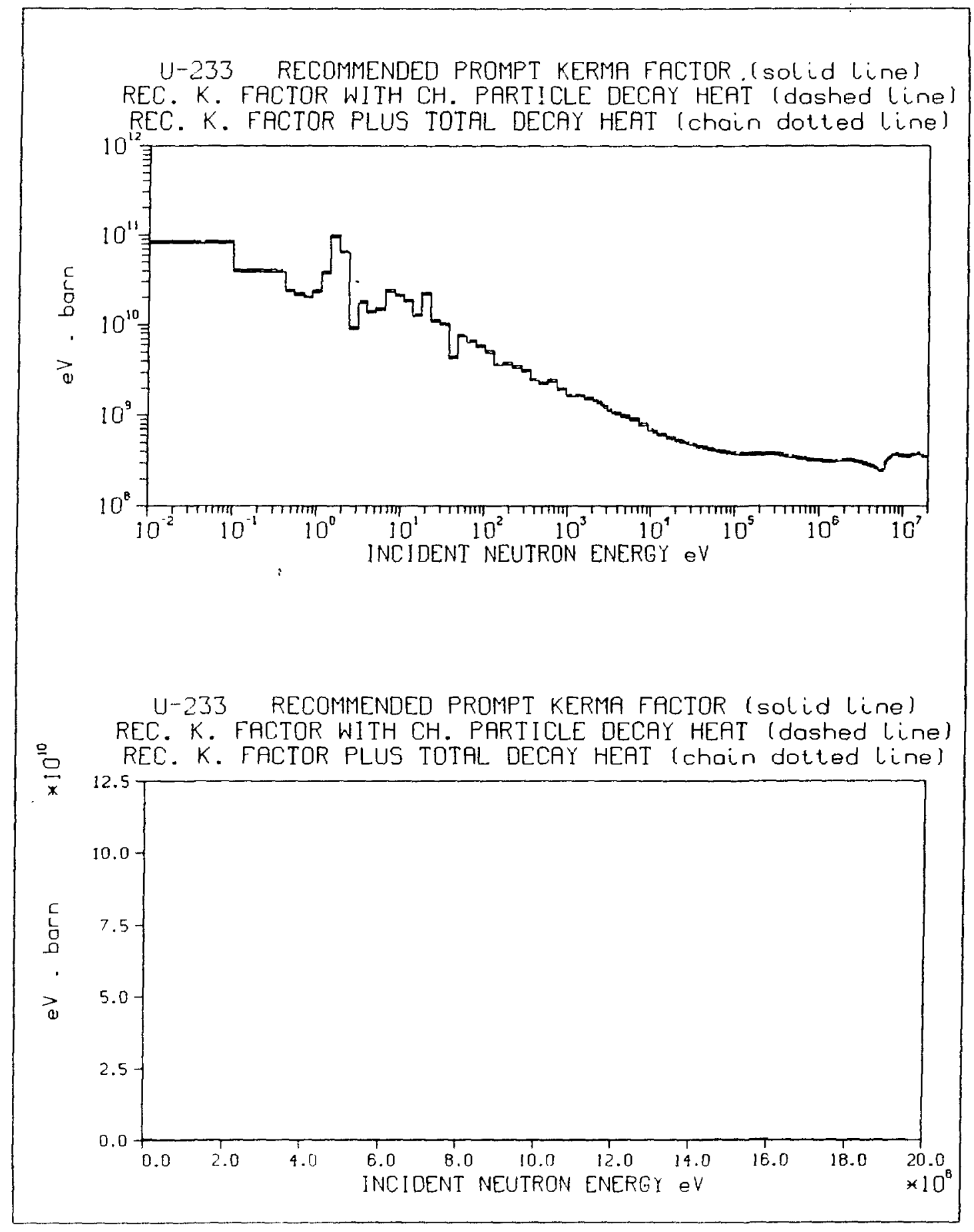




\section{U-233 PROMPT GRMMA ENERGY PRODUCTION}

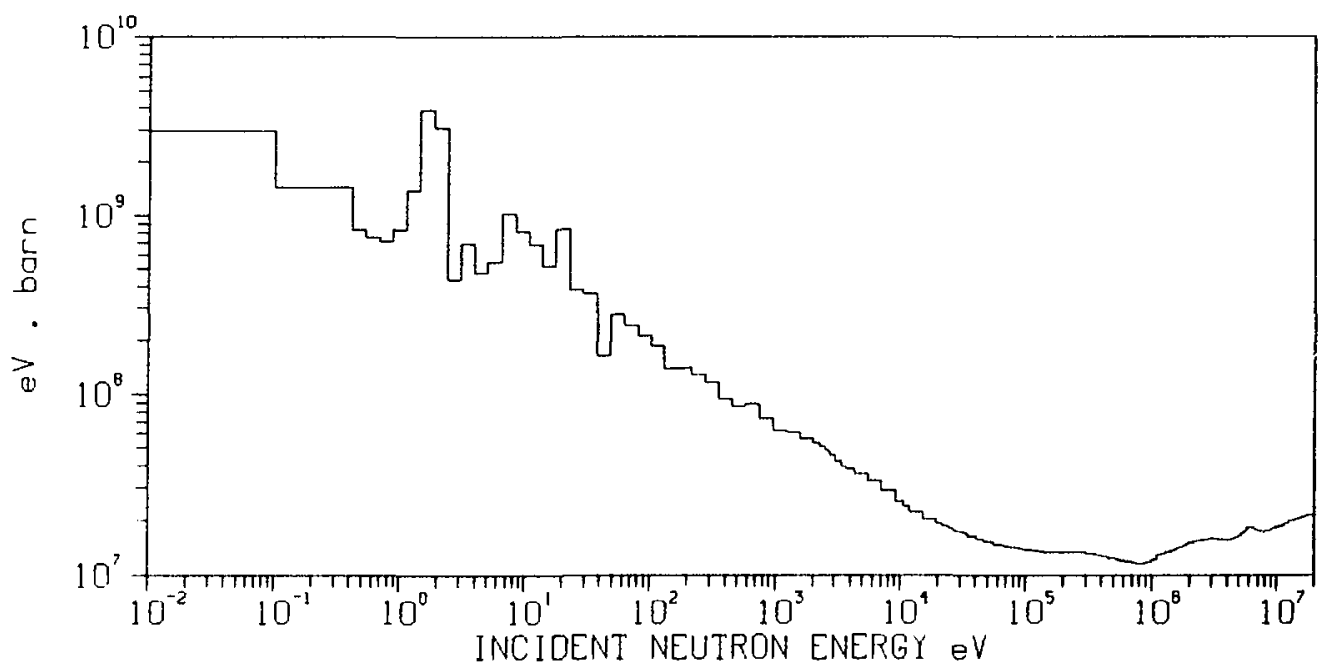

U-233 TOTAL CROSS SECTION

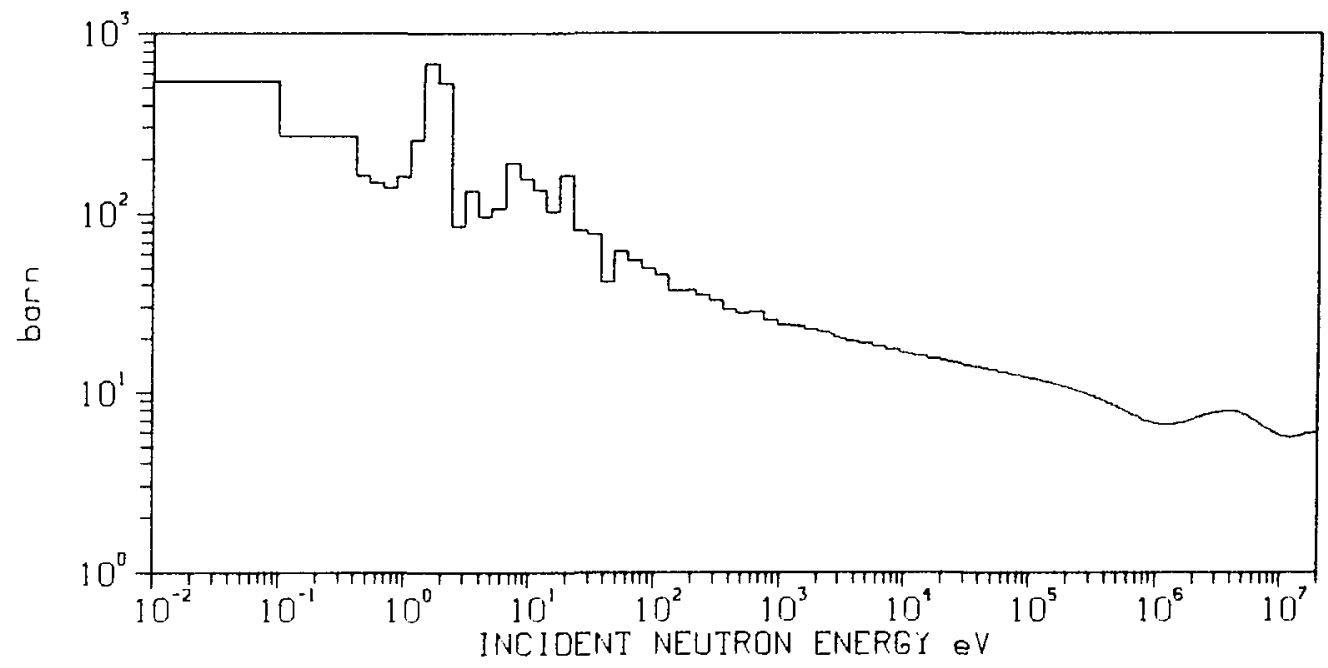




\section{U-233 ELASTIC CROSS SECTION}

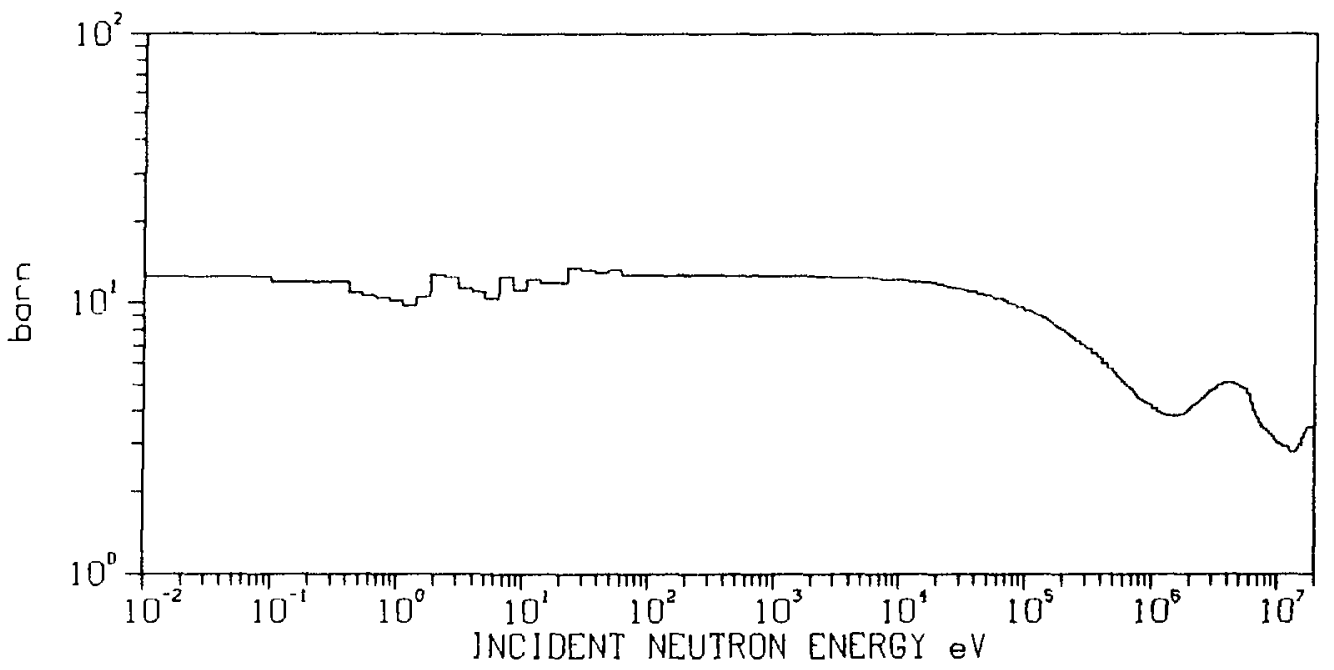

U-233 TOTAL INELASTIC CROSS SECTION

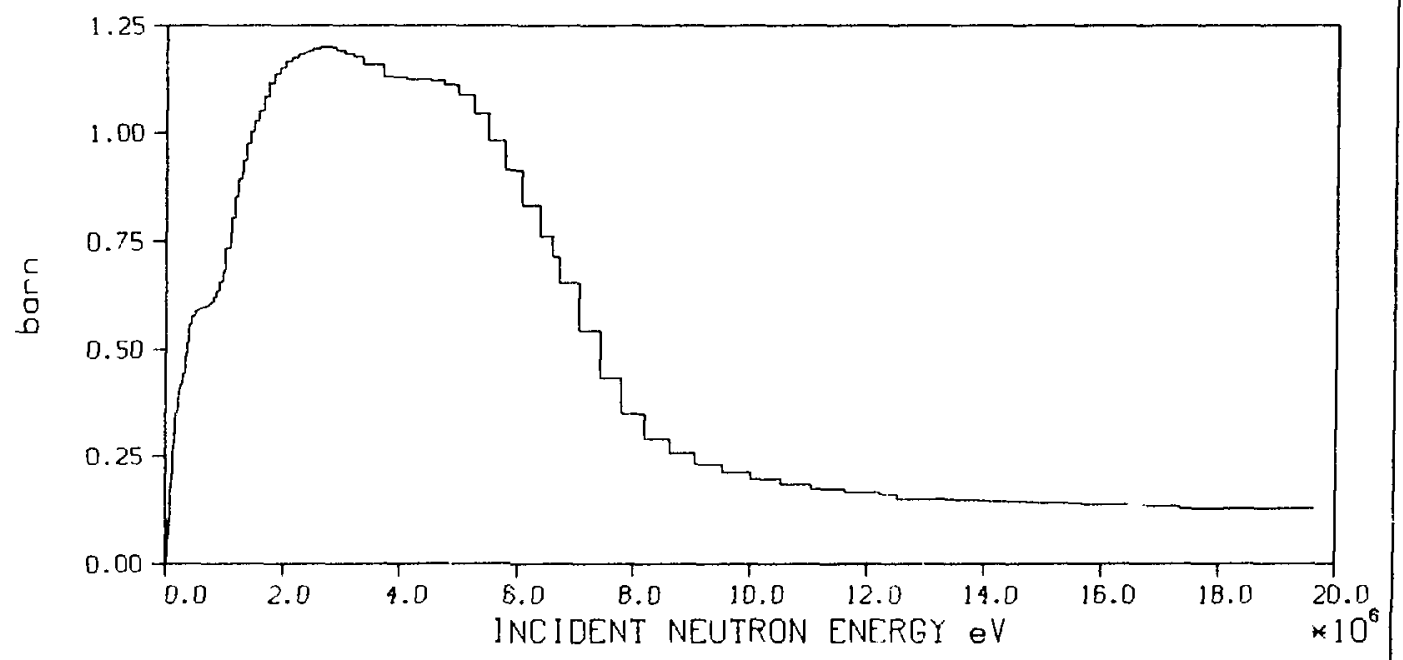


U-233 RADIATIVE CAPTURE CROSS SECTION

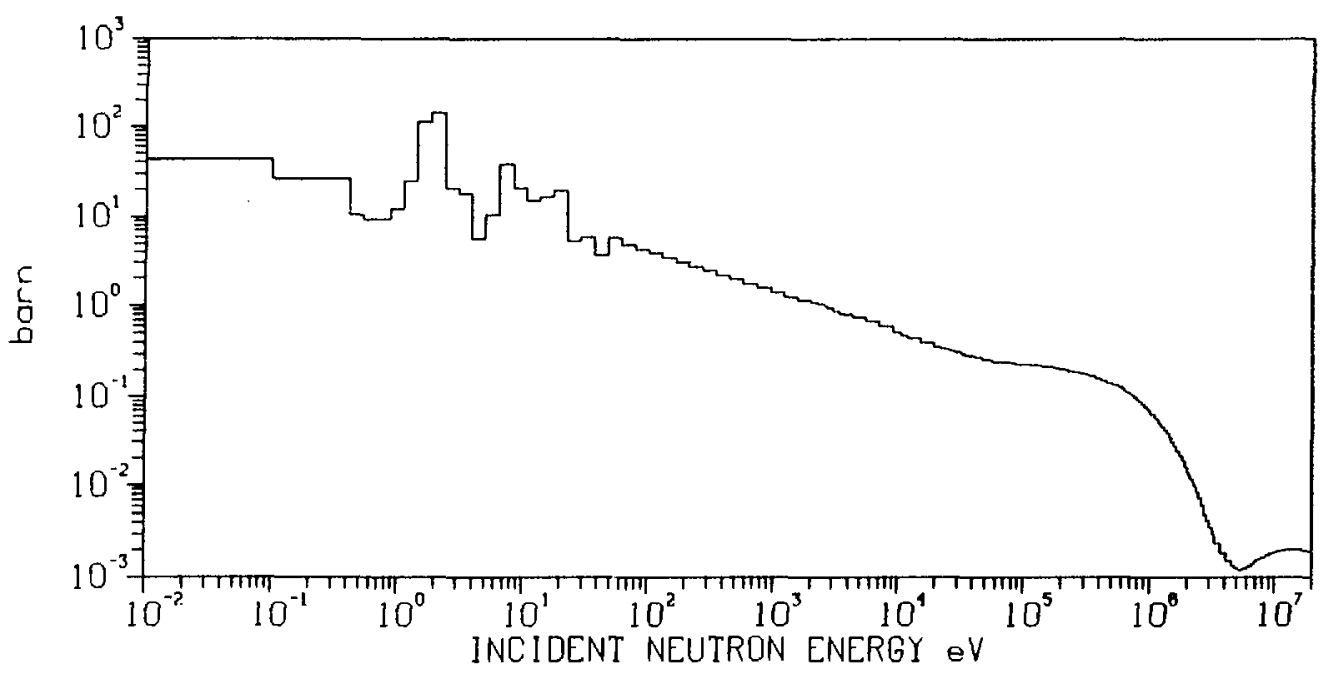

U-233 TOTAL $(n, 2 n)$ REACTION CROSS SECTION

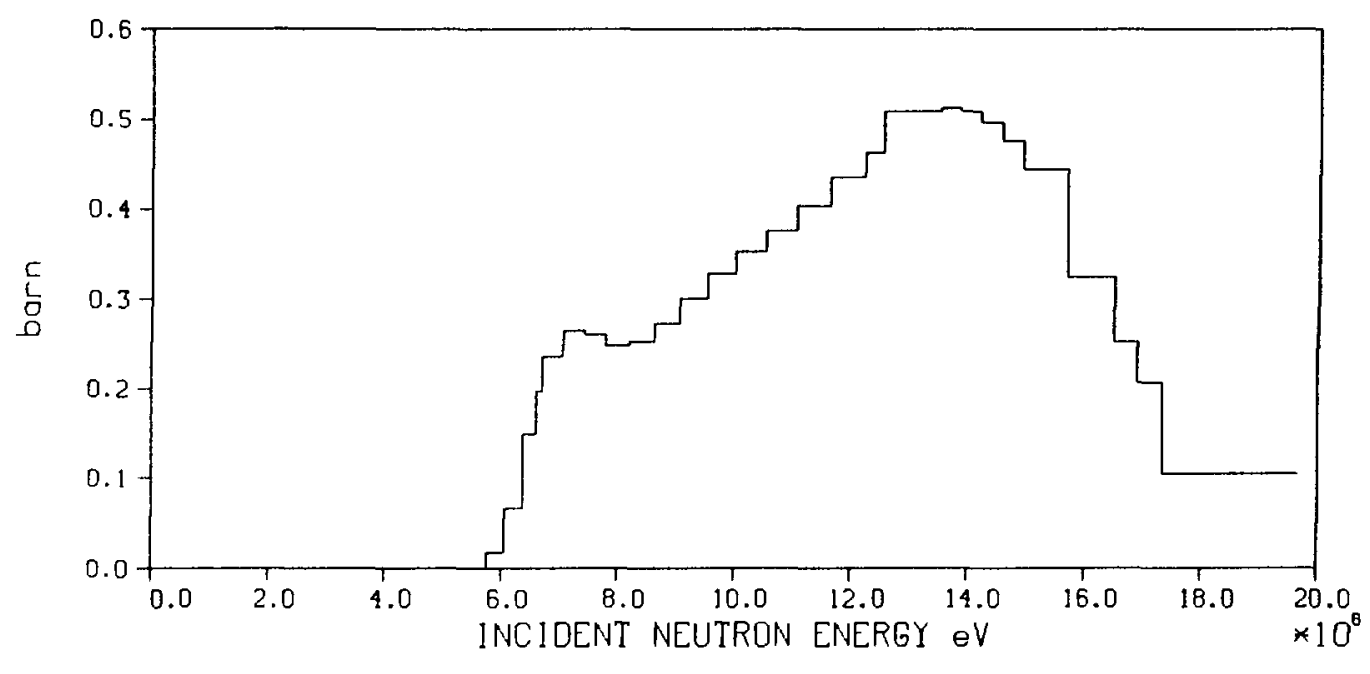


U-233 TOTAL $[n, 3 n]$ CROSS SECTION

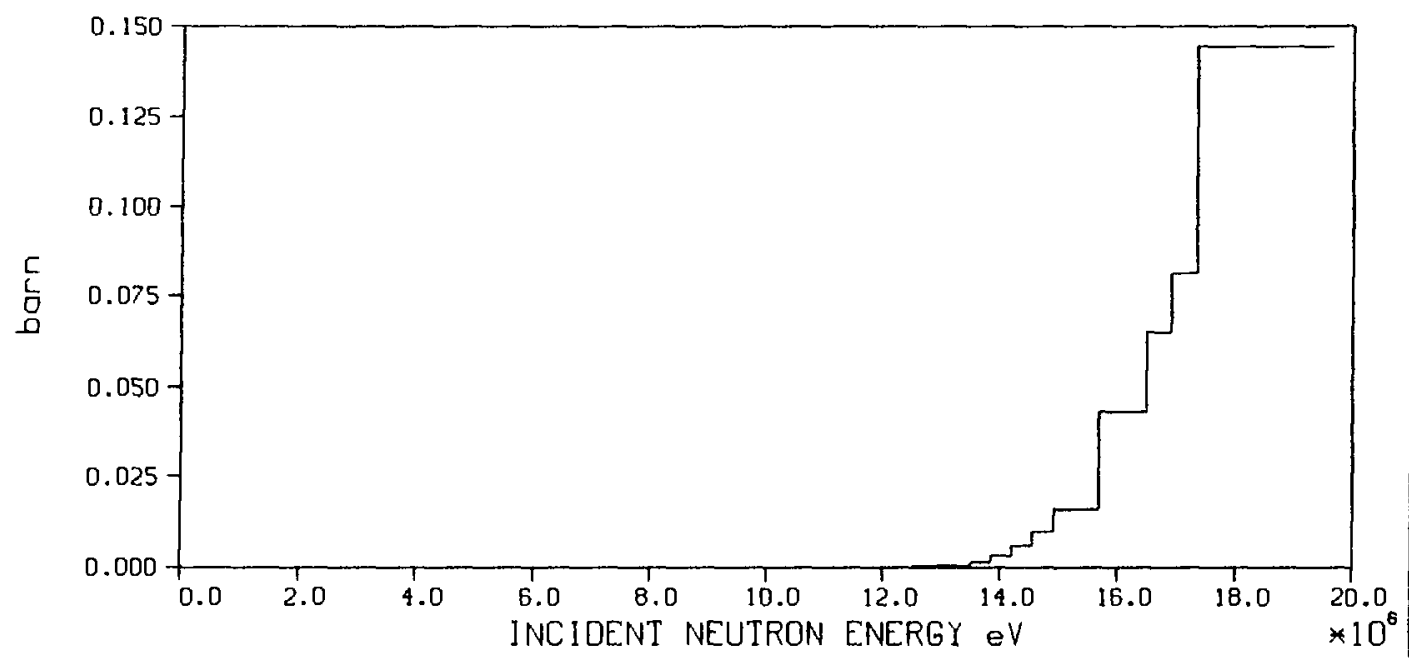

U-233 NUMBER OF FISSION NEUTRONS * EROSS SECTION

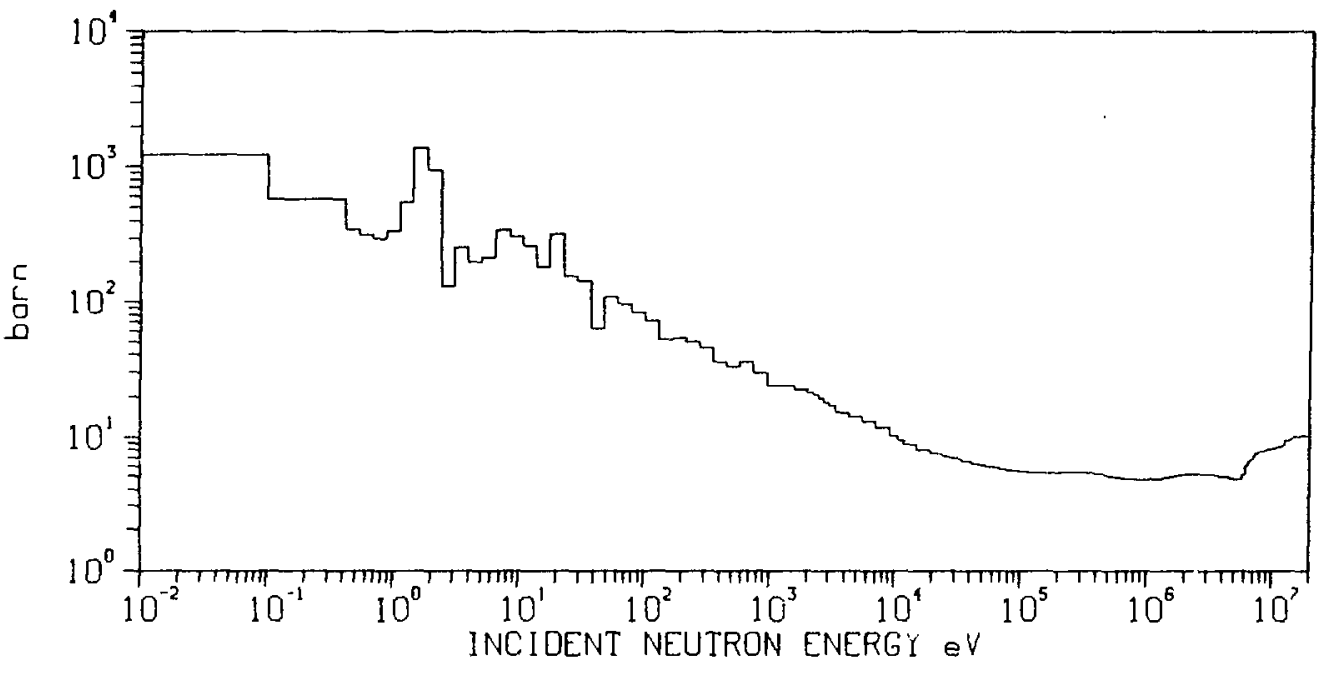


U-234 RECOMMENDED PROMPT KERMA FACTOR (solid line) REC. K. FACTOR WITH CH. FRRTICLE DECAY HEAT (dashed line)

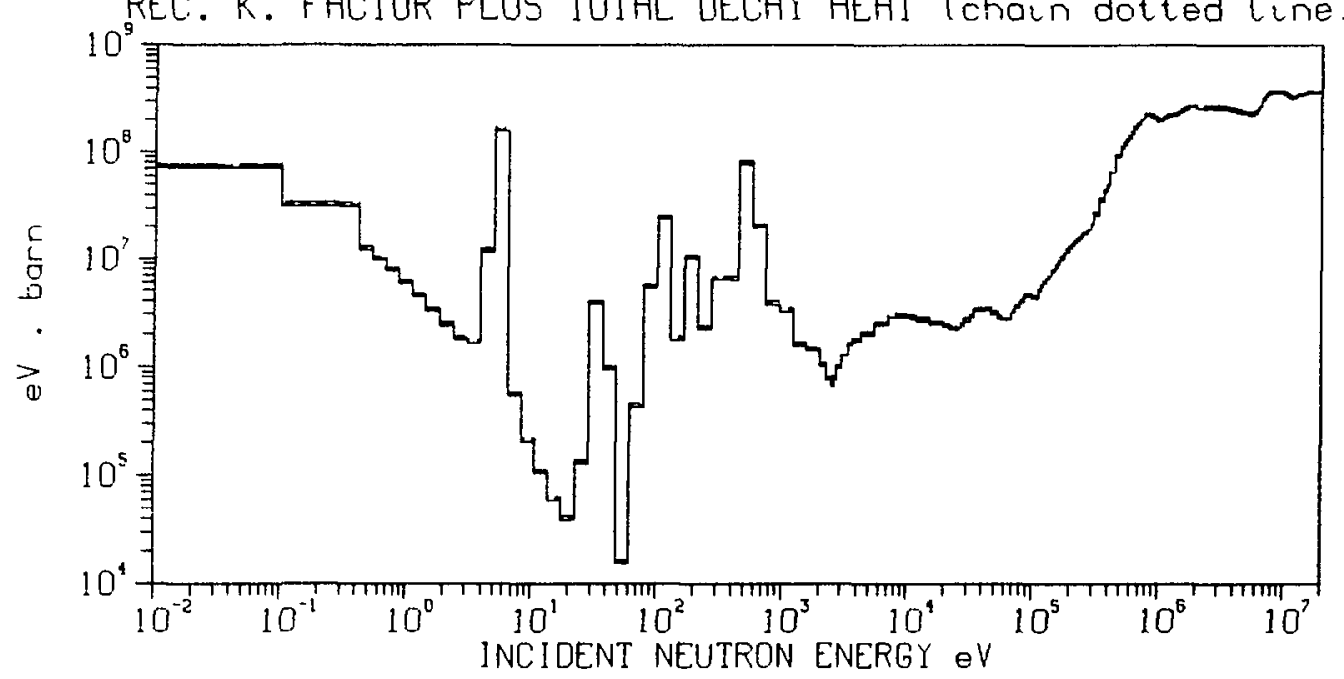

U-234 RECOMMENDED PROMPT KERMA FACTOR (solid line)

REC. K. FACTOR WITH CH. PARTICLE DECAY HEAT (dashed line)

$\infty$ REC. K. FACTOR PLUS TOTAL DECAY HEAT (chain dotted line)

$\bar{x}$

4.

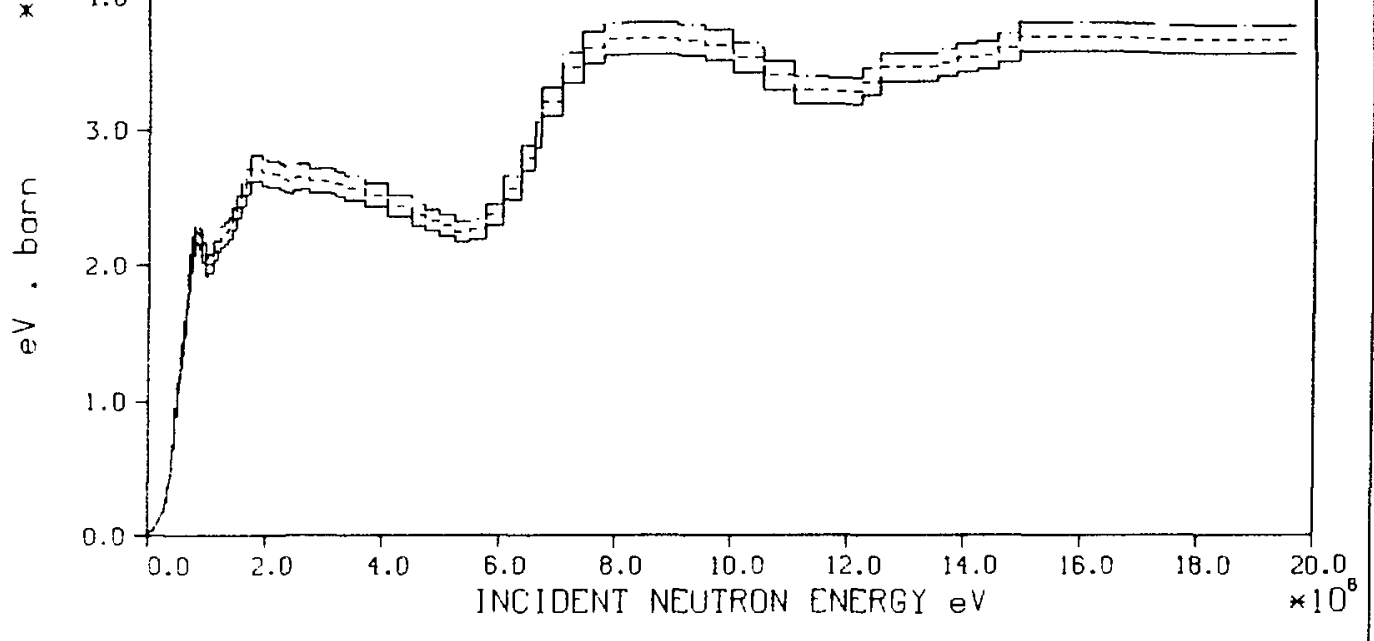


U-234 TOTAL CROSS SECTION

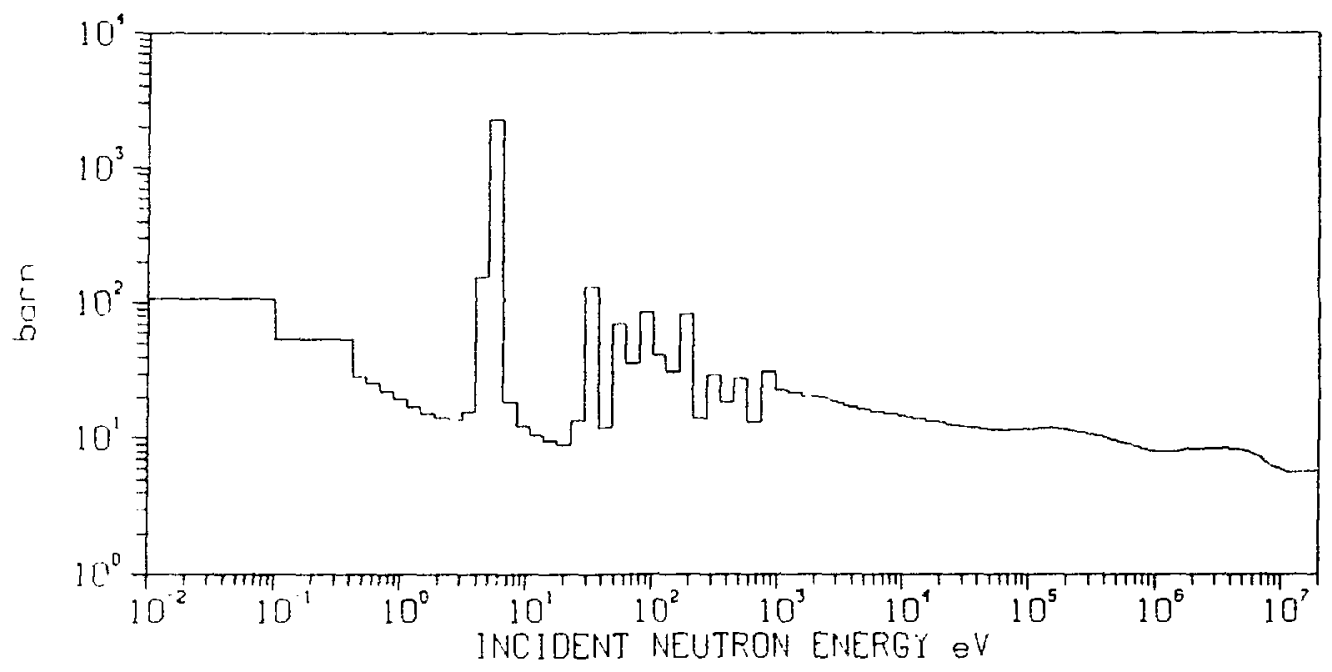

U-234 ELASTIC CROSS SECTION

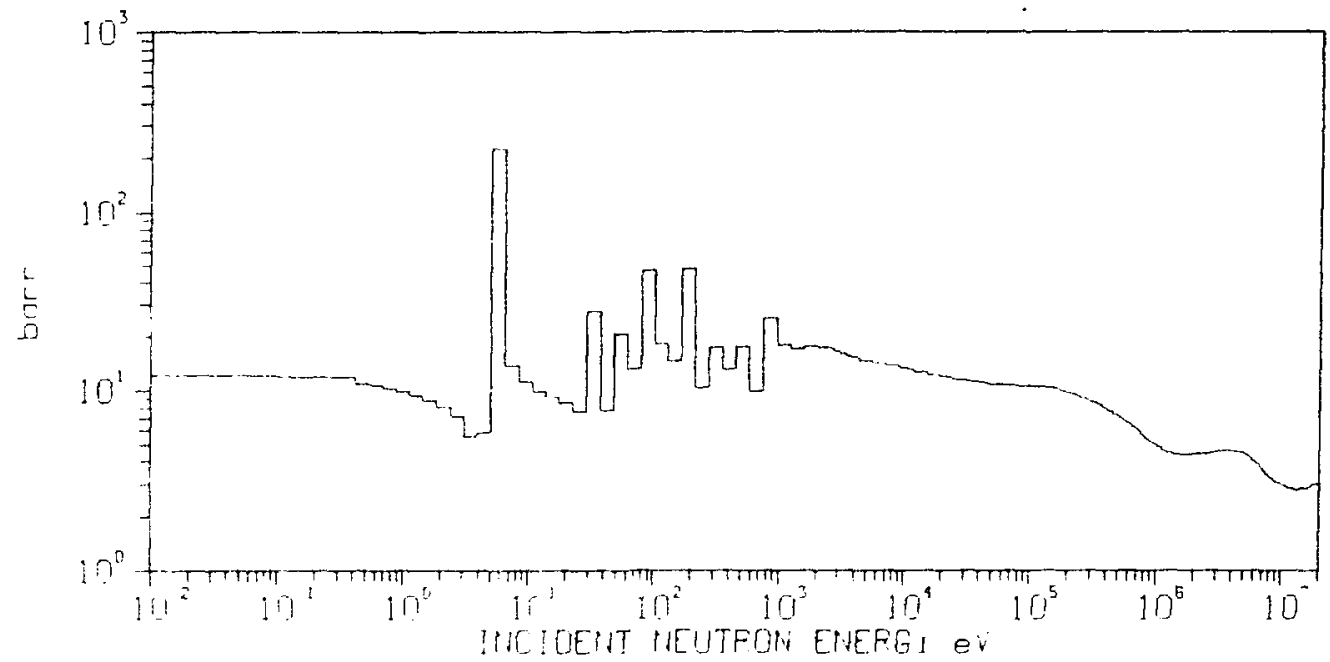




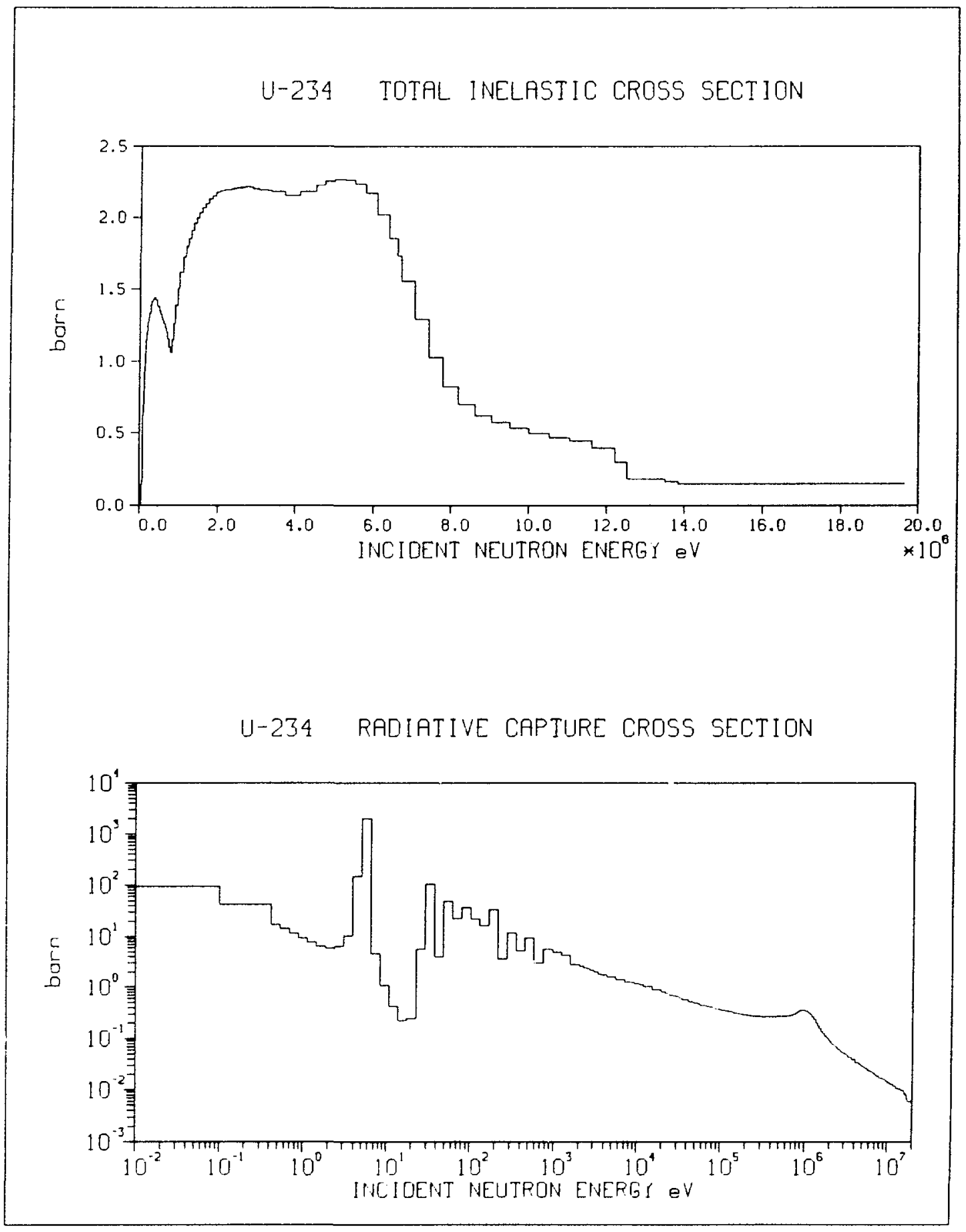




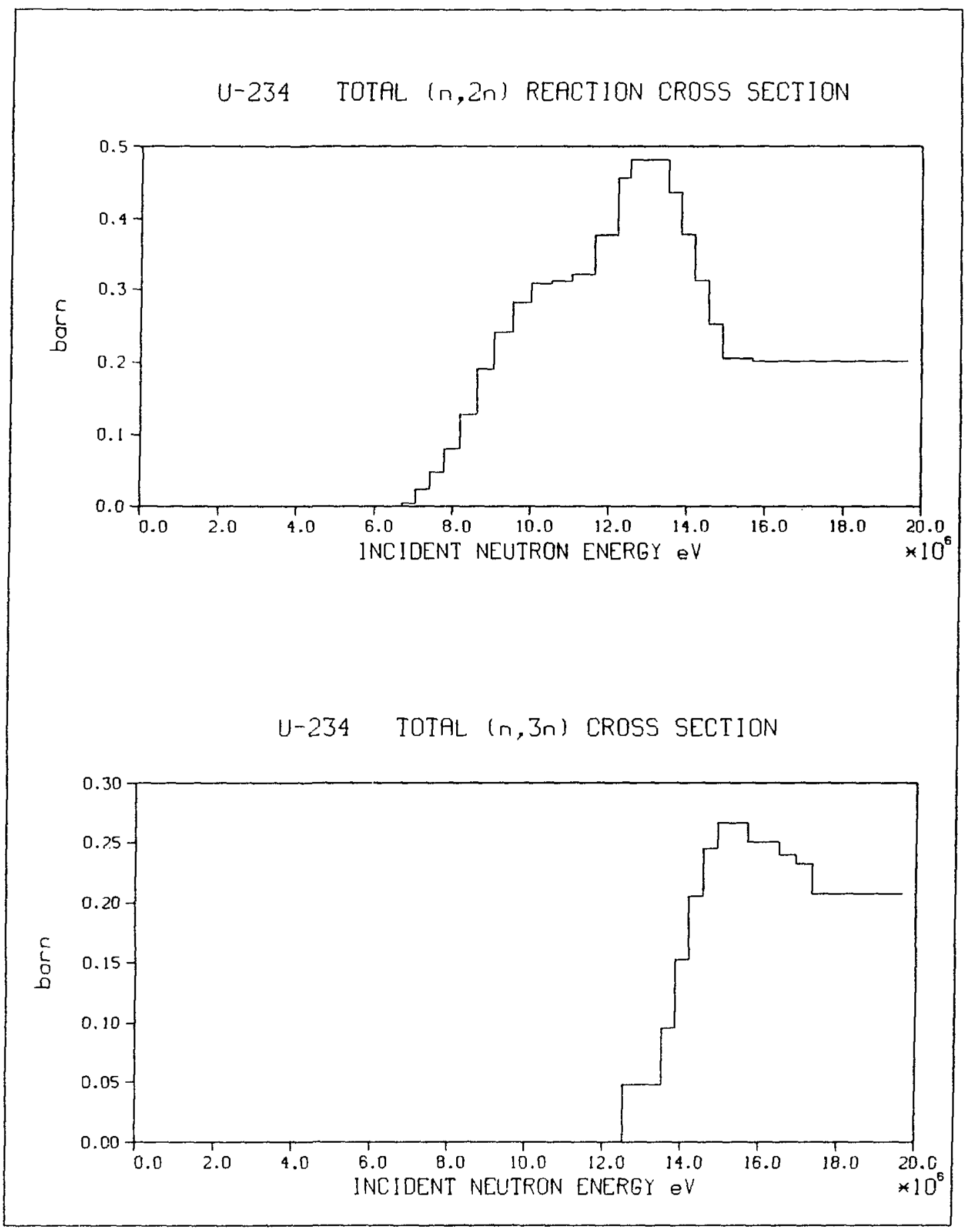




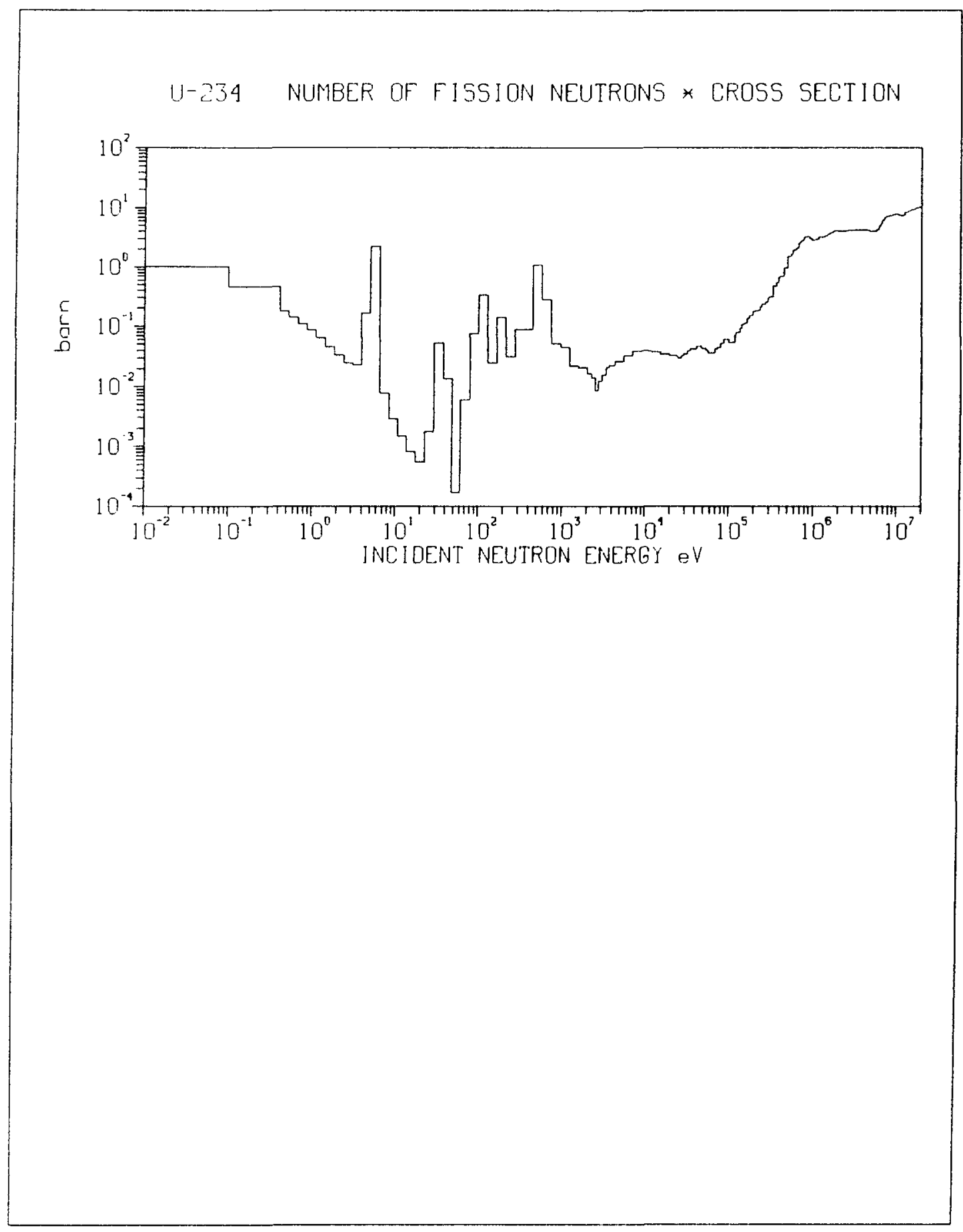




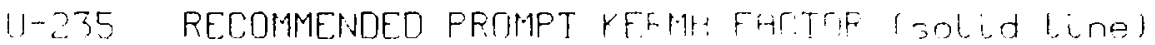

FEC. K. FACTOR WITH CH. PARTICLE TERA HF.AT ldashed linel REE. K. FACTOR PLUS TOTAL DECA! HEHT ! ghom dotted lune!

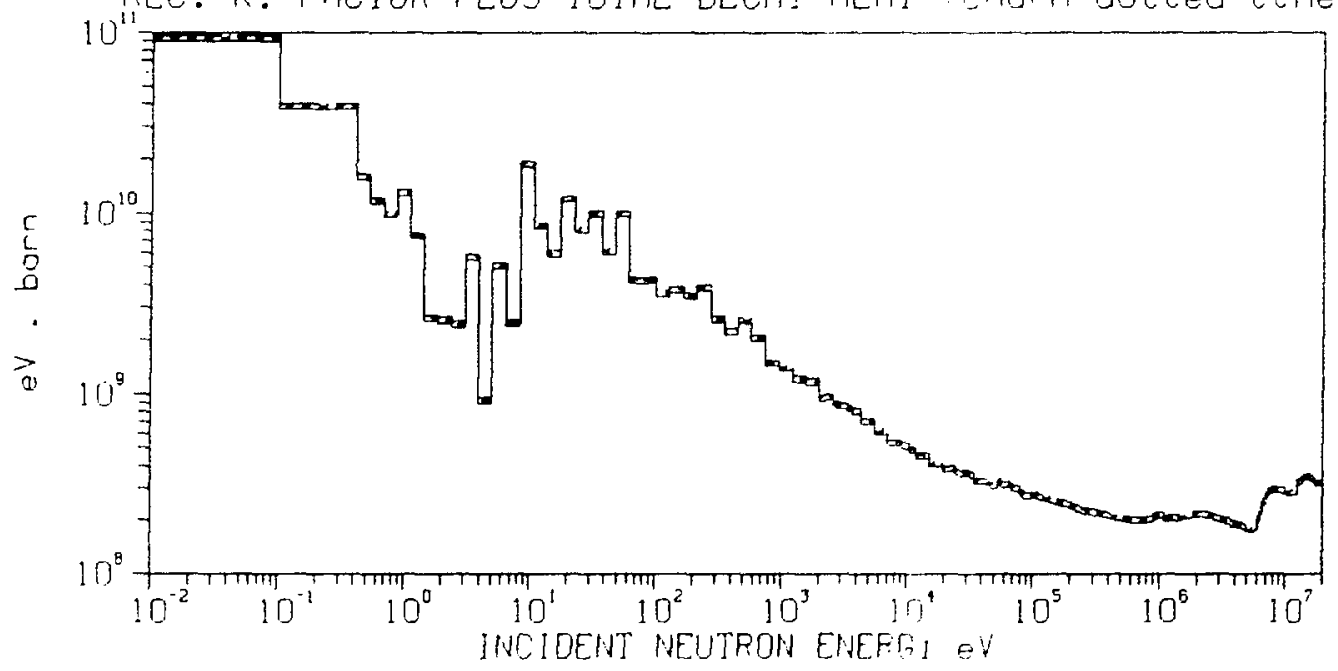

U-235 PECOMMEHOED PROMPT KEPHA FACTDR (solid line) PEC. K. FACIOF WITH CH. PARTICLE DECRI HEAT I doshed Lune $\because$ REC. K. FACTOR PLUS TOTFL DECAI HEFT I chain dotted Line) $\underset{x}{2}$

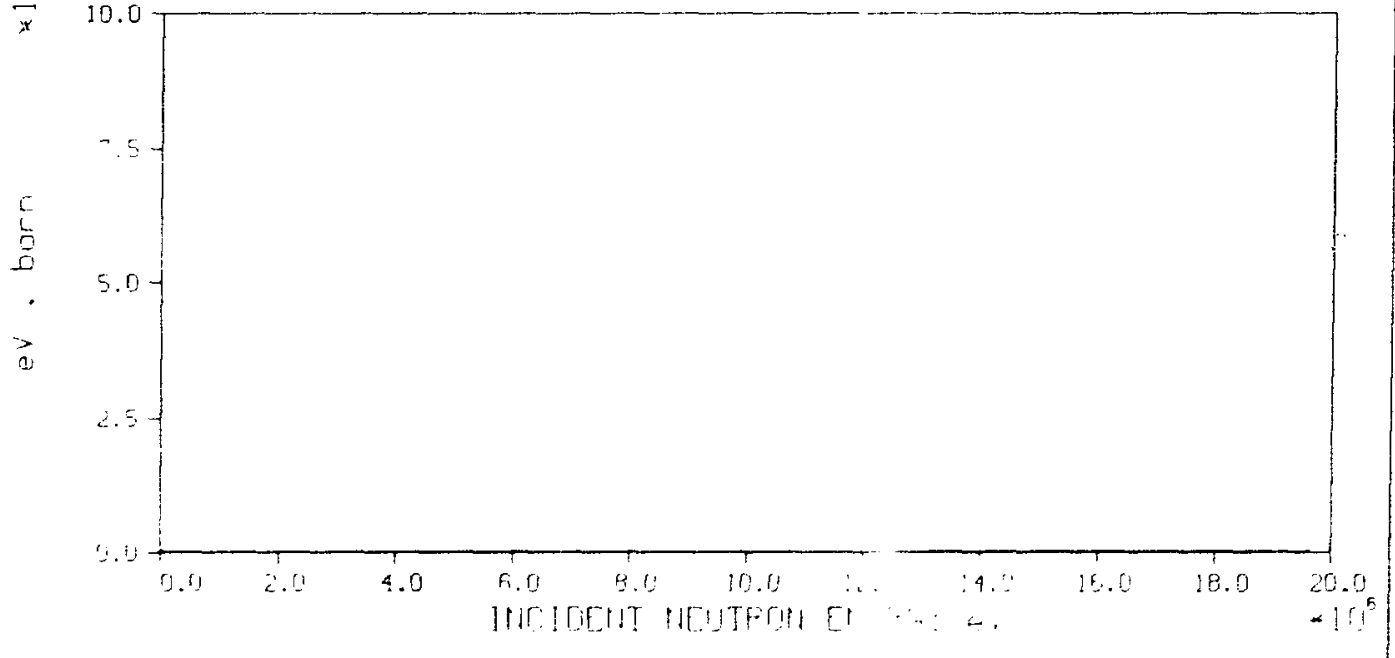




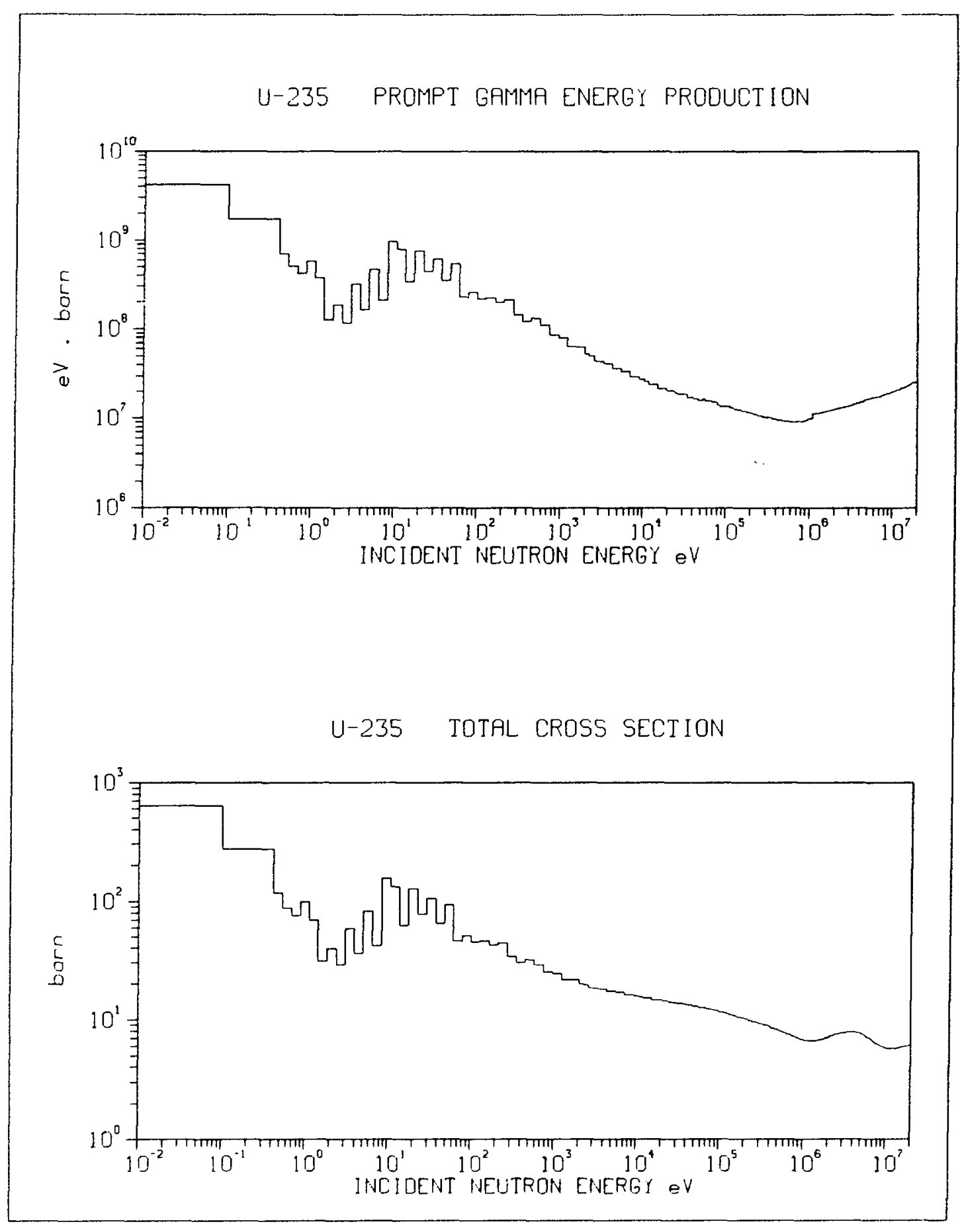




\section{U-235 ELASTIC CROSS SECTION}

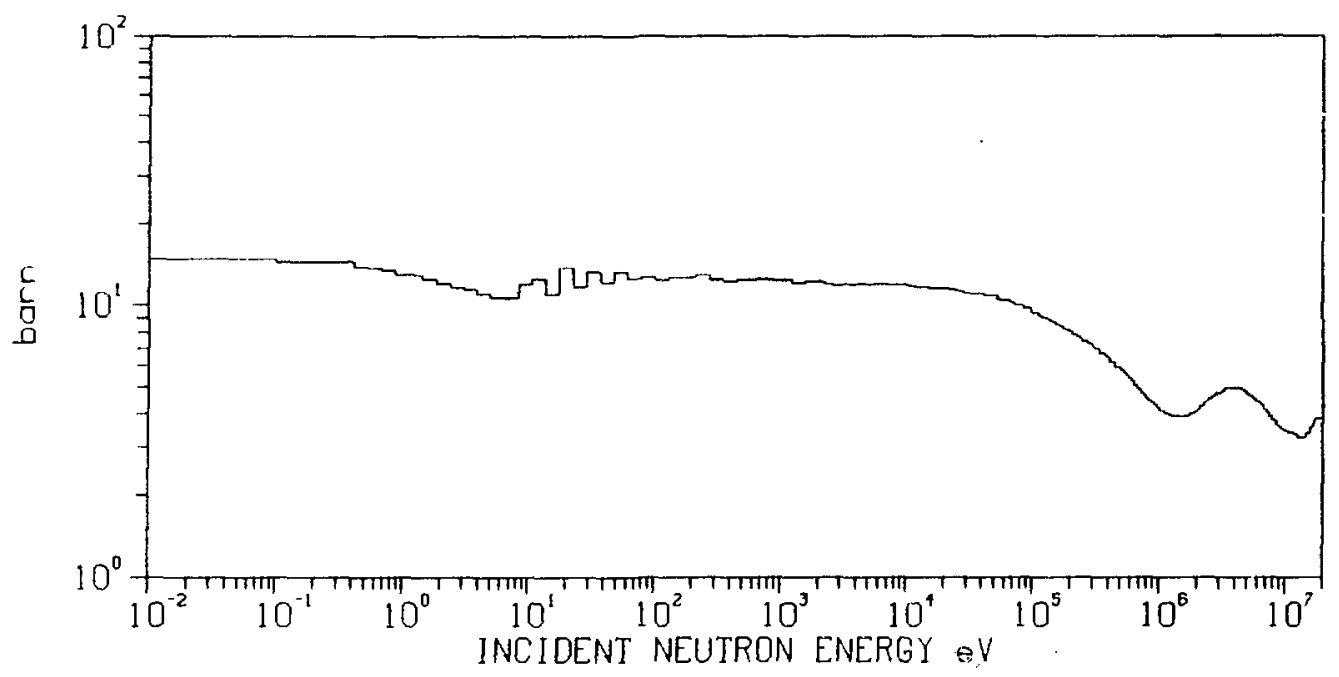

U-235 TOTAL INELASTIC CROSS SECTION

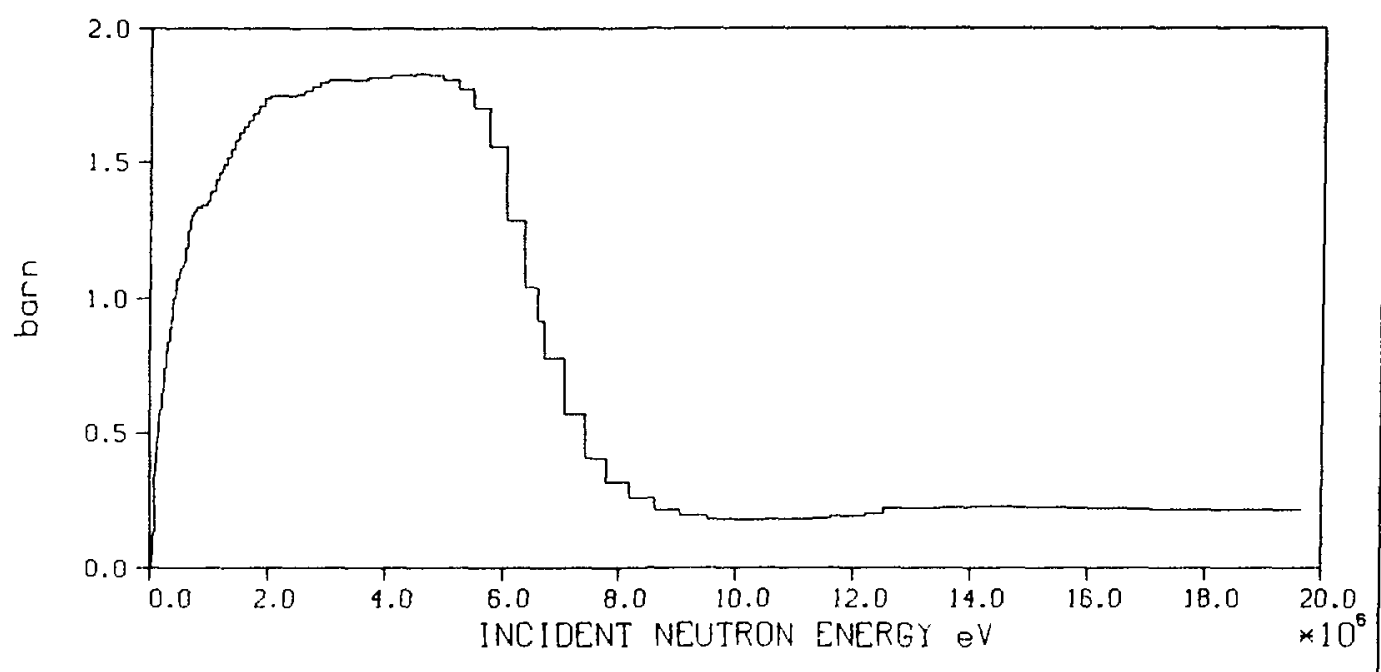




\section{U-235 RAOIATIVE CAPTURE CROSS SECTION}

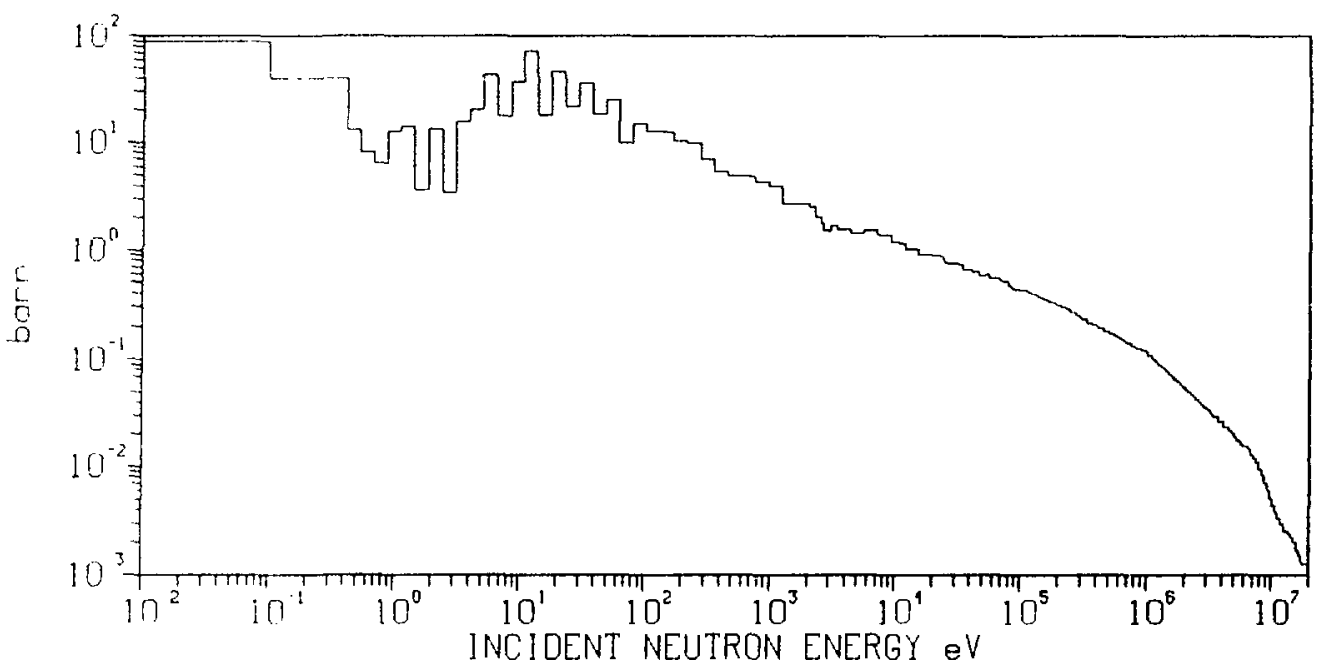

U-235 TOTAL $(n, 2 n)$ REACTION CROSS SECTION

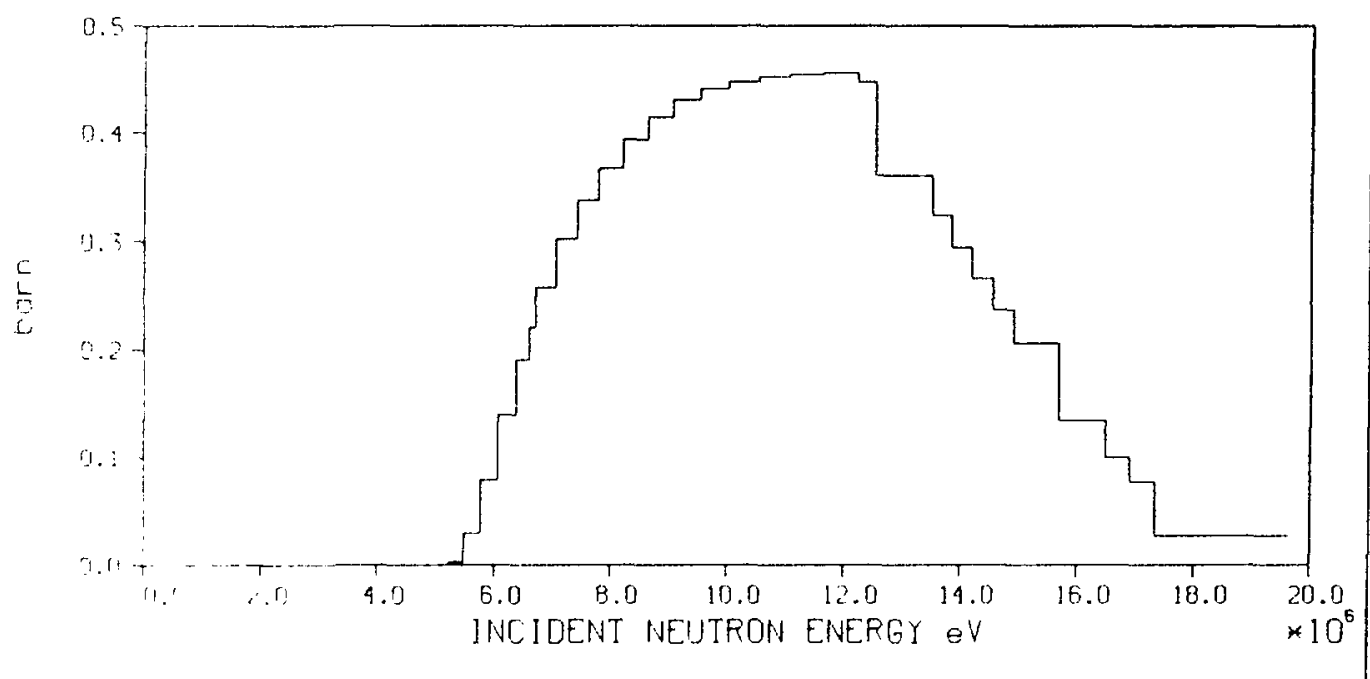


U-235 TOTAL $(n, 3 n)$ CROSS SECTION

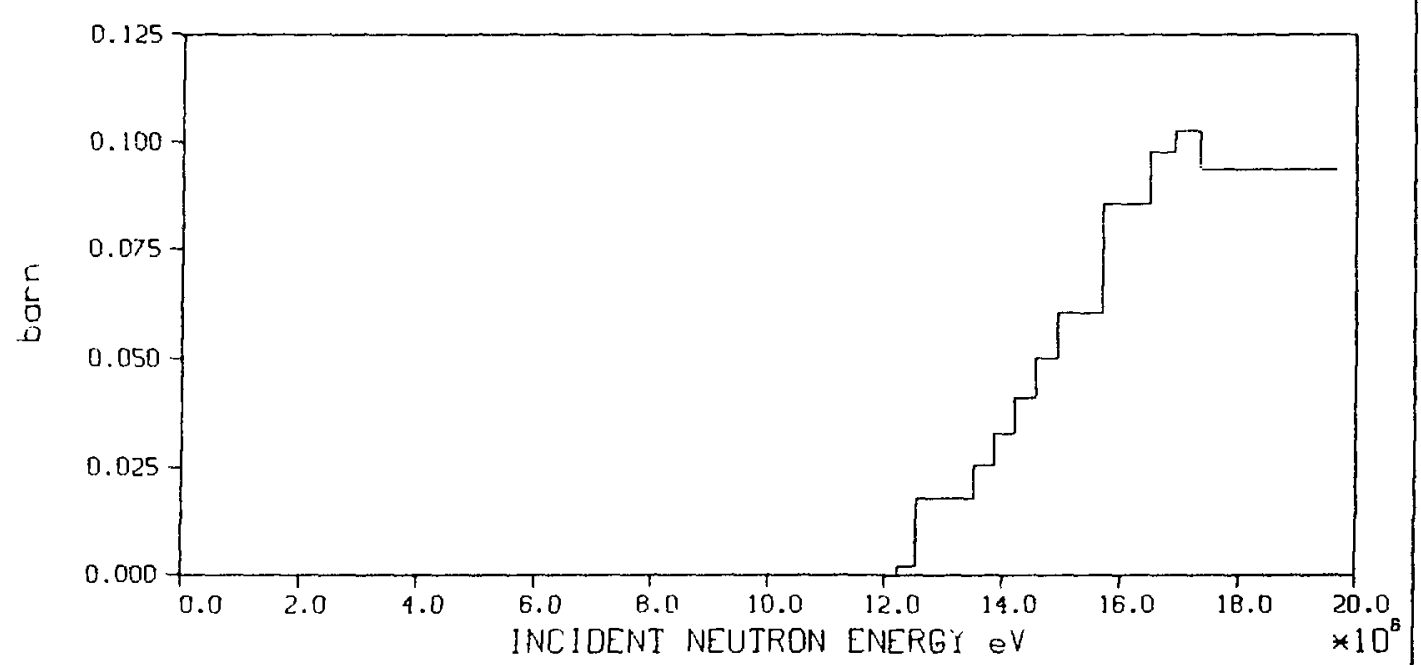

U-235 NUMBER OF FISSION NEUTRONS * CROSS SECTION

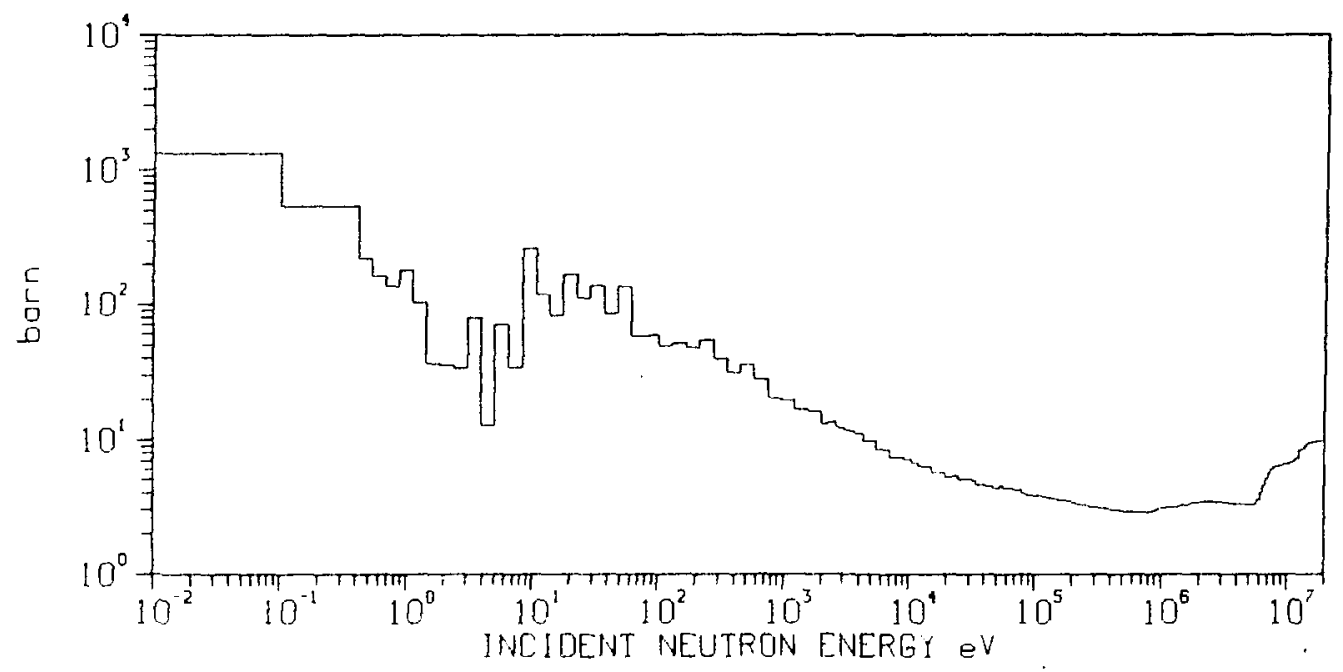




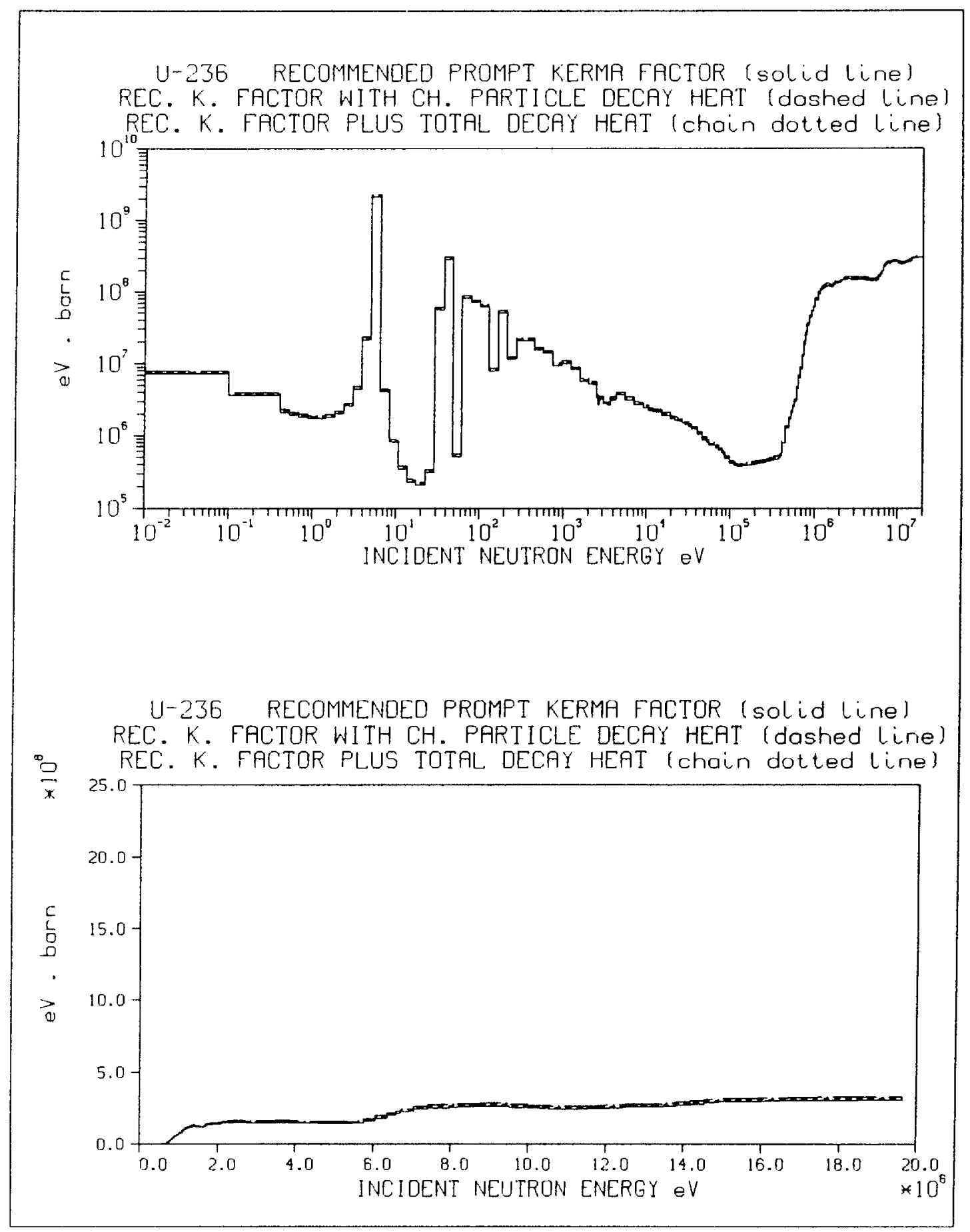


U-236 TOTAL CROSS SECTION

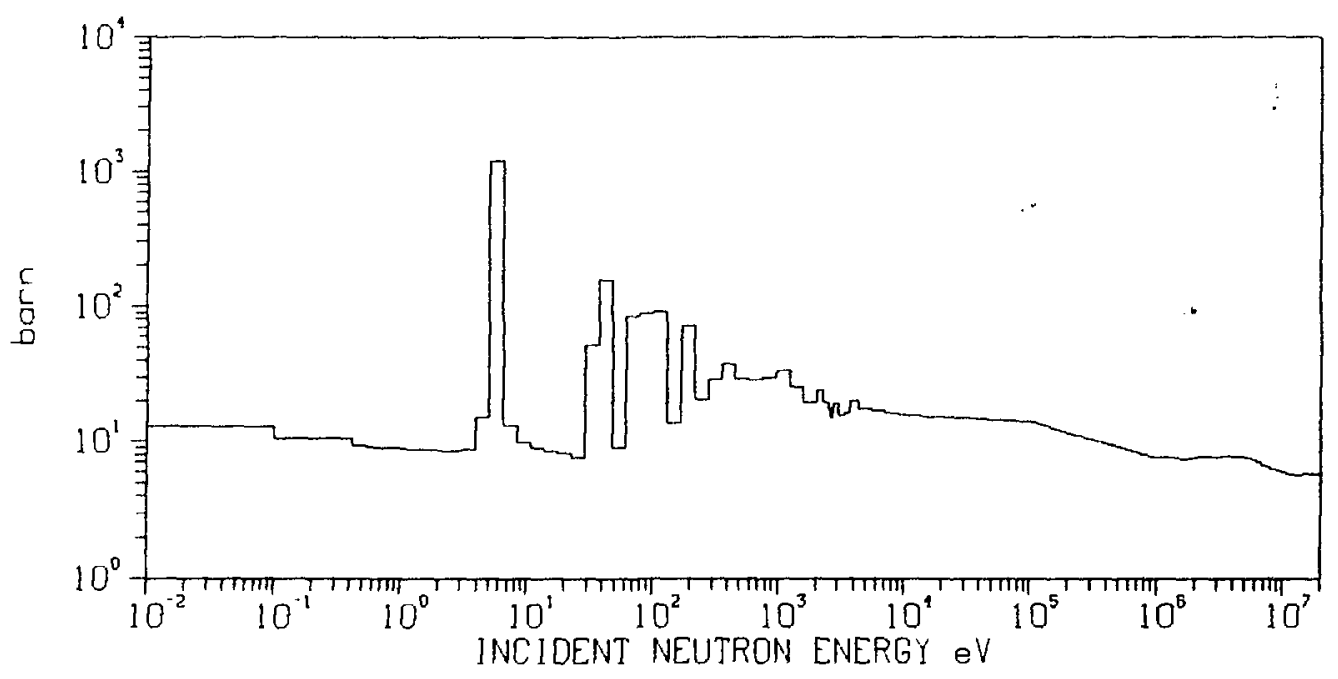

U-236 ELASTIC CROSS SECTION

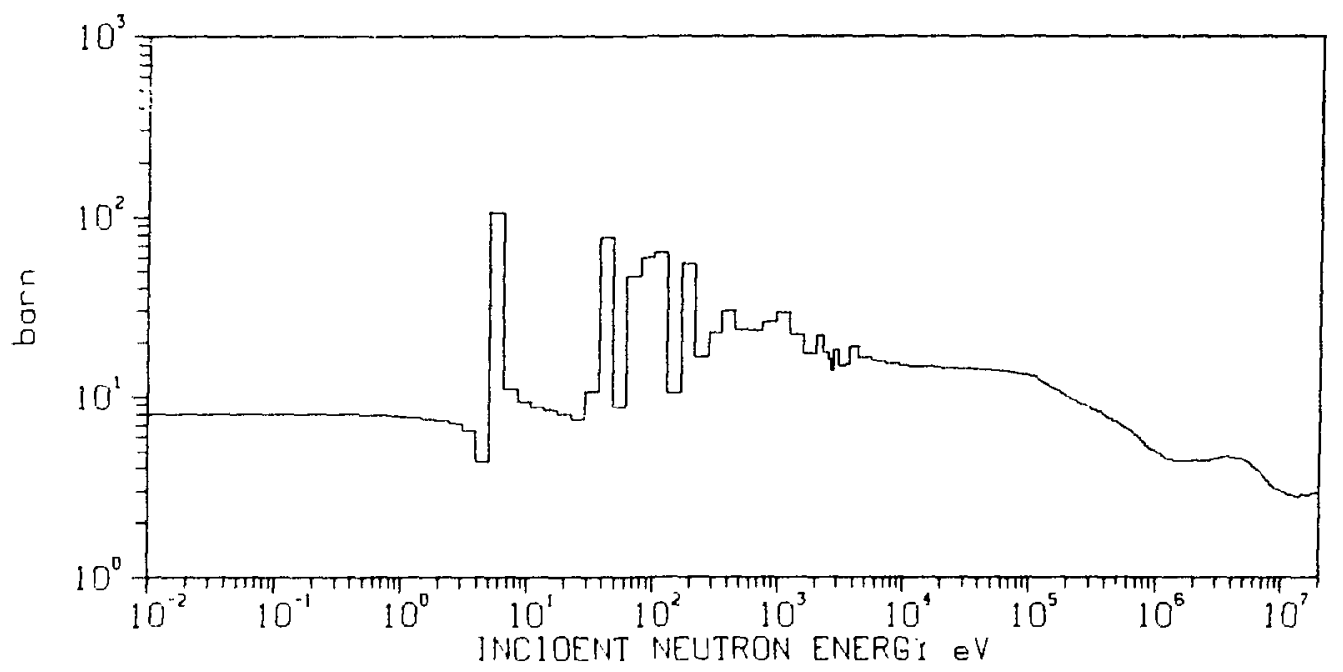




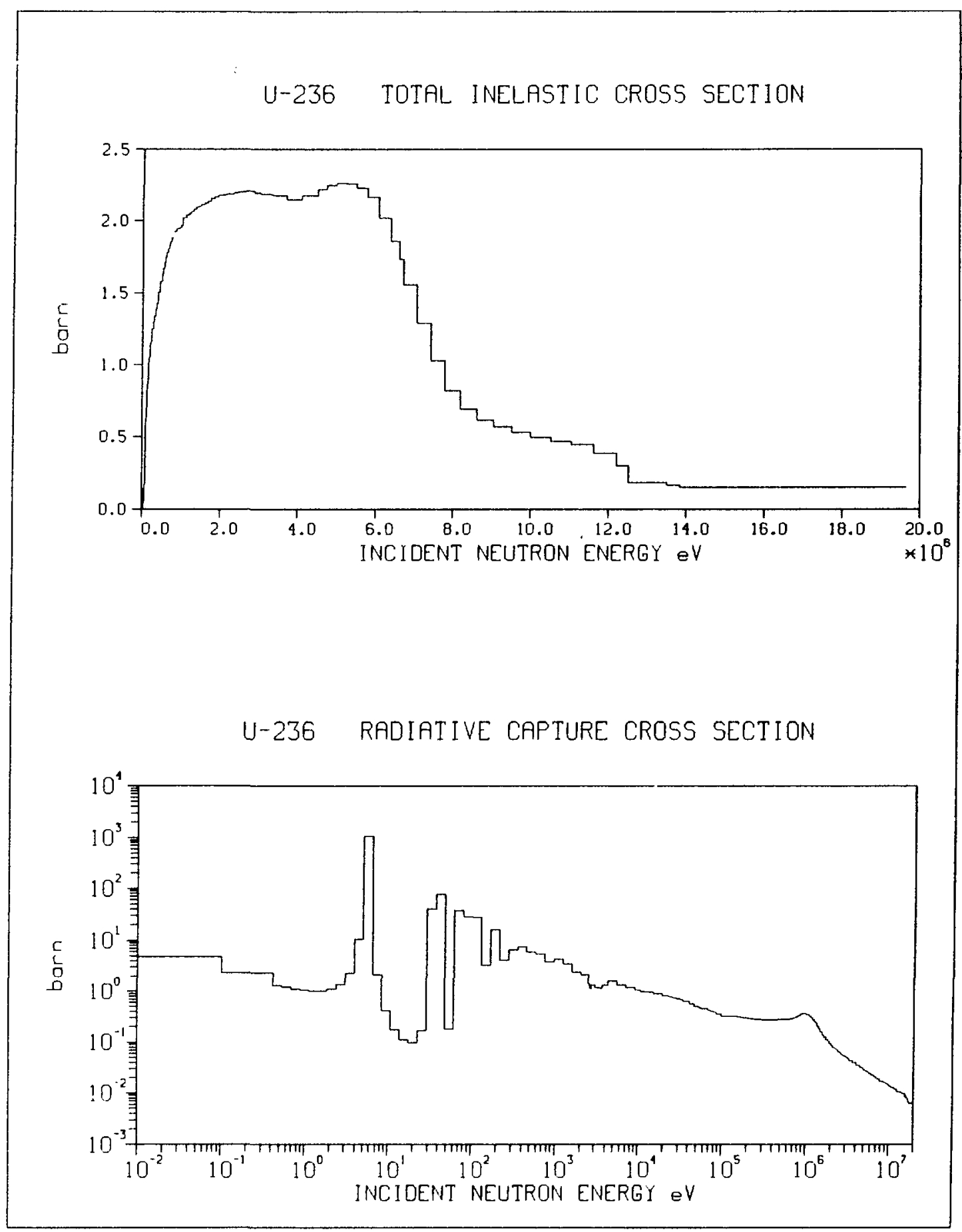


U-236 TOTAL $(n, 2 n)$ REACTION CROSS SECTION

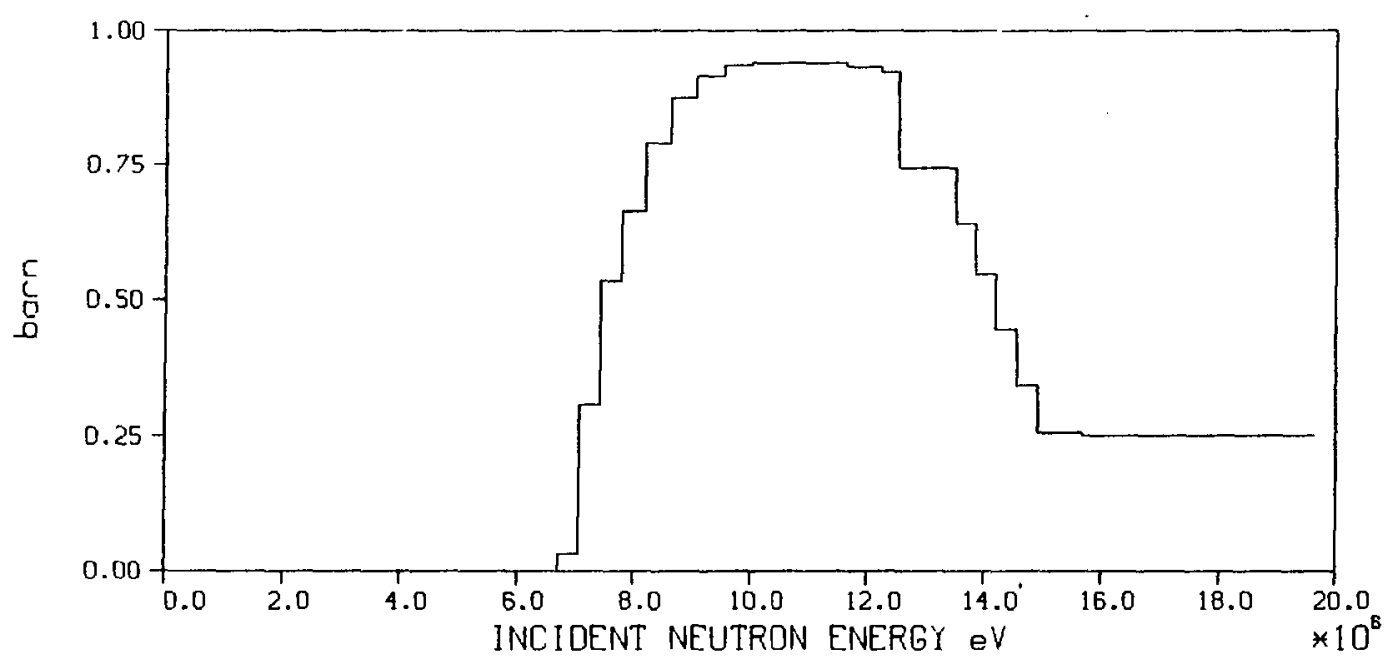

U-236 TOTAL $(n, 3 n)$ CROSS SECTION

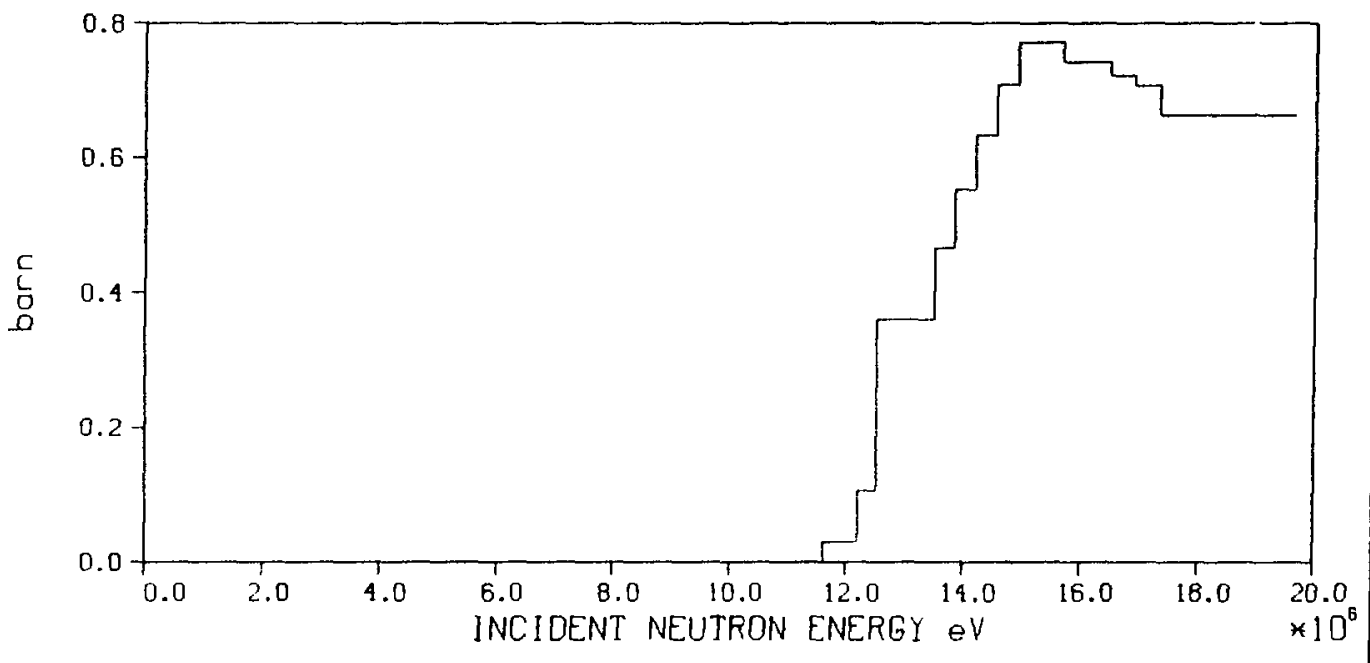




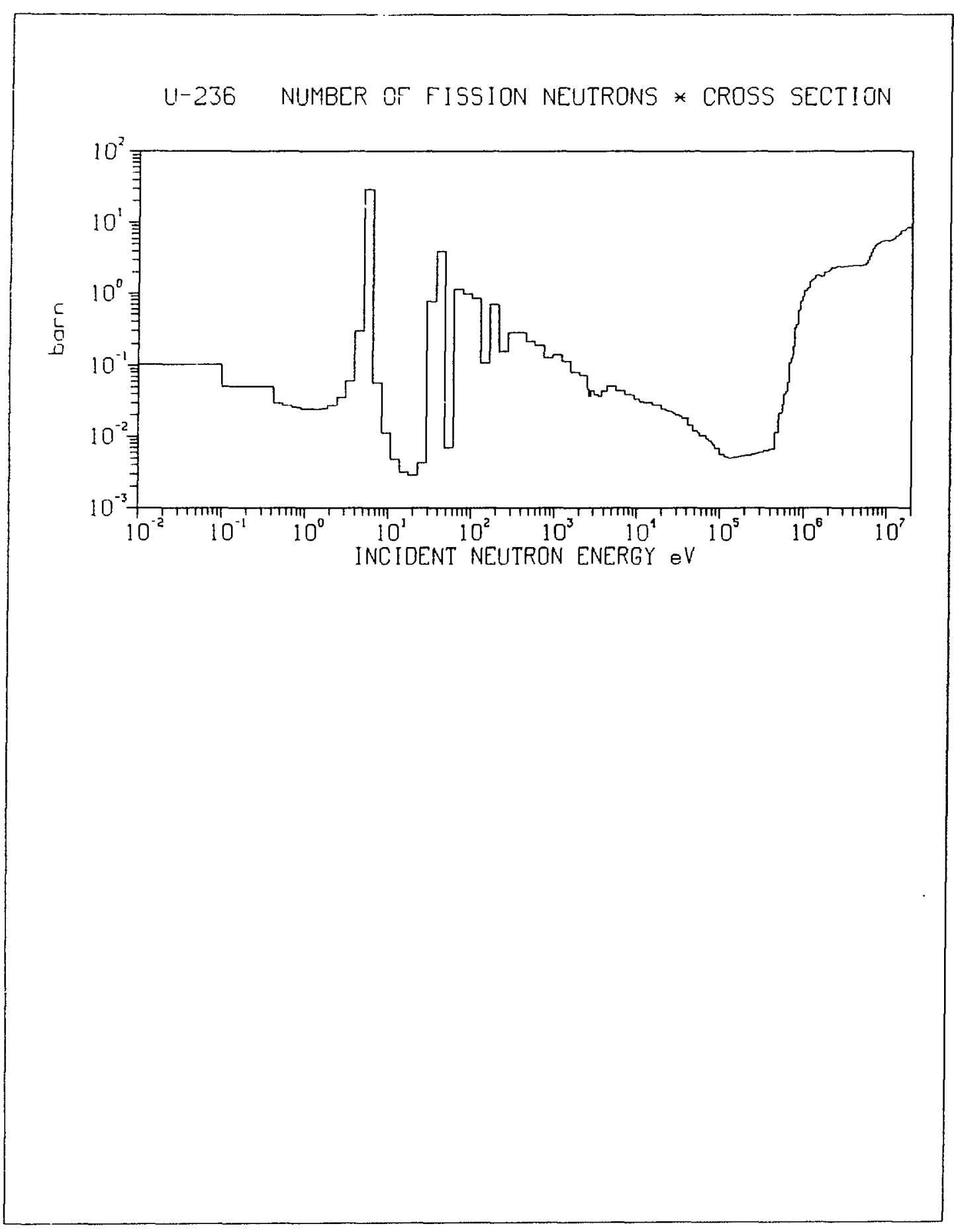


U-238 RECOMMENLEO PROMPT KERMA FACTOR (solid line) PEC. K. FACTDR WITH CH. PARTICLE DECAI HEAT (doshed line)

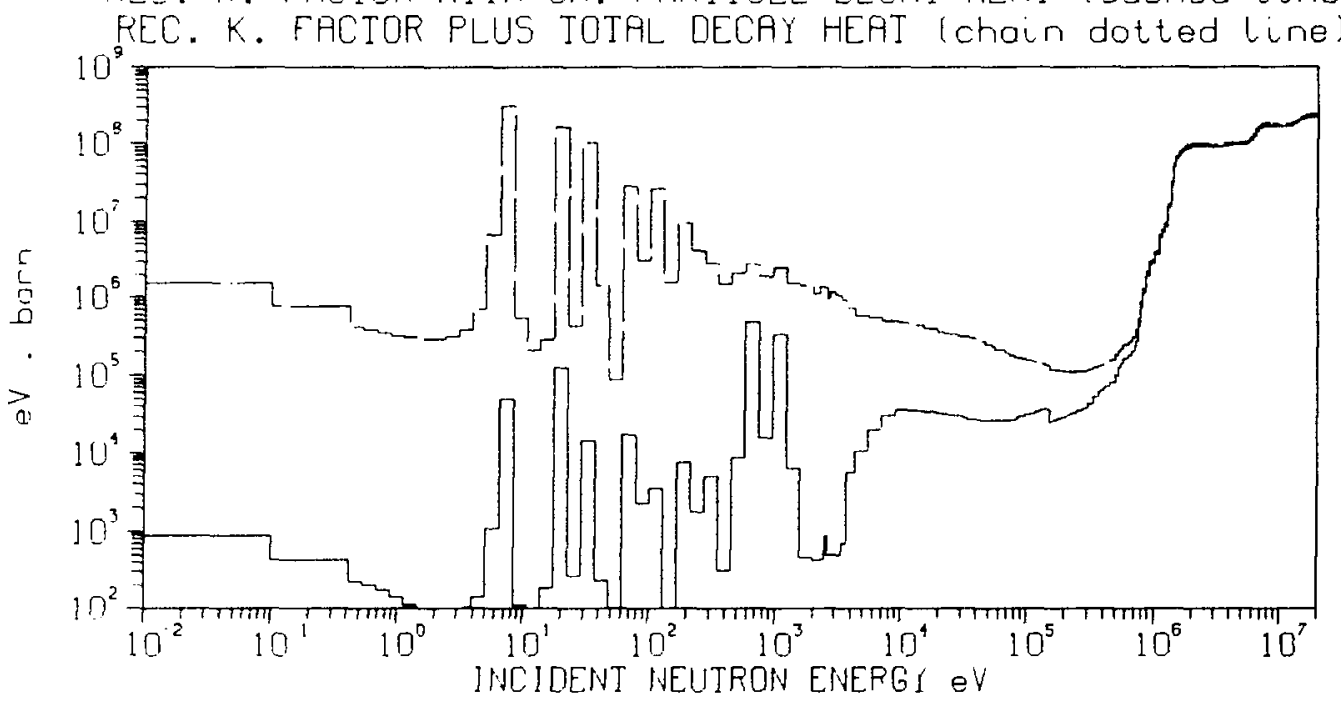

IJ-238 RECOMMENDED PROMPT KERMA FACTOR (solid line) REC. $K$. FACTOR WITH CH. PARTICLE OECAY HEAT (doshed line) $\because \quad$ PEC. K. FACTOR PLUS TOTAL DECAY HEAT (chain dotted line)

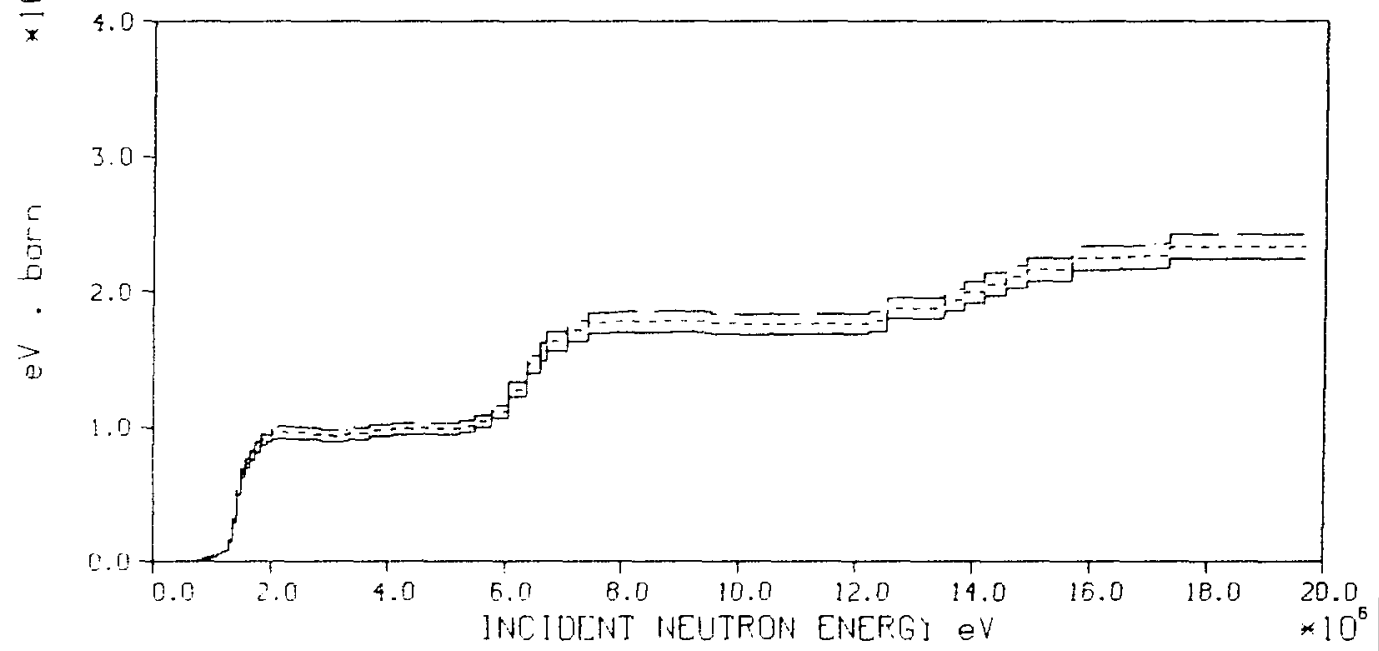




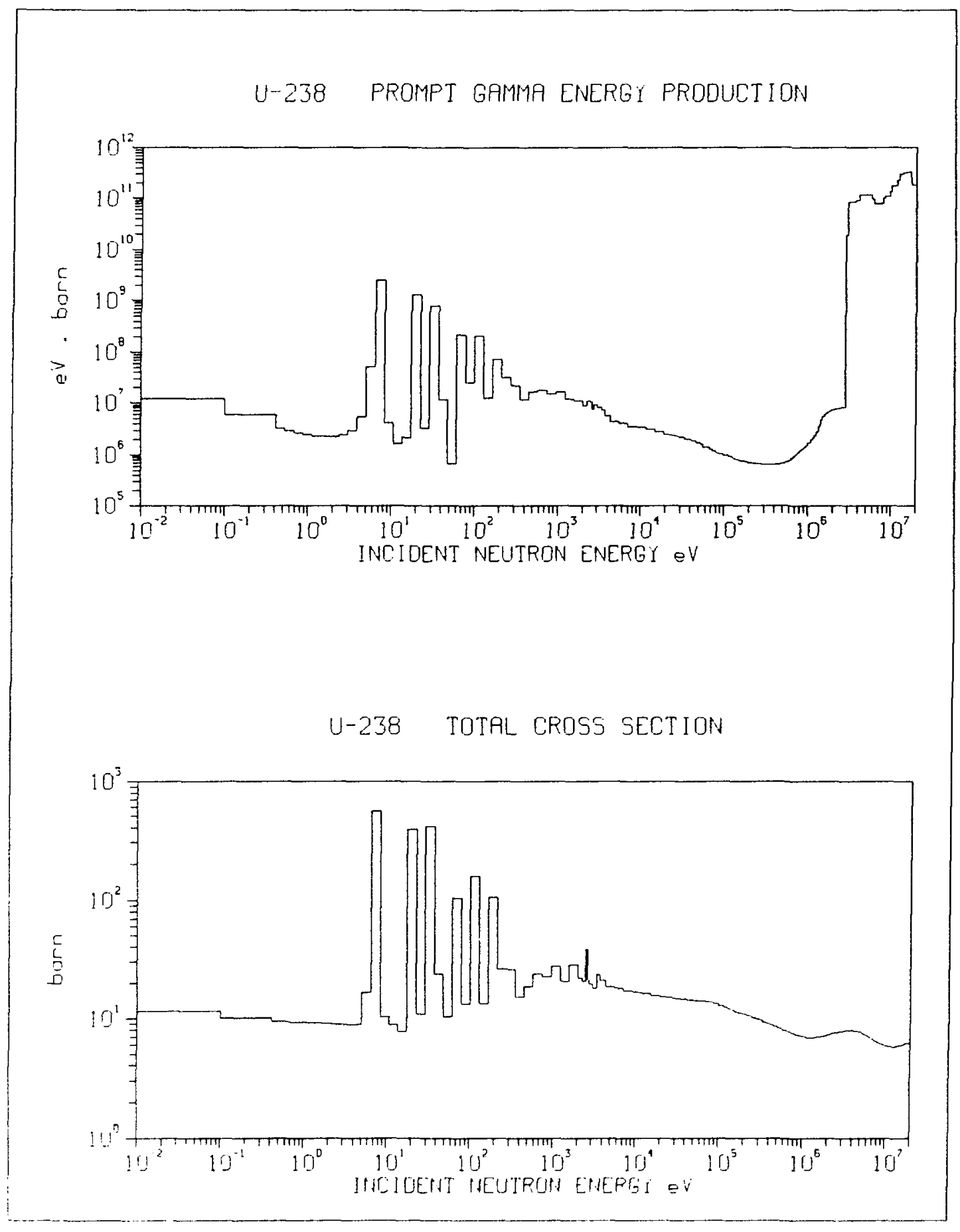


U-238 CLASTIC CROSS SECTION

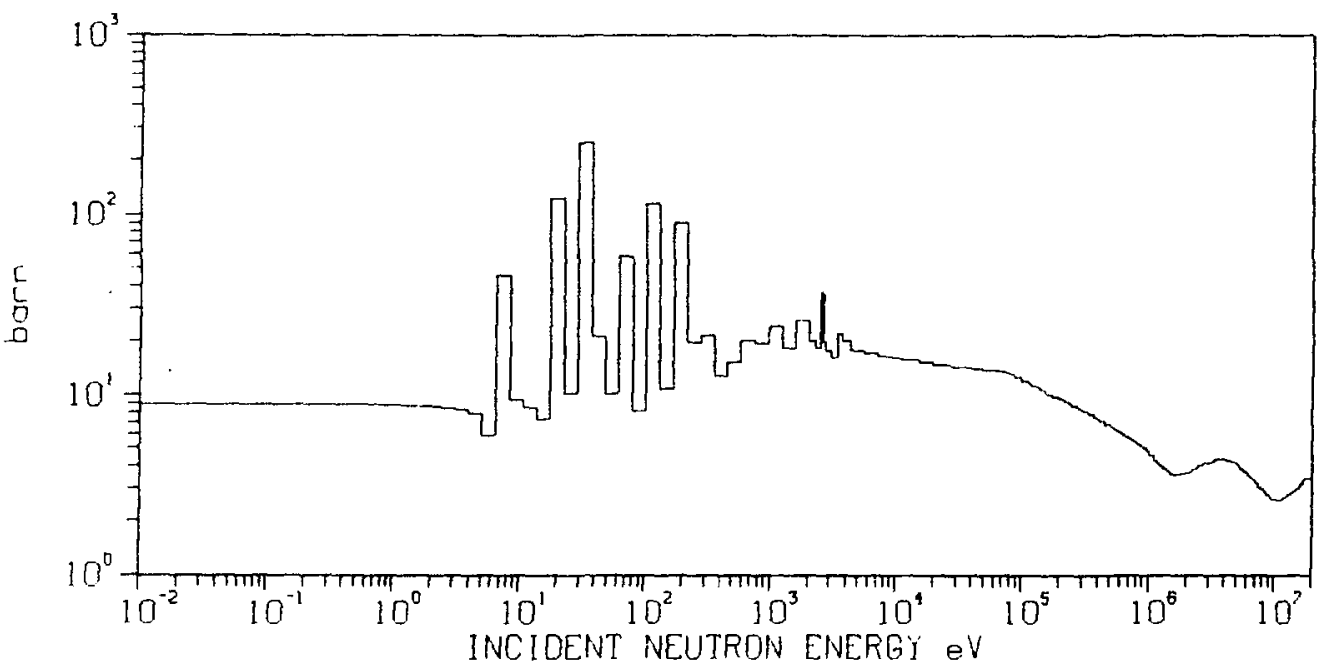

U-238 TOTAL INELASTIC CROSS SECTION

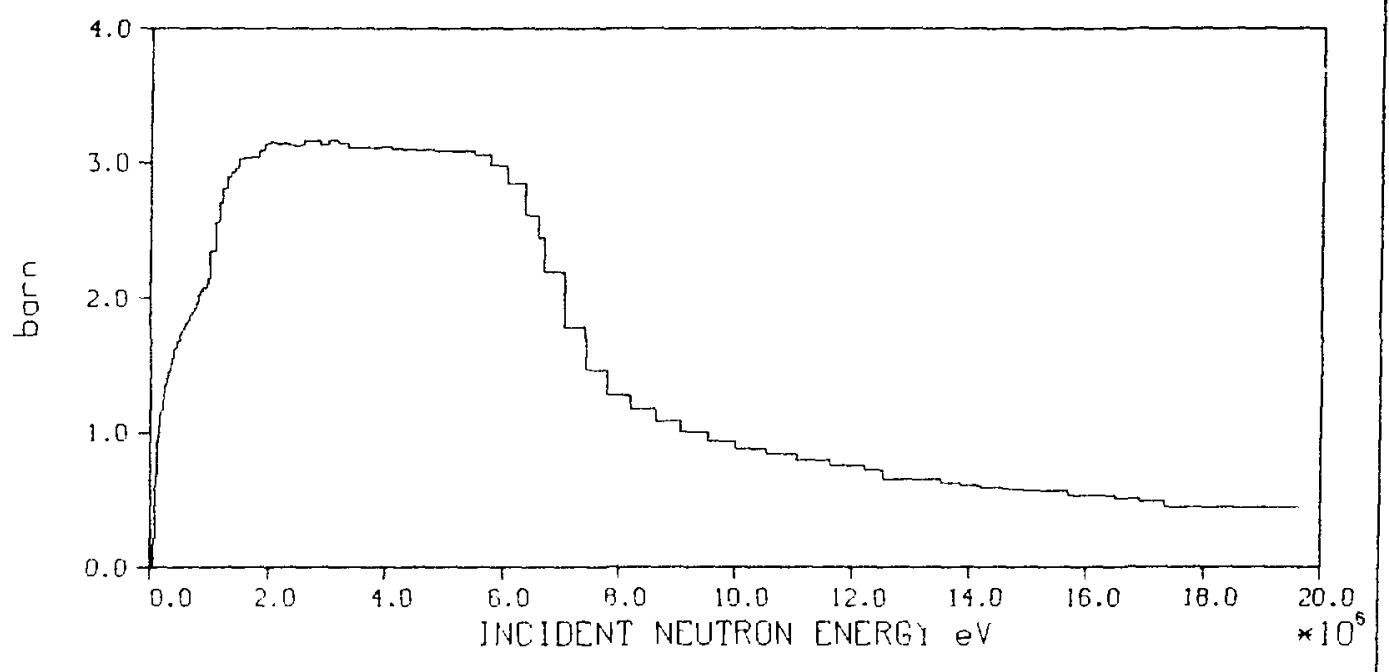




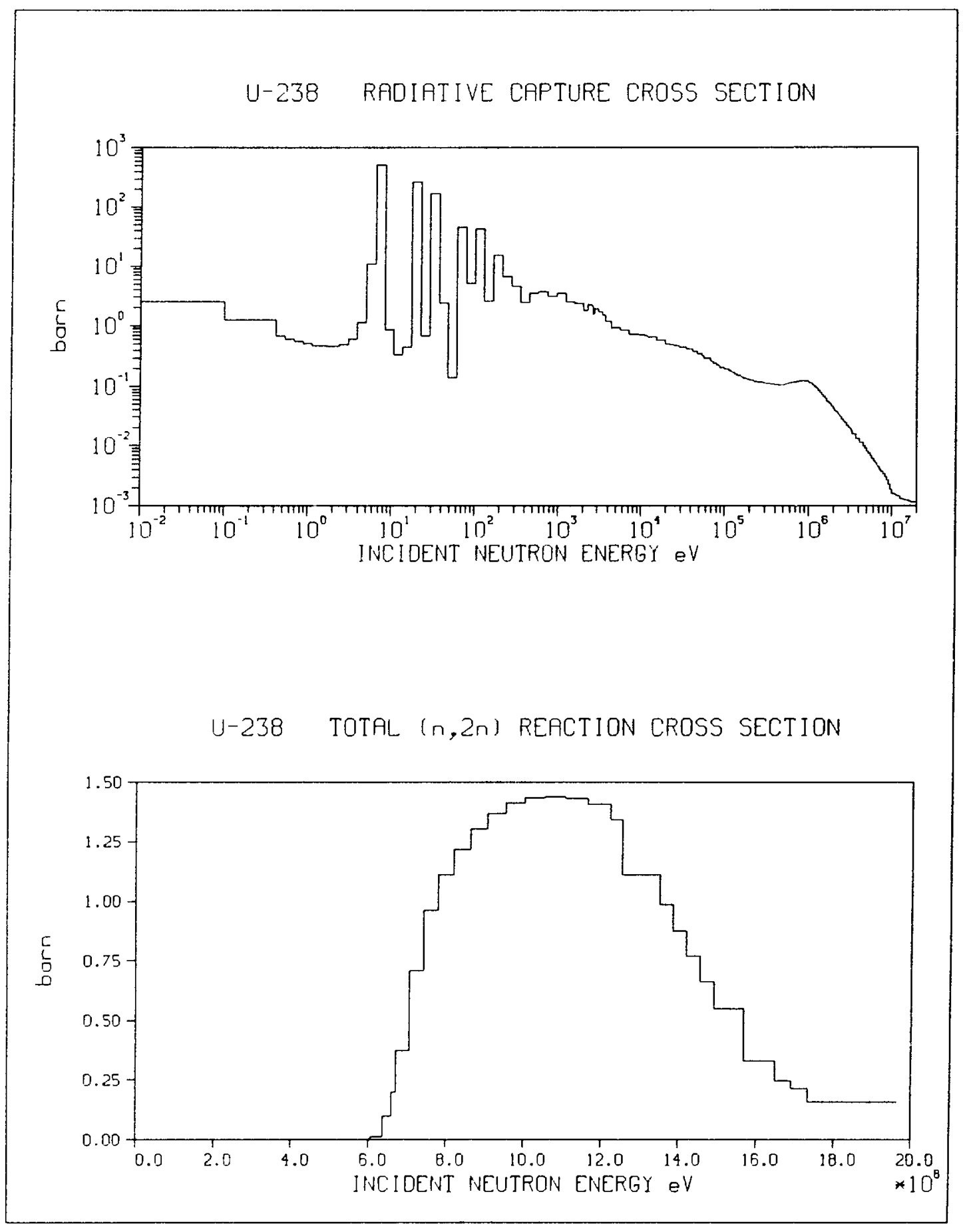


U-238 TOTAL $(n, 3 n)$ CROSS SECTION

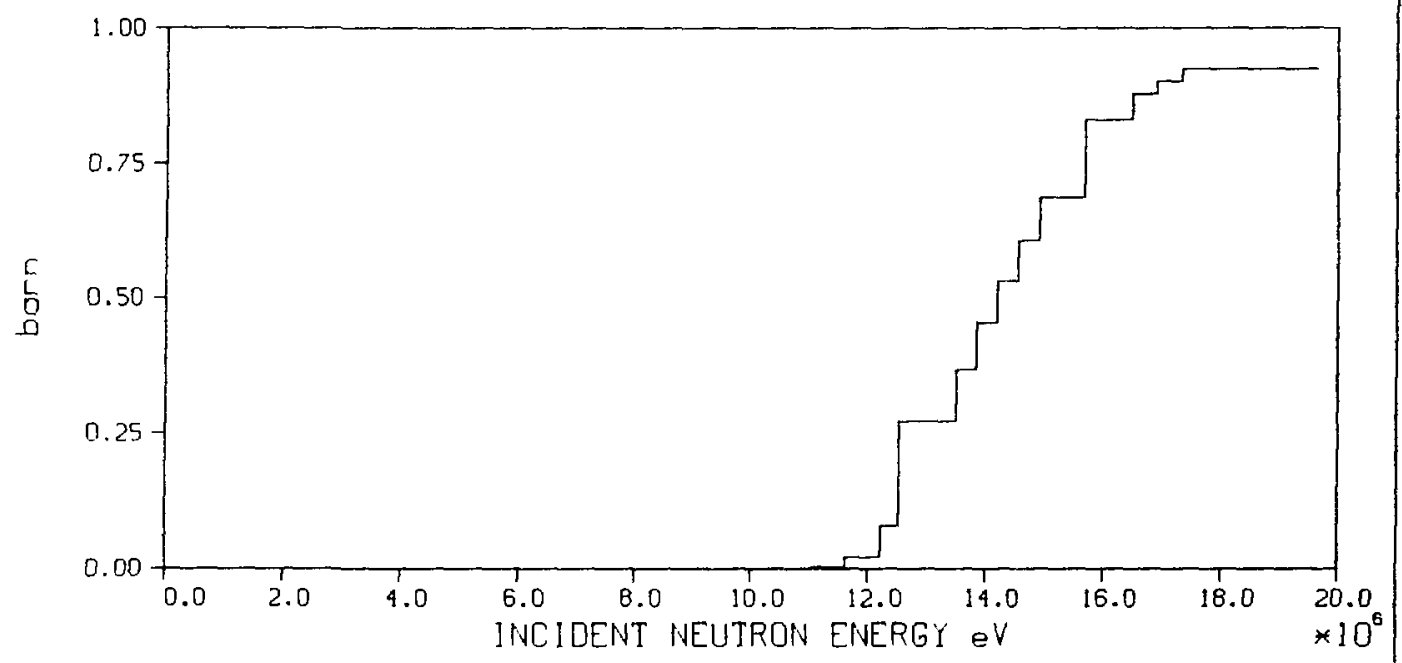

U-238 NUMBER OF FISSION NEUTRONS * CROSS SECTION

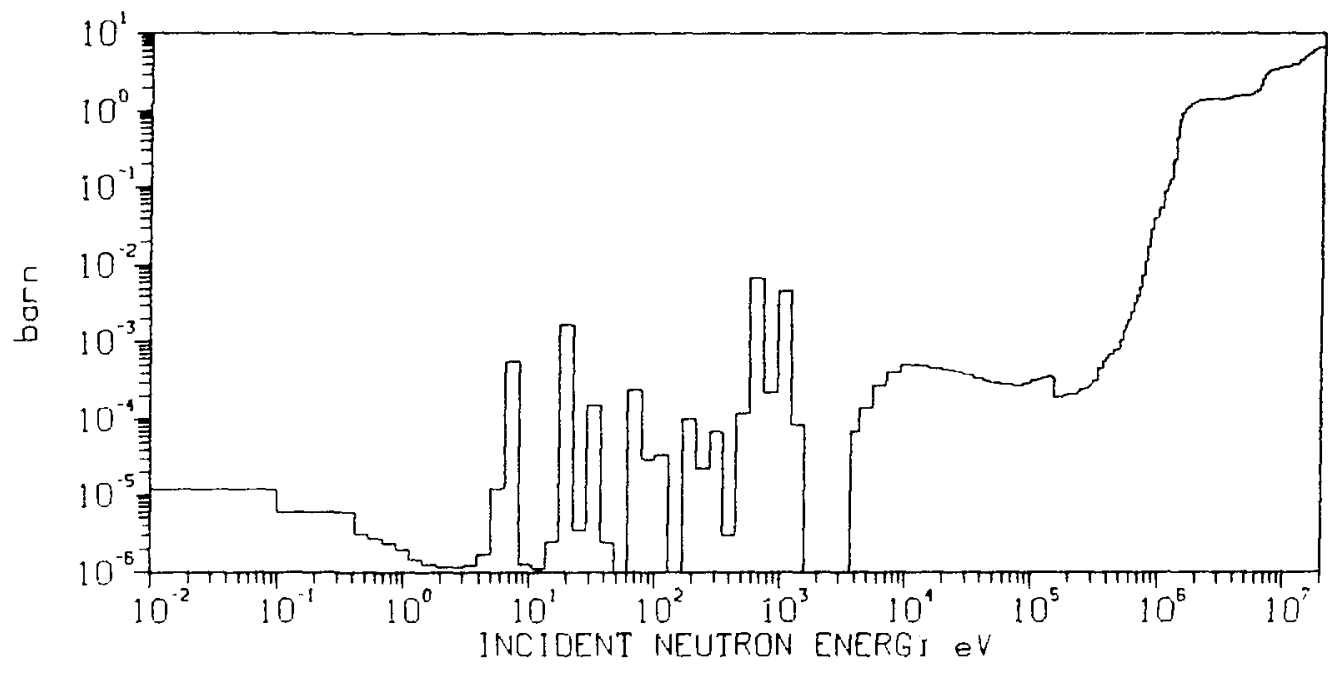


NF-237 RECOMMENDED PROMPT KERMA FACTOR (solid line)

REC. K. FACTOR WITH CH. PARTICLE DECAII HEAT (dashed line)

REC. K. FACTOR PLUS TOTAL OECAY HEFT lchain dotted lines

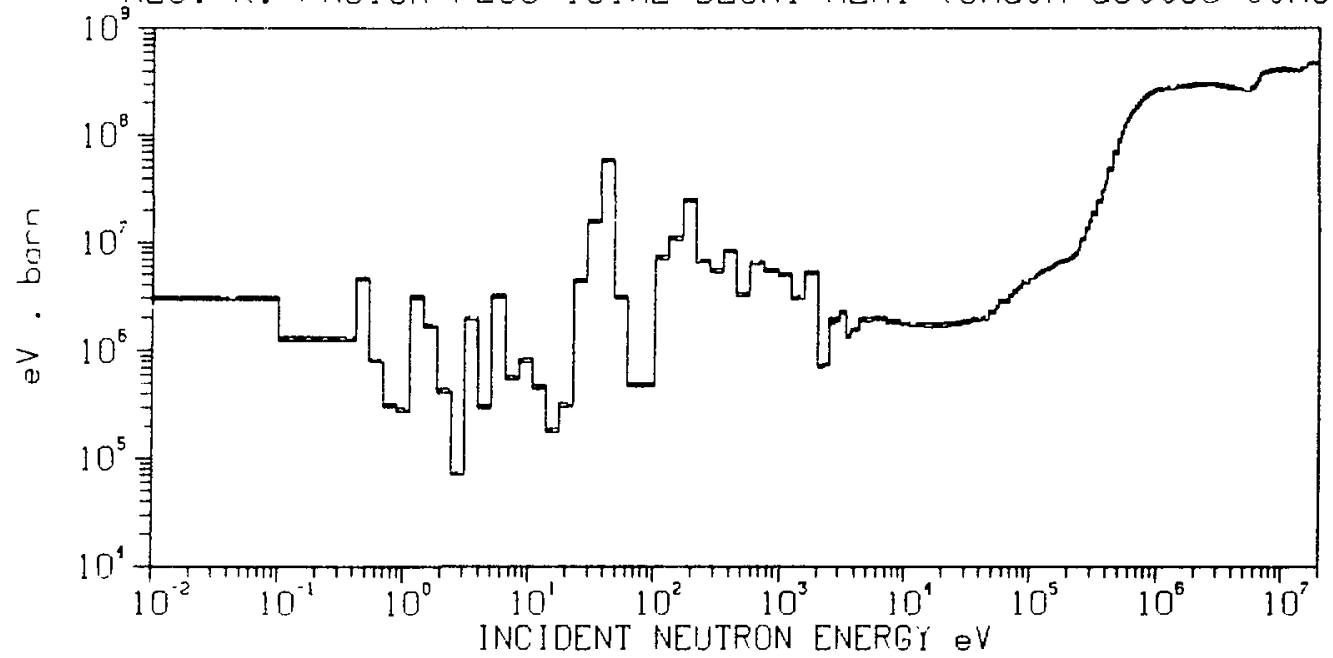

I.JP-237 RECOMMENDED PROMPT K.ERMA FACTOR (solid line) PEC. K. FACTOR WITH CH. PARTICLE DECAY HEAT (dashed line) $D \quad$ REC. K. FACTOR PLUS TOTAL DECAI HEAT (chain dotted line)

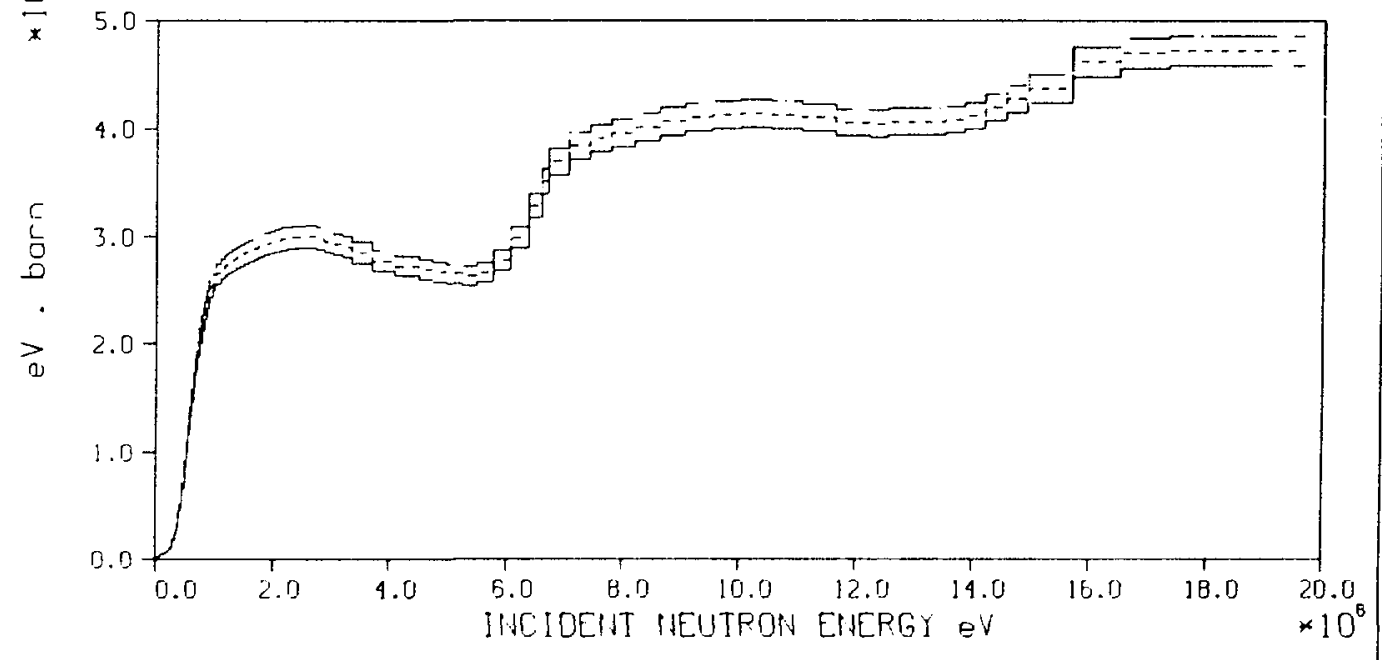


NP-237 TOTAL CROSS SECTION

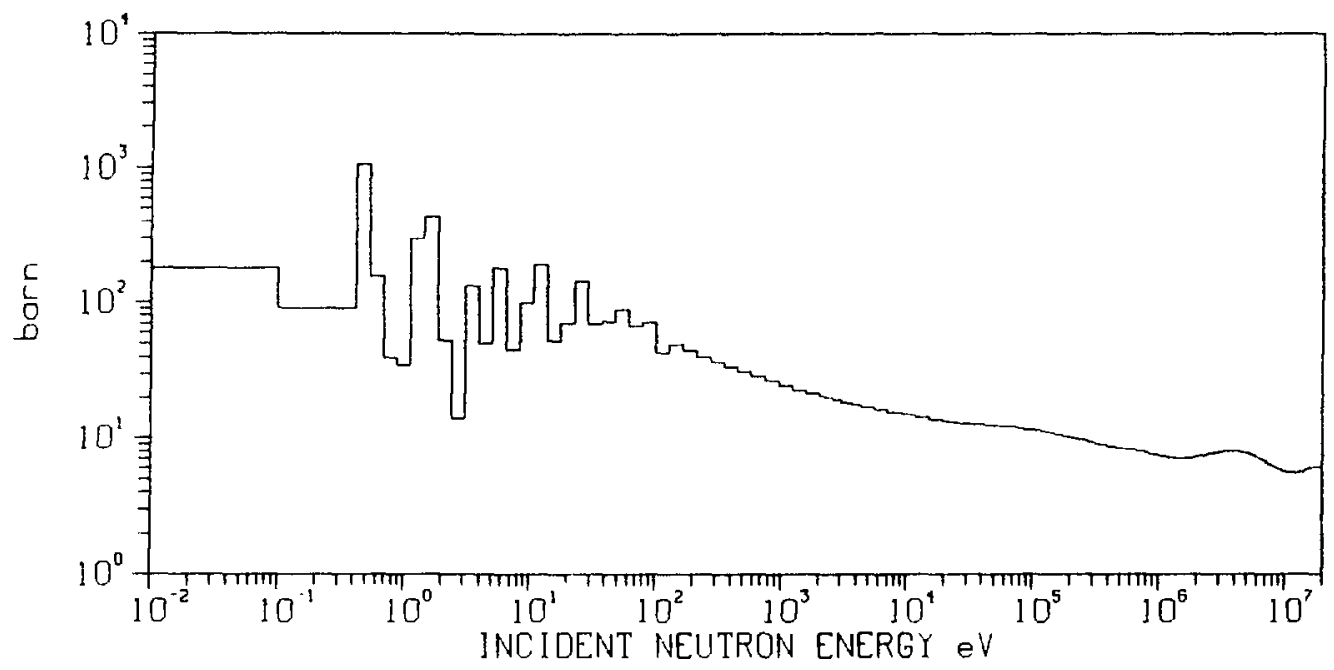

NP-237 ELASTIC CROSS SECTION

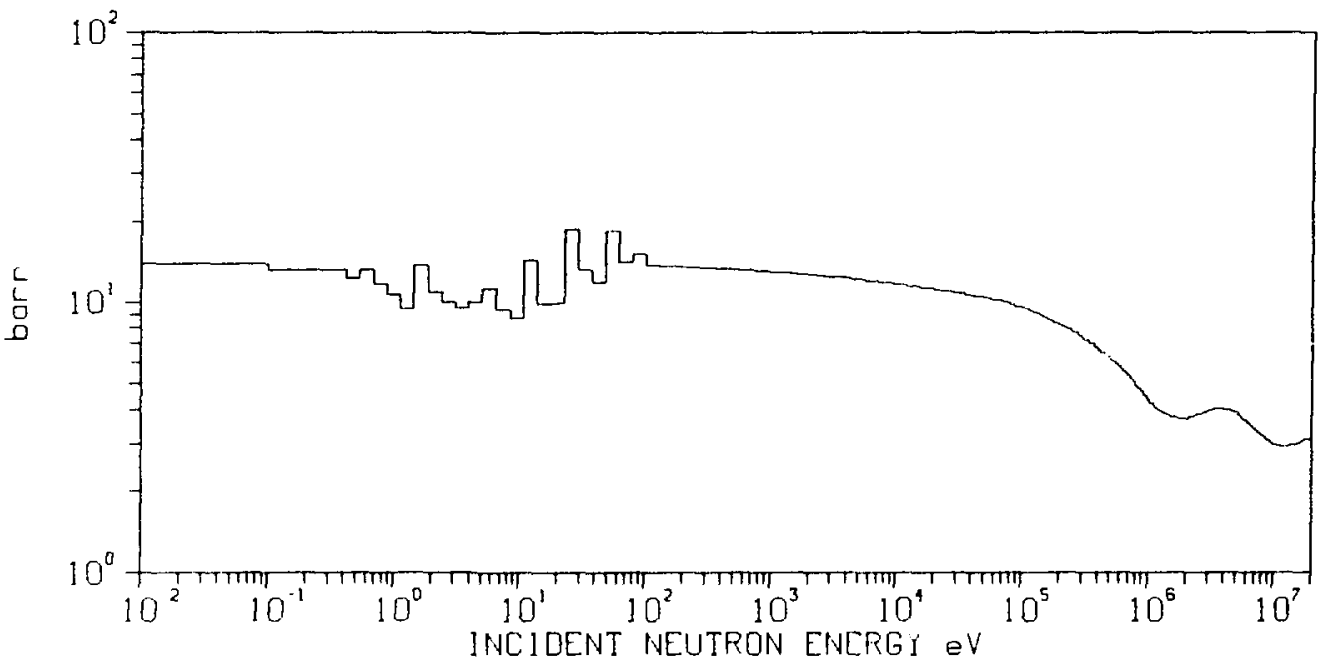


NP-237 TOTAL INELASTIC CROSS SECTION

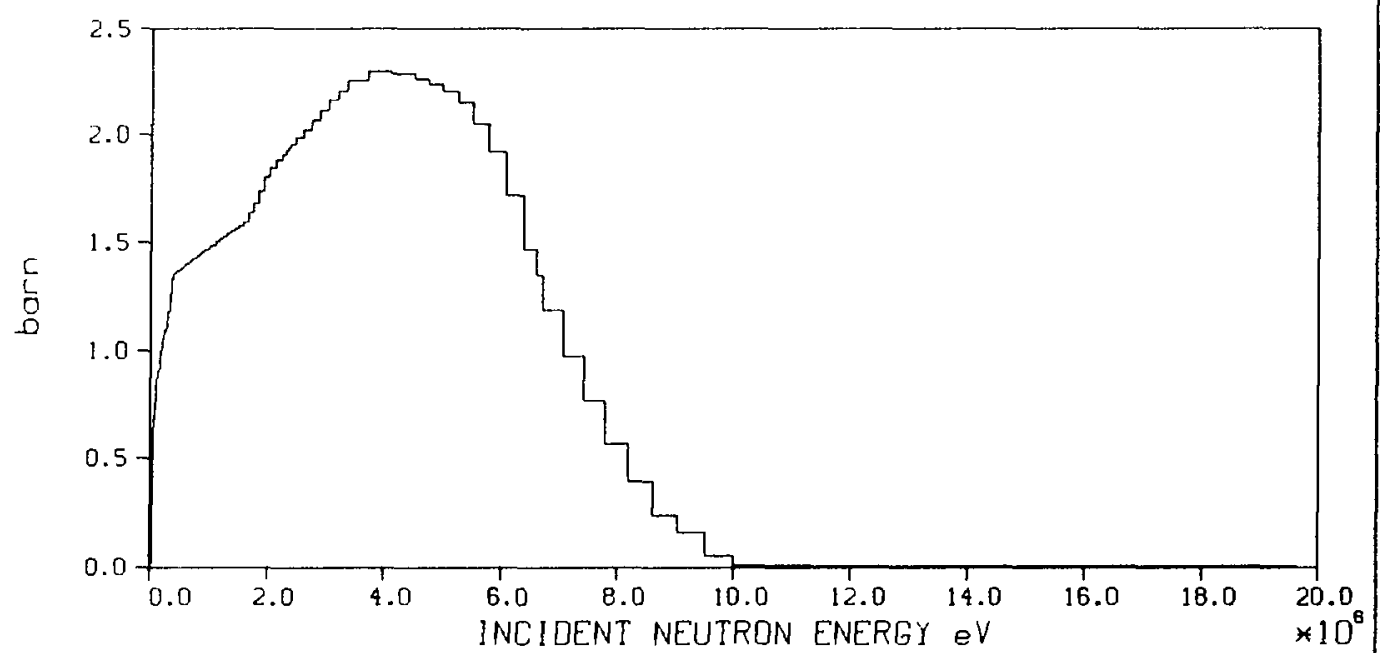

NP-237 RADIATIVE CAPTURE CROSS SECTION

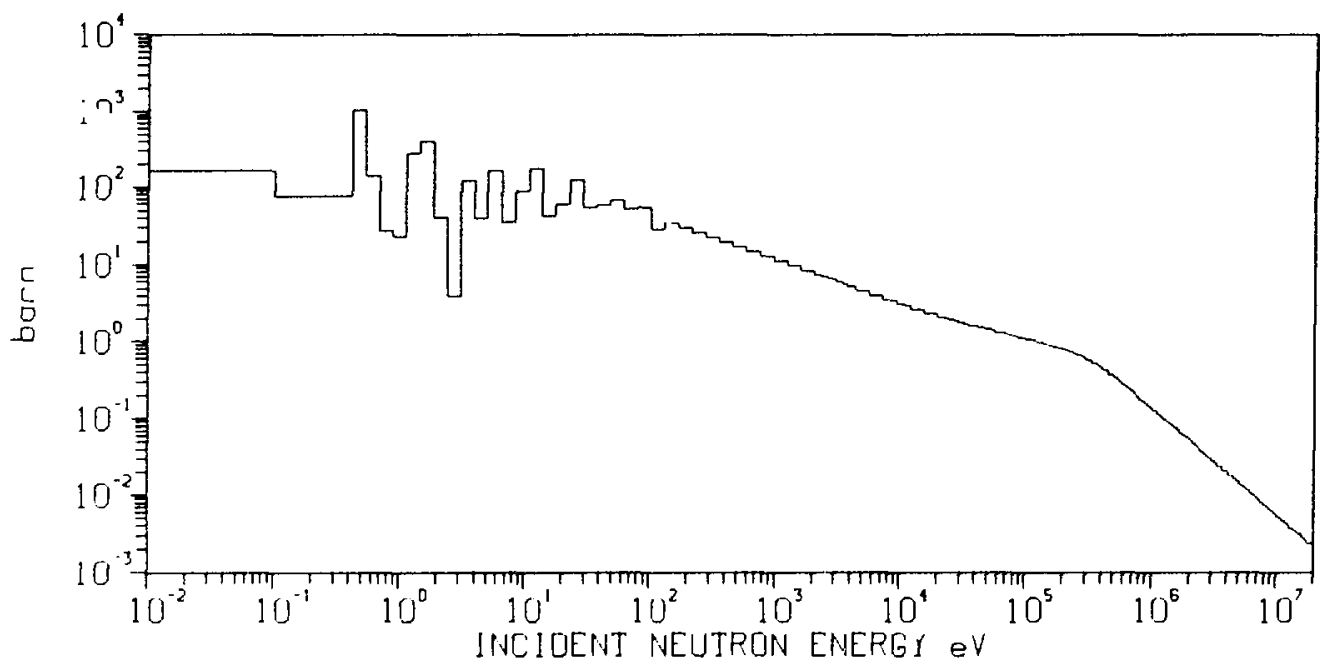




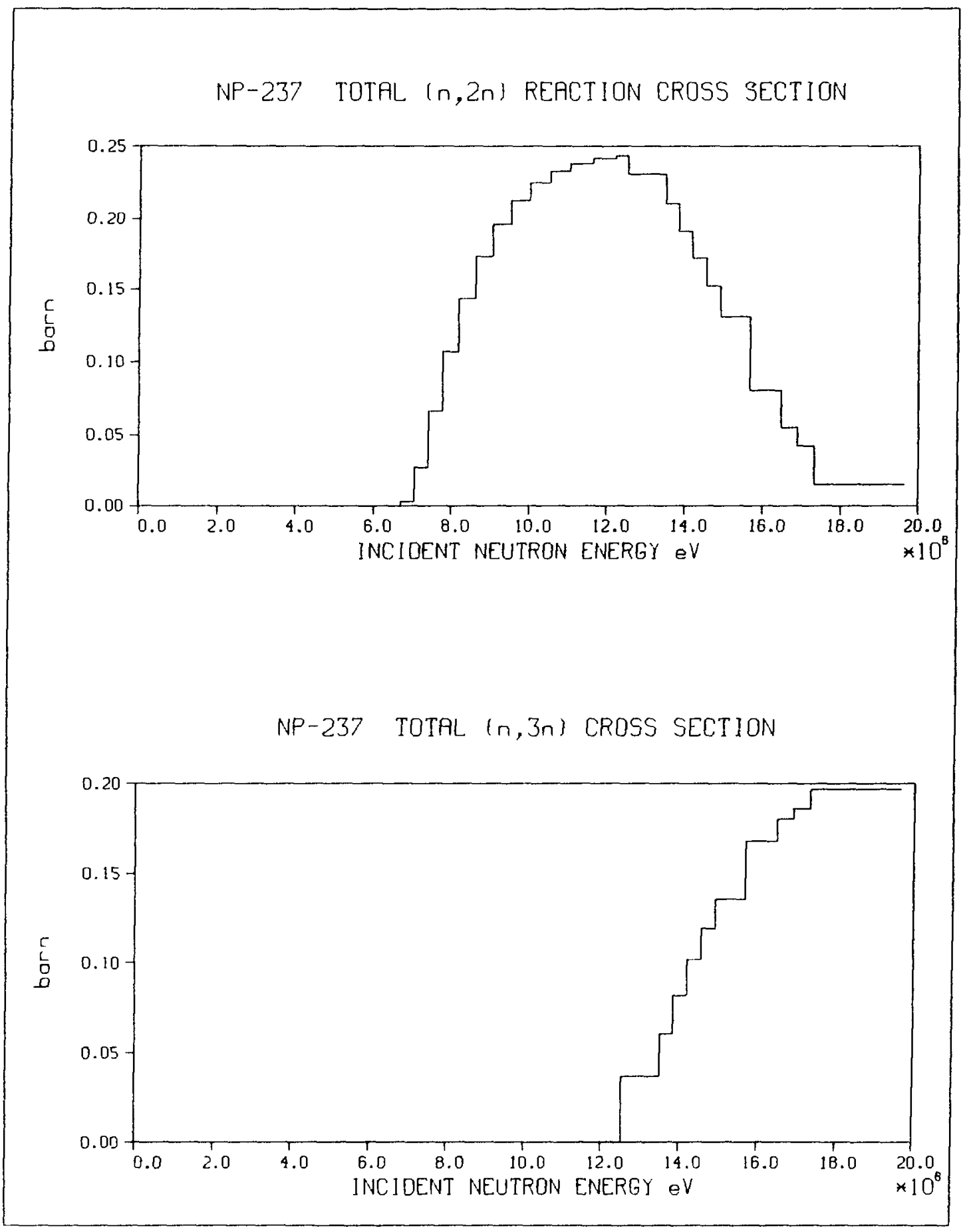




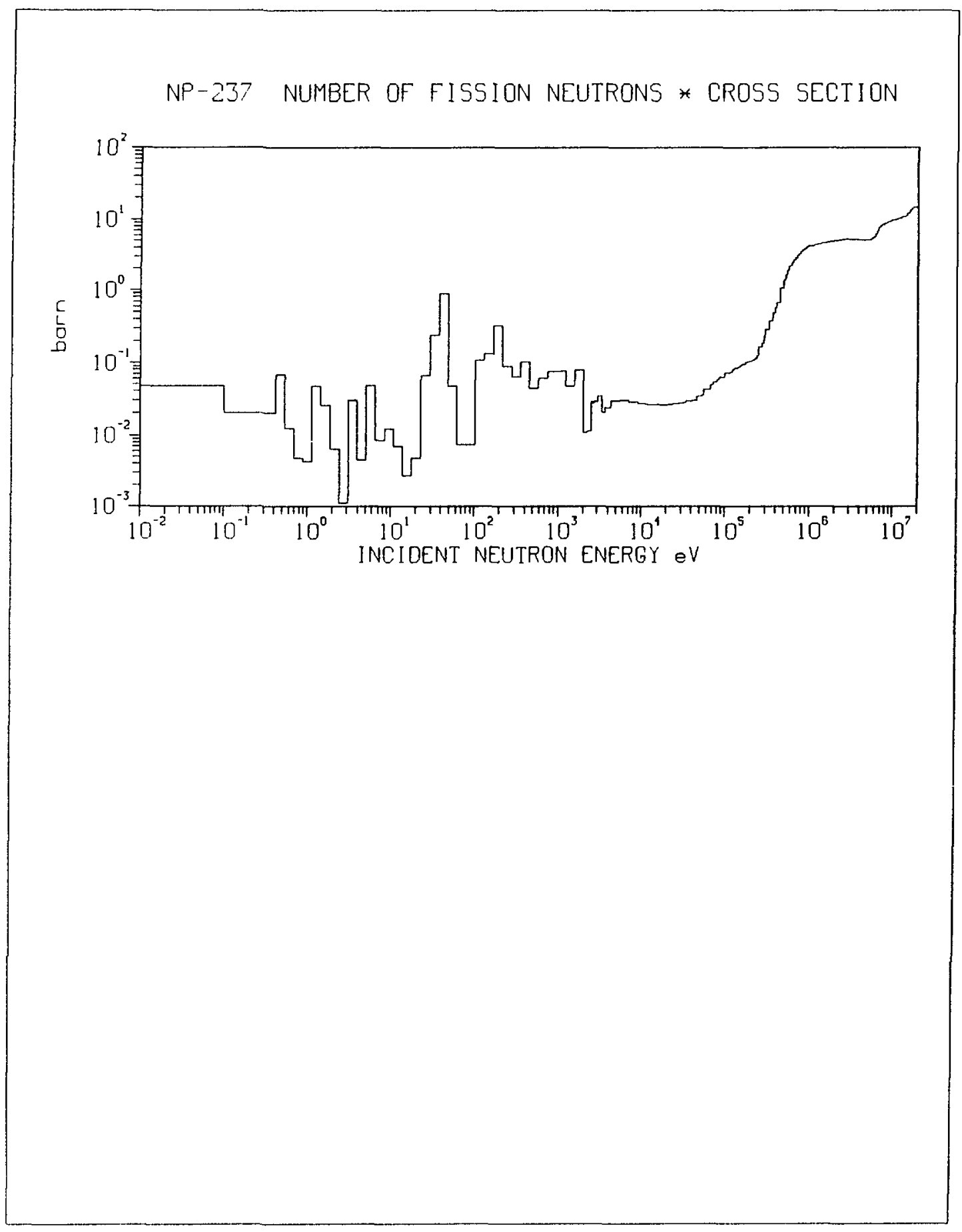


PU-238 RECOMMENDED PROMPT KERMA FACTOR (solid line) REC. K. FACTOR WITH CH. PARTICLE DECAY HERT (dashed line) REC. K. FACTOR PLUS TOTAL DECAY HEAT (chain dotted line)

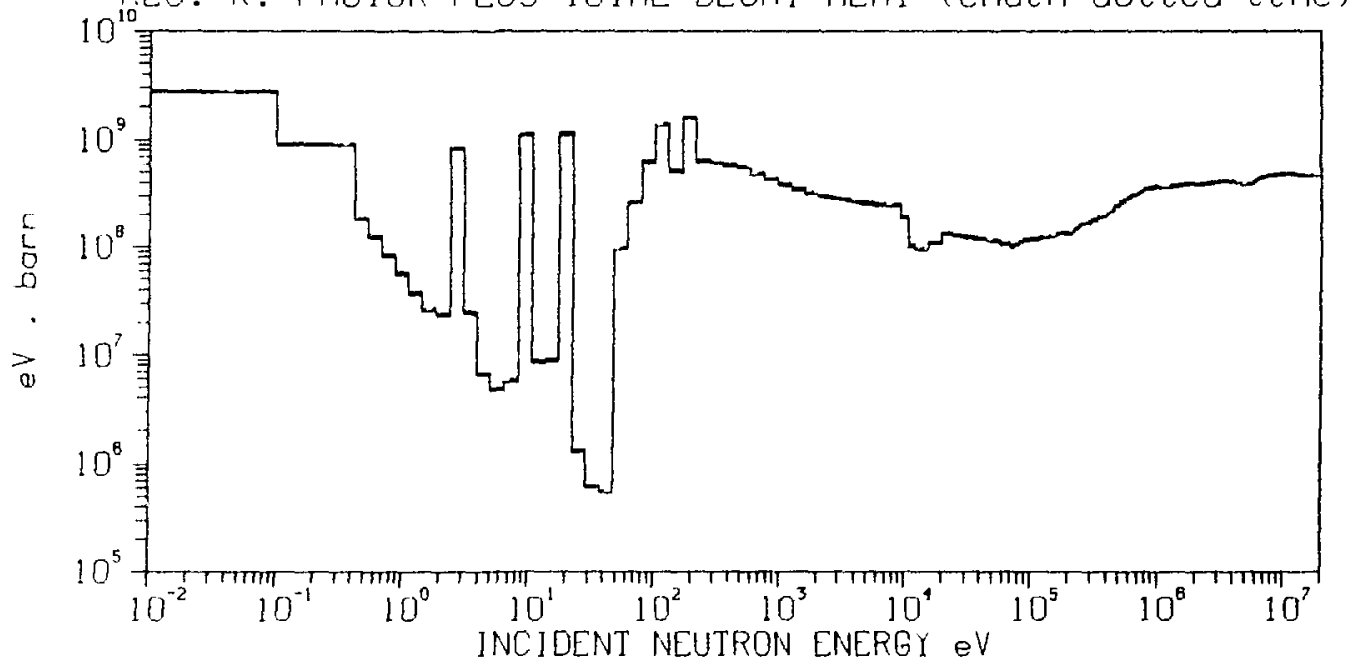

PU-238 RECOMMENDEO PROMPT KERMA FACTOR (solid line) FEC. $K$. FACTOR WITH CH. PARTICLE DECAY HEAT (dashed line) ¿ REC. K. FACTOR PLUS TOTAL DECAI HEAT (chain dotted line)

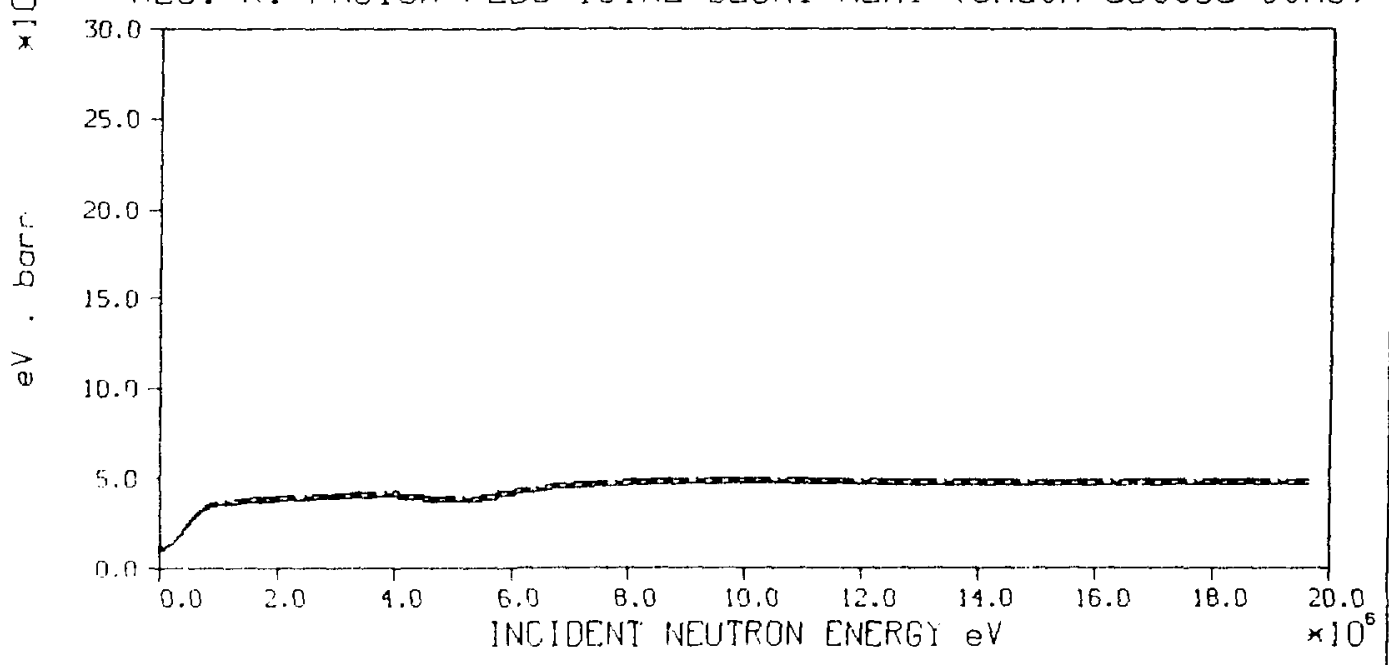


PU-238 TOTAL CROSS SECTION

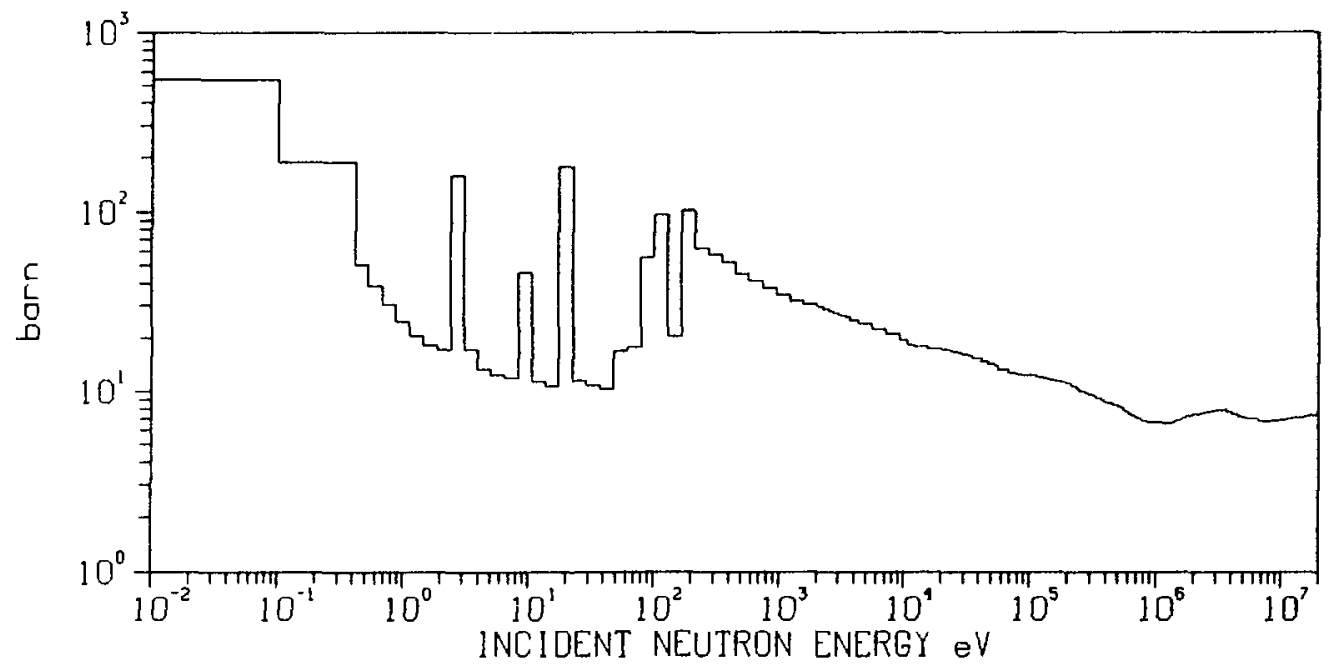

PU-238 ELASTIC CROSS SECTION

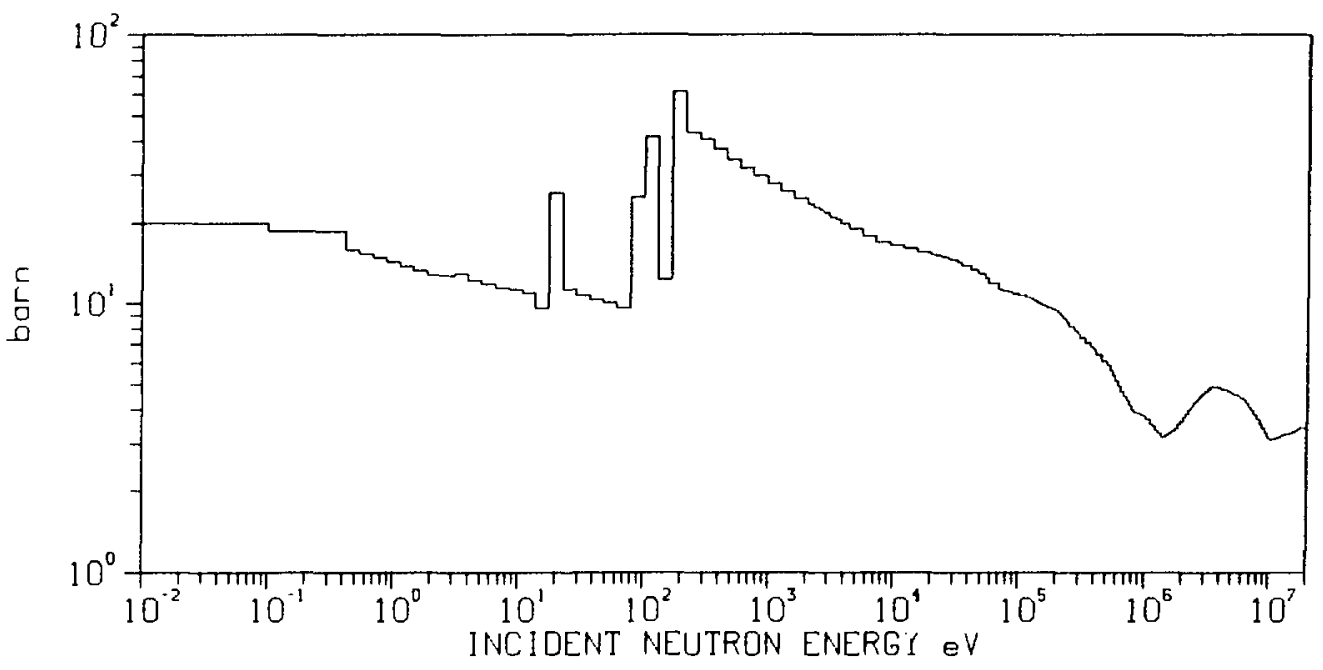


PU-238 TOTAL INELASTIC CROSS SECTION

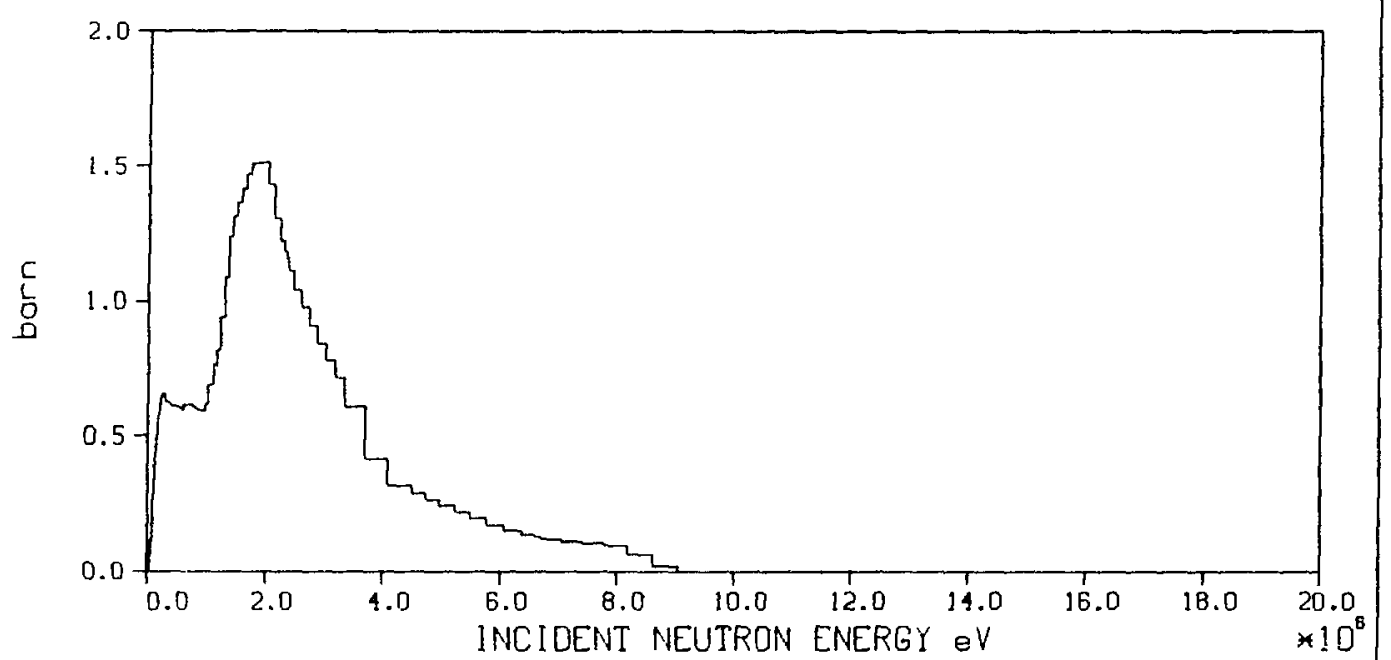

PU-238 RADIATIVE CAPTURE CROSS SECTION

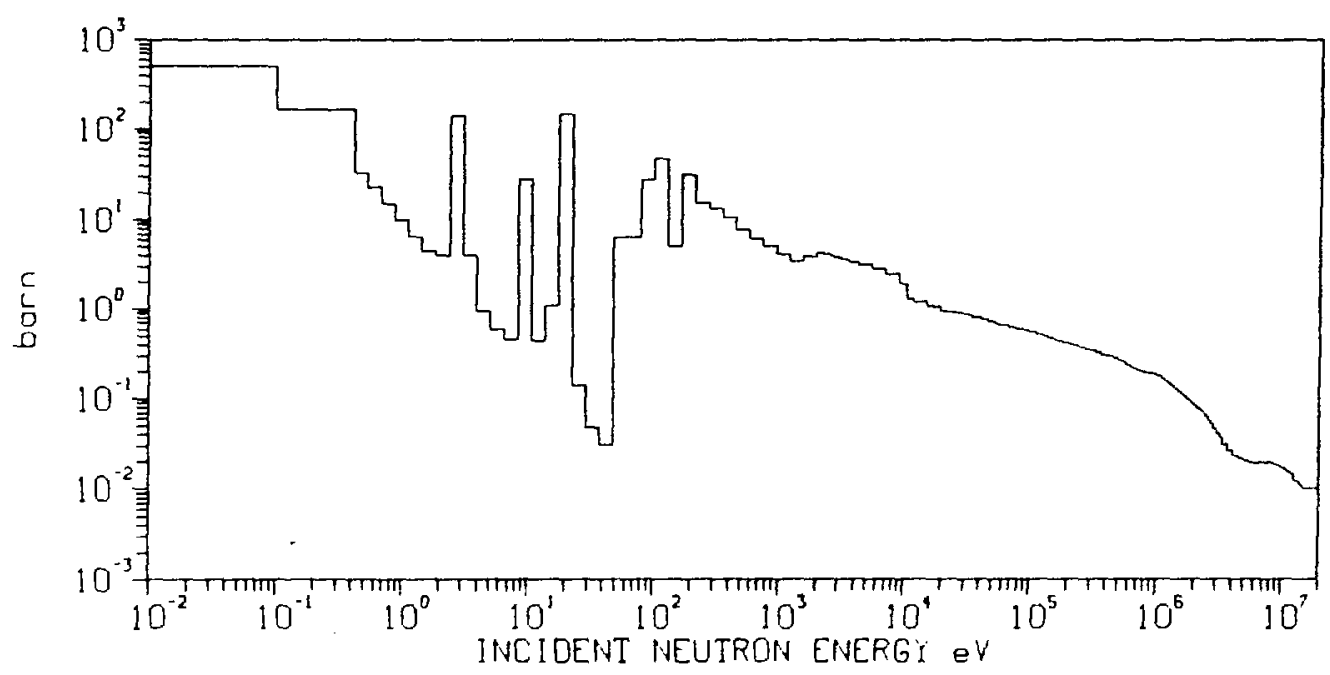




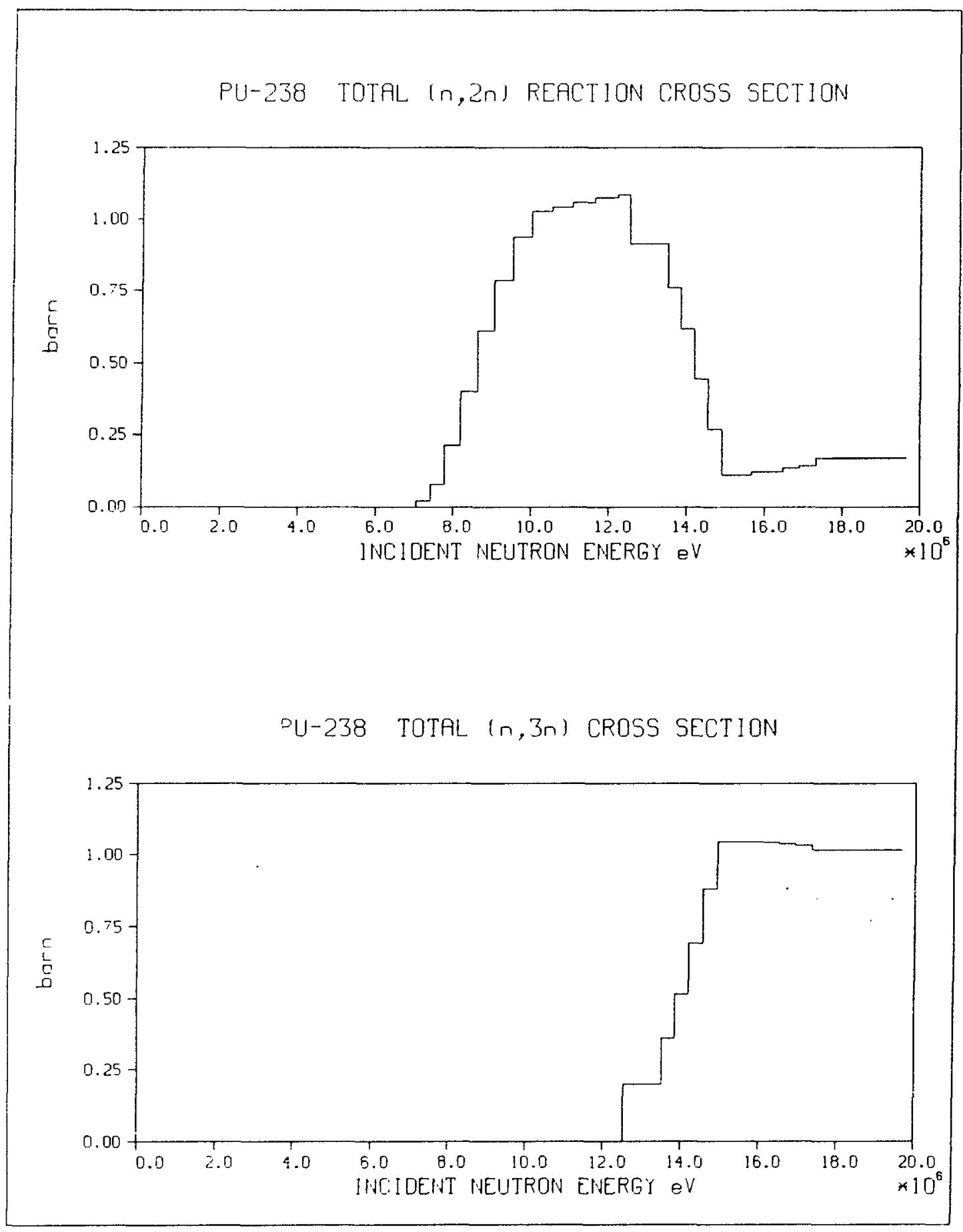


PU-238 NUMBER OF FISSION NEUTRONS * CROSS SECTION

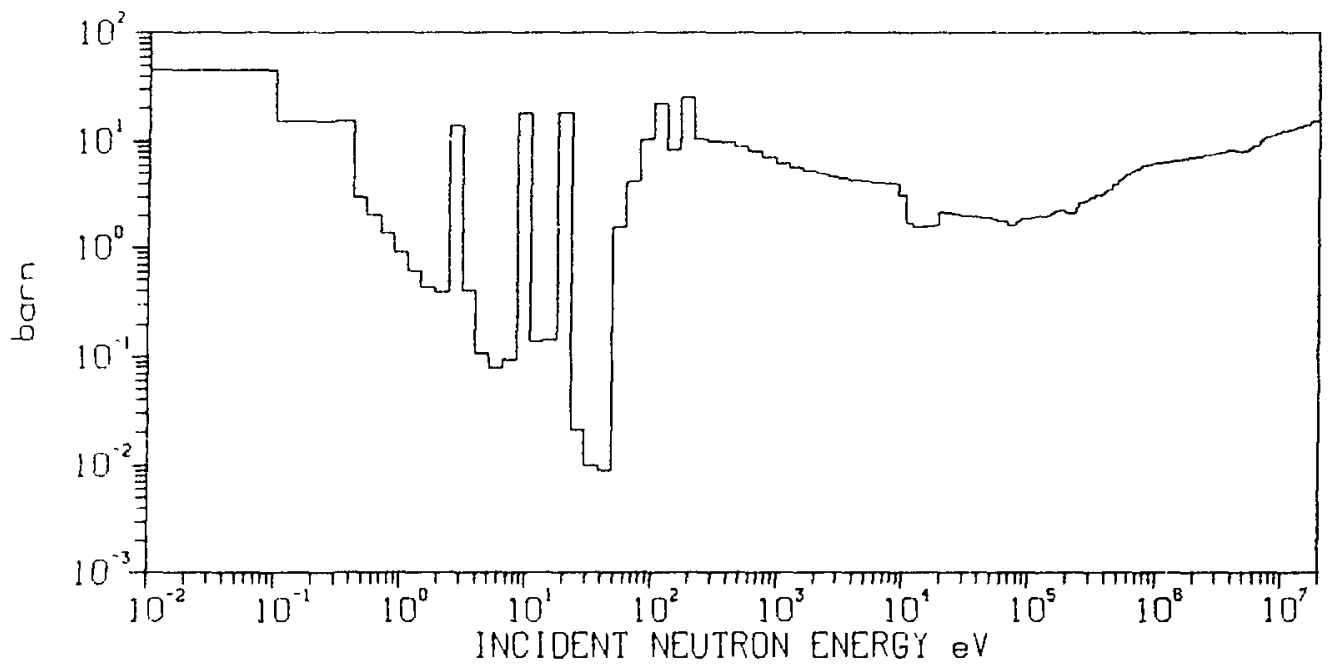




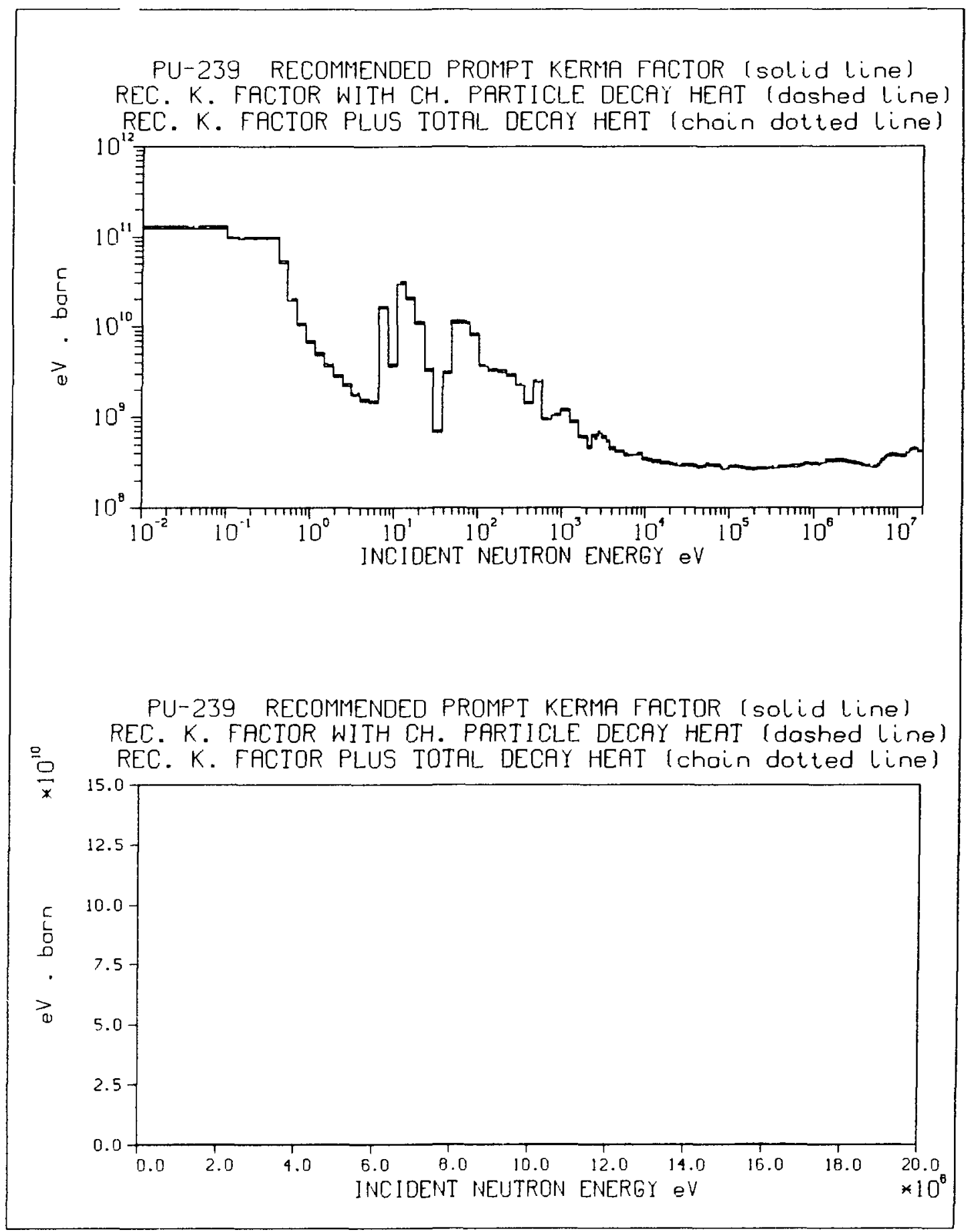




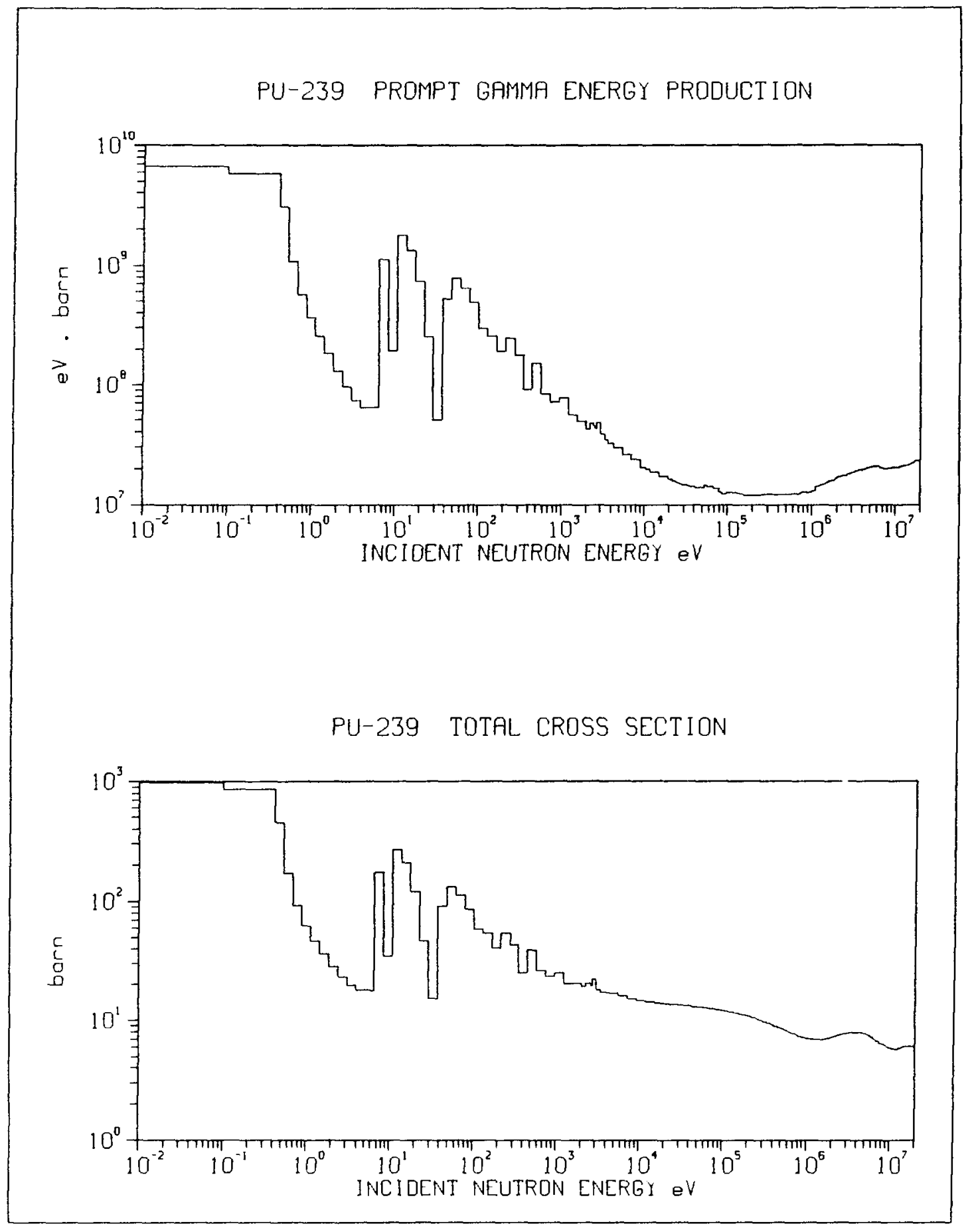




\section{PU-239 ELASTIC, CROSS SECTION}

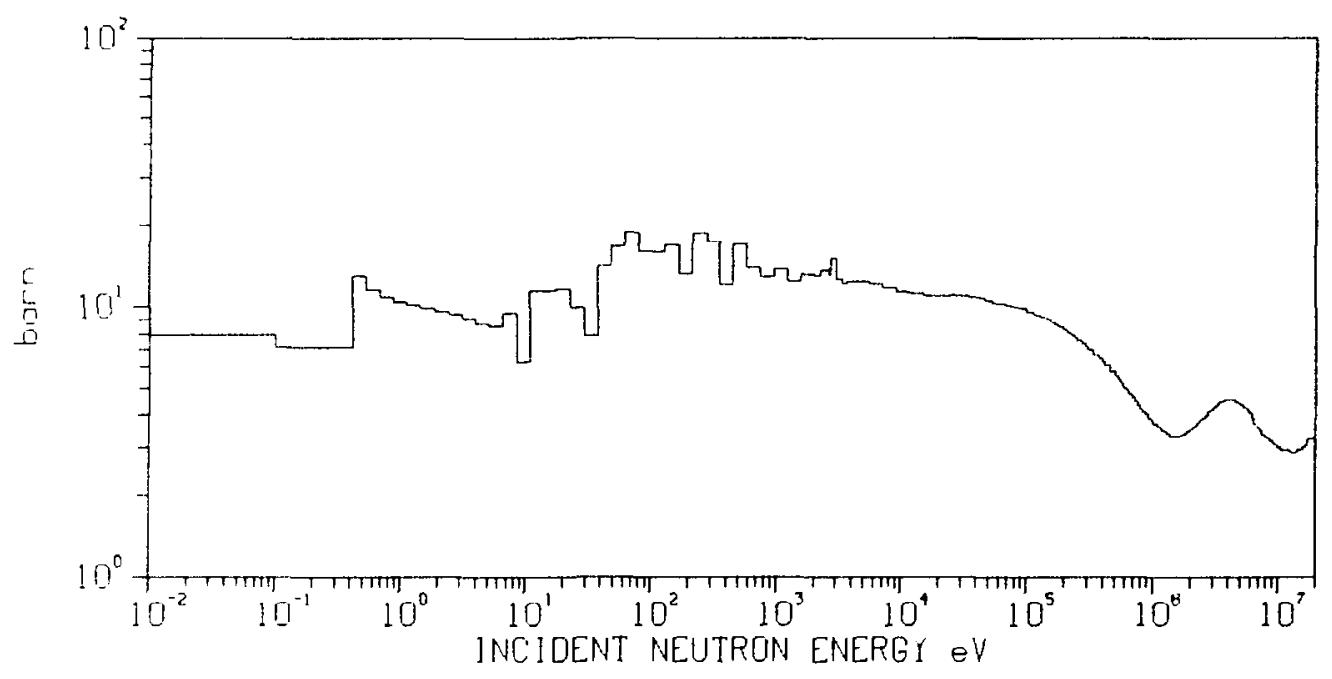

PU-239 TOTAL INELASTIC CROSS SECTION

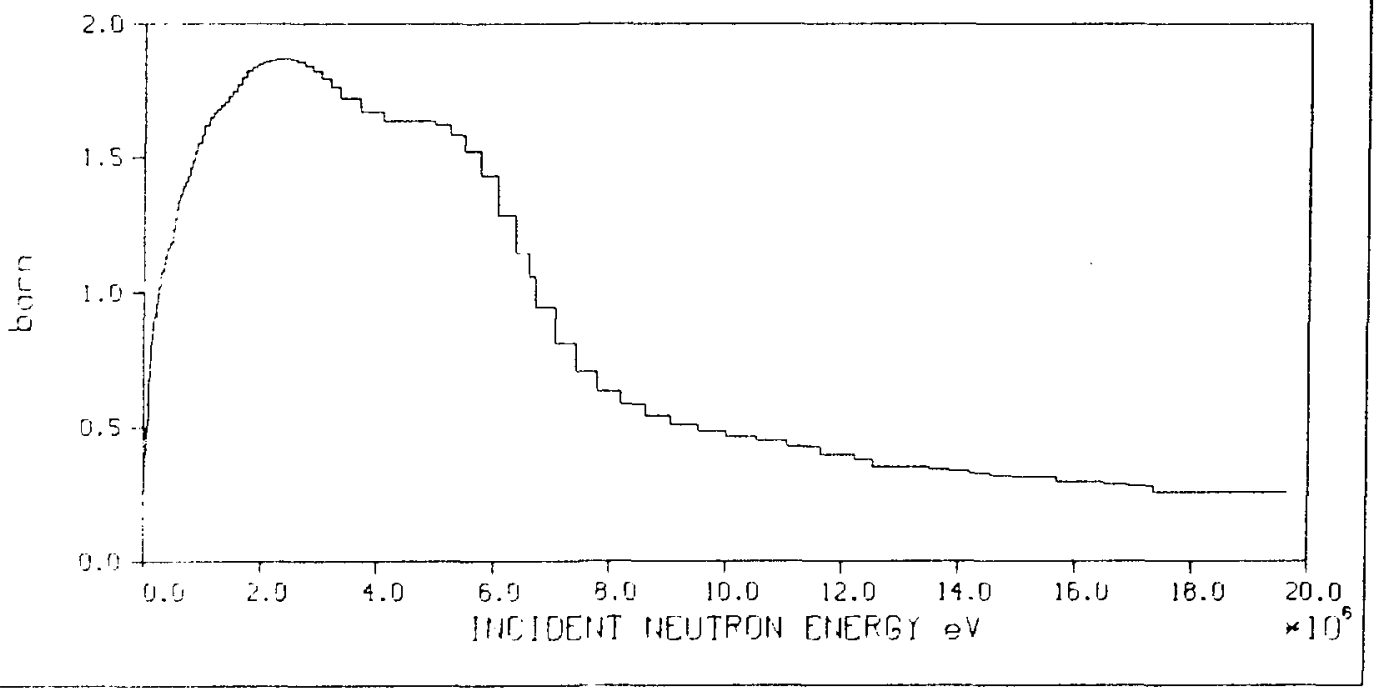




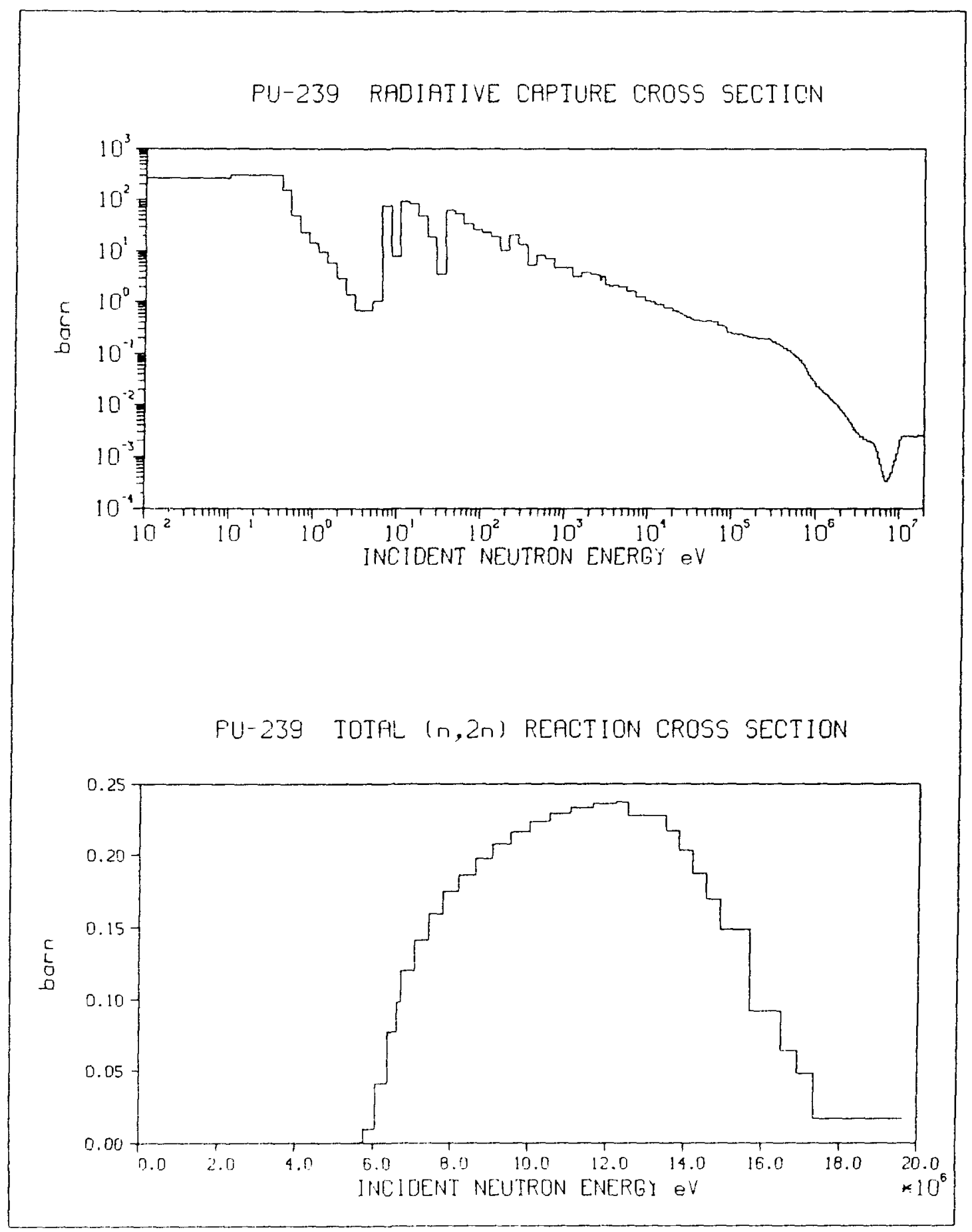


PU-239 IOTAL $(n, 3 n)$ CROSS SECTION

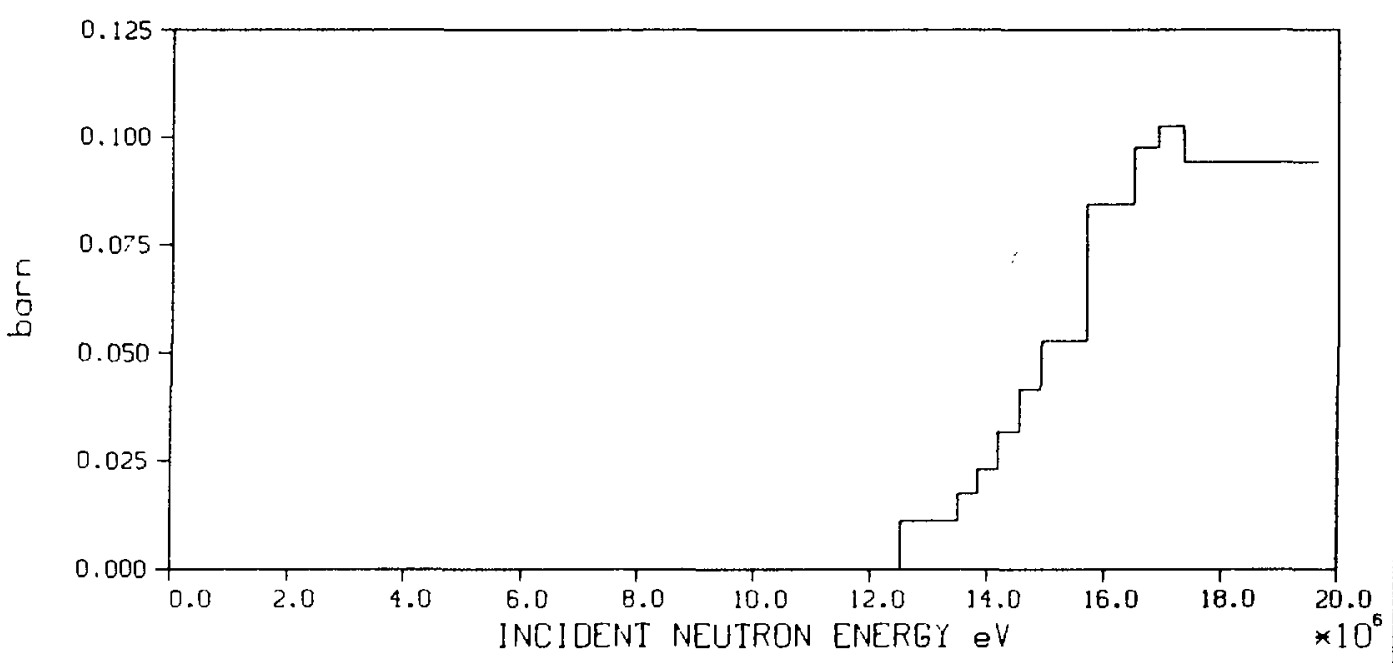

PU-239 NUMBER OF FISSION NEUTRONS * CROSS SECTION

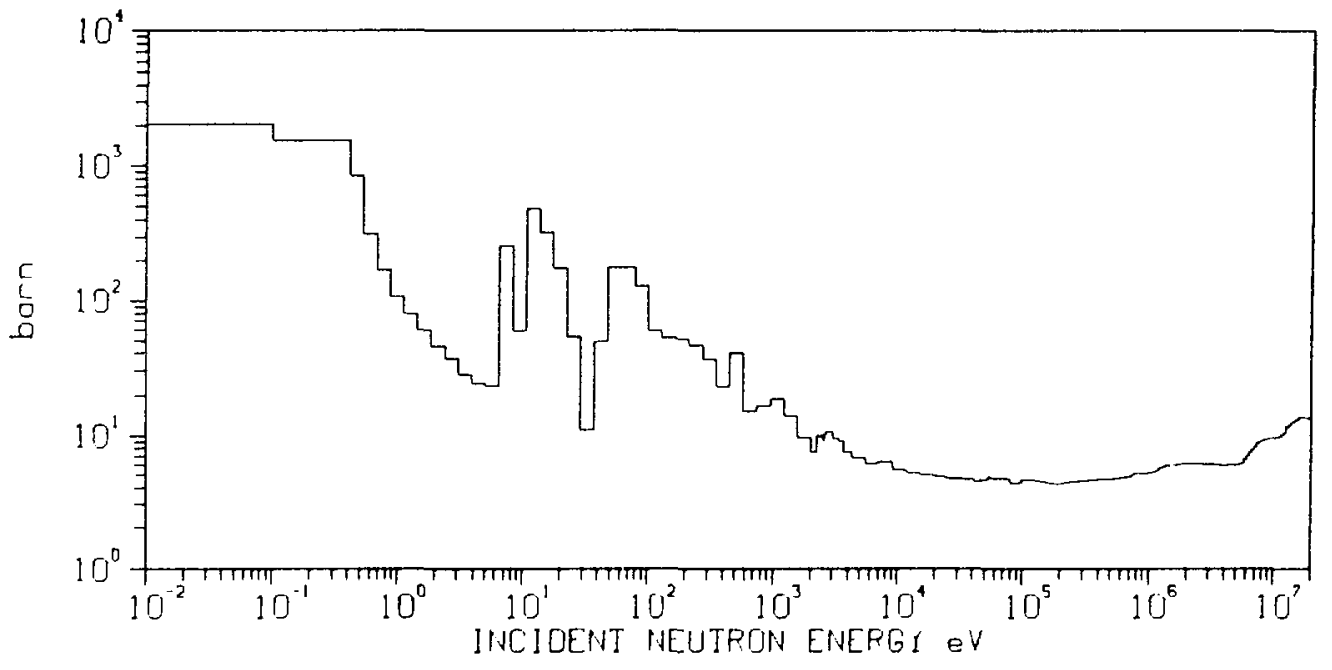




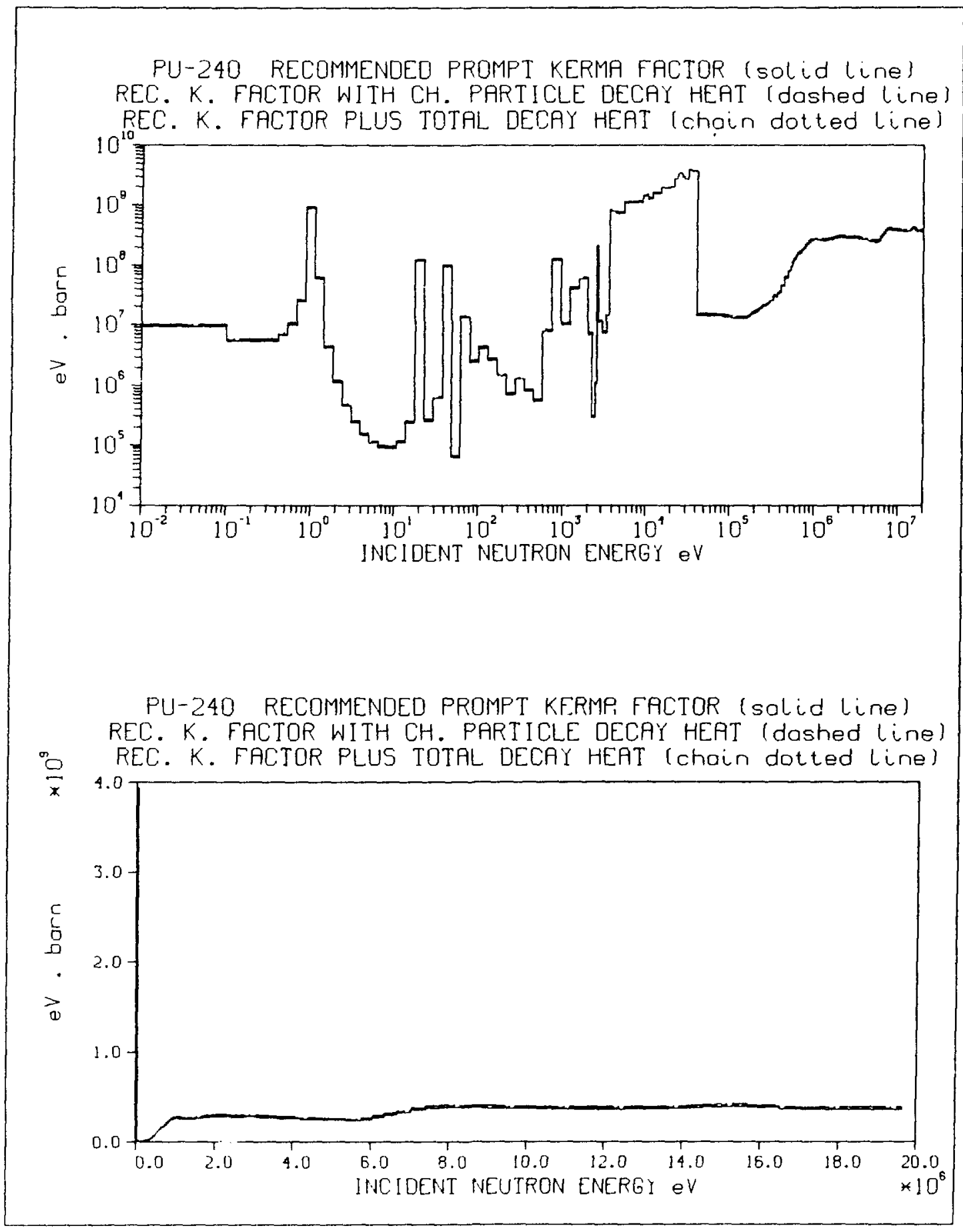




\section{PU-240 PROMPT GAMMA ENERGY PRODUCTION}

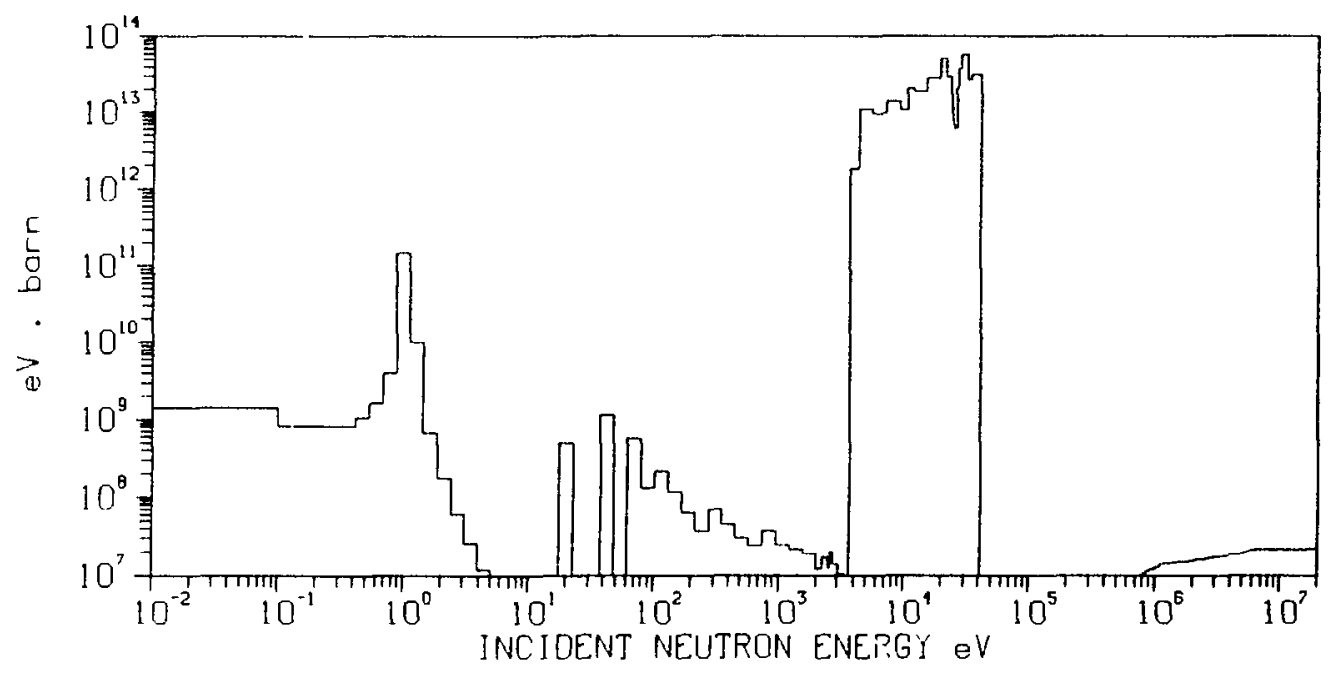

PU-240 TOTAL CROSS SECTION

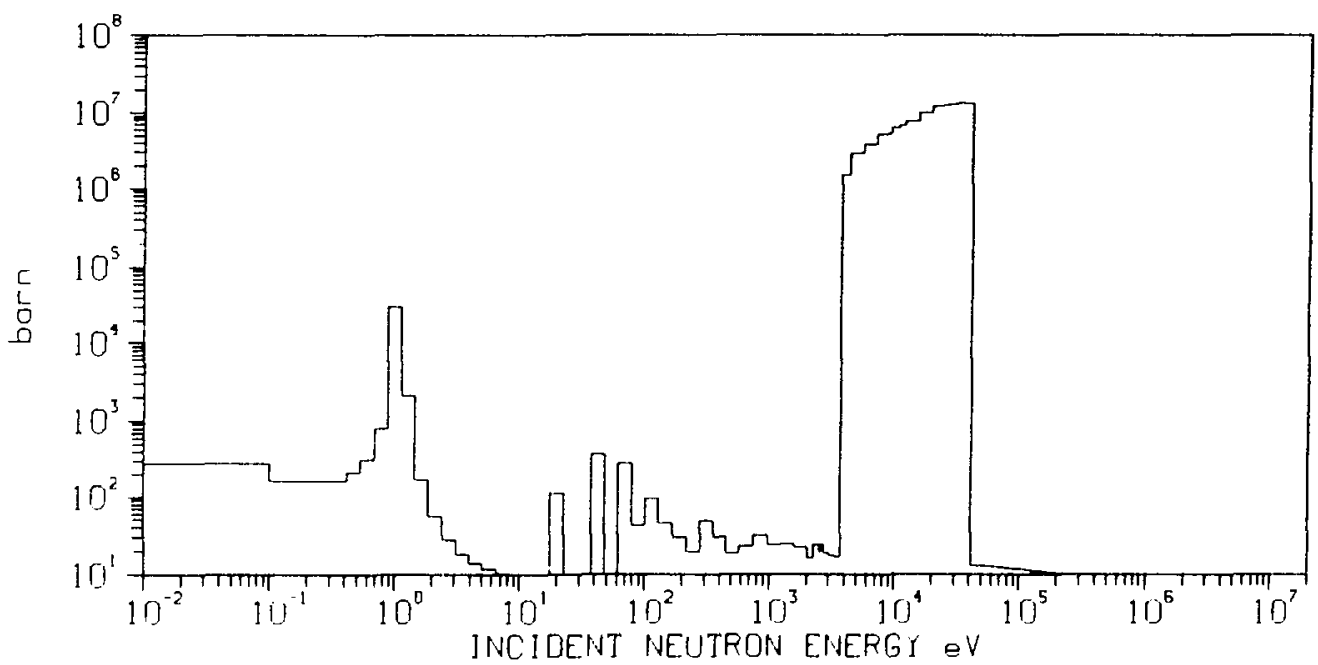


PU-240 ELASTIC CROSS SECTION

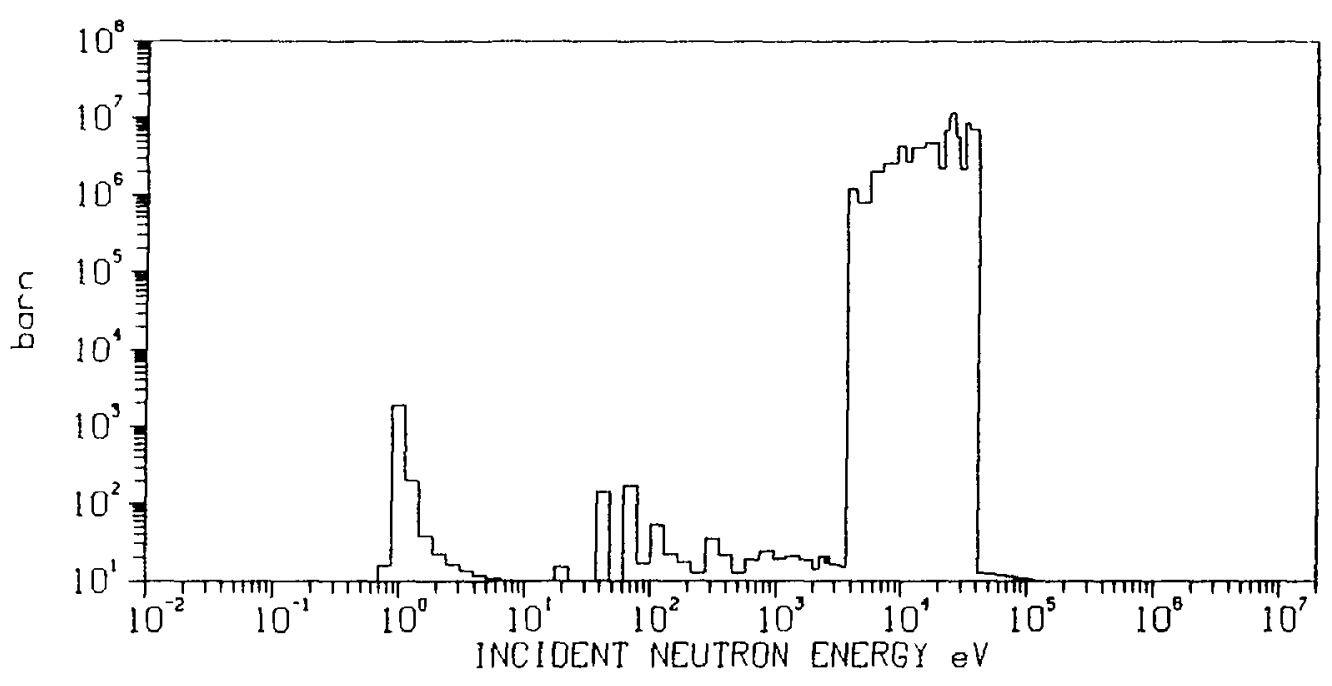

PU-240 TOTAL INELASTIC CROSS SECTION

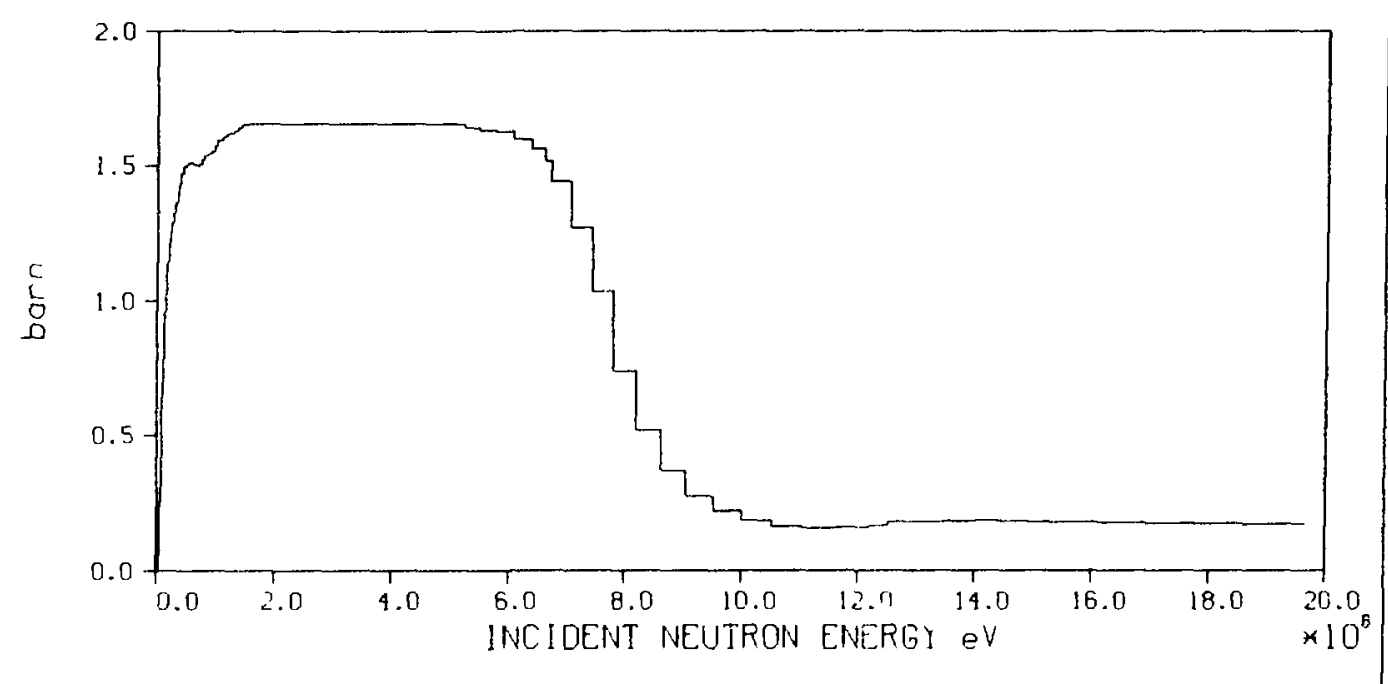




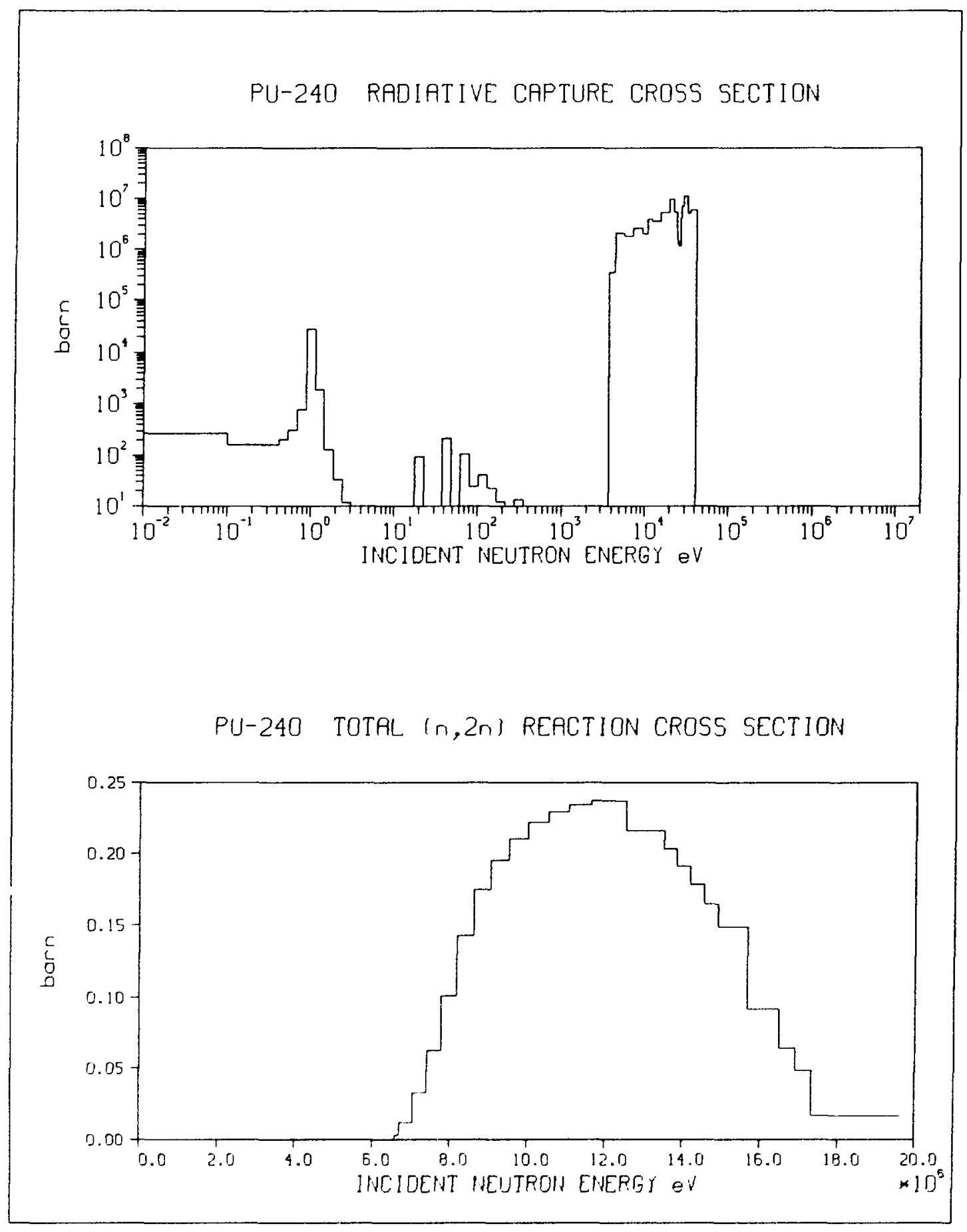


PU-240 TOTAL $(n, 3 n)$ CROSS SECT.ION

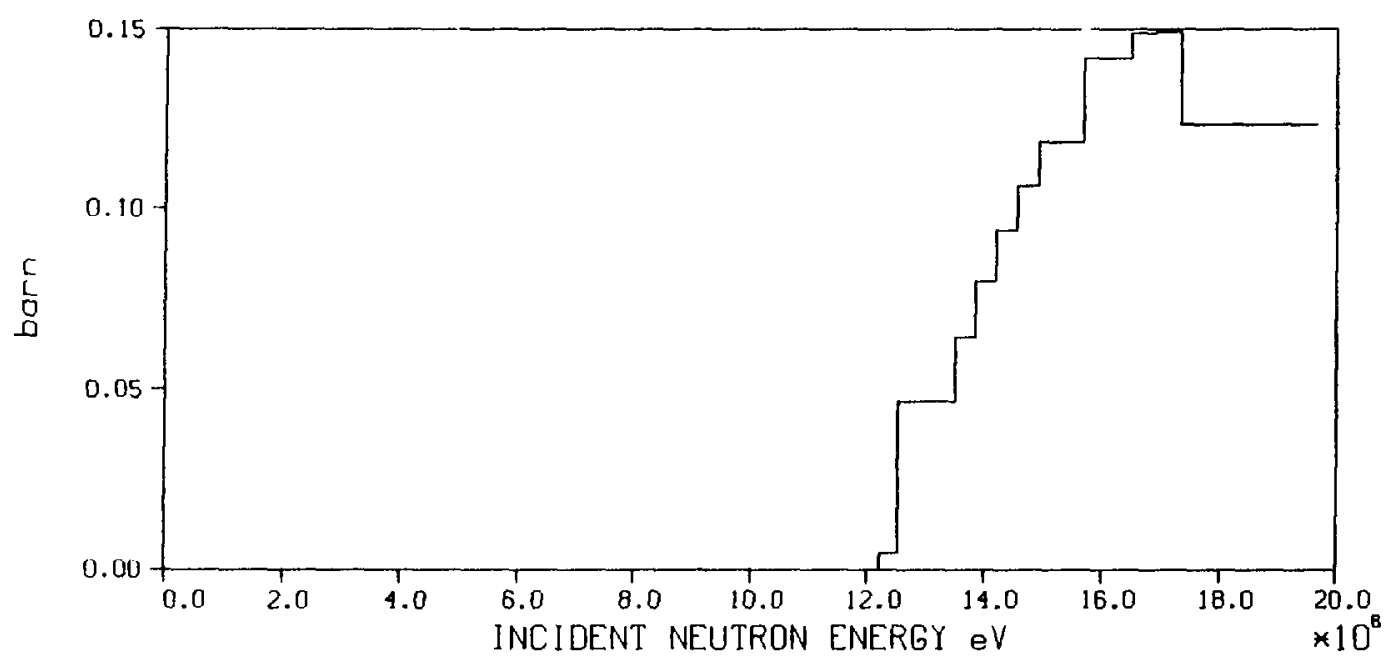

PU-240 NUMBER OF FISSION NEUTRONS * CROSS SECTION

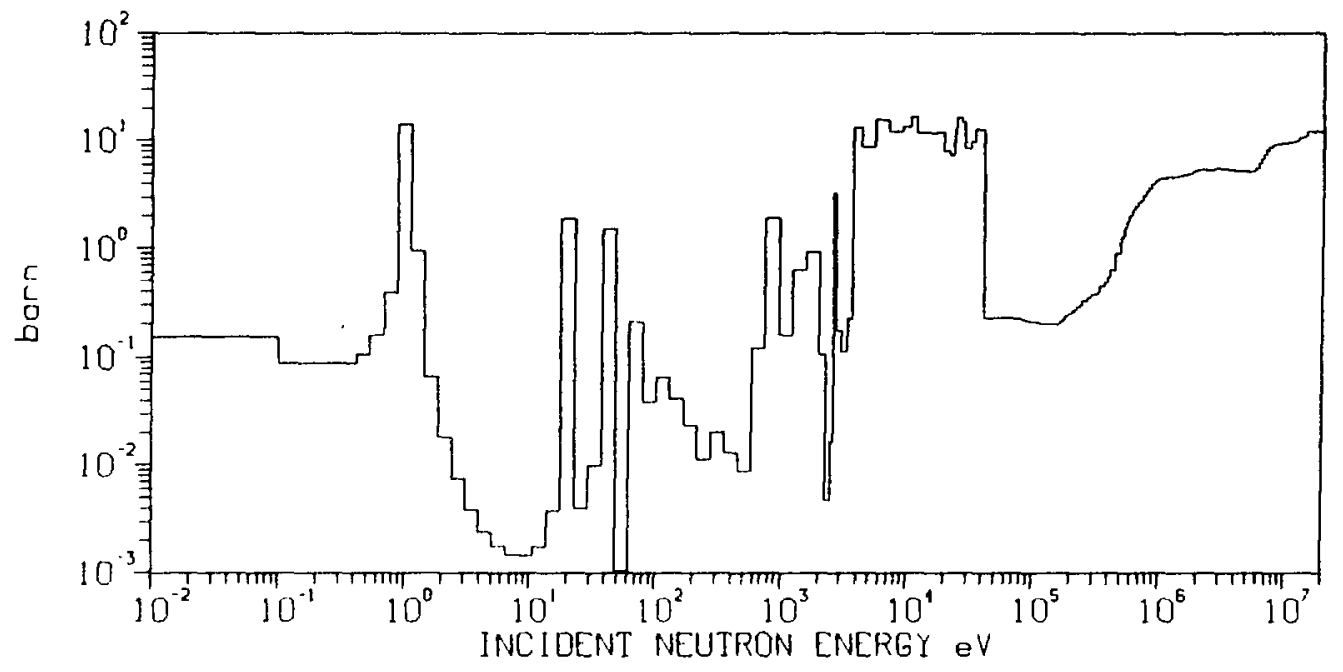


PU-241 RECOMMENDED PROMPT KERMA FACTOR (solid line)

REC. K. FACTOR WITH CH. PARTICLE DECAY HEAT (doshed Line)

REC. K. FACTOR PLUS TOTAL DECAY HEAT (choin dotted Line)

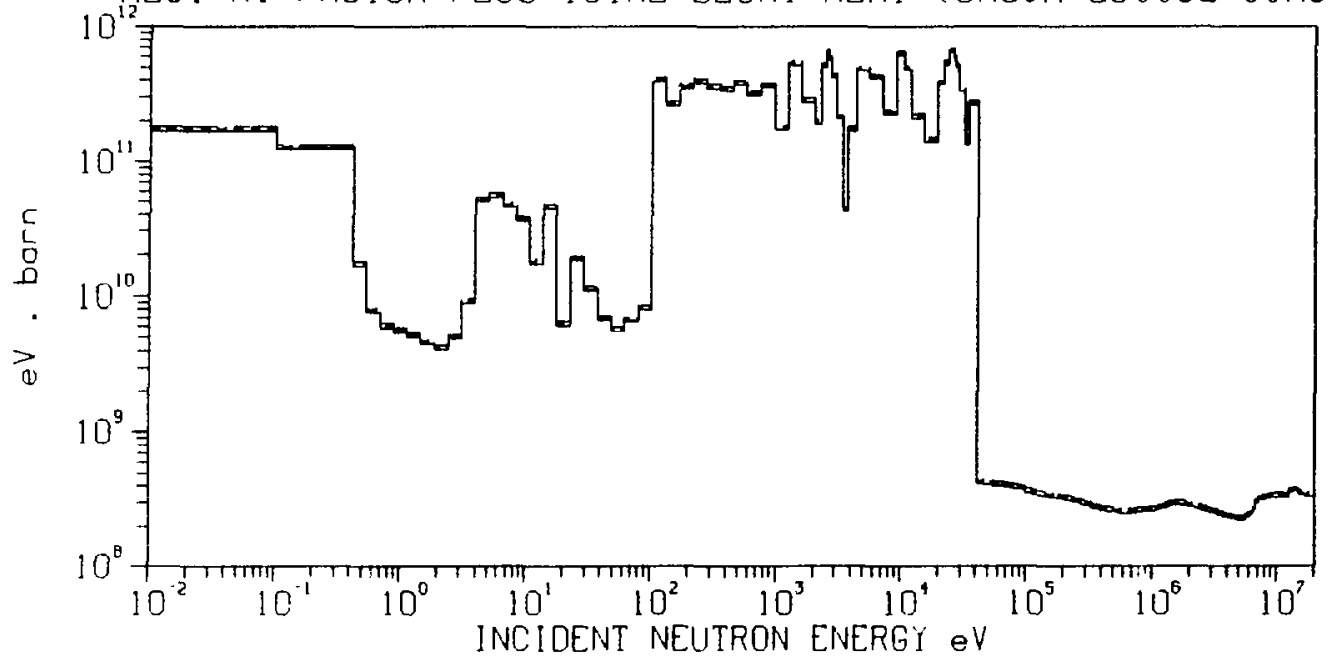

PU-241 RECOMMENDED PROMPT KERMA FACTOR (solid line) REC. K. FACTOR WITH CH. PARTICLE DECAY HEAT (doshed Line) $\because \quad$ REC. K. FACTOR PLUS TOTAL DECAY HEAT (chain dotted line)

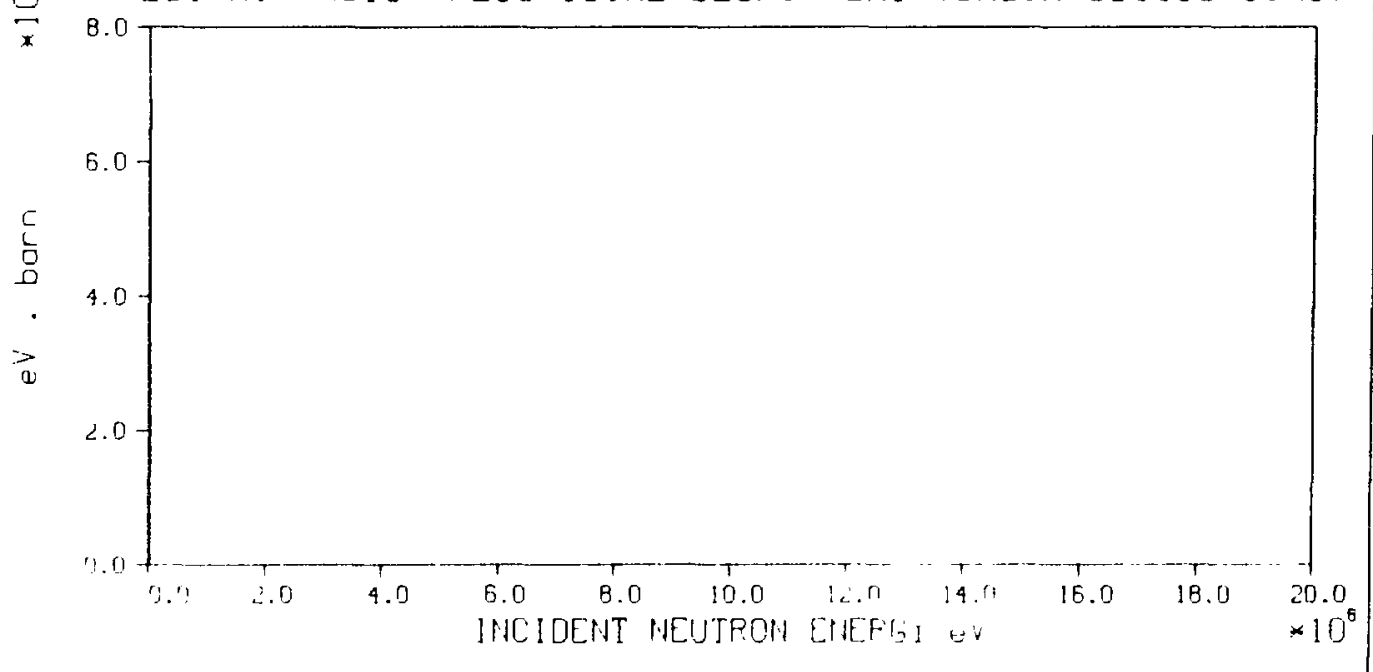




\section{PU-241 PROMPT GRMMA ENERGI PROCUCT ION}

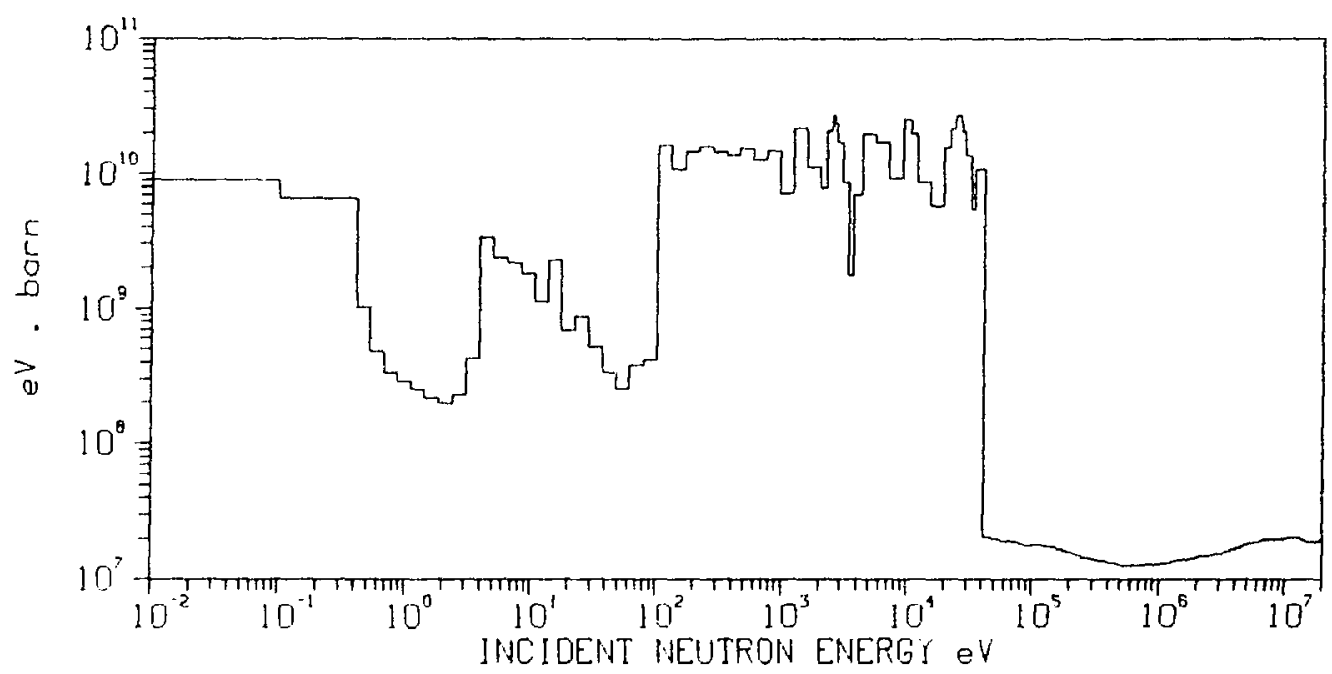

PU-241 TOTAL CROSS SECTION

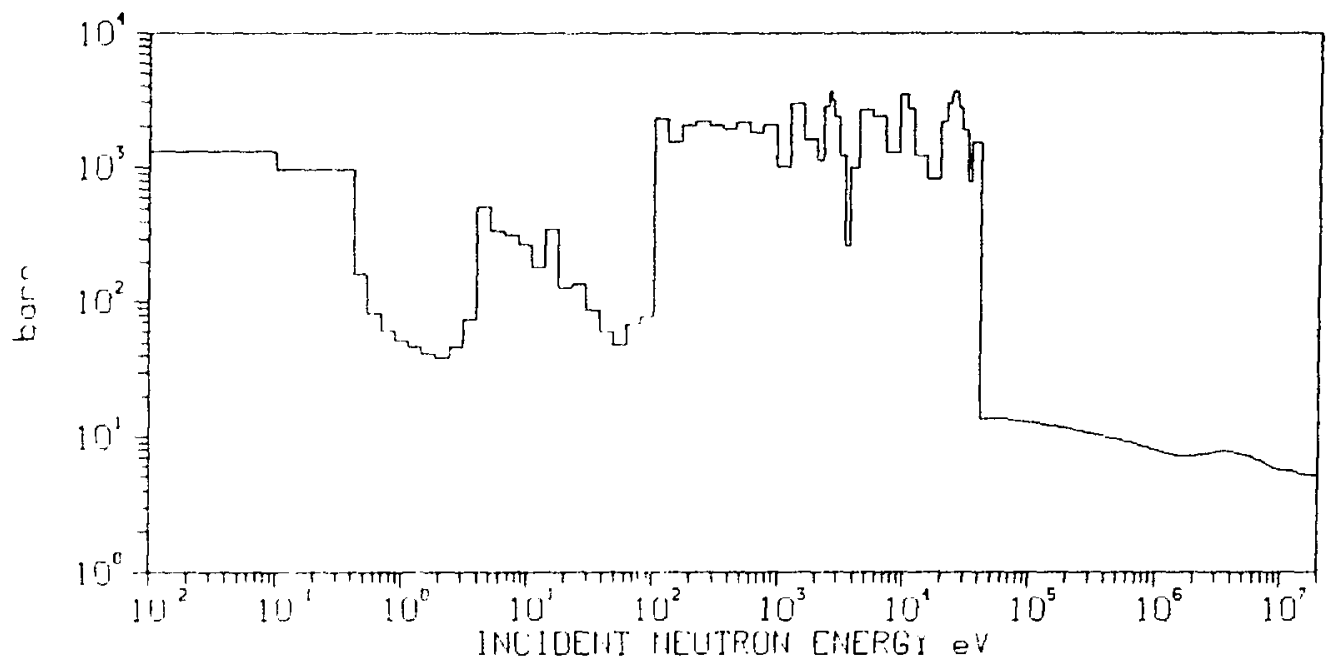




\section{PU-241 ELASTIC CROSS SECTION}

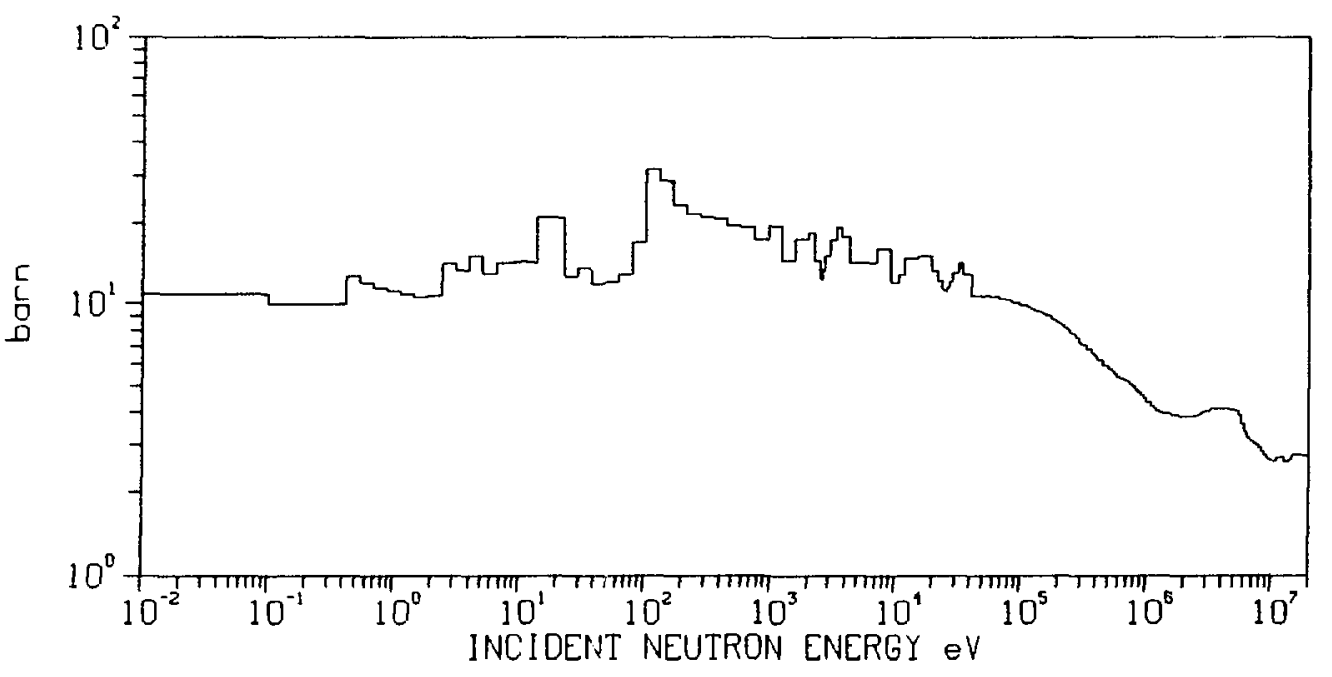

PU-241 TOTAL INELASTIC CROSS SECTION

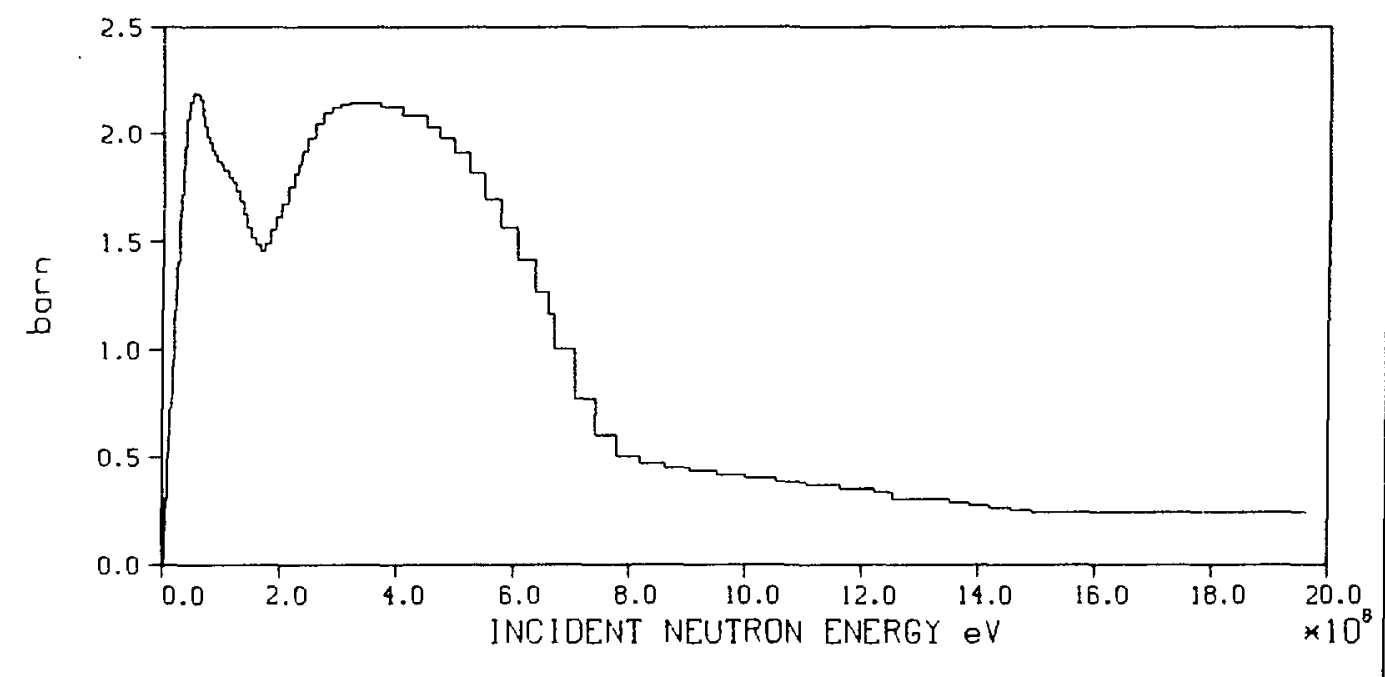


PU-241 RAIIATIVE CAPTURE CROSS SECTION

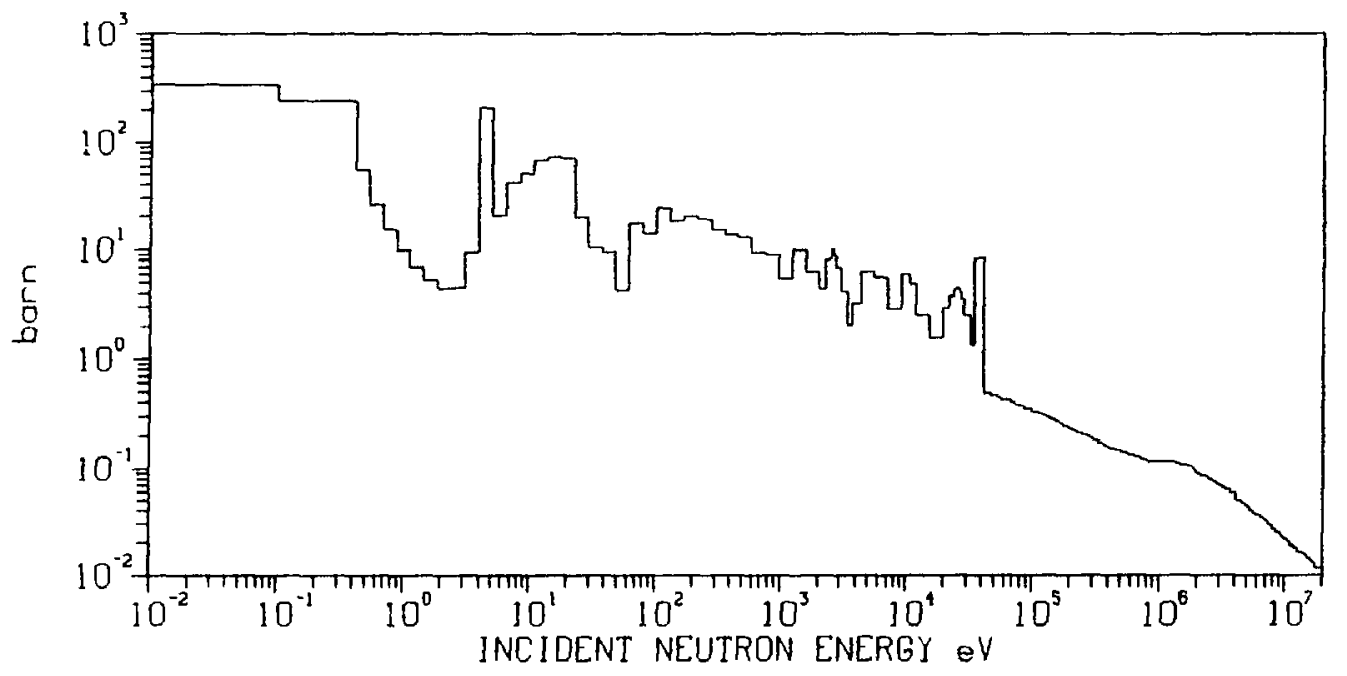

PU-241 TOTAL $(n, 2 n)$ REACTION CROSS SECTION

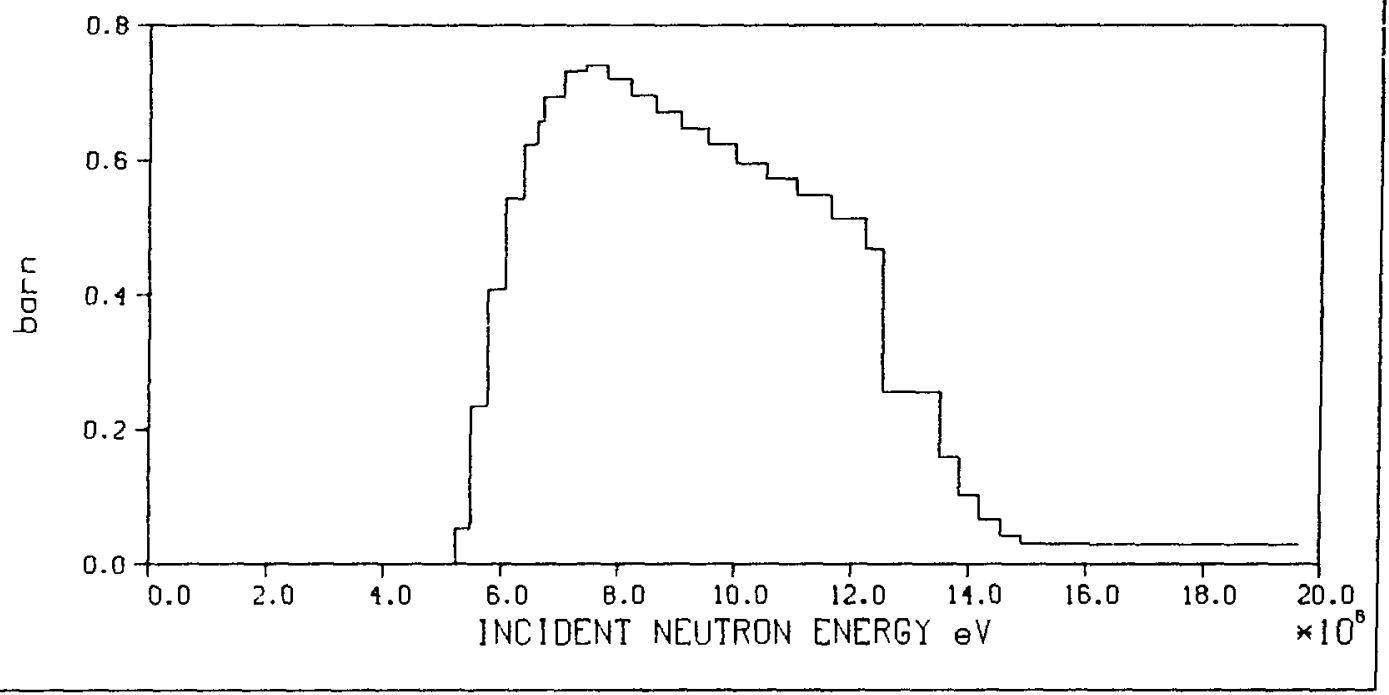


FU-241 TOTAL $(n, 3 n)$ CROSS SECTION

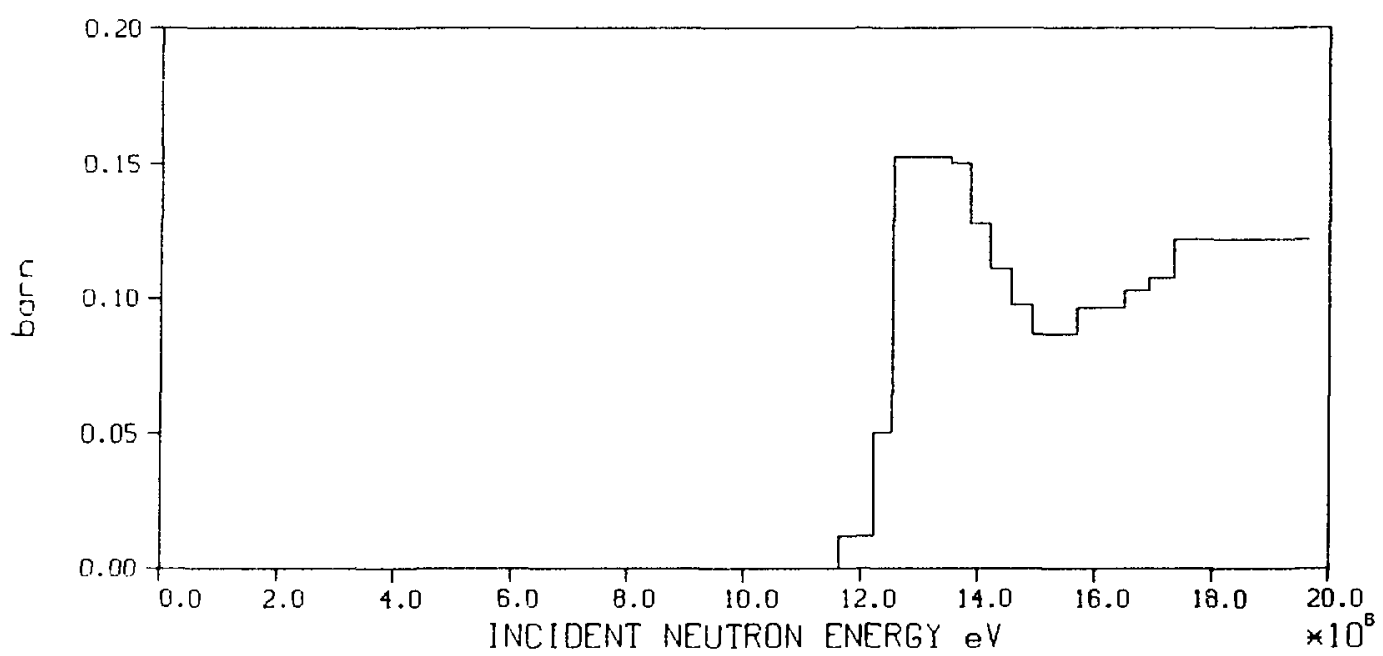

FU-241 NUMBER OF FISSION NEUTRONS * CROSS SECTION

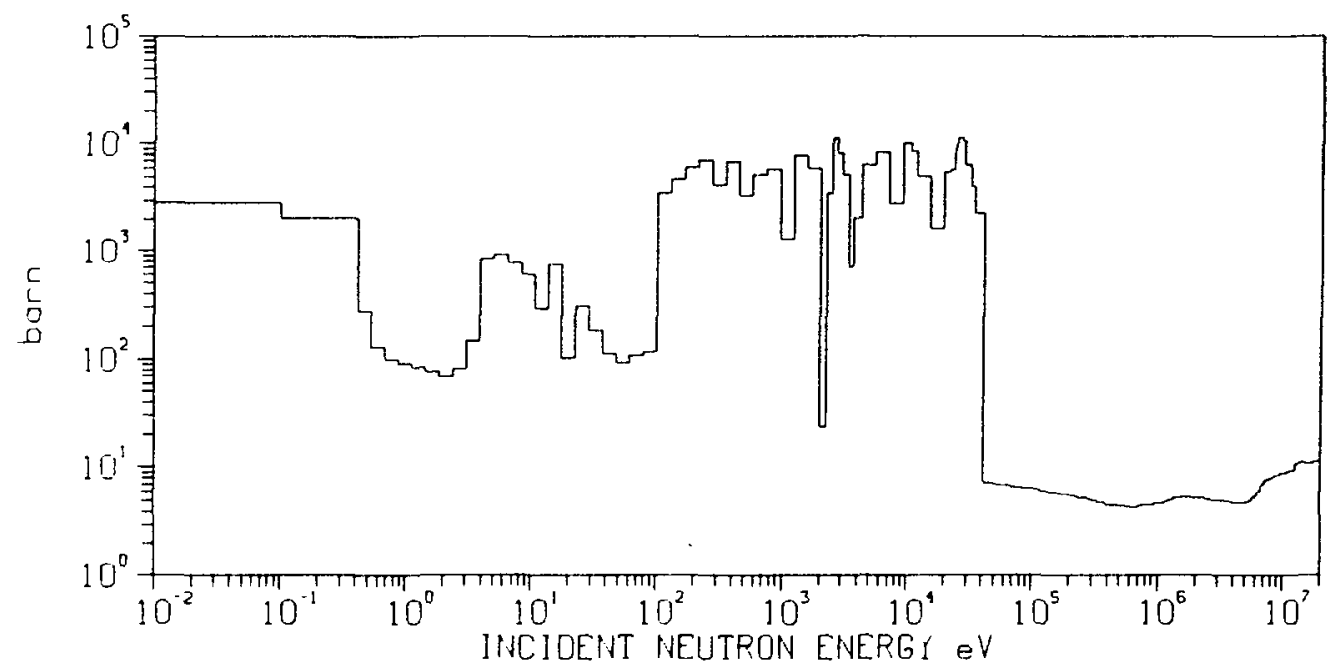




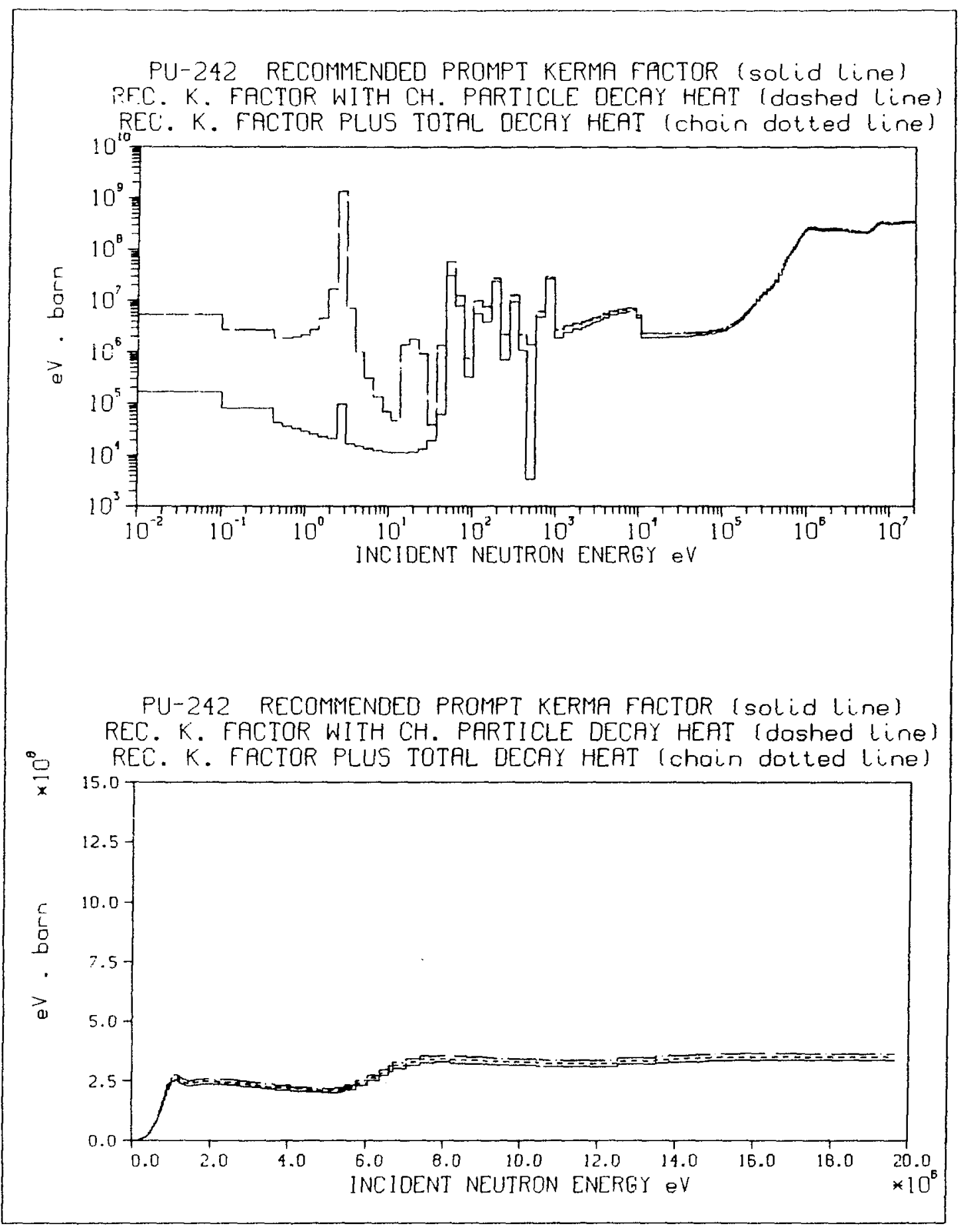




\section{PU-242 PROMPT GAMMA ENERGY PRODUCTION}

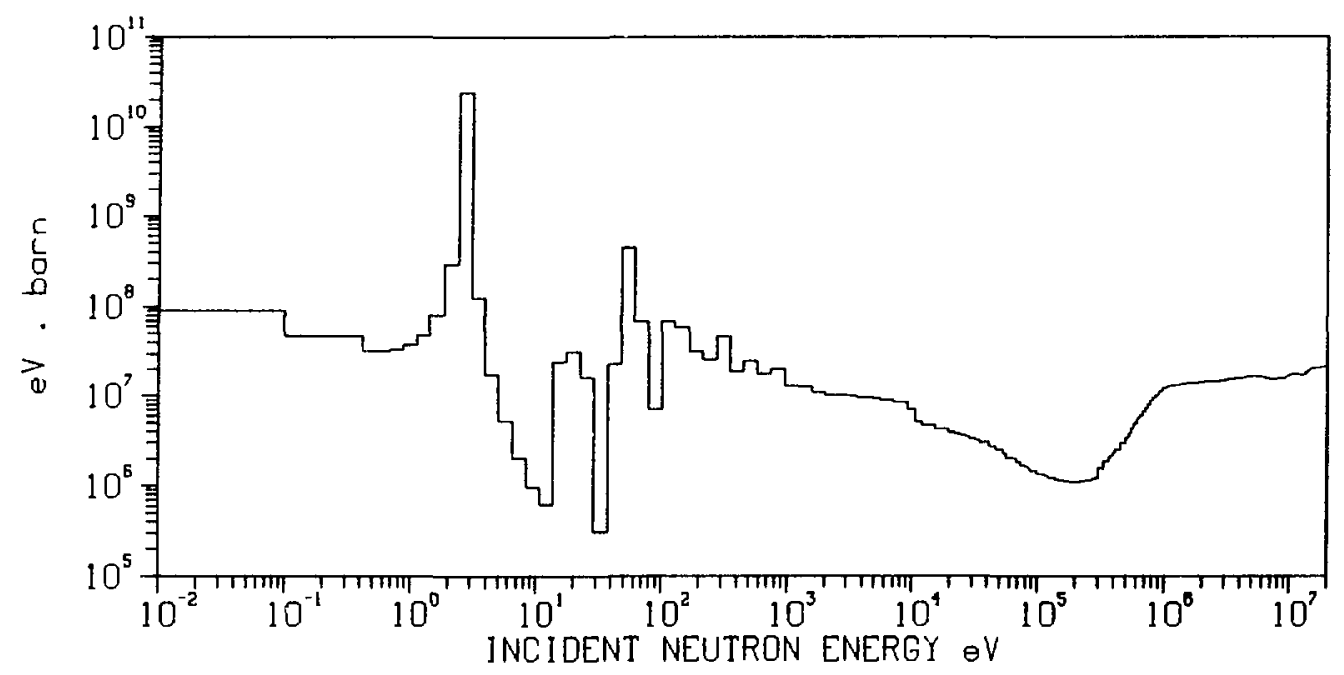

PU-242 TOTAL CROSS SECTION

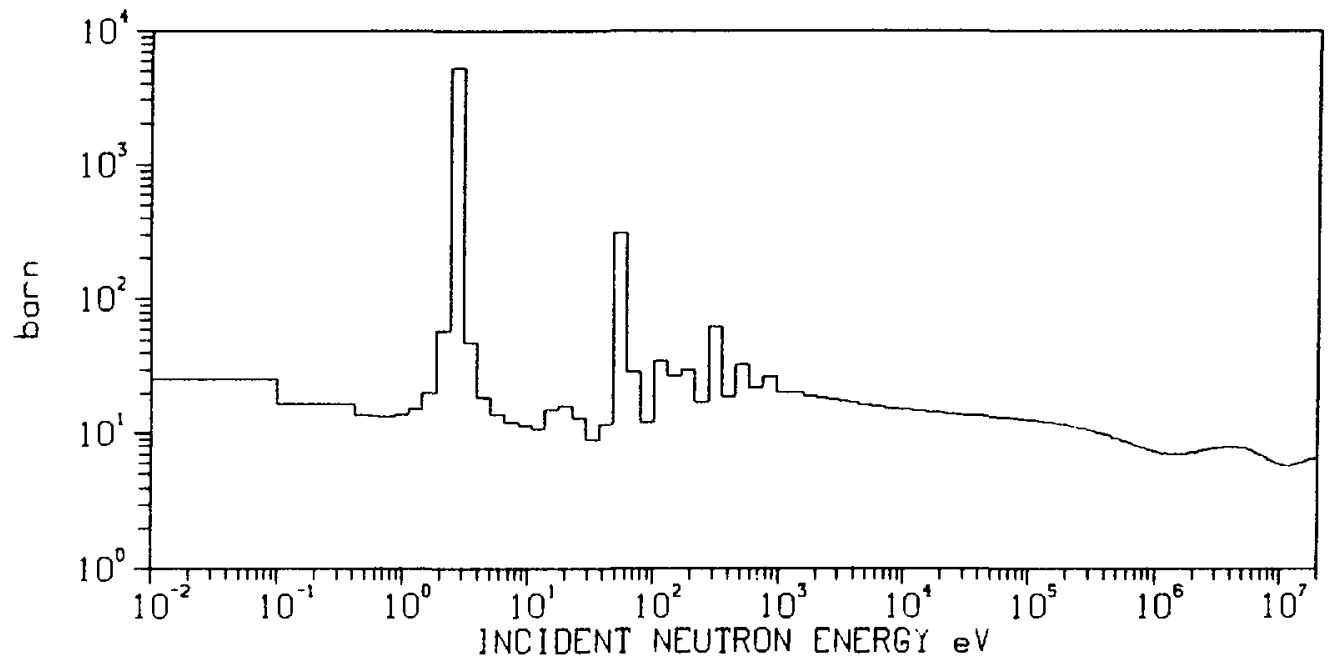




\section{PU-242 ELASTIC CROSS SÉCTION}

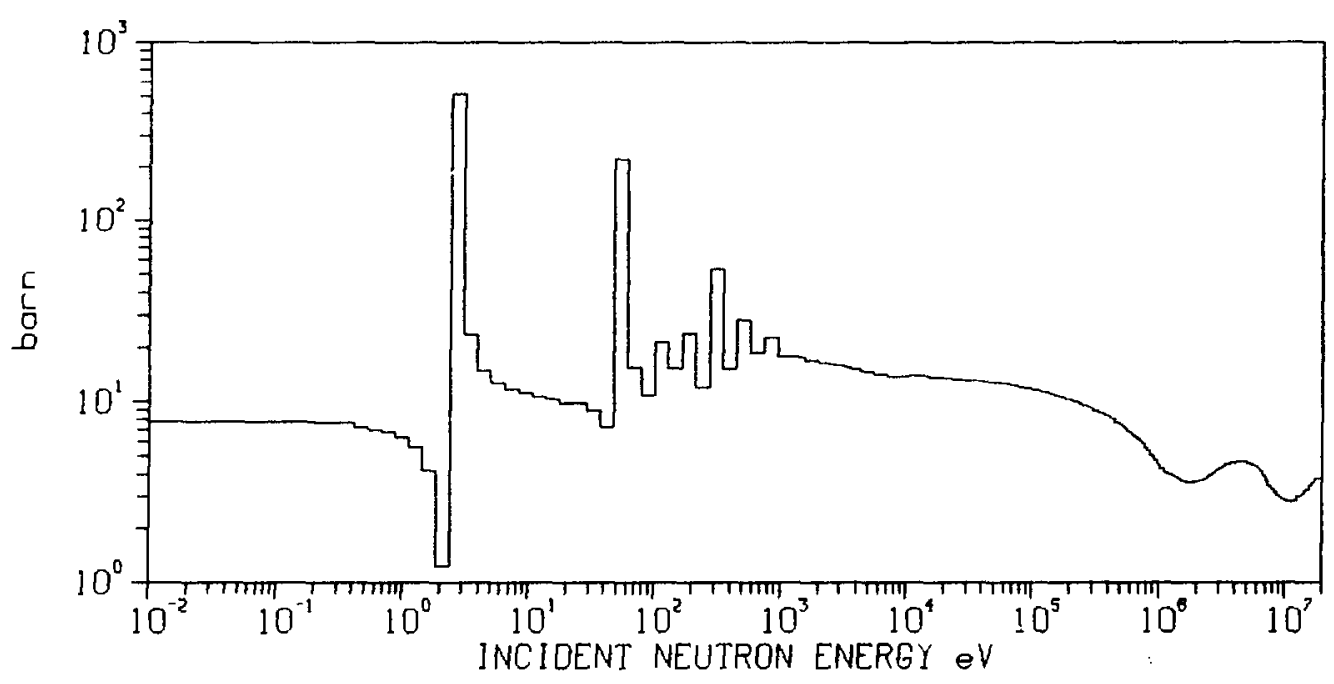

PU-242 TOTAL INELASTIC CROSS SECTION

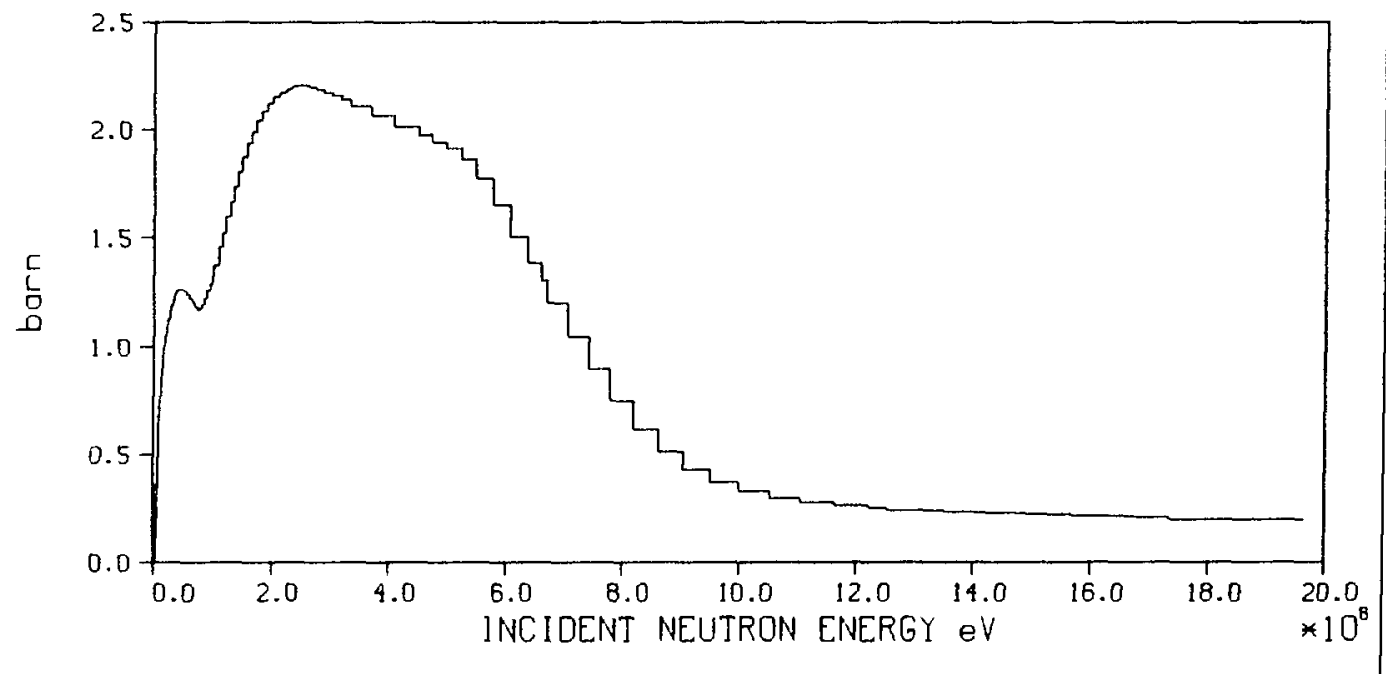


PU-242 RAOIATIVE CAPTURE CROSS SECTION

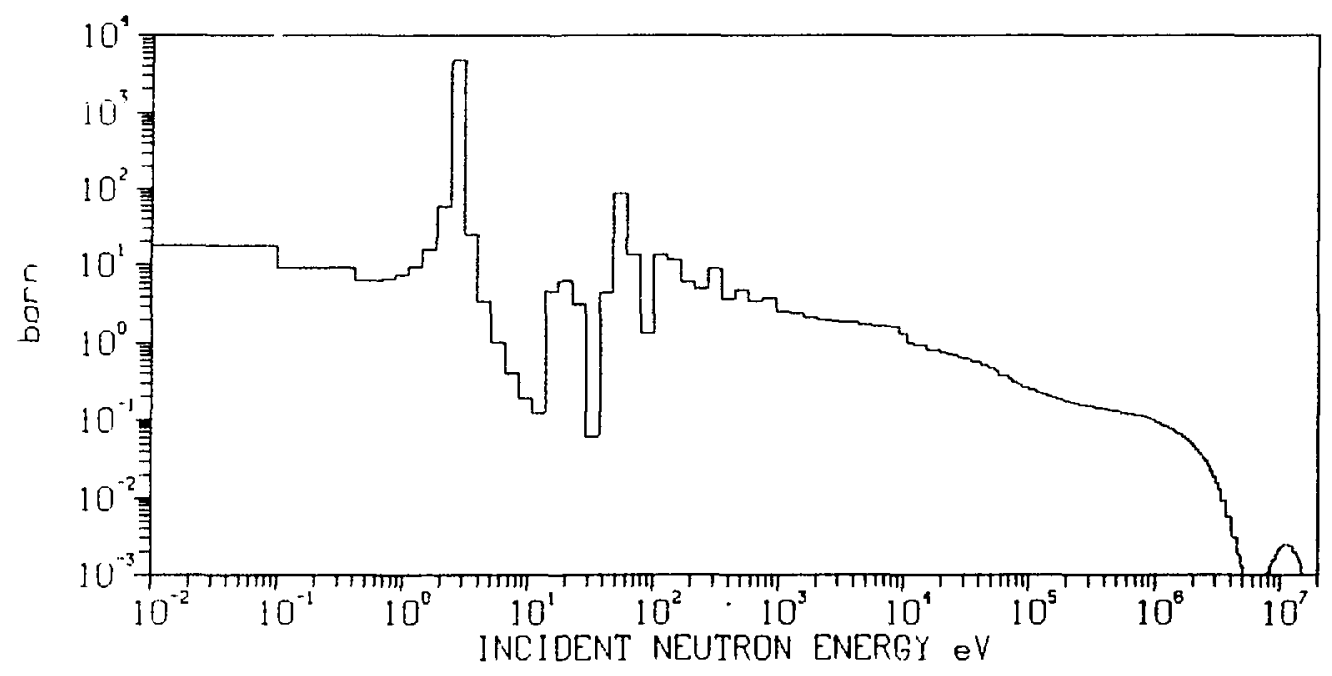

PU-242 TOTAL $(n, 2 n)$ REACTION CROSS SECTION

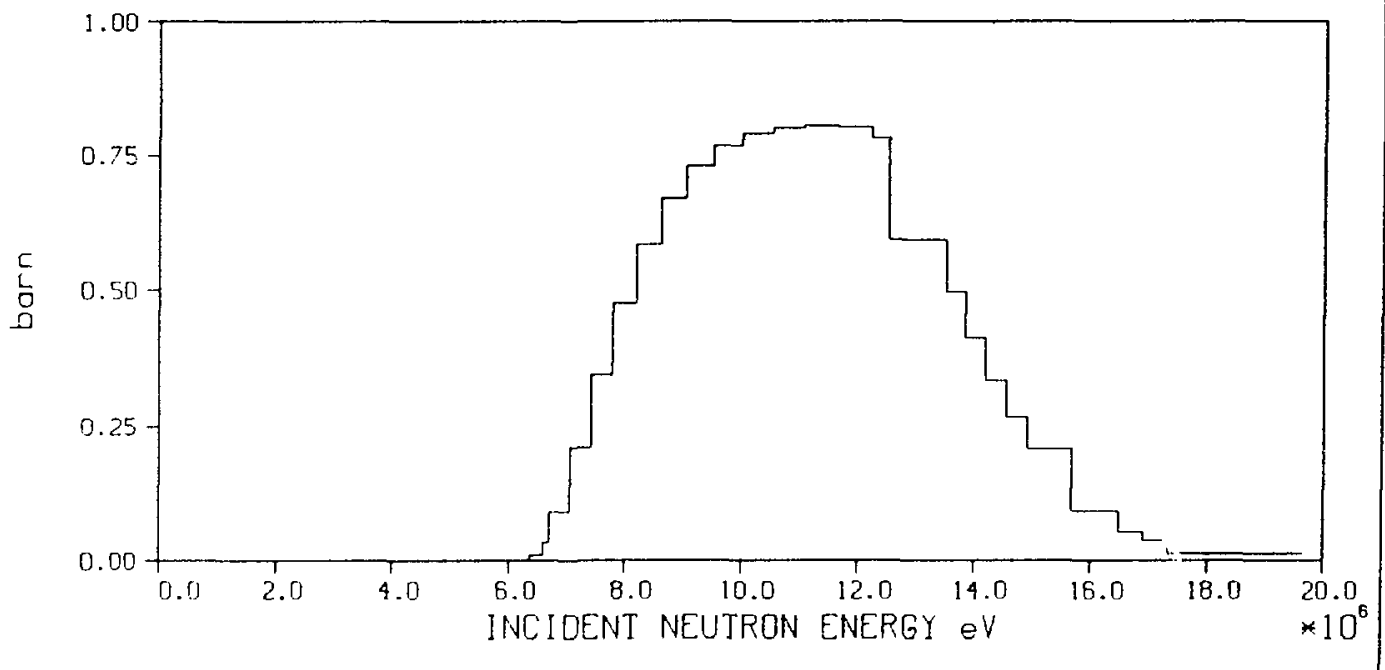


PU-242 TOTAL. In,3nI CROSS SECTION

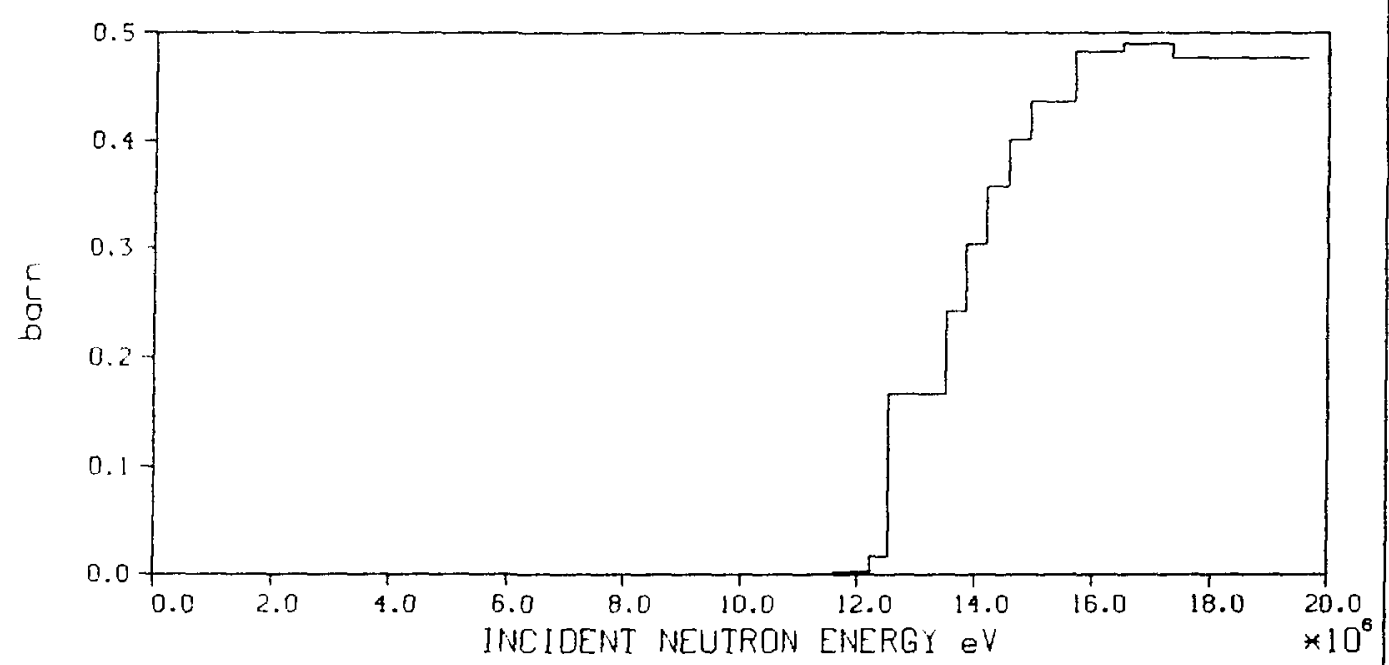

PU-242 NUMBER OF FISSION NEUTRONS * CROSS SECTION

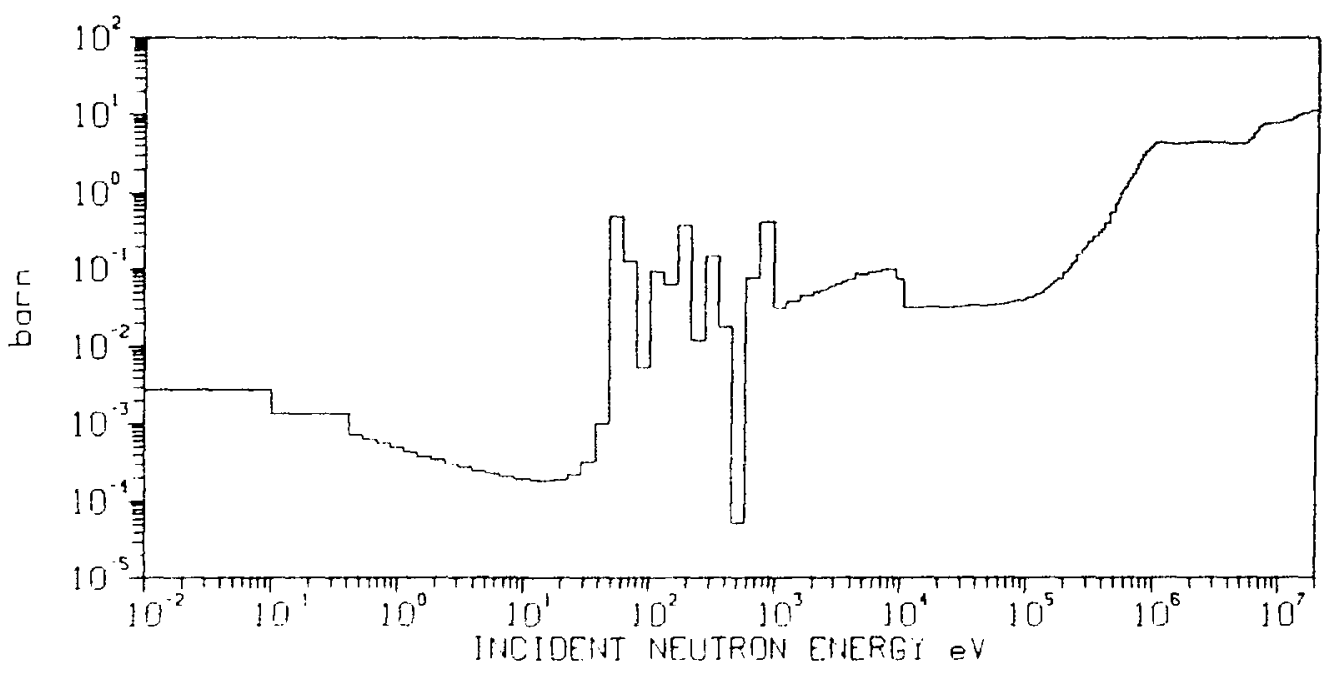




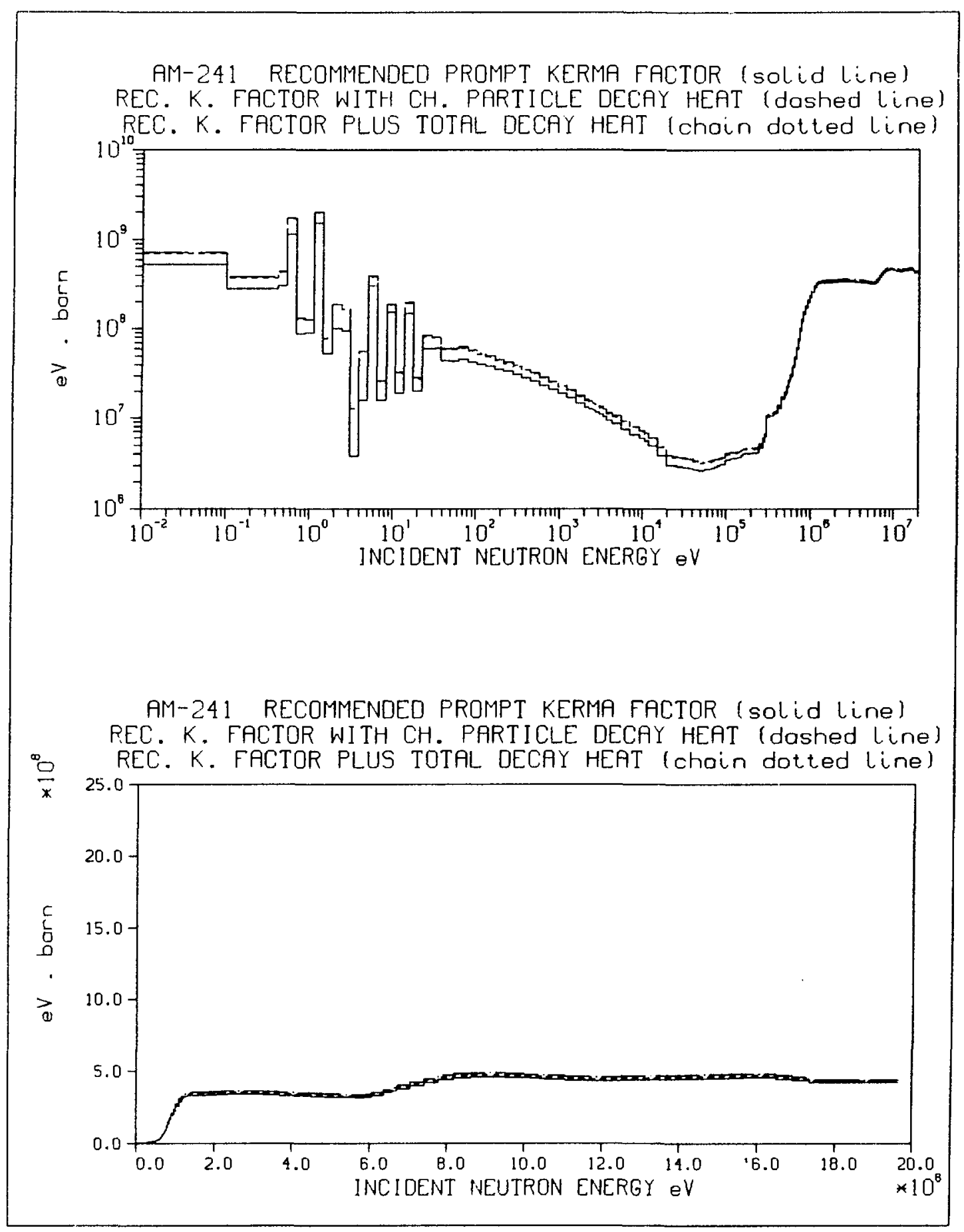


AM-241 PROMPT GAMMA ENERGY PRODUCTION

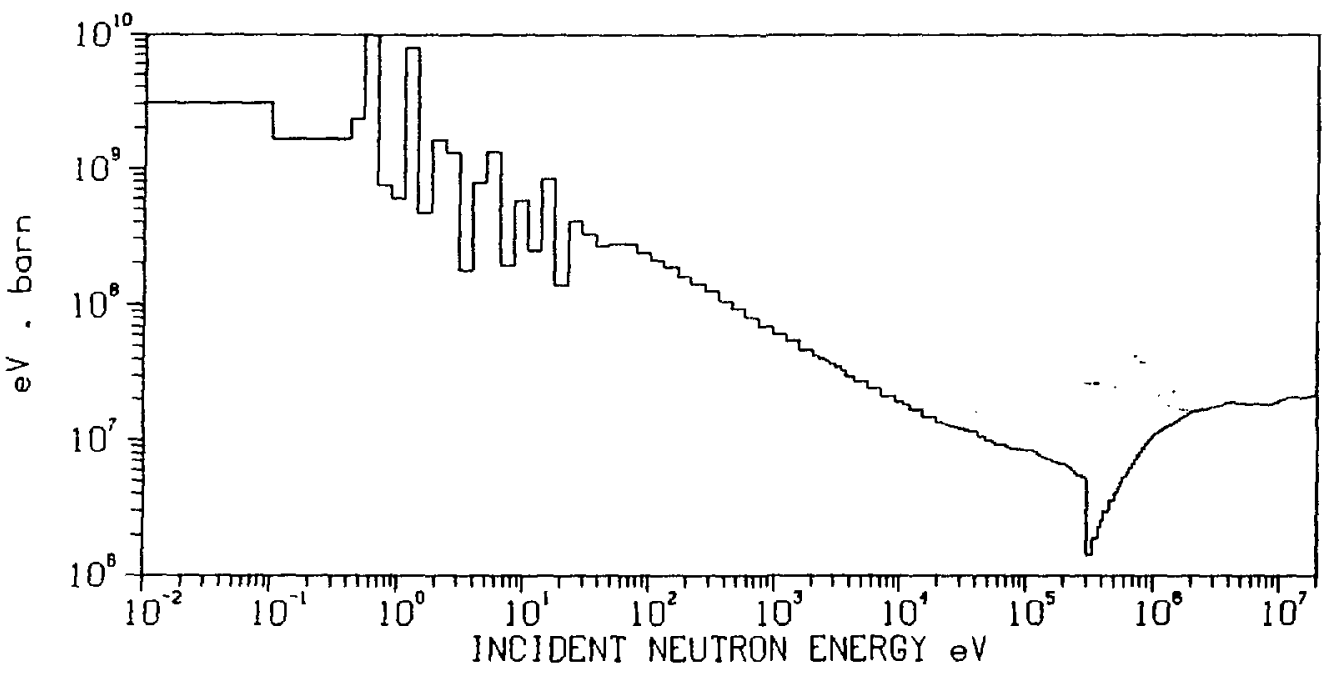

AM-241 TOTAL CROSS SECTION

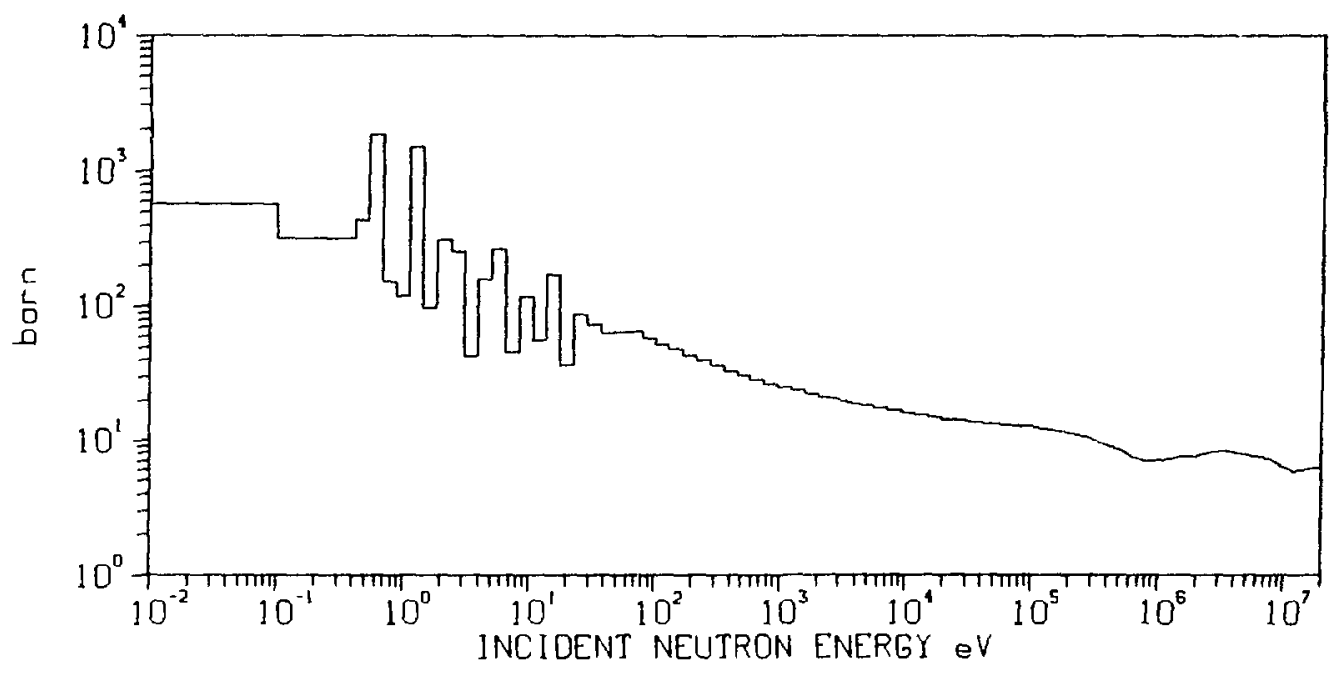




\section{AM-241 ELASTIC CROSS SECTION}

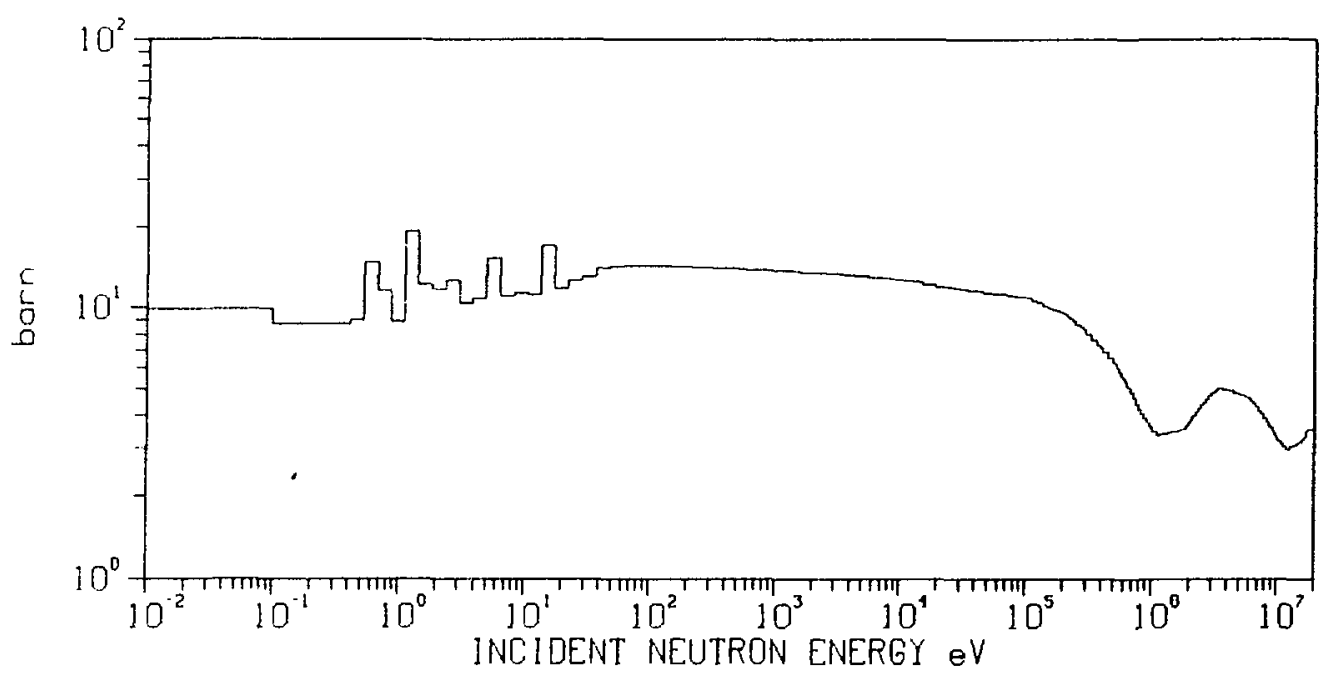

AM-241 TOTAL INELASTIC CROSS SECTION

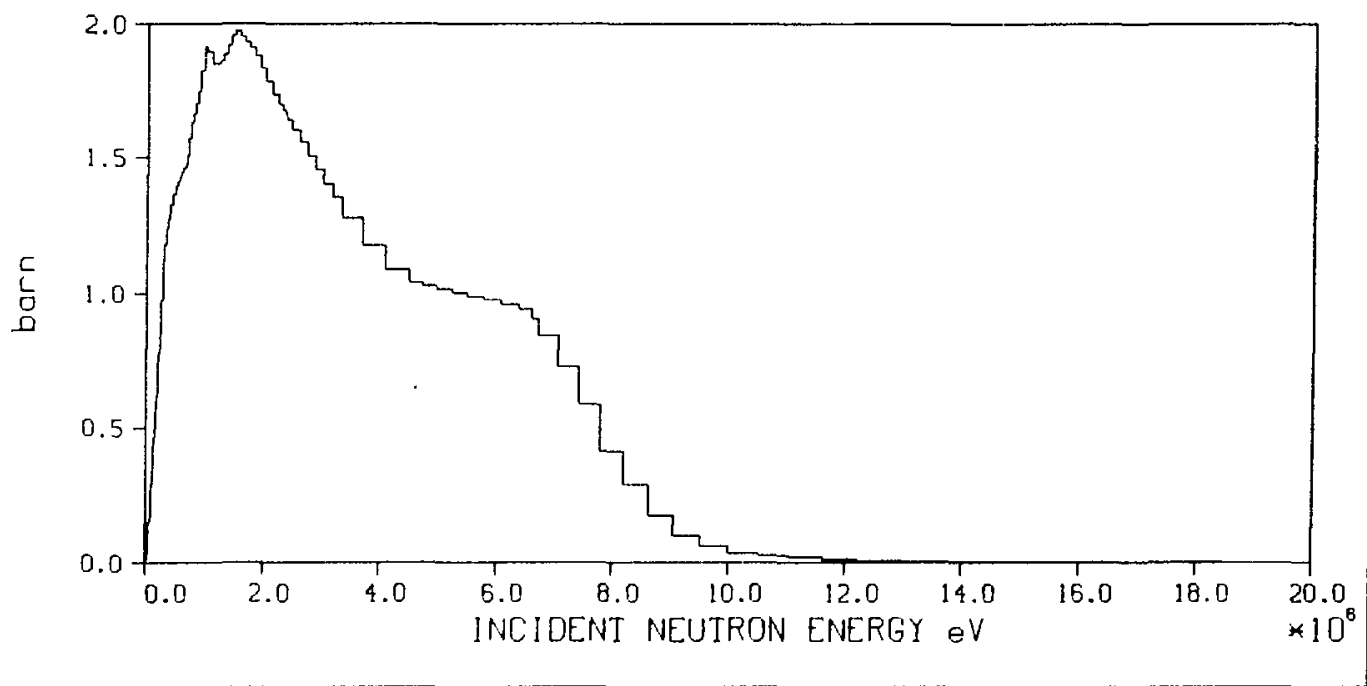


AM-241 RADIHITIVE CAPTURE CROSS SECTION

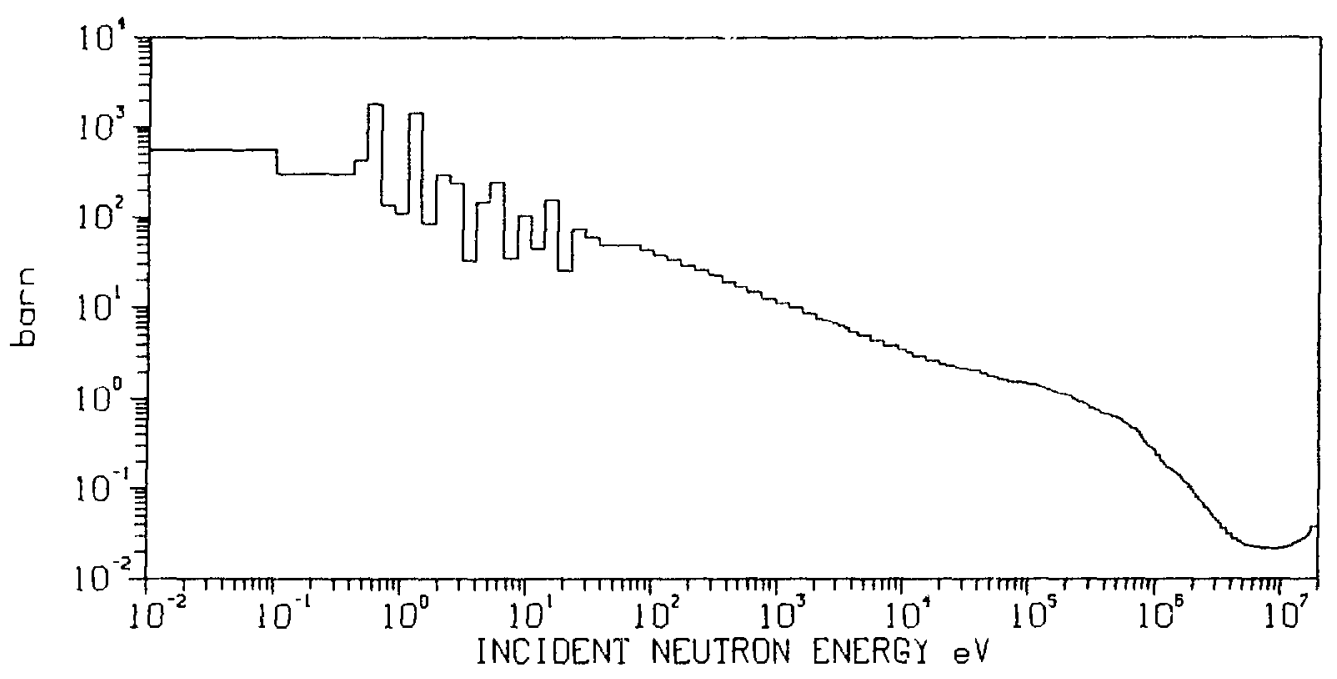

AM-241 TOTAL $(n, 2 n)$ REACTION CROSS SECTION

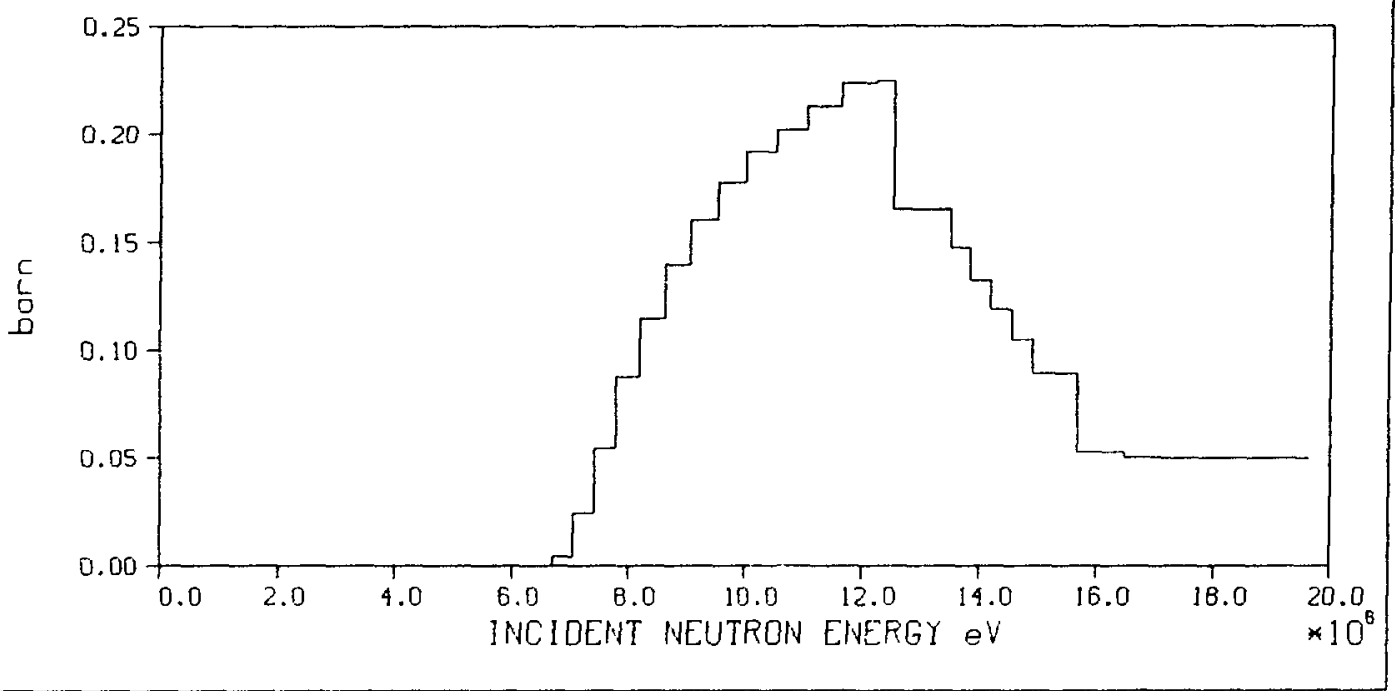


AM-241 TOTAL $(n, 3 n)$ CROSS SECTION

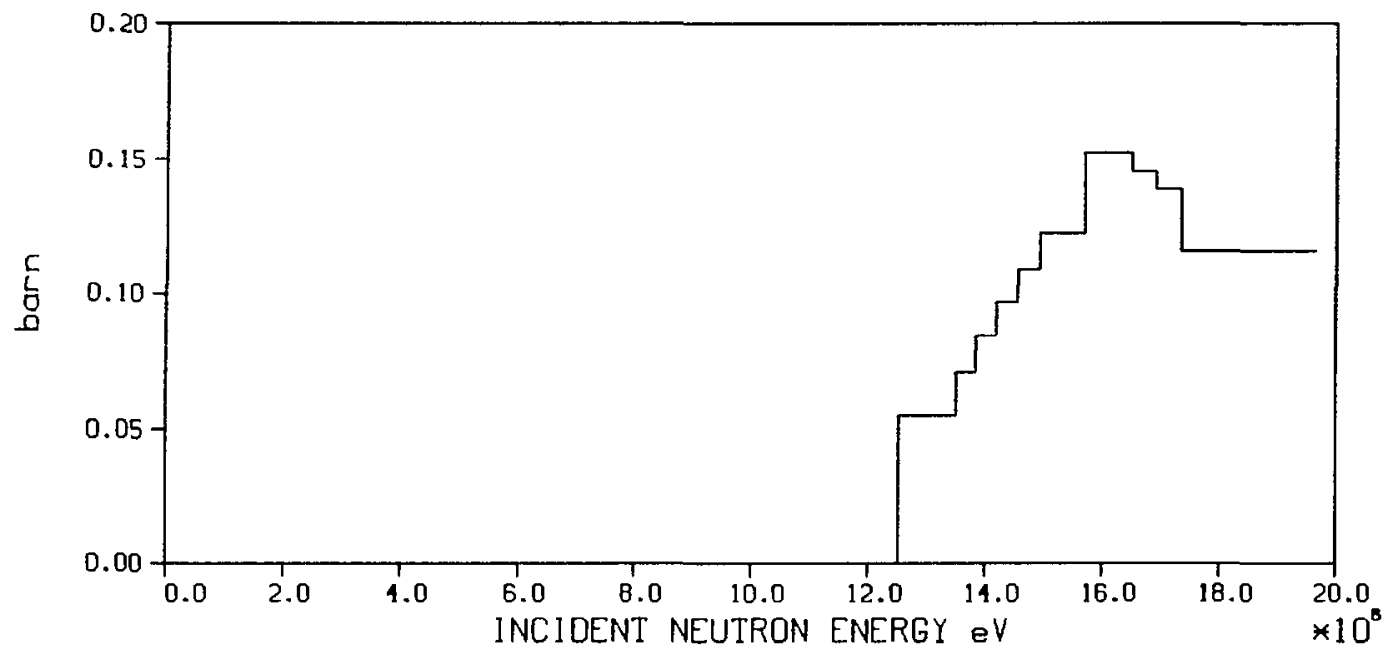

AM-241 NUMBER OF FISSION NEUTRONS * CROSS SECTION

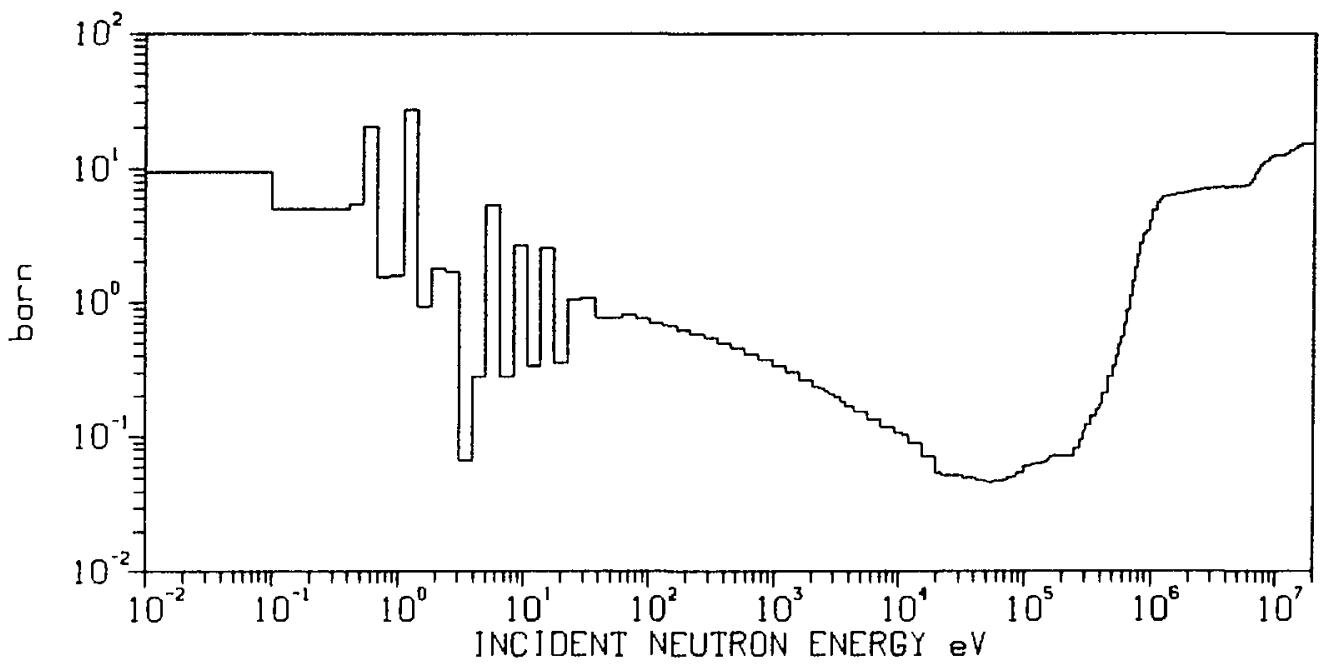


AM-243 RECOMMENDED PROMPT KERMA FACTOR (solid line) REC. K. FACTOR WITH CH. PARTICLE DECAY HEAT (dashed line)

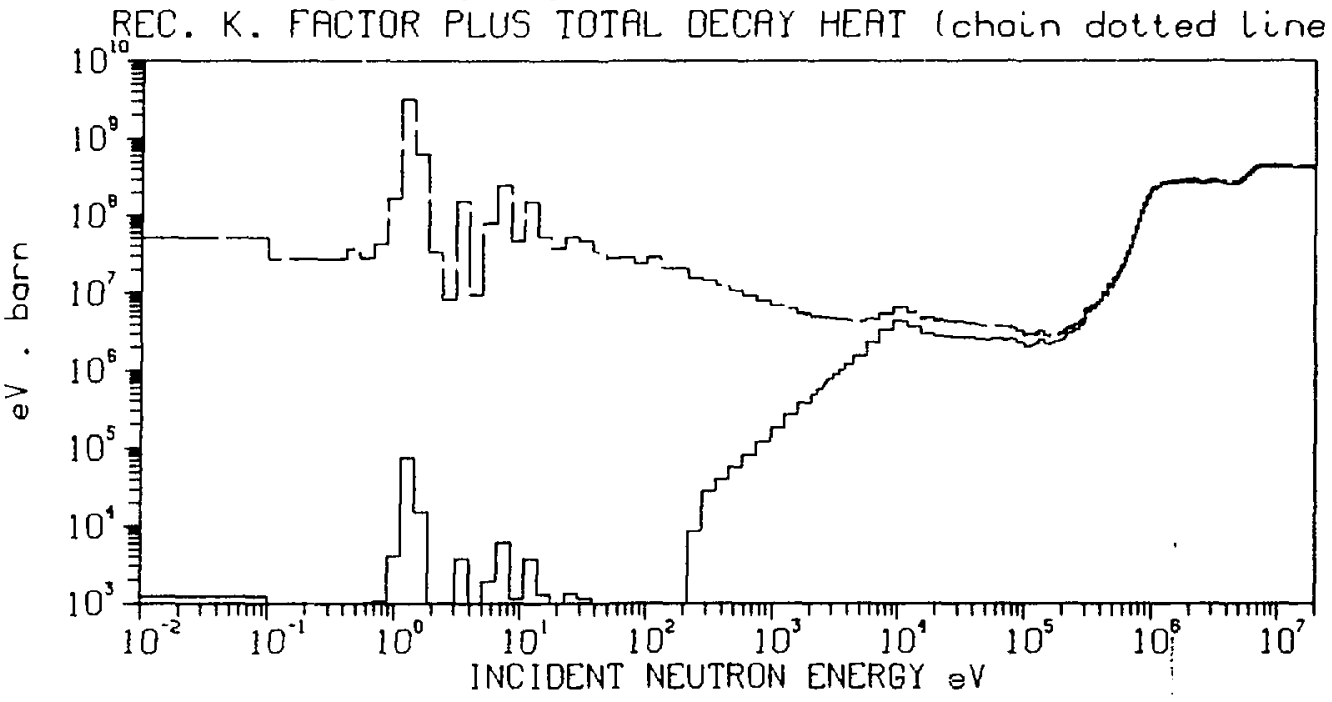

AM-243 RECOMMENDED PROMPT KERMA FACTOR (solid line) REC. K. FACTOR W]TH CH. PARTICLE DECAY HEAT (dashed Line) $\circ$ REC. K. FACTOR PLUS TOTAL DECAY HEAT (chain dotted line)

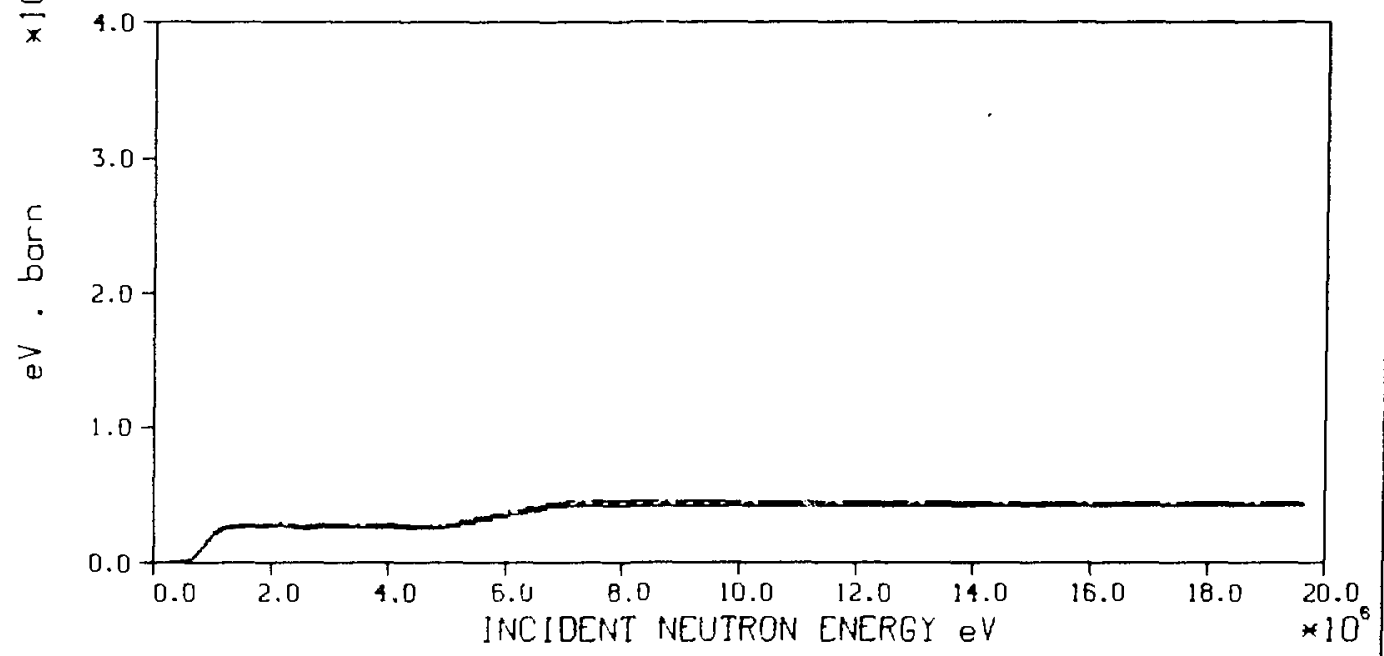


AM-243 PROMPT GRMMA ENERGI PRODUCTION

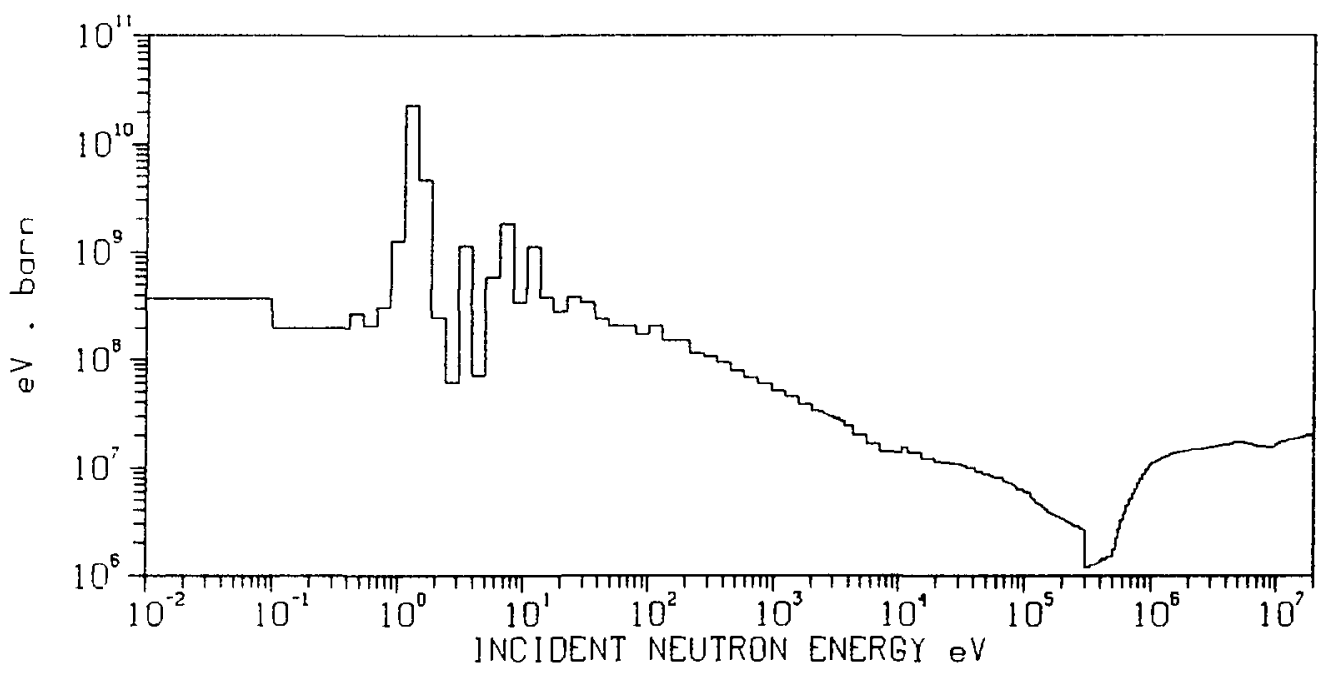

AM-243 TOTAL CROSS SECTION

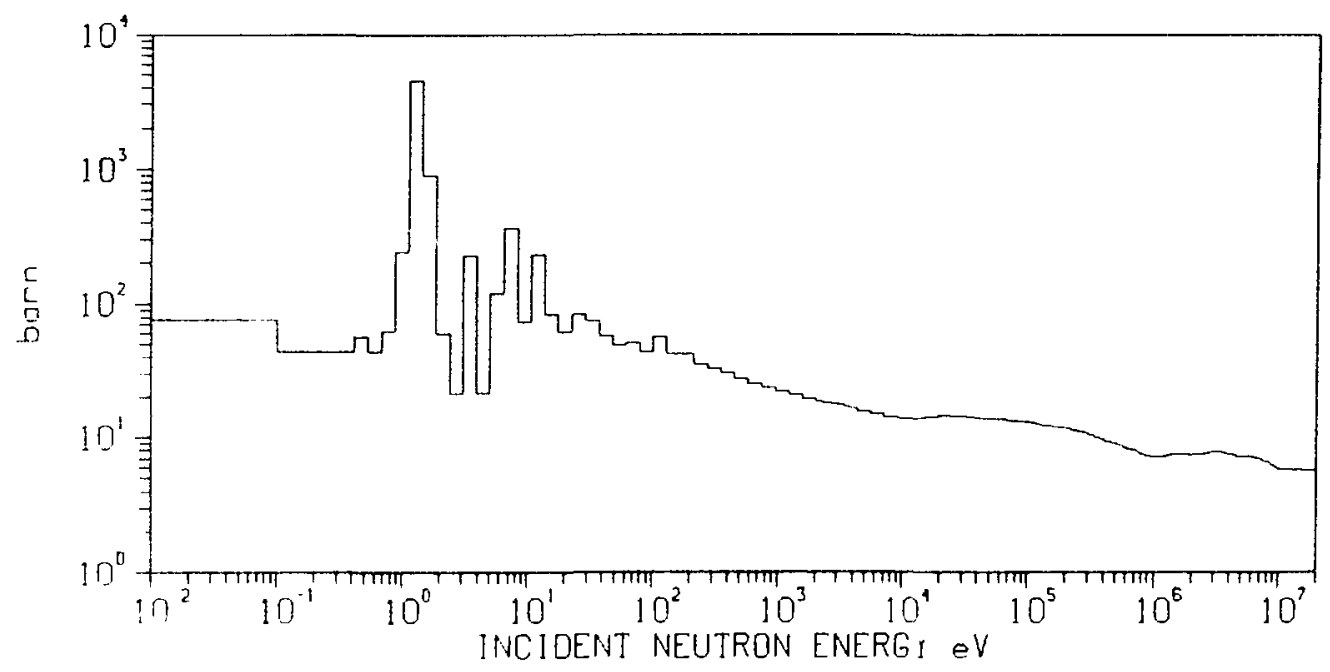




\section{AM-243 ELASTIC CROSS SECTION}

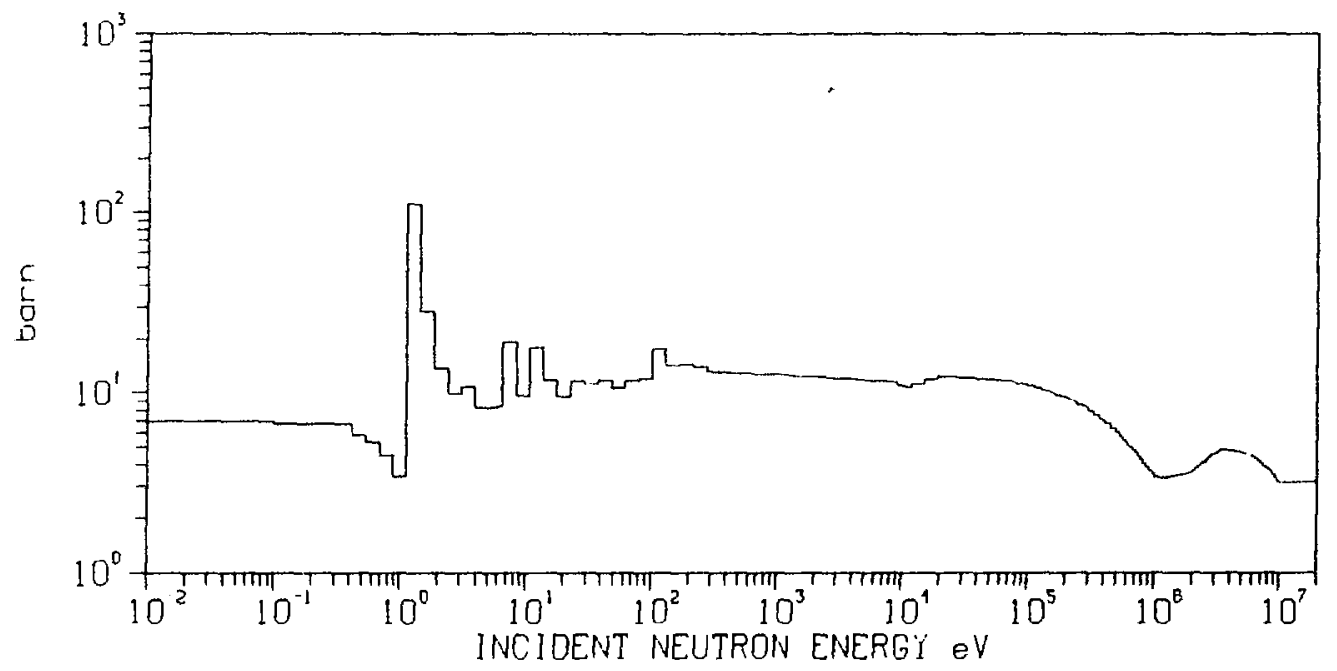

AM-243 TOTAL INELASTIC CROSS SECTION

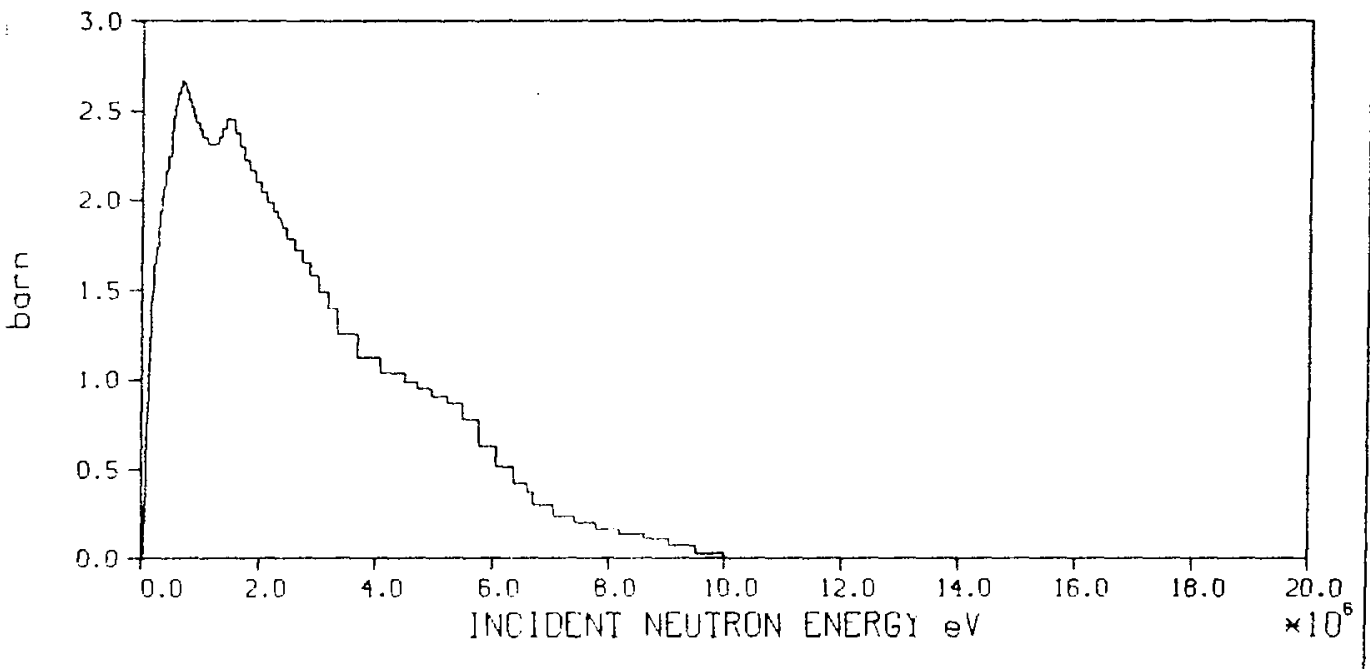


AM-243 RADIATIVE CAPTURE CROSS SECTION

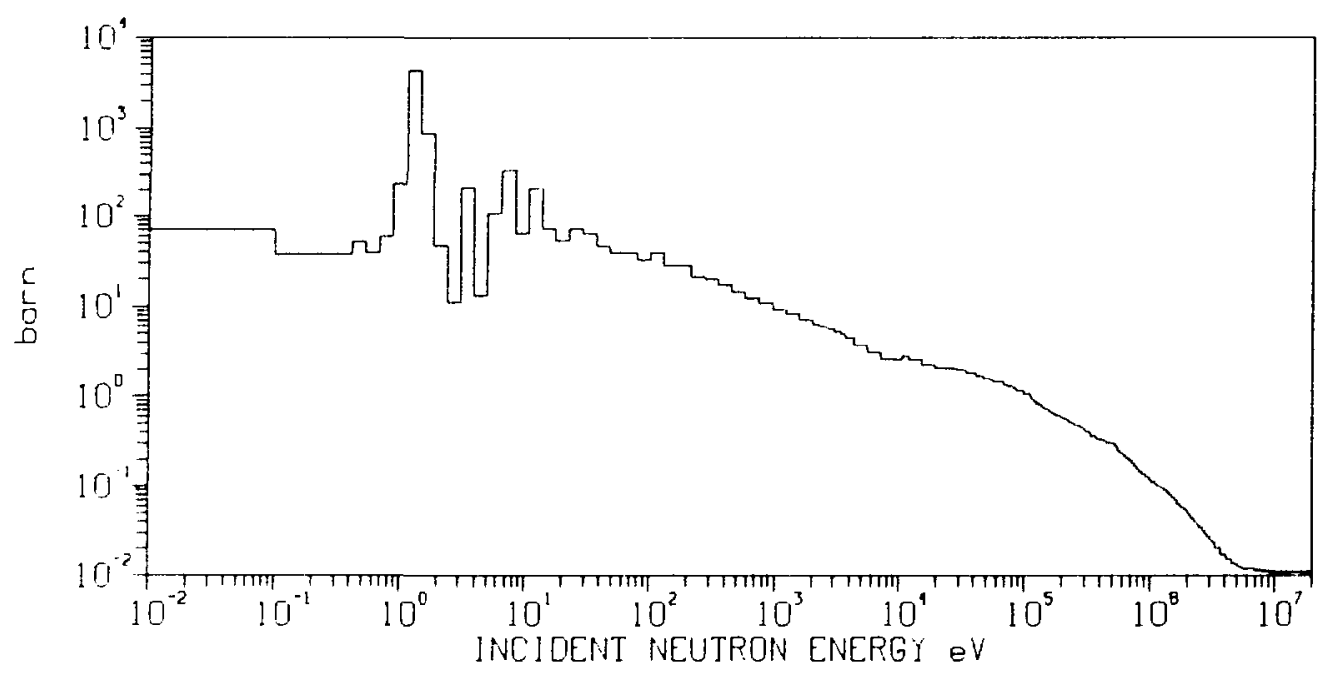

AM-243 TOTAL $(n, 2 n)$ REACTION CROSS SECTION

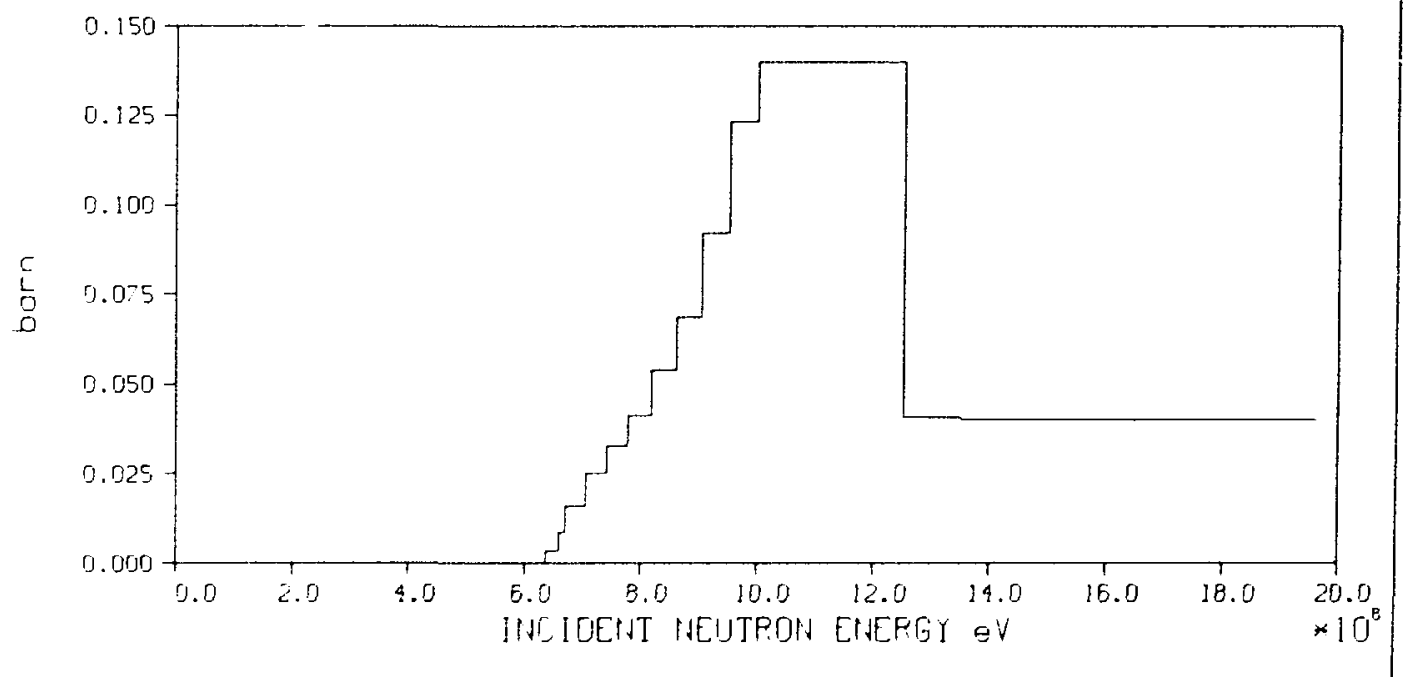


AM-243 TOTAL $(n, 3 n)$ CROSS SECTION

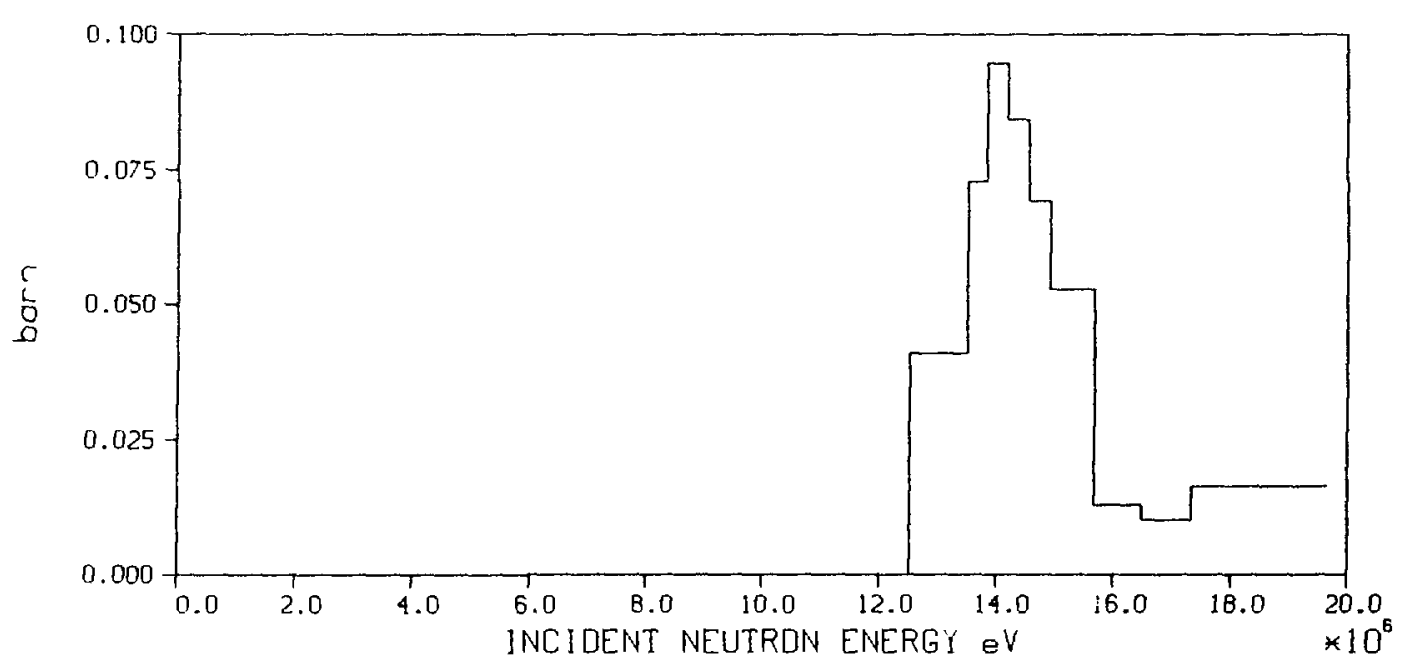

AM-243 NUMBER OF FISSION NEUTRONS * CROSS SECTION

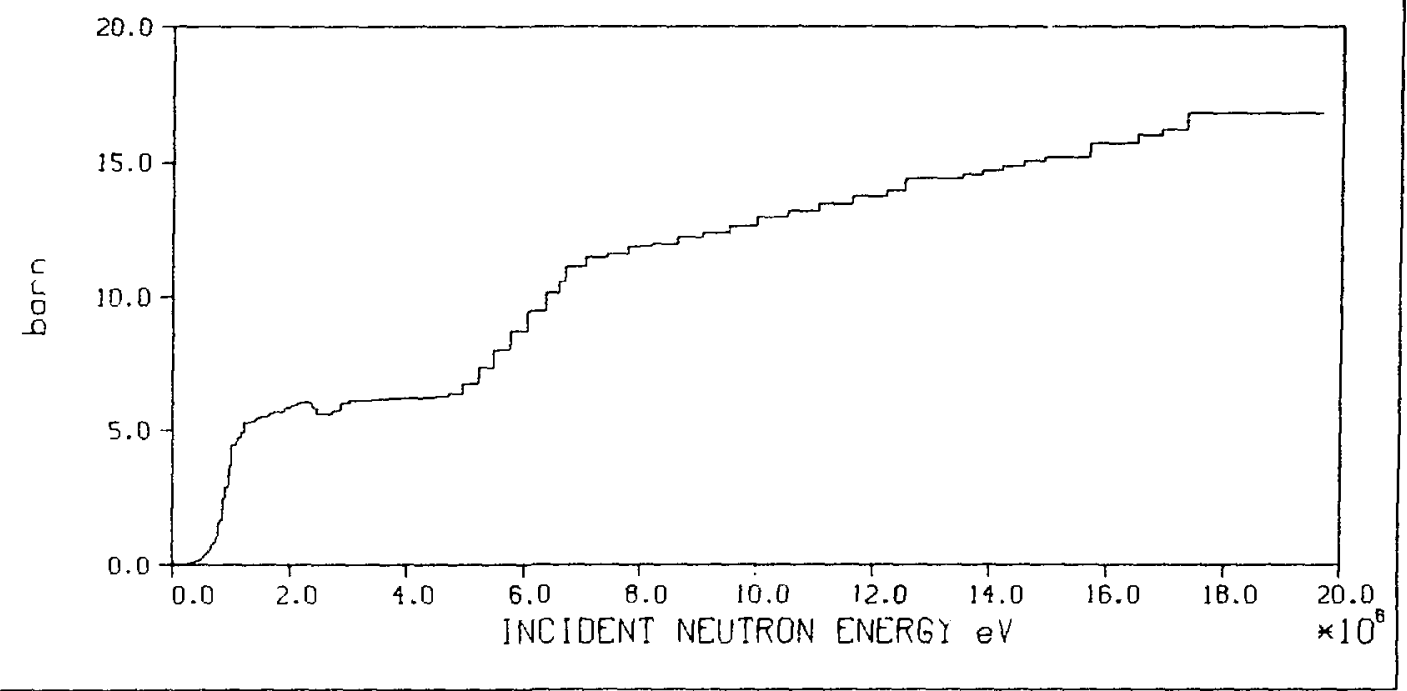




\section{REFERENCES:}

1- R. Kinsey, "ENDF-102 Data Formats and Procedures for the Evaluated Nuclear Data File, ENDF" BNL-NCS-50496 (ENDF 102) (Oct. 1979), Revised by B. Magurno (Nov. 1983).

2- R. Little, and R. Seamon, "Negative Heating Numbers", Los Alamos National Laboratory Memorandus to P. Young, and E. Arthur, CSEWG Evaluations Committee. (June 17, 1981).

3- R. Little, and R. Seamon; Los Alamos National Laboratory, Radiation Iransport Group, A Letter to J. Huang, University of Wisconsin-Madison (Nov. 1982).

4- Y. Farawila et al., "KAOS-V: A Program to Evaluate Neutron Kerma Factors and other Nuclear Responses" Argonne National Laboratory Report, ANL/FPP/TM-240 (1989).

5- R. MacFarlane, D. Muir, and R. Boicourt, "The NJoy Nuclear Data Processing System, Vol. I: User's manual" LA-9303-M, Vol. I (ENDF- 324).

6- R. Macfarlane, D. Muir, and R. Boicourt, "The NJOY Nuclear Data Processing System, Vol. II: The NJOY, RECONR, BROADR, HEATER, and THERMR Modules" LA-9303-M, Vol. II (ENDF-324).

7- W. W. Engle, Jr., "A User Manual for ANISN: A One Dimensional Discrete Ordinates Transport Code with Anisotropic Scattering" Oak Ridge National Laboratory Report K-1693 (1973).

8- R. W. Roussin et al., "Vitamin-E $174_{N}, 38_{G}$ General Purpose Cross-Section Library" Proceedings of the International Conference on Nuclear Data for Basic and Applied Science, Santa Fe, New Mexico (May 1985).

9- I. M. Bondarenko and Eh. E. Petrov, "Evaluation of Neutron- 6 Li and- $7_{L i}$ Interaction Cross-Sections for Calculating Kerma Factors" INDC(CCP)265/L, (Dec. 1986). 
10- P. Deluca, Jr., H. Barschall, M. Burhoe, and R. Haight, "Carbon Kerma Factor for 18- and 20-MeV Neutrons" Nuclear Science and Engineering: 94,192-198 (1986).

11- P. Deluca, Jr., H. Barscha11, R. Haight, and J. McDonald, "Kerma Factor of Carbon for 14.1-MeV Neutrons" Radiation Research 100, 78-86 (1984).

12- R. Haight, S. Grimes, R. Johnson, and $H$. Barschall, "The ${ }^{12} \mathrm{C}(n, a)$ Reaction and the Kerma Factor for Carbon at $E_{n}=14.1 \mathrm{MeV}$ " Nuclear Science and Engineering: 87,41-47 (1984).

13-P. DeLuca, Jr., H. Barschal1, R. Haight, and J. McDonald, "Measured Neutron Carbon Kerma Factors from $14.1 \mathrm{MeV}$ to $18 \mathrm{MeV}$ " 1984, Proc. 5th Symp. Neutron Dosimetry Munich/Neuherberg, FRG, EUR-9762, p.193, Commission of the European Communities, Luxembourg (1985).

14- B. Antolokovic and J. Hudomalj, " $3 a$ Decay of the 10.84 and $11.83 \mathrm{MeV}$ States of 12C" Nuclear Physics A237 (1975) 253-259.

15- G. M. Frye, Jr., L. Rosen, and L. Stewart, "Disintegration of Carbon into Three Alpha Particles by 12-20 Mev Neutrons" Physical Review Vol. 99, No. 5 (Sept. 1955).

16- B. Antolkovic and Z. Dolenec, "The Neutron-Induced ${ }^{12} \mathrm{C}\left(n, n^{\prime}\right) 3 \boldsymbol{\alpha}$ Reaction at $14.4 \mathrm{MeV}$ in a Kinematically Complete Experiment" Nuclear Physics A237 (1975) 235-252

17- E. A. Davis et al., "Disintegration of 016 and $C_{12}$ by Fast Neutrons" Nuclear Physics 48 (1963) 169-175.

18-R. A. Al-Kital and R. A. Peck, Jr., "C $\mathrm{C}^{12}(n, a) B \mathrm{Be}^{9}$ Reaction Induced by $14-$ MeV Neutrons" Physical Review Vol. 130, No. 4 (May 1963) 1500- 1503.

19- A. W. Obst, T.B. Grandy, and J.L. Weil, "Reaction $9_{B e}(a, n)^{12} \mathrm{C}$ from 1.7 to 6.4 MeV" Physical Review C, Vol.5 No.3 (March 1972). 
20- L. Van der Zwan and K.W. Geiger, "The ${ }^{9} \mathrm{Be}(a, n){ }^{12} \mathrm{C}$ Cross Section between 1.5 and 7.8 MeV" Nuclear Physics A152 (1970) 481-494.

21- V. Verbinski et a1., "Neutrons from ${ }^{9} \mathrm{Be}(\alpha, n)$ Reaction for $\mathrm{E}_{\boldsymbol{\alpha}}$ between 6 and 10 MeV" Physical Review Vol.170, No.4 (June 1968) 916- 923.

22- H. Schumacher, H. Menzel, and J. Coyne, "Critical Evaluation of Neutron Kerma Factors Using Theoretical and Experimental Ionization Yield Spectra" 1984, Proc. 5th Symp. Neutron Dosimetry Munich/Neuherberg, FRG, EUR-9762, p. 213, Commission of the European Communities, Luxembourg (1985).

23- R. MacFarlane, "Energy Balance of ENDF/B-V.2" Prepared for the meeting of CSEWG at BNL (May 1984).

24- C. Lederer, and V. Shirly, Eds "Table of Isotopes", 7th ed. (Wiley, New York, 1978).

25- C. Fu., Oak Ridge National Laboratory, Private Communications.

26- Yu. E. Kozy and G. A. Prokopets, "The Reactions $\left(n_{1} n^{\prime} \gamma\right),(n, n p)$, and $(n, p n)$ in $23 \mathrm{Na}, 27 \mathrm{Al}$, and $32 \mathrm{~S}$ at a Neutron Energy $14.6 \mathrm{MeV}$ " Sov. J. Nucl. Phys. 28(1), (July 1978).

27-R. J. Howerton and M. H. MacGregor, "The LLL Evaluated Nuclear Data Library (ENDL): Descriptions of Individual Evaluations for $Z=0-98$ " UCRL50400 Vol.15 Part D Rev.1 (May 1978).

28- G. L. Morgan, "Mn $(n, x y)$ Reaction Cross Section for Incident Neutron Energies between 0.2 and $20.0 \mathrm{MeV}$ "ORNL/TM-5531 (Aug. 1976).

29- D. E. Cullen et al., "Supplemental Neutron-Induced Interactions $(Z \leq 35)$ : Graphical, Experimental Data" UCRL-50400 Vol. 8 Rev. 1 Part A (July 1976). 
30- C. Philis et al., "Titanium-II: An Evaluated Nuclear Data File" ANL/NDM 28, (June 1977).

31- P. Guenther et al., "Fast Neutron Cross Sections of Vanadium and an Evaluated Neutronic File" ANL/NDM-24, (May 1977).

32- G. L. Morgan and E. Newman, "The $\operatorname{Cr}(n, x \gamma)$ Reaction Cross Section for Incident Neutron Energies Between 0.2 and $20.0 \mathrm{MeV}$ "ORNL-TM-5098 (ENDF222), (Jan. 1976).

33-E. Arthur, and P. Young, "Evaluated Neutron-Induced Cross Sections for $54,56 \mathrm{Fe}$ to $40 \mathrm{Mev"} \mathrm{Los} \mathrm{Alamos} \mathrm{National} \mathrm{Laboratory} \mathrm{report} \mathrm{LA-8626-MS}$ (ENDF-304) (Dec. 1980).

34- B. Magurno, and P. Young, "ENDF-201 Supplement I - ENDF/B-V.2 Summary Documentations" BNL-NCS-17541 (ENDF-201) (Jan. 1985).

35- D. M. Hetrick, C. Y. Fu, and D. C. Larson, "Calculated Neutron- Induced Cross Sections for $63,65 \mathrm{Cu}$ from 1 to $20 \mathrm{MeV}$ and Comparisons with Experiments" ORNL/TM-9083 (ENDF-337) (Aug. 1984). 


\section{Appendix A}

\section{DECAY ENERGY TABLES}

The decay energies for all the materials in the KAOS/LIB-V library are listed below. The materials that do not produce decay heat with half-life within the 1 day cutoff are not listed. For single-isotope materials, charged particles decay energy $E_{d}(c h)$ and gamma decay energy $E_{d}(\gamma)$ are listed. The producing reactions are identified using the ENDF/B-V MT number. For natural elements, the radioactive reaction products are also identified.

Single-Isotope Materials

MT

$E_{d}(c h)$

$E_{d}(\gamma)$

$3-L i-6$

103

1.568

0.0

3-Li-7

9.316

0.0

104

1.568

0.0

4-Be-9

$\begin{array}{lll}103 & 6.177 & 0.0 \\ 104 & 9.316 & 0.0 \\ 107 & 1.568 & 0.0\end{array}$




\begin{tabular}{|c|c|c|c|}
\hline & MT & $E_{d}(c h)$ & $E_{d}(\gamma)$ \\
\hline \multicolumn{4}{|l|}{$5-B-11$} \\
\hline & 102 & 6.3405 & 0.0577 \\
\hline & 103 & 4.825 & 1.351 \\
\hline & 107 & 9.3031 & 0.0 \\
\hline \multicolumn{4}{|l|}{$6-c-12$} \\
\hline & 103 & 6.4 & 0.0 \\
\hline \multicolumn{4}{|l|}{$7-N-14$} \\
\hline & 16 & 0.4794 & 1.022 \\
\hline \multicolumn{4}{|l|}{$8-0-16$} \\
\hline & 103 & 2.664 & 4.605 \\
\hline \multicolumn{4}{|l|}{$9-F-19$} \\
\hline & 16 & 0.2456 & 0.9903 \\
\hline & 102 & 2.481 & 1.6326 \\
\hline & 103 & 1.701 & 1.008 \\
\hline & 107 & 2.664 & 4.605 \\
\hline
\end{tabular}

$11-\mathrm{Na}-23$

$\begin{array}{lll}102 & 0.5626 & 4.1228 \\ 103 & 1.9011 & 0.14664 \\ 107 & 2.4808 & 1.6326\end{array}$

13-A] -27

$\begin{array}{lll}102 & 1.2315 & 1.7789 \\ 103 & 0.6884 & 0.8842 \\ 107 & 0.56265 & 4.1228\end{array}$




\begin{tabular}{|c|c|c|}
\hline \multirow{2}{*}{\multicolumn{3}{|c|}{$15-P-31$}} \\
\hline & & \\
\hline 16 & 1.44 & 1.022 \\
\hline 103 & 0.589 & 0.0 \\
\hline 107 & 1.2315 & 1.7789 \\
\hline
\end{tabular}

25-Mn-55

$\begin{array}{lll}102 & 0.833 & 2.047 \\ 103 & 1.093 & 0.0\end{array}$

27-Co-59

$\begin{array}{lll}106 & 1.1016 & 0.0655 \\ 107 & 0.833 & 2.047\end{array}$

$40-z r-92$

$\begin{array}{lll}103 & 1.48 & 0.246\end{array}$

$40-2 r-94$

$\begin{array}{lll}103 & 1.9 & 0.6 \\ 107 & 0.615 & 1.124\end{array}$

$40-z r-96$

$\begin{array}{lll}102 & 1.184 & 1.532 \\ 107 & 1.74 & 1.19\end{array}$

72-Hf-178

$\begin{array}{lll}103 & 0.8584 & 0.0\end{array}$

72-Hf-179

$\begin{array}{lll}103 & 0.4877 & 0.214\end{array}$

72-Hf-180

103

$0.5652 \quad 1.62$ 
$74-W-184$
MT
$E_{d}(c h)$
$E_{d}(\gamma)$

103

0.385

2.481

$74^{\circ}-W-186$

$\begin{array}{lll}28 & 0.677 & 0.298 \\ 102 & 0.521 & 0.2727 \\ 103 & 1.71 & 0.885 \\ 107 & 0.4684 & 0.722\end{array}$

$83-B \mathfrak{j}-209$

107

0.5845

0.0

Natural Elements Decay Data

$\begin{array}{llll}\text { MT } & \text { Product } & E_{d}(c h) & E_{d}(\gamma)\end{array}$

12-Mg-nat

$\begin{array}{llll}16 & \mathrm{Mg}-23 & 1.334 & 1.06 \\ 28 & \mathrm{Na}-24 & 0.5626 & 4.1228 \\ & \mathrm{Na}-25 & 1.494 & 0.4244 \\ 102 & \mathrm{Mg}-27 & 0.6884 & 0.8842 \\ 103 & \mathrm{Na}-24 & 0.5626 & 4.1228 \\ & \mathrm{Na}-25 & 1.494 & 0.4244 \\ & \mathrm{Na}-26 & 3.3565 & 2.176 \\ 107 & \mathrm{Ne}-23 & 1.9011 & 0.14664\end{array}$

14-Si-nat

103

A) -28

1.255

1.779 
16-5-nat

16

$S-31$

2.00

1.022

103

$\mathrm{P}-34$

2.3

0.319

105

P-30

1.44

1.022

107

Si-31

0.589

0.0

111

Si-31

0.589

0.0

17-Cl-nat

16

102

103

107

Cl -34

1.524

1.022

1.51

1.419

$\mathrm{S}-37$

0.780

2.9295

2.30

0.319

19-K-nat

16
10
103
107

$k-38$

1.2115

3.1896

102

$k-42$

1.4185

0.2744

103

Ar -41

0.4492

1.294

1.51

1.419

20-Ca-nat

16

Ca-39

2.58

1.022

105

K-38

1.2115

3.1896

22-Ti-nat

16

$T i-45$

0.3785

0.879

Sc-49

0.8292

0.0

102

Ti-51

0.8678

0.3688

103

Sc -49

0.8292

0.0

Sc -50

1.658

3.125

104

Sc -49

0.8292

0.0

105

Sc -44

0.6029

2.07 
MT

Product

$E_{d}(c h)$

$E_{d}(\gamma)$

23-v-nat

102

V-52

1.0931

1.434

103

Ti-51

0.8673

0.3687

24-Cr-nat

16

$\mathrm{Cr}-49$

0.645

1.127

28

V -52

1.093

1.434

$v-53$

0.998

1.037

102

$\mathrm{Cr}-55$

1.12

0.0

103

$v-52$

1.093

1.434

$v-53$

0.998

1.037

$v-54$

1.313

3.944

104

$V-52$

1.093

1.434

$v-53$

0.998

1.037

105

V -52

1.093

1.434

106

$\mathrm{Ti}-51$

0.867

0.369

$\mathrm{Ti}-52$

0.72

0.142

107

$\mathrm{Ti}-51$

0.867

0.369

26-Fe-nat

16

$\mathrm{Fe}-53$

1.153

1.1808

103

Mn-56

0.833

2.047

$\mathrm{Mn}-58$

2.907

0.0

107

$\mathrm{Cr}-55$

1.094

0.0

28-Ni-nat

28

Co-61

0.4522

0.09163

102

$\mathrm{Ni}-65$

0.6364

0.5427

103

Co-61

0.4522

0.09163

Co-62

1.664

1.512

Co-64

3.329

0.181

107

$\mathrm{Fe}-61$

1.581

1.129 


\begin{tabular}{|c|c|c|c|}
\hline MT & Product & $E_{d}(c h)$ & $E_{d}(\gamma)$ \\
\hline \multicolumn{4}{|l|}{ 29-Cu-nat } \\
\hline 16 & $\mathrm{Cu}-62$ & 1.288 & 0.9995 \\
\hline & $\mathrm{Cu}-64$ & 0.1297 & 0.2053 \\
\hline 22 & $\mathrm{Co}-61$ & 0.5816 & 0.09771 \\
\hline 102 & $\mathrm{Cu}-64$ & 0.1297 & 0.2053 \\
\hline & $\mathrm{Cu}-66$ & 1.071 & 0.08315 \\
\hline 103 & $\mathrm{Ni}-65$ & 0.6364 & 0.5413 \\
\hline 106 & Co-61 & 0.5816 & 0.09771 \\
\hline & Co-63 & 1.549 & 0.1362 \\
\hline 107 & $\mathrm{Co}-62$ & 1.664 & 1.512 \\
\hline
\end{tabular}

42-Mo-nat

$\begin{array}{llll}16 & \text { Mo-91 } & 1.47 & 0.9576 \\ 102 & \text { Mo-101 } & 0.505 & 1.5\end{array}$

82-Pb-nat

$\begin{array}{llll}16 & \mathrm{~Pb}-203 & 0.0 & 0.3 \\ 102 & \mathrm{~Pb}-209 & 0.2095 & 0.0\end{array}$

Decay Energy for Non-fission Reactions in Fissionable Materials

$\begin{array}{llll}\text { Material } & \text { MT } & E_{d}(c h) & E_{d}(\boldsymbol{\gamma}) \\ \text { Th-232 } & 102 & 0.603 & 0.0 \\ \text { Pa-233 } & 102 & 0.291 & 1.646 \\ \text { U-238 } & 102 & 0.6174 & 0.0 \\ \text { Pu-242 } & 102 & 0.29 & 0.0 \\ \text { Am-241 } & 102 & 0.2734 & 0.0 \\ \text { Am-243 } & 102 & 0.72 & 0.0\end{array}$


Appendix B

Vitamin-E 174 Group Structure

\begin{tabular}{|c|c|c|c|}
\hline group & Top Energy eV & group & Top Energy eV \\
\hline 1 & $1.00001 E-01$ & 31 & $5.82947 E+02$ \\
\hline 2 & $4.13994 E-01$ & 32 & $7: 48518 E+02$ \\
\hline 3 & $5.31578 E-01$ & 33 & $9.61116 \mathrm{E}+02$ \\
\hline 4 & $6.82560 E-01$ & 34 & $1.23410 E+03$ \\
\hline 5 & $8.76425 E-01$ & 35 & $1.58461 E+03$ \\
\hline 6 & $1.12535 \mathrm{E}+00$ & 36 & $2.03468 E+03$ \\
\hline 7 & $1.44498 E+00$ & 37 & $2.24867 E+03$ \\
\hline 8 & $1.85539 \mathrm{E}+00$ & 38 & $2.48517 E+03$ \\
\hline 9 & $2.38237 E+00$ & 39 & $2.61259 E+03$ \\
\hline 10 & $3.05902 E+00$ & 40 & $2.74654 E+03$ \\
\hline 11 & $3.92786 E+00$ & 41 & $3.03539 E+03$ \\
\hline 12 & $5.04348 E+00$ & 42 & $3.35463 E+03$ \\
\hline 13 & $6.47595 E+00$ & 43 & $3.70744 E+03$ \\
\hline 14 & $8.31529 E+00$ & 44 & $4.30742 E+03$ \\
\hline 15 & $1.06770 \mathrm{E}+01$ & 45 & $5.53084 E+03$ \\
\hline 16 & $1.37096 \mathrm{E}+01$ & 46 & $7.10174 \mathrm{E}+03$ \\
\hline 17 & $1.76035 \mathrm{E}+01$ & 47 & $9.11882 E+03$ \\
\hline 18 & $2.26033 E+01$ & 48 & $1.05946 \mathrm{E}+04$ \\
\hline 19 & $2.90232 E+01$ & 49 & $1.17088 \mathrm{E}+04$ \\
\hline 20 & $3.72665 E+01$ & 50 & $1.50344 E+04$ \\
\hline 21 & $4.78512 E+01$ & 51 & $1.93045 E+04$ \\
\hline 22 & $6.14421 E+01$ & 52 & $2.18749 E+04$ \\
\hline 23 & $7.88932 E+01$ & 53 & $2.35786 E+04$ \\
\hline 24 & $1.01301 \mathrm{E}+02$ & 54 & $2.41755 \mathrm{E}+04$ \\
\hline 25 & $1.30073 E+02$ & 55 & $2.47875 E+04$ \\
\hline 26 & $1.67017 \mathrm{E}+02$ & 56 & $2.60584 E+04$ \\
\hline 27 & $2.14454 \mathrm{E}+02$ & 57 & $2.70001 E+04$ \\
\hline 28 & $2.75364 E+02$ & 58 & $2.85011 E+04$ \\
\hline 29 & $3.53575 E+02$ & 59 & $3.18278 E+04$ \\
\hline 30 & $4.53999 E+02$ & 60 & $3.43067 E+04$ \\
\hline
\end{tabular}


2. $97211 E+05$

62

4. $63092 E+04$

92

2. $98491 E+05$

63

$5.24752 E+04$

93

3. $01974 E+05$

64

$5.65622 E+04$

94

3. $33733 E+05$

65

$6.73794 E+04$

95

3. $68832 E+05$

66

$7.19981 E+04$

96

$3.87742 \mathrm{E}+05$

67

$7.94987 E+04$

97

$4.07622 \mathrm{E}+05$

68

8.25034E+04

98

$4.50492 \mathrm{E}+05$

69

8. $65169 E+04$

99

$4.97871 \mathrm{E}+05$

70

$9.80366 \mathrm{E}+04$

100

$5.23397 E+05$

71

$1.11090 E+05$

101

$5.50232 E+05$

72

1. $16786 E+05$

102

$5.78444 \mathrm{E}+05$

1.22773E+05

103

$6.08101 E+05$

74

$1.29068 \mathrm{E}+05$

104

$6.39279 \mathrm{E}+05$

75

$1.35686 \mathrm{E}+05$

105

$6.72055 E+05$

76

1. $42642 E+05$

106

$7.06512 E+05$

77

$1.49956 E+05$

107

$7.42736 \mathrm{E}+05$

78

1. $57644 E+05$

108

$7.80817 E+05$

79

$1.65727 E+05$

109

$8.20850 E+05$

80

1. $74224 \mathrm{E}+05$

110

$8.62936 E+05$

81

$1.83156 E+05$

111

9.07180E+05

82

$1.92547 \mathrm{E}+05$

112

$9.61640 \mathrm{E}+05$

83

$2.02419 E+05$

113

$1.00259 \mathrm{E}+06$

84

2. $12797 E+05$

114

$1.10803 E+06$

85

2. $23708 E+05$

115

$1.16484 E+06$

85

2. $35177 E+05$

116

$1.22456 \mathrm{E}+06$

87

$2.47235 E+05$

117

$1.28735 E+06$

88

2. $73237 E+05$

118

$1.35335 E+06$

89

2. $87247 E+05$

119

$1.42274 \mathrm{E}+06$

90

$2.94518 E+05$

120

$1.49569 \mathrm{E}+06$ 


\begin{tabular}{|c|c|c|c|}
\hline Group & Top Energy eV & Group & Top Energy eV \\
\hline 121 & $1.57237 E+06$ & 151 & $6.70320 E+06$ \\
\hline 122 & $1.65299 E+06$ & 152 & $7.04688 E+06$ \\
\hline 123 & $1.73774 E+06$ & 153 & $7.40818 E+06$ \\
\hline 124 & $1.82684 E+06$ & 154 & $7.78801 E+06$ \\
\hline 125 & $1.92050 E+06$ & 155 & $8.18731 E+06$ \\
\hline 126 & $2.01897 E+06$ & 156 & $8.60708 E+06$ \\
\hline 127 & $2.12248 E+06$ & 157 & $9.04837 E+06$ \\
\hline 128 & $2.23130 E+06$ & 158 & $9.51229 E+06$ \\
\hline 129 & $2.30686 E+06$ & 159 & $1.00000 E+07$ \\
\hline 130 & $2.34570 E+06$ & 160 & $1.05127 E+07$ \\
\hline 131 & $2.36525 E+06$ & 161 & $1.10517 E+07$ \\
\hline 132 & $2.38521 E+06$ & 162 & $1.16183 E+07$ \\
\hline 133 & $2.46597 E+06$ & 163 & $1.22140 \mathrm{E}+07$ \\
\hline 134 & $2.59240 E+06$ & 164 & $1.25232 E+07$ \\
\hline 135 & $2.72532 E+06$ & 165 & $1.34986 E+07$ \\
\hline 136 & $2.86505 E+06$ & 166 & $1.38403 E+07$ \\
\hline 137 & $3.01194 \mathrm{E}+06$ & 167 & $1.41907 E+07$ \\
\hline 138 & $3.16637 E+06$ & 168 & $1.45499 \mathrm{E}+07$ \\
\hline 139 & $3.32871 E+06$ & 169 & $1.49183 E+07$ \\
\hline 140 & $3.67879 E+06$ & 170 & $1.56831 E+07$ \\
\hline 141 & $4.06570 E+06$ & 171 & $1.64872 E+07$ \\
\hline 142 & $4.49329 E+06$ & 172 & $1.69046 E+07$ \\
\hline 143 & $4.72367 E+06$ & 173 & $1.73325 \mathrm{E}+07$ \\
\hline 144 & $4.96585 E+06$ & 174 & $1.96403 E+07$ \\
\hline 145 & $5.22046 E+06$ & & \\
\hline 146 & $5.48812 E+06$ & & \\
\hline 147 & $5.76950 E+06$ & & \\
\hline 148 & $6.06531 E+06$ & & \\
\hline 149 & $6.37628 E+06$ & & \\
\hline 150 & $6.59238 E+06$ & & \\
\hline
\end{tabular}




\section{Internal:}

C. Adams

M. Billone

R. Blomquist

J. Brooks

Y. Chang

R. Clemmer

D. Ehst

K. Evans

P. Finn

E. Fujita

E. Gelbard

Y. Gohar (10)

L. Greenwood
D. Gruen

P. Guenther

A. Hassanein

T. Hua

C. Johnson

A. Krauss

R. Lawson

L. LeSage

B. Loomis

S. Ma jumdar

R. Mattas

B. Picologlou

K. Porges
C. Reed

A. Smith

D. Smith

D. Sze

C. Till

L. Turner

$S$. Yang

FPP Files (25)

ANL Contract File

ANL Libraries

ANL Patent Dept.

TIS Files (3)

\section{External:}

DOE-OSTI, for distribution per UC-420 (37)

Manager, Chicago Operations office

\section{U.S. Department of Energy, Office of Fusion Energy}

S. Berk

M. Cohen

R.J. Dowling

G. Haas

G.R. Nardella

A.L. Opdenaker

R. Price

T. Reuther

S. Staten

U.S. Department of Energy, Division of Nuclear Physics

S.L. Whetstone

Battelle Pacific Northwest Laboratory

L. Carter

D.G. Doran

G. Hollenberg

Brookhaven National Laboratory

S. Baron

M.R. Bhat

M. Divadeenam

C.L. Dunford

N.E. Holden

R.R. Kinsey

V. McLane

S.F. Mughabghab

S. Pearlstein

P.F. Rose

J.R. Tuli 
Central Institute of Physies, Bucharest v. Zoita

Columbia University R.A. Gross

Commission of the European Communities

G. Casini

J. Daruis

C. Ponti

D. Wat tecamps

Culham Laboratory

G.J. Butterworth

Duke University

R. Walter

EG\&G Idaho, Inc.

J. Crocker

D. Holland

S. Piet

C.W. Reich

J. Smith

Fusion Engineering Design Center

C. Flanagan

$T$. Shannon

Fusion Power Associates

S. Dean

GA Technologies, Inc.

E. Cheng

K. Schultz

Georgia Institute of Technology

Y. Farawila (10)

W. Stacey, Jr.

Grumman Space Systems

L. Deutsch

J. Rathke

M. Stauber

A. Tobin

I.V. Kurchatov Institute of Atomic Energy
D.V. Markovskiy
O. Schipakin
G. Shatalov
L.V. Tocheniy
S. Zimin 
Japan Atomic Energy Research Institute

T. Kuroda

K. Maki

T. Nakamura

Y. Seki

T. Tomabech i

Kernforschungszentrum Karlsrune

J.E. Vetter

Lawrence Berkeley Laboratory

B. Cooper

W. Lindquist

Lawrence Livermore National Laboratory

J. Doggett

J. Gilleland

M.W. Guinan

R. Haight

L. Harisen

C. Henring

R. Howerton

S. I wasak $\mathrm{I}$

J.j. Lee

G. Logan

J.K. Miller

L.J. Perkins

F. Moir

P.D. Sorar

K.I. Thomassen

Los Alamos Natjonal Laboratory

R.E. Al couffe

J. Anderson

E. Arthur

L. Booth

J. Briesmeister

E.W. Brinkiley

k. Brown

F. Clinard

J.W. Davidson

[j. Dudziak

J. Hendricks

Ni. Jarmie

R.J. Juzaitis

R. Krakowsti $i$

P. Lisowski

R.C. Littie

H.J. Livar

H. MacFarlarie

h. Miller

U. Muir

K.L. O'vell

K.E. Seamor 
W.L. Thompson

J.T. West I1]

P. Young

Massachusetts Institute of Technology

L. Bromberg

D. Cohn

M. Kazimi

D. Montgomery

Max-Planck Institute fur Hlasmaphysik

J.L. Boutard

M. Chazalon

W. Daenner

H. Kranse

A. Knoblock

E. Salpietro

R. Tosch $\mathrm{i}$

K. Verschuur

G. Vieider

Mclonnel1 Doug Jas Astronautics Compary

J. Davis

D.A. DeFreece

National Bureau of Standards

R. Reed

0 . Wasson

North Carolina State University

C. Gould

Oak Ridge Natjoral Laboratory

R.G. Alsmiller

C.C. Baker

V.C. Baker

L.A. Berry

B.L. Bishop

J.A. Blair

E.E. Bloom

U. Campbel1

J. Cannon

C. Fu

'T. Gabriej

G. Haste

D. Hetrick

D. Larson

R.A. Lillie

M.J. Martin

H. MeCurdy

r. Koussin, RSIC

R.'I'. Santoro

J. Scott

J. Stout

1.W. Swain 
Ohio University

S.M. Grimes

R.0. Lane

Ontar io Hydro, Canada

B. Stasko

Osaka University

K. Sumita

A. Takahash i

Princeton Plasma Physics Laboratory

H. Furth

D. Jassby

L.P. Ku

R. Little

P.H. Rutherford

J. Schmidt

Purdue University

P. Lykoudis

Rensselaer Polytechnic Institute

M.J. Embrechts

D. Steiner

Sandia National Laboratories

W. Bauer

M.A. Sweeney

SIN, Villigen, Switzerland

C. Marinucci

TRW, Inc.

J. Garner

J. Gordon

J.A. Maniscalco

University of California-Los Angeles

M. Abdou

R. Conn

N. Ghoneim

Y. Ikeda

A. Kumar

F. Na jmabodi

M.2. Youssef

University of Illinois-Urbana

G.H. Miley

M.H. Ragheb

University of Michigan-Ann Arbor

G. Knoll 
Univer'sity of Missouri-Columbia

A. Bolon

University of Oregon

A. Klein

University of Tennessee-Knoxville

T.W. Kerlin

P. Stevens

University of Tokyo, Japan

Y. Oka

University of Washington-Seattle

F.L. Ribe

G.L. Woodruff

University of Wisconsin-Madison

G. Kulcinski

C. Maynard (10)

M. Sawan

W. Vogelsang

West inghouse Hanford Company

F.M. Mann

R.J. Puigh 